\title{
Analytical Data Report for Sediment Samples Collected From 200 BP 5 OU, C7515 (299-E24-25) M-Well
}

Michael Lindberg

June 2010

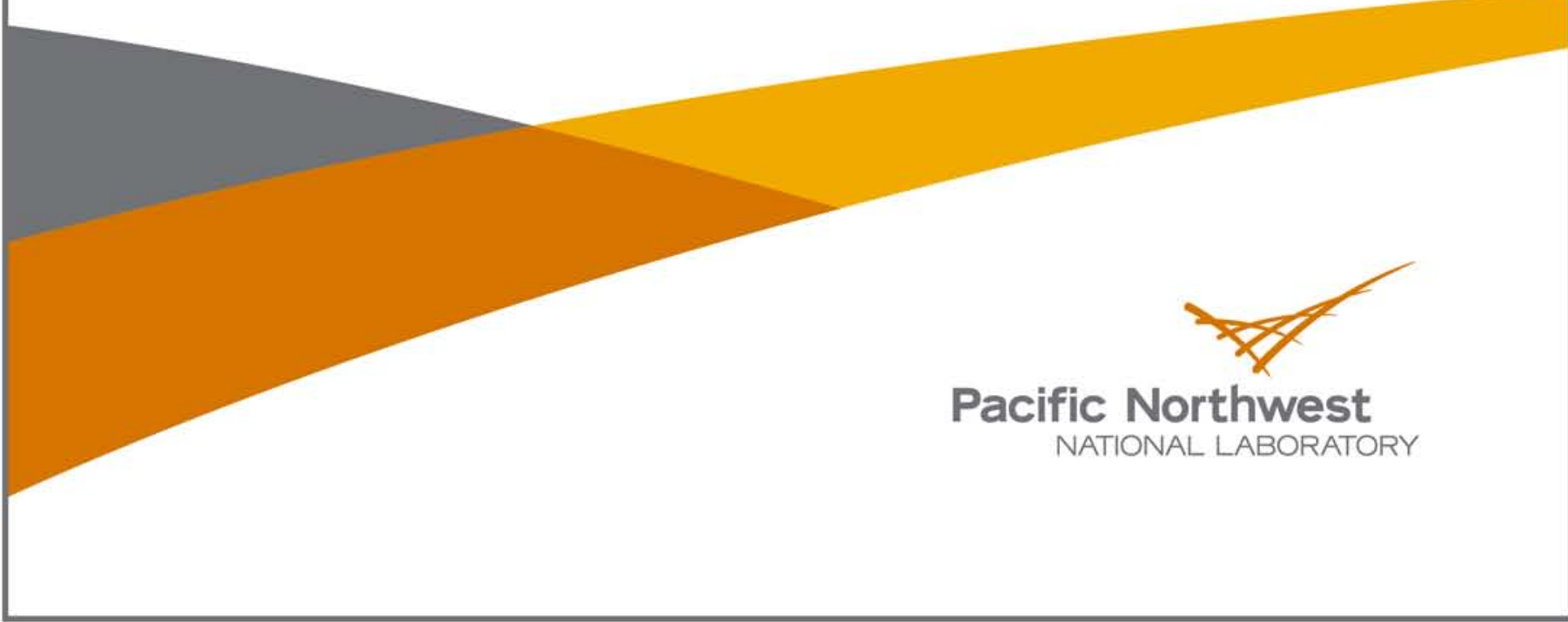




\title{
DISCLAIMER
}

This report was prepared as an account of work sponsored by an agency of the United States Government. Neither the United States Government nor any agency thereof, nor Battelle Memorial Institute, nor any of their employees, makes any warranty, express or implied, or assumes any legal liability or responsibility for the accuracy, completeness, or usefulness of any information, apparatus, product, or process disclosed, or represents that its use would not infringe privately owned rights. Reference herein to any specific commercial product, process, or service by trade name, trademark, manufacturer, or otherwise does not necessarily constitute or imply its endorsement, recommendation, or favoring by the United States Government or any agency thereof, or Battelle Memorial Institute. The views and opinions of authors expressed herein do not necessarily state or reflect those of the United States Government or any agency thereof.

\author{
PACIFIC NORTHWEST NATIONAL LABORATORY \\ operated by \\ BATTELLE \\ for the \\ UNITED STATES DEPARTMENT OF ENERGY \\ under Contract DE-AC05-76RL01830
}

Printed in the United States of America
Available to DOE and DOE contractors from the Office of Scientific and Technical Information,
P.O. Box 62, Oak Ridge, TN 37831-0062;
ph: (865) 576-8401
fax: $(865)$ 576-5728
email: reports@adonis.osti.gov

\begin{abstract}
Available to the public from the National Technical Information Service, U.S. Department of Commerce, 5285 Port Royal Rd., Springfield, VA 22161 ph: (800) 553-6847 fax: $(703) 605-6900$ email: orders@ntis.fedworld.gov online ordering: http://www.ntis.gov/ordering.htm
\end{abstract}

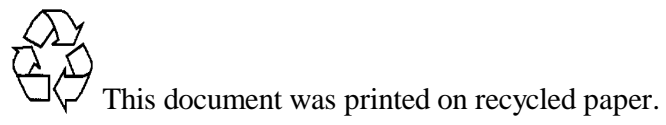


$06 / 15 / 1011: 57$

To: Dale Dyekman

From: M ichael J. Lindberg

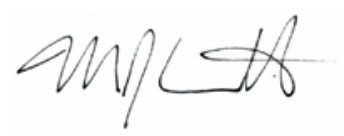

Environmental Sciences Laboratory

Energy and Environment Directorate, Pacific Northwest National Laboratory

Subject: Analytical Data Report for Sediment Samples Collected From 200 BP 5 OU, C7515 (299-E24-25)

M-Well, Sample Delivery Group ESL090014, SAF Number F10-045

This letter contains the following information for sample delivery group ESL090014

- Cover Sheet

- Narrative

- Analytical Results

- Quality Control

- Geologic Logs

- Geologic Photos

- Chain of Custodies 


\section{Introduction}

Between January 14, 2010 and February 9, 2010 sediment samples were received from 200 BP 5 OU, C7515 (299-E24-25) M -Well for geochemical studies.

\section{Analytical Results/Methodology}

The analyses for this project were performed at the 325 building located in the 300 Area of the Hanford Site. The analyses were performed according to Pacific Northwest National Laboratory (PNNL) approved procedures and/or nationally recognized test procedures. The data sets include the sample identification numbers, analytical results, estimated quantification limits (EQL), and quality control data.

\section{Quality Control}

The preparatory and analytical quality control requirements, calibration requirements, acceptance criteria, and failure actions are defined in the on-line QA plan "Conducting Analytical Work in Support of Regulatory Programs" (CAW). This QA plan implements the Hanford Analytical Services Quality Assurance Requirements Documents (HASQARD) for PNNL.

\section{Definitions}

Dup Duplicate

RPD Relative Percent Difference

NR No Recovery (percent recovery less than zero)

ND Non-Detectable

\%REC Percent Recovery

\section{Sample Receipt}

Samples were received with a chain of custody (COC) and were analyzed according to the sample identification numbers supplied by the client. All Samples were refrigerated upon receipt until prepared for analysis.

All samples were received with custody seals intact unless noted in the Case Narrative.

\section{Holding Times}

Holding time is defined as the time from sample preparation to the time of analyses. The prescribed holding times were met for all analytes unless noted in the Case Narrative.

\section{Analytical Results}

All reported analytical results meet the requirements of the CAW or client specified SOW unless noted in the case narrative. 


\section{Case Narrative Report}

\section{Hold Time:}

Due to the requirements of the statement of work and sampling events in the field, the 28 day and the 48 hr requirements can not be met. The statement of work requires samples to be selected at the completion of the borehole. It is not always possible to complete a borehole and have the samples shipped to the laboratory within the hold time requirements.

\section{Preparation Blank (PB):}

No discrepancies noted.

\section{Duplicate (DUP):}

No discrepancies noted.

\section{Laboratory Control Samples (LCS):}

LCS Recovery for Silver (43.8\%) was outside acceptance limits (80-120) in 0C11002-BS1 for ICP-OES Vadose-AE Samples results associated with this batch were not reported.

\section{Post Spike (PS):}

Post-Spike Recovery for Calcium (562\%) was outside acceptance limits (75-125) in 0C11001-PS1 for ICP-OES Vadose-WE The native sample concentration was greater than 5 times the spike concentration. There should be no impact to data as reported.

Post-Spike Recovery for Magnesium (207\%) was outside acceptance limits (75-125) in 0C11001-PS1 for ICP-OES Vadose-WE The native sample concentration was greater than 5 times the spike concentration. There should be no impact to data as reported.

Post-Spike Recovery for Iron (24.8\%) was outside acceptance limits (75-125) in 0C11002-PS1 for ICP-OES Vadose-AE The native sample concentration was greater than 5 times the spike concentration. There should be no impact to data as reported.

Post-Spike Recovery for Uranium (36.4\%) was outside acceptance limits (75-125) in 0C17003-PS1 for ICP-ICPMS-Tc_U WE The native sample concentration was greater than 5 times the spike concentration. There should be no impact to data as reported.

\section{Matrix Spike (MS):}

Matrix Spike Recovery for Chromium, Hexavalent (47.4\%) was outside acceptance limits (75-125) in 0D09005-MS1 for Hexavalent Chromium/Soil

Potential Matrix interference. Sample results associated with this batch are below the EQL. There should be no impact to the data as reported.

\section{Other QC Criteria:}

No discrepancies noted. 
I certify that this data package is in compliance with the SOW, both technically and for completeness, for other than the conditions detailed above. Release of the data contained in this hard copy data package has been authorized by the Laboratory Analytical Manager as verified by this signature.

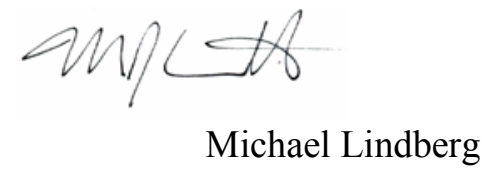

\section{DISCLAIMER}

This report was prepared as an account of work sponsored by an agency of the United States Government. Neither the United States Government nor any agency thereof, nor Battelle Memorial Institute, nor any of their employees, makes any warranty, express or implied, or assumes any legal liability or responsibility for the accuracy, completeness, or usefulness of any information, apparatus, product, or process disclosed, or represents that its use would not infringe privately owned rights. Reference herein to any specific commercial product, process, or service by trade name, trademark, manufacturer, or otherwise does not necessarily constitute or imply its endorsement, recommendation, or favoring by the United States Government or any agency thereof, or Battelle Memorial Institute. The views and opinions of authors expressed herein do not necessarily state or reflect those of the United States Government or any agency thereof.

\section{SAMPLES INCLUDED IN THIS REPORT}

200 BP 5 OU, C7515 (299-E28-30) M-Well

$\begin{array}{lll}\text { HEIS No. } & \text { Laboratory ID } & \text { Matrix } \\ \text { B235H1 } & 1001004-04 & \text { SOIL } \\ \text { B235J0 } & 1001004-22 & \text { SOIL } \\ \text { B235J6 } & 1001004-34 & \text { SOIL } \\ \text { B235K1 } & 1001004-42 & \text { SOIL } \\ \text { B235K5 } & 1001004-46 & \text { SOIL } \\ \text { B235L9 } & 1001004-98 & \text { SOIL } \\ \text { B235N9 } & 1001004-B C & \text { SOIL } \\ \text { B235R2 } & 1001004-B V & \text { SOIL } \\ \text { B235R9 } & 1001004-C C & \text { SOIL } \\ \text { B235T8 } & 1001004-D A & \text { SOIL }\end{array}$

\begin{tabular}{|c|c|}
\hline Date Collected & Date Received \\
\hline $1 / 13 / 10 \quad 12: 45$ & $1 / 14 / 10 \quad 11: 30$ \\
\hline $1 / 14 / 10 \quad 14: 20$ & $1 / 18 / 10 \quad 11: 40$ \\
\hline $1 / 15 / 10 \quad 09: 50$ & $1 / 18 / 10 \quad 11: 40$ \\
\hline 1/19/10 11:55 & $1 / 20 / 10 \quad 11: 50$ \\
\hline $1 / 19 / 10 \quad 14: 20$ & $1 / 20 / 10 \quad 11: 50$ \\
\hline $1 / 25 / 10 \quad 13: 05$ & $1 / 26 / 10 \quad 11: 15$ \\
\hline $1 / 28 / 10 \quad 10: 48$ & $2 / 1 / 10 \quad 12: 00$ \\
\hline 2/1/10 09:08 & $2 / 2 / 10 \quad 12: 10$ \\
\hline $2 / 3 / 10 \quad 10: 35$ & $2 / 4 / 10 \quad 09: 30$ \\
\hline $2 / 8 / 10 \quad 10: 15$ & $2 / 9 / 10 \quad 10: 00$ \\
\hline
\end{tabular}


The following analyses were performed on the following samples included in this report:

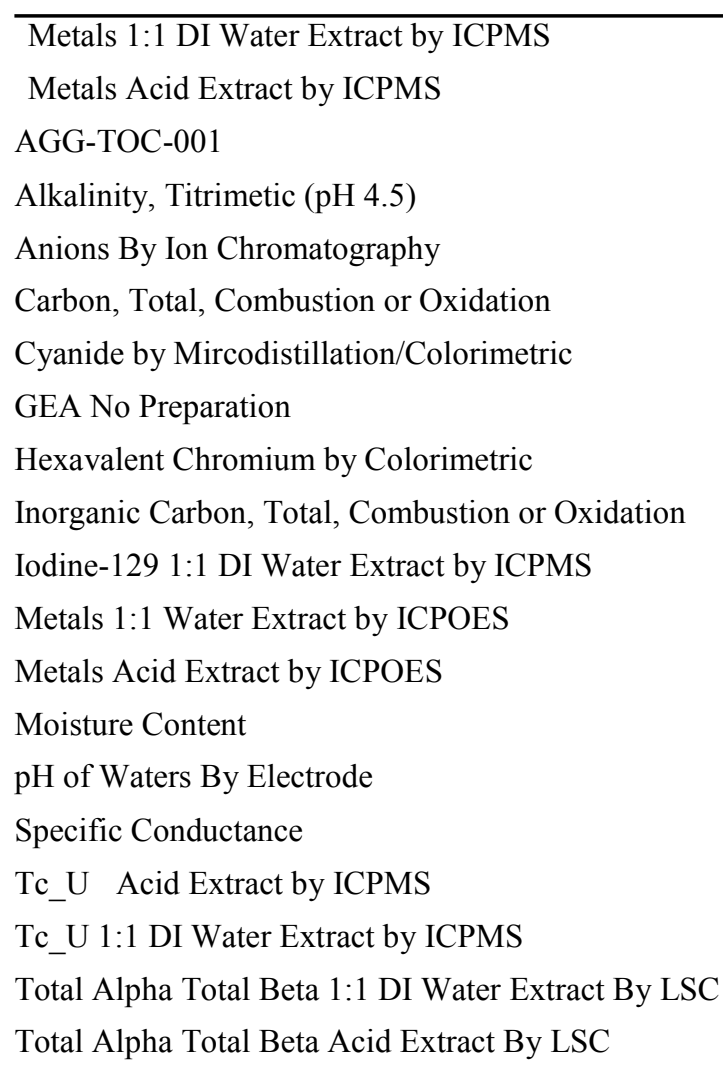

SAMPLES ANALYZED IN THIS REPORT

$\begin{array}{lll}\text { HEIS No. } & \begin{array}{l}\text { Laboratory ID } \\ 1001004-04\end{array} & \text { Matrix } \\ \text { B235H1 } & \text { SOIL } \\ \text { B235J0 } & 1001004-22 & \text { SOIL } \\ \text { B235J6 } & 1001004-34 & \text { SOIL } \\ \text { B235K1 } & 1001004-42 & \text { SOIL } \\ \text { B235K5 } & 1001004-46 & \text { SOIL } \\ \text { B235L9 } & 1001004-98 & \text { SOIL } \\ \text { B235N9 } & 1001004-B C & \text { SOIL } \\ \text { B235R2 } & 1001004-B V & \text { SOIL } \\ \text { B235R9 } & 1001004-C C & \text { SOIL } \\ \text { B235T8 } & 1001004-D A & \text { SOIL }\end{array}$

\begin{tabular}{|c|c|}
\hline $\begin{array}{l}\text { Date Collected } \\
1 / 13 / 10 \quad 12: 45\end{array}$ & $\begin{array}{l}\text { Date Received } \\
1 / 14 / 10 \quad 11: 30\end{array}$ \\
\hline $1 / 14 / 10 \quad 14: 20$ & 1/18/10 $11: 40$ \\
\hline $\begin{array}{ll}1 / 15 / 10 & 09: 50\end{array}$ & 1/18/10 $\quad 11: 40$ \\
\hline 1/19/10 $11: 55$ & $1 / 20 / 10 \quad 11: 50$ \\
\hline $\begin{array}{ll}1 / 19 / 10 & 14: 20\end{array}$ & $1 / 20 / 10 \quad 11: 50$ \\
\hline 1/25/10 $13: 05$ & $1 / 26 / 10 \quad 11: 15$ \\
\hline $\begin{array}{ll}1 / 28 / 10 & 10: 48\end{array}$ & 2/1/10 $12: 00$ \\
\hline 2/1/10 09:08 & $2 / 2 / 10 \quad 12: 10$ \\
\hline $2 / 3 / 10 \quad 10: 35$ & 2/4/10 09:30 \\
\hline 2/8/10 $\quad 10: 15$ & $2 / 9 / 10 \quad 10: 00$ \\
\hline
\end{tabular}




\section{Wet Chemistry}

\begin{tabular}{|c|c|c|c|c|c|}
\hline $\begin{array}{l}\text { Alkalinity } \\
\text { Lab ID }\end{array}$ & $\begin{array}{c}\text { CaCO3 ( } \\
\text { HEIS No. }\end{array}$ & $\begin{array}{l}\text { Methods } \\
\text { Results }\end{array}$ & $\begin{array}{l}\text { EB } \\
\text { EQL }\end{array}$ & Analyzed & Batch \\
\hline 1001004-04 & B235H1 & $2.65 \mathrm{E} 1$ & $2.34 \mathrm{E} 1$ & $4 / 08 / 10$ & 0D01005 \\
\hline 1001004-22 & B235J0 & $2.53 \mathrm{E} 1$ & $2.37 \mathrm{E} 1$ & $4 / 08 / 10$ & 0D01005 \\
\hline 1001004-34 & B235J6 & $3.67 \mathrm{E} 1$ & $2.35 \mathrm{E} 1$ & $4 / 08 / 10$ & 0D01005 \\
\hline $1001004-42$ & B235K1 & $<2.35 \mathrm{E} 1$ & $2.35 \mathrm{E} 1$ & $4 / 08 / 10$ & 0D01005 \\
\hline $1001004-46$ & B235K5 & $<2.35 \mathrm{E} 1$ & $2.35 \mathrm{E} 1$ & $4 / 08 / 10$ & 0D01005 \\
\hline $1001004-98$ & B235L9 & $<2.35 \mathrm{E} 1$ & $2.35 \mathrm{E} 1$ & $4 / 08 / 10$ & 0D01005 \\
\hline 1001004-BC & B235N9 & $3.17 \mathrm{E} 1$ & $2.34 \mathrm{E} 1$ & $4 / 08 / 10$ & 0D01005 \\
\hline 1001004-BV & B235R2 & 2.96E1 & $2.35 \mathrm{E} 1$ & $4 / 08 / 10$ & 0D01005 \\
\hline 1001004-CC & B235R9 & $3.79 \mathrm{E} 1$ & $2.35 \mathrm{E} 1$ & 4/08/10 & 0D01005 \\
\hline 1001004-DA & B235T8 & $5.92 \mathrm{E} 1$ & $2.35 \mathrm{E} 1$ & $4 / 08 / 10$ & 0D01005 \\
\hline
\end{tabular}




\section{Wet Chemistry}

Specific Conductance (EC) (mS/cm) by EPA 120.1

\begin{tabular}{llcccc|} 
Lab ID & HEIS No. & Results & EQL & Analyzed & Batch \\
\hline $1001004-04$ & B235H1 & $8.40 \mathrm{E}-1$ & $1.00 \mathrm{E}-2$ & $3 / 10 / 10$ & $0 \mathrm{C} 10003$ \\
$1001004-22$ & B235J0 & $1.60 \mathrm{E} 0$ & $1.00 \mathrm{E}-2$ & $3 / 10 / 10$ & $0 \mathrm{C} 10003$ \\
$1001004-34$ & B235J6 & $2.81 \mathrm{E} 0$ & $1.00 \mathrm{E}-2$ & $3 / 10 / 10$ & $0 \mathrm{C} 10003$ \\
$1001004-42$ & B235K1 & $4.36 \mathrm{E} 0$ & $1.00 \mathrm{E}-2$ & $3 / 10 / 10$ & $0 \mathrm{C} 10003$ \\
$1001004-46$ & B235K5 & $1.85 \mathrm{E} 0$ & $1.00 \mathrm{E}-2$ & $3 / 10 / 10$ & $0 \mathrm{C} 10003$ \\
$1001004-98$ & B235L9 & $1.06 \mathrm{E} 0$ & $1.00 \mathrm{E}-2$ & $3 / 10 / 10$ & $0 \mathrm{C} 10003$ \\
$1001004-\mathrm{BC}$ & B235N9 & $1.84 \mathrm{E}-1$ & $1.00 \mathrm{E}-2$ & $3 / 10 / 10$ & $0 \mathrm{C} 10003$ \\
$1001004-\mathrm{BV}$ & B235R2 & $2.34 \mathrm{E}-1$ & $1.00 \mathrm{E}-2$ & $3 / 10 / 10$ & $0 \mathrm{C} 10003$ \\
$1001004-\mathrm{CC}$ & B235R9 & $2.34 \mathrm{E}-1$ & $1.00 \mathrm{E}-2$ & $3 / 10 / 10$ & $0 \mathrm{C} 10003$ \\
$1001004-\mathrm{DA}$ & B235T8 & $2.41 \mathrm{E}-1$ & $1.00 \mathrm{E}-2$ & $3 / 10 / 10$ & $0 \mathrm{C} 10003$ \\
\hline
\end{tabular}




\section{Wet Chemistry}

Moisture Content (\% by Weight) by AGG-WC-001

\begin{tabular}{llclcc} 
Lab ID & HEIS No. & Results & EQL & Analyzed & Batch \\
\hline $1001004-04$ & B235H1 & $6.98 \mathrm{E} 0$ & N/A & $2 / 24 / 10$ & $0 \mathrm{~B} 12007$ \\
$1001004-22$ & B235J0 & $4.18 \mathrm{E} 0$ & N/A & $2 / 24 / 10$ & $0 \mathrm{~B} 12007$ \\
$1001004-34$ & B235J6 & $4.52 \mathrm{E} 0$ & N/A & $2 / 24 / 10$ & $0 \mathrm{~B} 12007$ \\
$1001004-42$ & B235K1 & $6.57 \mathrm{E} 0$ & N/A & $2 / 24 / 10$ & $0 \mathrm{~B} 12007$ \\
$1001004-46$ & B235K5 & $5.22 \mathrm{E} 0$ & N/A & $2 / 24 / 10$ & $0 \mathrm{~B} 12007$ \\
$1001004-98$ & B235L9 & $5.10 \mathrm{E} 0$ & N/A & $2 / 24 / 10$ & $0 \mathrm{~B} 12007$ \\
$1001004-\mathrm{BC}$ & B235N9 & $2.89 \mathrm{E} 0$ & N/A & $2 / 24 / 10$ & $0 \mathrm{~B} 12007$ \\
$1001004-\mathrm{BV}$ & B235R2 & $3.29 \mathrm{E} 0$ & N/A & $2 / 24 / 10$ & $0 \mathrm{~B} 12007$ \\
$1001004-\mathrm{CC}$ & B235R9 & $4.44 \mathrm{E} 0$ & N/A & $2 / 24 / 10$ & $0 \mathrm{~B} 12007$ \\
$1001004-\mathrm{DA}$ & B235T8 & $4.97 \mathrm{E} 0$ & N/A & & $0 \mathrm{~B} 12007$ \\
\hline
\end{tabular}




\section{Wet Chemistry}

pH (pH Units) by AGG-pH-001

\begin{tabular}{llcccc} 
Lab ID & HEIS No. & Results & EQL & Analyzed & Batch \\
\hline $1001004-04$ & B235H1 & $7.01 \mathrm{E} 0$ & N/A & $3 / 10 / 10$ & $0 \mathrm{C} 10004$ \\
$1001004-22$ & B235J0 & $6.96 \mathrm{E} 0$ & N/A & $3 / 10 / 10$ & $0 \mathrm{C} 10004$ \\
$1001004-34$ & B235J6 & $7.69 \mathrm{E} 0$ & N/A & $3 / 10 / 10$ & $0 \mathrm{C} 10004$ \\
$1001004-42$ & B235K1 & $6.98 \mathrm{E} 0$ & N/A & $3 / 10 / 10$ & $0 \mathrm{C} 10004$ \\
$1001004-46$ & B235K5 & $7.08 \mathrm{E} 0$ & N/A & $3 / 10 / 10$ & $0 \mathrm{C} 10004$ \\
$1001004-98$ & B235L9 & $7.11 \mathrm{E} 0$ & N/A & $3 / 10 / 10$ & $0 \mathrm{C} 10004$ \\
$1001004-B C$ & B235N9 & $7.93 \mathrm{E} 0$ & N/A & $3 / 10 / 10$ & $0 \mathrm{C} 10004$ \\
$1001004-B V$ & B235R2 & $8.90 \mathrm{E} 0$ & N/A & $3 / 10 / 10$ & $0 \mathrm{C} 10004$ \\
$1001004-C C$ & B235R9 & $8.26 \mathrm{E} 0$ & N/A & $3 / 10 / 10$ & $0 \mathrm{C} 10004$ \\
$1001004-D A$ & B235T8 & $8.01 \mathrm{E} 0$ & N/A & & $0 \mathrm{C} 10004$ \\
\hline
\end{tabular}


Anions by Ion Chromatography

\begin{tabular}{|c|c|c|c|c|c|c|c|}
\hline CAS \# & Analyte & Results & Units & EQL & Analyzed & Batch & Method \\
\hline HEIS No. & B235H1 & \multicolumn{3}{|c|}{ Lab ID: $\quad$ 1001004-04 } & & & \\
\hline $16984-48-8$ & Fluoride & $<1.99 \mathrm{E} 0$ & \multicolumn{2}{|c|}{ ug/g dry $1.99 \mathrm{E} 0$} & $3 / 11 / 10$ & $0 \mathrm{C} 11004$ & AGG-IC-001 \\
\hline $16887-00-6$ & Chloride & 7.57E0 & \multicolumn{2}{|c|}{$\mathrm{ug} / \mathrm{g}$ dry $\quad 4.97 \mathrm{E} 0$} & $3 / 11 / 10$ & 0C11004 & AGG-IC-001 \\
\hline $14797-65-0$ & Nitrite & $<9.95 \mathrm{E} 0$ & ug/g dry & $9.95 \mathrm{E} 0$ & $3 / 11 / 10$ & 0C11004 & AGG-IC-001 \\
\hline $24959-67-9$ & Bromide & $<9.95 \mathrm{E} 0$ & ug/g dry & $9.95 \mathrm{E} 0$ & $3 / 11 / 10$ & 0C11004 & AGG-IC-001 \\
\hline $14797-55-8$ & Nitrate & $3.39 \mathrm{E} 2$ & ug/g dry & $9.95 \mathrm{E} 0$ & $3 / 11 / 10$ & 0C11004 & AGG-IC-001 \\
\hline $14808-79-8$ & Sulfate & $4.99 \mathrm{E} 1$ & ug/g dry & $1.49 \mathrm{E} 1$ & $3 / 11 / 10$ & 0C11004 & AGG-IC-001 \\
\hline $14265-44-2$ & Phosphate & $<1.49 \mathrm{E} 1$ & ug/g dry & $1.49 \mathrm{E} 1$ & $3 / 11 / 10$ & 0C11004 & AGG-IC-001 \\
\hline HEIS No. & B235J0 & \multicolumn{3}{|c|}{ Lab ID: $\quad$ 1001004-22 } & & & \\
\hline $16984-48-8$ & Fluoride & $<2.02 \mathrm{E} 0$ & ug/g dry & $2.02 \mathrm{E} 0$ & $3 / 11 / 10$ & $0 \mathrm{C} 11004$ & AGG-IC-001 \\
\hline $16887-00-6$ & Chloride & $<5.04 \mathrm{E} 0$ & ug/g dry & $5.04 \mathrm{E} 0$ & $3 / 11 / 10$ & 0C11004 & AGG-IC-001 \\
\hline $14797-65-0$ & Nitrite & $<1.01 \mathrm{E} 1$ & ug/g dry & $1.01 \mathrm{E} 1$ & $3 / 11 / 10$ & 0C11004 & AGG-IC-001 \\
\hline 24959-67-9 & Bromide & $<1.01 \mathrm{E} 1$ & ug/g dry & $1.01 \mathrm{E} 1$ & $3 / 11 / 10$ & $0 \mathrm{C} 11004$ & AGG-IC-001 \\
\hline $14797-55-8$ & Nitrate & $7.92 \mathrm{E} 2$ & ug/g dry & $1.01 \mathrm{E} 1$ & $3 / 11 / 10$ & 0C11004 & AGG-IC-001 \\
\hline $14808-79-8$ & Sulfate & $1.51 \mathrm{E} 1$ & ug/g dry & $1.51 \mathrm{E} 1$ & $3 / 11 / 10$ & 0C11004 & AGG-IC-001 \\
\hline $14265-44-2$ & Phosphate & $<1.51 \mathrm{E} 1$ & ug/g dry & $1.51 \mathrm{E} 1$ & $3 / 11 / 10$ & 0C11004 & AGG-IC-001 \\
\hline HEIS No. & B235J6 & \multicolumn{3}{|c|}{ Lab ID: $\quad$ 1001004-34 } & & & \\
\hline $16984-48-8$ & Fluoride & $<2.00 \mathrm{E} 1$ & \multirow{2}{*}{$\begin{array}{l}\text { ug/g dry } \\
\text { ug/g dry }\end{array}$} & $2.00 \mathrm{E} 1$ & $3 / 11 / 10$ & 0C11004 & AGG-IC-001 \\
\hline $16887-00-6$ & Chloride & $<5.00 \mathrm{E} 1$ & & $5.00 \mathrm{E} 1$ & $3 / 11 / 10$ & 0C11004 & AGG-IC-001 \\
\hline $14797-65-0$ & Nitrite & $<1.00 \mathrm{E} 2$ & ug/g dry & $1.00 \mathrm{E} 2$ & $3 / 11 / 10$ & 0C11004 & AGG-IC-001 \\
\hline $24959-67-9$ & Bromide & $<1.00 \mathrm{E} 2$ & ug/g dry & $1.00 \mathrm{E} 2$ & $3 / 11 / 10$ & 0C11004 & AGG-IC-001 \\
\hline $14797-55-8$ & Nitrate & $1.40 \mathrm{E} 3$ & ug/g dry & $1.00 \mathrm{E} 2$ & $3 / 11 / 10$ & $0 \mathrm{C} 11004$ & AGG-IC-001 \\
\hline $14808-79-8$ & Sulfate & $<1.50 \mathrm{E} 2$ & ug/g dry & $1.50 \mathrm{E} 2$ & $3 / 11 / 10$ & 0C11004 & AGG-IC-001 \\
\hline $14265-44-2$ & Phosphate & $<1.50 \mathrm{E} 2$ & ug/g dry & $1.50 \mathrm{E} 2$ & $3 / 11 / 10$ & 0C11004 & AGG-IC-001 \\
\hline HEIS No. & B235K1 & & ID: $\quad 10($ & 1004-42 & & & \\
\hline $16984-48-8$ & Fluoride & $<2.00 \mathrm{E} 1$ & ug/g dry & $2.00 \mathrm{E} 1$ & $3 / 11 / 10$ & 0C11004 & AGG-IC-001 \\
\hline $16887-00-6$ & Chloride & $<5.01 \mathrm{E} 1$ & ug/g dry & $5.01 \mathrm{E} 1$ & $3 / 11 / 10$ & $0 \mathrm{C} 11004$ & AGG-IC-001 \\
\hline $14797-65-0$ & Nitrite & $<1.00 \mathrm{E} 2$ & ug/g dry & $1.00 \mathrm{E} 2$ & $3 / 11 / 10$ & 0C11004 & AGG-IC-001 \\
\hline $24959-67-9$ & Bromide & $<1.00 \mathrm{E} 2$ & ug/g dry & $1.00 \mathrm{E} 2$ & $3 / 11 / 10$ & 0C11004 & AGG-IC-001 \\
\hline $14797-55-8$ & Nitrate & $2.57 \mathrm{E} 3$ & $\mathrm{ug} / \mathrm{g}$ dry & $1.00 \mathrm{E} 2$ & $3 / 11 / 10$ & $0 \mathrm{C} 11004$ & AGG-IC-001 \\
\hline $14808-79-8$ & Sulfate & $<1.50 \mathrm{E} 2$ & ug/g dry & $1.50 \mathrm{E} 2$ & $3 / 11 / 10$ & 0C11004 & AGG-IC-001 \\
\hline $14265-44-2$ & Phosphate & $<1.50 \mathrm{E} 2$ & ug/g dry & $1.50 \mathrm{E} 2$ & $3 / 11 / 10$ & 0C11004 & AGG-IC-001 \\
\hline HEIS No. & B235K5 & & ID: $\quad 10$ & 1004-46 & & & \\
\hline $16984-48-8$ & Fluoride & $<2.00 \mathrm{E} 0$ & ug/g dry & $2.00 \mathrm{E} 0$ & $3 / 11 / 10$ & 0C11004 & AGG-IC-001 \\
\hline $16887-00-6$ & Chloride & $<5.01 \mathrm{E} 0$ & ug/g dry & $5.01 \mathrm{E} 0$ & $3 / 11 / 10$ & $0 \mathrm{C} 11004$ & AGG-IC-001 \\
\hline $14797-65-0$ & Nitrite & $<1.00 \mathrm{E} 1$ & ug/g dry & $1.00 \mathrm{E} 1$ & $3 / 11 / 10$ & 0C11004 & AGG-IC-001 \\
\hline $24959-67-9$ & Bromide & $<1.00 \mathrm{E} 1$ & ug/g dry & $1.00 \mathrm{E} 1$ & $3 / 11 / 10$ & 0C11004 & AGG-IC-001 \\
\hline $14797-55-8$ & Nitrate & 9.72E2 & $\mathrm{ug} / \mathrm{g}$ dry & $1.00 \mathrm{E} 1$ & $3 / 11 / 10$ & 0C11004 & AGG-IC-001 \\
\hline $14808-79-8$ & Sulfate & $<1.50 \mathrm{E} 1$ & ug/g dry & $1.50 \mathrm{E} 1$ & $3 / 11 / 10$ & 0C11004 & AGG-IC-001 \\
\hline $14265-44-2$ & Phosphate & $<1.50 \mathrm{E} 1$ & ug/g dry & $1.50 \mathrm{E} 1$ & $3 / 11 / 10$ & 0C11004 & AGG-IC-001 \\
\hline HEIS No. & B235L9 & & ID: $\quad 10$ & 1004-98 & & & \\
\hline $16984-48-8$ & Fluoride & $<2.00 \mathrm{E} 0$ & ug/g dry & $2.00 \mathrm{E} 0$ & $3 / 11 / 10$ & 0C11004 & AGG-IC-001 \\
\hline $16887-00-6$ & Chloride & $<5.00 \mathrm{E} 0$ & $\mathrm{ug} / \mathrm{g}$ dry & $5.00 \mathrm{E} 0$ & $3 / 11 / 10$ & 0C11004 & AGG-IC-001 \\
\hline $14797-65-0$ & Nitrite & $<1.00 \mathrm{E} 1$ & ug/g dry & $1.00 \mathrm{E} 1$ & $3 / 11 / 10$ & 0C11004 & AGG-IC-001 \\
\hline $24959-67-9$ & Bromide & $<1.00 \mathrm{E} 1$ & ug/g dry & $1.00 \mathrm{E} 1$ & $3 / 11 / 10$ & 0C11004 & AGG-IC-001 \\
\hline $14797-55-8$ & Nitrate & $5.28 \mathrm{E} 2$ & ug/g dry & $1.00 \mathrm{E} 1$ & $3 / 11 / 10$ & 0C11004 & AGG-IC-001 \\
\hline $14808-79-8$ & Sulfate & $<1.50 \mathrm{E} 1$ & ug/g dry & $1.50 \mathrm{E} 1$ & $3 / 11 / 10$ & $0 \mathrm{C} 11004$ & AGG-IC-001 \\
\hline $14265-44-2$ & Phosphate & $<1.50 \mathrm{E} 1$ & ug/g dry & $1.50 \mathrm{E} 1$ & $3 / 11 / 10$ & 0C11004 & AGG-IC-001 \\
\hline HEIS No. & B235N9 & La & ID: & 1004-BC & & & \\
\hline
\end{tabular}




\section{Anions by Ion Chromatography}

\begin{tabular}{|c|c|c|c|c|c|c|c|}
\hline CAS \# & Analyte & Results & Units & EQL & Analyzed & Batch & Method \\
\hline HEIS No. & B235N9 & \multicolumn{3}{|c|}{ Lab ID: $\quad$ 1001004-BC } & & & \\
\hline $16984-48-8$ & Fluoride & $4.20 \mathrm{E}-1$ & ug/g dry & $2.00 \mathrm{E}-1$ & $3 / 11 / 10$ & 0C11004 & AGG-IC-001 \\
\hline $16887-00-6$ & Chloride & $9.07 \mathrm{E}-1$ & ug/g dry & $4.99 \mathrm{E}-1$ & $3 / 11 / 10$ & 0C11004 & AGG-IC-001 \\
\hline $14797-65-0$ & Nitrite & $<9.98 \mathrm{E}-1$ & $u g / g$ dry & $9.98 \mathrm{E}-1$ & $3 / 11 / 10$ & 0C11004 & AGG-IC-001 \\
\hline $24959-67-9$ & Bromide & $<9.98 \mathrm{E}-1$ & ug/g dry & $9.98 \mathrm{E}-1$ & $3 / 11 / 10$ & 0C11004 & AGG-IC-001 \\
\hline $14797-55-8$ & Nitrate & 2.22E1 & $u g / g$ dry & $9.98 \mathrm{E}-1$ & $3 / 11 / 10$ & 0C11004 & AGG-IC-001 \\
\hline $14808-79-8$ & Sulfate & $2.79 \mathrm{E} 1$ & $\mathrm{ug} / \mathrm{g}$ dry & $1.50 \mathrm{E} 0$ & $3 / 11 / 10$ & 0C11004 & AGG-IC-001 \\
\hline $14265-44-2$ & Phosphate & $<1.50 \mathrm{E} 0$ & $u g / g$ dry & $1.50 \mathrm{E} 0$ & $3 / 11 / 10$ & 0C11004 & AGG-IC-001 \\
\hline HEIS No. & B235R2 & \multicolumn{3}{|c|}{ Lab ID: $1001004-B V$} & & & \\
\hline $16984-48-8$ & Fluoride & 7.03E-1 & ug/g dry & $2.00 \mathrm{E}-1$ & $3 / 11 / 10$ & 0C11004 & AGG-IC-001 \\
\hline $16887-00-6$ & Chloride & $1.21 \mathrm{E} 0$ & $u g / g$ dry & $5.00 \mathrm{E}-1$ & $3 / 11 / 10$ & 0C11004 & AGG-IC-001 \\
\hline $14797-65-0$ & Nitrite & $3.87 \mathrm{E} 0$ & $\mathrm{ug} / \mathrm{g}$ dry & $9.99 \mathrm{E}-1$ & $3 / 11 / 10$ & 0C11004 & AGG-IC-001 \\
\hline $24959-67-9$ & Bromide & $<9.99 \mathrm{E}-1$ & ug/g dry & $9.99 \mathrm{E}-1$ & $3 / 11 / 10$ & 0C11004 & AGG-IC-001 \\
\hline $14797-55-8$ & Nitrate & $1.65 \mathrm{E} 1$ & $u g / g$ dry & $9.99 \mathrm{E}-1$ & $3 / 11 / 10$ & 0C11004 & AGG-IC-001 \\
\hline $14808-79-8$ & Sulfate & $5.25 \mathrm{E} 1$ & ug/g dry & $1.50 \mathrm{E} 0$ & $3 / 11 / 10$ & 0C11004 & AGG-IC-001 \\
\hline $14265-44-2$ & Phosphate & $<1.50 \mathrm{E} 0$ & ug/g dry & $1.50 \mathrm{E} 0$ & $3 / 11 / 10$ & 0C11004 & AGG-IC-001 \\
\hline HEIS No. & B235R9 & \multicolumn{3}{|c|}{ Lab ID: $\quad$ 1001004-CC } & & & \\
\hline $16984-48-8$ & Fluoride & $5.71 \mathrm{E}-1$ & ug/g dry & $2.00 \mathrm{E}-1$ & $3 / 11 / 10$ & 0C11004 & AGG-IC-001 \\
\hline $16887-00-6$ & Chloride & $1.56 \mathrm{E} 0$ & ug/g dry & $5.00 \mathrm{E}-1$ & $3 / 11 / 10$ & 0C11004 & AGG-IC-001 \\
\hline $14797-65-0$ & Nitrite & $<1.00 \mathrm{E} 0$ & ug/g dry & $1.00 \mathrm{E} 0$ & $3 / 11 / 10$ & 0C11004 & AGG-IC-001 \\
\hline $24959-67-9$ & Bromide & $<1.00 \mathrm{E} 0$ & ug/g dry & $1.00 \mathrm{E} 0$ & $3 / 11 / 10$ & 0C11004 & AGG-IC-001 \\
\hline $14797-55-8$ & Nitrate & $1.09 \mathrm{E} 1$ & ug/g dry & $1.00 \mathrm{E} 0$ & $3 / 11 / 10$ & 0C11004 & AGG-IC-001 \\
\hline $14808-79-8$ & Sulfate & $5.91 \mathrm{E} 1$ & ug/g dry & $1.50 \mathrm{E} 0$ & $3 / 11 / 10$ & 0C11004 & AGG-IC-001 \\
\hline $14265-44-2$ & Phosphate & $<1.50 \mathrm{E} 0$ & ug/g dry & $1.50 \mathrm{E} 0$ & $3 / 11 / 10$ & 0C11004 & AGG-IC-001 \\
\hline HEIS No. & В235T8 & \multicolumn{3}{|c|}{ Lab ID: $1001004-D A$} & & & \\
\hline $16984-48-8$ & Fluoride & 7.14E-1 & ug/g dry & $2.00 \mathrm{E}-1$ & $3 / 11 / 10$ & 0C11004 & AGG-IC-001 \\
\hline $16887-00-6$ & Chloride & $2.20 \mathrm{E} 0$ & ug/g dry & $4.99 \mathrm{E}-1$ & $3 / 11 / 10$ & 0C11004 & AGG-IC-001 \\
\hline $14797-65-0$ & Nitrite & $<9.98 \mathrm{E}-1$ & $\mathrm{ug} / \mathrm{g}$ dry & $9.98 \mathrm{E}-1$ & $3 / 11 / 10$ & 0C11004 & AGG-IC-001 \\
\hline $24959-67-9$ & Bromide & $<9.98 \mathrm{E}-1$ & $\mathrm{ug} / \mathrm{g}$ dry & $9.98 \mathrm{E}-1$ & $3 / 11 / 10$ & 0C11004 & AGG-IC-001 \\
\hline $14797-55-8$ & Nitrate & $1.54 \mathrm{E} 0$ & ug/g dry & $9.98 \mathrm{E}-1$ & $3 / 11 / 10$ & 0C11004 & AGG-IC-001 \\
\hline $14808-79-8$ & Sulfate & $4.66 \mathrm{E} 1$ & $\mathrm{ug} / \mathrm{g}$ dry & $1.50 \mathrm{E} 0$ & $3 / 11 / 10$ & 0C11004 & AGG-IC-001 \\
\hline $14265-44-2$ & Phosphate & $<1.50 \mathrm{E} 0$ & ug/g dry & $1.50 \mathrm{E} 0$ & $3 / 11 / 10$ & 0C11004 & AGG-IC-001 \\
\hline
\end{tabular}




\begin{tabular}{|c|c|c|c|c|c|}
\hline \multicolumn{6}{|c|}{ Cyanide by Mircodistillation/Colorimetric } \\
\hline \multicolumn{6}{|c|}{ Cyanide (ug/g dry) by MICRODIST Cyanide Method } \\
\hline Lab ID & HEIS No. & Results & EQL & Analyzed & Batch \\
\hline 1001004-04 & B235H1 & $<1.07 \mathrm{E}-1$ & $1.07 \mathrm{E}-1$ & $4 / 09 / 10$ & 0D08003 \\
\hline 1001004-22 & B235J0 & $<1.03 \mathrm{E}-1$ & $1.03 \mathrm{E}-1$ & $4 / 09 / 10$ & 0D08003 \\
\hline 1001004-34 & B235J6 & $<1.05 \mathrm{E}-1$ & $1.05 \mathrm{E}-1$ & $4 / 09 / 10$ & 0D08003 \\
\hline $1001004-42$ & B235K1 & $<1.06 \mathrm{E}-1$ & $1.06 \mathrm{E}-1$ & $4 / 09 / 10$ & 0D08003 \\
\hline $1001004-46$ & B235K5 & $<1.05 \mathrm{E}-1$ & $1.05 \mathrm{E}-1$ & $4 / 09 / 10$ & 0D08003 \\
\hline 1001004-98 & B235L9 & $<1.06 \mathrm{E}-1$ & $1.06 \mathrm{E}-1$ & $4 / 09 / 10$ & 0D08003 \\
\hline 1001004-BC & B235N9 & $<1.01 \mathrm{E}-1$ & $1.01 \mathrm{E}-1$ & $4 / 09 / 10$ & 0D08003 \\
\hline 1001004-BV & $\mathrm{B} 235 \mathrm{R} 2$ & $<6.43 \mathrm{E}-2$ & $6.43 \mathrm{E}-2$ & $4 / 09 / 10$ & 0D08003 \\
\hline 1001004-CC & B235R9 & $<1.06 \mathrm{E}-1$ & $1.06 \mathrm{E}-1$ & $4 / 09 / 10$ & 0D08003 \\
\hline 1001004-DA & В235T8 & $<1.06 \mathrm{E}-1$ & $1.06 \mathrm{E}-1$ & $4 / 09 / 10$ & 0D08003 \\
\hline
\end{tabular}


Hexavalent Chromium/Soil

Chromium, Hexavalent (ug/g dry) by Colorimetric Determination

\begin{tabular}{llcccc} 
Lab ID & HEIS No. & Results & EQL & Analyzed & Batch \\
\hline $1001004-04$ & B235H1 & $<5.22 \mathrm{E}-1$ & $5.22 \mathrm{E}-1$ & $3 / 25 / 10$ & $0 \mathrm{D} 09005$ \\
$1001004-22$ & B235J0 & $<5.17 \mathrm{E}-1$ & $5.17 \mathrm{E}-1$ & $3 / 25 / 10$ & $0 \mathrm{D} 09005$ \\
$1001004-34$ & B235J6 & $<5.18 \mathrm{E}-1$ & $5.18 \mathrm{E}-1$ & $3 / 25 / 10$ & 0 D09005 \\
$1001004-42$ & B235K1 & $<5.20 \mathrm{E}-1$ & $5.20 \mathrm{E}-1$ & $3 / 25 / 10$ & 0 D09005 \\
$1001004-46$ & B235K5 & $<5.21 \mathrm{E}-1$ & $5.21 \mathrm{E}-1$ & $3 / 25 / 10$ & 0 D09005 \\
$1001004-98$ & B235L9 & $<5.25 \mathrm{E}-1$ & $5.25 \mathrm{E}-1$ & $3 / 25 / 10$ & 0 D09005 \\
$1001004-B C$ & B235N9 & $<5.04 \mathrm{E}-1$ & $5.04 \mathrm{E}-1$ & $3 / 25 / 10$ & $0 \mathrm{D} 09005$ \\
$1001004-\mathrm{BV}$ & B235R2 & $<5.08 \mathrm{E}-1$ & $5.08 \mathrm{E}-1$ & $3 / 25 / 10$ & $0 \mathrm{D} 09005$ \\
$1001004-\mathrm{CC}$ & B235R9 & $<5.06 \mathrm{E}-1$ & $5.06 \mathrm{E}-1$ & $3 / 25 / 10$ & 0 D09005 \\
$1001004-\mathrm{DA}$ & B235T8 & $<5.25 \mathrm{E}-1$ & $5.25 \mathrm{E}-1$ & $3 / 25 / 10$ & 0 D09005 \\
\hline
\end{tabular}


Total Metals by PNNL-AGG-ICP-AES/Water Extract

\begin{tabular}{|c|c|c|c|c|c|c|c|}
\hline CAS \# & Analyte & Results & Units & EQL & Analyzed & Batch & Method \\
\hline HEIS No. & B235H1 & \multicolumn{3}{|c|}{ Lab ID: $\quad$ 1001004-04 } & & & \\
\hline $7429-90-5$ & Aluminum & $<9.32 \mathrm{E}-2$ & ug/g dry & $9.32 \mathrm{E}-2$ & $3 / 11 / 10$ & 0C11001 & PNNL-AGG-ICP-AES \\
\hline $7440-38-2$ & Arsenic & $<3.10 \mathrm{E}-1$ & ug/g dry & $3.10 \mathrm{E}-1$ & $3 / 11 / 10$ & 0C11001 & PNNL-AGG-ICP-AES \\
\hline $7440-70-2$ & Calcium & $7.45 \mathrm{E} 1$ & $u g / g$ dry & $4.50 \mathrm{E}-1$ & $3 / 11 / 10$ & 0C11001 & PNNL-AGG-ICP-AES \\
\hline $7440-43-9$ & Cadmium & $<1.03 \mathrm{E}-1$ & ug/g dry & $1.03 \mathrm{E}-1$ & $3 / 11 / 10$ & 0C11001 & PNNL-AGG-ICP-AES \\
\hline $7440-47-3$ & Chromium & $<2.82 \mathrm{E}-2$ & $u g / g$ dry & $2.82 \mathrm{E}-2$ & $3 / 11 / 10$ & 0C11001 & PNNL-AGG-ICP-AES \\
\hline $7440-50-8$ & Copper & $<8.74 \mathrm{E}-2$ & ug/g dry & $8.74 \mathrm{E}-2$ & $3 / 11 / 10$ & 0C11001 & PNNL-AGG-ICP-AES \\
\hline $7439-89-6$ & Iron & $<1.04 \mathrm{E}-1$ & $u g / g$ dry & $1.04 \mathrm{E}-1$ & $3 / 11 / 10$ & 0C11001 & PNNL-AGG-ICP-AES \\
\hline 7440-09-7 & Potassium & 8.67E0 & ug/g dry & $1.28 \mathrm{E} 0$ & $3 / 11 / 10$ & 0C11001 & PNNL-AGG-ICP-AES \\
\hline $7439-95-4$ & Magnesium & $2.64 \mathrm{E} 1$ & $u g / g$ dry & $4.86 \mathrm{E}-2$ & $3 / 11 / 10$ & 0C11001 & PNNL-AGG-ICP-AES \\
\hline $7439-96-5$ & Manganese & $<6.21 \mathrm{E}-2$ & ug/g dry & $6.21 \mathrm{E}-2$ & $3 / 11 / 10$ & 0C11001 & PNNL-AGG-ICP-AES \\
\hline $7440-02-0$ & Nickel & $<9.62 \mathrm{E}-2$ & ug/g dry & $9.62 \mathrm{E}-2$ & $3 / 11 / 10$ & 0C11001 & PNNL-AGG-ICP-AES \\
\hline $7439-92-1$ & Lead & $<1.60 \mathrm{E}-1$ & ug/g dry & $1.60 \mathrm{E}-1$ & $3 / 11 / 10$ & 0C11001 & PNNL-AGG-ICP-AES \\
\hline $7782-49-2$ & Selenium & $<1.20 \mathrm{E} 0$ & ug/g dry & $1.20 \mathrm{E} 0$ & $3 / 11 / 10$ & 0C11001 & PNNL-AGG-ICP-AES \\
\hline $7440-28-0$ & Thallium & $<9.83 \mathrm{E}-1$ & $\mathrm{ug} / \mathrm{g}$ dry & $9.83 \mathrm{E}-1$ & $3 / 11 / 10$ & 0C11001 & PNNL-AGG-ICP-AES \\
\hline $7440-23-5$ & Sodium & $1.77 \mathrm{E} 1$ & ug/g dry & 7.93E-1 & $3 / 11 / 10$ & 0C11001 & PNNL-AGG-ICP-AES \\
\hline $7440-22-4$ & Silver & $<5.65 \mathrm{E}-2$ & $\mathrm{ug} / \mathrm{g}$ dry & $5.65 \mathrm{E}-2$ & $3 / 11 / 10$ & 0C11001 & PNNL-AGG-ICP-AES \\
\hline HEIS No. & B235J0 & \multicolumn{3}{|c|}{ Lab ID: $\quad 1001004-22$} & & & \\
\hline $7429-90-5$ & Aluminum & $<9.45 \mathrm{E}-2$ & ug/g dry & $9.45 \mathrm{E}-2$ & $3 / 11 / 10$ & 0C11001 & PNNL-AGG-ICP-AES \\
\hline $7440-38-2$ & Arsenic & $<3.14 \mathrm{E}-1$ & $u g / g$ dry & $3.14 \mathrm{E}-1$ & $3 / 11 / 10$ & 0C11001 & PNNL-AGG-ICP-AES \\
\hline $7440-70-2$ & Calcium & $2.03 \mathrm{E} 2$ & ug/g dry & $4.56 \mathrm{E}-1$ & $3 / 11 / 10$ & 0C11001 & PNNL-AGG-ICP-AES \\
\hline $7440-43-9$ & Cadmium & $<1.05 \mathrm{E}-1$ & $u g / g$ dry & $1.05 \mathrm{E}-1$ & $3 / 11 / 10$ & 0C11001 & PNNL-AGG-ICP-AES \\
\hline $7440-47-3$ & Chromium & $<2.86 \mathrm{E}-2$ & ug/g dry & $2.86 \mathrm{E}-2$ & $3 / 11 / 10$ & 0C11001 & PNNL-AGG-ICP-AES \\
\hline $7440-50-8$ & Copper & $<8.86 \mathrm{E}-2$ & $u g / g$ dry & $8.86 \mathrm{E}-2$ & $3 / 11 / 10$ & 0C11001 & PNNL-AGG-ICP-AES \\
\hline $7439-89-6$ & Iron & $<1.06 \mathrm{E}-1$ & ug/g dry & $1.06 \mathrm{E}-1$ & $3 / 11 / 10$ & 0C11001 & PNNL-AGG-ICP-AES \\
\hline $7440-09-7$ & Potassium & $1.16 \mathrm{E} 1$ & $u g / g$ dry & $1.30 \mathrm{E} 0$ & $3 / 11 / 10$ & 0C11001 & PNNL-AGG-ICP-AES \\
\hline $7439-95-4$ & Magnesium & $2.84 \mathrm{E} 1$ & ug/g dry & $4.93 \mathrm{E}-2$ & $3 / 11 / 10$ & 0C11001 & PNNL-AGG-ICP-AES \\
\hline $7439-96-5$ & Manganese & $9.15 \mathrm{E}-2$ & $u g / g$ dry & $6.30 \mathrm{E}-2$ & $3 / 11 / 10$ & 0C11001 & PNNL-AGG-ICP-AES \\
\hline $7440-02-0$ & Nickel & $<9.75 \mathrm{E}-2$ & $u g / g$ dry & $9.75 \mathrm{E}-2$ & $3 / 11 / 10$ & 0C11001 & PNNL-AGG-ICP-AES \\
\hline $7439-92-1$ & Lead & $<1.62 \mathrm{E}-1$ & $u g / g$ dry & $1.62 \mathrm{E}-1$ & $3 / 11 / 10$ & 0C11001 & PNNL-AGG-ICP-AES \\
\hline $7782-49-2$ & Selenium & $<1.21 \mathrm{E} 0$ & $u g / g$ dry & $1.21 \mathrm{E} 0$ & $3 / 11 / 10$ & 0C11001 & PNNL-AGG-ICP-AES \\
\hline $7440-28-0$ & Thallium & $<9.96 \mathrm{E}-1$ & ug/g dry & $9.96 \mathrm{E}-1$ & $3 / 11 / 10$ & 0C11001 & PNNL-AGG-ICP-AES \\
\hline $7440-23-5$ & Sodium & 5.81E0 & ug/g dry & $8.04 \mathrm{E}-1$ & $3 / 11 / 10$ & 0C11001 & PNNL-AGG-ICP-AES \\
\hline $7440-22-4$ & Silver & $<5.73 \mathrm{E}-2$ & ug/g dry & $5.73 \mathrm{E}-2$ & $3 / 11 / 10$ & 0C11001 & PNNL-AGG-ICP-AES \\
\hline HEIS No. & B235J6 & \multicolumn{3}{|c|}{ Lab ID: $\quad 1001004-34$} & & & \\
\hline $7429-90-5$ & Aluminum & $<9.37 \mathrm{E}-2$ & ug/g dry & $9.37 \mathrm{E}-2$ & $3 / 11 / 10$ & 0C11001 & PNNL-AGG-ICP-AES \\
\hline $7440-38-2$ & Arsenic & $<3.11 \mathrm{E}-1$ & ug/g dry & $3.11 \mathrm{E}-1$ & $3 / 11 / 10$ & 0C11001 & PNNL-AGG-ICP-AES \\
\hline $7440-70-2$ & Calcium & 2.27E2 & $u g / g$ dry & $4.52 \mathrm{E}-1$ & $3 / 11 / 10$ & 0C11001 & PNNL-AGG-ICP-AES \\
\hline $7440-43-9$ & Cadmium & $<1.04 \mathrm{E}-1$ & ug/g dry & $1.04 \mathrm{E}-1$ & $3 / 11 / 10$ & 0C11001 & PNNL-AGG-ICP-AES \\
\hline $7440-47-3$ & Chromium & $<2.84 \mathrm{E}-2$ & ug/g dry & $2.84 \mathrm{E}-2$ & $3 / 11 / 10$ & 0C11001 & PNNL-AGG-ICP-AES \\
\hline $7440-50-8$ & Copper & $<8.78 \mathrm{E}-2$ & ug/g dry & $8.78 \mathrm{E}-2$ & $3 / 11 / 10$ & 0C11001 & PNNL-AGG-ICP-AES \\
\hline $7439-89-6$ & Iron & $<1.05 \mathrm{E}-1$ & $u g / g$ dry & $1.05 \mathrm{E}-1$ & $3 / 11 / 10$ & 0C11001 & PNNL-AGG-ICP-AES \\
\hline $7440-09-7$ & Potassium & $3.58 \mathrm{E} 1$ & ug/g dry & $1.29 \mathrm{E} 0$ & $3 / 11 / 10$ & 0C11001 & PNNL-AGG-ICP-AES \\
\hline $7439-95-4$ & Magnesium & $3.87 \mathrm{E} 1$ & $u g / g$ dry & $4.88 \mathrm{E}-2$ & $3 / 11 / 10$ & 0C11001 & PNNL-AGG-ICP-AES \\
\hline $7439-96-5$ & Manganese & $3.63 \mathrm{E}-1$ & ug/g dry & $6.24 \mathrm{E}-2$ & $3 / 11 / 10$ & 0C11001 & PNNL-AGG-ICP-AES \\
\hline $7440-02-0$ & Nickel & $<9.67 \mathrm{E}-2$ & $u g / g$ dry & $9.67 \mathrm{E}-2$ & $3 / 11 / 10$ & 0C11001 & PNNL-AGG-ICP-AES \\
\hline $7439-92-1$ & Lead & $<1.61 \mathrm{E}-1$ & $u g / g$ dry & $1.61 \mathrm{E}-1$ & $3 / 11 / 10$ & 0C11001 & PNNL-AGG-ICP-AES \\
\hline $7782-49-2$ & Selenium & $<1.20 \mathrm{E} 0$ & ug/g dry & $1.20 \mathrm{E} 0$ & $3 / 11 / 10$ & 0C11001 & PNNL-AGG-ICP-AES \\
\hline $7440-28-0$ & Thallium & $<9.88 \mathrm{E}-1$ & ug/g dry & $9.88 \mathrm{E}-1$ & $3 / 11 / 10$ & 0C11001 & PNNL-AGG-ICP-AES \\
\hline
\end{tabular}


Total Metals by PNNL-AGG-ICP-AES/Water Extract

\begin{tabular}{|c|c|c|c|c|c|c|c|}
\hline CAS \# & Analyte & Results & Units & EQL & Analyzed & Batch & Method \\
\hline HEIS No. & B235J6 & \multicolumn{3}{|c|}{ Lab ID: $\quad 1001004-34$} & & & \\
\hline $7440-23-5$ & Sodium & $1.17 \mathrm{E} 1$ & ug/g dry & $7.97 \mathrm{E}-1$ & $3 / 11 / 10$ & 0C11001 & PNNL-AGG-ICP-AES \\
\hline $7440-22-4$ & Silver & $<5.68 \mathrm{E}-2$ & ug/g dry & $5.68 \mathrm{E}-2$ & $3 / 11 / 10$ & 0C11001 & PNNL-AGG-ICP-AES \\
\hline HEIS No. & B235K1 & \multicolumn{3}{|c|}{ Lab ID: $\quad 1001004-42$} & & & \\
\hline $7429-90-5$ & Aluminum & $<9.38 \mathrm{E}-2$ & ug/g dry & $9.38 \mathrm{E}-2$ & $3 / 11 / 10$ & 0C11001 & PNNL-AGG-ICP-AES \\
\hline $7440-38-2$ & Arsenic & $<3.12 \mathrm{E}-1$ & ug/g dry & $3.12 \mathrm{E}-1$ & $3 / 11 / 10$ & 0C11001 & PNNL-AGG-ICP-AES \\
\hline $7440-70-2$ & Calcium & $6.93 \mathrm{E} 2$ & ug/g dry & $4.53 \mathrm{E}-1$ & $3 / 11 / 10$ & 0C11001 & PNNL-AGG-ICP-AES \\
\hline $7440-43-9$ & Cadmium & $<1.04 \mathrm{E}-1$ & ug/g dry & $1.04 \mathrm{E}-1$ & $3 / 11 / 10$ & 0C11001 & PNNL-AGG-ICP-AES \\
\hline $7440-47-3$ & Chromium & $<2.84 \mathrm{E}-2$ & ug/g dry & $2.84 \mathrm{E}-2$ & $3 / 11 / 10$ & 0C11001 & PNNL-AGG-ICP-AES \\
\hline $7440-50-8$ & Copper & $<8.80 \mathrm{E}-2$ & ug/g dry & $8.80 \mathrm{E}-2$ & $3 / 11 / 10$ & 0C11001 & PNNL-AGG-ICP-AES \\
\hline $7439-89-6$ & Iron & $<1.05 \mathrm{E}-1$ & ug/g dry & $1.05 \mathrm{E}-1$ & $3 / 11 / 10$ & 0C11001 & PNNL-AGG-ICP-AES \\
\hline $7440-09-7$ & Potassium & 2.34E1 & ug/g dry & $1.29 \mathrm{E} 0$ & $3 / 11 / 10$ & 0C11001 & PNNL-AGG-ICP-AES \\
\hline $7439-95-4$ & Magnesium & $6.82 \mathrm{E} 1$ & ug/g dry & $4.89 \mathrm{E}-2$ & $3 / 11 / 10$ & 0C11001 & PNNL-AGG-ICP-AES \\
\hline $7439-96-5$ & Manganese & $2.72 \mathrm{E}-1$ & ug/g dry & $6.25 \mathrm{E}-2$ & $3 / 11 / 10$ & 0C11001 & PNNL-AGG-ICP-AES \\
\hline $7440-02-0$ & Nickel & $<9.68 \mathrm{E}-2$ & ug/g dry & $9.68 \mathrm{E}-2$ & $3 / 11 / 10$ & 0C11001 & PNNL-AGG-ICP-AES \\
\hline $7439-92-1$ & Lead & $<1.61 \mathrm{E}-1$ & ug/g dry & $1.61 \mathrm{E}-1$ & $3 / 11 / 10$ & 0C11001 & PNNL-AGG-ICP-AES \\
\hline $7782-49-2$ & Selenium & $<1.20 \mathrm{E} 0$ & $\mathrm{ug} / \mathrm{g}$ dry & $1.20 \mathrm{E} 0$ & $3 / 11 / 10$ & 0C11001 & PNNL-AGG-ICP-AES \\
\hline $7440-28-0$ & Thallium & $<9.89 \mathrm{E}-1$ & ug/g dry & $9.89 \mathrm{E}-1$ & $3 / 11 / 10$ & 0C11001 & PNNL-AGG-ICP-AES \\
\hline $7440-23-5$ & Sodium & $1.88 \mathrm{E} 1$ & ug/g dry & $7.98 \mathrm{E}-1$ & $3 / 11 / 10$ & 0C11001 & PNNL-AGG-ICP-AES \\
\hline $7440-22-4$ & Silver & $<5.69 \mathrm{E}-2$ & ug/g dry & $5.69 \mathrm{E}-2$ & $3 / 11 / 10$ & 0C11001 & PNNL-AGG-ICP-AES \\
\hline HEIS No. & B235K5 & \multicolumn{3}{|c|}{ Lab ID: $1001004-46$} & & & \\
\hline $7429-90-5$ & Aluminum & $<9.38 \mathrm{E}-2$ & ug/g dry & $9.38 \mathrm{E}-2$ & $3 / 11 / 10$ & 0C11001 & PNNL-AGG-ICP-AES \\
\hline $7440-38-2$ & Arsenic & $<3.12 \mathrm{E}-1$ & ug/g dry & $3.12 \mathrm{E}-1$ & $3 / 11 / 10$ & 0C11001 & PNNL-AGG-ICP-AES \\
\hline $7440-70-2$ & Calcium & $2.35 \mathrm{E} 2$ & ug/g dry & $4.53 \mathrm{E}-1$ & $3 / 11 / 10$ & 0C11001 & PNNL-AGG-ICP-AES \\
\hline $7440-43-9$ & Cadmium & $<1.04 \mathrm{E}-1$ & ug/g dry & $1.04 \mathrm{E}-1$ & $3 / 11 / 10$ & 0C11001 & PNNL-AGG-ICP-AES \\
\hline $7440-47-3$ & Chromium & $<2.84 \mathrm{E}-2$ & ug/g dry & $2.84 \mathrm{E}-2$ & $3 / 11 / 10$ & 0C11001 & PNNL-AGG-ICP-AES \\
\hline $7440-50-8$ & Copper & $<8.80 \mathrm{E}-2$ & ug/g dry & $8.80 \mathrm{E}-2$ & $3 / 11 / 10$ & 0C11001 & PNNL-AGG-ICP-AES \\
\hline $7439-89-6$ & Iron & $<1.05 \mathrm{E}-1$ & ug/g dry & $1.05 \mathrm{E}-1$ & $3 / 11 / 10$ & 0C11001 & PNNL-AGG-ICP-AES \\
\hline $7440-09-7$ & Potassium & $9.95 \mathrm{E} 0$ & ug/g dry & $1.29 \mathrm{E} 0$ & $3 / 11 / 10$ & 0C11001 & PNNL-AGG-ICP-AES \\
\hline $7439-95-4$ & Magnesium & $4.31 \mathrm{E} 1$ & ug/g dry & $4.89 \mathrm{E}-2$ & $3 / 11 / 10$ & $0 \mathrm{C} 11001$ & PNNL-AGG-ICP-AES \\
\hline $7439-96-5$ & Manganese & $<6.25 \mathrm{E}-2$ & ug/g dry & $6.25 \mathrm{E}-2$ & $3 / 11 / 10$ & 0C11001 & PNNL-AGG-ICP-AES \\
\hline $7440-02-0$ & Nickel & $<9.68 \mathrm{E}-2$ & ug/g dry & $9.68 \mathrm{E}-2$ & $3 / 11 / 10$ & 0C11001 & PNNL-AGG-ICP-AES \\
\hline $7439-92-1$ & Lead & $<1.61 \mathrm{E}-1$ & ug/g dry & $1.61 \mathrm{E}-1$ & $3 / 11 / 10$ & 0C11001 & PNNL-AGG-ICP-AES \\
\hline $7782-49-2$ & Selenium & $<1.20 \mathrm{E} 0$ & ug/g dry & $1.20 \mathrm{E} 0$ & $3 / 11 / 10$ & 0C11001 & PNNL-AGG-ICP-AES \\
\hline $7440-28-0$ & Thallium & $<9.89 \mathrm{E}-1$ & ug/g dry & $9.89 \mathrm{E}-1$ & $3 / 11 / 10$ & 0C11001 & PNNL-AGG-ICP-AES \\
\hline $7440-23-5$ & Sodium & $9.54 \mathrm{E} 0$ & ug/g dry & $7.98 \mathrm{E}-1$ & $3 / 11 / 10$ & $0 \mathrm{C} 11001$ & PNNL-AGG-ICP-AES \\
\hline $7440-22-4$ & Silver & $<5.69 \mathrm{E}-2$ & ug/g dry & $5.69 \mathrm{E}-2$ & $3 / 11 / 10$ & 0C11001 & PNNL-AGG-ICP-AES \\
\hline HEIS No. & B235L9 & \multicolumn{3}{|c|}{ Lab ID: $\quad 1001004-98$} & & & \\
\hline $7429-90-5$ & Aluminum & $<9.38 \mathrm{E}-2$ & ug/g dry & $9.38 \mathrm{E}-2$ & $3 / 11 / 10$ & 0C11001 & PNNL-AGG-ICP-AES \\
\hline $7440-38-2$ & Arsenic & $<3.12 \mathrm{E}-1$ & ug/g dry & $3.12 \mathrm{E}-1$ & $3 / 11 / 10$ & 0C11001 & PNNL-AGG-ICP-AES \\
\hline $7440-70-2$ & Calcium & $1.12 \mathrm{E} 2$ & $\mathrm{ug} / \mathrm{g}$ dry & $4.52 \mathrm{E}-1$ & $3 / 11 / 10$ & 0C11001 & PNNL-AGG-ICP-AES \\
\hline $7440-43-9$ & Cadmium & $<1.04 \mathrm{E}-1$ & ug/g dry & $1.04 \mathrm{E}-1$ & $3 / 11 / 10$ & 0C11001 & PNNL-AGG-ICP-AES \\
\hline $7440-47-3$ & Chromium & $<2.84 \mathrm{E}-2$ & ug/g dry & $2.84 \mathrm{E}-2$ & $3 / 11 / 10$ & 0C11001 & PNNL-AGG-ICP-AES \\
\hline $7440-50-8$ & Copper & $<8.79 \mathrm{E}-2$ & ug/g dry & $8.79 \mathrm{E}-2$ & $3 / 11 / 10$ & 0C11001 & PNNL-AGG-ICP-AES \\
\hline $7439-89-6$ & Iron & $<1.05 \mathrm{E}-1$ & ug/g dry & $1.05 \mathrm{E}-1$ & $3 / 11 / 10$ & 0C11001 & PNNL-AGG-ICP-AES \\
\hline $7440-09-7$ & Potassium & 7.63E0 & ug/g dry & $1.29 \mathrm{E} 0$ & $3 / 11 / 10$ & 0C11001 & PNNL-AGG-ICP-AES \\
\hline $7439-95-4$ & Magnesium & $3.24 \mathrm{E} 1$ & ug/g dry & $4.89 \mathrm{E}-2$ & $3 / 11 / 10$ & 0C11001 & PNNL-AGG-ICP-AES \\
\hline $7439-96-5$ & Manganese & $<6.25 \mathrm{E}-2$ & ug/g dry & $6.25 \mathrm{E}-2$ & $3 / 11 / 10$ & 0C11001 & PNNL-AGG-ICP-AES \\
\hline $7440-02-0$ & Nickel & $<9.68 \mathrm{E}-2$ & ug/g dry & $9.68 \mathrm{E}-2$ & $3 / 11 / 10$ & $0 \mathrm{C} 11001$ & PNNL-AGG-ICP-AES \\
\hline
\end{tabular}


Total Metals by PNNL-AGG-ICP-AES/Water Extract

\begin{tabular}{|c|c|c|c|c|c|c|c|}
\hline CAS \# & Analyte & Results & Units & EQL & Analyzed & Batch & Method \\
\hline HEIS No. & B235L9 & \multicolumn{3}{|c|}{ Lab ID: $\quad 1001004-98$} & & & \\
\hline 7439-92-1 & Lead & $<1.61 \mathrm{E}-1$ & ug/g dry & $1.61 \mathrm{E}-1$ & $3 / 11 / 10$ & 0C11001 & PNNL-AGG-ICP-AES \\
\hline $7782-49-2$ & Selenium & $<1.20 \mathrm{E} 0$ & ug/g dry & $1.20 \mathrm{E} 0$ & $3 / 11 / 10$ & 0C11001 & PNNL-AGG-ICP-AES \\
\hline $7440-28-0$ & Thallium & $<9.88 \mathrm{E}-1$ & ug/g dry & $9.88 \mathrm{E}-1$ & $3 / 11 / 10$ & 0C11001 & PNNL-AGG-ICP-AES \\
\hline $7440-23-5$ & Sodium & 9.04E0 & ug/g dry & $7.98 \mathrm{E}-1$ & $3 / 11 / 10$ & 0C11001 & PNNL-AGG-ICP-AES \\
\hline $7440-22-4$ & Silver & $<5.68 \mathrm{E}-2$ & ug/g dry & $5.68 \mathrm{E}-2$ & $3 / 11 / 10$ & 0C11001 & PNNL-AGG-ICP-AES \\
\hline HEIS No. & B235N9 & \multicolumn{3}{|c|}{ Lab ID: $\quad$ 1001004-BC } & & & \\
\hline $7429-90-5$ & Aluminum & $1.58 \mathrm{E}-1$ & ug/g dry & $9.35 \mathrm{E}-2$ & $3 / 11 / 10$ & 0C11001 & PNNL-AGG-ICP-AES \\
\hline $7440-38-2$ & Arsenic & $<3.11 \mathrm{E}-1$ & ug/g dry & $3.11 \mathrm{E}-1$ & $3 / 11 / 10$ & 0C11001 & PNNL-AGG-ICP-AES \\
\hline $7440-70-2$ & Calcium & $1.12 \mathrm{E} 1$ & ug/g dry & $4.51 \mathrm{E}-1$ & $3 / 11 / 10$ & $0 \mathrm{C} 11001$ & PNNL-AGG-ICP-AES \\
\hline $7440-43-9$ & Cadmium & $<1.04 \mathrm{E}-1$ & ug/g dry & $1.04 \mathrm{E}-1$ & $3 / 11 / 10$ & 0C11001 & PNNL-AGG-ICP-AES \\
\hline $7440-47-3$ & Chromium & $<2.83 \mathrm{E}-2$ & ug/g dry & $2.83 \mathrm{E}-2$ & $3 / 11 / 10$ & 0C11001 & PNNL-AGG-ICP-AES \\
\hline $7440-50-8$ & Copper & $<8.76 \mathrm{E}-2$ & ug/g dry & $8.76 \mathrm{E}-2$ & $3 / 11 / 10$ & 0C11001 & PNNL-AGG-ICP-AES \\
\hline $7439-89-6$ & Iron & $<1.05 \mathrm{E}-1$ & ug/g dry & $1.05 \mathrm{E}-1$ & $3 / 11 / 10$ & 0C11001 & PNNL-AGG-ICP-AES \\
\hline $7440-09-7$ & Potassium & $5.16 \mathrm{E} 0$ & ug/g dry & $1.29 \mathrm{E} 0$ & $3 / 11 / 10$ & 0C11001 & PNNL-AGG-ICP-AES \\
\hline $7439-95-4$ & Magnesium & $3.37 \mathrm{E} 0$ & ug/g dry & $4.88 \mathrm{E}-2$ & $3 / 11 / 10$ & 0C11001 & PNNL-AGG-ICP-AES \\
\hline $7439-96-5$ & Manganese & $<6.23 \mathrm{E}-2$ & ug/g dry & $6.23 \mathrm{E}-2$ & $3 / 11 / 10$ & 0C11001 & PNNL-AGG-ICP-AES \\
\hline $7440-02-0$ & Nickel & $<9.65 \mathrm{E}-2$ & ug/g dry & $9.65 \mathrm{E}-2$ & $3 / 11 / 10$ & $0 \mathrm{C} 11001$ & PNNL-AGG-ICP-AES \\
\hline $7439-92-1$ & Lead & $<1.61 \mathrm{E}-1$ & ug/g dry & $1.61 \mathrm{E}-1$ & $3 / 11 / 10$ & 0C11001 & PNNL-AGG-ICP-AES \\
\hline $7782-49-2$ & Selenium & $<1.20 \mathrm{E} 0$ & ug/g dry & $1.20 \mathrm{E} 0$ & $3 / 11 / 10$ & 0C11001 & PNNL-AGG-ICP-AES \\
\hline $7440-28-0$ & Thallium & $<9.86 \mathrm{E}-1$ & ug/g dry & $9.86 \mathrm{E}-1$ & $3 / 11 / 10$ & 0C11001 & PNNL-AGG-ICP-AES \\
\hline $7440-23-5$ & Sodium & $1.32 \mathrm{E} 1$ & ug/g dry & $7.95 \mathrm{E}-1$ & $3 / 11 / 10$ & $0 \mathrm{C} 11001$ & PNNL-AGG-ICP-AES \\
\hline $7440-22-4$ & Silver & $<5.67 \mathrm{E}-2$ & ug/g dry & $5.67 \mathrm{E}-2$ & $3 / 11 / 10$ & 0C11001 & PNNL-AGG-ICP-AES \\
\hline HEIS No. & B235R2 & \multicolumn{3}{|c|}{ Lab ID: $\quad$ 1001004-BV } & & & \\
\hline $7429-90-5$ & Aluminum & $1.61 \mathrm{E}-1$ & ug/g dry & $9.36 \mathrm{E}-2$ & $3 / 11 / 10$ & 0C11001 & PNNL-AGG-ICP-AES \\
\hline $7440-38-2$ & Arsenic & $<3.11 \mathrm{E}-1$ & ug/g dry & $3.11 \mathrm{E}-1$ & $3 / 11 / 10$ & 0C11001 & PNNL-AGG-ICP-AES \\
\hline $7440-70-2$ & Calcium & $1.65 \mathrm{E} 1$ & ug/g dry & $4.52 \mathrm{E}-1$ & $3 / 11 / 10$ & 0C11001 & PNNL-AGG-ICP-AES \\
\hline $7440-43-9$ & Cadmium & $<1.04 \mathrm{E}-1$ & ug/g dry & $1.04 \mathrm{E}-1$ & $3 / 11 / 10$ & 0C11001 & PNNL-AGG-ICP-AES \\
\hline $7440-47-3$ & Chromium & $<2.84 \mathrm{E}-2$ & ug/g dry & $2.84 \mathrm{E}-2$ & $3 / 11 / 10$ & 0C11001 & PNNL-AGG-ICP-AES \\
\hline $7440-50-8$ & Copper & $<8.78 \mathrm{E}-2$ & ug/g dry & $8.78 \mathrm{E}-2$ & $3 / 11 / 10$ & 0C11001 & PNNL-AGG-ICP-AES \\
\hline $7439-89-6$ & Iron & $<1.05 \mathrm{E}-1$ & ug/g dry & $1.05 \mathrm{E}-1$ & $3 / 11 / 10$ & 0C11001 & PNNL-AGG-ICP-AES \\
\hline 7440-09-7 & Potassium & $5.30 \mathrm{E} 0$ & ug/g dry & $1.29 \mathrm{E} 0$ & $3 / 11 / 10$ & 0C11001 & PNNL-AGG-ICP-AES \\
\hline $7439-95-4$ & Magnesium & $3.69 \mathrm{E} 0$ & ug/g dry & $4.88 \mathrm{E}-2$ & $3 / 11 / 10$ & 0C11001 & PNNL-AGG-ICP-AES \\
\hline $7439-96-5$ & Manganese & $<6.24 \mathrm{E}-2$ & ug/g dry & $6.24 \mathrm{E}-2$ & $3 / 11 / 10$ & 0C11001 & PNNL-AGG-ICP-AES \\
\hline $7440-02-0$ & Nickel & $<9.66 \mathrm{E}-2$ & ug/g dry & $9.66 \mathrm{E}-2$ & $3 / 11 / 10$ & 0C11001 & PNNL-AGG-ICP-AES \\
\hline $7439-92-1$ & Lead & $<1.61 \mathrm{E}-1$ & ug/g dry & $1.61 \mathrm{E}-1$ & $3 / 11 / 10$ & 0C11001 & PNNL-AGG-ICP-AES \\
\hline $7782-49-2$ & Selenium & $<1.20 \mathrm{E} 0$ & ug/g dry & $1.20 \mathrm{E} 0$ & $3 / 11 / 10$ & 0C11001 & PNNL-AGG-ICP-AES \\
\hline $7440-28-0$ & Thallium & $<9.87 \mathrm{E}-1$ & ug/g dry & $9.87 \mathrm{E}-1$ & $3 / 11 / 10$ & 0C11001 & PNNL-AGG-ICP-AES \\
\hline $7440-23-5$ & Sodium & $1.50 \mathrm{E} 1$ & ug/g dry & 7.96E-1 & $3 / 11 / 10$ & 0C11001 & PNNL-AGG-ICP-AES \\
\hline $7440-22-4$ & Silver & $<5.67 \mathrm{E}-2$ & ug/g dry & $5.67 \mathrm{E}-2$ & $3 / 11 / 10$ & 0C11001 & PNNL-AGG-ICP-AES \\
\hline HEIS No. & B235R9 & \multicolumn{3}{|c|}{ Lab ID: 1001004-CC } & & & \\
\hline $7429-90-5$ & Aluminum & $1.89 \mathrm{E}-1$ & ug/g dry & $9.37 \mathrm{E}-2$ & $3 / 11 / 10$ & 0C11001 & PNNL-AGG-ICP-AES \\
\hline $7440-38-2$ & Arsenic & $<3.11 \mathrm{E}-1$ & ug/g dry & $3.11 \mathrm{E}-1$ & $3 / 11 / 10$ & 0C11001 & PNNL-AGG-ICP-AES \\
\hline $7440-70-2$ & Calcium & $1.73 \mathrm{E} 1$ & ug/g dry & $4.52 \mathrm{E}-1$ & $3 / 11 / 10$ & 0C11001 & PNNL-AGG-ICP-AES \\
\hline $7440-43-9$ & Cadmium & $<1.04 \mathrm{E}-1$ & ug/g dry & $1.04 \mathrm{E}-1$ & $3 / 11 / 10$ & 0C11001 & PNNL-AGG-ICP-AES \\
\hline $7440-47-3$ & Chromium & $<2.84 \mathrm{E}-2$ & ug/g dry & $2.84 \mathrm{E}-2$ & $3 / 11 / 10$ & 0C11001 & PNNL-AGG-ICP-AES \\
\hline $7440-50-8$ & Copper & $<8.78 \mathrm{E}-2$ & ug/g dry & $8.78 \mathrm{E}-2$ & $3 / 11 / 10$ & 0C11001 & PNNL-AGG-ICP-AES \\
\hline $7439-89-6$ & Iron & $<1.05 \mathrm{E}-1$ & ug/g dry & $1.05 \mathrm{E}-1$ & $3 / 11 / 10$ & 0C11001 & PNNL-AGG-ICP-AES \\
\hline $7440-09-7$ & Potassium & $5.01 \mathrm{E} 0$ & ug/g dry & $1.29 \mathrm{E} 0$ & $3 / 11 / 10$ & 0C11001 & PNNL-AGG-ICP-AES \\
\hline
\end{tabular}


Total Metals by PNNL-AGG-ICP-AES/Water Extract

\begin{tabular}{|c|c|c|c|c|c|c|c|}
\hline CAS \# & Analyte & Results & Units & EQL & Analyzed & Batch & Method \\
\hline HEIS No. & B235R9 & \multicolumn{3}{|c|}{ Lab ID: $1001004-C C$} & & & \\
\hline $7439-95-4$ & Magnesium & $5.51 \mathrm{E} 0$ & ug/g dry & 4.89E-2 & $3 / 11 / 10$ & 0C11001 & PNNL-AGG-ICP-AES \\
\hline $7439-96-5$ & Manganese & $<6.24 \mathrm{E}-2$ & ug/g dry & $6.24 \mathrm{E}-2$ & $3 / 11 / 10$ & 0C11001 & PNNL-AGG-ICP-AES \\
\hline $7440-02-0$ & Nickel & $<9.67 \mathrm{E}-2$ & $u g / g$ dry & $9.67 \mathrm{E}-2$ & $3 / 11 / 10$ & 0C11001 & PNNL-AGG-ICP-AES \\
\hline $7439-92-1$ & Lead & $<1.61 \mathrm{E}-1$ & ug/g dry & $1.61 \mathrm{E}-1$ & $3 / 11 / 10$ & 0C11001 & PNNL-AGG-ICP-AES \\
\hline $7782-49-2$ & Selenium & $<1.20 \mathrm{E} 0$ & ug/g dry & $1.20 \mathrm{E} 0$ & $3 / 11 / 10$ & 0C11001 & PNNL-AGG-ICP-AES \\
\hline $7440-28-0$ & Thallium & $<9.88 \mathrm{E}-1$ & ug/g dry & $9.88 \mathrm{E}-1$ & $3 / 11 / 10$ & 0C11001 & PNNL-AGG-ICP-AES \\
\hline $7440-23-5$ & Sodium & $1.27 \mathrm{E} 1$ & ug/g dry & 7.97E-1 & $3 / 11 / 10$ & 0C11001 & PNNL-AGG-ICP-AES \\
\hline $7440-22-4$ & Silver & $<5.68 \mathrm{E}-2$ & ug/g dry & $5.68 \mathrm{E}-2$ & $3 / 11 / 10$ & 0C11001 & PNNL-AGG-ICP-AES \\
\hline HEIS No. & B235T8 & \multicolumn{3}{|c|}{ Lab ID: 1001004-DA } & & & \\
\hline $7429-90-5$ & Aluminum & $1.79 \mathrm{E}-1$ & ug/g dry & $9.36 \mathrm{E}-2$ & $3 / 11 / 10$ & 0C11001 & PNNL-AGG-ICP-AES \\
\hline $7440-38-2$ & Arsenic & $<3.11 \mathrm{E}-1$ & ug/g dry & $3.11 \mathrm{E}-1$ & $3 / 11 / 10$ & 0C11001 & PNNL-AGG-ICP-AES \\
\hline $7440-70-2$ & Calcium & $1.55 \mathrm{E} 1$ & ug/g dry & $4.52 \mathrm{E}-1$ & $3 / 11 / 10$ & 0C11001 & PNNL-AGG-ICP-AES \\
\hline $7440-43-9$ & Cadmium & $<1.04 \mathrm{E}-1$ & $\mathrm{ug} / \mathrm{g}$ dry & $1.04 \mathrm{E}-1$ & $3 / 11 / 10$ & 0C11001 & PNNL-AGG-ICP-AES \\
\hline $7440-47-3$ & Chromium & $<2.83 \mathrm{E}-2$ & ug/g dry & $2.83 \mathrm{E}-2$ & $3 / 11 / 10$ & 0C11001 & PNNL-AGG-ICP-AES \\
\hline $7440-50-8$ & Copper & $<8.77 \mathrm{E}-2$ & ug/g dry & $8.77 \mathrm{E}-2$ & $3 / 11 / 10$ & 0C11001 & PNNL-AGG-ICP-AES \\
\hline $7439-89-6$ & Iron & $<1.05 \mathrm{E}-1$ & ug/g dry & $1.05 \mathrm{E}-1$ & $3 / 11 / 10$ & 0C11001 & PNNL-AGG-ICP-AES \\
\hline $7440-09-7$ & Potassium & $6.77 \mathrm{E} 0$ & ug/g dry & $1.29 \mathrm{E} 0$ & $3 / 11 / 10$ & 0C11001 & PNNL-AGG-ICP-AES \\
\hline $7439-95-4$ & Magnesium & 4.70E0 & ug/g dry & $4.88 \mathrm{E}-2$ & $3 / 11 / 10$ & 0C11001 & PNNL-AGG-ICP-AES \\
\hline $7439-96-5$ & Manganese & $<6.24 \mathrm{E}-2$ & ug/g dry & $6.24 \mathrm{E}-2$ & $3 / 11 / 10$ & 0C11001 & PNNL-AGG-ICP-AES \\
\hline $7440-02-0$ & Nickel & $<9.66 \mathrm{E}-2$ & ug/g dry & $9.66 \mathrm{E}-2$ & $3 / 11 / 10$ & 0C11001 & PNNL-AGG-ICP-AES \\
\hline $7439-92-1$ & Lead & $<1.61 \mathrm{E}-1$ & ug/g dry & $1.61 \mathrm{E}-1$ & $3 / 11 / 10$ & 0C11001 & PNNL-AGG-ICP-AES \\
\hline $7782-49-2$ & Selenium & $<1.20 \mathrm{E} 0$ & ug/g dry & $1.20 \mathrm{E} 0$ & $3 / 11 / 10$ & 0C11001 & PNNL-AGG-ICP-AES \\
\hline $7440-28-0$ & Thallium & $<9.86 \mathrm{E}-1$ & ug/g dry & $9.86 \mathrm{E}-1$ & $3 / 11 / 10$ & 0C11001 & PNNL-AGG-ICP-AES \\
\hline $7440-23-5$ & Sodium & $1.92 \mathrm{E} 1$ & ug/g dry & 7.96E-1 & $3 / 11 / 10$ & 0C11001 & PNNL-AGG-ICP-AES \\
\hline $7440-22-4$ & Silver & $<5.67 \mathrm{E}-2$ & ug/g dry & $5.67 \mathrm{E}-2$ & $3 / 11 / 10$ & 0C11001 & PNNL-AGG-ICP-AES \\
\hline
\end{tabular}


Total Metals by PNNL-AGG-ICP-AES/Acid Extract

\begin{tabular}{|c|c|c|c|c|c|c|c|}
\hline CAS \# & Analyte & Results & Units & EQL & Analyzed & Batch & Method \\
\hline HEIS No. & B235H1 & \multicolumn{3}{|c|}{ Lab ID: $\quad$ 1001004-04 } & & & \\
\hline $7429-90-5$ & Aluminum & $5.51 \mathrm{E} 3$ & ug/g dry & $2.43 \mathrm{E} 1$ & $3 / 11 / 10$ & 0C11002 & PNNL-AGG-ICP-AES \\
\hline $7440-70-2$ & Calcium & 7.34E3 & ug/g dry & $2.52 \mathrm{E} 1$ & $3 / 11 / 10$ & 0C11002 & PNNL-AGG-ICP-AES \\
\hline $7440-43-9$ & Cadmium & $<1.32 \mathrm{E} 0$ & ug/g dry & $1.32 \mathrm{E} 0$ & $3 / 11 / 10$ & 0C11002 & PNNL-AGG-ICP-AES \\
\hline $7440-47-3$ & Chromium & $8.21 \mathrm{E} 0$ & ug/g dry & $2.12 \mathrm{E} 0$ & $3 / 11 / 10$ & 0C11002 & PNNL-AGG-ICP-AES \\
\hline $7440-50-8$ & Copper & $<6.95 \mathrm{E} 0$ & ug/g dry & $6.95 \mathrm{E} 0$ & $3 / 11 / 10$ & 0C11002 & PNNL-AGG-ICP-AES \\
\hline $7439-89-6$ & Iron & $1.07 \mathrm{E} 4$ & ug/g dry & $6.53 \mathrm{E} 0$ & $3 / 11 / 10$ & 0C11002 & PNNL-AGG-ICP-AES \\
\hline $7440-09-7$ & Potassium & $1.36 \mathrm{E} 3$ & ug/g dry & $9.30 \mathrm{E} 1$ & $3 / 11 / 10$ & 0C11002 & PNNL-AGG-ICP-AES \\
\hline $7439-95-4$ & Magnesium & $3.94 \mathrm{E} 3$ & ug/g dry & $6.92 \mathrm{E} 0$ & $3 / 11 / 10$ & 0C11002 & PNNL-AGG-ICP-AES \\
\hline $7439-96-5$ & Manganese & $2.09 \mathrm{E} 2$ & ug/g dry & $2.17 \mathrm{E} 0$ & $3 / 11 / 10$ & 0C11002 & PNNL-AGG-ICP-AES \\
\hline $7440-02-0$ & Nickel & $8.66 \mathrm{E} 0$ & ug/g dry & $5.04 \mathrm{E} 0$ & $3 / 11 / 10$ & 0C11002 & PNNL-AGG-ICP-AES \\
\hline $7439-92-1$ & Lead & $<1.09 \mathrm{E} 1$ & ug/g dry & $1.09 \mathrm{E} 1$ & $3 / 11 / 10$ & 0C11002 & PNNL-AGG-ICP-AES \\
\hline $7782-49-2$ & Selenium & $<6.16 \mathrm{E} 1$ & ug/g dry & $6.16 \mathrm{E} 1$ & $3 / 11 / 10$ & 0C11002 & PNNL-AGG-ICP-AES \\
\hline $7440-28-0$ & Thallium & $<3.48 \mathrm{E} 1$ & ug/g dry & $3.48 \mathrm{E} 1$ & $3 / 11 / 10$ & 0C11002 & PNNL-AGG-ICP-AES \\
\hline $7440-23-5$ & Sodium & $1.12 \mathrm{E} 2$ & ug/g dry & $6.84 \mathrm{E} 1$ & $3 / 11 / 10$ & 0C11002 & PNNL-AGG-ICP-AES \\
\hline HEIS No. & B235J0 & \multicolumn{3}{|c|}{ Lab ID: $\quad 1001004-22$} & & & \\
\hline $7429-90-5$ & Aluminum & 4.73E3 & ug/g dry & $2.38 \mathrm{E} 1$ & $3 / 11 / 10$ & 0C11002 & PNNL-AGG-ICP-AES \\
\hline $7440-70-2$ & Calcium & 4.37E3 & $\mathrm{ug} / \mathrm{g}$ dry & $2.47 \mathrm{E} 1$ & $3 / 11 / 10$ & $0 \mathrm{C} 11002$ & PNNL-AGG-ICP-AES \\
\hline $7440-43-9$ & Cadmium & $<1.30 \mathrm{E} 0$ & $\mathrm{ug} / \mathrm{g}$ dry & $1.30 \mathrm{E} 0$ & $3 / 11 / 10$ & 0C11002 & PNNL-AGG-ICP-AES \\
\hline $7440-47-3$ & Chromium & 7.89E0 & ug/g dry & $2.08 \mathrm{E} 0$ & $3 / 11 / 10$ & 0C11002 & PNNL-AGG-ICP-AES \\
\hline $7440-50-8$ & Copper & $<6.81 \mathrm{E} 0$ & ug/g dry & $6.81 \mathrm{E} 0$ & $3 / 11 / 10$ & 0C11002 & PNNL-AGG-ICP-AES \\
\hline $7439-89-6$ & Iron & $1.05 \mathrm{E} 4$ & ug/g dry & $6.40 \mathrm{E} 0$ & $3 / 11 / 10$ & 0C11002 & PNNL-AGG-ICP-AES \\
\hline $7440-09-7$ & Potassium & $9.00 \mathrm{E} 2$ & $\mathrm{ug} / \mathrm{g}$ dry & $9.11 \mathrm{E} 1$ & $3 / 11 / 10$ & 0C11002 & PNNL-AGG-ICP-AES \\
\hline $7439-95-4$ & Magnesium & $3.32 \mathrm{E} 3$ & ug/g dry & $6.78 \mathrm{E} 0$ & $3 / 11 / 10$ & 0C11002 & PNNL-AGG-ICP-AES \\
\hline $7439-96-5$ & Manganese & $1.98 \mathrm{E} 2$ & ug/g dry & $2.13 \mathrm{E} 0$ & $3 / 11 / 10$ & 0C11002 & PNNL-AGG-ICP-AES \\
\hline $7440-02-0$ & Nickel & $8.52 \mathrm{E} 0$ & ug/g dry & $4.94 \mathrm{E} 0$ & $3 / 11 / 10$ & 0C11002 & PNNL-AGG-ICP-AES \\
\hline $7439-92-1$ & Lead & $<1.06 \mathrm{E} 1$ & ug/g dry & $1.06 \mathrm{E} 1$ & $3 / 11 / 10$ & 0C11002 & PNNL-AGG-ICP-AES \\
\hline $7782-49-2$ & Selenium & $<6.04 \mathrm{E} 1$ & $\mathrm{ug} / \mathrm{g}$ dry & $6.04 \mathrm{E} 1$ & $3 / 11 / 10$ & $0 \mathrm{C} 11002$ & PNNL-AGG-ICP-AES \\
\hline $7440-28-0$ & Thallium & $<3.42 \mathrm{E} 1$ & ug/g dry & $3.42 \mathrm{E} 1$ & $3 / 11 / 10$ & 0C11002 & PNNL-AGG-ICP-AES \\
\hline $7440-23-5$ & Sodium & $9.78 \mathrm{E} 1$ & ug/g dry & $6.71 \mathrm{E} 1$ & $3 / 11 / 10$ & 0C11002 & PNNL-AGG-ICP-AES \\
\hline HEIS No. & B235J6 & \multicolumn{3}{|c|}{ Lab ID: $\quad$ 1001004-34 } & & & \\
\hline $7429-90-5$ & Aluminum & $4.58 \mathrm{E} 3$ & ug/g dry & $2.39 \mathrm{E} 1$ & $3 / 11 / 10$ & 0C11002 & PNNL-AGG-ICP-AES \\
\hline $7440-70-2$ & Calcium & $3.88 \mathrm{E} 3$ & ug/g dry & $2.48 \mathrm{E} 1$ & $3 / 11 / 10$ & 0C11002 & PNNL-AGG-ICP-AES \\
\hline $7440-43-9$ & Cadmium & $<1.30 \mathrm{E} 0$ & ug/g dry & $1.30 \mathrm{E} 0$ & $3 / 11 / 10$ & 0C11002 & PNNL-AGG-ICP-AES \\
\hline $7440-47-3$ & Chromium & $8.10 \mathrm{E} 0$ & ug/g dry & $2.08 \mathrm{E} 0$ & $3 / 11 / 10$ & 0C11002 & PNNL-AGG-ICP-AES \\
\hline $7440-50-8$ & Copper & $<6.84 \mathrm{E} 0$ & $\mathrm{ug} / \mathrm{g}$ dry & $6.84 \mathrm{E} 0$ & $3 / 11 / 10$ & 0C11002 & PNNL-AGG-ICP-AES \\
\hline $7439-89-6$ & Iron & $9.52 \mathrm{E} 3$ & ug/g dry & $6.43 \mathrm{E} 0$ & $3 / 11 / 10$ & 0C11002 & PNNL-AGG-ICP-AES \\
\hline $7440-09-7$ & Potassium & $9.48 \mathrm{E} 2$ & ug/g dry & $9.15 \mathrm{E} 1$ & $3 / 11 / 10$ & $0 \mathrm{C} 11002$ & PNNL-AGG-ICP-AES \\
\hline $7439-95-4$ & Magnesium & $3.18 \mathrm{E} 3$ & ug/g dry & $6.81 \mathrm{E} 0$ & $3 / 11 / 10$ & 0C11002 & PNNL-AGG-ICP-AES \\
\hline $7439-96-5$ & Manganese & $1.88 \mathrm{E} 2$ & $\mathrm{ug} / \mathrm{g}$ dry & $2.14 \mathrm{E} 0$ & $3 / 11 / 10$ & 0C11002 & PNNL-AGG-ICP-AES \\
\hline $7440-02-0$ & Nickel & $8.40 \mathrm{E} 0$ & $\mathrm{ug} / \mathrm{g}$ dry & 4.96E0 & $3 / 11 / 10$ & 0C11002 & PNNL-AGG-ICP-AES \\
\hline $7439-92-1$ & Lead & $<1.07 \mathrm{E} 1$ & ug/g dry & $1.07 \mathrm{E} 1$ & $3 / 11 / 10$ & 0C11002 & PNNL-AGG-ICP-AES \\
\hline $7782-49-2$ & Selenium & $<6.07 \mathrm{E} 1$ & ug/g dry & $6.07 \mathrm{E} 1$ & $3 / 11 / 10$ & 0C11002 & PNNL-AGG-ICP-AES \\
\hline $7440-28-0$ & Thallium & $<3.43 \mathrm{E} 1$ & $\mathrm{ug} / \mathrm{g}$ dry & $3.43 \mathrm{E} 1$ & $3 / 11 / 10$ & 0C11002 & PNNL-AGG-ICP-AES \\
\hline $7440-23-5$ & Sodium & $9.12 \mathrm{E} 1$ & ug/g dry & $6.73 \mathrm{E} 1$ & $3 / 11 / 10$ & 0C11002 & PNNL-AGG-ICP-AES \\
\hline HEIS No. & B235K1 & \multicolumn{3}{|c|}{ Lab ID: $\quad 1001004-42$} & & & \\
\hline $7429-90-5$ & Aluminum & $3.87 \mathrm{E} 3$ & ug/g dry & $2.48 \mathrm{E} 1$ & $3 / 11 / 10$ & 0C11002 & PNNL-AGG-ICP-AES \\
\hline $7440-70-2$ & Calcium & $4.59 \mathrm{E} 3$ & ug/g dry & $2.58 \mathrm{E} 1$ & $3 / 11 / 10$ & 0C11002 & PNNL-AGG-ICP-AES \\
\hline $7440-43-9$ & Cadmium & $<1.35 \mathrm{E} 0$ & ug/g dry & $1.35 \mathrm{E} 0$ & $3 / 11 / 10$ & 0C11002 & PNNL-AGG-ICP-AES \\
\hline
\end{tabular}


Total Metals by PNNL-AGG-ICP-AES/Acid Extract

\begin{tabular}{|c|c|c|c|c|c|c|c|}
\hline CAS \# & Analyte & Results & Units & EQL & Analyzed & Batch & Method \\
\hline HEIS No. & B235K1 & \multicolumn{3}{|c|}{ Lab ID: $\quad 1001004-42$} & & & \\
\hline $7440-47-3$ & Chromium & $8.70 \mathrm{E} 0$ & ug/g dry & $2.16 \mathrm{E} 0$ & $3 / 11 / 10$ & $0 \mathrm{C} 11002$ & PNNL-AGG-ICP-AES \\
\hline $7440-50-8$ & Copper & $<7.09 \mathrm{E} 0$ & $\mathrm{ug} / \mathrm{g}$ dry & 7.09E0 & $3 / 11 / 10$ & 0C11002 & PNNL-AGG-ICP-AES \\
\hline $7439-89-6$ & Iron & $9.15 \mathrm{E} 3$ & ug/g dry & $6.67 \mathrm{E} 0$ & $3 / 11 / 10$ & 0C11002 & PNNL-AGG-ICP-AES \\
\hline $7440-09-7$ & Potassium & $9.35 \mathrm{E} 2$ & ug/g dry & $9.49 \mathrm{E} 1$ & $3 / 11 / 10$ & 0C11002 & PNNL-AGG-ICP-AES \\
\hline $7439-95-4$ & Magnesium & 3.37E3 & ug/g dry & $7.06 \mathrm{E} 0$ & $3 / 11 / 10$ & 0C11002 & PNNL-AGG-ICP-AES \\
\hline $7439-96-5$ & Manganese & $1.77 \mathrm{E} 2$ & $\mathrm{ug} / \mathrm{g}$ dry & $2.22 \mathrm{E} 0$ & $3 / 11 / 10$ & 0C11002 & PNNL-AGG-ICP-AES \\
\hline $7440-02-0$ & Nickel & $9.21 \mathrm{E} 0$ & ug/g dry & $5.14 \mathrm{E} 0$ & $3 / 11 / 10$ & 0C11002 & PNNL-AGG-ICP-AES \\
\hline $7439-92-1$ & Lead & $<1.11 \mathrm{E} 1$ & $\mathrm{ug} / \mathrm{g}$ dry & $1.11 \mathrm{E} 1$ & $3 / 11 / 10$ & 0C11002 & PNNL-AGG-ICP-AES \\
\hline $7782-49-2$ & Selenium & $<6.29 \mathrm{E} 1$ & ug/g dry & $6.29 \mathrm{E} 1$ & $3 / 11 / 10$ & 0C11002 & PNNL-AGG-ICP-AES \\
\hline $7440-28-0$ & Thallium & $<3.56 \mathrm{E} 1$ & $\mathrm{ug} / \mathrm{g}$ dry & $3.56 \mathrm{E} 1$ & $3 / 11 / 10$ & 0C11002 & PNNL-AGG-ICP-AES \\
\hline $7440-23-5$ & Sodium & $9.80 \mathrm{E} 1$ & ug/g dry & $6.99 \mathrm{E} 1$ & $3 / 11 / 10$ & 0C11002 & PNNL-AGG-ICP-AES \\
\hline HEIS No. & B235K5 & \multicolumn{3}{|c|}{ Lab ID: $\quad 1001004-46$} & & & \\
\hline $7429-90-5$ & Aluminum & $5.29 \mathrm{E} 3$ & ug/g dry & $2.43 \mathrm{E} 1$ & $3 / 11 / 10$ & 0C11002 & PNNL-AGG-ICP-AES \\
\hline $7440-70-2$ & Calcium & $7.00 \mathrm{E} 3$ & ug/g dry & $2.52 \mathrm{E} 1$ & $3 / 11 / 10$ & 0C11002 & PNNL-AGG-ICP-AES \\
\hline $7440-43-9$ & Cadmium & $<1.32 \mathrm{E} 0$ & ug/g dry & $1.32 \mathrm{E} 0$ & $3 / 11 / 10$ & 0C11002 & PNNL-AGG-ICP-AES \\
\hline $7440-47-3$ & Chromium & $7.66 \mathrm{E} 0$ & ug/g dry & $2.12 \mathrm{E} 0$ & $3 / 11 / 10$ & 0C11002 & PNNL-AGG-ICP-AES \\
\hline $7440-50-8$ & Copper & $<6.94 \mathrm{E} 0$ & ug/g dry & $6.94 \mathrm{E} 0$ & $3 / 11 / 10$ & 0C11002 & PNNL-AGG-ICP-AES \\
\hline $7439-89-6$ & Iron & $1.09 \mathrm{E} 4$ & ug/g dry & $6.53 \mathrm{E} 0$ & $3 / 11 / 10$ & 0C11002 & PNNL-AGG-ICP-AES \\
\hline $7440-09-7$ & Potassium & $1.17 \mathrm{E} 3$ & ug/g dry & $9.29 \mathrm{E} 1$ & $3 / 11 / 10$ & 0C11002 & PNNL-AGG-ICP-AES \\
\hline $7439-95-4$ & Magnesium & $3.54 \mathrm{E} 3$ & ug/g dry & $6.91 \mathrm{E} 0$ & $3 / 11 / 10$ & 0C11002 & PNNL-AGG-ICP-AES \\
\hline $7439-96-5$ & Manganese & $2.10 \mathrm{E} 2$ & ug/g dry & $2.17 \mathrm{E} 0$ & $3 / 11 / 10$ & 0C11002 & PNNL-AGG-ICP-AES \\
\hline $7440-02-0$ & Nickel & $7.54 \mathrm{E} 0$ & $\mathrm{ug} / \mathrm{g}$ dry & $5.03 \mathrm{E} 0$ & $3 / 11 / 10$ & 0C11002 & PNNL-AGG-ICP-AES \\
\hline $7439-92-1$ & Lead & $<1.09 \mathrm{E} 1$ & ug/g dry & $1.09 \mathrm{E} 1$ & $3 / 11 / 10$ & 0C11002 & PNNL-AGG-ICP-AES \\
\hline $7782-49-2$ & Selenium & $<6.16 \mathrm{E} 1$ & $\mathrm{ug} / \mathrm{g}$ dry & $6.16 \mathrm{E} 1$ & $3 / 11 / 10$ & $0 \mathrm{C} 11002$ & PNNL-AGG-ICP-AES \\
\hline $7440-28-0$ & Thallium & $<3.48 \mathrm{E} 1$ & ug/g dry & $3.48 \mathrm{E} 1$ & $3 / 11 / 10$ & 0C11002 & PNNL-AGG-ICP-AES \\
\hline $7440-23-5$ & Sodium & $1.08 \mathrm{E} 2$ & ug/g dry & $6.84 \mathrm{E} 1$ & $3 / 11 / 10$ & 0C11002 & PNNL-AGG-ICP-AES \\
\hline HEIS No. & B235L9 & \multicolumn{3}{|c|}{ Lab ID: $\quad 1001004-98$} & & & \\
\hline $7429-90-5$ & Aluminum & $4.43 \mathrm{E} 3$ & ug/g dry & $2.40 \mathrm{E} 1$ & $3 / 11 / 10$ & 0C11002 & PNNL-AGG-ICP-AES \\
\hline $7440-70-2$ & Calcium & $7.13 \mathrm{E} 3$ & ug/g dry & $2.50 \mathrm{E} 1$ & $3 / 11 / 10$ & $0 \mathrm{C} 11002$ & PNNL-AGG-ICP-AES \\
\hline $7440-43-9$ & Cadmium & $<1.31 \mathrm{E} 0$ & $\mathrm{ug} / \mathrm{g}$ dry & $1.31 \mathrm{E} 0$ & $3 / 11 / 10$ & 0C11002 & PNNL-AGG-ICP-AES \\
\hline $7440-47-3$ & Chromium & $8.06 \mathrm{E} 0$ & ug/g dry & $2.10 \mathrm{E} 0$ & $3 / 11 / 10$ & 0C11002 & PNNL-AGG-ICP-AES \\
\hline $7440-50-8$ & Copper & $<6.87 \mathrm{E} 0$ & $\mathrm{ug} / \mathrm{g}$ dry & $6.87 \mathrm{E} 0$ & $3 / 11 / 10$ & 0C11002 & PNNL-AGG-ICP-AES \\
\hline $7439-89-6$ & Iron & $8.64 \mathrm{E} 3$ & ug/g dry & $6.46 \mathrm{E} 0$ & $3 / 11 / 10$ & 0C11002 & PNNL-AGG-ICP-AES \\
\hline $7440-09-7$ & Potassium & $8.81 \mathrm{E} 2$ & ug/g dry & $9.20 \mathrm{E} 1$ & $3 / 11 / 10$ & 0C11002 & PNNL-AGG-ICP-AES \\
\hline $7439-95-4$ & Magnesium & $3.32 \mathrm{E} 3$ & ug/g dry & $6.85 \mathrm{E} 0$ & $3 / 11 / 10$ & 0C11002 & PNNL-AGG-ICP-AES \\
\hline $7439-96-5$ & Manganese & $1.83 \mathrm{E} 2$ & ug/g dry & $2.15 \mathrm{E} 0$ & $3 / 11 / 10$ & 0C11002 & PNNL-AGG-ICP-AES \\
\hline $7440-02-0$ & Nickel & $9.19 \mathrm{E} 0$ & ug/g dry & 4.98E0 & $3 / 11 / 10$ & 0C11002 & PNNL-AGG-ICP-AES \\
\hline $7439-92-1$ & Lead & $<1.08 \mathrm{E} 1$ & $\mathrm{ug} / \mathrm{g}$ dry & $1.08 \mathrm{E} 1$ & $3 / 11 / 10$ & 0C11002 & PNNL-AGG-ICP-AES \\
\hline $7782-49-2$ & Selenium & $<6.10 \mathrm{E} 1$ & $\mathrm{ug} / \mathrm{g}$ dry & $6.10 \mathrm{E} 1$ & $3 / 11 / 10$ & 0C11002 & PNNL-AGG-ICP-AES \\
\hline $7440-28-0$ & Thallium & $<3.45 \mathrm{E} 1$ & ug/g dry & $3.45 \mathrm{E} 1$ & $3 / 11 / 10$ & 0C11002 & PNNL-AGG-ICP-AES \\
\hline $7440-23-5$ & Sodium & $8.61 \mathrm{E} 1$ & ug/g dry & $6.77 \mathrm{E} 1$ & $3 / 11 / 10$ & 0C11002 & PNNL-AGG-ICP-AES \\
\hline HEIS No. & B235N9 & \multicolumn{3}{|c|}{ Lab ID: $\quad$ 1001004-BC } & & & \\
\hline $7429-90-5$ & Aluminum & 4.14E3 & ug/g dry & $2.35 \mathrm{E} 1$ & $3 / 11 / 10$ & $0 \mathrm{C} 11002$ & PNNL-AGG-ICP-AES \\
\hline $7440-70-2$ & Calcium & $5.38 \mathrm{E} 3$ & $\mathrm{ug} / \mathrm{g}$ dry & $2.44 \mathrm{E} 1$ & $3 / 11 / 10$ & 0C11002 & PNNL-AGG-ICP-AES \\
\hline $7440-43-9$ & Cadmium & $<1.28 \mathrm{E} 0$ & ug/g dry & $1.28 \mathrm{E} 0$ & $3 / 11 / 10$ & 0C11002 & PNNL-AGG-ICP-AES \\
\hline $7440-47-3$ & Chromium & $5.71 \mathrm{E} 0$ & $\mathrm{ug} / \mathrm{g}$ dry & $2.05 \mathrm{E} 0$ & $3 / 11 / 10$ & 0C11002 & PNNL-AGG-ICP-AES \\
\hline $7440-50-8$ & Copper & $<6.73 \mathrm{E} 0$ & ug/g dry & $6.73 \mathrm{E} 0$ & $3 / 11 / 10$ & 0C11002 & PNNL-AGG-ICP-AES \\
\hline $7439-89-6$ & Iron & $1.00 \mathrm{E} 4$ & $\mathrm{ug} / \mathrm{g}$ dry & $6.33 \mathrm{E} 0$ & $3 / 11 / 10$ & $0 \mathrm{C} 11002$ & PNNL-AGG-ICP-AES \\
\hline
\end{tabular}


Total Metals by PNNL-AGG-ICP-AES/Acid Extract

\begin{tabular}{|c|c|c|c|c|c|c|c|}
\hline CAS \# & Analyte & Results & Units & EQL & Analyzed & Batch & Method \\
\hline HEIS No. & B235N9 & \multicolumn{3}{|c|}{ Lab ID: $\quad$ 1001004-BC } & & & \\
\hline $7440-09-7$ & Potassium & $7.31 \mathrm{E} 2$ & ug/g dry & $9.01 \mathrm{E} 1$ & $3 / 11 / 10$ & 0C11002 & PNNL-AGG-ICP-AES \\
\hline $7439-95-4$ & Magnesium & $2.82 \mathrm{E} 3$ & $\mathrm{ug} / \mathrm{g}$ dry & $6.70 \mathrm{E} 0$ & $3 / 11 / 10$ & 0C11002 & PNNL-AGG-ICP-AES \\
\hline $7439-96-5$ & Manganese & $1.73 \mathrm{E} 2$ & ug/g dry & $2.10 \mathrm{E} 0$ & $3 / 11 / 10$ & 0C11002 & PNNL-AGG-ICP-AES \\
\hline $7440-02-0$ & Nickel & $6.78 \mathrm{E} 0$ & ug/g dry & 4.88E0 & $3 / 11 / 10$ & 0C11002 & PNNL-AGG-ICP-AES \\
\hline $7439-92-1$ & Lead & $<1.05 \mathrm{E} 1$ & ug/g dry & $1.05 \mathrm{E} 1$ & $3 / 11 / 10$ & 0C11002 & PNNL-AGG-ICP-AES \\
\hline $7782-49-2$ & Selenium & $<5.97 \mathrm{E} 1$ & $\mathrm{ug} / \mathrm{g}$ dry & $5.97 \mathrm{E} 1$ & $3 / 11 / 10$ & 0C11002 & PNNL-AGG-ICP-AES \\
\hline $7440-28-0$ & Thallium & $<3.38 \mathrm{E} 1$ & ug/g dry & $3.38 \mathrm{E} 1$ & $3 / 11 / 10$ & 0C11002 & PNNL-AGG-ICP-AES \\
\hline $7440-23-5$ & Sodium & $1.43 \mathrm{E} 2$ & $\mathrm{ug} / \mathrm{g}$ dry & $6.63 \mathrm{E} 1$ & $3 / 11 / 10$ & 0C11002 & PNNL-AGG-ICP-AES \\
\hline HEIS No. & B235R2 & \multicolumn{3}{|c|}{ Lab ID: $1001004-B V$} & & & \\
\hline $7429-90-5$ & Aluminum & $4.15 \mathrm{E} 3$ & ug/g dry & $2.34 \mathrm{E} 1$ & $3 / 11 / 10$ & 0C11002 & PNNL-AGG-ICP-AES \\
\hline $7440-70-2$ & Calcium & $6.13 \mathrm{E} 3$ & ug/g dry & $2.43 \mathrm{E} 1$ & $3 / 11 / 10$ & 0C11002 & PNNL-AGG-ICP-AES \\
\hline $7440-43-9$ & Cadmium & $<1.27 \mathrm{E} 0$ & ug/g dry & $1.27 \mathrm{E} 0$ & $3 / 11 / 10$ & 0C11002 & PNNL-AGG-ICP-AES \\
\hline $7440-47-3$ & Chromium & $6.22 \mathrm{E} 0$ & ug/g dry & $2.04 \mathrm{E} 0$ & $3 / 11 / 10$ & 0C11002 & PNNL-AGG-ICP-AES \\
\hline $7440-50-8$ & Copper & $<6.68 \mathrm{E} 0$ & ug/g dry & $6.68 \mathrm{E} 0$ & $3 / 11 / 10$ & 0C11002 & PNNL-AGG-ICP-AES \\
\hline $7439-89-6$ & Iron & $1.15 \mathrm{E} 4$ & ug/g dry & $6.28 \mathrm{E} 0$ & $3 / 11 / 10$ & 0C11002 & PNNL-AGG-ICP-AES \\
\hline $7440-09-7$ & Potassium & $6.68 \mathrm{E} 2$ & ug/g dry & $8.94 \mathrm{E} 1$ & $3 / 11 / 10$ & 0C11002 & PNNL-AGG-ICP-AES \\
\hline $7439-95-4$ & Magnesium & $2.71 \mathrm{E} 3$ & ug/g dry & $6.65 \mathrm{E} 0$ & $3 / 11 / 10$ & 0C11002 & PNNL-AGG-ICP-AES \\
\hline $7439-96-5$ & Manganese & $1.72 \mathrm{E} 2$ & ug/g dry & 2.09E0 & $3 / 11 / 10$ & 0C11002 & PNNL-AGG-ICP-AES \\
\hline $7440-02-0$ & Nickel & $6.22 \mathrm{E} 0$ & ug/g dry & $4.84 \mathrm{E} 0$ & $3 / 11 / 10$ & 0C11002 & PNNL-AGG-ICP-AES \\
\hline $7439-92-1$ & Lead & $<1.04 \mathrm{E} 1$ & ug/g dry & $1.04 \mathrm{E} 1$ & $3 / 11 / 10$ & 0C11002 & PNNL-AGG-ICP-AES \\
\hline $7782-49-2$ & Selenium & $<5.93 \mathrm{E} 1$ & ug/g dry & $5.93 \mathrm{E} 1$ & $3 / 11 / 10$ & 0C11002 & PNNL-AGG-ICP-AES \\
\hline $7440-28-0$ & Thallium & $<3.35 \mathrm{E} 1$ & $\mathrm{ug} / \mathrm{g}$ dry & $3.35 \mathrm{E} 1$ & $3 / 11 / 10$ & 0C11002 & PNNL-AGG-ICP-AES \\
\hline $7440-23-5$ & Sodium & $2.35 \mathrm{E} 2$ & ug/g dry & $6.58 \mathrm{E} 1$ & $3 / 11 / 10$ & 0C11002 & PNNL-AGG-ICP-AES \\
\hline HEIS No. & B235R9 & \multicolumn{3}{|c|}{ Lab ID: $1001004-C C$} & & & \\
\hline $7429-90-5$ & Aluminum & $4.53 \mathrm{E} 3$ & ug/g dry & $2.39 \mathrm{E} 1$ & $3 / 11 / 10$ & 0C11002 & PNNL-AGG-ICP-AES \\
\hline $7440-70-2$ & Calcium & $4.49 \mathrm{E} 3$ & ug/g dry & $2.48 \mathrm{E} 1$ & $3 / 11 / 10$ & 0C11002 & PNNL-AGG-ICP-AES \\
\hline $7440-43-9$ & Cadmium & $<1.30 \mathrm{E} 0$ & ug/g dry & $1.30 \mathrm{E} 0$ & $3 / 11 / 10$ & 0C11002 & PNNL-AGG-ICP-AES \\
\hline $7440-47-3$ & Chromium & $1.54 \mathrm{E} 1$ & ug/g dry & $2.08 \mathrm{E} 0$ & $3 / 11 / 10$ & 0C11002 & PNNL-AGG-ICP-AES \\
\hline $7440-50-8$ & Copper & $<6.83 \mathrm{E} 0$ & ug/g dry & $6.83 \mathrm{E} 0$ & $3 / 11 / 10$ & $0 \mathrm{C} 11002$ & PNNL-AGG-ICP-AES \\
\hline $7439-89-6$ & Iron & $1.67 \mathrm{E} 4$ & $\mathrm{ug} / \mathrm{g}$ dry & $6.43 \mathrm{E} 0$ & $3 / 11 / 10$ & 0C11002 & PNNL-AGG-ICP-AES \\
\hline $7440-09-7$ & Potassium & 6.92E2 & ug/g dry & $9.15 \mathrm{E} 1$ & $3 / 11 / 10$ & 0C11002 & PNNL-AGG-ICP-AES \\
\hline $7439-95-4$ & Magnesium & 2.91E3 & $\mathrm{ug} / \mathrm{g}$ dry & $6.81 \mathrm{E} 0$ & $3 / 11 / 10$ & 0C11002 & PNNL-AGG-ICP-AES \\
\hline $7439-96-5$ & Manganese & $2.53 \mathrm{E} 2$ & ug/g dry & $2.14 \mathrm{E} 0$ & $3 / 11 / 10$ & 0C11002 & PNNL-AGG-ICP-AES \\
\hline $7440-02-0$ & Nickel & $6.75 \mathrm{E} 0$ & $\mathrm{ug} / \mathrm{g}$ dry & 4.96E0 & $3 / 11 / 10$ & 0C11002 & PNNL-AGG-ICP-AES \\
\hline $7439-92-1$ & Lead & $<1.07 \mathrm{E} 1$ & ug/g dry & $1.07 \mathrm{E} 1$ & $3 / 11 / 10$ & 0C11002 & PNNL-AGG-ICP-AES \\
\hline $7782-49-2$ & Selenium & $<6.06 \mathrm{E} 1$ & $\mathrm{ug} / \mathrm{g}$ dry & $6.06 \mathrm{E} 1$ & $3 / 11 / 10$ & $0 \mathrm{C} 11002$ & PNNL-AGG-ICP-AES \\
\hline $7440-28-0$ & Thallium & $<3.43 \mathrm{E} 1$ & ug/g dry & $3.43 \mathrm{E} 1$ & $3 / 11 / 10$ & 0C11002 & PNNL-AGG-ICP-AES \\
\hline $7440-23-5$ & Sodium & $2.60 \mathrm{E} 2$ & $\mathrm{ug} / \mathrm{g}$ dry & $6.73 \mathrm{E} 1$ & $3 / 11 / 10$ & 0C11002 & PNNL-AGG-ICP-AES \\
\hline HEIS No. & B235T8 & \multicolumn{3}{|c|}{ Lab ID: 1001004-DA } & & & \\
\hline $7429-90-5$ & Aluminum & $4.37 \mathrm{E} 3$ & ug/g dry & $2.43 \mathrm{E} 1$ & $3 / 11 / 10$ & 0C11002 & PNNL-AGG-ICP-AES \\
\hline $7440-70-2$ & Calcium & $3.58 \mathrm{E} 3$ & ug/g dry & $2.52 \mathrm{E} 1$ & $3 / 11 / 10$ & 0C11002 & PNNL-AGG-ICP-AES \\
\hline $7440-43-9$ & Cadmium & $<1.32 \mathrm{E} 0$ & ug/g dry & $1.32 \mathrm{E} 0$ & $3 / 11 / 10$ & 0C11002 & PNNL-AGG-ICP-AES \\
\hline $7440-47-3$ & Chromium & $9.80 \mathrm{E} 0$ & ug/g dry & $2.12 \mathrm{E} 0$ & $3 / 11 / 10$ & 0C11002 & PNNL-AGG-ICP-AES \\
\hline $7440-50-8$ & Copper & 7.34E0 & $\mathrm{ug} / \mathrm{g}$ dry & $6.95 \mathrm{E} 0$ & $3 / 11 / 10$ & 0C11002 & PNNL-AGG-ICP-AES \\
\hline $7439-89-6$ & Iron & $1.06 \mathrm{E} 4$ & ug/g dry & $6.53 \mathrm{E} 0$ & $3 / 11 / 10$ & 0C11002 & PNNL-AGG-ICP-AES \\
\hline $7440-09-7$ & Potassium & $7.28 \mathrm{E} 2$ & $\mathrm{ug} / \mathrm{g}$ dry & $9.30 \mathrm{E} 1$ & $3 / 11 / 10$ & 0C11002 & PNNL-AGG-ICP-AES \\
\hline $7439-95-4$ & Magnesium & $2.76 \mathrm{E} 3$ & ug/g dry & $6.92 \mathrm{E} 0$ & $3 / 11 / 10$ & 0C11002 & PNNL-AGG-ICP-AES \\
\hline $7439-96-5$ & Manganese & $1.87 \mathrm{E} 2$ & $\mathrm{ug} / \mathrm{g}$ dry & $2.17 \mathrm{E} 0$ & $3 / 11 / 10$ & $0 \mathrm{C} 11002$ & PNNL-AGG-ICP-AES \\
\hline
\end{tabular}


Total Metals by PNNL-AGG-ICP-AES/Acid Extract

\begin{tabular}{|c|c|c|c|c|c|c|c|}
\hline CAS \# & Analyte & Results & Units & EQL & Analyzed & Batch & Method \\
\hline HEIS No. & B235T8 & \multicolumn{3}{|c|}{ Lab ID: $\quad$ 1001004-DA } & & & \\
\hline $7440-02-0$ & Nickel & $7.46 \mathrm{E} 0$ & ug/g dry & $5.04 \mathrm{E} 0$ & $3 / 11 / 10$ & 0C11002 & PNNL-AGG-ICP-AES \\
\hline 7439-92-1 & Lead & $<1.09 \mathrm{E} 1$ & ug/g dry & $1.09 \mathrm{E} 1$ & $3 / 11 / 10$ & 0C11002 & PNNL-AGG-ICP-AES \\
\hline $7782-49-2$ & Selenium & $<6.17 \mathrm{E} 1$ & ug/g dry & $6.17 \mathrm{E} 1$ & $3 / 11 / 10$ & 0C11002 & PNNL-AGG-ICP-AES \\
\hline $7440-28-0$ & Thallium & $<3.49 \mathrm{E} 1$ & ug/g dry & $3.49 \mathrm{E} 1$ & $3 / 11 / 10$ & $0 \mathrm{C} 11002$ & PNNL-AGG-ICP-AES \\
\hline $7440-23-5$ & Sodium & $2.46 \mathrm{E} 2$ & ug/g dry & $6.84 \mathrm{E} 1$ & $3 / 11 / 10$ & 0C11002 & PNNL-AGG-ICP-AES \\
\hline
\end{tabular}




\section{Radionuclides by ICP-MS/Acid Extract}

\begin{tabular}{|c|c|c|c|c|c|c|c|}
\hline CAS \# & Analyte & Results & Units & EQL & Analyzed & Batch & Method \\
\hline HEIS No. & B235H1 & \multicolumn{3}{|c|}{ Lab ID: $\quad$ 1001004-04 } & & & \\
\hline \multirow[t]{2}{*}{$14133-76-7$} & Technetium-99 & $<5.28 \mathrm{E}-3$ & ug/g dry & $5.28 \mathrm{E}-3$ & $4 / 04 / 10$ & 0C17004 & PNNL-AGG-415 \\
\hline & Uranium 238 & $3.28 \mathrm{E} 0$ & ug/g dry & $3.60 \mathrm{E}-2$ & $3 / 22 / 10$ & 0C17004 & PNNL-AGG-415 \\
\hline HEIS No. & B235J0 & \multicolumn{3}{|c|}{ Lab ID: $\quad 1001004-22$} & & & \\
\hline \multirow[t]{2}{*}{$14133-76-7$} & Technetium-99 & $<5.18 \mathrm{E}-3$ & ug/g dry & $5.18 \mathrm{E}-3$ & $4 / 04 / 10$ & 0C17004 & PNNL-AGG-415 \\
\hline & Uranium 238 & $5.20 \mathrm{E} 0$ & ug/g dry & $3.52 \mathrm{E}-2$ & $3 / 22 / 10$ & 0C17004 & PNNL-AGG-415 \\
\hline HEIS No. & B235J6 & \multicolumn{3}{|c|}{ Lab ID: $\quad$ 1001004-34 } & & & \\
\hline \multirow[t]{2}{*}{$14133-76-7$} & Technetium-99 & $<5.20 \mathrm{E}-3$ & ug/g dry & $5.20 \mathrm{E}-3$ & $4 / 04 / 10$ & 0C17004 & PNNL-AGG-415 \\
\hline & Uranium 238 & 8.84E0 & ug/g dry & $3.54 \mathrm{E}-2$ & $3 / 22 / 10$ & 0C17004 & PNNL-AGG-415 \\
\hline HEIS No. & B235K1 & \multicolumn{3}{|c|}{ Lab ID: $\quad 1001004-42$} & & & \\
\hline \multirow[t]{2}{*}{$14133-76-7$} & Technetium-99 & $<5.39 \mathrm{E}-3$ & ug/g dry & $5.39 \mathrm{E}-3$ & $4 / 04 / 10$ & 0C17004 & PNNL-AGG-415 \\
\hline & Uranium 238 & $1.55 \mathrm{E} 1$ & ug/g dry & $3.67 \mathrm{E}-2$ & $3 / 22 / 10$ & 0C17004 & PNNL-AGG-415 \\
\hline HEIS No. & B235K5 & \multicolumn{3}{|c|}{ Lab ID: $\quad$ 1001004-46 } & & & \\
\hline \multirow[t]{2}{*}{$14133-76-7$} & Technetium-99 & $<5.28 \mathrm{E}-3$ & ug/g dry & $5.28 \mathrm{E}-3$ & $4 / 04 / 10$ & 0C17004 & PNNL-AGG-415 \\
\hline & Uranium 238 & $5.07 \mathrm{E} 0$ & ug/g dry & $3.60 \mathrm{E}-2$ & $3 / 22 / 10$ & 0C17004 & PNNL-AGG-415 \\
\hline HEIS No. & B235L9 & \multicolumn{3}{|c|}{ Lab ID: $\quad$ 1001004-98 } & & & \\
\hline \multirow[t]{2}{*}{$14133-76-7$} & Technetium-99 & $<5.23 \mathrm{E}-3$ & ug/g dry & $5.23 \mathrm{E}-3$ & $4 / 04 / 10$ & 0C17004 & PNNL-AGG-415 \\
\hline & Uranium 238 & $2.43 \mathrm{E}-1$ & ug/g dry & $3.56 \mathrm{E}-2$ & $3 / 22 / 10$ & 0C17004 & PNNL-AGG-415 \\
\hline HEIS No. & B235N9 & \multicolumn{3}{|c|}{ Lab ID: $\quad$ 1001004-BC } & & & \\
\hline \multirow[t]{2}{*}{$14133-76-7$} & Technetium-99 & $<5.12 \mathrm{E}-3$ & ug/g dry & $5.12 \mathrm{E}-3$ & $4 / 04 / 10$ & 0C17004 & PNNL-AGG-415 \\
\hline & Uranium 238 & $2.03 \mathrm{E}-1$ & ug/g dry & $3.49 \mathrm{E}-2$ & $3 / 22 / 10$ & 0C17004 & PNNL-AGG-415 \\
\hline HEIS No. & B235R2 & \multicolumn{3}{|c|}{ Lab ID: 1001004-BV } & & & \\
\hline \multirow[t]{2}{*}{$14133-76-7$} & Technetium-99 & $<5.08 \mathrm{E}-3$ & ug/g dry & $5.08 \mathrm{E}-3$ & $4 / 04 / 10$ & 0C17004 & PNNL-AGG-415 \\
\hline & Uranium 238 & $2.43 \mathrm{E}-1$ & ug/g dry & $3.46 \mathrm{E}-2$ & $3 / 22 / 10$ & 0C17004 & PNNL-AGG-415 \\
\hline HEIS No. & B235R9 & \multicolumn{3}{|c|}{ Lab ID: $\quad$ 1001004-CC } & & & \\
\hline \multirow[t]{2}{*}{$14133-76-7$} & Technetium-99 & $<5.20 \mathrm{E}-3$ & ug/g dry & $5.20 \mathrm{E}-3$ & $4 / 04 / 10$ & 0C17004 & PNNL-AGG-415 \\
\hline & Uranium 238 & $2.68 \mathrm{E}-1$ & ug/g dry & $3.54 \mathrm{E}-2$ & $3 / 22 / 10$ & 0C17004 & PNNL-AGG-415 \\
\hline HEIS No. & В235T8 & \multicolumn{3}{|c|}{ Lab ID: $\quad$ 1001004-DA } & & & \\
\hline \multirow[t]{2}{*}{$14133-76-7$} & Technetium-99 & $<5.28 \mathrm{E}-3$ & ug/g dry & $5.28 \mathrm{E}-3$ & $4 / 04 / 10$ & 0C17004 & PNNL-AGG-415 \\
\hline & Uranium 238 & $2.11 \mathrm{E}-1$ & ug/g dry & $3.60 \mathrm{E}-2$ & $3 / 22 / 10$ & 0C17004 & PNNL-AGG-415 \\
\hline
\end{tabular}




\section{Radionuclides by ICP-MS/Water Extract}

\begin{tabular}{|c|c|c|c|c|c|c|}
\hline CAS \# & Analyte & Results & Units & Analyzed & Batch & Method \\
\hline HEIS No. & B235H1 & \multicolumn{2}{|c|}{ Lab ID: $\quad$ 1001004-04 } & & & \\
\hline $15046-84-1$ & Iodine-129 & $<4.97 \mathrm{E}-5$ & ug/g dry & $5 / 03 / 10$ & 0C31001 & PNNL-AGG-415 \\
\hline HEIS No. & B235J0 & \multicolumn{2}{|c|}{ Lab ID: $\quad 1001004-22$} & & & \\
\hline $15046-84-1$ & Iodine-129 & $<5.04 \mathrm{E}-5$ & $\mathrm{ug} / \mathrm{g}$ dry $\quad 5.04 \mathrm{E}-5$ & $5 / 03 / 10$ & 0C31001 & PNNL-AGG-415 \\
\hline HEIS No. & B235J6 & \multicolumn{2}{|c|}{ Lab ID: $\quad$ 1001004-34 } & & & \\
\hline $15046-84-1$ & Iodine-129 & $<5.00 \mathrm{E}-5$ & $\mathrm{ug} / \mathrm{g}$ dry $\quad 5.00 \mathrm{E}-5$ & $5 / 03 / 10$ & 0C31001 & PNNL-AGG-415 \\
\hline HEIS No. & B235K1 & \multicolumn{2}{|c|}{ Lab ID: $\quad$ 1001004-42 } & & & \\
\hline $15046-84-1$ & Iodine-129 & $8.39 \mathrm{E}-5$ & ug/g dry $\quad 5.01 \mathrm{E}-5$ & $5 / 03 / 10$ & 0C31001 & PNNL-AGG-415 \\
\hline HEIS No. & B235K5 & \multicolumn{2}{|c|}{ Lab ID: $\quad 1001004-46$} & & & \\
\hline $15046-84-1$ & Iodine-129 & $<5.01 \mathrm{E}-5$ & ug/g dry $\quad 5.01 \mathrm{E}-5$ & $5 / 03 / 10$ & 0C31001 & PNNL-AGG-415 \\
\hline HEIS No. & B235L9 & \multicolumn{2}{|c|}{ Lab ID: $\quad 1001004-98$} & & & \\
\hline $15046-84-1$ & Iodine-129 & $<5.00 \mathrm{E}-5$ & ug/g dry $\quad 5.00 \mathrm{E}-5$ & $5 / 03 / 10$ & 0C31001 & PNNL-AGG-415 \\
\hline HEIS No. & B235N9 & \multicolumn{2}{|c|}{ Lab ID: $1001004-B C$} & & & \\
\hline $15046-84-1$ & Iodine-129 & $<4.99 \mathrm{E}-5$ & ug/g dry $\quad 4.99 E-5$ & $5 / 03 / 10$ & 0C31001 & PNNL-AGG-415 \\
\hline HEIS No. & B235R2 & \multicolumn{2}{|c|}{ Lab ID: $\quad$ 1001004-BV } & & & \\
\hline $15046-84-1$ & Iodine-129 & $<5.00 \mathrm{E}-5$ & ug/g dry $\quad 5.00 \mathrm{E}-5$ & $5 / 03 / 10$ & 0C31001 & PNNL-AGG-415 \\
\hline HEIS No. & B235R9 & \multicolumn{2}{|c|}{ Lab ID: $\quad$ 1001004-CC } & & & \\
\hline $15046-84-1$ & Iodine-129 & $<5.00 \mathrm{E}-5$ & ug/g dry $\quad 5.00 \mathrm{E}-5$ & $5 / 03 / 10$ & 0C31001 & PNNL-AGG-415 \\
\hline HEIS No. & B235T8 & \multicolumn{2}{|c|}{ Lab ID: $\quad$ 1001004-DA } & & & \\
\hline $15046-84-1$ & Iodine-129 & $<4.99 \mathrm{E}-5$ & $\mathrm{ug} / \mathrm{g}$ dry $\quad 4.99 \mathrm{E}-5$ & $5 / 03 / 10$ & 0C31001 & PNNL-AGG-415 \\
\hline
\end{tabular}




\section{Radionuclides by ICP-MS/Water Extract}

\begin{tabular}{|c|c|c|c|c|c|c|c|}
\hline CAS \# & Analyte & Results & Units & EQL & Analyzed & Batch & Method \\
\hline \multirow{3}{*}{$\begin{array}{l}\text { HEIS No. } \\
14133-76-7\end{array}$} & B235H1 & \multicolumn{3}{|c|}{ Lab ID: $\quad$ 1001004-04 } & & & \\
\hline & Technetium-99 & $<3.88 \mathrm{E}-5$ & ug/g dry & $3.88 \mathrm{E}-5$ & $3 / 22 / 10$ & $0 \mathrm{C} 17003$ & PNNL-AGG-415 \\
\hline & Uranium 238 & $1.42 \mathrm{E}-1$ & ug/g dry & $7.96 \mathrm{E}-5$ & $3 / 22 / 10$ & $0 \mathrm{C} 17003$ & PNNL-AGG-415 \\
\hline \multirow{3}{*}{$\begin{array}{l}\text { HEIS No. } \\
14133-76-7\end{array}$} & B235J0 & \multicolumn{3}{|c|}{ Lab ID: $\quad 1001004-22$} & & & \\
\hline & Technetium-99 & $<3.93 \mathrm{E}-5$ & ug/g dry & $3.93 \mathrm{E}-5$ & $3 / 22 / 10$ & $0 \mathrm{C} 17003$ & PNNL-AGG-415 \\
\hline & Uranium 238 & $3.87 \mathrm{E}-1$ & ug/g dry & $8.06 \mathrm{E}-5$ & $3 / 22 / 10$ & 0C17003 & PNNL-AGG-415 \\
\hline \multirow{3}{*}{$\begin{array}{l}\text { HEIS No. } \\
14133-76-7\end{array}$} & B235J6 & \multicolumn{3}{|c|}{ Lab ID: $\quad$ 1001004-34 } & & & \\
\hline & Technetium-99 & $<3.90 \mathrm{E}-5$ & ug/g dry & $3.90 \mathrm{E}-5$ & $3 / 22 / 10$ & 0C17003 & PNNL-AGG-415 \\
\hline & Uranium 238 & $2.19 \mathrm{E}-1$ & ug/g dry & $8.00 \mathrm{E}-5$ & $3 / 22 / 10$ & 0C17003 & PNNL-AGG-415 \\
\hline \multirow{3}{*}{$\begin{array}{l}\text { HEIS No. } \\
14133-76-7\end{array}$} & B235K1 & \multicolumn{3}{|c|}{ Lab ID: $\quad$ 1001004-42 } & & & \\
\hline & Technetium-99 & $<3.91 \mathrm{E}-5$ & ug/g dry & $3.91 \mathrm{E}-5$ & $3 / 22 / 10$ & $0 \mathrm{C} 17003$ & PNNL-AGG-415 \\
\hline & Uranium 238 & $1.54 \mathrm{E} 0$ & ug/g dry & $1.60 \mathrm{E}-3$ & $3 / 22 / 10$ & $0 \mathrm{C} 17003$ & PNNL-AGG-415 \\
\hline \multirow{3}{*}{$\begin{array}{l}\text { HEIS No. } \\
14133-76-7\end{array}$} & B235K5 & \multicolumn{3}{|c|}{ Lab ID: $\quad$ 1001004-46 } & & & \\
\hline & Technetium-99 & $<3.91 \mathrm{E}-5$ & ug/g dry & $3.91 \mathrm{E}-5$ & $3 / 22 / 10$ & $0 \mathrm{C} 17003$ & PNNL-AGG-415 \\
\hline & Uranium 238 & $2.28 \mathrm{E}-1$ & ug/g dry & $8.01 \mathrm{E}-5$ & $3 / 22 / 10$ & 0C17003 & PNNL-AGG-415 \\
\hline \multirow{3}{*}{$\begin{array}{l}\text { HEIS No. } \\
14133-76-7\end{array}$} & B235L9 & \multicolumn{3}{|c|}{ Lab ID: $\quad 1001004-98$} & & & \\
\hline & Technetium-99 & $<3.90 \mathrm{E}-5$ & ug/g dry & $3.90 \mathrm{E}-5$ & $3 / 22 / 10$ & 0C17003 & PNNL-AGG-415 \\
\hline & Uranium 238 & $2.18 \mathrm{E}-3$ & ug/g dry & $8.00 \mathrm{E}-5$ & $3 / 22 / 10$ & 0C17003 & PNNL-AGG-415 \\
\hline \multirow{3}{*}{$\begin{array}{l}\text { HEIS No. } \\
14133-76-7\end{array}$} & B235N9 & \multicolumn{3}{|c|}{ Lab ID: $1001004-B C$} & & & \\
\hline & Technetium-99 & $<3.89 \mathrm{E}-5$ & ug/g dry & $3.89 \mathrm{E}-5$ & $3 / 22 / 10$ & $0 \mathrm{C} 17003$ & PNNL-AGG-415 \\
\hline & Uranium 238 & $1.63 \mathrm{E}-4$ & ug/g dry & $7.98 \mathrm{E}-5$ & $3 / 22 / 10$ & 0C17003 & PNNL-AGG-415 \\
\hline \multirow{3}{*}{$\begin{array}{l}\text { HEIS No. } \\
14133-76-7\end{array}$} & B235R2 & \multicolumn{3}{|c|}{ Lab ID: $1001004-B V$} & & & \\
\hline & Technetium-99 & $<3.90 \mathrm{E}-5$ & ug/g dry & $3.90 \mathrm{E}-5$ & $3 / 22 / 10$ & $0 \mathrm{C} 17003$ & PNNL-AGG-415 \\
\hline & Uranium 238 & $1.07 \mathrm{E}-4$ & ug/g dry & $7.99 \mathrm{E}-5$ & $3 / 22 / 10$ & $0 \mathrm{C} 17003$ & PNNL-AGG-415 \\
\hline \multirow{3}{*}{$\begin{array}{l}\text { HEIS No. } \\
14133-76-7\end{array}$} & B235R9 & \multicolumn{3}{|c|}{ Lab ID: $1001004-C C$} & & & \\
\hline & Technetium-99 & $<3.90 \mathrm{E}-5$ & ug/g dry & $3.90 \mathrm{E}-5$ & $3 / 22 / 10$ & $0 \mathrm{C} 17003$ & PNNL-AGG-415 \\
\hline & Uranium 238 & $2.71 \mathrm{E}-4$ & ug/g dry & $8.00 \mathrm{E}-5$ & $3 / 22 / 10$ & 0C17003 & PNNL-AGG-415 \\
\hline \multirow{3}{*}{$\begin{array}{l}\text { HEIS No. } \\
14133-76-7\end{array}$} & B235T8 & \multicolumn{3}{|c|}{ Lab ID: 1001004-DA } & & & \\
\hline & Technetium-99 & $<3.89 \mathrm{E}-5$ & ug/g dry & $3.89 \mathrm{E}-5$ & $3 / 22 / 10$ & 0C17003 & PNNL-AGG-415 \\
\hline & Uranium 238 & 3.61E-4 & ug/g dry & $7.99 \mathrm{E}-5$ & $3 / 22 / 10$ & 0C17003 & PNNL-AGG-415 \\
\hline
\end{tabular}


RCRA Metals By PNNL-AGG-415/Water Extract

\begin{tabular}{|c|c|c|c|c|c|c|c|}
\hline CAS \# & Analyte & Results & Units & EQL & Analyzed & Batch & Method \\
\hline HEIS No. & B235H1 & \multicolumn{3}{|c|}{ Lab ID: $\quad$ 1001004-04 } & & & \\
\hline $14265-72-6$ & Antimony & $<6.55 \mathrm{E}-4$ & ug/g dry & $6.55 \mathrm{E}-4$ & $3 / 10 / 10$ & 0C10007 & PNNL-AGG-415 \\
\hline $15756-10-2$ & Mercury & $<6.51 \mathrm{E}-4$ & $\mathrm{ug} / \mathrm{g}$ dry & $6.51 \mathrm{E}-4$ & $3 / 10 / 10$ & 0C10007 & PNNL-AGG-415 \\
\hline HEIS No. & B235J0 & \multicolumn{3}{|c|}{ Lab ID: $1001004-22$} & & & \\
\hline $14265-72-6$ & Antimony & $<6.64 \mathrm{E}-4$ & ug/g dry & $6.64 \mathrm{E}-4$ & $3 / 10 / 10$ & 0C10007 & PNNL-AGG-415 \\
\hline $15756-10-2$ & Mercury & $<6.59 \mathrm{E}-4$ & ug/g dry & $6.59 \mathrm{E}-4$ & $3 / 10 / 10$ & 0C10007 & PNNL-AGG-415 \\
\hline HEIS No. & B235J6 & \multicolumn{3}{|c|}{ Lab ID: $\quad$ 1001004-34 } & & & \\
\hline $14265-72-6$ & Antimony & $9.04 \mathrm{E}-4$ & ug/g dry & $6.58 \mathrm{E}-4$ & $3 / 10 / 10$ & 0C10007 & PNNL-AGG-415 \\
\hline $15756-10-2$ & Mercury & $<6.54 \mathrm{E}-4$ & ug/g dry & $6.54 \mathrm{E}-4$ & $3 / 10 / 10$ & 0C10007 & PNNL-AGG-415 \\
\hline HEIS No. & B235K1 & \multicolumn{3}{|c|}{ Lab ID: $\quad 1001004-42$} & & & \\
\hline $14265-72-6$ & Antimony & $<6.59 \mathrm{E}-4$ & ug/g dry & $6.59 \mathrm{E}-4$ & $3 / 10 / 10$ & 0C10007 & PNNL-AGG-415 \\
\hline $15756-10-2$ & Mercury & $<6.55 \mathrm{E}-4$ & ug/g dry & $6.55 \mathrm{E}-4$ & $3 / 10 / 10$ & 0C10007 & PNNL-AGG-415 \\
\hline HEIS No. & B235K5 & \multicolumn{3}{|c|}{ Lab ID: $\quad 1001004-46$} & & & \\
\hline $14265-72-6$ & Antimony & $<6.59 \mathrm{E}-4$ & ug/g dry & $6.59 \mathrm{E}-4$ & $3 / 10 / 10$ & 0C10007 & PNNL-AGG-415 \\
\hline $15756-10-2$ & Mercury & $1.05 \mathrm{E}-3$ & ug/g dry & $6.55 \mathrm{E}-4$ & $3 / 10 / 10$ & 0C10007 & PNNL-AGG-415 \\
\hline HEIS No. & B235L9 & \multicolumn{3}{|c|}{ Lab ID: $\quad 1001004-98$} & & & \\
\hline $14265-72-6$ & Antimony & $<6.59 \mathrm{E}-4$ & ug/g dry & $6.59 \mathrm{E}-4$ & $3 / 10 / 10$ & 0C10007 & PNNL-AGG-415 \\
\hline $15756-10-2$ & Mercury & $1.03 \mathrm{E}-3$ & ug/g dry & $6.54 \mathrm{E}-4$ & $3 / 10 / 10$ & 0C10007 & PNNL-AGG-415 \\
\hline HEIS No. & B235N9 & \multicolumn{3}{|c|}{ Lab ID: $\quad$ 1001004-BC } & & & \\
\hline $14265-72-6$ & Antimony & $<6.57 \mathrm{E}-4$ & ug/g dry & $6.57 \mathrm{E}-4$ & $3 / 10 / 10$ & 0C10007 & PNNL-AGG-415 \\
\hline $15756-10-2$ & Mercury & $<6.52 \mathrm{E}-4$ & $\mathrm{ug} / \mathrm{g}$ dry & $6.52 \mathrm{E}-4$ & $3 / 10 / 10$ & 0C10007 & PNNL-AGG-415 \\
\hline HEIS No. & B235R2 & \multicolumn{3}{|c|}{ Lab ID: $1001004-B V$} & & & \\
\hline $14265-72-6$ & Antimony & $1.20 \mathrm{E}-3$ & ug/g dry & $6.58 \mathrm{E}-4$ & $3 / 10 / 10$ & 0C10007 & PNNL-AGG-415 \\
\hline $15756-10-2$ & Mercury & $<6.53 \mathrm{E}-4$ & ug/g dry & $6.53 \mathrm{E}-4$ & $3 / 10 / 10$ & $0 \mathrm{C} 10007$ & PNNL-AGG-415 \\
\hline HEIS No. & B235R9 & \multicolumn{3}{|c|}{ Lab ID: $1001004-C C$} & & & \\
\hline $14265-72-6$ & Antimony & $<6.58 \mathrm{E}-4$ & ug/g dry & $6.58 \mathrm{E}-4$ & $3 / 10 / 10$ & 0C10007 & PNNL-AGG-415 \\
\hline $15756-10-2$ & Mercury & $8.61 \mathrm{E}-4$ & ug/g dry & $6.54 \mathrm{E}-4$ & $3 / 10 / 10$ & 0C10007 & PNNL-AGG-415 \\
\hline HEIS No. & B235T8 & \multicolumn{3}{|c|}{ Lab ID: $1001004-D A$} & & & \\
\hline $14265-72-6$ & Antimony & $8.17 \mathrm{E}-3$ & ug/g dry & $6.57 \mathrm{E}-4$ & $3 / 10 / 10$ & 0C10007 & PNNL-AGG-415 \\
\hline $15756-10-2$ & Mercury & $1.27 \mathrm{E}-3$ & ug/g dry & $6.53 \mathrm{E}-4$ & $3 / 10 / 10$ & 0C10007 & PNNL-AGG-415 \\
\hline
\end{tabular}


RCRA Metals By PNNL-AGG-415/Acid Extract

\begin{tabular}{|c|c|c|c|c|c|c|c|}
\hline CAS \# & Analyte & Results & Units & EQL & Analyzed & Batch & Method \\
\hline HEIS No. & B235H1 & \multicolumn{3}{|c|}{ Lab ID: $\quad$ 1001004-04 } & & & \\
\hline $7440-38-2$ & Arsenic & $1.50 \mathrm{E} 0$ & ug/g dry & $2.95 \mathrm{E}-1$ & $3 / 11 / 10$ & 0C10008 & PNNL-AGG-415 \\
\hline $14265-72-6$ & Antimony & $<1.89 \mathrm{E}-1$ & ug/g dry & $1.89 \mathrm{E}-1$ & $3 / 11 / 10$ & 0C10008 & PNNL-AGG-415 \\
\hline $15756-10-2$ & Mercury & $<1.64 \mathrm{E}-1$ & ug/g dry & $1.64 \mathrm{E}-1$ & $3 / 11 / 10$ & 0C10008 & PNNL-AGG-415 \\
\hline HEIS No. & B235J0 & \multicolumn{3}{|c|}{ Lab ID: $\quad 1001004-22$} & & & \\
\hline $7440-38-2$ & Arsenic & $1.87 \mathrm{E} 0$ & ug/g dry & $2.89 \mathrm{E}-1$ & $3 / 11 / 10$ & 0C10008 & PNNL-AGG-415 \\
\hline $14265-72-6$ & Antimony & $<1.85 \mathrm{E}-1$ & ug/g dry & $1.85 \mathrm{E}-1$ & $3 / 11 / 10$ & 0C10008 & PNNL-AGG-415 \\
\hline $15756-10-2$ & Mercury & $<1.61 \mathrm{E}-1$ & ug/g dry & $1.61 \mathrm{E}-1$ & $3 / 11 / 10$ & 0C10008 & PNNL-AGG-415 \\
\hline HEIS No. & B235J6 & \multicolumn{3}{|c|}{ Lab ID: $\quad$ 1001004-34 } & & & \\
\hline $7440-38-2$ & Arsenic & $1.90 \mathrm{E} 0$ & ug/g dry & $2.90 \mathrm{E}-1$ & $3 / 11 / 10$ & 0C10008 & PNNL-AGG-415 \\
\hline $14265-72-6$ & Antimony & $<1.86 \mathrm{E}-1$ & ug/g dry & $1.86 \mathrm{E}-1$ & $3 / 11 / 10$ & 0C10008 & PNNL-AGG-415 \\
\hline $15756-10-2$ & Mercury & $<1.62 \mathrm{E}-1$ & ug/g dry & $1.62 \mathrm{E}-1$ & $3 / 11 / 10$ & 0C10008 & PNNL-AGG-415 \\
\hline HEIS No. & B235K1 & \multicolumn{3}{|c|}{ Lab ID: $\quad 1001004-42$} & & & \\
\hline $7440-38-2$ & Arsenic & $2.78 \mathrm{E} 0$ & ug/g dry & $3.01 \mathrm{E}-1$ & $3 / 11 / 10$ & 0C10008 & PNNL-AGG-415 \\
\hline $14265-72-6$ & Antimony & $<1.93 \mathrm{E}-1$ & ug/g dry & $1.93 \mathrm{E}-1$ & $3 / 11 / 10$ & 0C10008 & PNNL-AGG-415 \\
\hline $15756-10-2$ & Mercury & $<1.68 \mathrm{E}-1$ & ug/g dry & $1.68 \mathrm{E}-1$ & $3 / 11 / 10$ & 0C10008 & PNNL-AGG-415 \\
\hline HEIS No. & B235K5 & \multicolumn{3}{|c|}{ Lab ID: $\quad$ 1001004-46 } & & & \\
\hline $7440-38-2$ & Arsenic & $1.66 \mathrm{E} 0$ & ug/g dry & $2.94 \mathrm{E}-1$ & $3 / 11 / 10$ & 0C10008 & PNNL-AGG-415 \\
\hline $14265-72-6$ & Antimony & $<1.89 \mathrm{E}-1$ & ug/g dry & $1.89 \mathrm{E}-1$ & $3 / 11 / 10$ & 0C10008 & PNNL-AGG-415 \\
\hline $15756-10-2$ & Mercury & $<1.64 \mathrm{E}-1$ & ug/g dry & $1.64 \mathrm{E}-1$ & $3 / 11 / 10$ & 0C10008 & PNNL-AGG-415 \\
\hline HEIS No. & B235L9 & \multicolumn{3}{|c|}{ Lab ID: $\quad 1001004-98$} & & & \\
\hline $7440-38-2$ & Arsenic & $2.84 \mathrm{E} 0$ & ug/g dry & $2.92 \mathrm{E}-1$ & $3 / 11 / 10$ & 0C10008 & PNNL-AGG-415 \\
\hline $14265-72-6$ & Antimony & $<1.87 \mathrm{E}-1$ & ug/g dry & $1.87 \mathrm{E}-1$ & $3 / 11 / 10$ & 0C10008 & PNNL-AGG-415 \\
\hline $15756-10-2$ & Mercury & $<1.63 \mathrm{E}-1$ & ug/g dry & $1.63 \mathrm{E}-1$ & $3 / 11 / 10$ & 0C10008 & PNNL-AGG-415 \\
\hline HEIS No. & B235N9 & \multicolumn{3}{|c|}{ Lab ID: $\quad$ 1001004-BC } & & & \\
\hline $7440-38-2$ & Arsenic & $9.98 \mathrm{E}-1$ & ug/g dry & $2.86 \mathrm{E}-1$ & $3 / 11 / 10$ & 0C10008 & PNNL-AGG-415 \\
\hline $14265-72-6$ & Antimony & $<1.83 \mathrm{E}-1$ & ug/g dry & $1.83 \mathrm{E}-1$ & $3 / 11 / 10$ & 0C10008 & PNNL-AGG-415 \\
\hline $15756-10-2$ & Mercury & $<1.59 \mathrm{E}-1$ & ug/g dry & $1.59 \mathrm{E}-1$ & $3 / 11 / 10$ & 0C10008 & PNNL-AGG-415 \\
\hline HEIS No. & B235R2 & \multicolumn{3}{|c|}{ Lab ID: $\quad$ 1001004-BV } & & & \\
\hline $7440-38-2$ & Arsenic & $6.59 \mathrm{E}-1$ & ug/g dry & $2.83 \mathrm{E}-1$ & $3 / 11 / 10$ & 0C10008 & PNNL-AGG-415 \\
\hline $14265-72-6$ & Antimony & $<1.82 \mathrm{E}-1$ & ug/g dry & $1.82 \mathrm{E}-1$ & $3 / 11 / 10$ & 0C10008 & PNNL-AGG-415 \\
\hline $15756-10-2$ & Mercury & $<1.58 \mathrm{E}-1$ & ug/g dry & $1.58 \mathrm{E}-1$ & $3 / 11 / 10$ & 0C10008 & PNNL-AGG-415 \\
\hline HEIS No. & B235R9 & \multicolumn{3}{|c|}{ Lab ID: $1001004-C C$} & & & \\
\hline $7440-38-2$ & Arsenic & 4.44E-1 & ug/g dry & $2.90 \mathrm{E}-1$ & $3 / 11 / 10$ & 0C10008 & PNNL-AGG-415 \\
\hline $14265-72-6$ & Antimony & $<1.86 \mathrm{E}-1$ & ug/g dry & $1.86 \mathrm{E}-1$ & $3 / 11 / 10$ & 0C10008 & PNNL-AGG-415 \\
\hline $15756-10-2$ & Mercury & $<1.62 \mathrm{E}-1$ & ug/g dry & $1.62 \mathrm{E}-1$ & $3 / 11 / 10$ & 0C10008 & PNNL-AGG-415 \\
\hline HEIS No. & В235T8 & \multicolumn{3}{|c|}{ Lab ID: $\quad$ 1001004-DA } & & & \\
\hline $7440-38-2$ & Arsenic & $7.16 \mathrm{E}-1$ & ug/g dry & $2.95 \mathrm{E}-1$ & $3 / 11 / 10$ & 0C10008 & PNNL-AGG-415 \\
\hline $14265-72-6$ & Antimony & $2.41 \mathrm{E}-1$ & ug/g dry & $1.89 \mathrm{E}-1$ & $3 / 11 / 10$ & 0C 10008 & PNNL-AGG-415 \\
\hline $15756-10-2$ & Mercury & $<1.65 \mathrm{E}-1$ & ug/g dry & $1.65 \mathrm{E}-1$ & $3 / 11 / 10$ & 0C10008 & PNNL-AGG-415 \\
\hline
\end{tabular}




\section{Carbon Analysis/Soil}

Total Organic Carbon (ug/g) by AGG-TOC-001

\begin{tabular}{|c|c|c|c|c|c|}
\hline Lab ID & HEIS No. & Results & EQL & Analyzed & Batch \\
\hline 1001004-04 & B235H1 & $<2.00 \mathrm{E} 2$ & $2.00 \mathrm{E} 2$ & $3 / 23 / 10$ & [CALC] \\
\hline $1001004-22$ & $\mathrm{~B} 235 \mathrm{~J} 0$ & $<2.00 \mathrm{E} 2$ & $2.00 \mathrm{E} 2$ & $3 / 23 / 10$ & [CALC] \\
\hline 1001004-34 & B235J6 & $2.78 \mathrm{E} 2$ & $2.00 \mathrm{E} 2$ & $3 / 23 / 10$ & [CALC] \\
\hline $1001004-42$ & B235K1 & $<2.00 \mathrm{E} 2$ & $2.00 \mathrm{E} 2$ & $3 / 23 / 10$ & [CALC] \\
\hline 1001004-46 & B235K5 & $<2.00 \mathrm{E} 2$ & $2.00 \mathrm{E} 2$ & $3 / 23 / 10$ & [CALC] \\
\hline 1001004-98 & B235L9 & $<2.00 \mathrm{E} 2$ & $2.00 \mathrm{E} 2$ & $3 / 23 / 10$ & [CALC] \\
\hline 1001004-BC & B235N9 & $<2.00 \mathrm{E} 2$ & $2.00 \mathrm{E} 2$ & $3 / 23 / 10$ & [CALC] \\
\hline 1001004-BV & $\mathrm{B} 235 \mathrm{R} 2$ & $<2.00 \mathrm{E} 2$ & $2.00 \mathrm{E} 2$ & $3 / 23 / 10$ & [CALC] \\
\hline 1001004-CC & B235R9 & $<2.00 \mathrm{E} 2$ & $2.00 \mathrm{E} 2$ & $3 / 23 / 10$ & [CALC] \\
\hline 1001004-DA & B235T8 & $8.52 \mathrm{E} 2$ & $2.00 \mathrm{E} 2$ & $3 / 23 / 10$ & [CALC] \\
\hline
\end{tabular}




\section{Carbon Analysis/Soil}

Total Carbon (ug/g) by AGG-TOC-001

\begin{tabular}{llcccc} 
Lab ID & HEIS No. & Results & EQL & Analyzed & Batch \\
\hline $1001004-04$ & B235H1 & $2.04 \mathrm{E} 3$ & $2.00 \mathrm{E} 2$ & $3 / 19 / 10$ & $0 \mathrm{C} 19002$ \\
$1001004-22$ & B235J0 & $9.63 \mathrm{E} 2$ & $2.00 \mathrm{E} 2$ & $3 / 19 / 10$ & $0 \mathrm{C} 19002$ \\
$1001004-34$ & B235J6 & $1.06 \mathrm{E} 3$ & $2.00 \mathrm{E} 2$ & $3 / 19 / 10$ & $0 \mathrm{C} 19002$ \\
$1001004-42$ & B235K1 & $1.01 \mathrm{E} 3$ & $2.00 \mathrm{E} 2$ & $3 / 19 / 10$ & $0 \mathrm{C} 19002$ \\
$1001004-46$ & B235K5 & $1.94 \mathrm{E} 3$ & $2.00 \mathrm{E} 2$ & $3 / 19 / 10$ & $0 \mathrm{C} 19002$ \\
$1001004-98$ & B235L9 & $1.99 \mathrm{E} 3$ & $2.00 \mathrm{E} 2$ & $3 / 19 / 10$ & $0 \mathrm{C} 19002$ \\
$1001004-\mathrm{BC}$ & B235N9 & $1.17 \mathrm{E} 3$ & $2.00 \mathrm{E} 2$ & $3 / 19 / 10$ & $0 \mathrm{C} 19002$ \\
$1001004-\mathrm{BV}$ & B235R2 & $1.55 \mathrm{E} 3$ & $2.00 \mathrm{E} 2$ & $3 / 19 / 10$ & $0 \mathrm{C} 19002$ \\
$1001004-\mathrm{CC}$ & B235R9 & $7.77 \mathrm{E} 2$ & $2.00 \mathrm{E} 2$ & $3 / 19 / 10$ & $0 \mathrm{C} 19002$ \\
$1001004-\mathrm{DA}$ & B235T8 & $1.41 \mathrm{E} 3$ & $2.00 \mathrm{E} 2$ & & $0 \mathrm{C} 19002$ \\
\hline
\end{tabular}




\begin{tabular}{|c|c|c|c|c|c|}
\hline \multicolumn{6}{|c|}{ Carbon Analysis/Soil } \\
\hline \multicolumn{6}{|c|}{ Total Inorganic Carbon (ug/g) by AGG-TOC-001 } \\
\hline Lab ID & HEIS No. & Results & EQL & Analyzed & Batch \\
\hline 1001004-04 & B235H1 & $2.17 \mathrm{E} 3$ & $2.00 \mathrm{E} 2$ & $3 / 23 / 10$ & $0 \mathrm{C} 23003$ \\
\hline 1001004-22 & B235J0 & 7.88E2 & $2.00 \mathrm{E} 2$ & $3 / 23 / 10$ & $0 \mathrm{C} 23003$ \\
\hline 1001004-34 & B235J6 & $7.86 \mathrm{E} 2$ & $2.00 \mathrm{E} 2$ & $3 / 23 / 10$ & $0 \mathrm{C} 23003$ \\
\hline $1001004-42$ & B235K1 & $9.40 \mathrm{E} 2$ & $2.00 \mathrm{E} 2$ & $3 / 23 / 10$ & 0C23003 \\
\hline $1001004-46$ & B235K5 & $2.10 \mathrm{E} 3$ & $2.00 \mathrm{E} 2$ & $3 / 23 / 10$ & 0C23003 \\
\hline $1001004-98$ & B235L9 & $2.17 \mathrm{E} 3$ & $2.00 \mathrm{E} 2$ & $3 / 23 / 10$ & 0C23003 \\
\hline 1001004-BC & B235N9 & $1.15 \mathrm{E} 3$ & $2.00 \mathrm{E} 2$ & $3 / 23 / 10$ & $0 \mathrm{C} 23003$ \\
\hline 1001004-BV & $\mathrm{B} 235 \mathrm{R} 2$ & $1.42 \mathrm{E} 3$ & $2.00 \mathrm{E} 2$ & $3 / 23 / 10$ & 0C23003 \\
\hline 1001004-CC & B235R9 & $6.99 \mathrm{E} 2$ & $2.00 \mathrm{E} 2$ & $3 / 23 / 10$ & $0 \mathrm{C} 23003$ \\
\hline 1001004-DA & В235T8 & $5.60 \mathrm{E} 2$ & $2.00 \mathrm{E} 2$ & $3 / 23 / 10$ & $0 \mathrm{C} 23003$ \\
\hline
\end{tabular}




\section{GEA/Soil}

\begin{tabular}{|c|c|c|c|c|c|c|c|c|}
\hline CAS \# & Analyte & Results & Units & MDA & UNC & Analyzed & Batch & Method \\
\hline HEIS No. & В235H1 & \multicolumn{3}{|c|}{ Lab ID: $\quad$ 1001004-04 } & & & & \\
\hline $10198-40-0$ & Cobalt-60 & $<2.18 \mathrm{E}-1$ & pCi/g dry & $2.18 \mathrm{E}-1$ & & $3 / 09 / 10$ & 0C09002 & AGG-RRL-001 \\
\hline $10045-97-3$ & Cesium-137 & $<2.78 \mathrm{E}-1$ & pCi/g dry & $2.78 \mathrm{E}-1$ & & $3 / 09 / 10$ & 0С09002 & AGG-RRL-001 \\
\hline $14683-23-9$ & Europium-152 & $<1.10 \mathrm{E} 0$ & pCi/g dry & $1.10 \mathrm{E} 0$ & & $3 / 09 / 10$ & 0C09002 & AGG-RRL-001 \\
\hline $15585-10-1$ & Europium-154 & $<6.35 \mathrm{E}-1$ & pCi/g dry & $6.35 \mathrm{E}-1$ & & $3 / 09 / 10$ & 0C09002 & AGG-RRL-001 \\
\hline $14391-16-3$ & Europium-155 & $<1.00 \mathrm{E} 0$ & pCi/g dry & $1.00 \mathrm{E} 0$ & & $3 / 09 / 10$ & 0C09002 & AGG-RRL-001 \\
\hline HEIS No. & B235J0 & \multicolumn{3}{|c|}{ Lab ID: $\quad 1001004-22$} & & & & \\
\hline $10198-40-0$ & Cobalt-60 & $<2.21 \mathrm{E}-1$ & pCi $/ g$ dry & $2.21 \mathrm{E}-1$ & & $3 / 09 / 10$ & 0C09002 & AGG-RRL-001 \\
\hline $10045-97-3$ & Cesium-137 & $<2.43 \mathrm{E}-1$ & pCi/g dry & $2.43 \mathrm{E}-1$ & & $3 / 09 / 10$ & 0C09002 & AGG-RRL-001 \\
\hline $14683-23-9$ & Europium-152 & $<9.16 \mathrm{E}-1$ & pCi/g dry & $9.16 \mathrm{E}-1$ & & $3 / 09 / 10$ & 0C09002 & AGG-RRL-001 \\
\hline $15585-10-1$ & Europium-154 & $<5.01 \mathrm{E}-1$ & pCi/g dry & $5.01 \mathrm{E}-1$ & & $3 / 09 / 10$ & 0C09002 & AGG-RRL-001 \\
\hline $14391-16-3$ & Europium-155 & $<8.92 \mathrm{E}-1$ & pCi/g dry & $8.92 \mathrm{E}-1$ & & $3 / 09 / 10$ & 0C09002 & AGG-RRL-001 \\
\hline HEIS No. & B235J6 & \multicolumn{3}{|c|}{ Lab ID: $\quad$ 1001004-34 } & & & & \\
\hline $10198-40-0$ & Cobalt-60 & $<2.08 \mathrm{E}-1$ & pCi $/ g$ dry & $2.08 \mathrm{E}-1$ & & $3 / 09 / 10$ & 0C09002 & AGG-RRL-001 \\
\hline $10045-97-3$ & Cesium-137 & $<2.63 \mathrm{E}-1$ & pCi/g dry & $2.63 \mathrm{E}-1$ & & $3 / 09 / 10$ & 0C09002 & AGG-RRL-001 \\
\hline $14683-23-9$ & Europium-152 & $<9.54 \mathrm{E}-1$ & pCi/g dry & $9.54 \mathrm{E}-1$ & & $3 / 09 / 10$ & 0C09002 & AGG-RRL-001 \\
\hline $15585-10-1$ & Europium-154 & $<6.07 \mathrm{E}-1$ & pCi/g dry & $6.07 \mathrm{E}-1$ & & $3 / 09 / 10$ & 0C09002 & AGG-RRL-001 \\
\hline $14391-16-3$ & Europium-155 & $<1.02 \mathrm{E} 0$ & pCi/g dry & $1.02 \mathrm{E} 0$ & & $3 / 09 / 10$ & 0C09002 & AGG-RRL-001 \\
\hline HEIS No. & B235K1 & \multicolumn{3}{|c|}{ Lab ID: $\quad$ 1001004-42 } & & & & \\
\hline $10198-40-0$ & Cobalt-60 & $<2.66 \mathrm{E}-1$ & pCi/g dry & $2.66 \mathrm{E}-1$ & & $3 / 09 / 10$ & 0C09002 & AGG-RRL-001 \\
\hline $10045-97-3$ & Cesium-137 & $<2.66 \mathrm{E}-1$ & pCi/g dry & $2.66 \mathrm{E}-1$ & & $3 / 09 / 10$ & 0C09002 & AGG-RRL-001 \\
\hline $14683-23-9$ & Europium-152 & $<1.03 \mathrm{E} 0$ & pCi/g dry & $1.03 \mathrm{E} 0$ & & $3 / 09 / 10$ & 0C09002 & AGG-RRL-001 \\
\hline $15585-10-1$ & Europium-154 & $<5.62 \mathrm{E}-1$ & $\mathrm{pCi} / \mathrm{g}$ dry & $5.62 \mathrm{E}-1$ & & $3 / 09 / 10$ & 0C09002 & AGG-RRL-001 \\
\hline $14391-16-3$ & Europium-155 & $<1.03 \mathrm{E} 0$ & pCi/g dry & $1.03 \mathrm{E} 0$ & & $3 / 09 / 10$ & 0C09002 & AGG-RRL-001 \\
\hline HEIS No. & B235K5 & \multicolumn{3}{|c|}{ Lab ID: $\quad 1001004-46$} & & & & \\
\hline $10198-40-0$ & Cobalt-60 & $<2.17 \mathrm{E}-1$ & pCi/g dry & $2.17 \mathrm{E}-1$ & & $3 / 10 / 10$ & 0C09002 & AGG-RRL-001 \\
\hline $10045-97-3$ & Cesium-137 & $<2.57 \mathrm{E}-1$ & pCi/g dry & $2.57 \mathrm{E}-1$ & & $3 / 10 / 10$ & 0C09002 & AGG-RRL-001 \\
\hline $14683-23-9$ & Europium-152 & $<9.80 \mathrm{E}-1$ & pCi/g dry & $9.80 \mathrm{E}-1$ & & $3 / 10 / 10$ & 0C09002 & AGG-RRL-001 \\
\hline $15585-10-1$ & Europium-154 & $<5.92 \mathrm{E}-1$ & pCi/g dry & $5.92 \mathrm{E}-1$ & & $3 / 10 / 10$ & 0C09002 & AGG-RRL-001 \\
\hline 14391-16-3 & Europium-155 & $<9.35 \mathrm{E}-1$ & $\mathrm{pCi} / \mathrm{g}$ dry & $9.35 \mathrm{E}-1$ & & $3 / 10 / 10$ & 0C09002 & AGG-RRL-001 \\
\hline HEIS No. & B235L9 & \multicolumn{3}{|c|}{ Lab ID: $\quad$ 1001004-98 } & & & & \\
\hline $10198-40-0$ & Cobalt-60 & $<2.42 \mathrm{E}-1$ & pCi/g dry & $2.42 \mathrm{E}-1$ & & $3 / 10 / 10$ & 0C09002 & AGG-RRL-001 \\
\hline $10045-97-3$ & Cesium-137 & $<3.07 \mathrm{E}-1$ & pCi/g dry & 3.07E-1 & & $3 / 10 / 10$ & 0C09002 & AGG-RRL-001 \\
\hline $14683-23-9$ & Europium-152 & $<1.10 \mathrm{E} 0$ & pCi/g dry & $1.10 \mathrm{E} 0$ & & $3 / 10 / 10$ & 0C09002 & AGG-RRL-001 \\
\hline $15585-10-1$ & Europium-154 & $<5.62 \mathrm{E}-1$ & pCi/g dry & $5.62 \mathrm{E}-1$ & & $3 / 10 / 10$ & 0C09002 & AGG-RRL-001 \\
\hline $14391-16-3$ & Europium-155 & $<9.44 \mathrm{E}-1$ & pCi/g dry & $9.44 \mathrm{E}-1$ & & $3 / 10 / 10$ & 0C09002 & AGG-RRL-001 \\
\hline HEIS No. & B235N9 & \multicolumn{3}{|c|}{ Lab ID: 1001004-BC } & & & & \\
\hline $10198-40-0$ & Cobalt-60 & $<1.71 \mathrm{E}-1$ & $\mathrm{pCi} / \mathrm{g}$ dry & $1.71 \mathrm{E}-1$ & & $3 / 11 / 10$ & 0C09002 & AGG-RRL-001 \\
\hline $10045-97-3$ & Cesium-137 & $<2.30 \mathrm{E}-1$ & $\mathrm{pCi} / \mathrm{g}$ dry & $2.30 \mathrm{E}-1$ & & $3 / 11 / 10$ & 0C09002 & AGG-RRL-001 \\
\hline $14683-23-9$ & Europium-152 & $<8.82 \mathrm{E}-1$ & pCi/g dry & $8.82 \mathrm{E}-1$ & & $3 / 11 / 10$ & 0C09002 & AGG-RRL-001 \\
\hline $15585-10-1$ & Europium-154 & $<5.21 \mathrm{E}-1$ & pCi/g dry & $5.21 \mathrm{E}-1$ & & $3 / 11 / 10$ & 0C09002 & AGG-RRL-001 \\
\hline 14391-16-3 & Europium-155 & $<8.01 \mathrm{E}-1$ & pCi/g dry & $8.01 \mathrm{E}-1$ & & $3 / 11 / 10$ & 0C09002 & AGG-RRL-001 \\
\hline HEIS No. & B235R2 & \multicolumn{3}{|c|}{ Lab ID: 1001004-BV } & & & & \\
\hline $10198-40-0$ & Cobalt-60 & $<1.81 \mathrm{E}-1$ & pCi/g dry & $1.81 \mathrm{E}-1$ & & $3 / 11 / 10$ & 0C09002 & AGG-RRL-001 \\
\hline $10045-97-3$ & Cesium-137 & $<1.99 \mathrm{E}-1$ & pCi/g dry & $1.99 \mathrm{E}-1$ & & $3 / 11 / 10$ & 0C09002 & AGG-RRL-001 \\
\hline $14683-23-9$ & Europium-152 & $<7.45 \mathrm{E}-1$ & pCi/g dry & 7.45E-1 & & $3 / 11 / 10$ & 0C09002 & AGG-RRL-001 \\
\hline $15585-10-1$ & Europium-154 & $<3.89 \mathrm{E}-1$ & $\mathrm{pCi} / \mathrm{g}$ dry & 3.89E-1 & & $3 / 11 / 10$ & 0C09002 & AGG-RRL-001 \\
\hline $14391-16-3$ & Europium-155 & $<6.15 \mathrm{E}-1$ & pCi/g dry & $6.15 \mathrm{E}-1$ & & $3 / 11 / 10$ & 0C09002 & AGG-RRL-001 \\
\hline
\end{tabular}




\section{GEA/Soil}

\begin{tabular}{|c|c|c|c|c|c|c|c|c|}
\hline CAS \# & Analyte & Results & Units & MDA & UNC & Analyzed & Batch & Method \\
\hline HEIS No. & B235R9 & \multicolumn{3}{|c|}{ Lab ID: $\quad$ 1001004-CC } & & & & \\
\hline $10198-40-0$ & Cobalt-60 & $<1.74 \mathrm{E}-1$ & $\mathrm{pCi} / \mathrm{g}$ dry & $1.74 \mathrm{E}-1$ & & $3 / 11 / 10$ & 0C09002 & AGG-RRL-001 \\
\hline $10045-97-3$ & Cesium-137 & $<2.07 \mathrm{E}-1$ & $\mathrm{pCi} / \mathrm{g}$ dry & $2.07 \mathrm{E}-1$ & & $3 / 11 / 10$ & 0C09002 & AGG-RRL-001 \\
\hline $14683-23-9$ & Europium-152 & $<8.09 \mathrm{E}-1$ & $\mathrm{pCi} / \mathrm{g}$ dry & 8.09E-1 & & $3 / 11 / 10$ & 0C09002 & AGG-RRL-001 \\
\hline $15585-10-1$ & Europium-154 & $<4.79 \mathrm{E}-1$ & $\mathrm{pCi} / \mathrm{g}$ dry & $4.79 \mathrm{E}-1$ & & $3 / 11 / 10$ & 0C09002 & AGG-RRL-001 \\
\hline $14391-16-3$ & Europium-155 & $<7.26 \mathrm{E}-1$ & pCi/g dry & $7.26 \mathrm{E}-1$ & & $3 / 11 / 10$ & 0C09002 & AGG-RRL-001 \\
\hline HEIS No. & В235T8 & \multicolumn{3}{|c|}{ Lab ID: 1001004-DA } & & & & \\
\hline $10198-40-0$ & Cobalt-60 & $<1.91 \mathrm{E}-1$ & $\mathrm{pCi} / \mathrm{g}$ dry & $1.91 \mathrm{E}-1$ & & $3 / 11 / 10$ & 0C09002 & AGG-RRL-001 \\
\hline $10045-97-3$ & Cesium-137 & $<2.24 \mathrm{E}-1$ & pCi/g dry & $2.24 \mathrm{E}-1$ & & $3 / 11 / 10$ & 0C09002 & AGG-RRL-001 \\
\hline $14683-23-9$ & Europium-152 & $<8.42 \mathrm{E}-1$ & pCi/g dry & $8.42 \mathrm{E}-1$ & & $3 / 11 / 10$ & 0C09002 & AGG-RRL-001 \\
\hline $15585-10-1$ & Europium-154 & $<4.39 \mathrm{E}-1$ & $\mathrm{pCi} / \mathrm{g}$ dry & $4.39 \mathrm{E}-1$ & & $3 / 11 / 10$ & 0C09002 & AGG-RRL-001 \\
\hline $14391-16-3$ & Europium-155 & $<7.10 \mathrm{E}-1$ & $\mathrm{pCi} / \mathrm{g}$ dry & $7.10 \mathrm{E}-1$ & & $3 / 11 / 10$ & 0C09002 & AGG-RRL-001 \\
\hline
\end{tabular}


Total Alpha Total Beta/Acid Extract

\begin{tabular}{|c|c|c|c|c|c|c|c|c|}
\hline CAS \# & Analyte & Results & Units & MDA & $\mathrm{UNC}$ & Analyzed & Batch & Method \\
\hline HEIS No. & В235H1 & \multicolumn{3}{|c|}{ Lab ID: $\quad$ 1001004-04 } & & & & \\
\hline $12587-47-2$ & Gross Beta & $<4.81 \mathrm{E} 1$ & pCi/g dry & $4.81 \mathrm{E} 1$ & & $3 / 17 / 10$ & 0C17002 & AGG-RRL-002 \\
\hline $12587-46-1$ & Gross Alpha & $<1.60 \mathrm{E} 1$ & pCi/g dry & $1.60 \mathrm{E} 1$ & & $3 / 17 / 10$ & $0 \mathrm{C} 17002$ & AGG-RRL-002 \\
\hline HEIS No. & B235J0 & \multicolumn{3}{|c|}{ Lab ID: $\quad 1001004-22$} & & & & \\
\hline $12587-47-2$ & Gross Beta & $<4.72 \mathrm{E} 1$ & pCi/g dry & $4.72 \mathrm{E} 1$ & & $3 / 17 / 10$ & $0 \mathrm{C} 17002$ & AGG-RRL-002 \\
\hline $12587-46-1$ & Gross Alpha & $<1.57 \mathrm{E} 1$ & pCi/g dry & $1.57 \mathrm{E} 1$ & & $3 / 17 / 10$ & 0C17002 & AGG-RRL-002 \\
\hline HEIS No. & B235J6 & \multicolumn{3}{|c|}{ Lab ID: $\quad$ 1001004-34 } & & & & \\
\hline $12587-47-2$ & Gross Beta & $<4.74 \mathrm{E} 1$ & pCi/g dry & 4.74E1 & & $3 / 17 / 10$ & 0C17002 & AGG-RRL-002 \\
\hline $12587-46-1$ & Gross Alpha & $<1.58 \mathrm{E} 1$ & pCi/g dry & $1.58 \mathrm{E} 1$ & & $3 / 17 / 10$ & 0C17002 & AGG-RRL-002 \\
\hline HEIS No. & B235K1 & \multicolumn{3}{|c|}{ Lab ID: $\quad 1001004-42$} & & & & \\
\hline $12587-47-2$ & Gross Beta & $<4.92 \mathrm{E} 1$ & pCi/g dry & 4.92E1 & & $3 / 17 / 10$ & 0C17002 & AGG-RRL-002 \\
\hline $12587-46-1$ & Gross Alpha & $<1.64 \mathrm{E} 1$ & pCi/g dry & $1.64 \mathrm{E} 1$ & & $3 / 17 / 10$ & $0 \mathrm{C} 17002$ & AGG-RRL-002 \\
\hline HEIS No. & B235K5 & \multicolumn{3}{|c|}{ Lab ID: $\quad 1001004-46$} & & & & \\
\hline $12587-47-2$ & Gross Beta & $<4.81 \mathrm{E} 1$ & pCi/g dry & $4.81 \mathrm{E} 1$ & & $3 / 17 / 10$ & 0C17002 & AGG-RRL-002 \\
\hline $12587-46-1$ & Gross Alpha & $<1.60 \mathrm{E} 1$ & pCi/g dry & $1.60 \mathrm{E} 1$ & & $3 / 17 / 10$ & 0C17002 & AGG-RRL-002 \\
\hline HEIS No. & B235L9 & \multicolumn{3}{|c|}{ Lab ID: $\quad 1001004-98$} & & & & \\
\hline $12587-47-2$ & Gross Beta & $<4.77 \mathrm{E} 1$ & pCi/g dry & $4.77 \mathrm{E} 1$ & & $3 / 17 / 10$ & 0C17002 & AGG-RRL-002 \\
\hline $12587-46-1$ & Gross Alpha & $<1.59 \mathrm{E} 1$ & pCi/g dry & $1.59 \mathrm{E} 1$ & & $3 / 17 / 10$ & 0C17002 & AGG-RRL-002 \\
\hline HEIS No. & B235N9 & \multicolumn{3}{|c|}{ Lab ID: $\quad$ 1001004-BC } & & & & \\
\hline $12587-47-2$ & Gross Beta & $<4.67 \mathrm{E} 1$ & pCi/g dry & 4.67E1 & & $3 / 17 / 10$ & 0C17002 & AGG-RRL-002 \\
\hline $12587-46-1$ & Gross Alpha & $<1.56 \mathrm{E} 1$ & pCi/g dry & $1.56 \mathrm{E} 1$ & & $3 / 17 / 10$ & 0C17002 & AGG-RRL-002 \\
\hline HEIS No. & B235R2 & \multicolumn{3}{|c|}{ Lab ID: $\quad$ 1001004-BV } & & & & \\
\hline $12587-47-2$ & Gross Beta & $<4.63 \mathrm{E} 1$ & pCi/g dry & 4.63E1 & & $3 / 17 / 10$ & 0C17002 & AGG-RRL-002 \\
\hline $12587-46-1$ & Gross Alpha & $<1.54 \mathrm{E} 1$ & pCi/g dry & $1.54 \mathrm{E} 1$ & & $3 / 17 / 10$ & 0C17002 & AGG-RRL-002 \\
\hline HEIS No. & B235R9 & \multicolumn{3}{|c|}{ Lab ID: $1001004-C C$} & & & & \\
\hline $12587-47-2$ & Gross Beta & $<4.74 \mathrm{E} 1$ & pCi/g dry & $4.74 \mathrm{E} 1$ & & $3 / 17 / 10$ & 0C17002 & AGG-RRL-002 \\
\hline $12587-46-1$ & Gross Alpha & $<1.58 \mathrm{E} 1$ & pCi/g dry & $1.58 \mathrm{E} 1$ & & $3 / 17 / 10$ & 0C17002 & AGG-RRL-002 \\
\hline HEIS No. & B235T8 & \multicolumn{3}{|c|}{ Lab ID: 1001004-DA } & & & & \\
\hline $12587-47-2$ & Gross Beta & $<4.82 \mathrm{E} 1$ & pCi/g dry & $4.82 \mathrm{E} 1$ & & $3 / 17 / 10$ & 0C17002 & AGG-RRL-002 \\
\hline $12587-46-1$ & Gross Alpha & $<1.61 \mathrm{E} 1$ & pCi/g dry & $1.61 \mathrm{E} 1$ & & $3 / 17 / 10$ & 0C17002 & AGG-RRL-002 \\
\hline
\end{tabular}


Total Alpha Total Beta/Water Extract

\begin{tabular}{|c|c|c|c|c|c|c|c|c|}
\hline CAS \# & Analyte & Results & Units & MDA & $\mathrm{UNC}$ & Analyzed & Batch & Method \\
\hline HEIS No. & B235H1 & \multicolumn{3}{|c|}{ Lab ID: $\quad 1001004-04$} & & & & \\
\hline $12587-47-2$ & Gross Beta & $<1.42 \mathrm{E} 1$ & pCi/g dry & $1.42 \mathrm{E} 1$ & & $3 / 17 / 10$ & 0C17001 & AGG-RRL-002 \\
\hline $12587-46-1$ & Gross Alpha & $<4.23 \mathrm{E} 0$ & pCi/g dry & 4.23E0 & & $3 / 17 / 10$ & 0C17001 & AGG-RRL-002 \\
\hline HEIS No. & B235J0 & \multicolumn{3}{|c|}{ Lab ID: $\quad 1001004-22$} & & & & \\
\hline $12587-47-2$ & Gross Beta & $<1.44 \mathrm{E} 1$ & pCi/g dry & $1.44 \mathrm{E} 1$ & & $3 / 17 / 10$ & 0C17001 & AGG-RRL-002 \\
\hline $12587-46-1$ & Gross Alpha & $<4.29 \mathrm{E} 0$ & $\mathrm{pCi} / \mathrm{g}$ dry & $4.29 \mathrm{E} 0$ & & $3 / 17 / 10$ & 0C17001 & AGG-RRL-002 \\
\hline HEIS No. & B235J6 & \multicolumn{3}{|c|}{ Lab ID: $\quad 1001004-34$} & & & & \\
\hline $12587-47-2$ & Gross Beta & $<1.42 \mathrm{E} 1$ & pCi/g dry & $1.42 \mathrm{E} 1$ & & $3 / 17 / 10$ & 0C17001 & AGG-RRL-002 \\
\hline $12587-46-1$ & Gross Alpha & $<4.25 \mathrm{E} 0$ & pCi/g dry & $4.25 \mathrm{E} 0$ & & $3 / 17 / 10$ & 0C17001 & AGG-RRL-002 \\
\hline HEIS No. & B235K1 & \multicolumn{3}{|c|}{ Lab ID: $\quad 1001004-42$} & & & & \\
\hline $12587-47-2$ & Gross Beta & $<1.43 \mathrm{E} 1$ & pCi/g dry & $1.43 \mathrm{E} 1$ & & $3 / 17 / 10$ & 0C17001 & AGG-RRL-002 \\
\hline $12587-46-1$ & Gross Alpha & $<4.26 \mathrm{E} 0$ & $\mathrm{pCi} / \mathrm{g}$ dry & $4.26 \mathrm{E} 0$ & & $3 / 17 / 10$ & 0C17001 & AGG-RRL-002 \\
\hline HEIS No. & 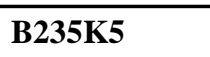 & \multicolumn{3}{|c|}{ Lab ID: $\quad 1001004-46$} & & & & \\
\hline $12587-47-2$ & Gross Beta & $<1.43 \mathrm{E} 1$ & pCi/g dry & $1.43 \mathrm{E} 1$ & & $3 / 17 / 10$ & 0C17001 & AGG-RRL-002 \\
\hline $12587-46-1$ & Gross Alpha & $<4.26 \mathrm{E} 0$ & $\mathrm{pCi} / \mathrm{g}$ dry & $4.26 \mathrm{E} 0$ & & $3 / 17 / 10$ & 0C17001 & AGG-RRL-002 \\
\hline HEIS No. & B235L9 & \multicolumn{3}{|c|}{$\begin{array}{ll}\text { Lab ID: } & 1001004-98\end{array}$} & & & & \\
\hline $12587-47-2$ & Gross Beta & $<1.43 \mathrm{E} 1$ & pCi/g dry & $1.43 \mathrm{E} 1$ & & $3 / 17 / 10$ & 0C17001 & AGG-RRL-002 \\
\hline $12587-46-1$ & Gross Alpha & $<4.26 \mathrm{E} 0$ & pCi/g dry & 4.26E0 & & $3 / 17 / 10$ & 0C17001 & AGG-RRL-002 \\
\hline HEIS No. & B235N9 & \multicolumn{3}{|c|}{ Lab ID: $1001004-B C$} & & & & \\
\hline $12587-47-2$ & Gross Beta & $<1.42 \mathrm{E} 1$ & pCi/g dry & $1.42 \mathrm{E} 1$ & & $3 / 17 / 10$ & 0C17001 & AGG-RRL-002 \\
\hline $12587-46-1$ & Gross Alpha & $<4.25 \mathrm{E} 0$ & pCi/g dry & $4.25 \mathrm{E} 0$ & & $3 / 17 / 10$ & 0C17001 & AGG-RRL-002 \\
\hline HEIS No. & 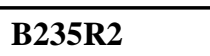 & \multicolumn{3}{|c|}{ Lab ID: $1001004-B V$} & & & & \\
\hline $12587-47-2$ & Gross Beta & $<1.42 \mathrm{E} 1$ & pCi/g dry & $1.42 \mathrm{E} 1$ & & $3 / 17 / 10$ & 0C17001 & AGG-RRL-002 \\
\hline $12587-46-1$ & Gross Alpha & $<4.25 \mathrm{E} 0$ & pCi/g dry & $4.25 \mathrm{E} 0$ & & $3 / 17 / 10$ & 0C17001 & AGG-RRL-002 \\
\hline HEIS No. & B235R9 & \multicolumn{3}{|c|}{ Lab ID: $1001004-C C$} & & & & \\
\hline $12587-47-2$ & Gross Beta & $<1.42 \mathrm{E} 1$ & pCi/g dry & $1.42 \mathrm{E} 1$ & & $3 / 17 / 10$ & 0C17001 & AGG-RRL-002 \\
\hline $12587-46-1$ & Gross Alpha & $<4.25 \mathrm{E} 0$ & pCi/g dry & $4.25 \mathrm{E} 0$ & & $3 / 17 / 10$ & 0C17001 & AGG-RRL-002 \\
\hline HEIS No. & 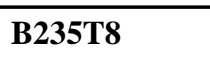 & \multicolumn{3}{|c|}{ Lab ID: $1001004-D A$} & & & & \\
\hline $12587-47-2$ & Gross Beta & $<1.42 \mathrm{E} 1$ & pCi/g dry & $1.42 \mathrm{E} 1$ & & $3 / 17 / 10$ & 0C17001 & AGG-RRL-002 \\
\hline $12587-46-1$ & Gross Alpha & $<4.25 \mathrm{E} 0$ & pCi/g dry & $4.25 \mathrm{E} 0$ & & $3 / 17 / 10$ & 0C17001 & AGG-RRL-002 \\
\hline
\end{tabular}


Wet Chemistry - Quality Control

Environmental Science Laboratory

\begin{tabular}{|lrrrrrrrrrrr}
\hline & & Reporting & & Spike & Source & & & $\%$ REC & & RPD & \\
Analyte & Result & Limit & Units & Level & Result & $\%$ REC & Limits & RPD & Limit & Notes \\
\hline
\end{tabular}

Batch 0C10003 - 1:1 Water Extract (pH_EC_Alk)

Blank (0C10003-BLK1)

Specific Conductance (EC)

$<1.00 \mathrm{E}-2 \quad 1.00 \mathrm{E}-2 \quad \mathrm{mS} / \mathrm{cm}$

Prepared \& Analyzed: 03/10/10

LCS (0C10003-BS1)

Specific Conductance (EC)

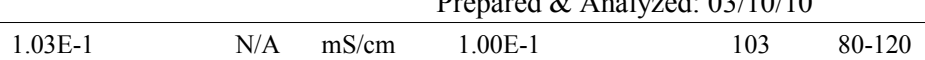

Duplicate (0C10003-DUP1)

Source: 1001004-42

Prepared \& Analyzed: 03/10/10

Specific Conductance (EC)

$\begin{array}{lll}4.51 \mathrm{E} 0 & 1.00 \mathrm{E}-2 \quad \mathrm{mS} / \mathrm{cm}\end{array}$

4.36E0

3.50

35

Batch 0C10004 - 1:1 Water Extract (pH_EC_Alk)

LCS (0C10004-BS1)

Prepared: 03/10/10 Analyzed: 03/19/10

$\mathrm{pH}$

$7.09 \mathrm{E} 0$

N/A $\quad$ pH Units $\quad 7.00 \mathrm{E} 0 \quad 101 \quad 80-120$

Duplicate (0C10004-DUP1)

Source: 1001004-42 Prepared \& Analyzed: 03/10/10

$\mathrm{pH}$

6.93E0 N/A pH Units

6.98E0

0.719

35

Batch 0D01005 - 1:1 Water Extract (pH_EC_Alk)

Blank (0D01005-BLK1)

Prepared: 04/01/10 Analyzed: 04/08/10

Alkalinity as $\mathrm{CaCO} 3$

$<2.35 \mathrm{E} 1$

$2.35 \mathrm{E} 1 \mathrm{ug} / \mathrm{g}$ wet

Duplicate (0D01005-DUP1)

Alkalinity as $\mathrm{CaCO} 3$

Source: 1001004-42

Prepared: 04/01/10 Analyzed: 04/08/10

$<2.41 \mathrm{E} 1 \quad 2.41 \mathrm{E} 1 \quad \mathrm{ug} / \mathrm{g}$ dry

ND

35 
Anions by Ion Chromatography - Quality Control

Environmental Science Laboratory

\begin{tabular}{|c|c|c|c|c|c|c|c|c|c|c|}
\hline Analyte & Result & $\begin{array}{r}\text { porting } \\
\text { Limit }\end{array}$ & Units & $\begin{array}{l}\text { Spike } \\
\text { Level }\end{array}$ & $\begin{array}{l}\text { Source } \\
\text { Result }\end{array}$ & $\%$ REC & $\begin{array}{l}\% \text { REC } \\
\text { Limits }\end{array}$ & RPD & $\begin{array}{l}\text { RPD } \\
\text { Limit }\end{array}$ & Notes \\
\hline
\end{tabular}

Batch 0C11004 - 1:1 Water Extract (IC)

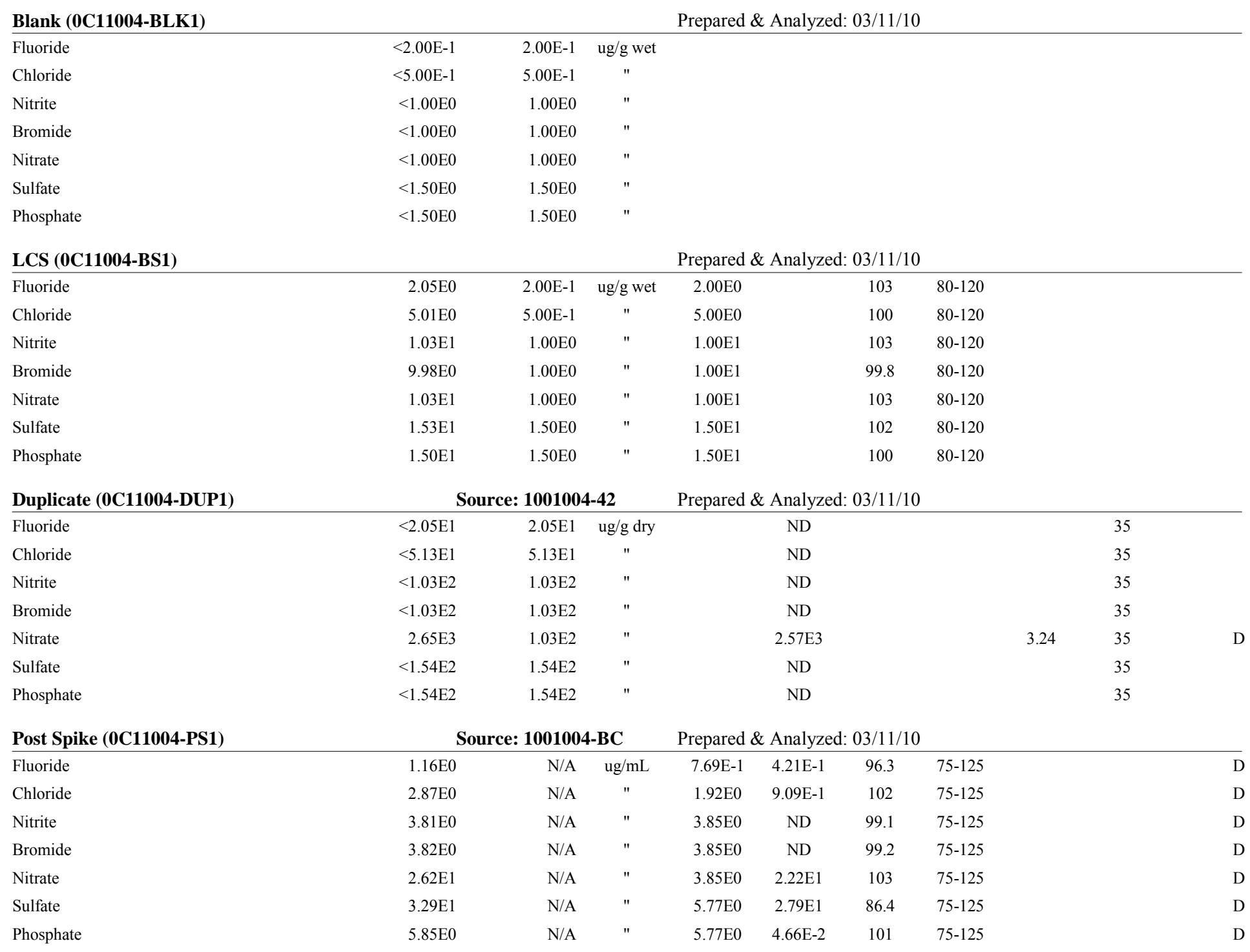


Cyanide by Mircodistillation/Colorimetric - Quality Control

Environmental Science Laboratory

\begin{tabular}{|lrrrrrrrrrrrr}
\hline & & Reporting & & Spike & Source & & & $\%$ REC & & RPD \\
Analyte & Result & Limit & Units & Level & Result & $\%$ REC & Limits & RPD & Limit & Notes
\end{tabular}

Batch 0D08003 - NO PREP

Blank (0D08003-BLK1)

\begin{tabular}{llll}
\hline Cyanide & $<1.00 \mathrm{E}-1$ & $1.00 \mathrm{E}-1$ & $\mathrm{ug} / \mathrm{g}$ wet
\end{tabular}

Prepared: 04/08/10 Analyzed: 04/09/10

LCS (0D08003-BS1)

Prepared: 04/08/10 Analyzed: 04/09/10

Cyanide

$1.00 \mathrm{E} 0 \quad 5.00 \mathrm{E}-1 \quad \mathrm{ug} / \mathrm{g}$ wet

$1.01 \mathrm{E} 0$

$99.5 \quad 80-120$

Duplicate (0D08003-DUP1)

Cyanide

Source: 1001004-DA

Prepared: 04/08/10 Analyzed: 04/09/10

$<1.06 \mathrm{E}-1 \quad 1.06 \mathrm{E}-1 \quad \mathrm{ug} / \mathrm{g}$ dry

ND

20 


\section{Hexavalent Chromium/Soil - Quality Control}

Environmental Science Laboratory

\begin{tabular}{|c|c|c|c|c|c|c|c|c|c|c|}
\hline & & orting & & Spike & Source & & $\%$ REC & & RPD & \\
\hline Analyte & Result & Limit & Units & Level & Result & $\%$ REC & Limits & RPD & Limit & Notes \\
\hline
\end{tabular}

Batch 0D09005 - Hexavalent Chromium Digest

Blank (0D09005-BLK1)

Chromium, Hexavalent Prepared: 03/19/10 Analyzed: 03/25/10

LCS (0D09005-BS1)

Chromium, Hexavalent

$<2.50 \mathrm{E} 0 \quad 2.50 \mathrm{E} 0 \quad \mathrm{ug} / \mathrm{g}$ wet

Prepared: 03/19/10 Analyzed: 03/25/10

Duplicate (0D09005-DUP1)

Chromium, Hexavalent Source: 1001004-04

Prepared: 03/19/10 Analyzed: 03/25/10

$<5.29 \mathrm{E}-1 \quad 5.29 \mathrm{E}-1 \quad \mathrm{ug} / \mathrm{g}$ dry

ND

20

Matrix Spike (0D09005-MS1)

Source: 1001004-22

Prepared: 03/19/10 Analyzed: 03/25/10

Chromium, Hexavalent

$\begin{array}{lllllll}2.43 \mathrm{E}-1 & 5.14 \mathrm{E}-1 & \mathrm{ug} / \mathrm{g} \text { dry } & 5.00 \mathrm{E}-1 & 6.00 \mathrm{E}-3 & 47.4 & 75-125\end{array}$


Total Metals by PNNL-AGG-ICP-AES/Water Extract - Quality Control

Environmental Science Laboratory

\begin{tabular}{|c|c|c|c|c|c|c|c|c|c|c|}
\hline Analyte & Result & $\begin{array}{r}\text { porting } \\
\text { Limit }\end{array}$ & Units & $\begin{array}{l}\text { Spike } \\
\text { Level }\end{array}$ & $\begin{array}{l}\text { Source } \\
\text { Result }\end{array}$ & $\%$ REC & $\begin{array}{l}\% \text { REC } \\
\text { Limits }\end{array}$ & RPD & $\begin{array}{l}\text { RPD } \\
\text { Limit }\end{array}$ & Notes \\
\hline
\end{tabular}

Batch 0C11001 - 1:1 Water Extract (ICP/ICPMS)

\begin{tabular}{|c|c|c|c|c|c|}
\hline Blank (0C11001-BLK1) & & & & Prepared: 03/10/10 & Analyzed: 03/11/10 \\
\hline Aluminum & $<9.37 \mathrm{E}-2$ & $9.37 \mathrm{E}-2$ & ug/g wet & & \\
\hline Arsenic & $<3.12 \mathrm{E}-1$ & $3.12 \mathrm{E}-1$ & $"$ & & \\
\hline Calcium & $<4.52 \mathrm{E}-1$ & $4.52 \mathrm{E}-1$ & $"$ & & \\
\hline Cadmium & $<1.04 \mathrm{E}-1$ & $1.04 \mathrm{E}-1$ & $"$ & & \\
\hline Chromium & $<2.84 \mathrm{E}-2$ & $2.84 \mathrm{E}-2$ & $"$ & & \\
\hline Copper & $<8.78 \mathrm{E}-2$ & $8.78 \mathrm{E}-2$ & $"$ & & \\
\hline Iron & $<1.05 \mathrm{E}-1$ & $1.05 \mathrm{E}-1$ & $"$ & & \\
\hline Potassium & $<1.29 \mathrm{E} 0$ & $1.29 \mathrm{E} 0$ & $"$ & & \\
\hline Magnesium & $<4.89 \mathrm{E}-2$ & 4.89E-2 & $"$ & & \\
\hline Manganese & $<6.25 \mathrm{E}-2$ & $6.25 \mathrm{E}-2$ & $"$ & & \\
\hline Nickel & $<9.67 \mathrm{E}-2$ & $9.67 \mathrm{E}-2$ & $"$ & & \\
\hline Lead & $<1.61 \mathrm{E}-1$ & $1.61 \mathrm{E}-1$ & $"$ & & \\
\hline Selenium & $<1.20 \mathrm{E} 0$ & $1.20 \mathrm{E} 0$ & $"$ & & \\
\hline Thallium & $<9.88 \mathrm{E}-1$ & $9.88 \mathrm{E}-1$ & $"$ & & \\
\hline Sodium & $<7.97 \mathrm{E}-1$ & 7.97E-1 & $"$ & & \\
\hline Silver & $<5.68 \mathrm{E}-2$ & $5.68 \mathrm{E}-2$ & $"$ & & \\
\hline LCS (0C11001-BS1) & & & & Prepared: 03/10/10 & Analyzed: 03/11/10 \\
\hline Aluminum & $4.74 \mathrm{E} 0$ & $9.37 \mathrm{E}-2$ & ug/g wet & $5.00 \mathrm{E} 0$ & $80-120$ \\
\hline Arsenic & $4.82 \mathrm{E} 0$ & $3.12 \mathrm{E}-1$ & $"$ & $5.00 \mathrm{E} 0$ & $80-120$ \\
\hline Calcium & 4.62E0 & $4.52 \mathrm{E}-1$ & $"$ & $5.00 \mathrm{E} 0$ & $80-120$ \\
\hline Cadmium & $4.94 \mathrm{E} 0$ & $1.04 \mathrm{E}-1$ & $"$ & $5.00 \mathrm{E} 0$ & $80-120$ \\
\hline Chromium & 4.98E0 & $2.84 \mathrm{E}-2$ & $"$ & $5.00 \mathrm{E} 0$ & $80-120$ \\
\hline Copper & 4.83E0 & $8.78 \mathrm{E}-2$ & $"$ & $5.00 \mathrm{E} 0$ & $80-120$ \\
\hline Iron & $4.76 \mathrm{E} 0$ & $1.05 \mathrm{E}-1$ & $"$ & $5.00 \mathrm{E} 0$ & $80-120$ \\
\hline Potassium & 4.63E1 & $1.29 \mathrm{E} 0$ & $"$ & $5.00 \mathrm{E} 1$ & $80-120$ \\
\hline Magnesium & $4.81 \mathrm{E} 0$ & $4.89 \mathrm{E}-2$ & $"$ & $5.00 \mathrm{E} 0$ & $80-120$ \\
\hline Manganese & $4.88 \mathrm{E} 0$ & $6.25 \mathrm{E}-2$ & $"$ & $5.00 \mathrm{E} 0$ & $80-120$ \\
\hline Nickel & 4.81E0 & $9.67 \mathrm{E}-2$ & $"$ & $5.00 \mathrm{E} 0$ & $80-120$ \\
\hline Lead & 4.92E0 & $1.61 \mathrm{E}-1$ & $"$ & $5.00 \mathrm{E} 0$ & $80-120$ \\
\hline Selenium & $5.01 \mathrm{E} 0$ & $1.20 \mathrm{E} 0$ & $"$ & $5.00 \mathrm{E} 0$ & $80-120$ \\
\hline Thallium & $4.84 \mathrm{E} 0$ & $9.88 \mathrm{E}-1$ & $"$ & $5.00 \mathrm{E} 0$ & $80-120$ \\
\hline Sodium & 4.88E0 & 7.97E-1 & $"$ & $5.00 \mathrm{E} 0$ & $80-120$ \\
\hline Silver & $4.88 \mathrm{E} 0$ & $5.68 \mathrm{E}-2$ & $"$ & $5.00 \mathrm{E} 0$ & $80-120$ \\
\hline
\end{tabular}


Total Metals by PNNL-AGG-ICP-AES/Water Extract - Quality Control

Environmental Science Laboratory

\begin{tabular}{|c|c|c|c|c|c|c|c|c|c|c|}
\hline Analyte & Result & $\begin{array}{r}\text { porting } \\
\text { Limit }\end{array}$ & Units & $\begin{array}{l}\text { Spike } \\
\text { Level }\end{array}$ & $\begin{array}{l}\text { Source } \\
\text { Result }\end{array}$ & $\%$ REC & $\begin{array}{l}\% \text { REC } \\
\text { Limits }\end{array}$ & RPD & $\begin{array}{l}\text { RPD } \\
\text { Limit }\end{array}$ & Notes \\
\hline
\end{tabular}

Batch 0C11001 - 1:1 Water Extract (ICP/ICPMS)

\begin{tabular}{|c|c|c|c|c|c|c|c|c|c|}
\hline \multirow{2}{*}{$\begin{array}{l}\text { Duplicate (0C11001-DUP1) } \\
\text { Aluminum }\end{array}$} & \multicolumn{3}{|c|}{ Source: 1001004-42 } & \multicolumn{2}{|c|}{ Prepared: 03/10/10 } & \multicolumn{2}{|c|}{ Analyzed: 03/11/10 } & & \multirow[b]{2}{*}{35} \\
\hline & $<9.61 \mathrm{E}-2$ & $9.61 \mathrm{E}-2$ & ug/g dry & & ND & & & & \\
\hline Arsenic & $<3.19 \mathrm{E}-1$ & $3.19 \mathrm{E}-1$ & $"$ & & ND & & & & 35 \\
\hline Calcium & 7.43E2 & $4.64 \mathrm{E}-1$ & $"$ & & $6.93 \mathrm{E} 2$ & & & 6.96 & 35 \\
\hline Cadmium & $<1.06 \mathrm{E}-1$ & $1.06 \mathrm{E}-1$ & $"$ & & ND & & & & 35 \\
\hline Chromium & $<2.91 \mathrm{E}-2$ & $2.91 \mathrm{E}-2$ & $"$ & & ND & & & & 35 \\
\hline Copper & $<9.01 \mathrm{E}-2$ & $9.01 \mathrm{E}-2$ & $"$ & & ND & & & & 35 \\
\hline Iron & $<1.08 \mathrm{E}-1$ & $1.08 \mathrm{E}-1$ & $"$ & & ND & & & & 35 \\
\hline Potassium & $2.43 \mathrm{E} 1$ & $1.32 \mathrm{E} 0$ & $"$ & & $2.34 \mathrm{E} 1$ & & & 3.97 & 35 \\
\hline Magnesium & $7.24 \mathrm{E} 1$ & $5.01 \mathrm{E}-2$ & $"$ & & $6.82 \mathrm{E} 1$ & & & 5.98 & 35 \\
\hline Manganese & $2.87 \mathrm{E}-1$ & $6.40 \mathrm{E}-2$ & $"$ & & $2.72 \mathrm{E}-1$ & & & 5.47 & 35 \\
\hline Nickel & $<9.92 \mathrm{E}-2$ & $9.92 \mathrm{E}-2$ & $"$ & & ND & & & & 35 \\
\hline Lead & $<1.65 \mathrm{E}-1$ & $1.65 \mathrm{E}-1$ & $"$ & & ND & & & & 35 \\
\hline Selenium & $<1.23 \mathrm{E} 0$ & $1.23 \mathrm{E} 0$ & $"$ & & ND & & & & 35 \\
\hline Thallium & $<1.01 \mathrm{E} 0$ & $1.01 \mathrm{E} 0$ & $"$ & & ND & & & & 35 \\
\hline Sodium & $1.96 \mathrm{E} 1$ & $8.17 \mathrm{E}-1$ & $"$ & & $1.88 \mathrm{E} 1$ & & & 3.99 & 35 \\
\hline Silver & $<5.82 \mathrm{E}-2$ & $5.82 \mathrm{E}-2$ & $"$ & & ND & & & & 35 \\
\hline Post Spike (0C11001-PS1) & \multicolumn{3}{|c|}{ Source: 1001004-42 } & \multicolumn{4}{|c|}{ Prepared \& Analyzed: 03/11/10 } & & \\
\hline Aluminum & $4.84 \mathrm{E} 2$ & N/A & $\mathrm{ug} / \mathrm{L}$ & $5.00 \mathrm{E} 2$ & ND & 102 & $75-125$ & & \\
\hline Arsenic & $4.94 \mathrm{E} 2$ & N/A & $"$ & $5.00 \mathrm{E} 2$ & ND & 103 & $75-125$ & & \\
\hline Calcium & $2.34 \mathrm{E} 5$ & N/A & $"$ & $5.00 \mathrm{E} 2$ & $2.31 \mathrm{E} 5$ & 562 & $75-125$ & & \\
\hline Cadmium & $2.49 \mathrm{E} 2$ & N/A & $"$ & $2.50 \mathrm{E} 2$ & ND & 100 & $75-125$ & & \\
\hline Chromium & $1.26 \mathrm{E} 2$ & N/A & $"$ & $1.25 \mathrm{E} 2$ & $1.57 \mathrm{E} 0$ & 99.3 & $75-125$ & & \\
\hline Copper & $5.09 \mathrm{E} 2$ & N/A & $"$ & $5.00 \mathrm{E} 2$ & $1.47 \mathrm{E} 0$ & 102 & $75-125$ & & \\
\hline Iron & $4.88 \mathrm{E} 2$ & N/A & $"$ & $5.00 \mathrm{E} 2$ & ND & 97.8 & $75-125$ & & \\
\hline Potassium & $9.19 \mathrm{E} 3$ & N/A & $"$ & $1.25 \mathrm{E} 3$ & $7.78 \mathrm{E} 3$ & 113 & $75-125$ & & \\
\hline Magnesium & $2.37 \mathrm{E} 4$ & N/A & $"$ & $5.00 \mathrm{E} 2$ & $2.27 \mathrm{E} 4$ & 207 & $75-125$ & & \\
\hline Manganese & $3.44 \mathrm{E} 2$ & N/A & $"$ & $2.50 \mathrm{E} 2$ & $9.04 \mathrm{E} 1$ & 101 & $75-125$ & & \\
\hline Nickel & $4.82 \mathrm{E} 2$ & N/A & $"$ & $5.00 \mathrm{E} 2$ & $1.00 \mathrm{E} 1$ & 94.4 & $75-125$ & & \\
\hline Lead & $4.44 \mathrm{E} 2$ & N/A & $"$ & $5.00 \mathrm{E} 2$ & ND & 101 & $75-125$ & & \\
\hline Selenium & $4.90 \mathrm{E} 2$ & N/A & $"$ & $5.00 \mathrm{E} 2$ & ND & 104 & $75-125$ & & \\
\hline Thallium & $2.04 \mathrm{E} 2$ & N/A & $"$ & $5.00 \mathrm{E} 2$ & ND & 100 & $75-125$ & & \\
\hline Sodium & $6.88 \mathrm{E} 3$ & N/A & $"$ & $5.00 \mathrm{E} 2$ & $6.27 \mathrm{E} 3$ & 123 & $75-125$ & & \\
\hline Silver & $5.11 \mathrm{E} 2$ & N/A & $"$ & $5.00 \mathrm{E} 2$ & ND & 103 & $75-125$ & & \\
\hline
\end{tabular}


Total Metals by PNNL-AGG-ICP-AES/Acid Extract - Quality Control

Environmental Science Laboratory

\begin{tabular}{|lrrrrrrrrrrrr}
\hline & & Reporting & & Spike & Source & & & $\%$ REC & & RPD \\
Analyte & Result & Limit & Units & Level & Result & $\%$ REC & Limits & RPD & Limit & Notes \\
\hline
\end{tabular}

Batch 0C11002 - ASTM D 5198 (ICP/ICPMS)

\begin{tabular}{|c|c|c|c|c|c|}
\hline Blank (0C11002-BLK1) & & & & Prepared: 03/08/10 & Analyzed: 03/11/10 \\
\hline Aluminum & $<6.76 \mathrm{E} 0$ & $6.76 \mathrm{E} 0$ & ug/g wet & & \\
\hline Calcium & $<7.02 \mathrm{E} 0$ & 7.02E0 & $"$ & & \\
\hline Cadmium & $<3.68 \mathrm{E}-1$ & $3.68 \mathrm{E}-1$ & $"$ & & \\
\hline Chromium & $<5.90 \mathrm{E}-1$ & $5.90 \mathrm{E}-1$ & $"$ & & \\
\hline Copper & $<1.93 \mathrm{E} 0$ & $1.93 \mathrm{E} 0$ & $"$ & & \\
\hline Iron & $<1.82 \mathrm{E} 0$ & $1.82 \mathrm{E} 0$ & $"$ & & \\
\hline Potassium & $<2.59 \mathrm{E} 1$ & $2.59 \mathrm{E} 1$ & $"$ & & \\
\hline Magnesium & $<1.92 \mathrm{E} 0$ & $1.92 \mathrm{E} 0$ & $"$ & & \\
\hline Manganese & $<6.04 \mathrm{E}-1$ & $6.04 \mathrm{E}-1$ & $"$ & & \\
\hline Nickel & $<1.40 \mathrm{E} 0$ & $1.40 \mathrm{E} 0$ & $"$ & & \\
\hline Lead & $<3.02 \mathrm{E} 0$ & $3.02 \mathrm{E} 0$ & $"$ & & \\
\hline Selenium & $<1.72 \mathrm{E} 1$ & $1.72 \mathrm{E} 1$ & $"$ & & \\
\hline Thallium & $<9.70 \mathrm{E} 0$ & $9.70 \mathrm{E} 0$ & $"$ & & \\
\hline Sodium & $<1.90 \mathrm{E} 1$ & $1.90 \mathrm{E} 1$ & $"$ & & \\
\hline LCS (0C11002-BS1) & & & & Prepared: 03/08/10 & Analyzed: 03/11/10 \\
\hline Aluminum & 5.31E0 & $6.76 \mathrm{E}-1$ & ug/g wet & $5.87 \mathrm{E} 0$ & $80-120$ \\
\hline Calcium & $5.68 \mathrm{E} 0$ & $7.02 \mathrm{E}-1$ & $"$ & $5.87 \mathrm{E} 0$ & $80-120$ \\
\hline Cadmium & 5.49E0 & $3.68 \mathrm{E}-2$ & $"$ & $5.87 \mathrm{E} 0$ & $80-120$ \\
\hline Chromium & $5.87 \mathrm{E} 0$ & $5.90 \mathrm{E}-2$ & $"$ & $5.87 \mathrm{E} 0$ & $80-120$ \\
\hline Copper & $5.65 \mathrm{E} 0$ & $1.93 \mathrm{E}-1$ & $"$ & $5.87 \mathrm{E} 0$ & $80-120$ \\
\hline Iron & $5.69 \mathrm{E} 0$ & $1.82 \mathrm{E}-1$ & $"$ & $5.87 \mathrm{E} 0$ & $80-120$ \\
\hline Potassium & $5.58 \mathrm{E} 1$ & $2.59 \mathrm{E} 0$ & $"$ & $5.87 \mathrm{E} 1$ & $80-120$ \\
\hline Magnesium & $5.40 \mathrm{E} 0$ & $1.92 \mathrm{E}-1$ & $"$ & $5.87 \mathrm{E} 0$ & $80-120$ \\
\hline Manganese & $5.72 \mathrm{E} 0$ & $6.04 \mathrm{E}-2$ & $"$ & $5.87 \mathrm{E} 0$ & $80-120$ \\
\hline Nickel & $5.61 \mathrm{E} 0$ & $1.40 \mathrm{E}-1$ & $"$ & $5.87 \mathrm{E} 0$ & $80-120$ \\
\hline Lead & $5.69 \mathrm{E} 0$ & $3.02 \mathrm{E}-1$ & $"$ & $5.87 \mathrm{E} 0$ & $80-120$ \\
\hline Selenium & $5.34 \mathrm{E} 0$ & $1.72 \mathrm{E} 0$ & $"$ & $5.87 \mathrm{E} 0$ & $80-120$ \\
\hline Thallium & $5.67 \mathrm{E} 0$ & $9.70 \mathrm{E}-1$ & $"$ & $5.87 \mathrm{E} 0$ & $80-120$ \\
\hline Sodium & 5.89E0 & $1.90 \mathrm{E} 0$ & $"$ & $5.87 \mathrm{E} 0$ & $80-120$ \\
\hline
\end{tabular}


Total Metals by PNNL-AGG-ICP-AES/Acid Extract - Quality Control

Environmental Science Laboratory

\begin{tabular}{|lrrrrrrrrrrrr}
\hline & & Reporting & & Spike & Source & & & $\%$ REC & & RPD \\
Analyte & Result & Limit & Units & Level & Result & $\%$ REC & Limits & RPD & Limit & Notes \\
\hline
\end{tabular}

Batch 0C11002 - ASTM D 5198 (ICP/ICPMS)

\begin{tabular}{|c|c|c|c|c|c|c|c|c|c|}
\hline \multirow{2}{*}{$\begin{array}{l}\text { Duplicate (0C11002-DUP1) } \\
\text { Aluminum }\end{array}$} & \multicolumn{3}{|c|}{ Source: $1001004-42$} & \multicolumn{2}{|c|}{ Prepared: 03/08/10 } & \multicolumn{2}{|c|}{ Analyzed: 03/11/10 } & \multirow[b]{2}{*}{14.7} & \multirow[b]{2}{*}{35} \\
\hline & $4.48 \mathrm{E} 3$ & $2.44 \mathrm{E} 1$ & ug/g dry & & $3.87 \mathrm{E} 3$ & & & & \\
\hline Calcium & $4.45 \mathrm{E} 3$ & $2.54 \mathrm{E} 1$ & $"$ & & $4.59 \mathrm{E} 3$ & & & 2.95 & 35 \\
\hline Cadmium & $<1.33 \mathrm{E} 0$ & $1.33 \mathrm{E} 0$ & $"$ & & ND & & & & 35 \\
\hline Chromium & $8.68 \mathrm{E} 0$ & $2.13 \mathrm{E} 0$ & $"$ & & $8.70 \mathrm{E} 0$ & & & 0.217 & 35 \\
\hline Copper & $<6.99 \mathrm{E} 0$ & $6.99 \mathrm{E} 0$ & $"$ & & ND & & & & 35 \\
\hline Iron & $8.96 \mathrm{E} 3$ & $6.57 \mathrm{E} 0$ & $"$ & & $9.15 \mathrm{E} 3$ & & & 2.14 & 35 \\
\hline Potassium & $9.12 \mathrm{E} 2$ & 9.35E1 & $"$ & & $9.35 \mathrm{E} 2$ & & & 2.49 & 35 \\
\hline Magnesium & $3.33 \mathrm{E} 3$ & $6.96 \mathrm{E} 0$ & $"$ & & 3.37E3 & & & 1.18 & 35 \\
\hline Manganese & $1.72 \mathrm{E} 2$ & $2.18 \mathrm{E} 0$ & $"$ & & $1.77 \mathrm{E} 2$ & & & 2.88 & 35 \\
\hline Nickel & $9.10 \mathrm{E} 0$ & $5.07 \mathrm{E} 0$ & $"$ & & $9.21 \mathrm{E} 0$ & & & 1.22 & 35 \\
\hline Lead & $<1.09 \mathrm{E} 1$ & $1.09 \mathrm{E} 1$ & $"$ & & ND & & & & 35 \\
\hline Selenium & $<6.20 \mathrm{E} 1$ & $6.20 \mathrm{E} 1$ & $"$ & & ND & & & & 35 \\
\hline Thallium & $<3.51 \mathrm{E} 1$ & $3.51 \mathrm{E} 1$ & $"$ & & ND & & & & 35 \\
\hline Sodium & $9.88 \mathrm{E} 1$ & $6.89 \mathrm{E} 1$ & $"$ & & $9.80 \mathrm{E} 1$ & & & 0.876 & 35 \\
\hline Post Spike (0C11002-PS1) & \multicolumn{3}{|c|}{ Source: 1001004-42 } & \multicolumn{4}{|c|}{ Prepared \& Analyzed: 03/11/10 } & & \\
\hline Aluminum & $2.16 \mathrm{E} 4$ & N/A & $\mathrm{ug} / \mathrm{L}$ & $5.00 \mathrm{E} 2$ & $2.11 \mathrm{E} 4$ & 107 & $75-125$ & & \\
\hline Calcium & $2.55 \mathrm{E} 4$ & N/A & $"$ & $5.00 \mathrm{E} 2$ & $2.50 \mathrm{E} 4$ & 101 & $75-125$ & & \\
\hline Cadmium & $2.44 \mathrm{E} 2$ & N/A & $"$ & $2.50 \mathrm{E} 2$ & ND & 97.8 & $75-125$ & & \\
\hline Chromium & $1.72 \mathrm{E} 2$ & N/A & $"$ & $1.25 \mathrm{E} 2$ & 4.74E1 & 100 & $75-125$ & & \\
\hline Copper & $5.21 \mathrm{E} 2$ & N/A & " & $5.00 \mathrm{E} 2$ & 3.03E1 & 98.2 & $75-125$ & & \\
\hline Iron & $5.00 \mathrm{E} 4$ & N/A & $"$ & $5.00 \mathrm{E} 2$ & 4.99E4 & 24.8 & $75-125$ & & \\
\hline Potassium & $6.31 \mathrm{E} 3$ & N/A & $"$ & $1.25 \mathrm{E} 3$ & $5.09 \mathrm{E} 3$ & 97.1 & $75-125$ & & \\
\hline Magnesium & $1.88 \mathrm{E} 4$ & N/A & $"$ & $5.00 \mathrm{E} 2$ & $1.84 \mathrm{E} 4$ & 90.7 & $75-125$ & & \\
\hline Manganese & $1.22 \mathrm{E} 3$ & N/A & $"$ & $2.50 \mathrm{E} 2$ & 9.67E2 & 101 & $75-125$ & & \\
\hline Nickel & 5.27E2 & N/A & $"$ & $5.00 \mathrm{E} 2$ & $5.02 \mathrm{E} 1$ & 95.4 & $75-125$ & & \\
\hline Lead & 4.93E2 & N/A & $"$ & $5.00 \mathrm{E} 2$ & ND & 100 & $75-125$ & & \\
\hline Selenium & $4.38 \mathrm{E} 2$ & N/A & $"$ & $5.00 \mathrm{E} 2$ & ND & 90.1 & $75-125$ & & \\
\hline Thallium & $4.01 \mathrm{E} 2$ & N/A & $"$ & $5.00 \mathrm{E} 2$ & ND & 97.5 & $75-125$ & & \\
\hline Sodium & $1.05 \mathrm{E} 3$ & N/A & $"$ & $5.00 \mathrm{E} 2$ & $5.34 \mathrm{E} 2$ & 102 & $75-125$ & & \\
\hline
\end{tabular}


Radionuclides by ICP-MS/Acid Extract - Quality Control

Environmental Science Laboratory

\begin{tabular}{|c|c|c|c|c|c|c|c|c|c|c|}
\hline & & orting & & Spike & Source & & $\%$ REC & & RPD & \\
\hline Analyte & Result & Limit & Units & Level & Result & $\%$ REC & Limits & RPD & Limit & Notes \\
\hline
\end{tabular}

Batch 0C17004 - ASTM D 5198 (ICP/ICPMS)

Blank (0C17004-BLK1)

Technetium-99

Uranium 238

$<1.47 \mathrm{E}-3 \quad 1.47 \mathrm{E}-3 \quad$ ug/g wet

Prepared: 03/12/10 Analyzed: 04/04/10

Duplicate (0C17004-DUP1)

$<1.00 \mathrm{E}-2 \quad 1.00 \mathrm{E}-2 \quad "$

Technetium-99

Source: 1001004-42

Prepared: 03/12/10 Analyzed: 04/04/10

Uranium 238

$<2.66 \mathrm{E}-2 \quad 2.66 \mathrm{E}-2 \quad \mathrm{ug} / \mathrm{g}$ dry

$1.57 \mathrm{E} 1$

3.62E-2

$\mathrm{ND}$

35

Post Spike (0C17004-PS1)

Source: 1001004-42

Prepared: 03/12/10 Analyzed: 04/04/10

Technetium-99

5.84E-1

N/A ug/L

$5.00 \mathrm{E}-1$

$117 \quad 75-125$

Uranium 238

$4.25 \mathrm{E} 1$

N/A

$5.00 \mathrm{E}-1 \quad 4.21 \mathrm{E} 1$

84.7

$75-125$




\section{Radionuclides by ICP-MS/Water Extract - Quality Control \\ Environmental Science Laboratory}

\begin{tabular}{|c|c|c|c|c|c|c|c|c|c|c|}
\hline & & oorting & & Spike & Source & & $\%$ REC & & RPD & \\
\hline Analyte & Result & Limit & Units & Level & Result & $\%$ REC & Limits & RPD & Limit & Notes \\
\hline
\end{tabular}

\section{Batch 0C17003 - 1:1 Water Extract (ICP/ICPMS)}

Blank (0C17003-BLK1)

Technetium-99

Uranium 238

Duplicate (0C17003-DUP1)

Technetium-99

Uranium 238

Post Spike (0C17003-PS1)

Technetium-99

Uranium 238

Batch 0C31001 - 1:1 Water Extract (ICP/ICPMS)

Duplicate (0C31001-DUP1)

Iodine-129

Post Spike (0C31001-PS1)

Iodine-129
Prepared: 03/12/10 Analyzed: 03/22/10

$\begin{array}{llc}<3.90 \mathrm{E}-5 & 3.90 \mathrm{E}-5 & \text { ug } / \mathrm{g} \text { wet } \\ <8.00 \mathrm{E}-5 & 8.00 \mathrm{E}-5 & "\end{array}$

Source: 1001004-42

Prepared: 03/12/10 Analyzed: 03/22/10

$\begin{array}{cccccc}<4.00 \mathrm{E}-5 & 4.00 \mathrm{E}-5 & \text { ug/g dry } & \text { ND } & 35 \\ 1.77 \mathrm{E} 0 & 1.64 \mathrm{E}-3 & \mu & 154 \mathrm{E} 0 & 13.8 & 35\end{array}$

Source: 1001004-42 Prepared: 03/12/10 Analyzed: 03/22/10

$\begin{array}{lllllll}5.20 \mathrm{E}-1 & \mathrm{~N} / \mathrm{A} & \mathrm{ug} / \mathrm{L} & 5.00 \mathrm{E}-1 & \mathrm{ND} & 110 & 75-125\end{array}$

$\begin{array}{llllll}1.63 \mathrm{E} 1 & \mathrm{~N} / \mathrm{A} & \quad & 2.50 \mathrm{E}-2 & 1.54 \mathrm{E} 1 \quad \mathrm{NR} & 75-125\end{array}$

Source: 1001004-42 Prepared \& Analyzed: 05/03/10

$\begin{array}{llllll}1.01 \mathrm{E}-4 & 5.13 \mathrm{E}-5 & \mathrm{ug} / \mathrm{g} \text { dry } & 8.39 \mathrm{E}-5 & 18.8 & 35\end{array}$

Source: 1001004-42 Prepared \& Analyzed: 05/03/10

$\begin{array}{lllllll}1.06 \mathrm{E}-1 & \mathrm{~N} / \mathrm{A} & \mathrm{ug} / \mathrm{L} & 1.00 \mathrm{E}-1 & 1.68 \mathrm{E}-2 & 89 & 75-125\end{array}$ 
RCRA Metals By PNNL-AGG-415/Water Extract - Quality Control

Environmental Science Laboratory

\begin{tabular}{|c|c|c|c|c|c|c|c|c|c|c|}
\hline & & oorting & & Spike & Source & & $\%$ REC & & RPD & \\
\hline Analyte & Result & Limit & Units & Level & Result & $\%$ REC & Limits & RPD & Limit & Notes \\
\hline
\end{tabular}

Batch 0C10007 - 1:1 Water Extract (ICP/ICPMS)

Blank (0C10007-BLK1)

Antimony

Mercury

Duplicate (0C10007-DUP1)

Antimony

Mercury

Post Spike (0C10007-PS1)

Antimony

Mercury

$\begin{array}{ccc}<6.58 \mathrm{E}-4 & 6.58 \mathrm{E}-4 & \text { ug/g wet } \\ <6.54 \mathrm{E}-4 & 6.54 \mathrm{E}-4 & "\end{array}$

Prepared \& Analyzed: 03/10/10

Source: 1001004-42

$<6.75 \mathrm{E}-4 \quad 6.75 \mathrm{E}-4 \quad$ ug/g dry

$1.47 \mathrm{E}-3$

Source: 1001004-42

$5.16 \mathrm{E} 0$

$5.39 \mathrm{E} 0$

\begin{tabular}{lccccc}
\multicolumn{1}{c}{ Prepared \& } & Analyzed: $03 / 10 / 10$ \\
N/A & ug/L & $5.00 \mathrm{E} 0$ & $2.42 \mathrm{E}-2$ & 103 & $75-125$ \\
N/A & $"$ & $5.00 \mathrm{E} 0$ & ND & 108 & $75-125$
\end{tabular}

Prepared \& Analyzed: 03/10/10

$\begin{array}{ll}\mathrm{ND} & 35 \\ \mathrm{ND} & 35\end{array}$


RCRA Metals By PNNL-AGG-415/Acid Extract - Quality Control

Environmental Science Laboratory

\begin{tabular}{|lrrrrrrrrrrr}
\hline & & Reporting & & Spike & Source & & & $\%$ REC & & RPD & \\
Analyte & Result & Limit & Units & Level & Result & $\%$ REC & Limits & RPD & Limit & Notes \\
\hline
\end{tabular}

Batch 0C10008 - ASTM D 5198 (ICP/ICPMS)

\begin{tabular}{|c|c|c|c|c|c|c|c|c|}
\hline \multirow{2}{*}{$\begin{array}{l}\text { Blank (0C10008-BLK1) } \\
\text { Arsenic }\end{array}$} & \multicolumn{8}{|c|}{ Prepared \& Analyzed: 03/11/10 } \\
\hline & $<8.20 \mathrm{E}-2$ & $8.20 \mathrm{E}-2$ & ug/g wet & & & & & \\
\hline Antimony & $<5.26 \mathrm{E}-2$ & $5.26 \mathrm{E}-2$ & $"$ & & & & & \\
\hline Mercury & $<4.58 \mathrm{E}-2$ & $4.58 \mathrm{E}-2$ & $"$ & & & & & \\
\hline LCS (0C10008-BS1) & \multicolumn{8}{|c|}{ Prepared \& Analyzed: 03/11/10 } \\
\hline Arsenic & 5.49E0 & $4.10 \mathrm{E}-1$ & $\mathrm{ug} / \mathrm{g}$ wet & $5.87 \mathrm{E} 0$ & & 93.5 & $80-120$ & \\
\hline Duplicate (0C10008-DUP1) & \multicolumn{3}{|c|}{ Source: $1001004-42$} & \multicolumn{5}{|c|}{ Prepared \& Analyzed: 03/11/10 } \\
\hline Arsenic & $2.68 \mathrm{E} 0$ & $2.96 \mathrm{E}-1$ & ug/g dry & & $2.78 \mathrm{E} 0$ & & 3.91 & 35 \\
\hline Antimony & $<1.90 \mathrm{E}-1$ & $1.90 \mathrm{E}-1$ & $"$ & & ND & & & 35 \\
\hline Mercury & $<1.66 \mathrm{E}-1$ & $1.66 \mathrm{E}-1$ & $"$ & & ND & & & 35 \\
\hline Post Spike (0C10008-PS1) & \multicolumn{3}{|c|}{ Source: 1001004-42 } & \multicolumn{4}{|c|}{ Prepared \& Analyzed: 03/11/10 } & \\
\hline Arsenic & $1.15 \mathrm{E} 1$ & $\mathrm{~N} / \mathrm{A}$ & $\mathrm{ug} / \mathrm{L}$ & $5.00 \mathrm{E} 0$ & $7.58 \mathrm{E} 0$ & 78.2 & $75-125$ & \\
\hline Antimony & $5.35 \mathrm{E} 0$ & $\mathrm{~N} / \mathrm{A}$ & $"$ & $5.00 \mathrm{E} 0$ & $1.38 \mathrm{E}-1$ & 104 & $75-125$ & \\
\hline Mercury & $4.55 \mathrm{E} 0$ & $\mathrm{~N} / \mathrm{A}$ & $"$ & $5.00 \mathrm{E} 0$ & $2.97 \mathrm{E}-2$ & 90.4 & $75-125$ & \\
\hline
\end{tabular}


Total Alpha Total Beta/Acid Extract - Quality Control

Environmental Science Laboratory

\begin{tabular}{|c|c|c|c|c|c|c|c|c|c|c|}
\hline Analyte & Result & $\begin{array}{r}\text { porting } \\
\text { Limit }\end{array}$ & Units & $\begin{array}{l}\text { Spike } \\
\text { Level }\end{array}$ & $\begin{array}{l}\text { Source } \\
\text { Result }\end{array}$ & $\%$ REC & $\begin{array}{l}\text { \% REC } \\
\text { Limits }\end{array}$ & RPD & $\begin{array}{l}\text { RPD } \\
\text { Limit }\end{array}$ & Notes \\
\hline
\end{tabular}

Batch 0C17002 - ASTM D 5198 (RadChem)

Blank (0C17002-BLK1)

Gross Beta

Gross Alpha

Duplicate (0C17002-DUP1)

Gross Beta

Gross Alpha

\section{(1)

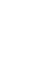

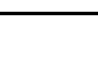

\begin{tabular}{ccc}
$<1.34 \mathrm{E} 1$ & \multicolumn{2}{c}{ Prer } \\
$<4.47 \mathrm{E} 0$ & $4.34 \mathrm{E} 1$ & $\mathrm{pCi} / \mathrm{g}$ wet
\end{tabular}

Prepared \& Analyzed: 03/17/10

Source: 1001004-42 Prepared \& Analyzed: 03/17/10

\begin{tabular}{|c|c|c|c|c|}
\hline \multicolumn{3}{|c|}{ Source: 1001004-42 } & \multicolumn{2}{|l|}{ Prepared \& Analyzed: 03/17/10 } \\
\hline$<4.84 \mathrm{E} 1$ & 4.84E1 & $\mathrm{pCi} / \mathrm{g}$ dry & ND & 35 \\
\hline$<1.61 \mathrm{E} 1$ & $1.61 \mathrm{E} 1$ & $"$ & ND & 35 \\
\hline
\end{tabular}


Total Alpha Total Beta/Water Extract - Quality Control

Environmental Science Laboratory

\begin{tabular}{|c|c|c|c|c|c|c|c|c|c|c|}
\hline & & oorting & & Spike & Source & & $\%$ REC & & RPD & \\
\hline Analyte & Result & Limit & Units & Level & Result & $\%$ REC & Limits & RPD & Limit & Notes \\
\hline
\end{tabular}

Batch 0C17001 - 1:1 Water Extract (RadChem)

Blank (0C17001-BLK1)

Gross Beta

Gross Alpha

$\begin{array}{llc}<1.43 \mathrm{E} 1 & 1.43 \mathrm{E} 1 & \text { pCi } / \text { g wet } \\ <4.26 \mathrm{E} 0 & 4.26 \mathrm{E} 0 & "\end{array}$

Prepared: 03/16/10 Analyzed: 03/17/10

Duplicate (0C17001-DUP1)

Gross Beta

Source: 1001004-42

Prepared: 03/16/10 Analyzed: 03/17/10

Gross Alpha

$<1.46 \mathrm{E} 1$

$1.46 \mathrm{E} 1 \mathrm{pCi} / \mathrm{g}$ dry

ND

35

$<4.36 \mathrm{E} 0$

$4.36 \mathrm{E} 0$

ND

35 


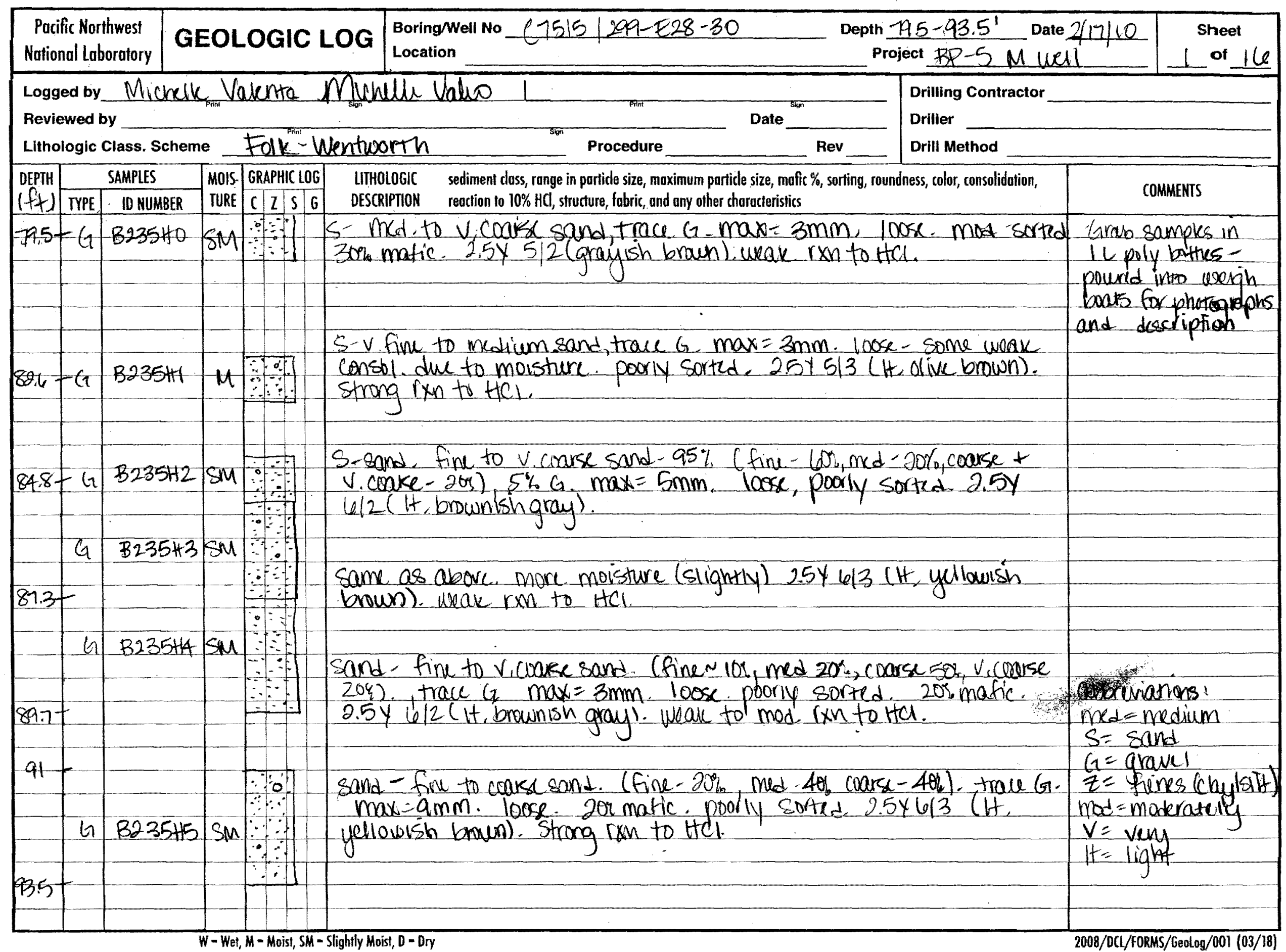




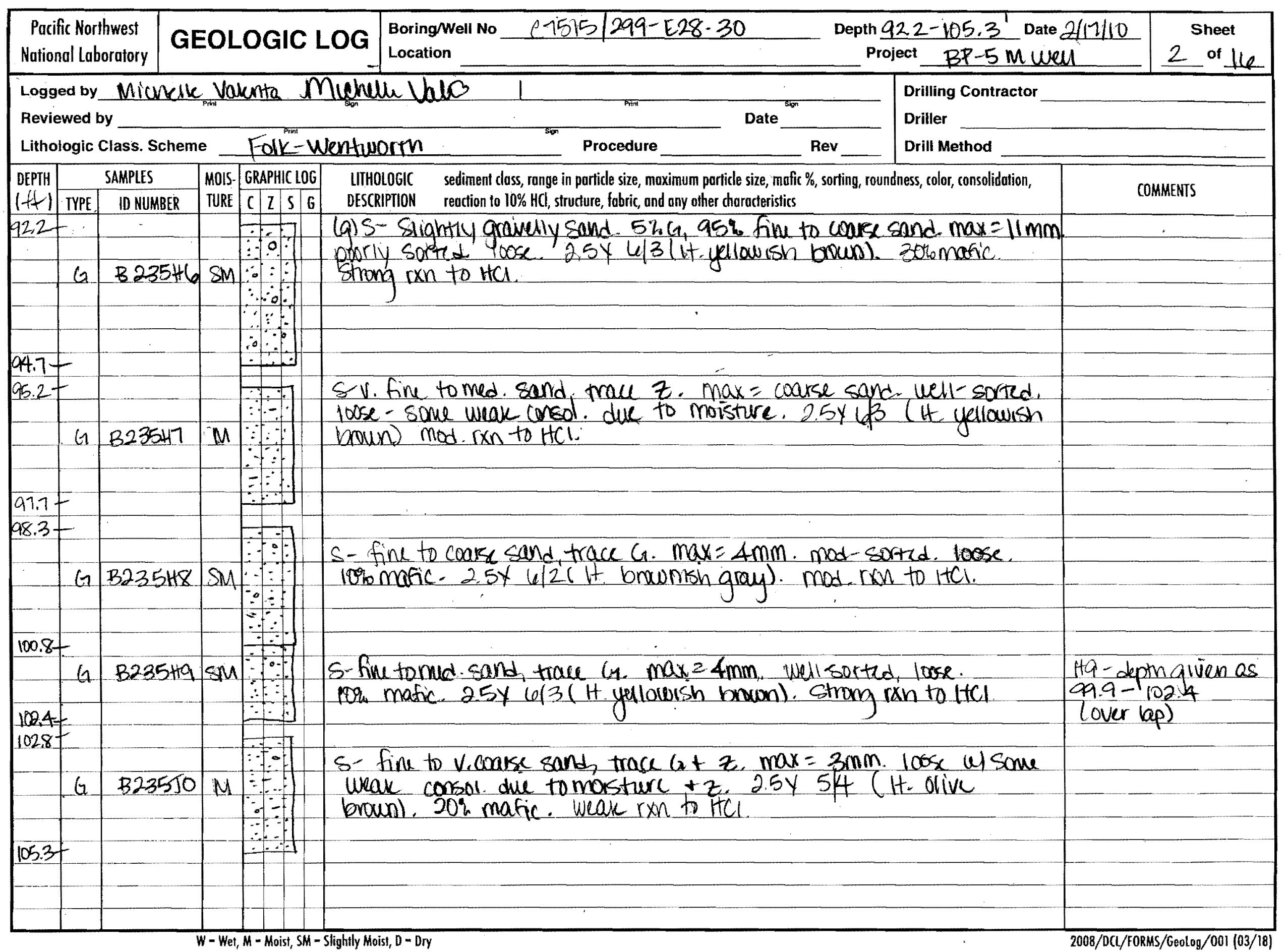




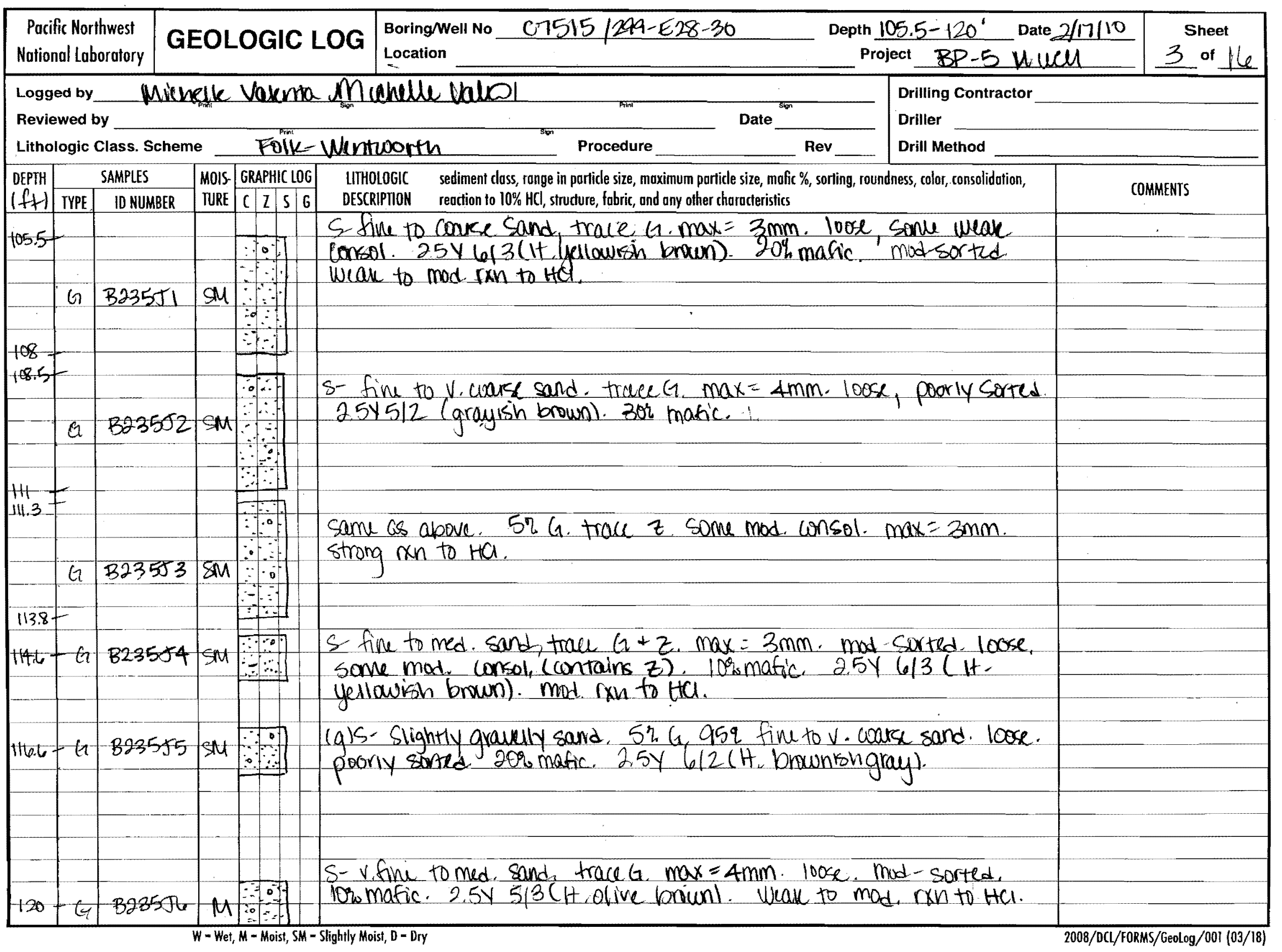




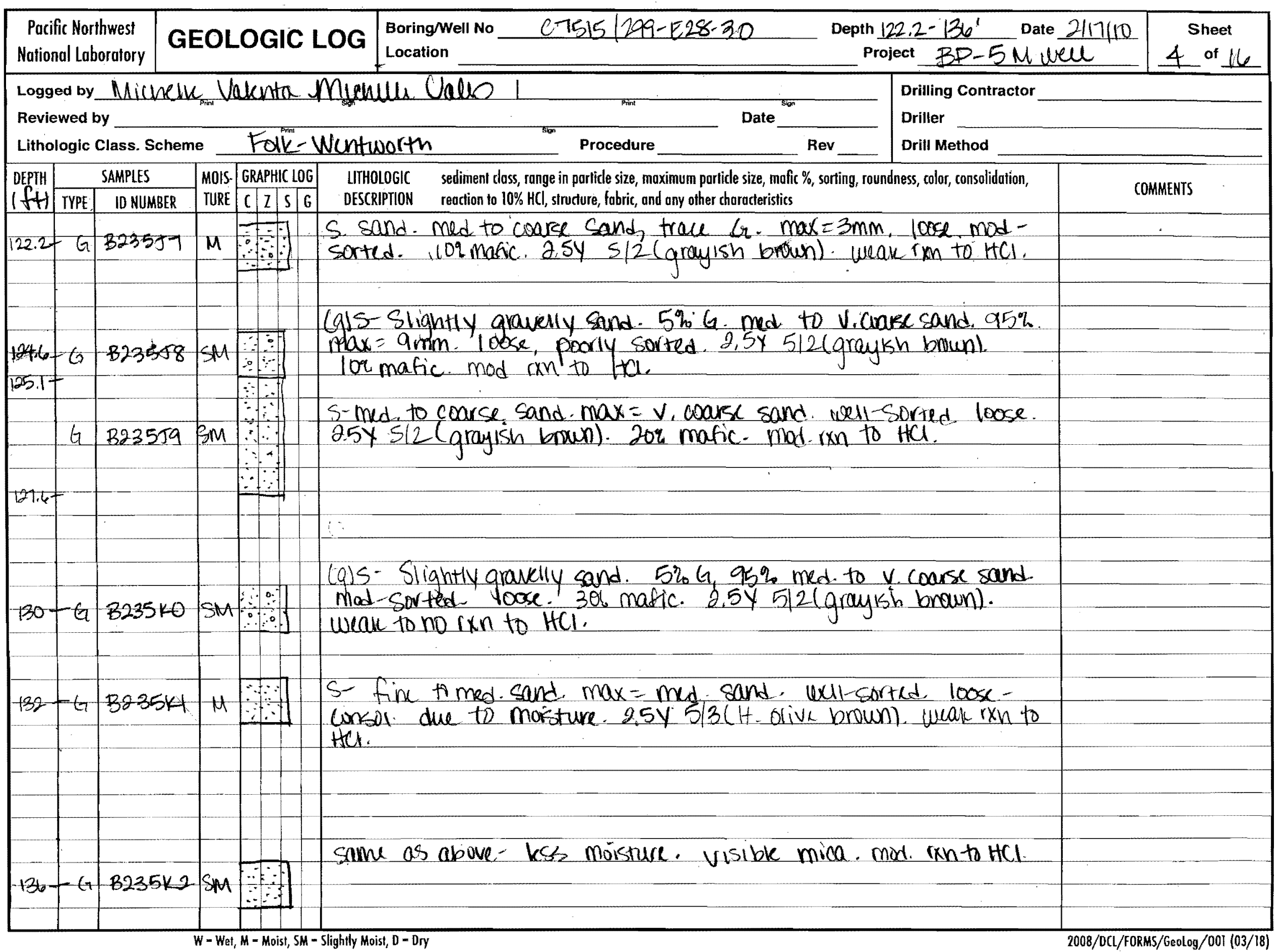




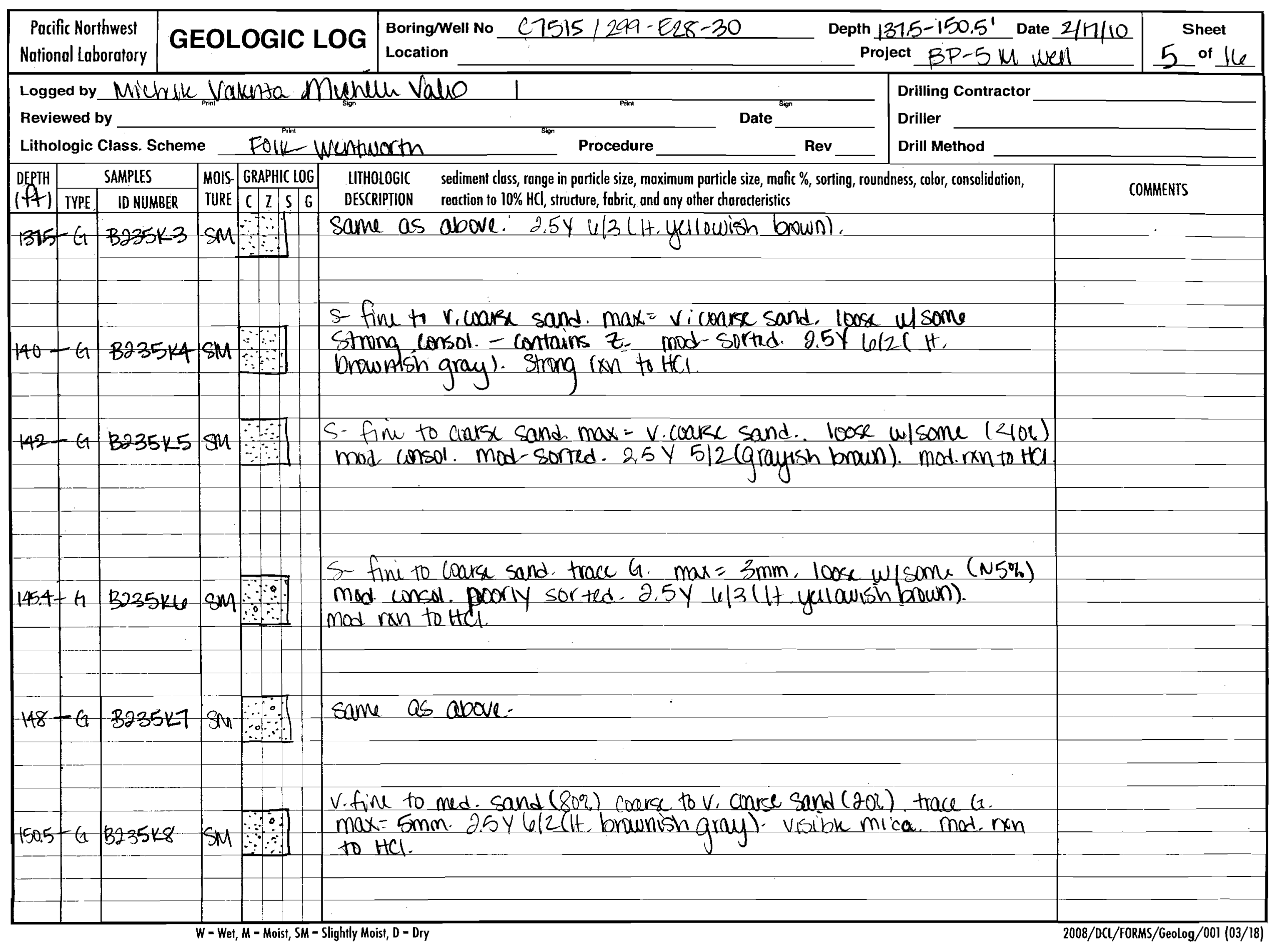




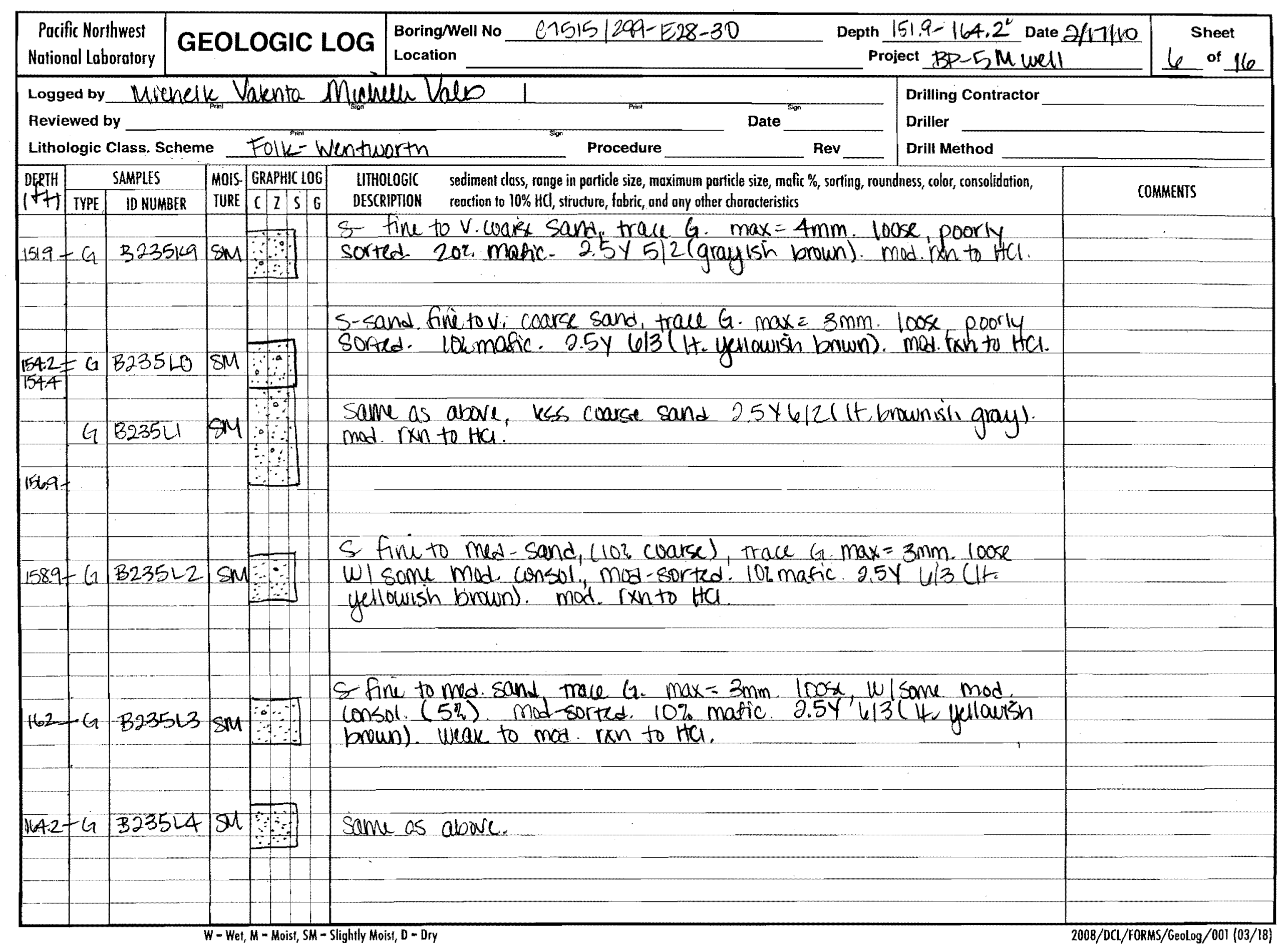




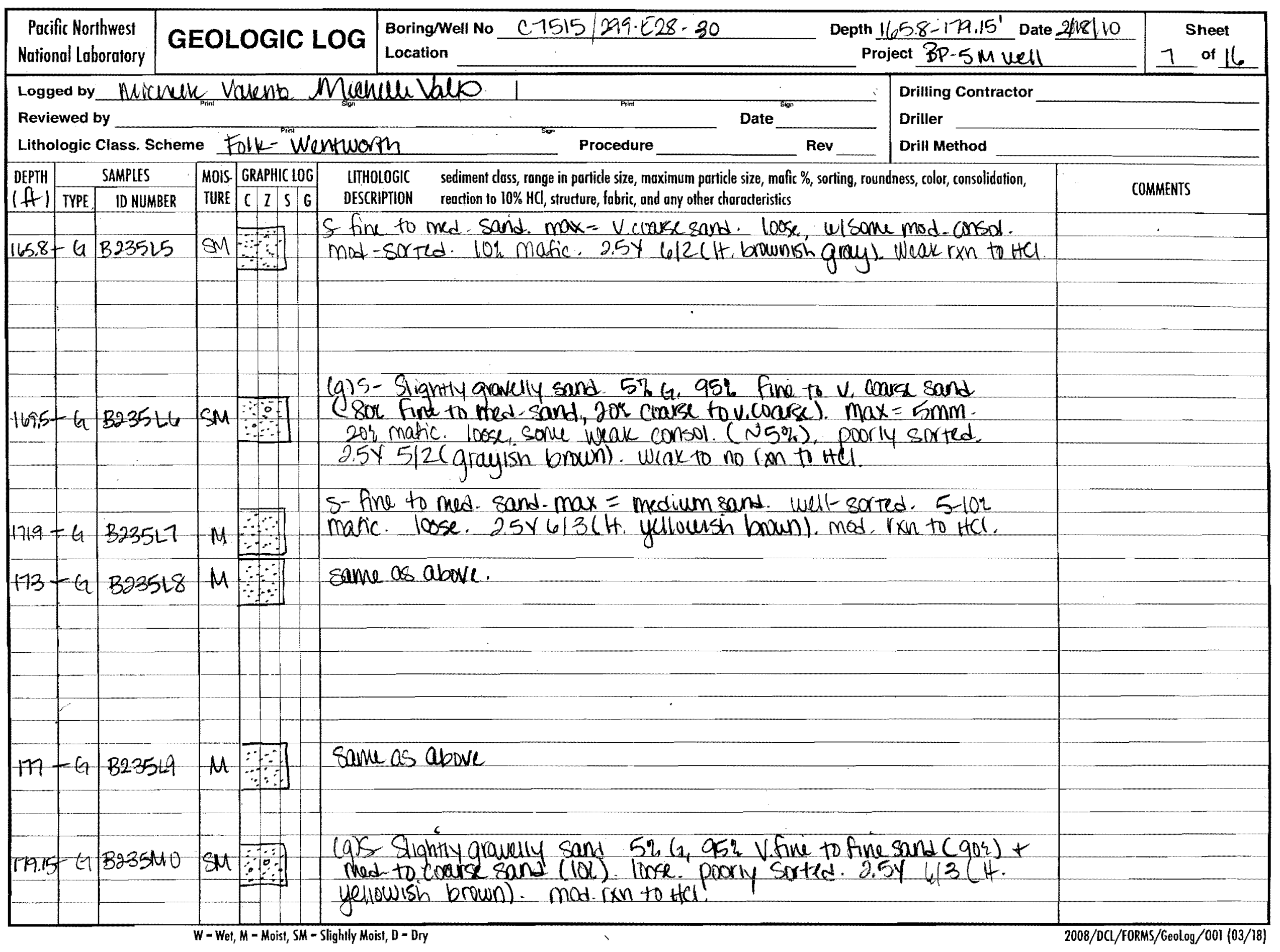




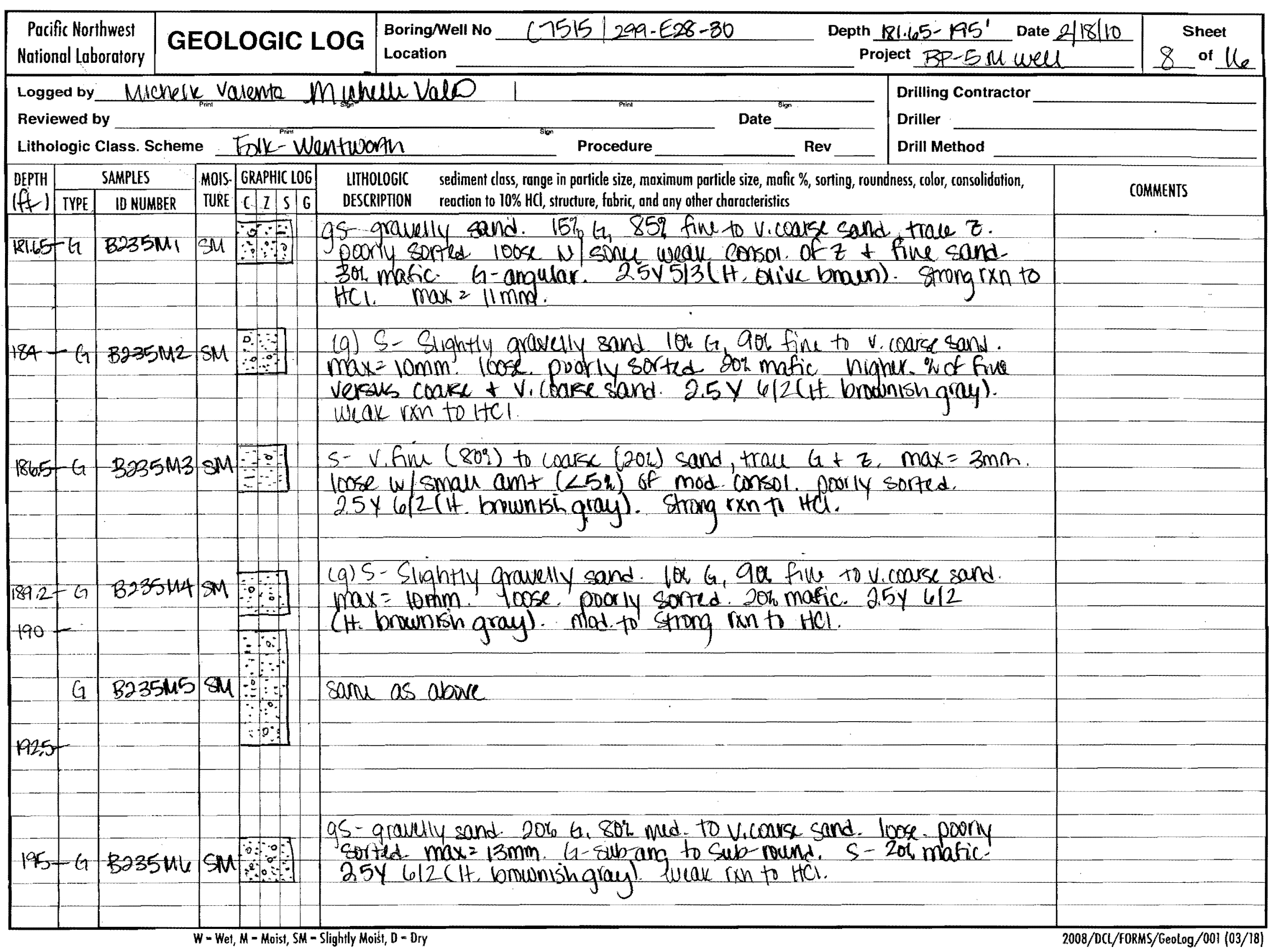




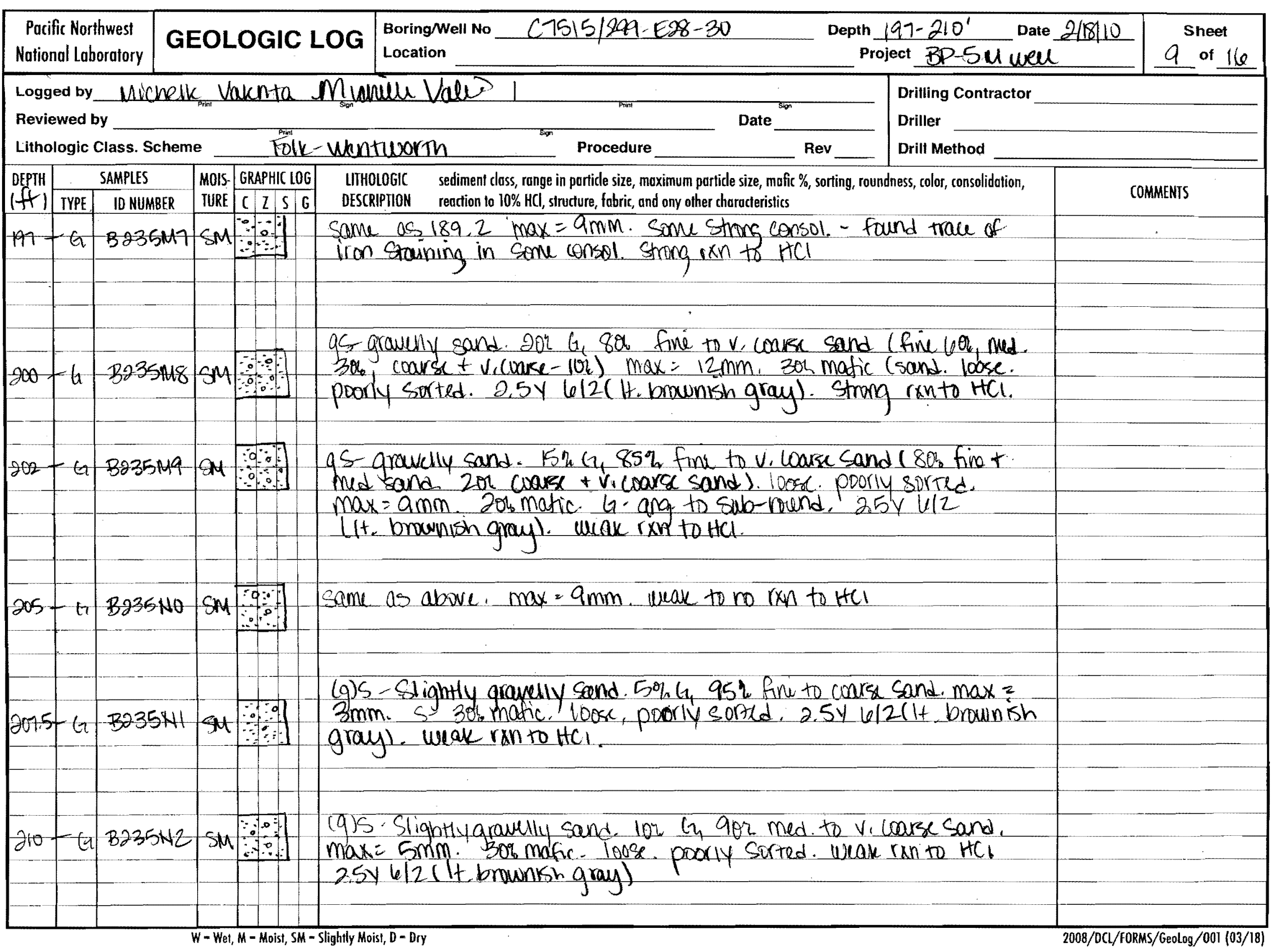




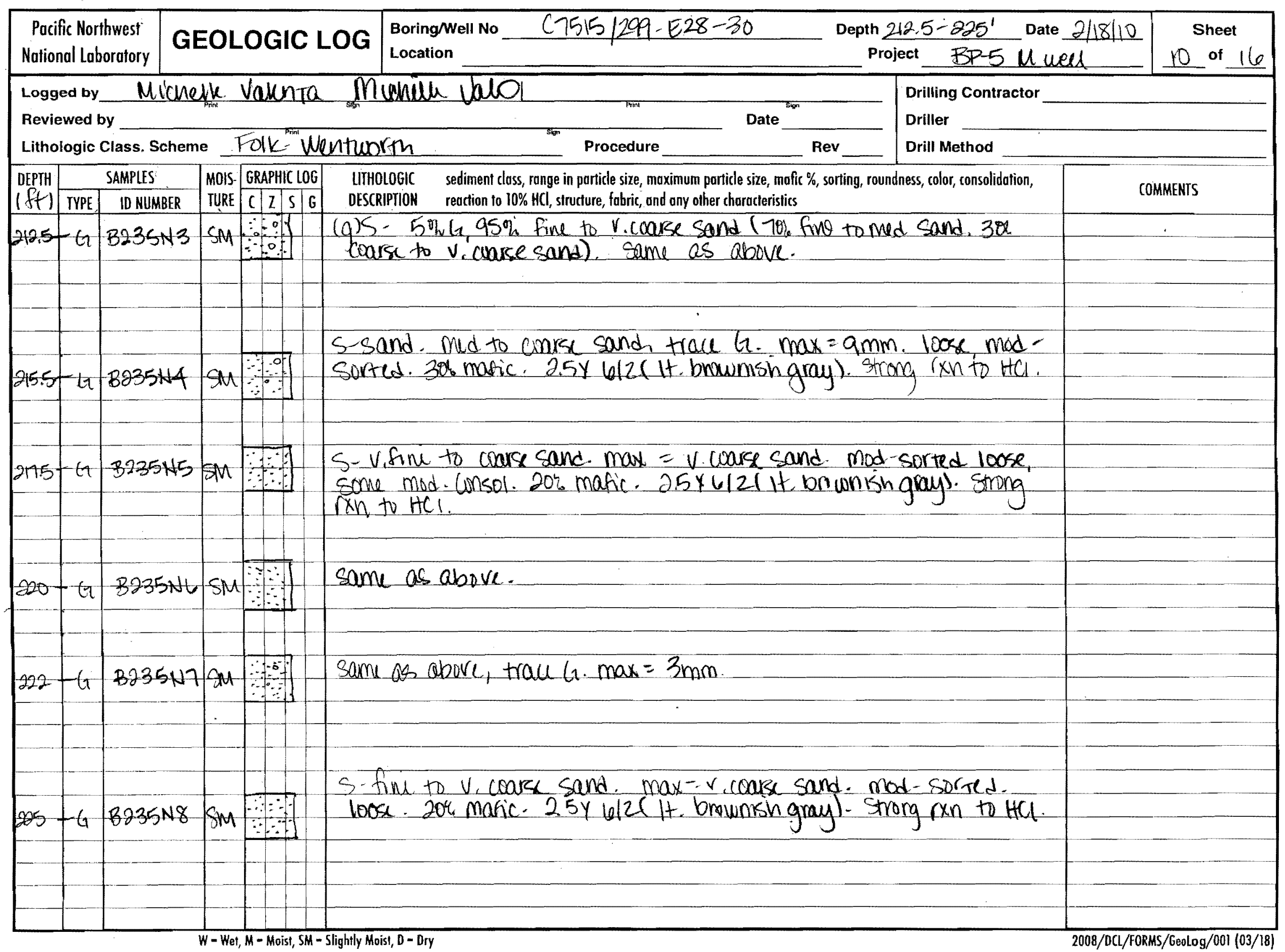




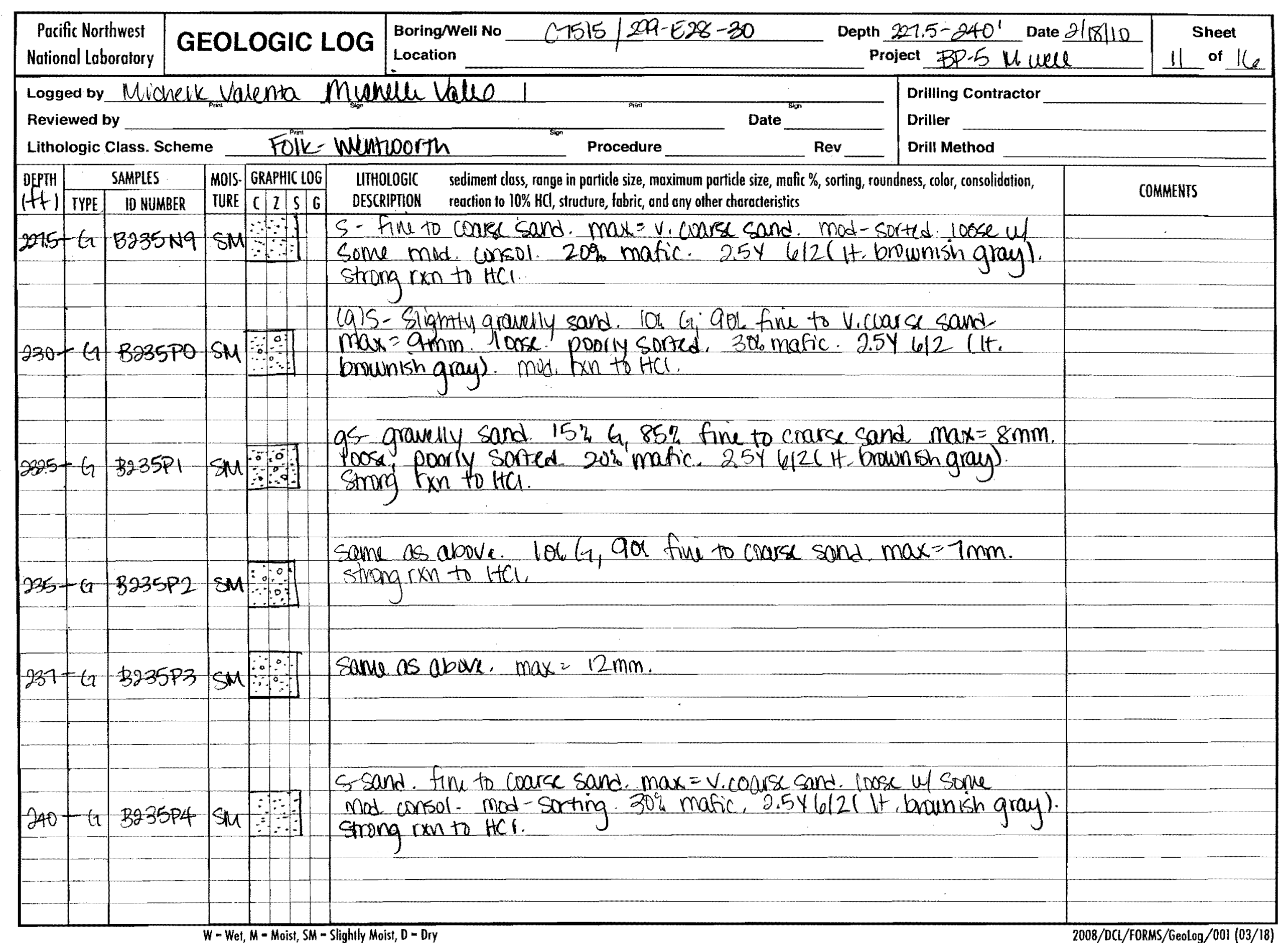




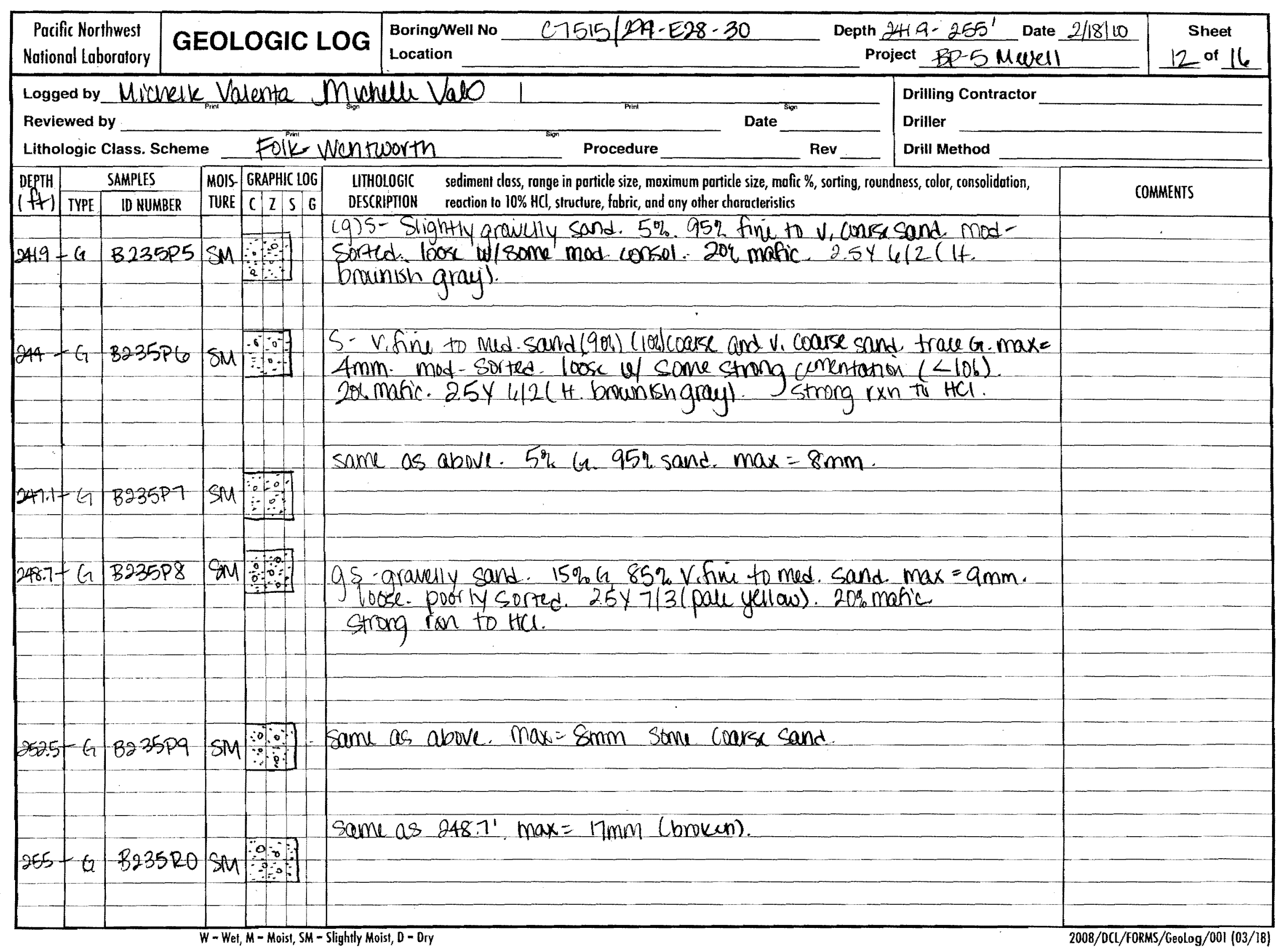




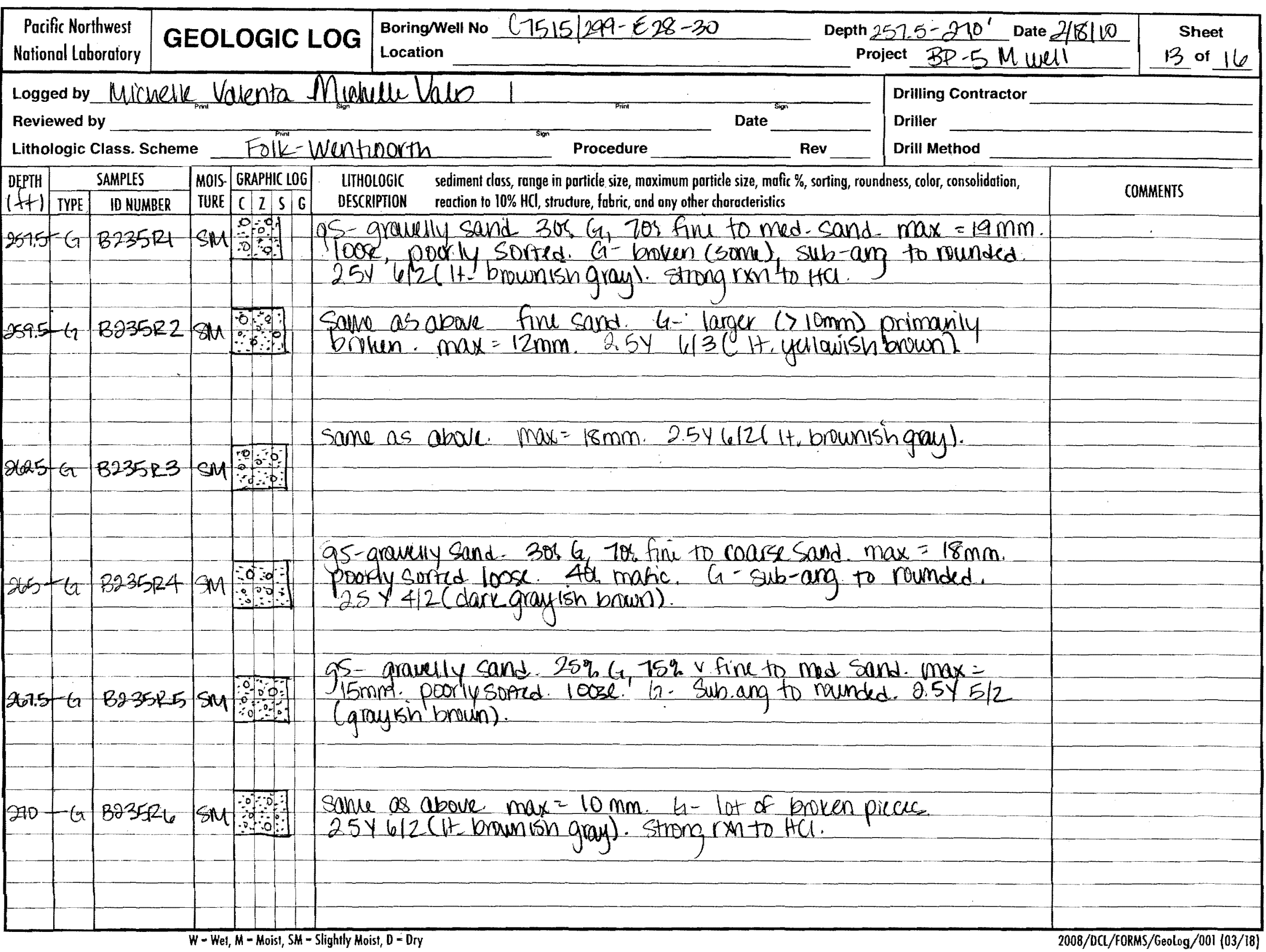




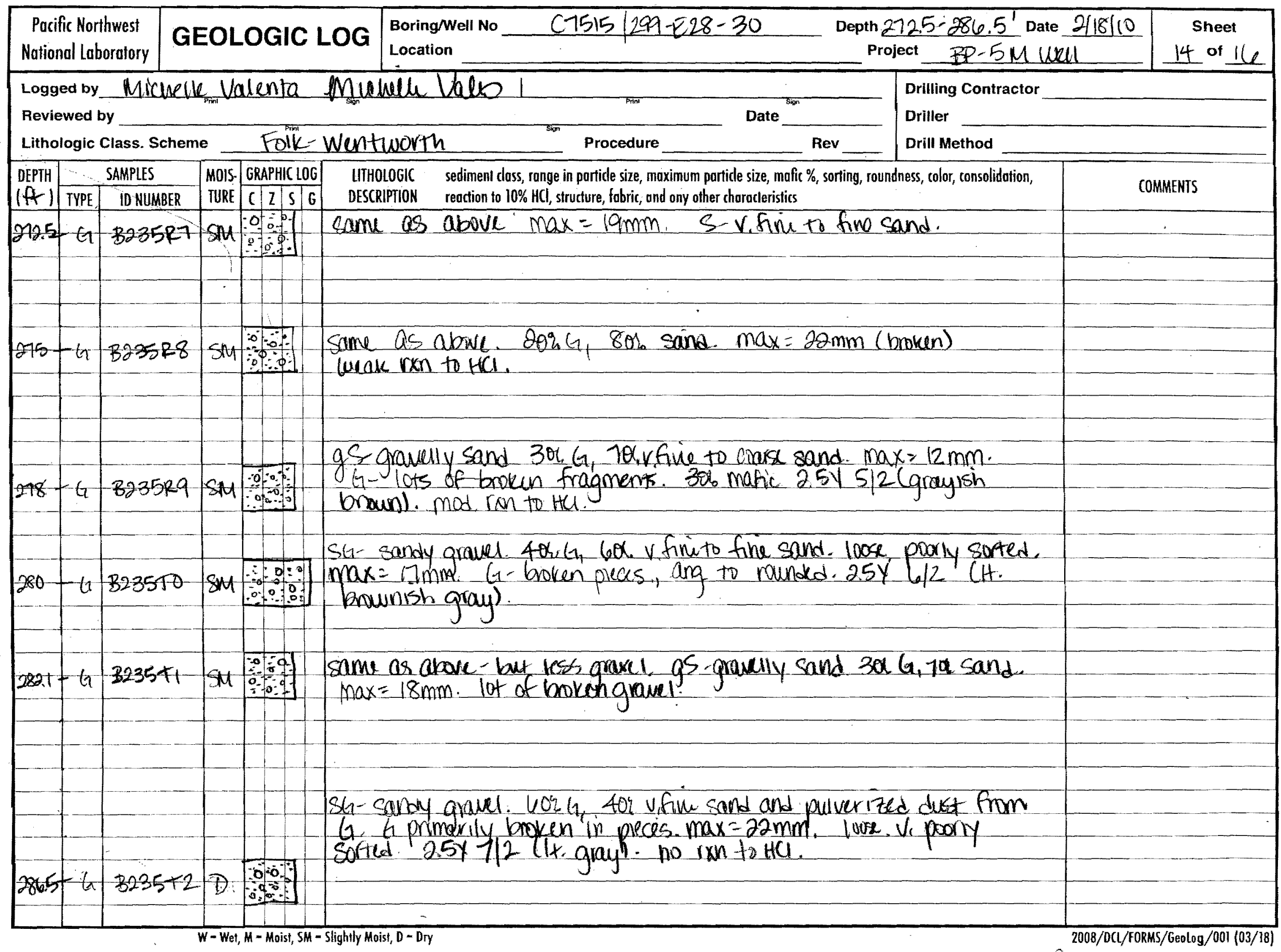




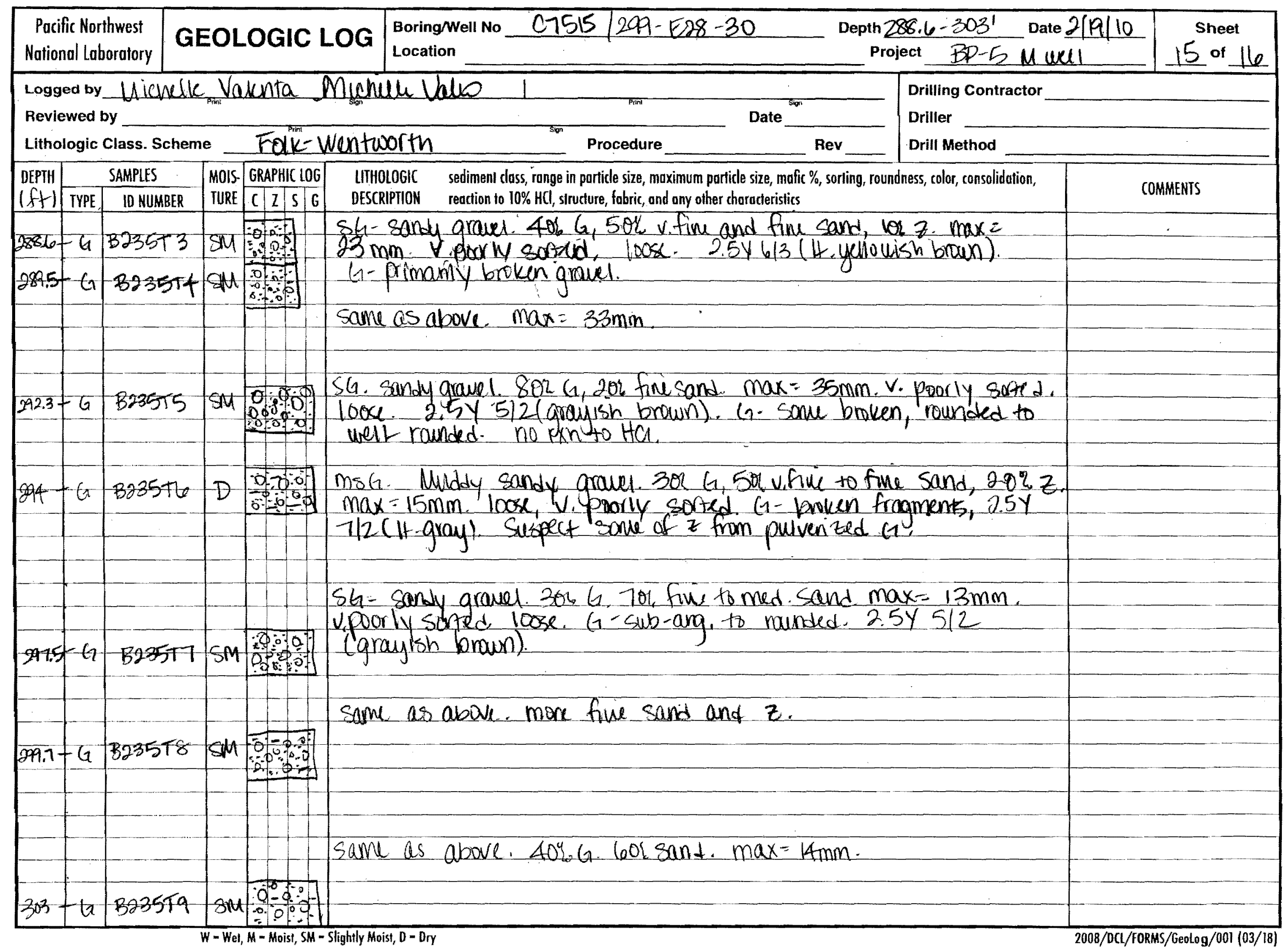




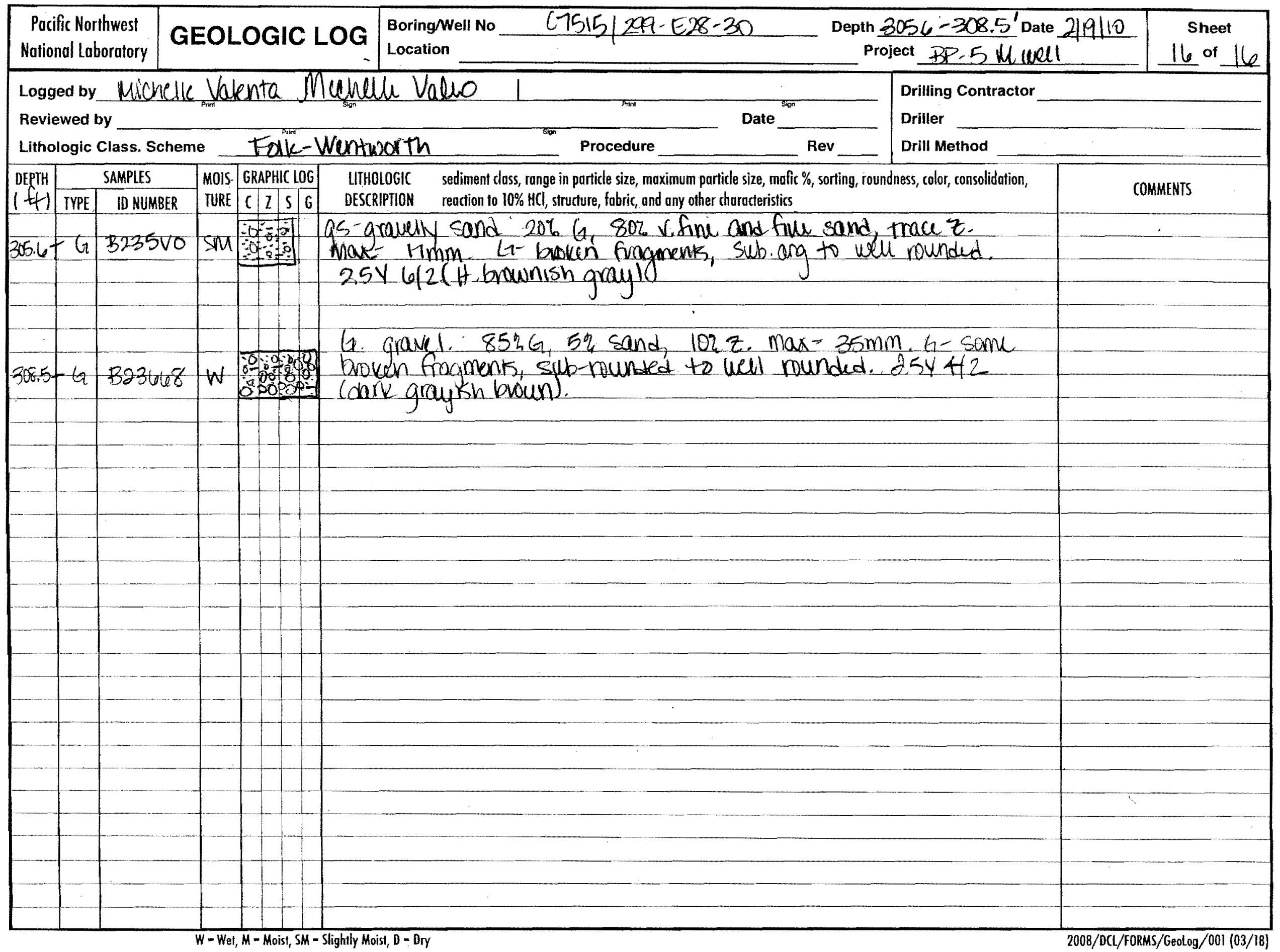




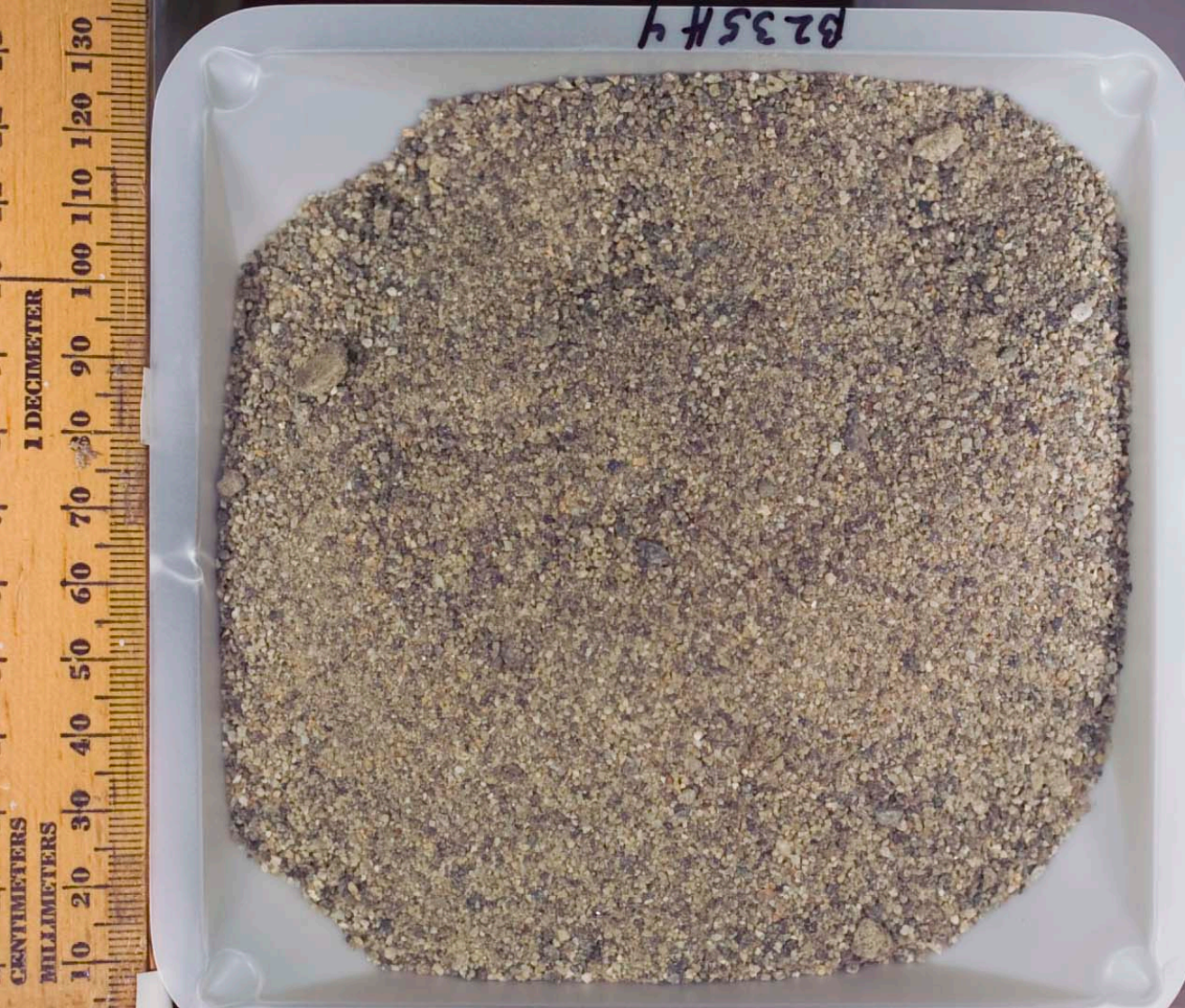

C7515

$\mathrm{B} 235 \mathrm{H} 4$ Sample ID Borehole ID
87.2-89.7 ft Depth from Chain-of-Custody
Grab 

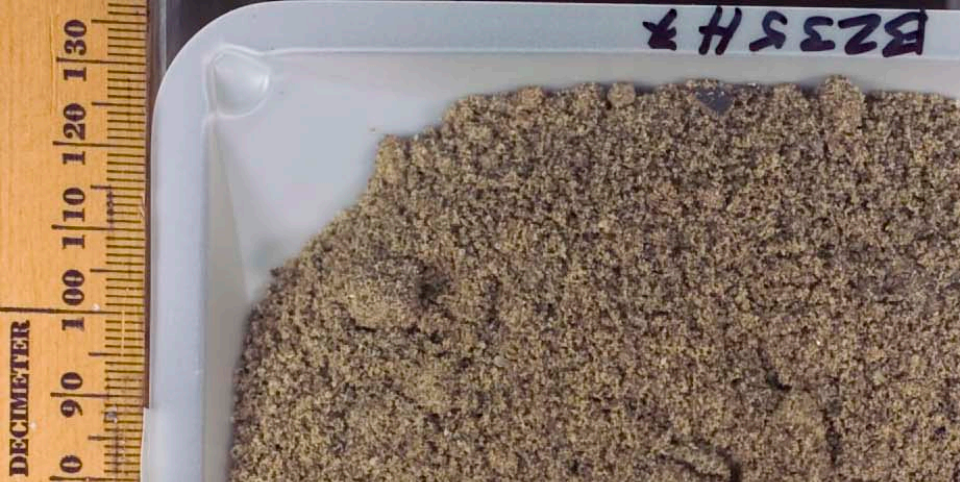

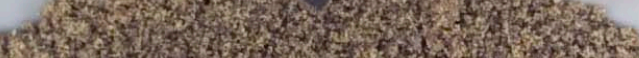

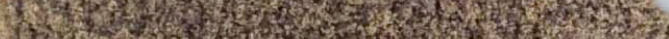

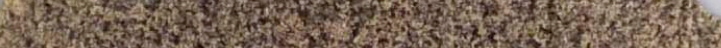

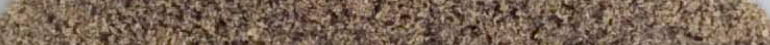

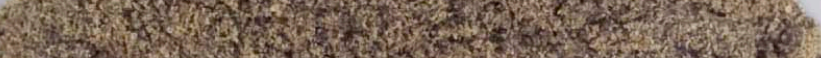

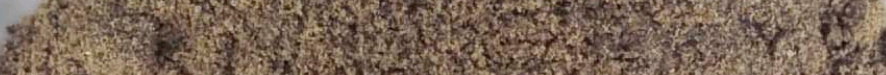

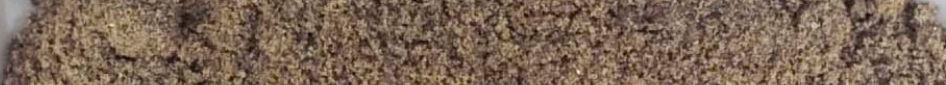

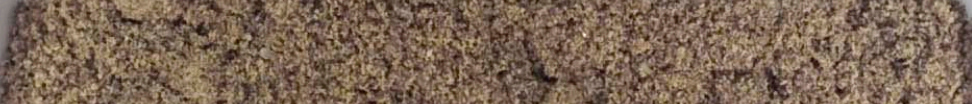

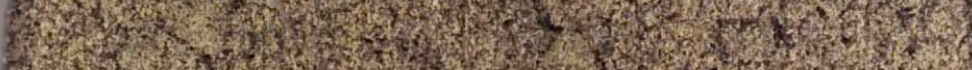

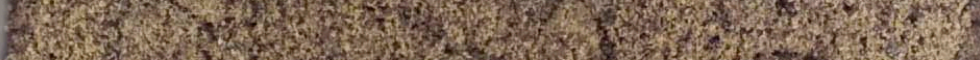

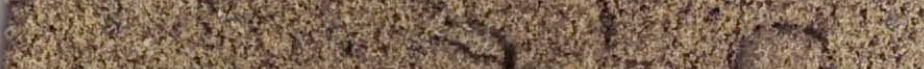

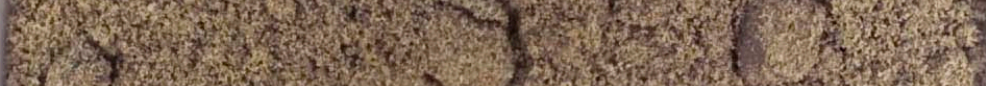

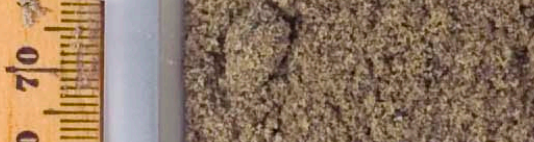

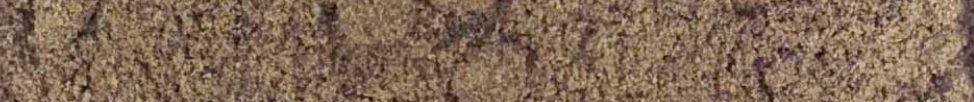

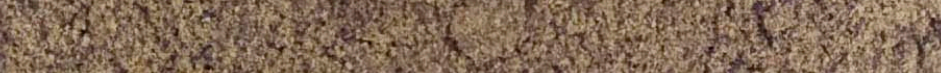
150. W.

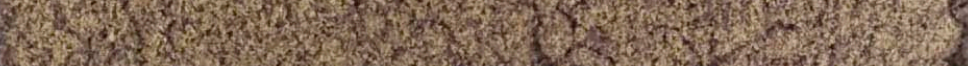

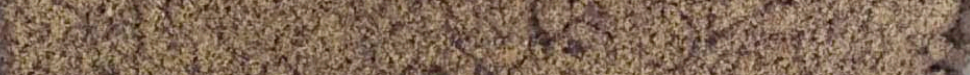

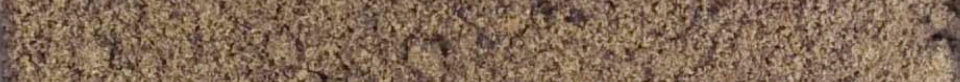
9.

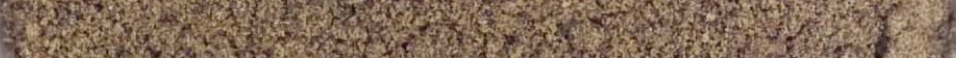

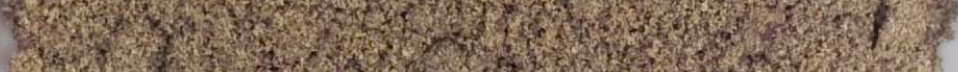

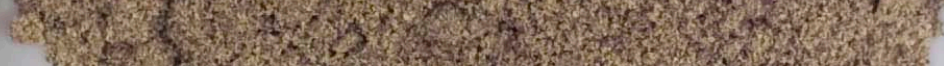

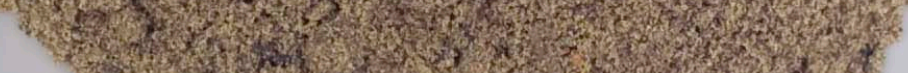

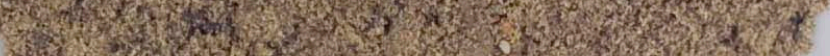
3 tow 


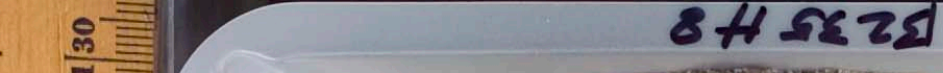




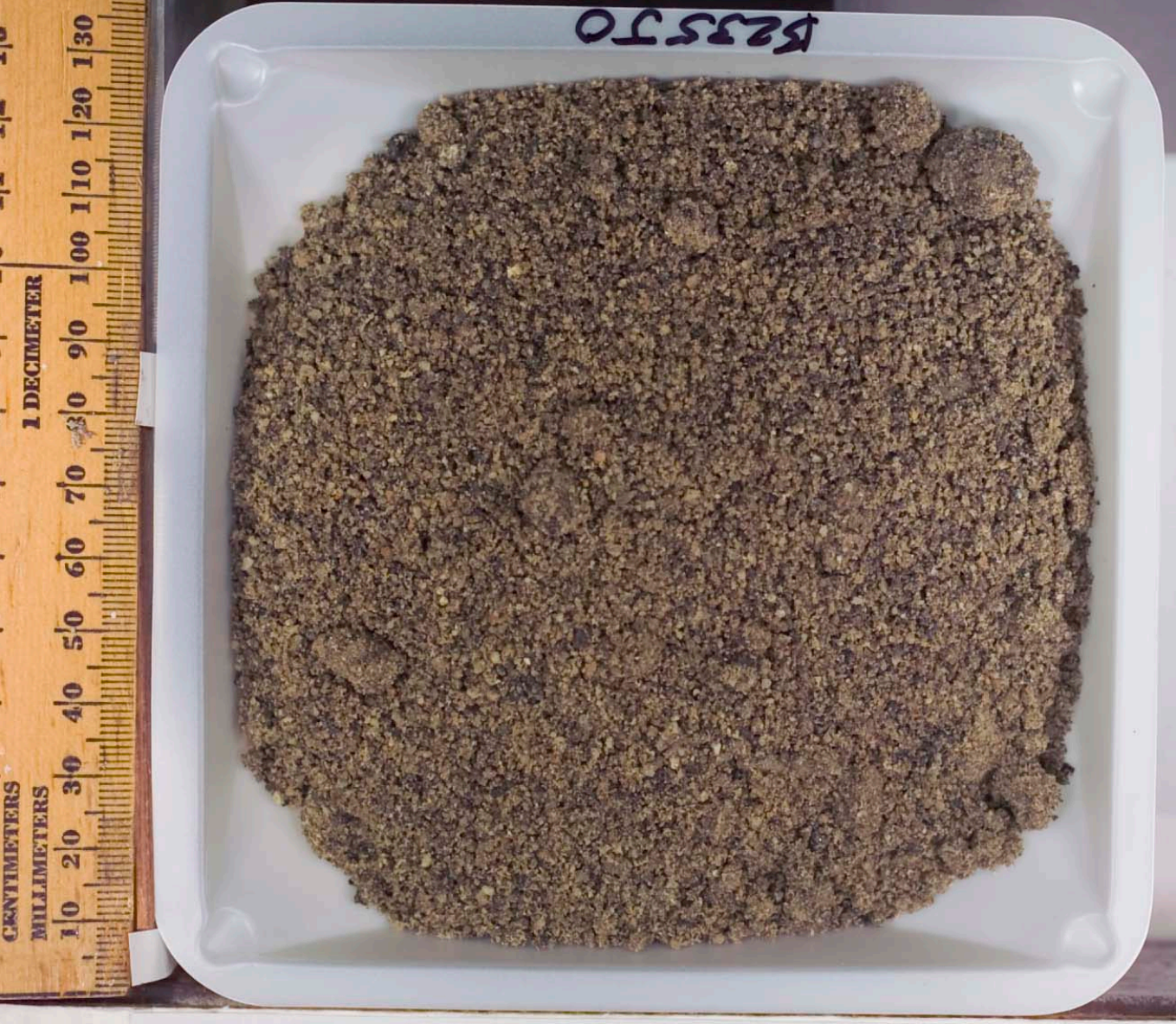

C7515 B235JO 102.8-105.3 ft

Grab

Borehole ID Sample ID

Depth from Chain-of-Custody

Sample 
栗

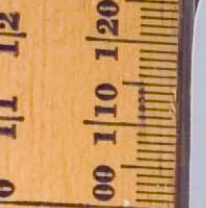

要

중

四三

$\theta \stackrel{\equiv}{= \pm}$

$\theta+$

corse 10. 0 (n)

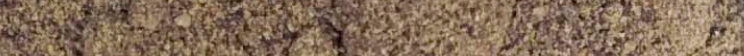

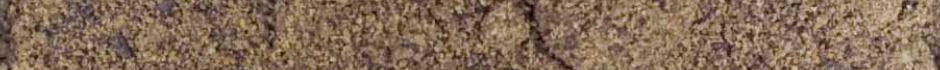

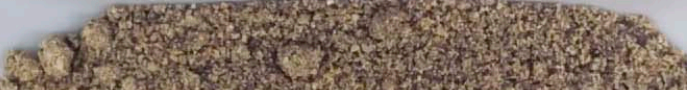

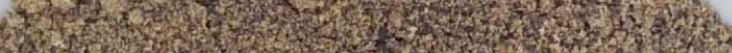

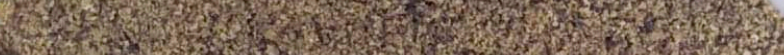
3f

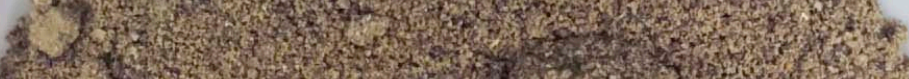

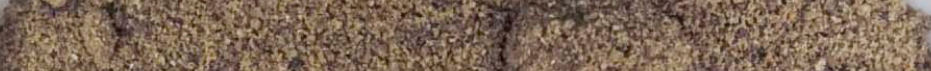
4. 4 f

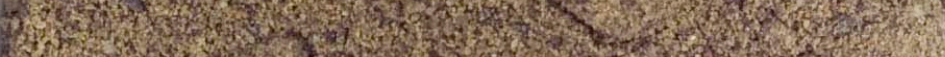

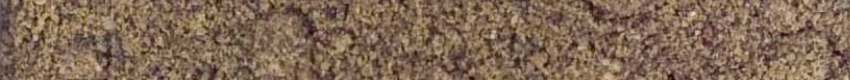
30
3

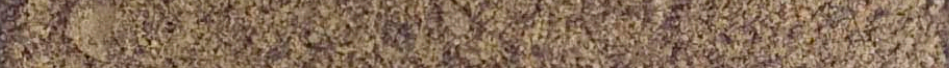
H.

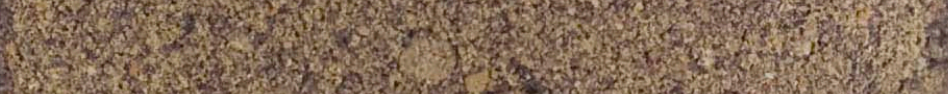
F.

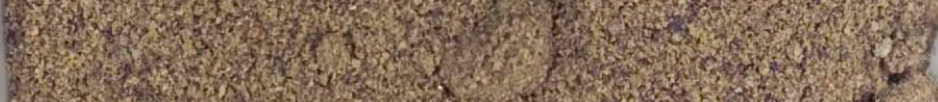

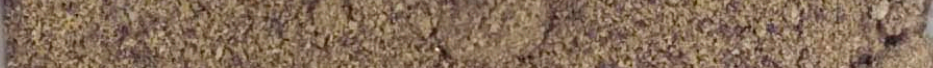

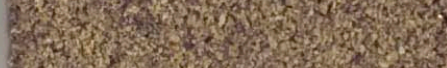

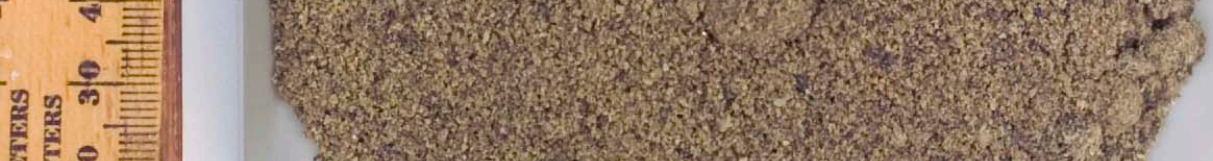

.

$\div$

A. $x^{2}$.

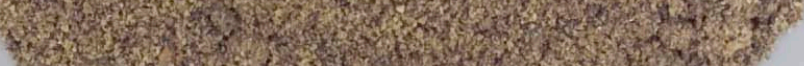

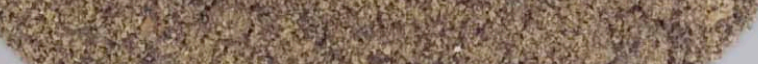

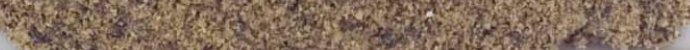

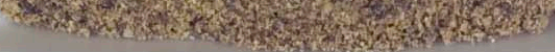

C7515

Borehole ID
B235J1

Sample ID
$105.5-108.0 \mathrm{ft}$

Depth from Chain-of-Custody
Grab

Sample 


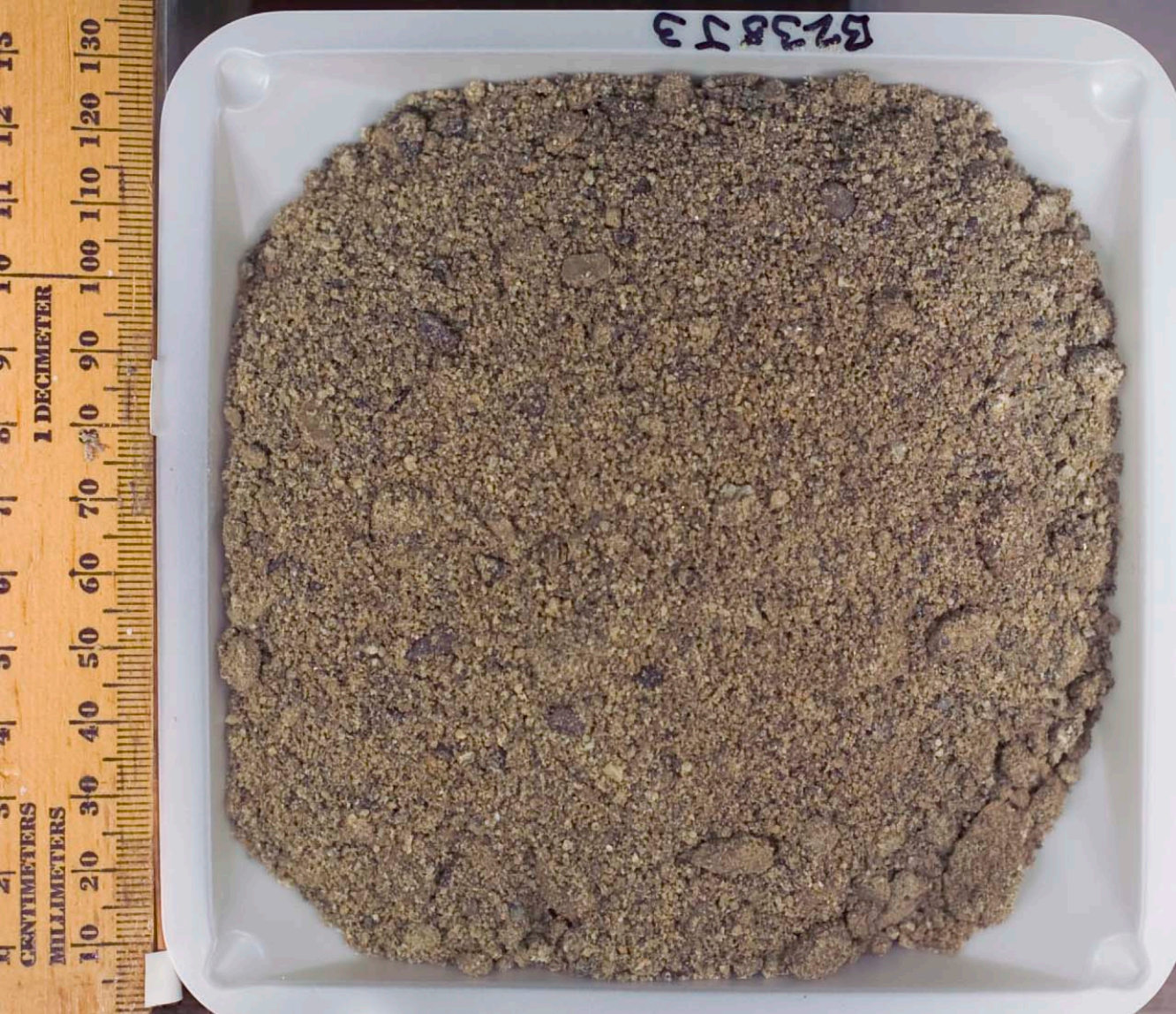




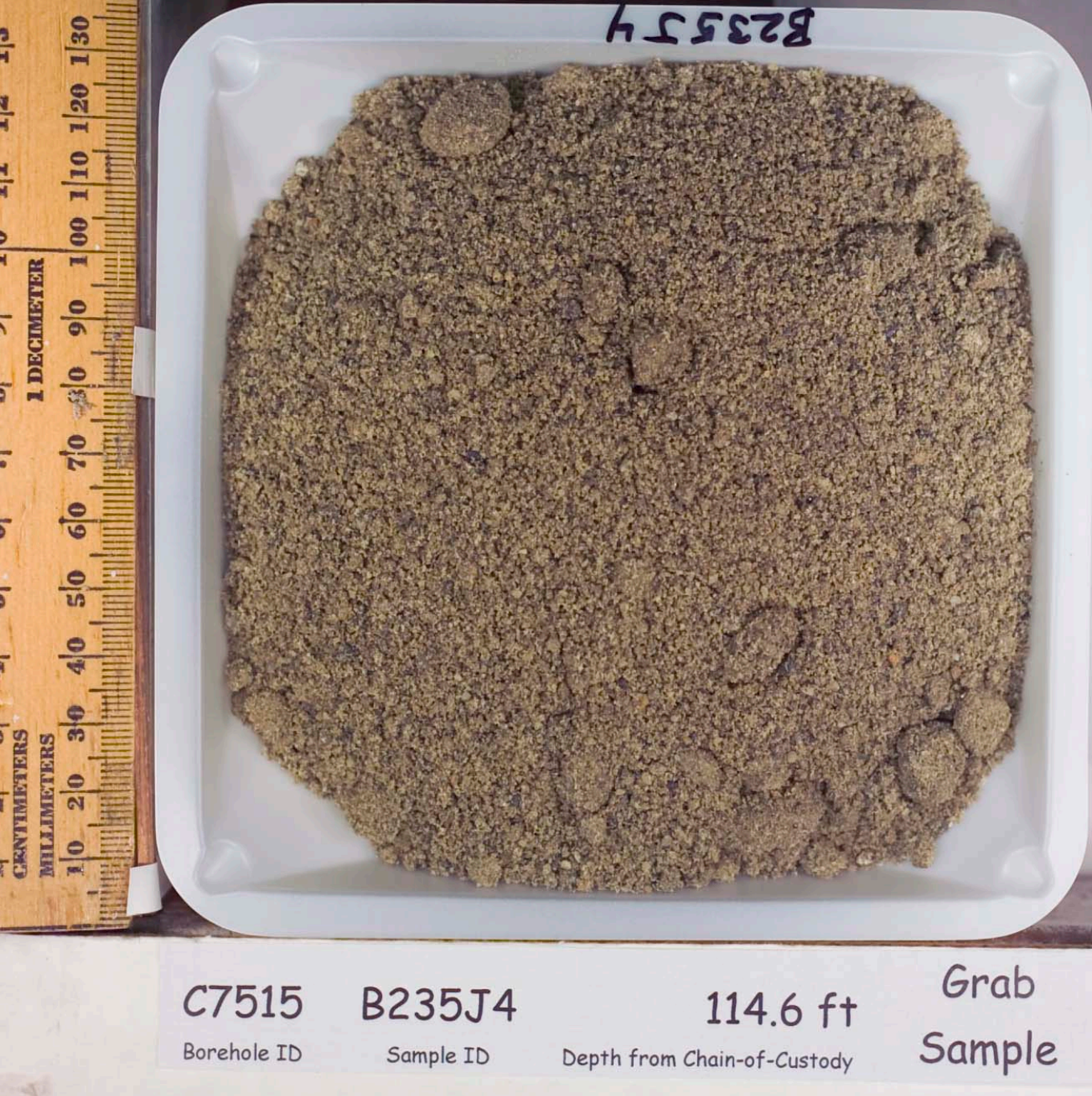




\section{5ร5รZ2}

en

s

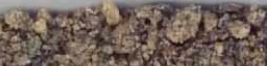

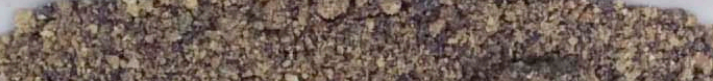

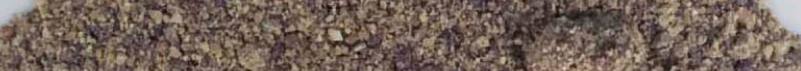

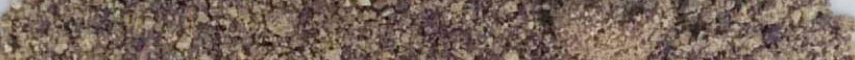
S.

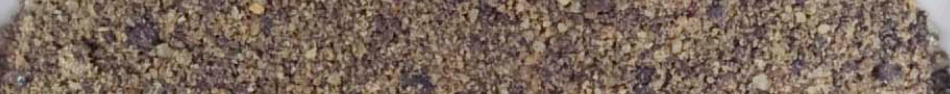

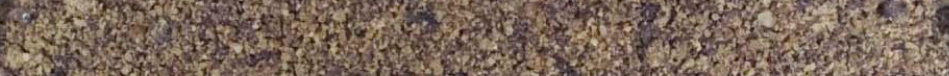

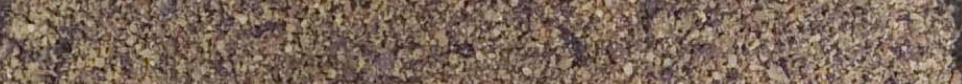

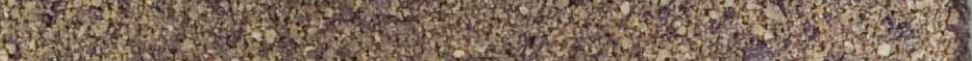

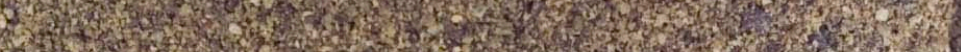

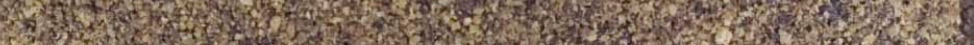

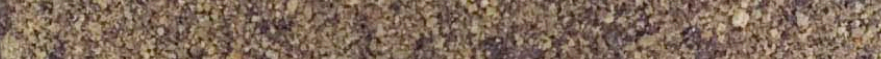

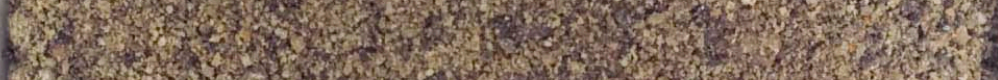

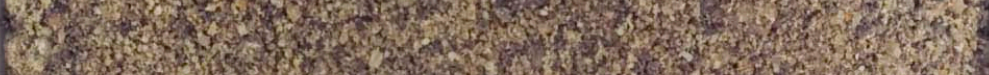

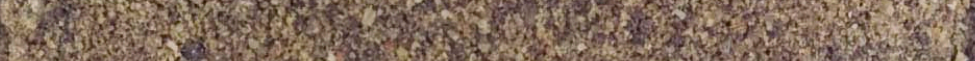

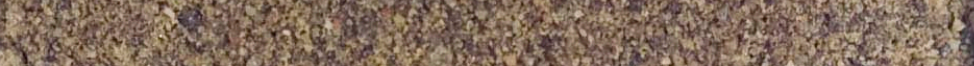

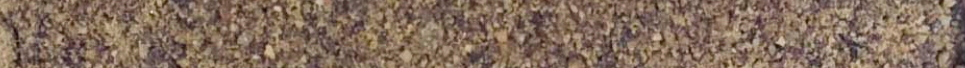

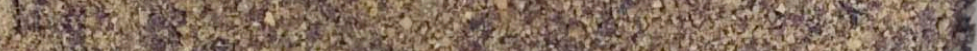

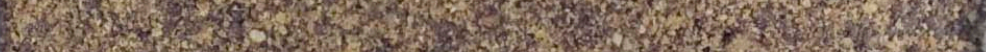

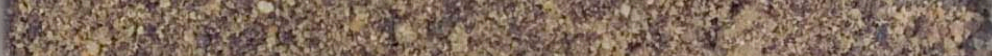

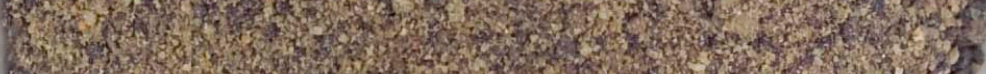
1.5. 3.5. 3
3

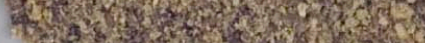

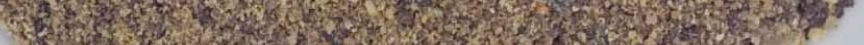

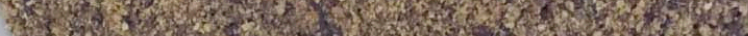

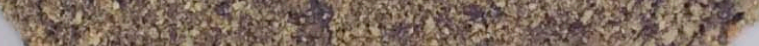

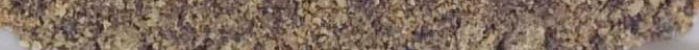

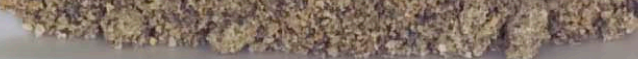

C7515

Borehole ID
B235J5

Sample ID
$116.6 \mathrm{ft}$

Depth from Chain-of-Custody
Grab Sample 


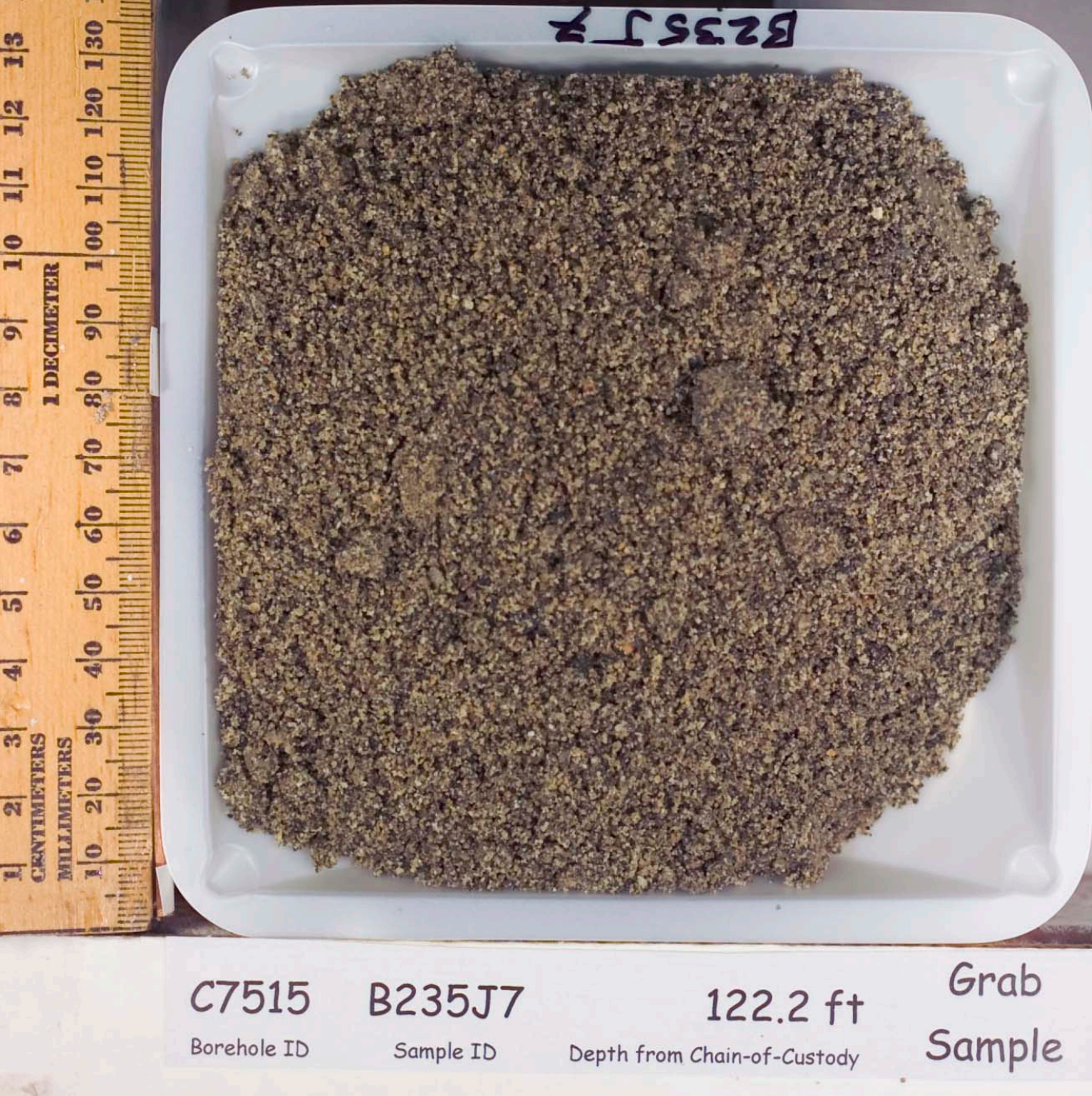


8ร5รZ

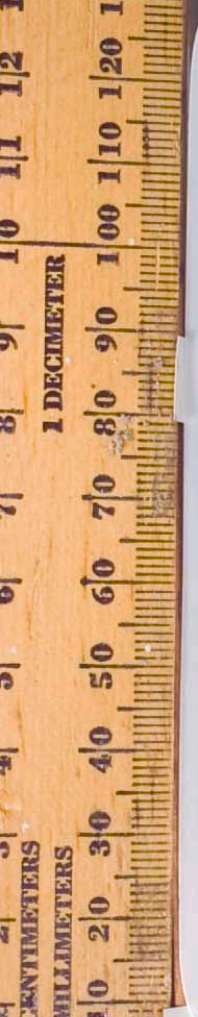

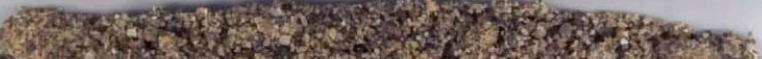

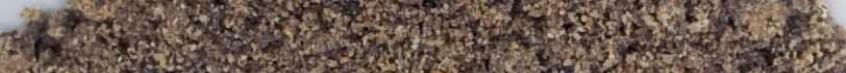

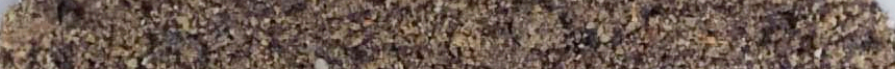

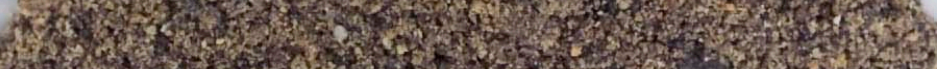

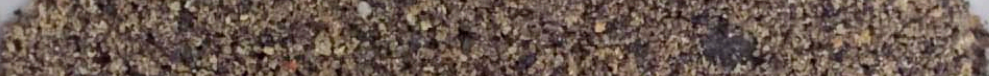

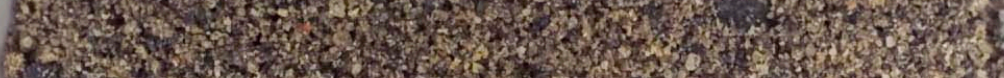

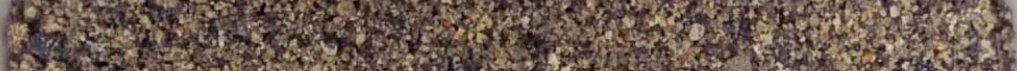

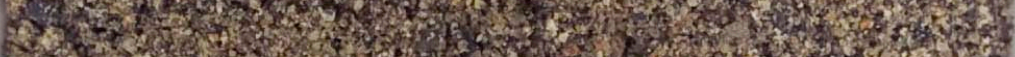

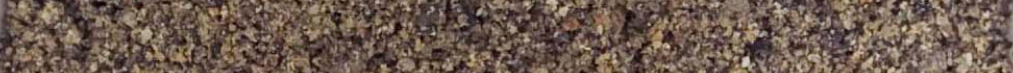

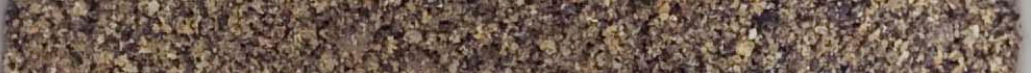

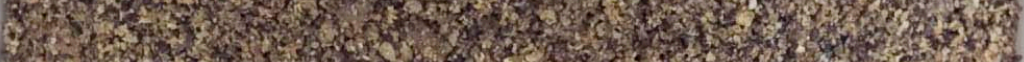

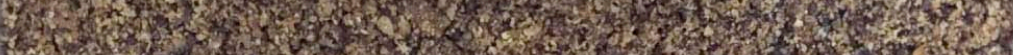

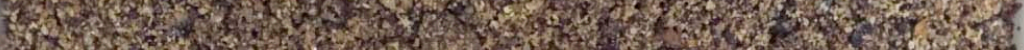

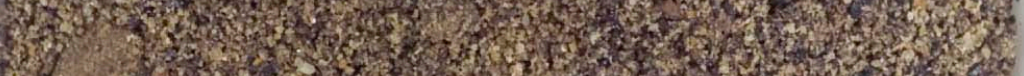

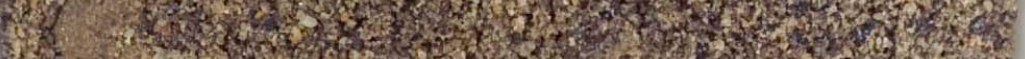

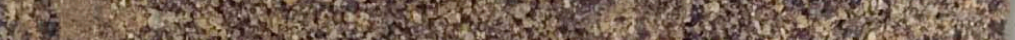

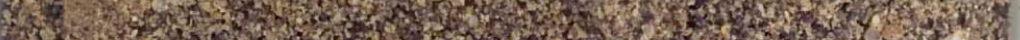

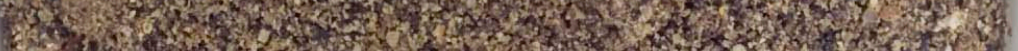

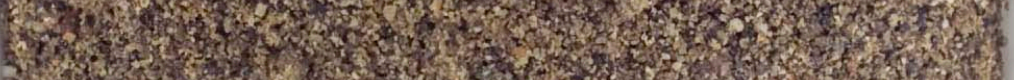
S.5. A. 1 .

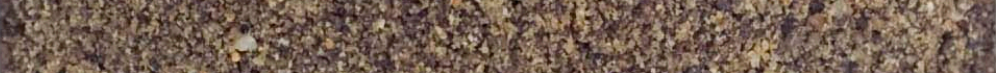
33
3

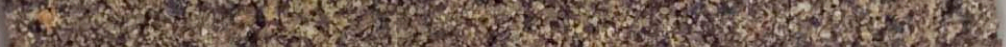
2
2

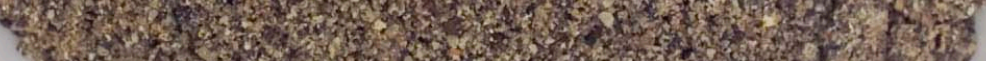

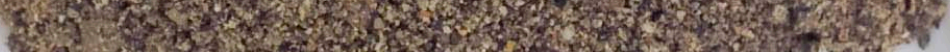

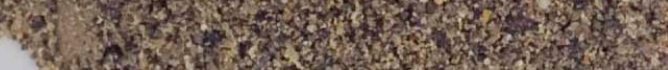
If 35 . 3.

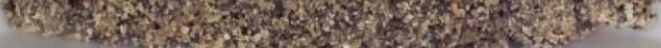

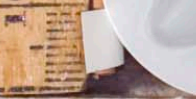

C7515

Borehole ID
B235J8

Sample ID
$124.6 \mathrm{ft}$

Depth from Chain-of-Custody
Sample 




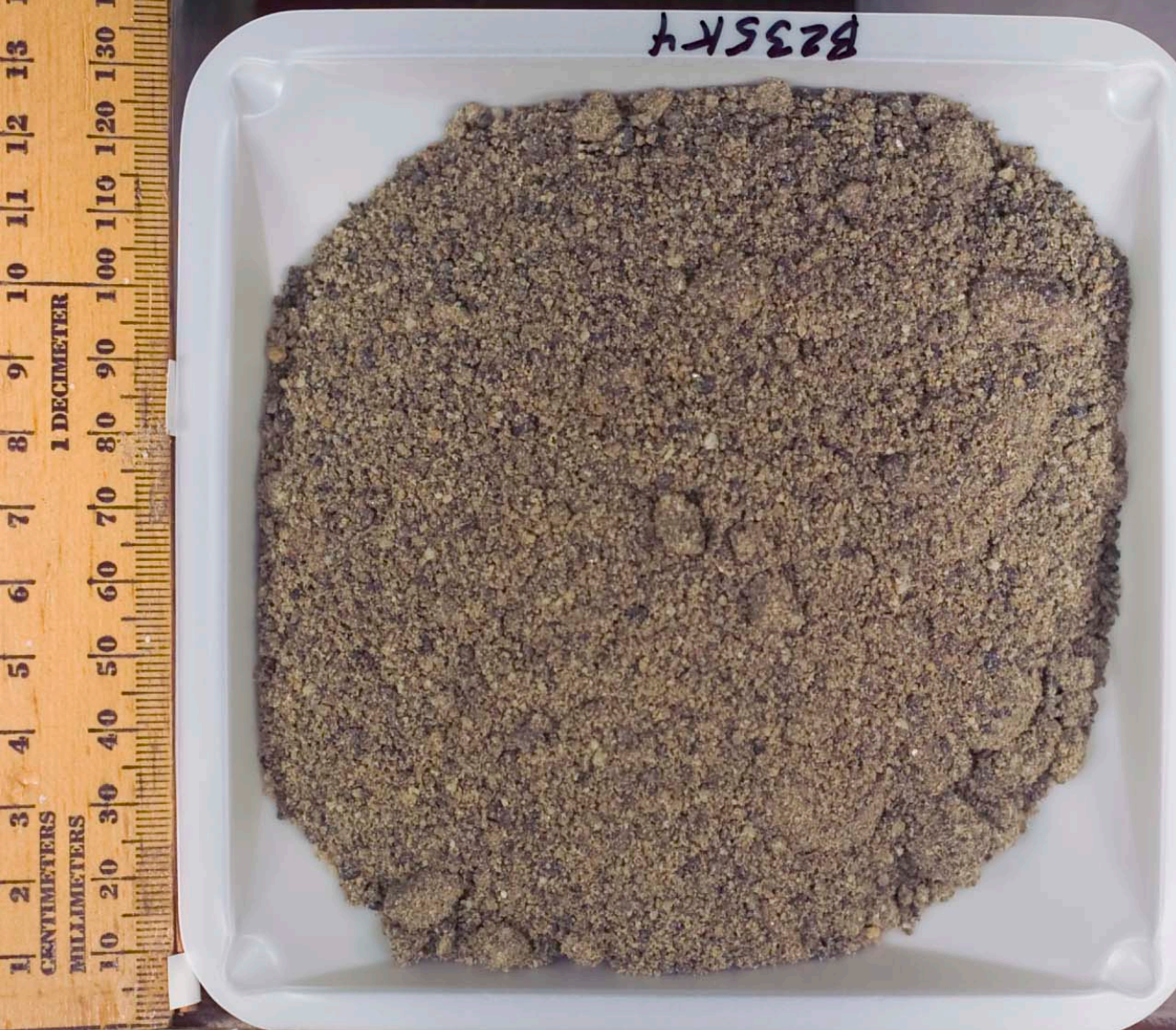




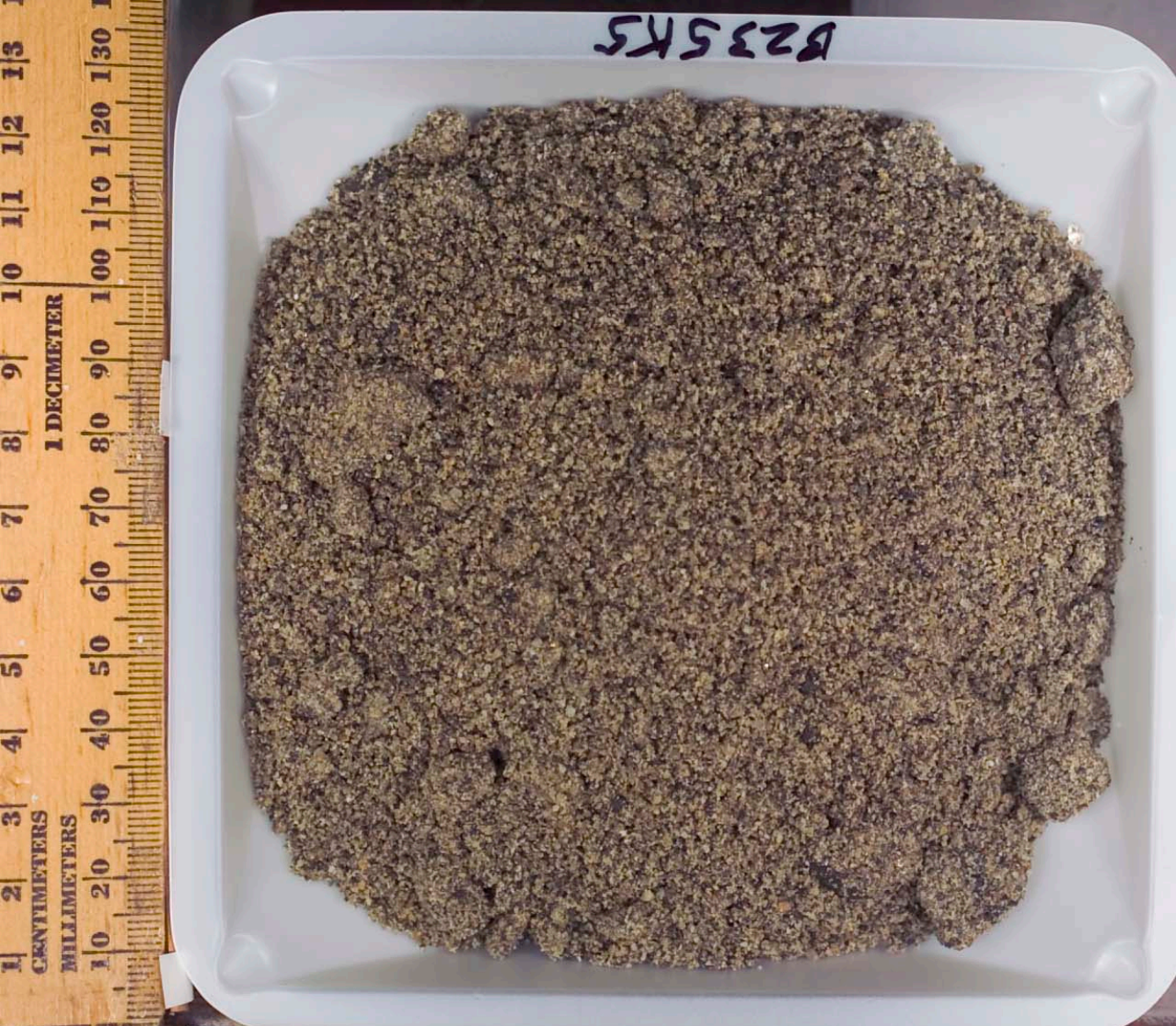




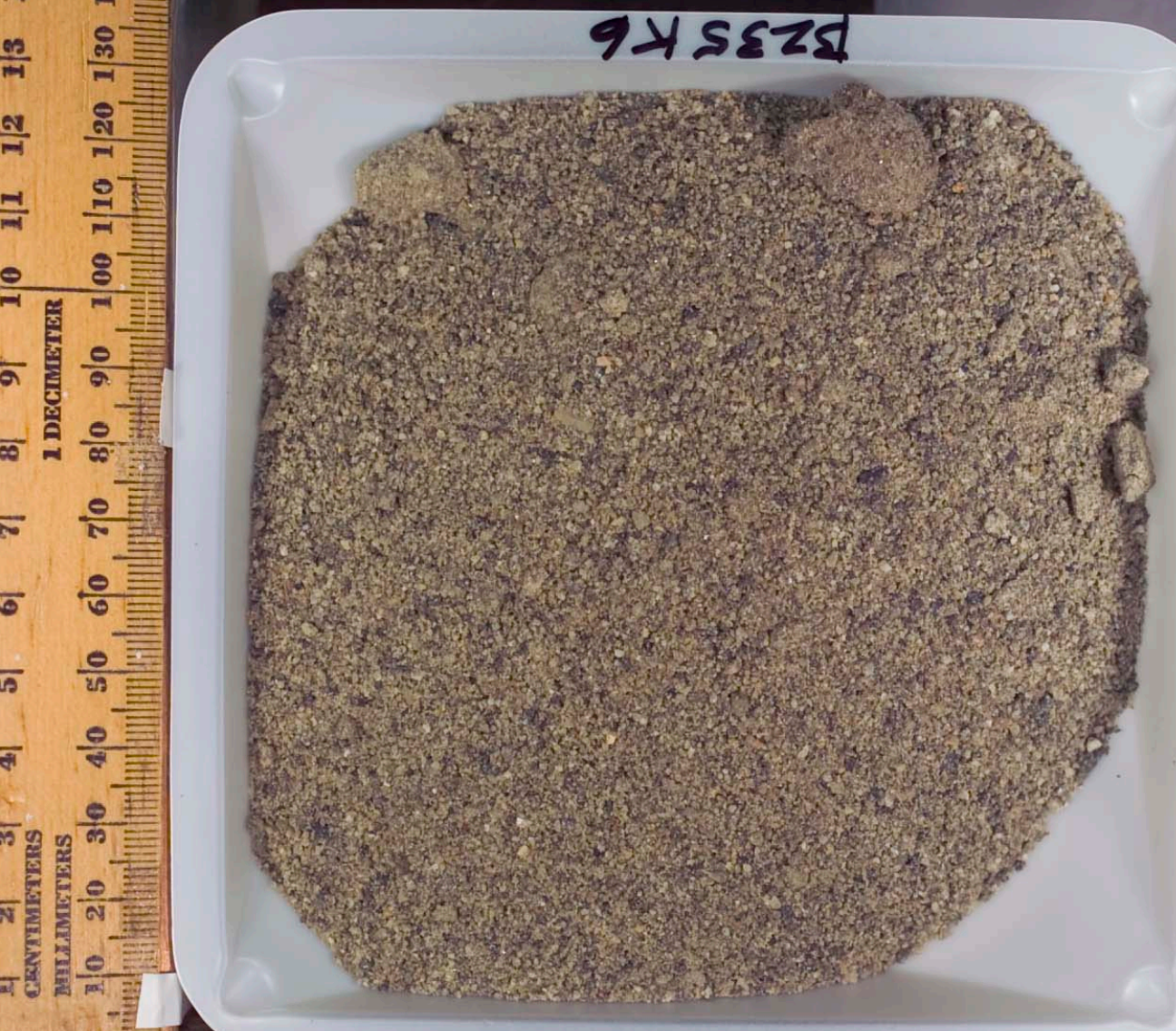




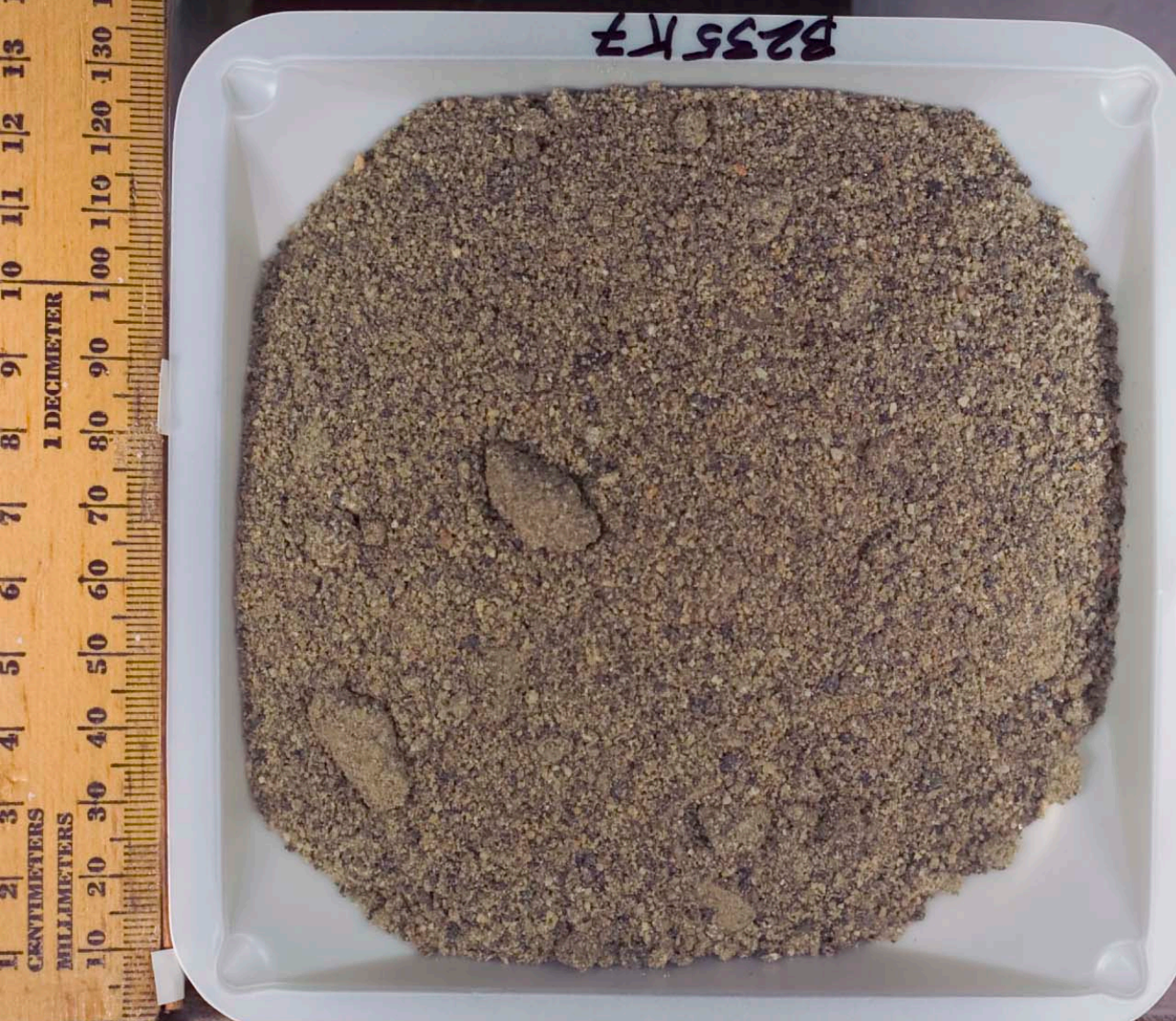

C7515 B235K7

Borehole ID
Sample ID
$148.0 \mathrm{ft}$

Depth from Chain-of-Custody
Grab

Sample 


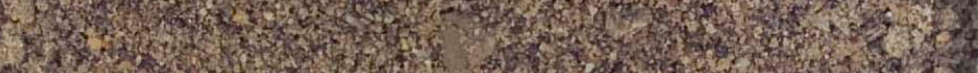

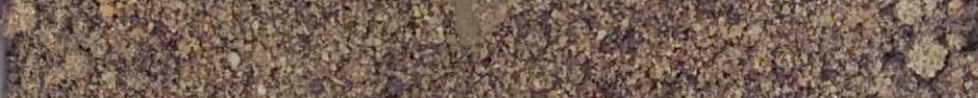
3.

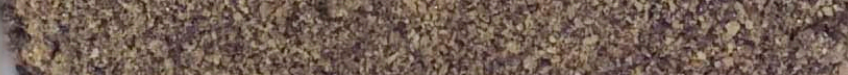

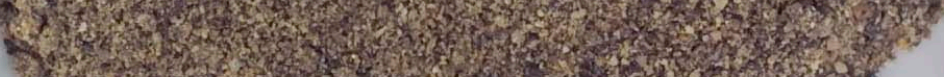

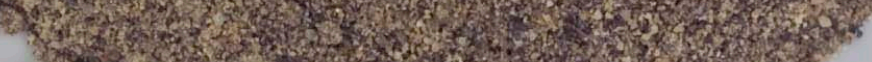

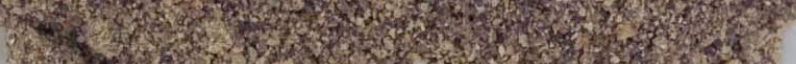

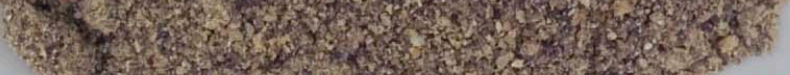

$151.9 \mathrm{ft}$ Depth from Chain-of-Custody
Grab Sample 


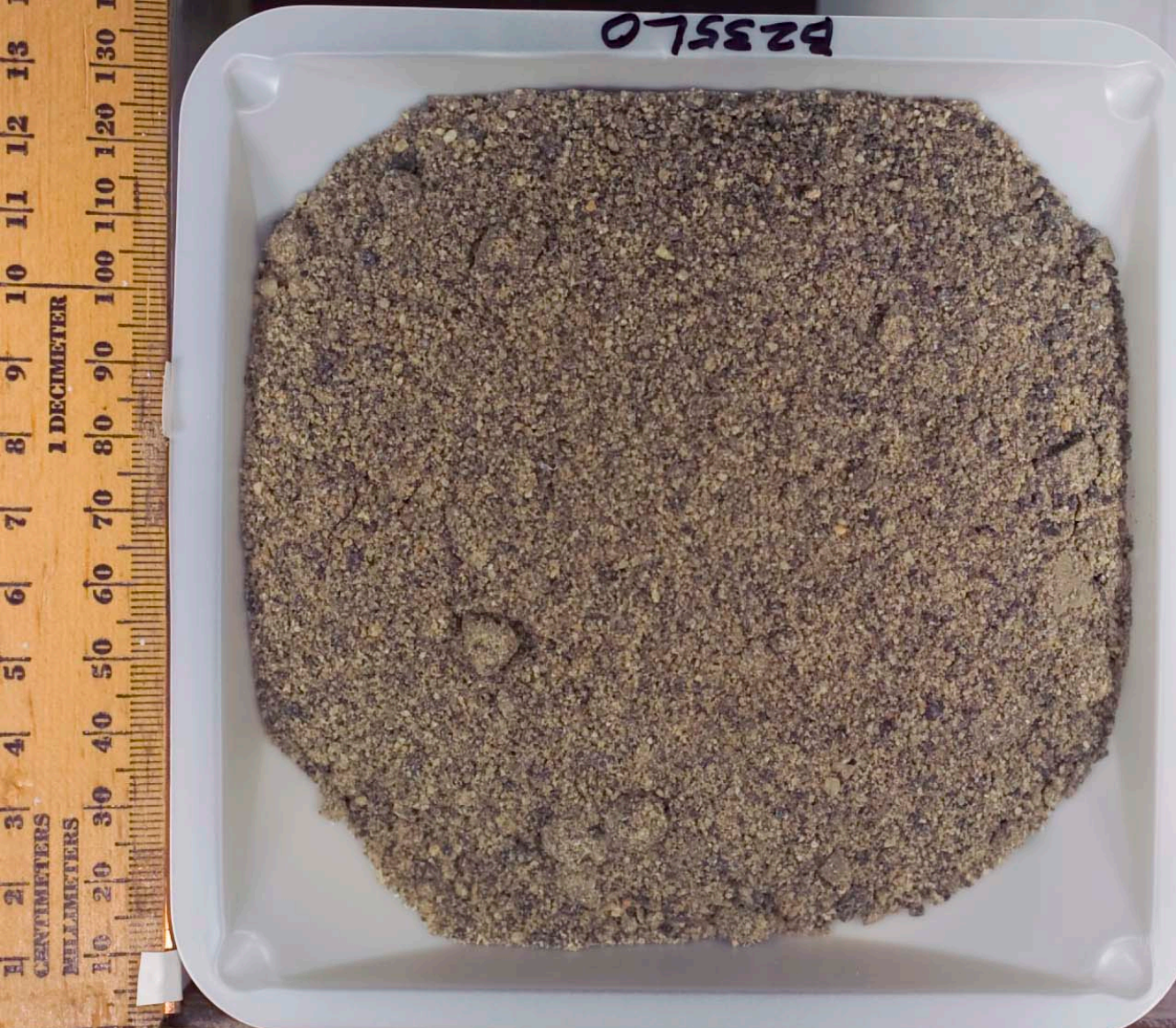




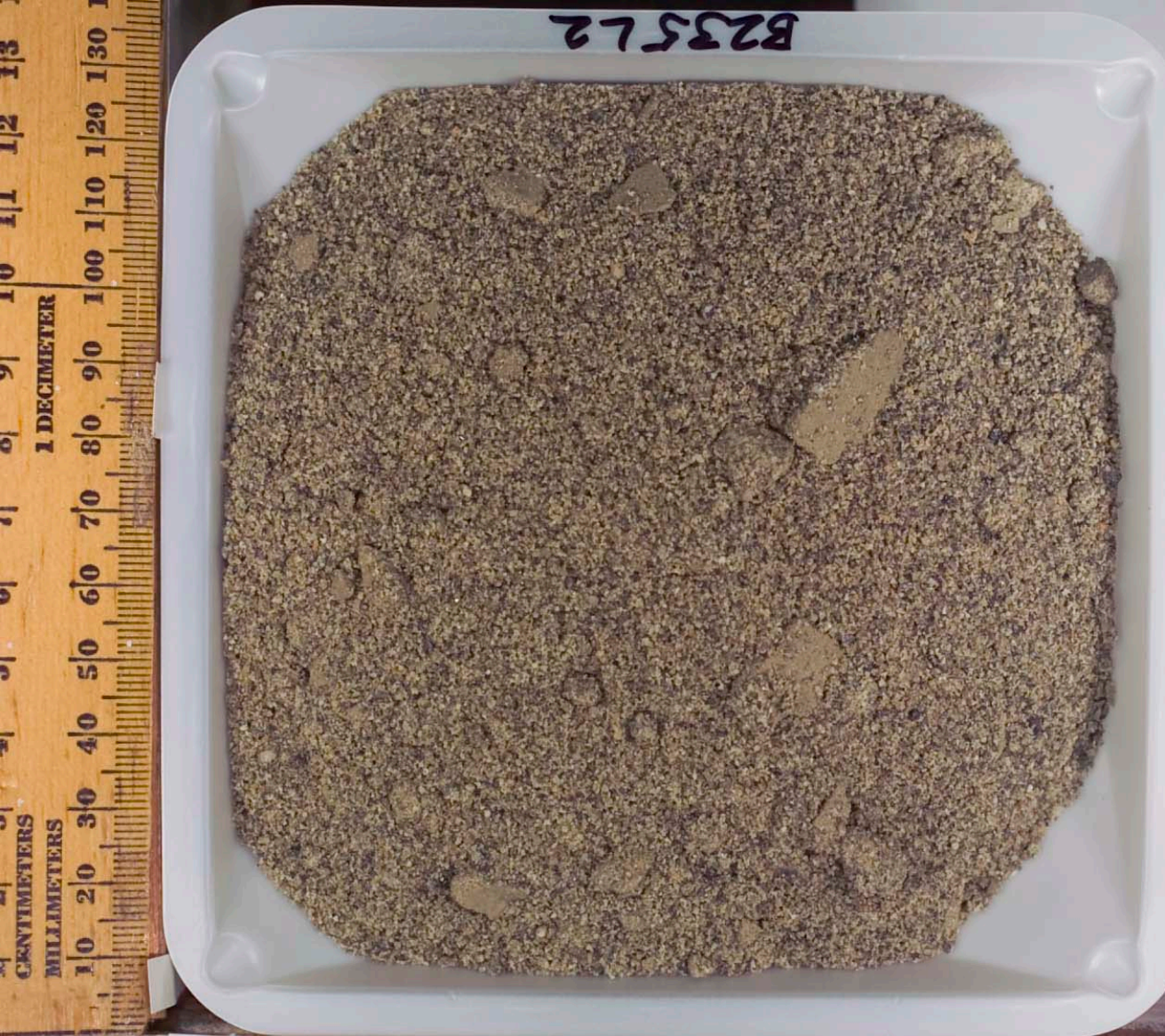




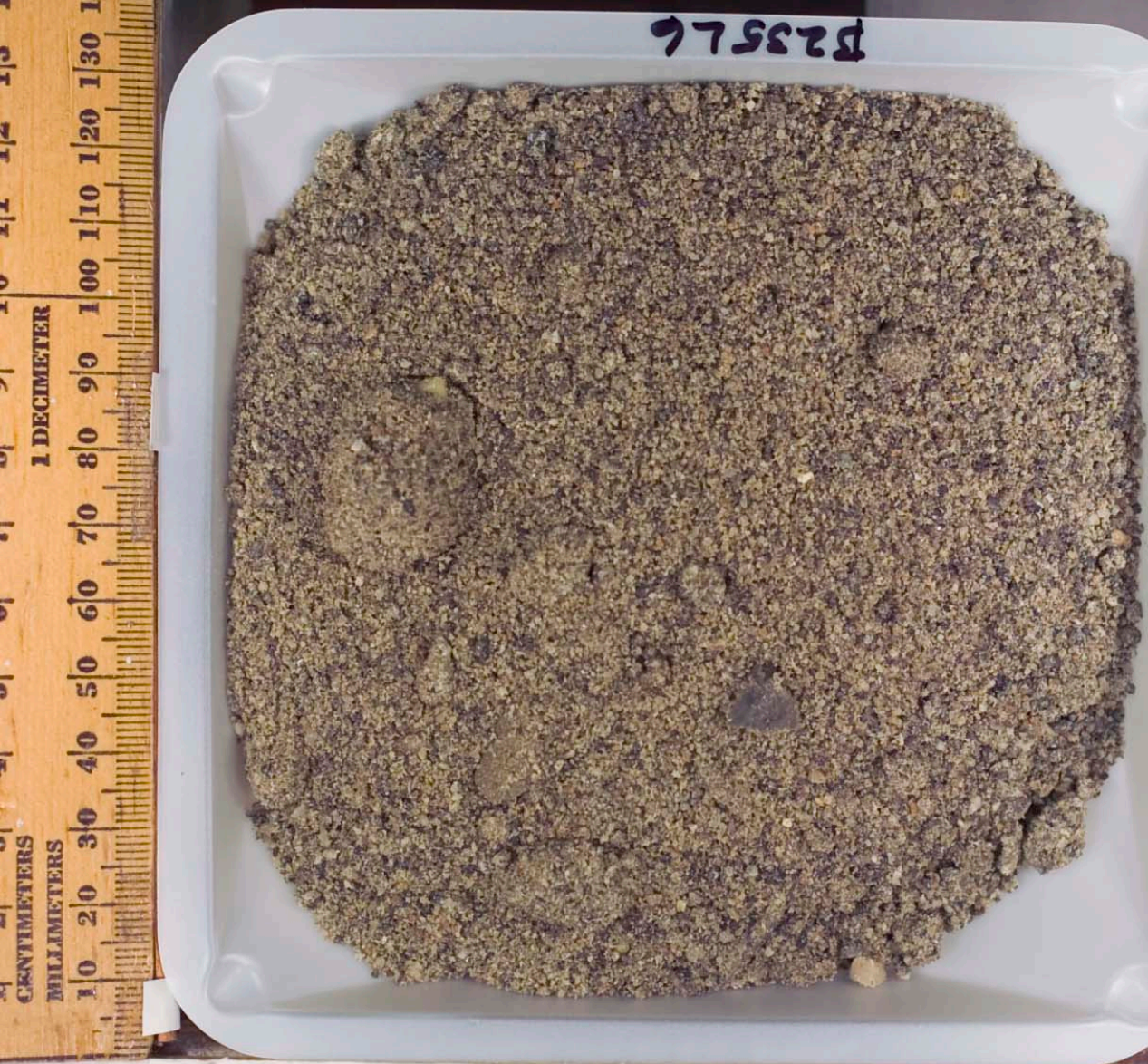



875ह2d

$\frac{\rho^{2}}{P}$

$\frac{1}{4}$

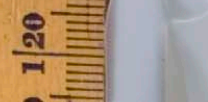

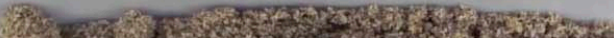

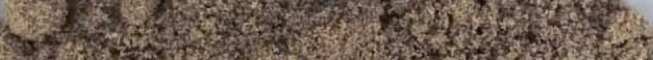

Ifs 5 (5)

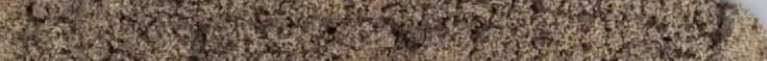

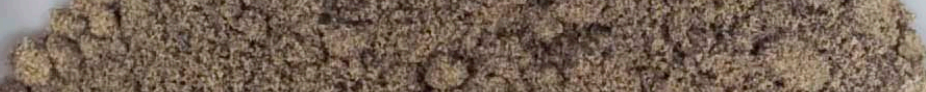

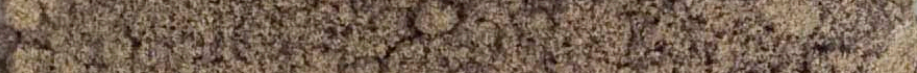

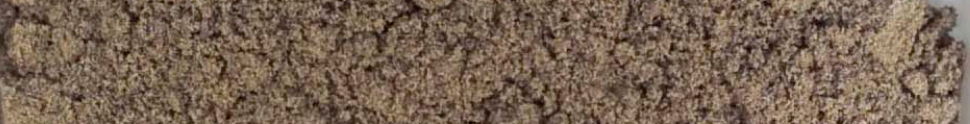
19 (3)

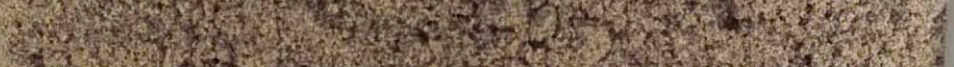

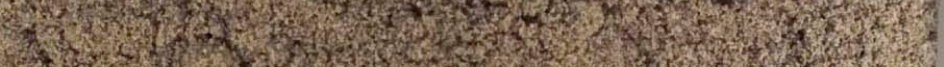

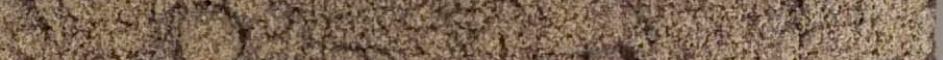

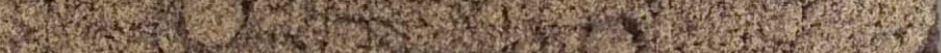
W.

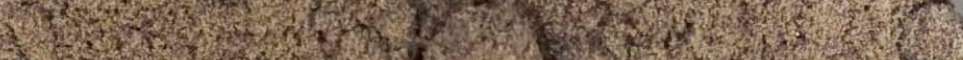
7.5. 3
3

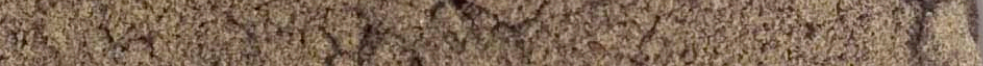

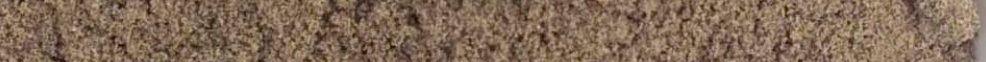

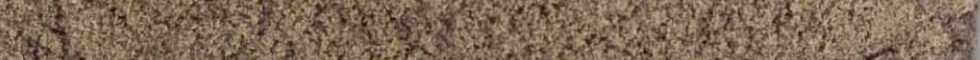

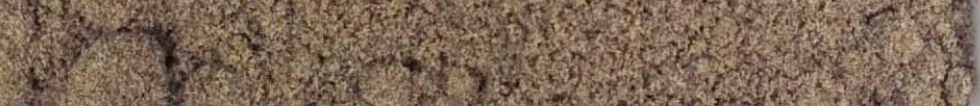

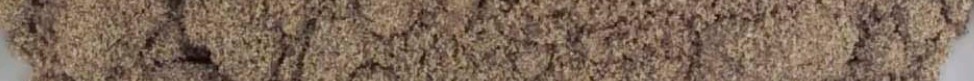

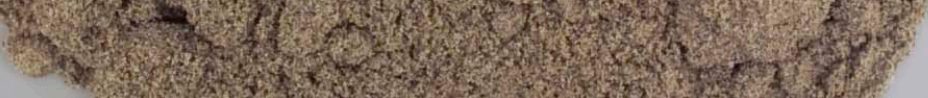

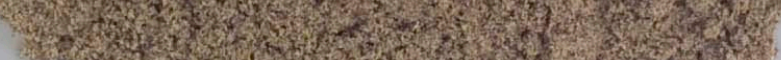

$\theta \equiv$

mis

C7515 B235L8

Borehole ID
Sample ID
$173.0 \mathrm{ft}$

Depth from Chain-of-Custody
Grab

Sample 


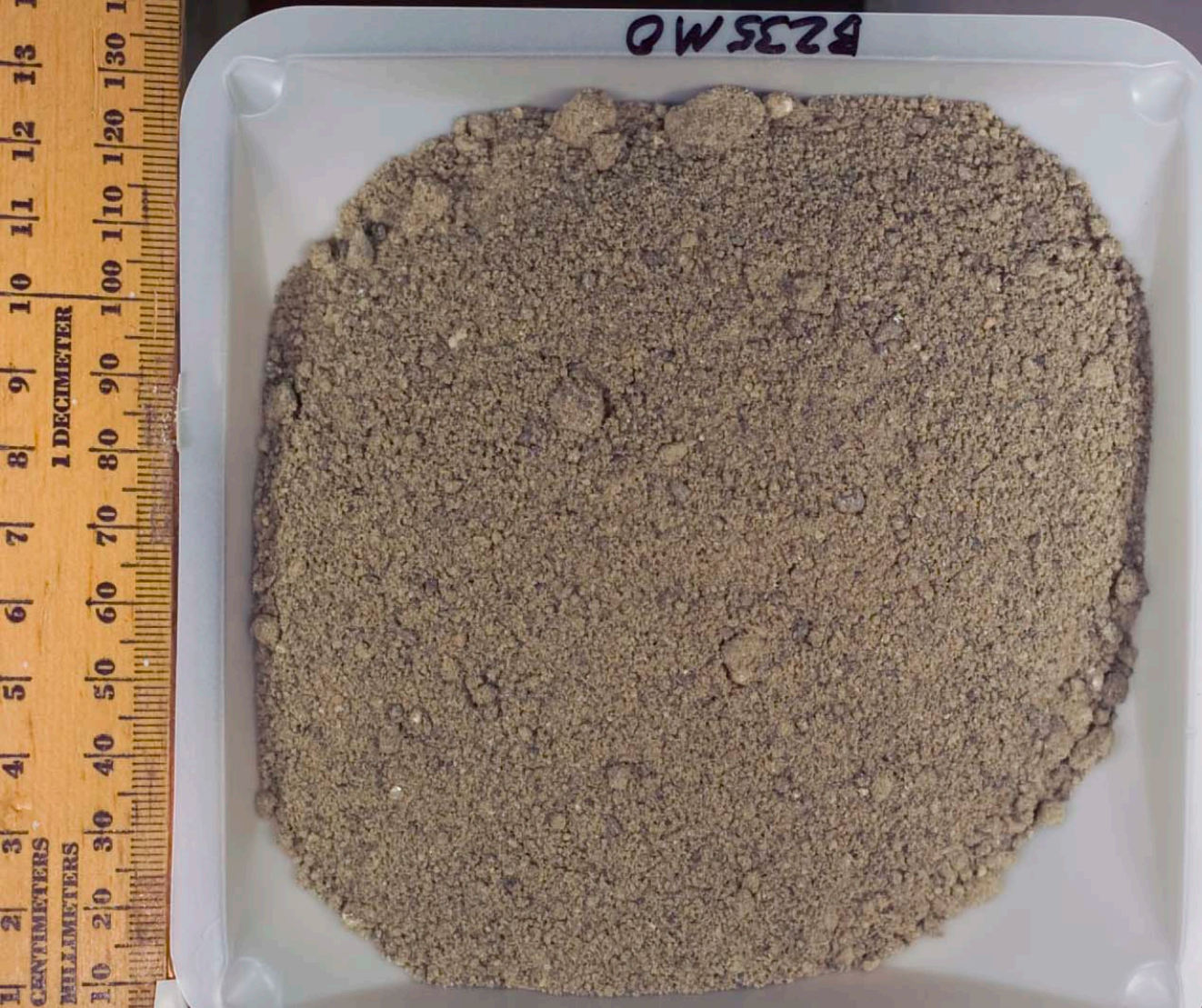


हW इड द्व

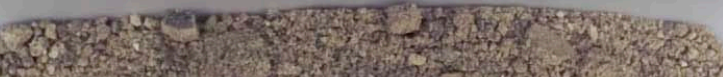

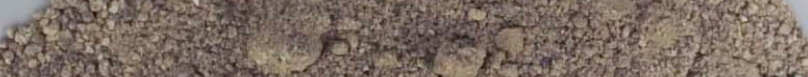

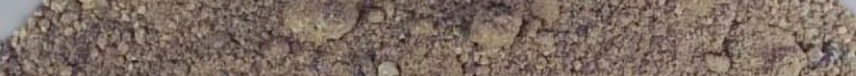

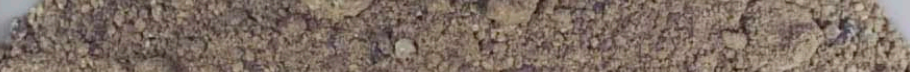

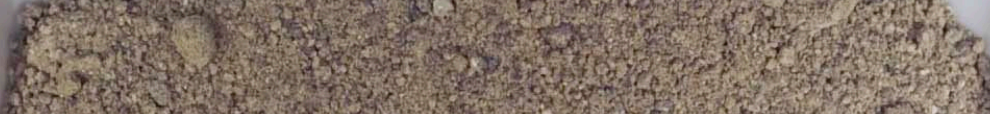

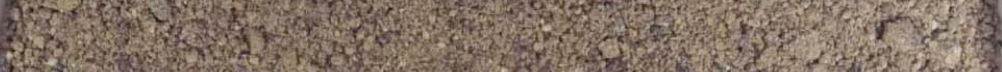
Q

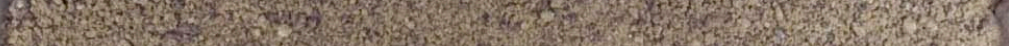
If 2 (1)

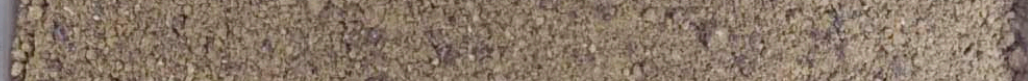

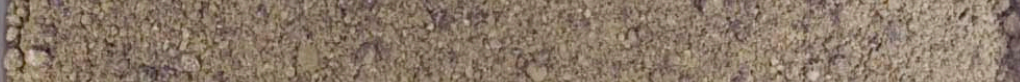

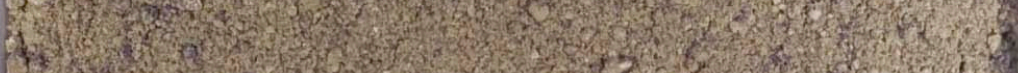

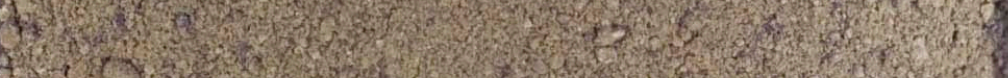
7. 3 (3)

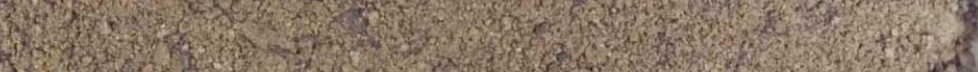

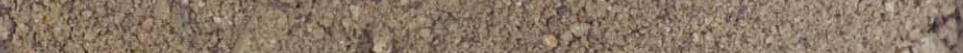

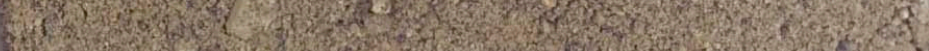

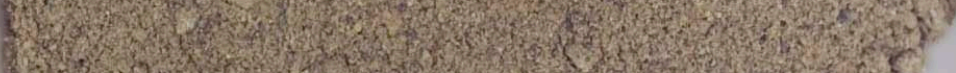

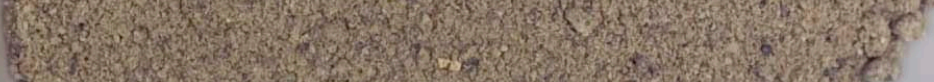

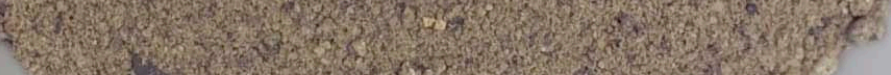

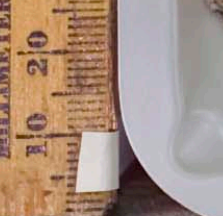

C7515 В235M3

$186.5 \mathrm{ft}$

Grab

Borehole ID

Sample ID

Depth from Chain-of-Custody

Sample 
hW SERQ

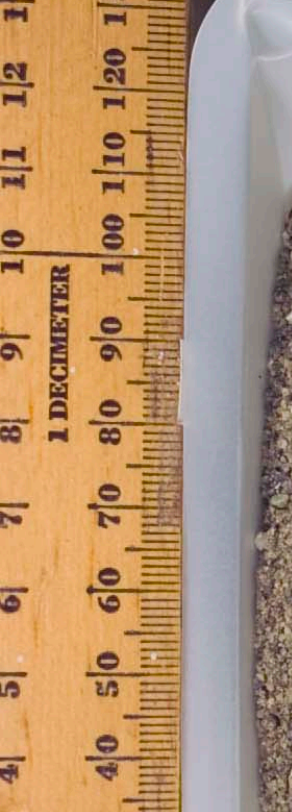

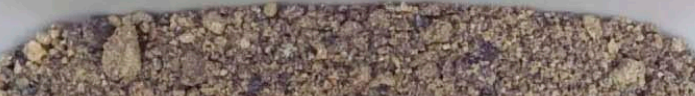
17.

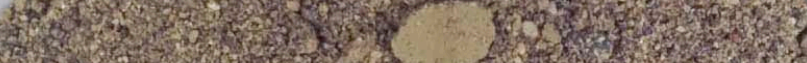

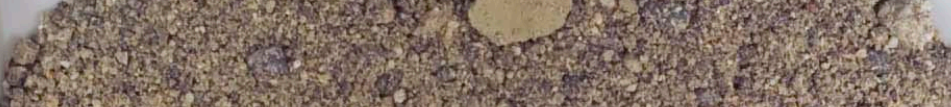

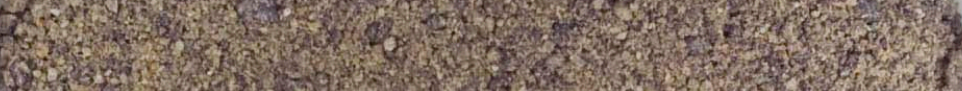

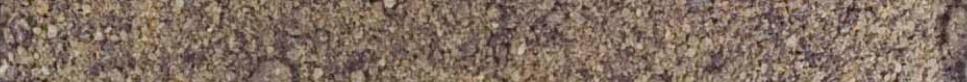

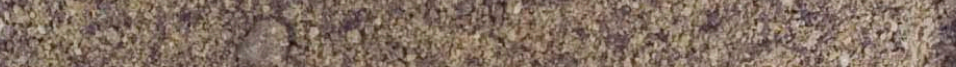

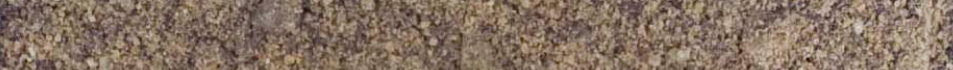

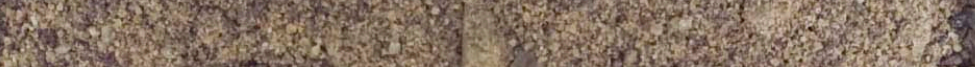
5.

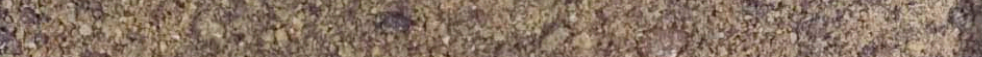

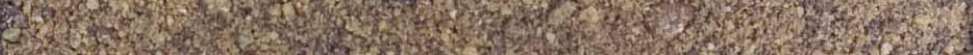

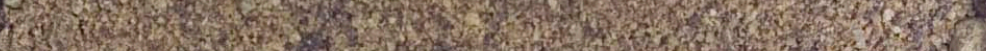
W.

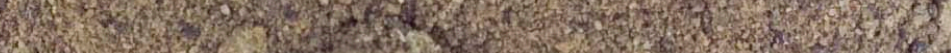

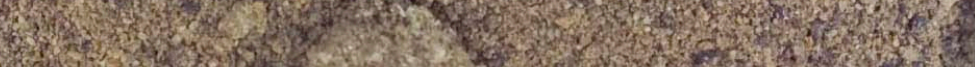
W.

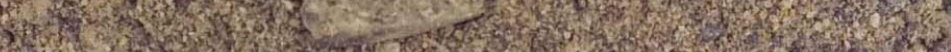

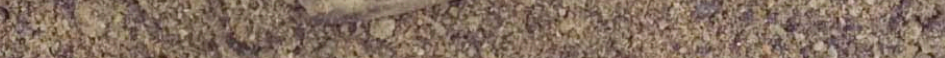

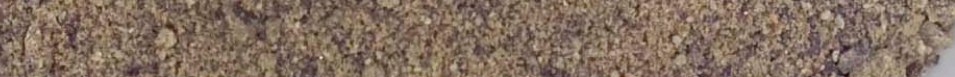

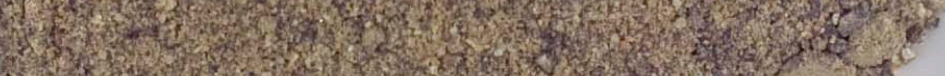

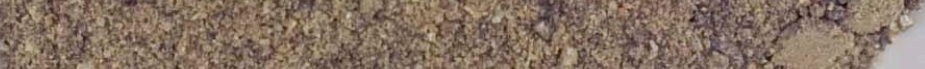
$4 \mathrm{c}^{2} \mathrm{~s}$.

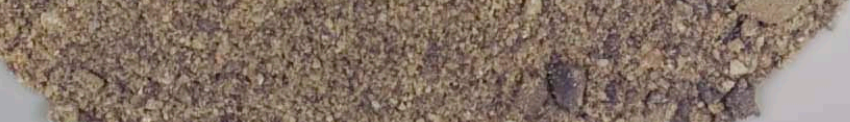

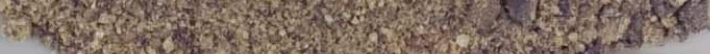

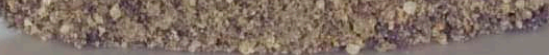

C7515 B235M4

Borehole ID
Sample ID
$189.2 \mathrm{ft}$

Depth from Chain-of-Custody
Grab

Sample 


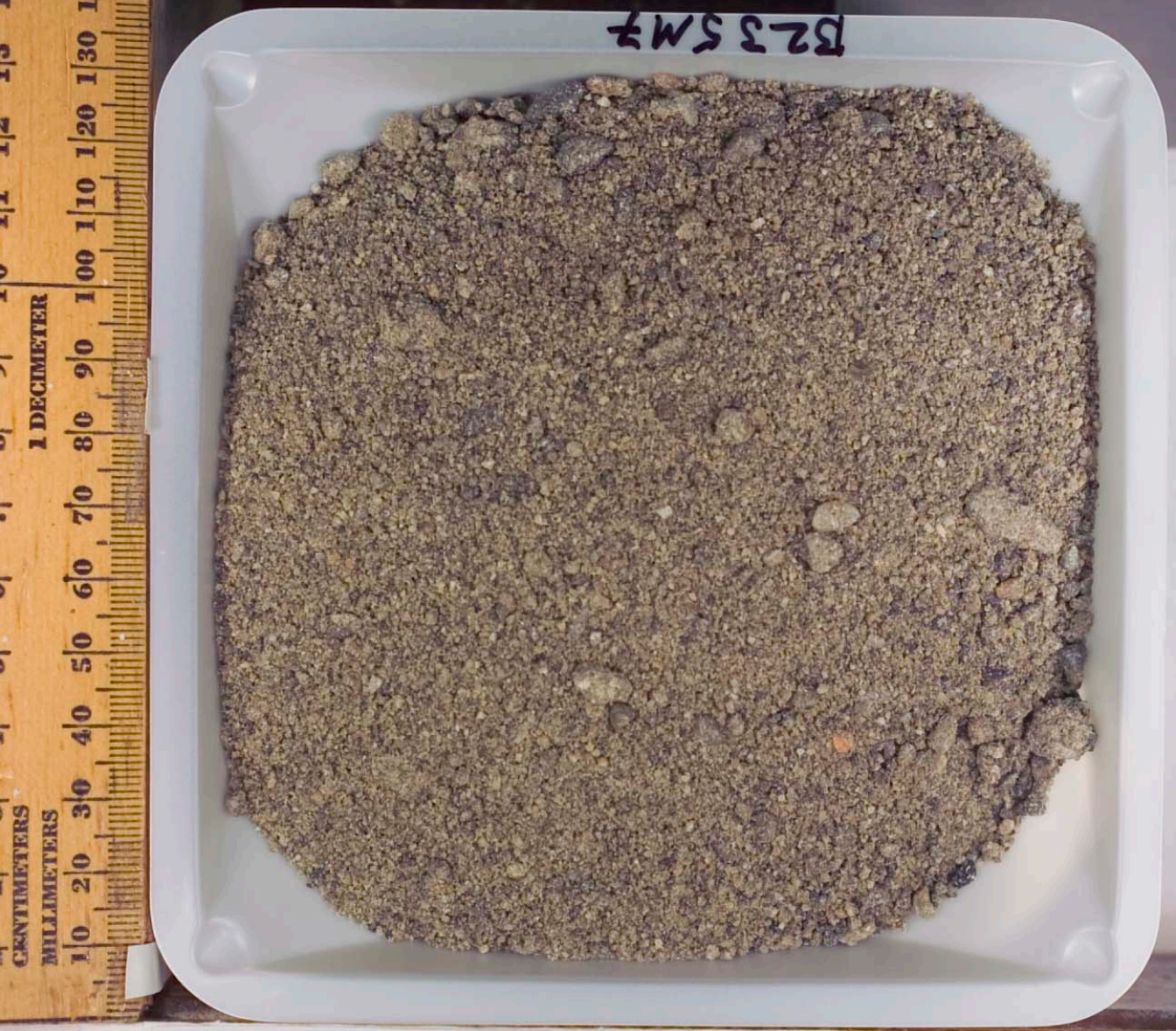

C7515 B235M7

Borehole ID
Sample ID
$197.0 \mathrm{ft}$

Depth from Chain-of-Custody
Grab Sample 


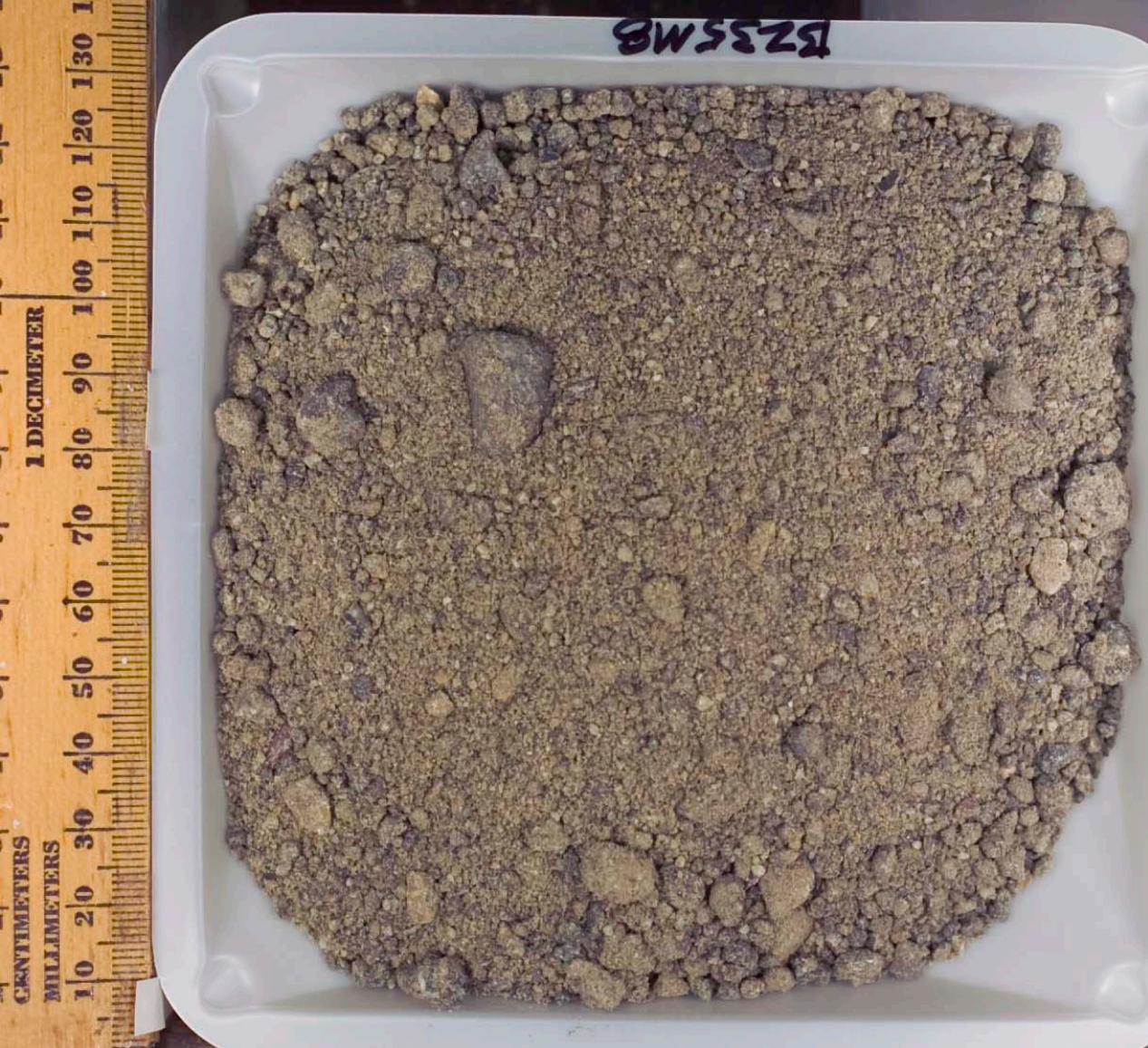




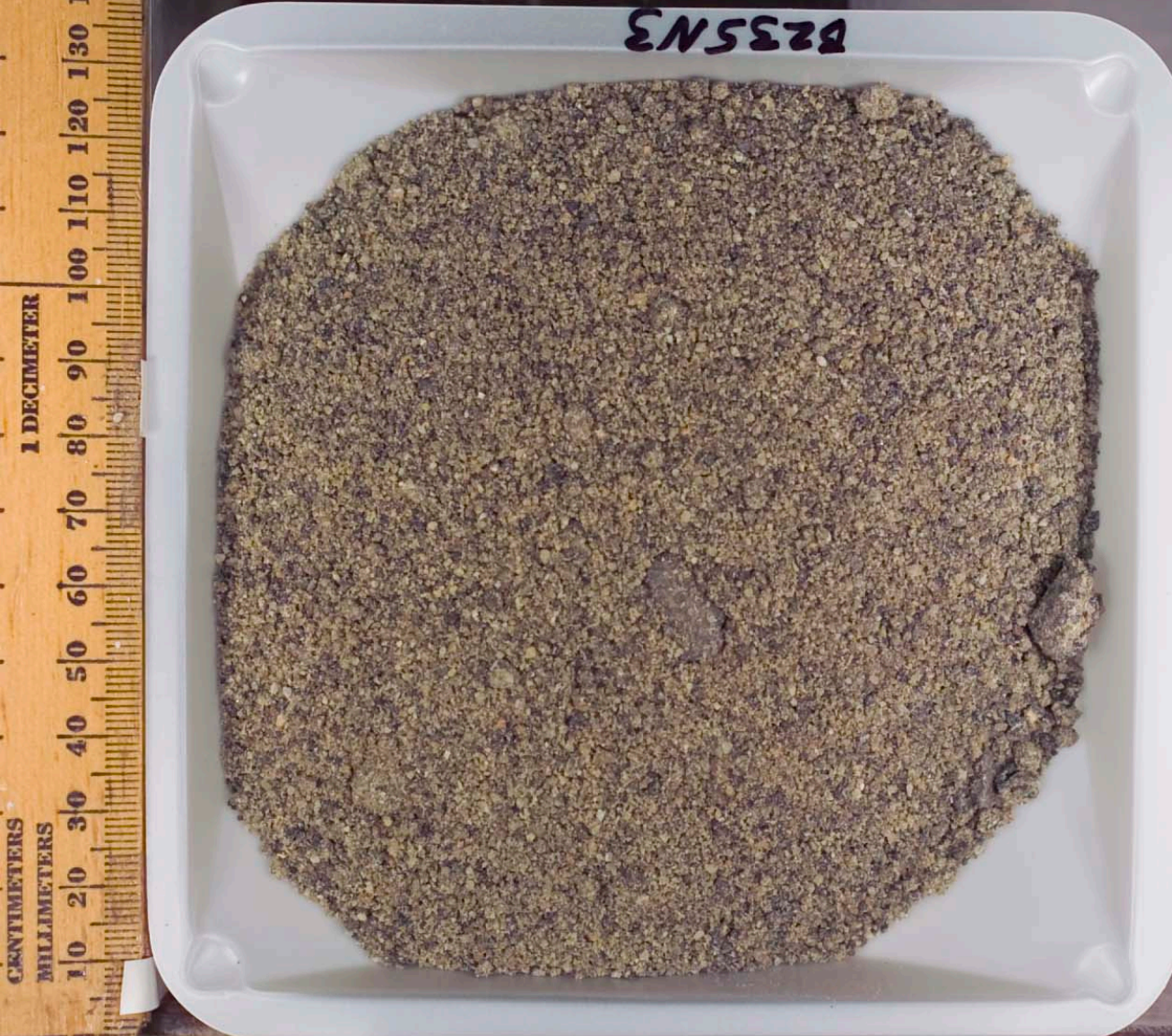





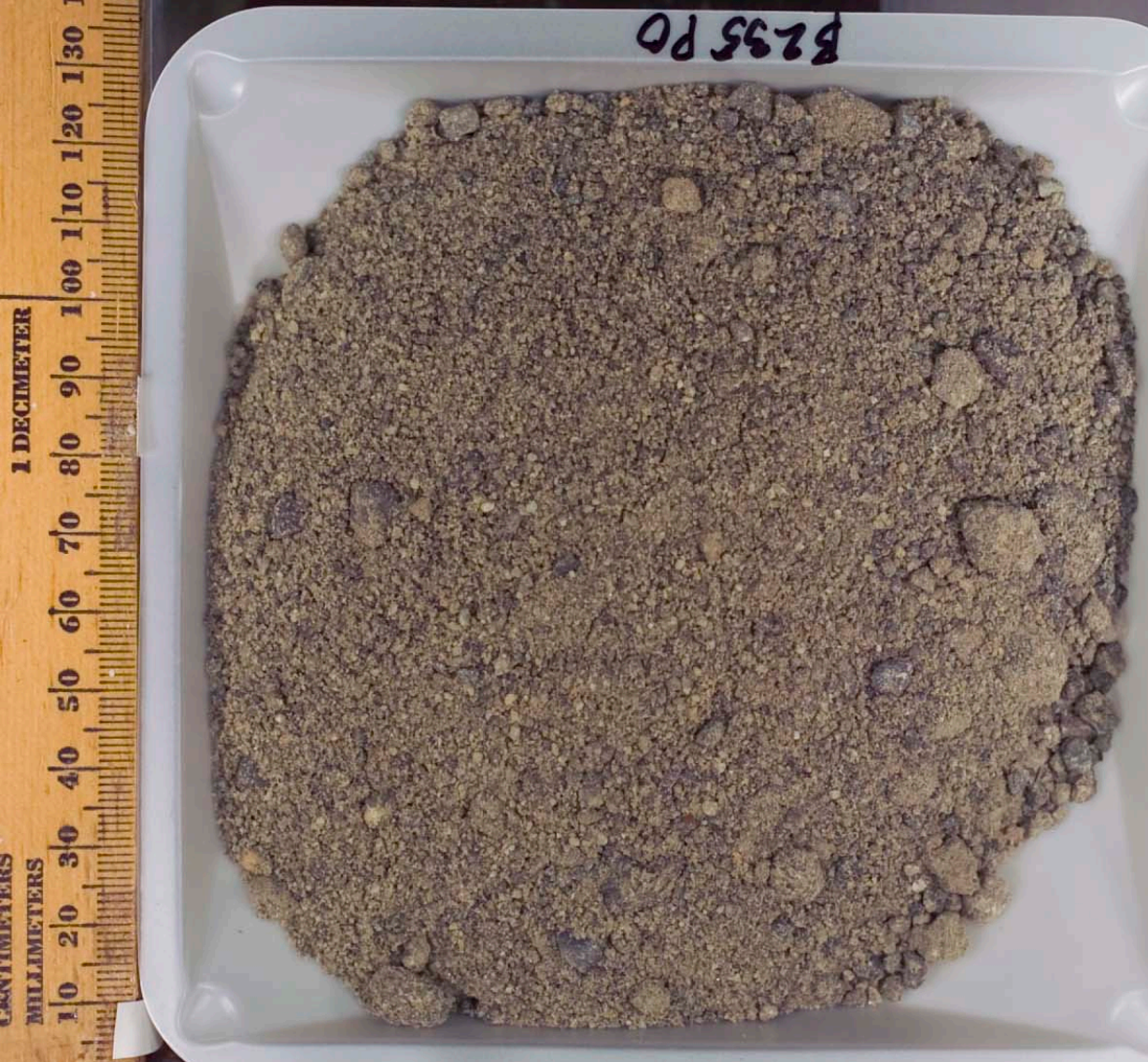




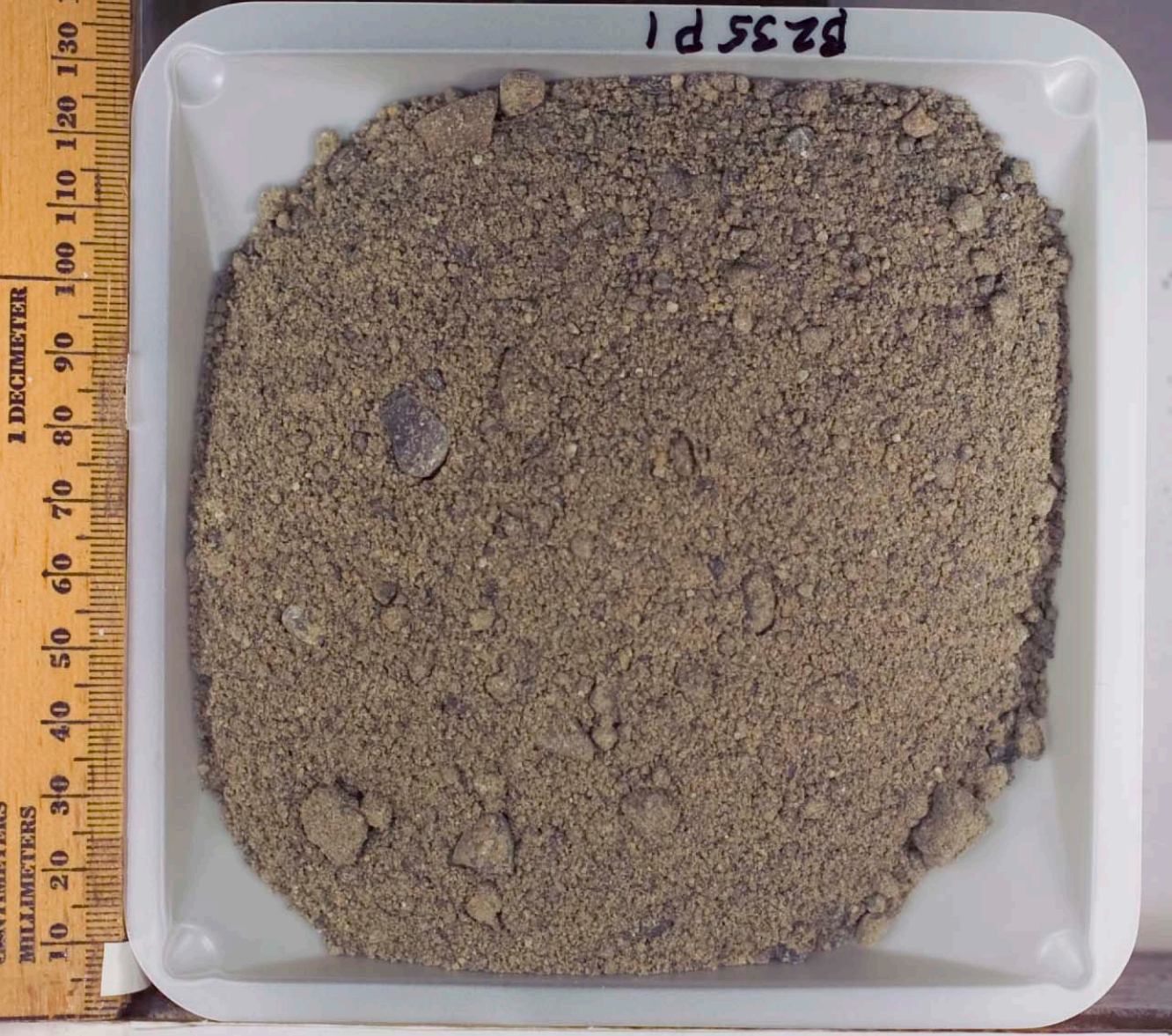

$\begin{array}{lll}C 7515 & B 235 P 1 & 232.5 \mathrm{ft}\end{array}$ Borehole ID Sample ID Depth from Chain-of-Custody Grab Sample 


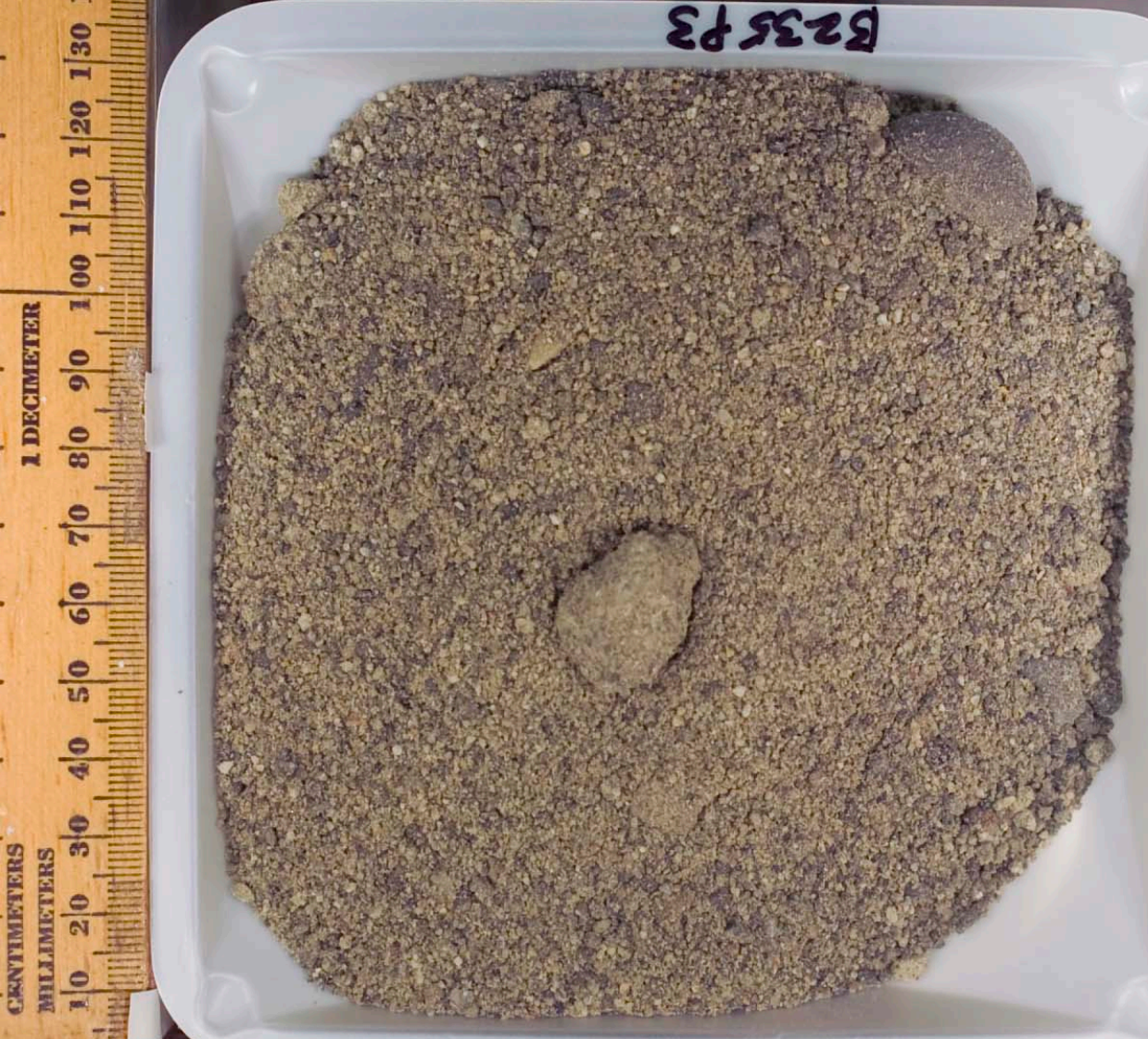



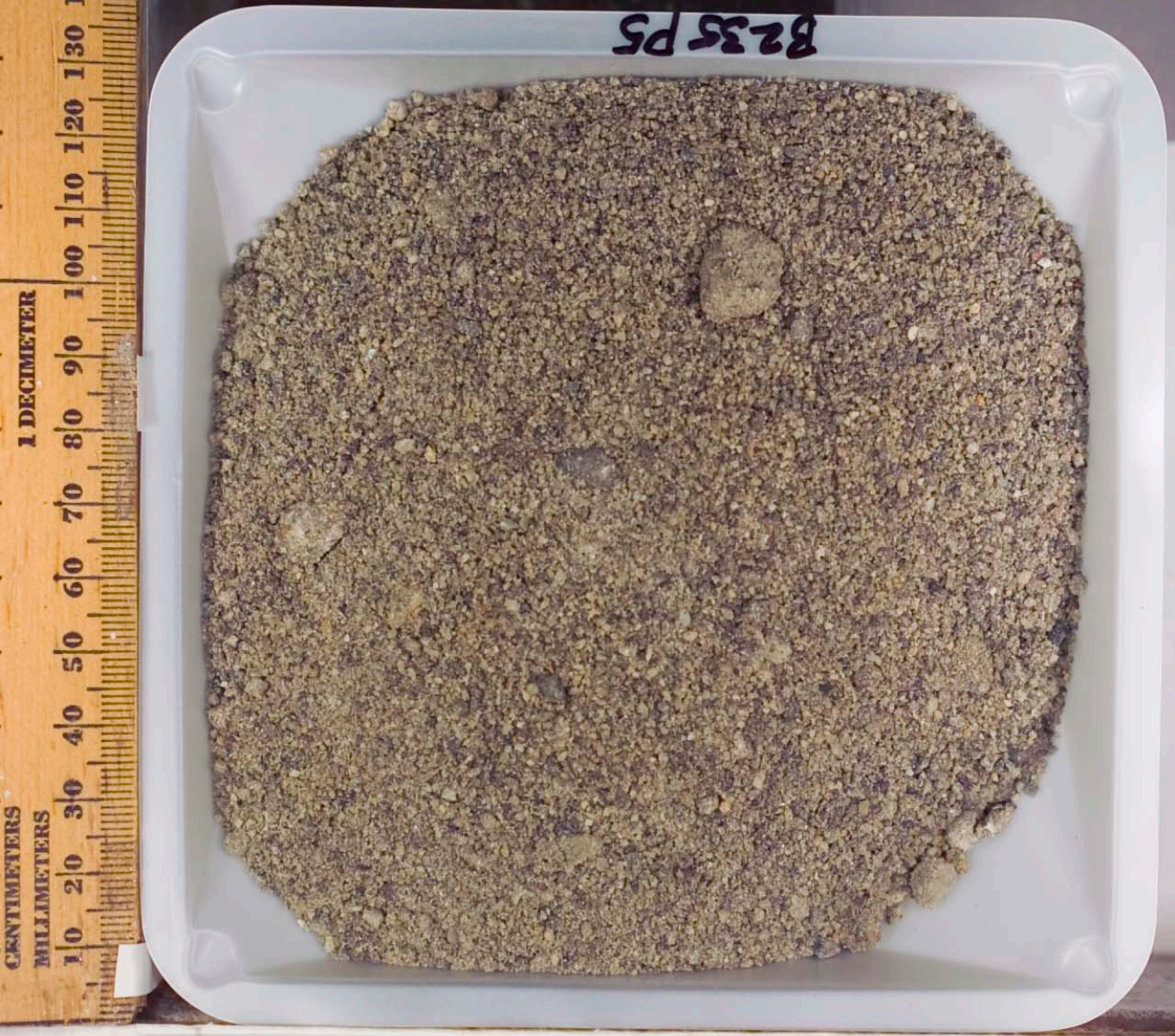
C7515
B235P5
$241.9 \mathrm{ft}$
Borehole ID
Sample ID
Depth from Chain-of-Custody
Sample

Grab 


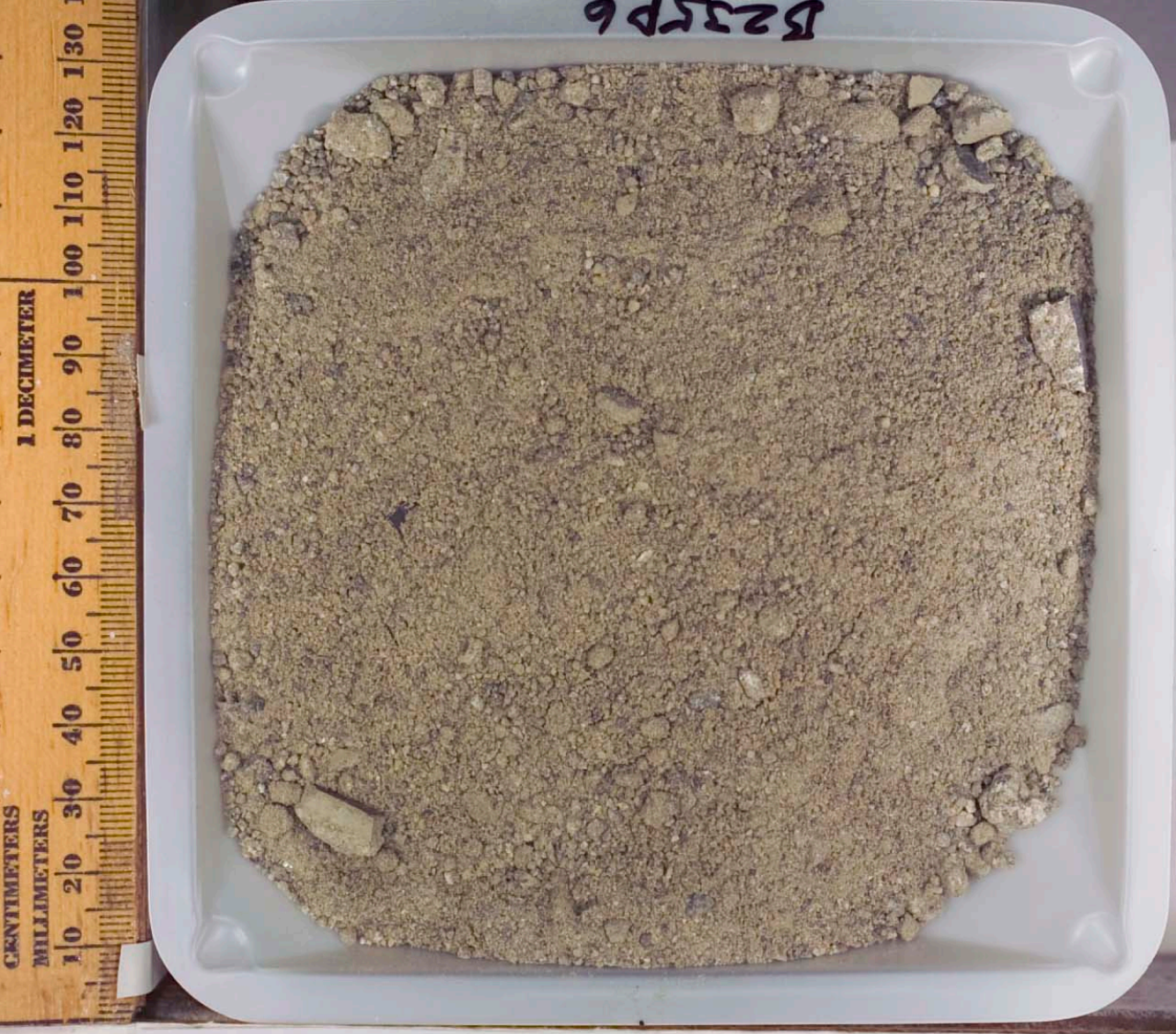

C7515 B235P6

$244.0 \mathrm{ft}$

Grab

Borehole ID Sample ID

Depth from Chain-of-Custody

Sample 


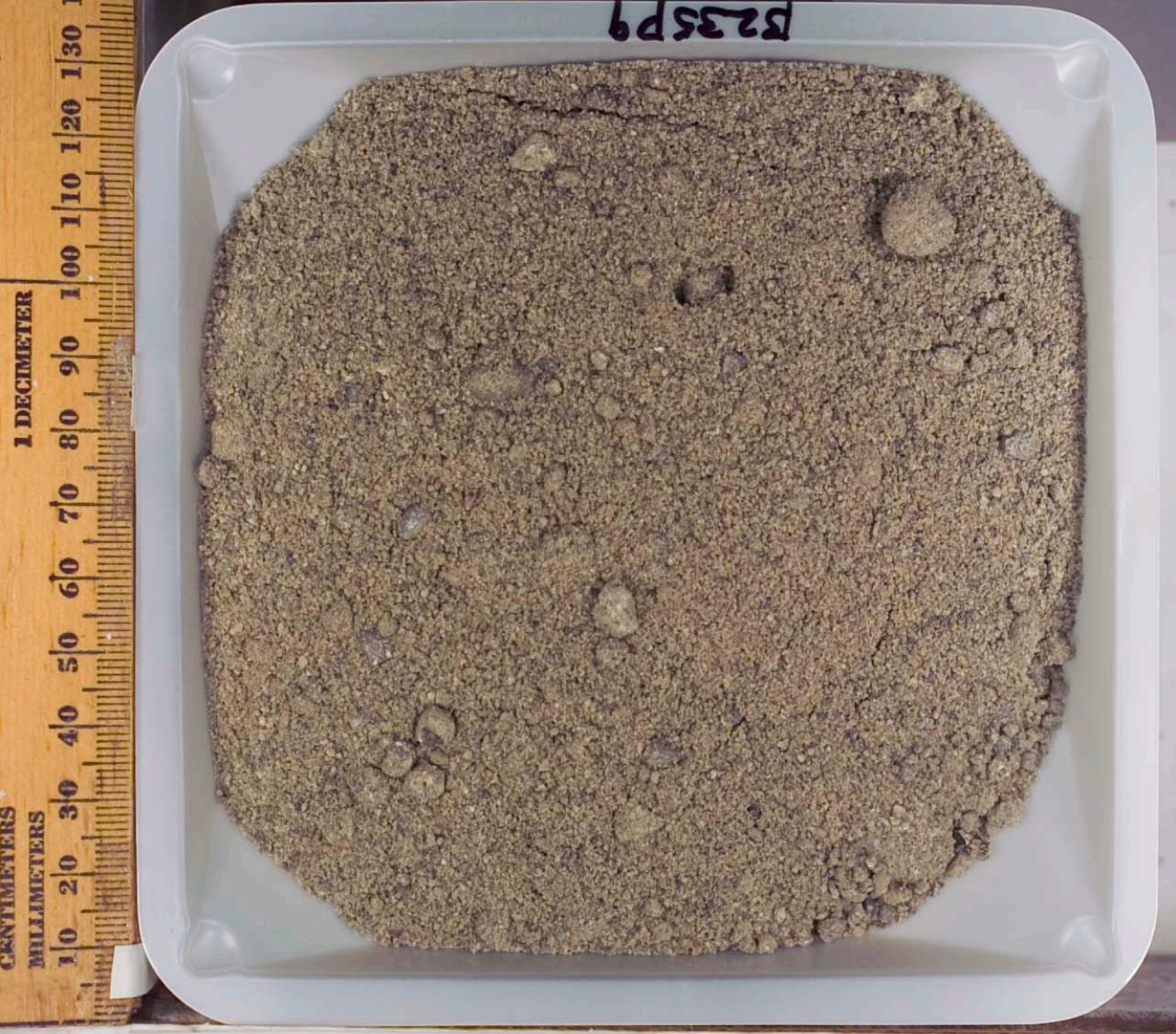

$\begin{array}{lll}C 7515 & \text { B235P9 } \quad 252.5 \mathrm{ft}\end{array}$

Borehole ID Sample ID Depth from Chain-of-Custody

Grab Sample 


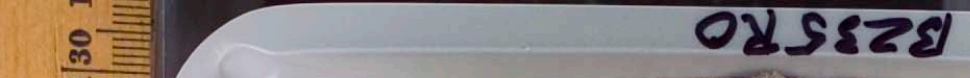




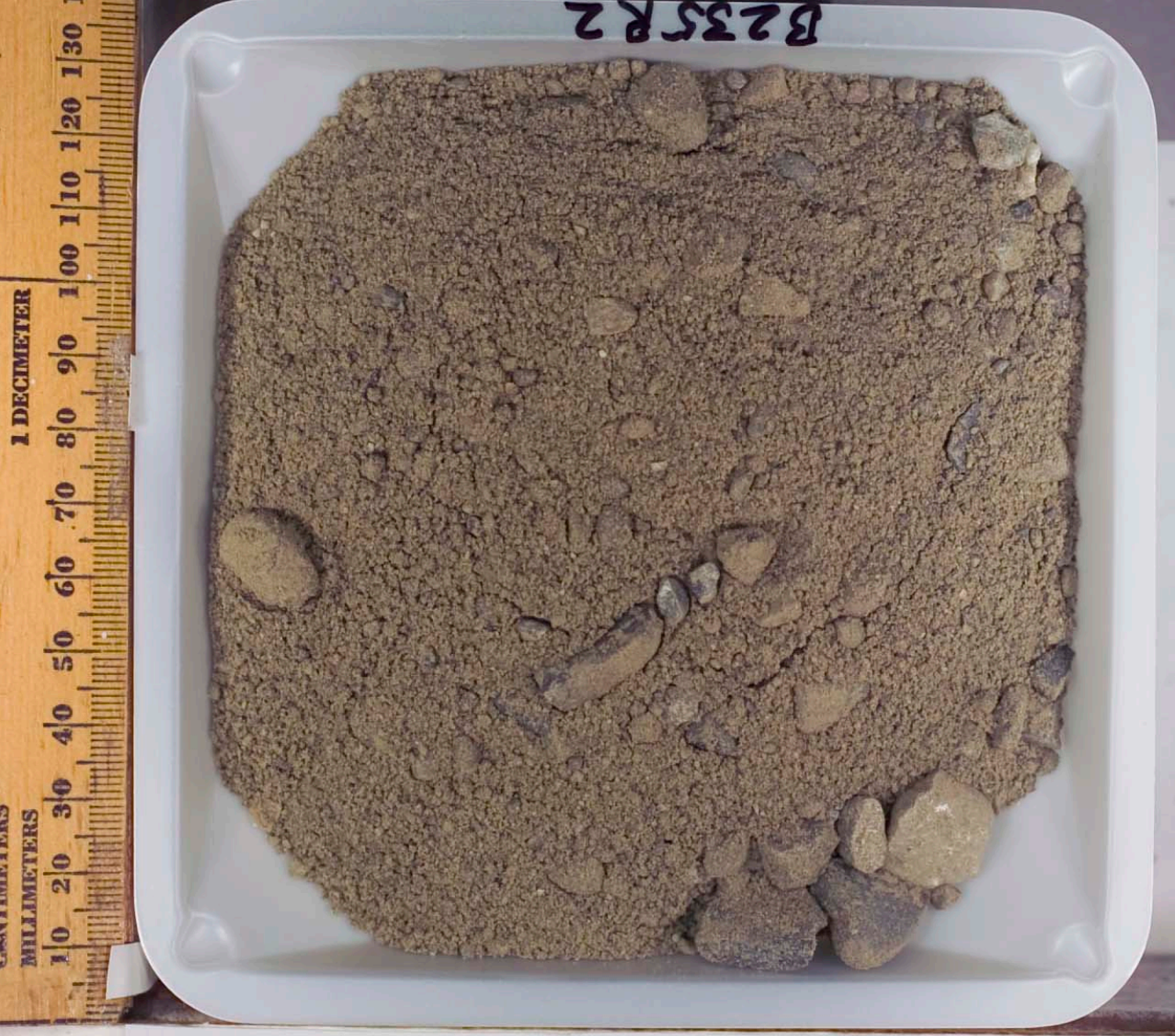

C7515 B235R2

Borehole ID
Sample ID
$259.5 \mathrm{ft}$

Grab Depth from Chain-of-Custody
Sample 


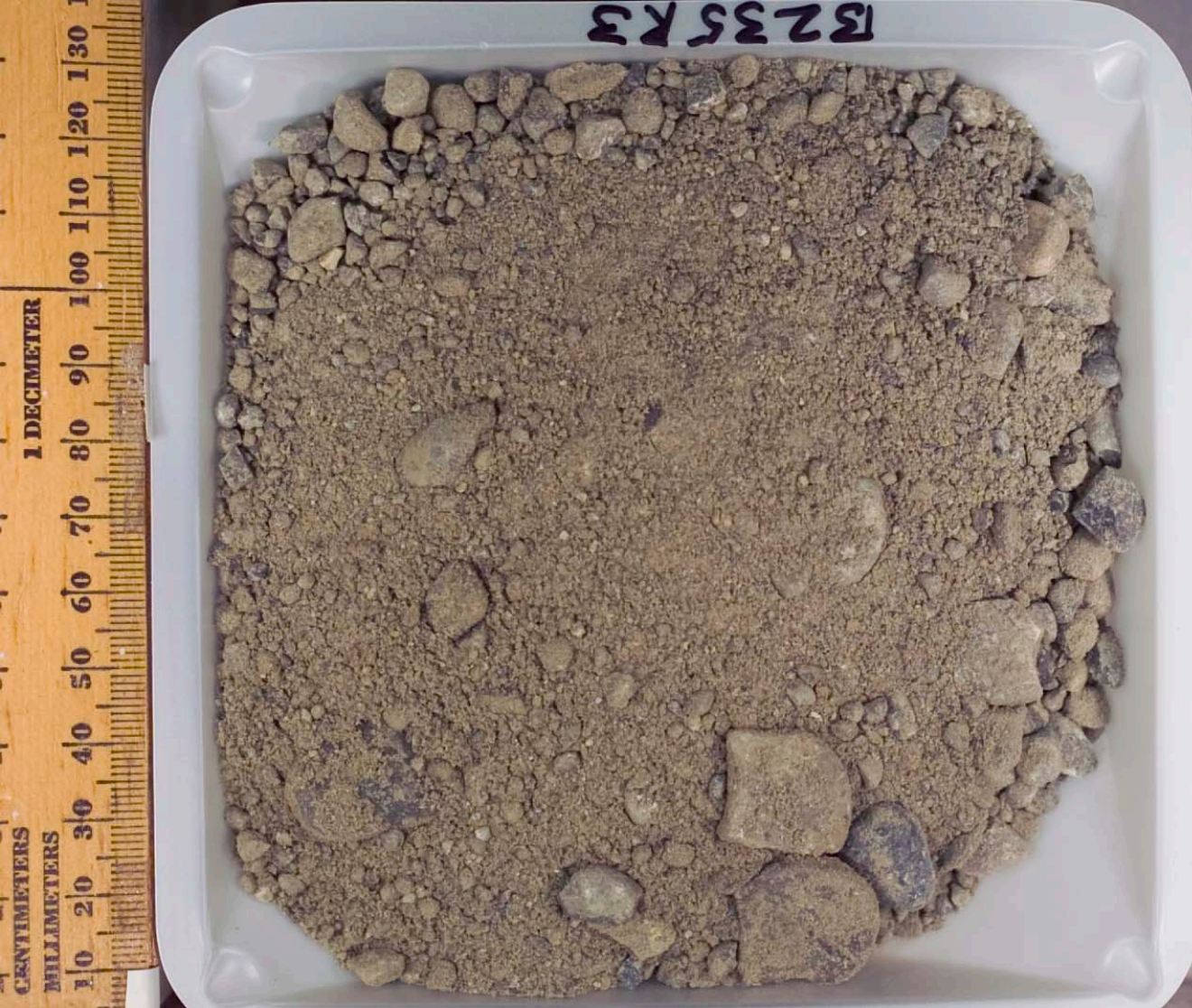

C7515 B235R3

Borehole ID 


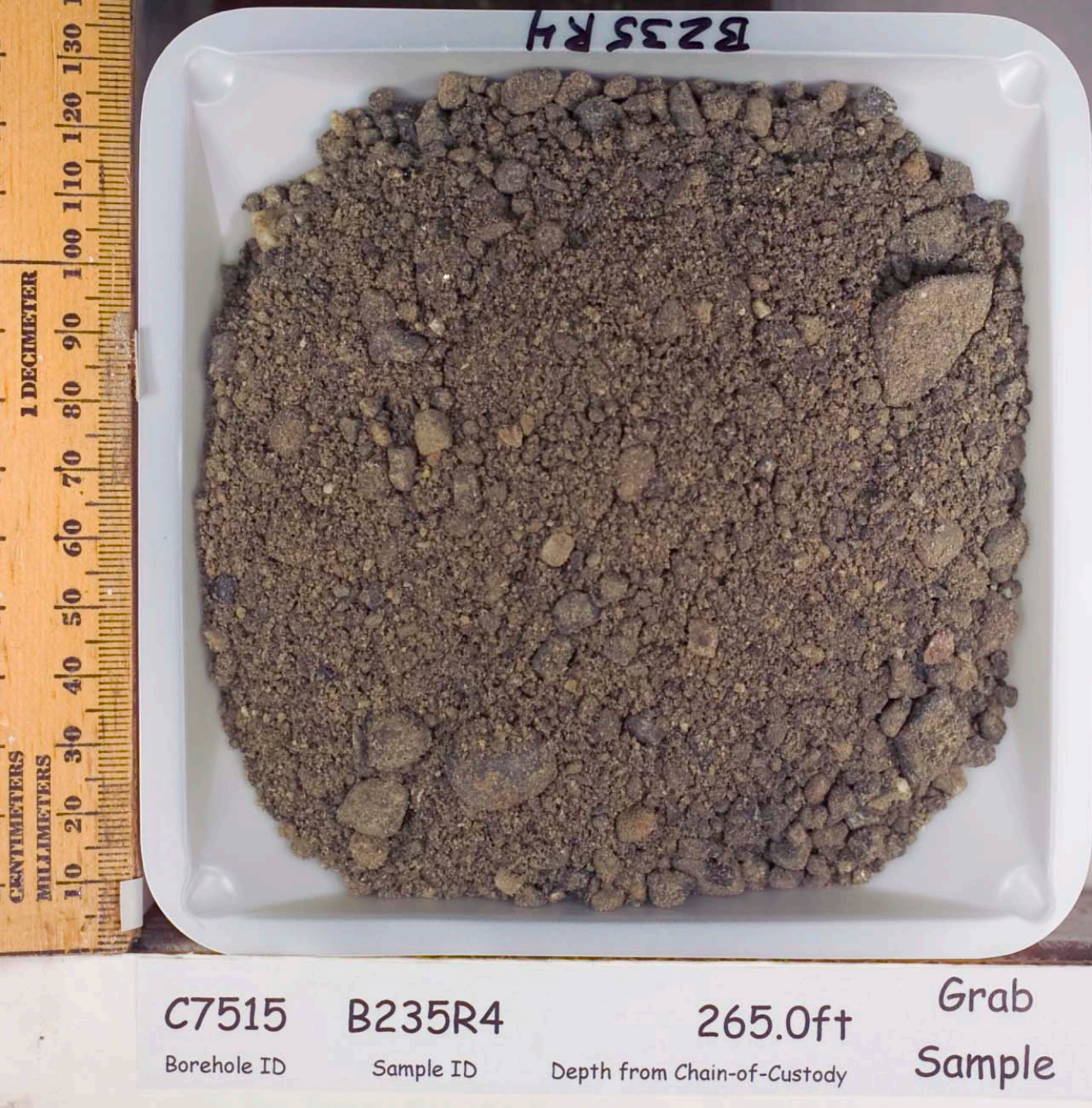




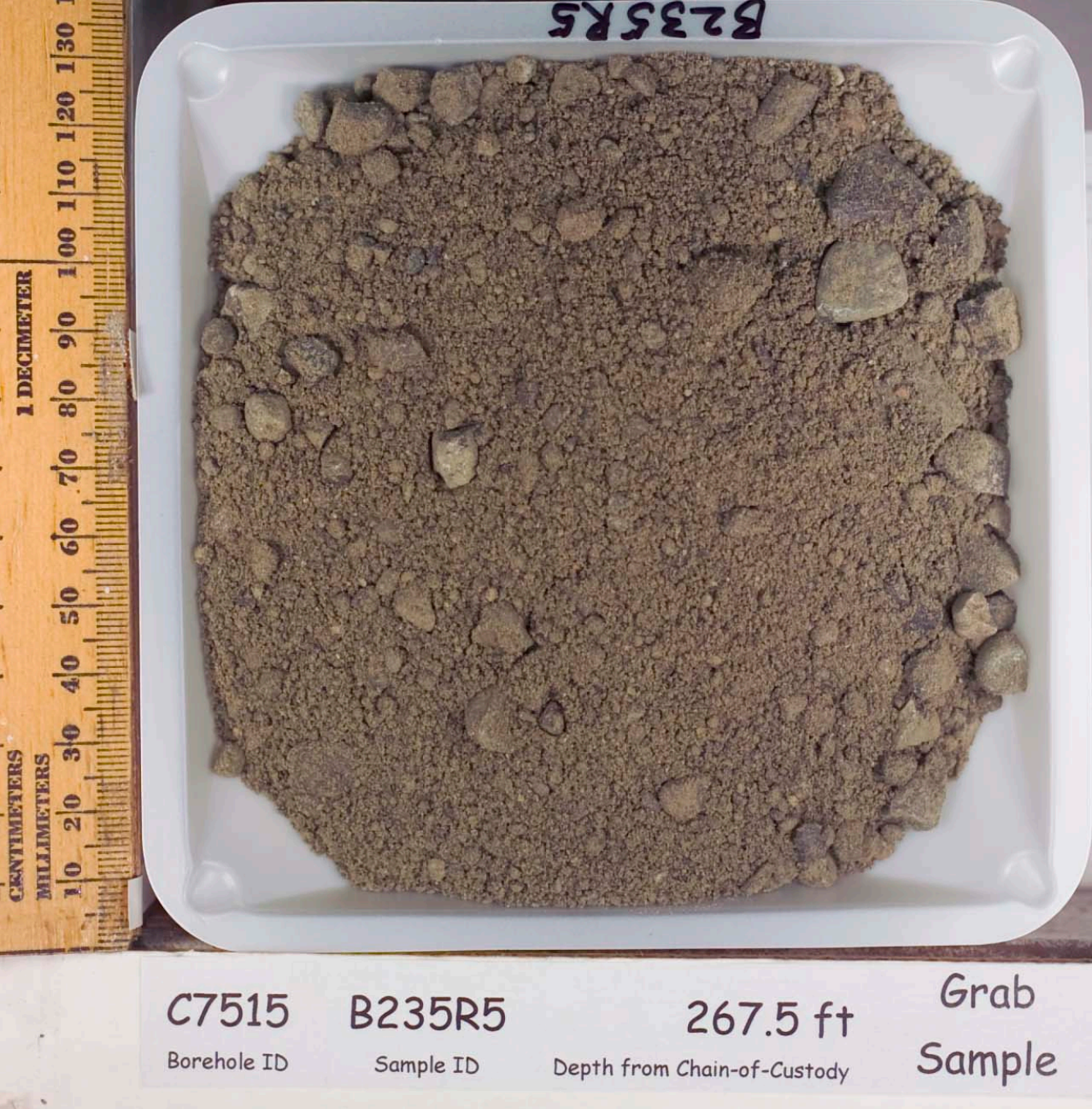




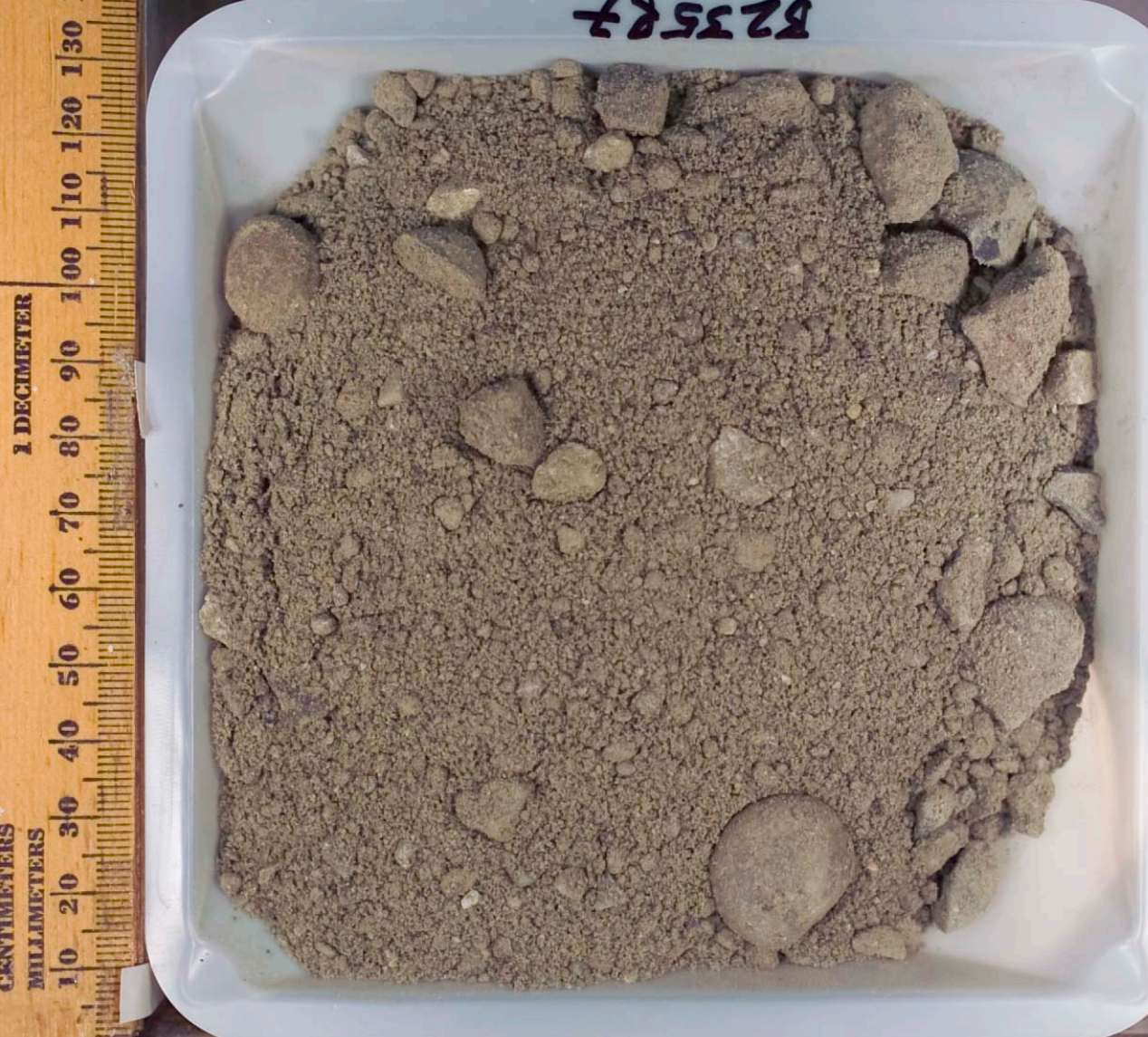




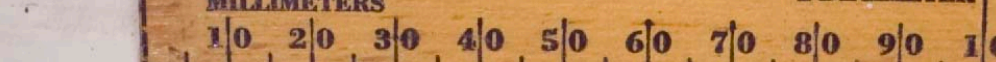

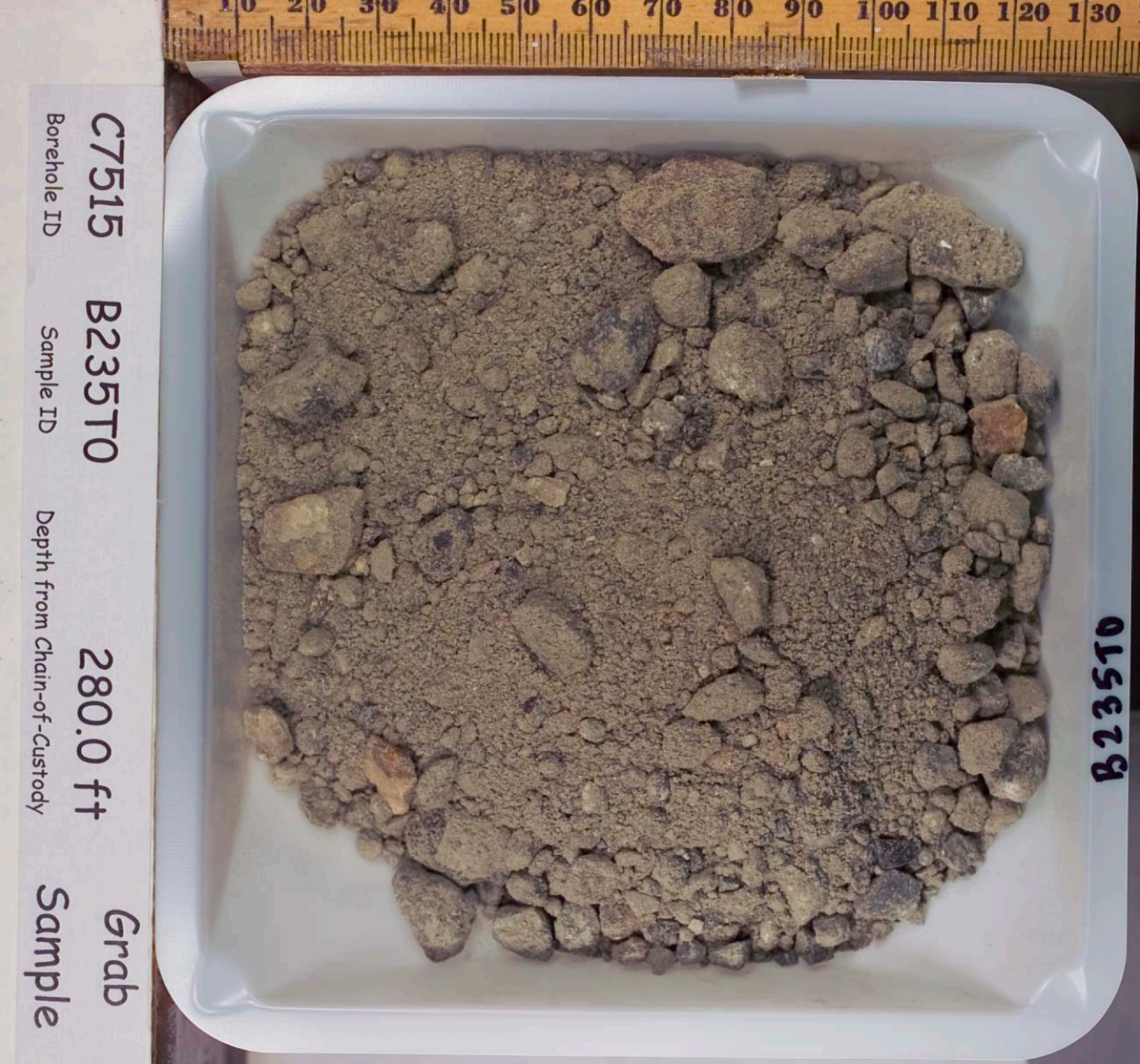


밤

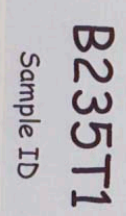

0
$\frac{8}{0}$
$\frac{1}{3}$
$\frac{1}{3}$
$\frac{1}{3}$

1

No

Do

N

กิ

亭 -

$\frac{3}{0} \frac{0}{0}$
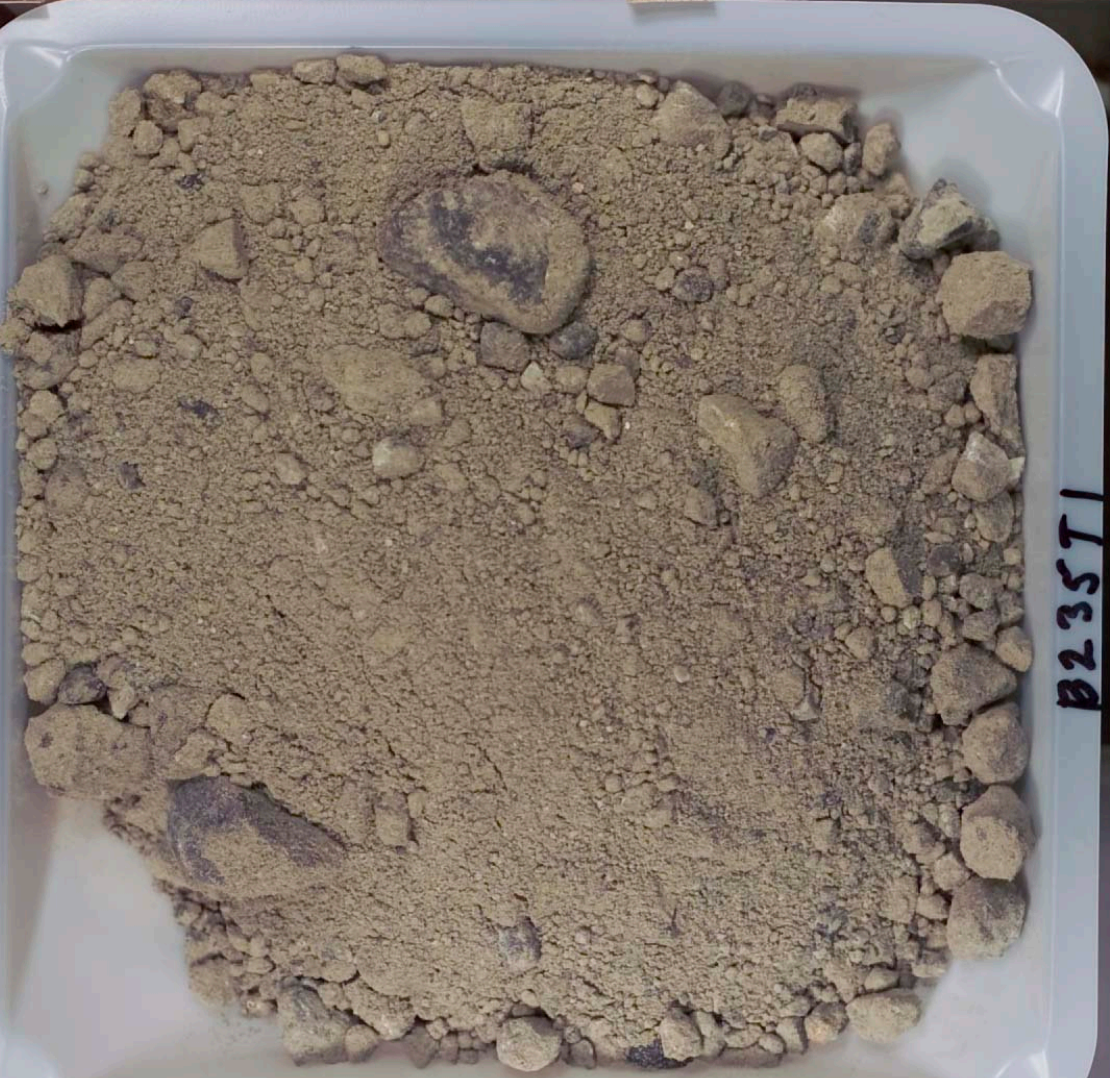


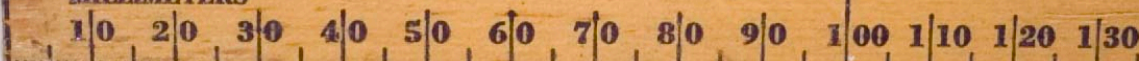
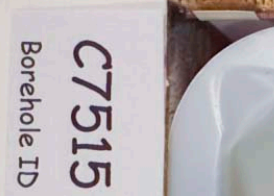

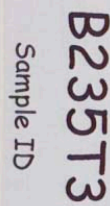

일

更

N

$\infty$

O0

O

$\rightarrow$

$\frac{3}{0} \frac{0}{0}$

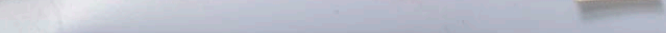

3.

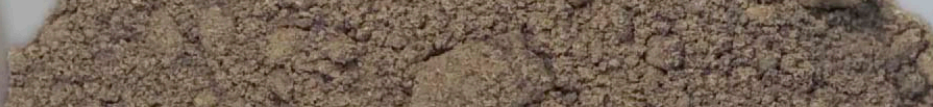

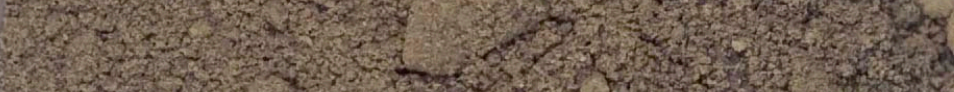

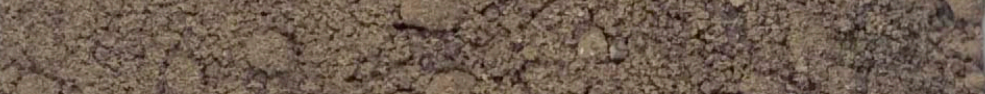

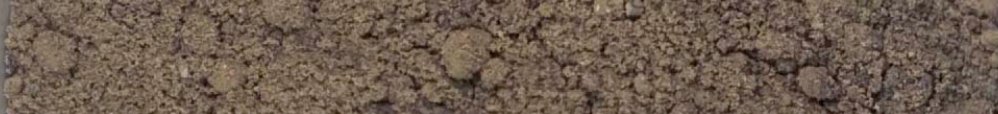

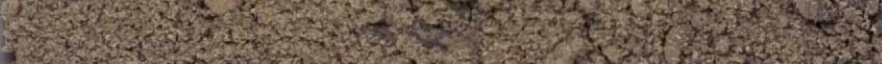

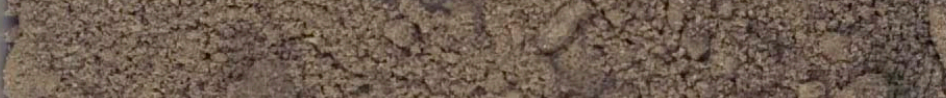

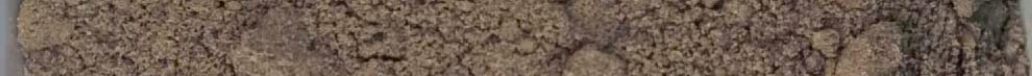

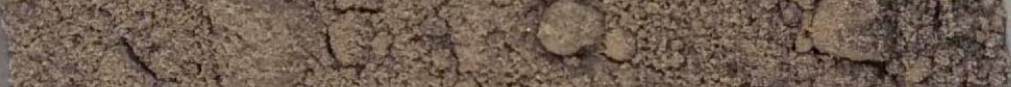

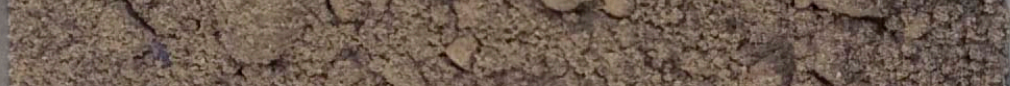

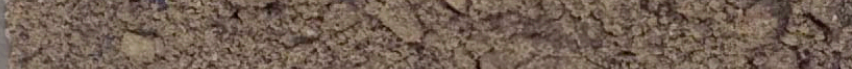

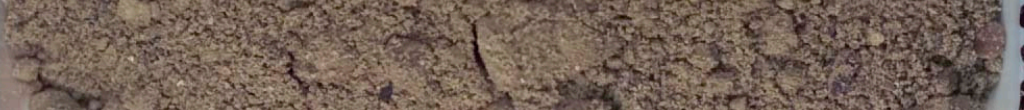
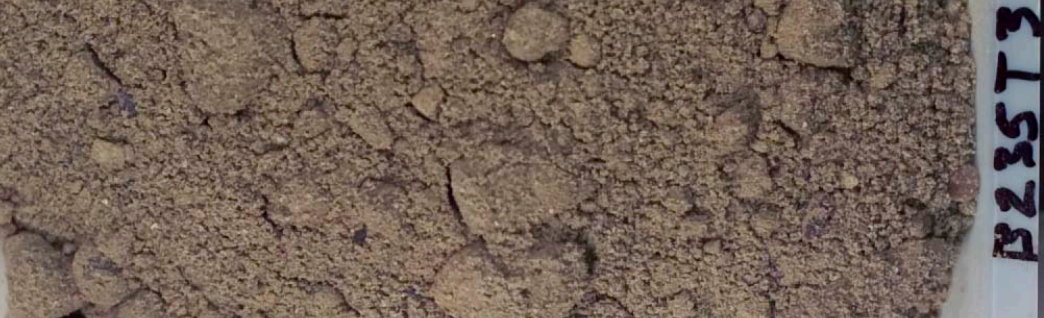


\section{MIUUMIETERS}

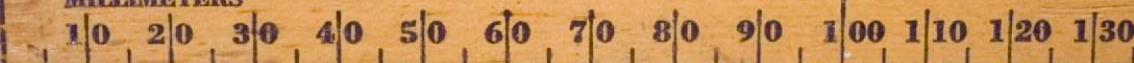
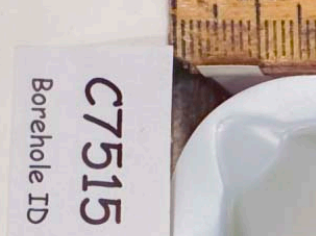

$\begin{array}{ll}0 \\ \text { n } \\ \frac{0}{3} & 0 \\ \frac{0}{3} & 0\end{array}$

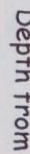

要

n

OO

6

01

$\frac{5}{4}$
aे
암

$\begin{array}{ll}\frac{3}{3} & 0 \\ \frac{0}{0} & 0\end{array}$

(
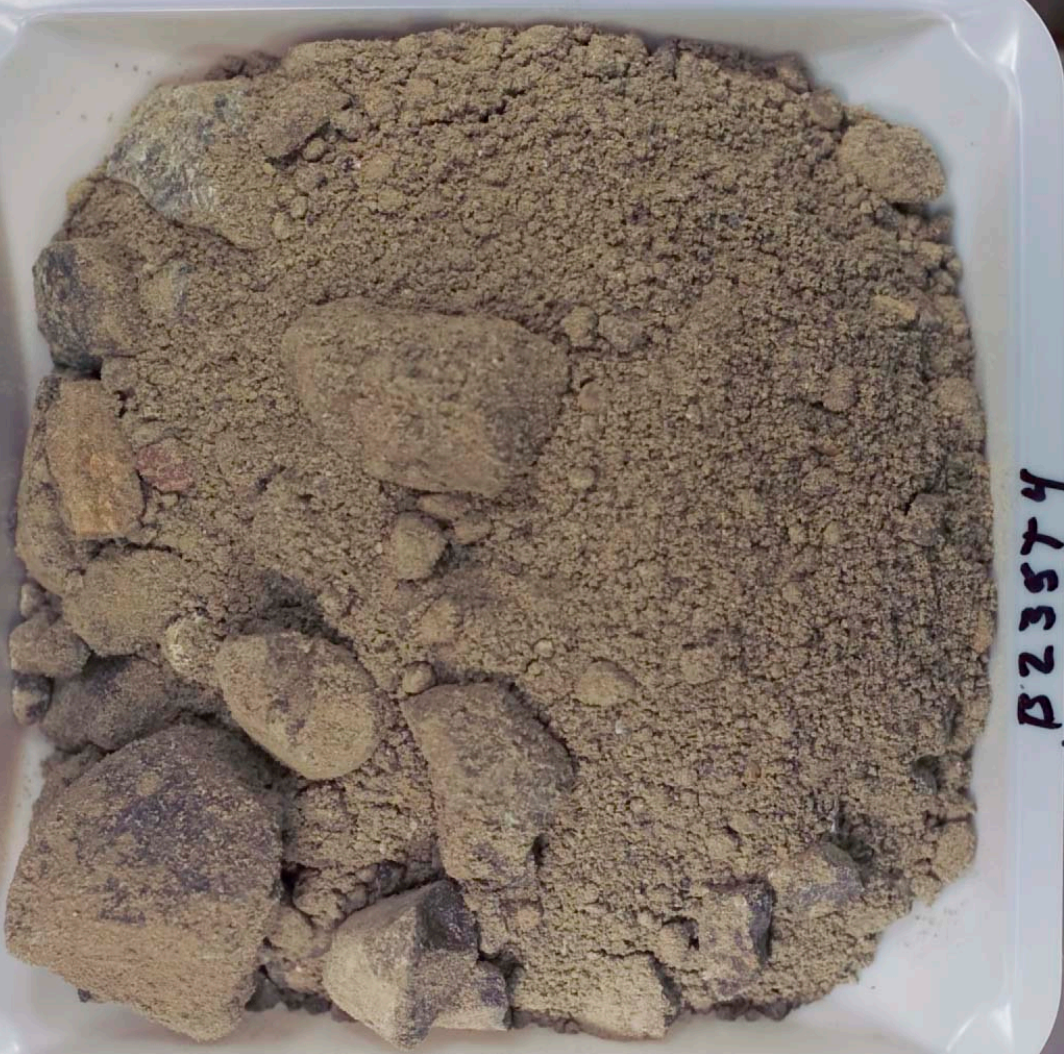


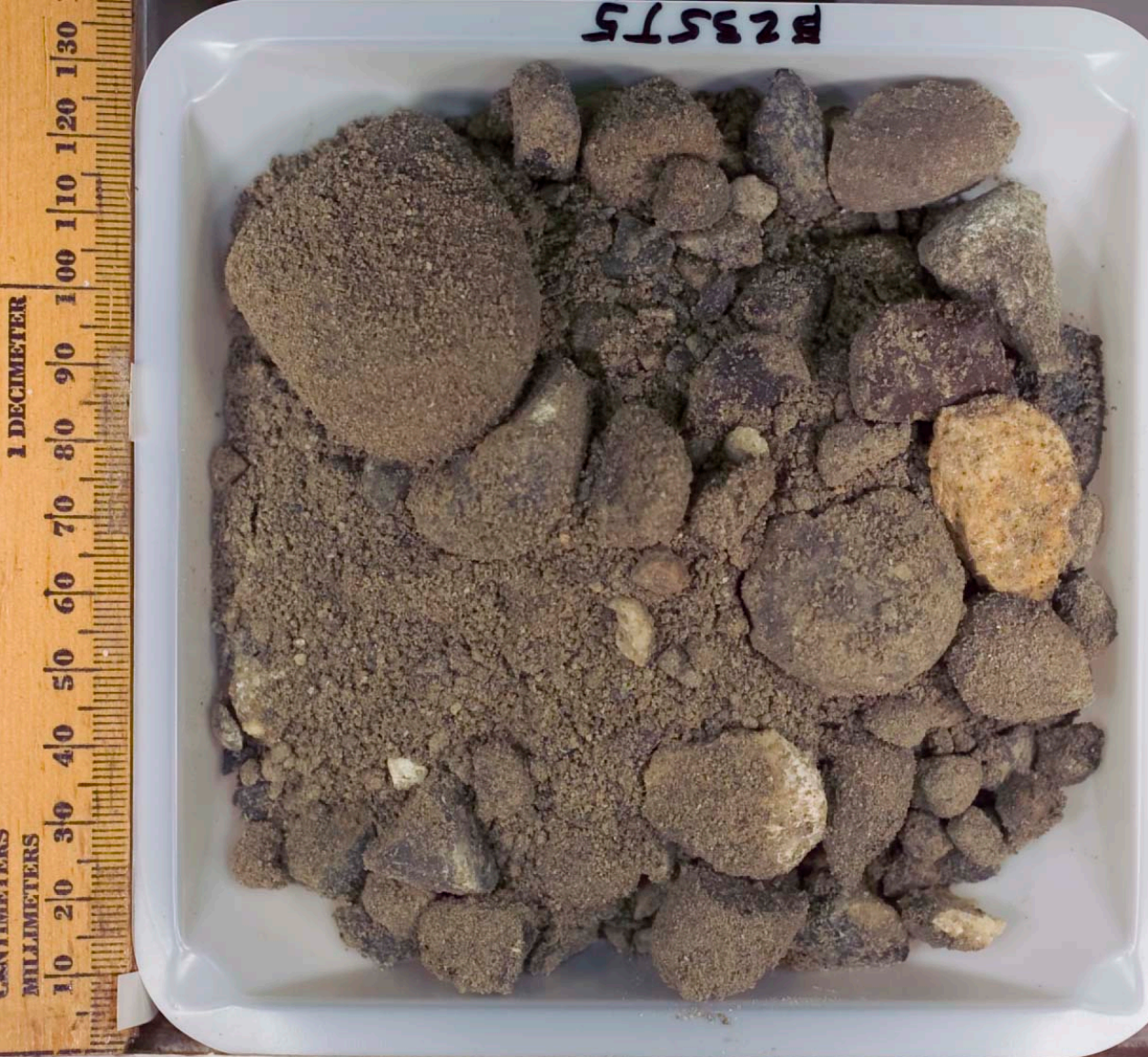




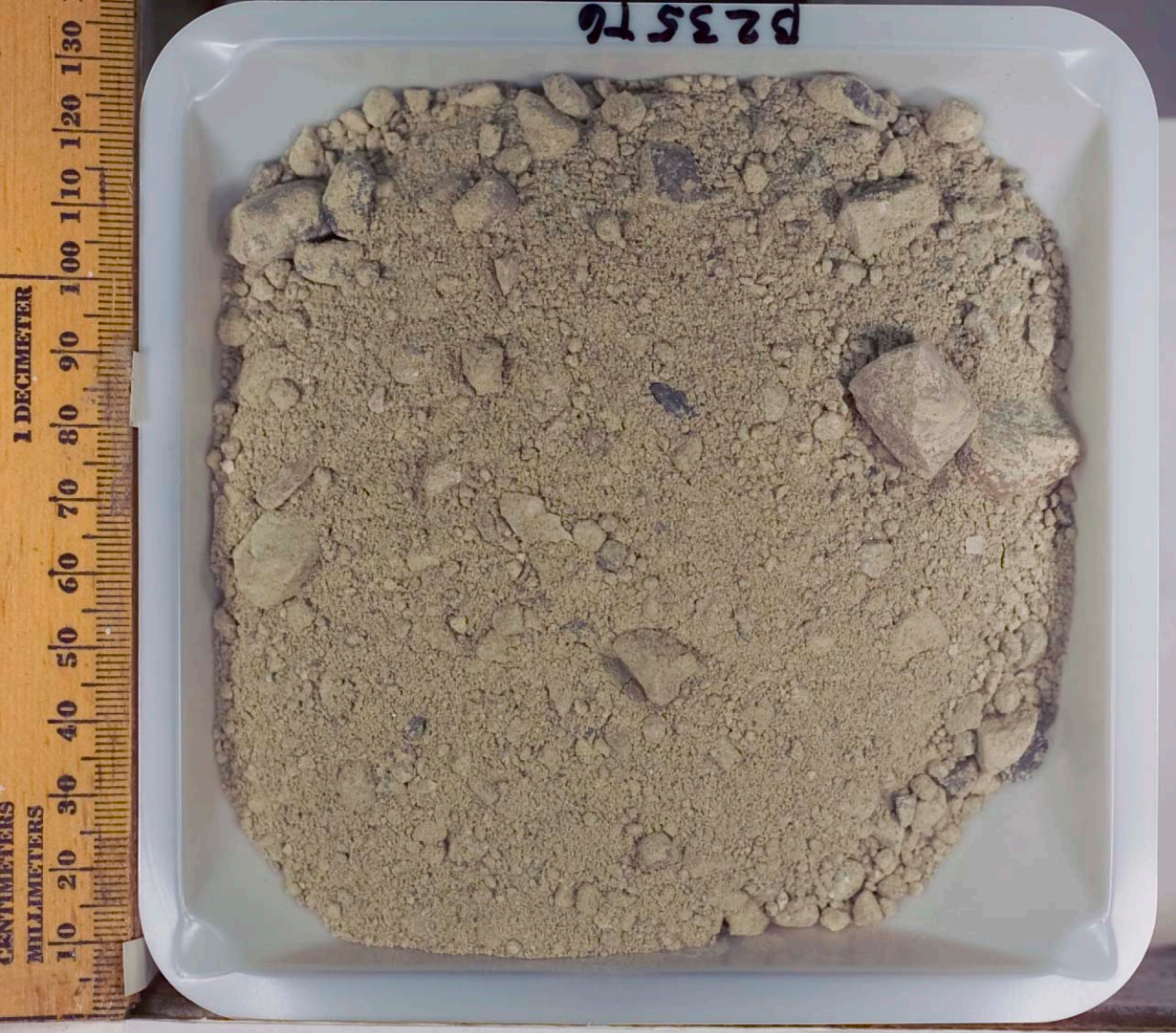

C7515 B235T6 Borehole ID
Sample ID
Grab Sample 


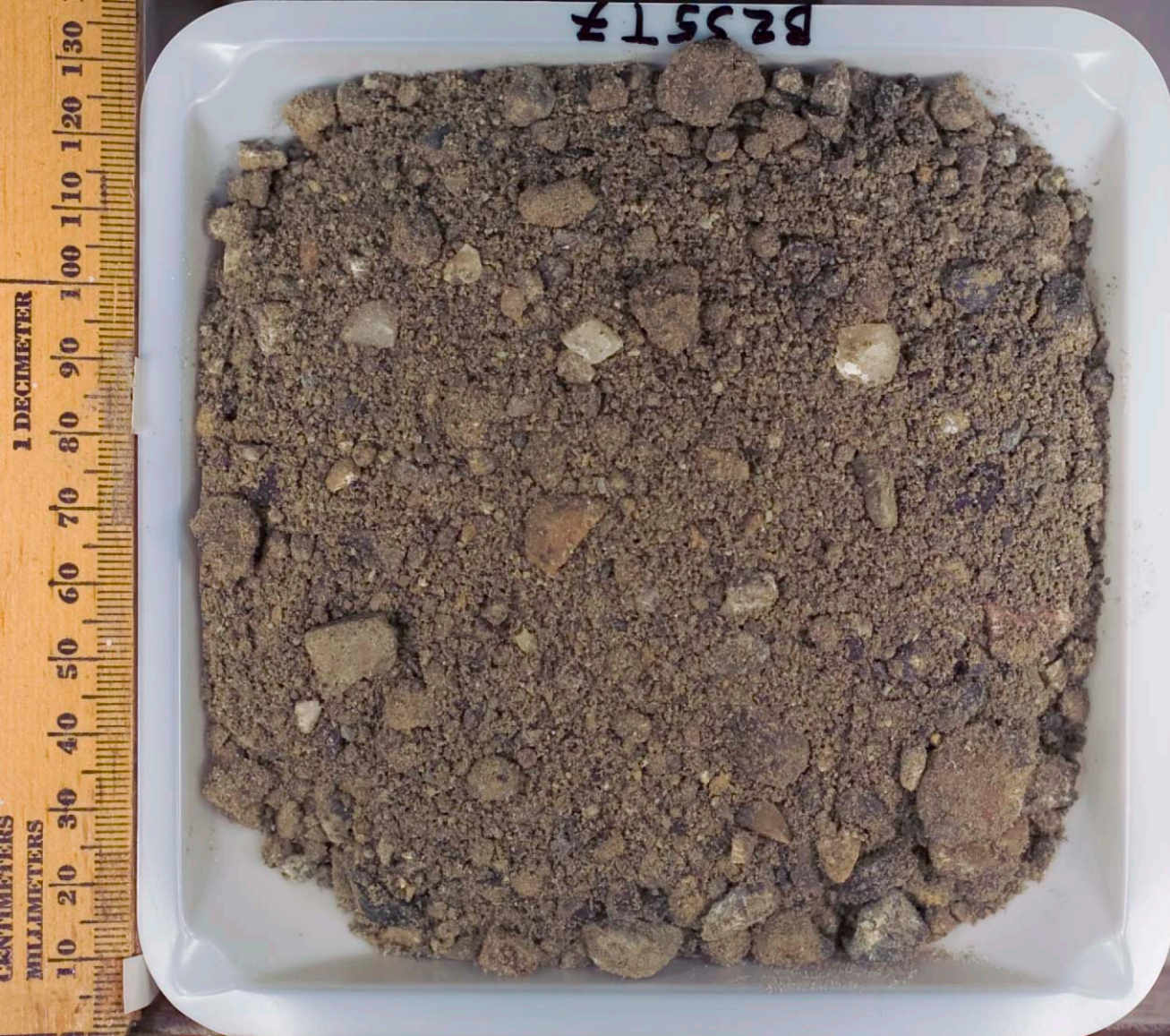

C7515

Borehole ID
B235T7 Sample ID
$297.5 \mathrm{ft}$ Depth from Chain-of-Custody
Grab Sample 


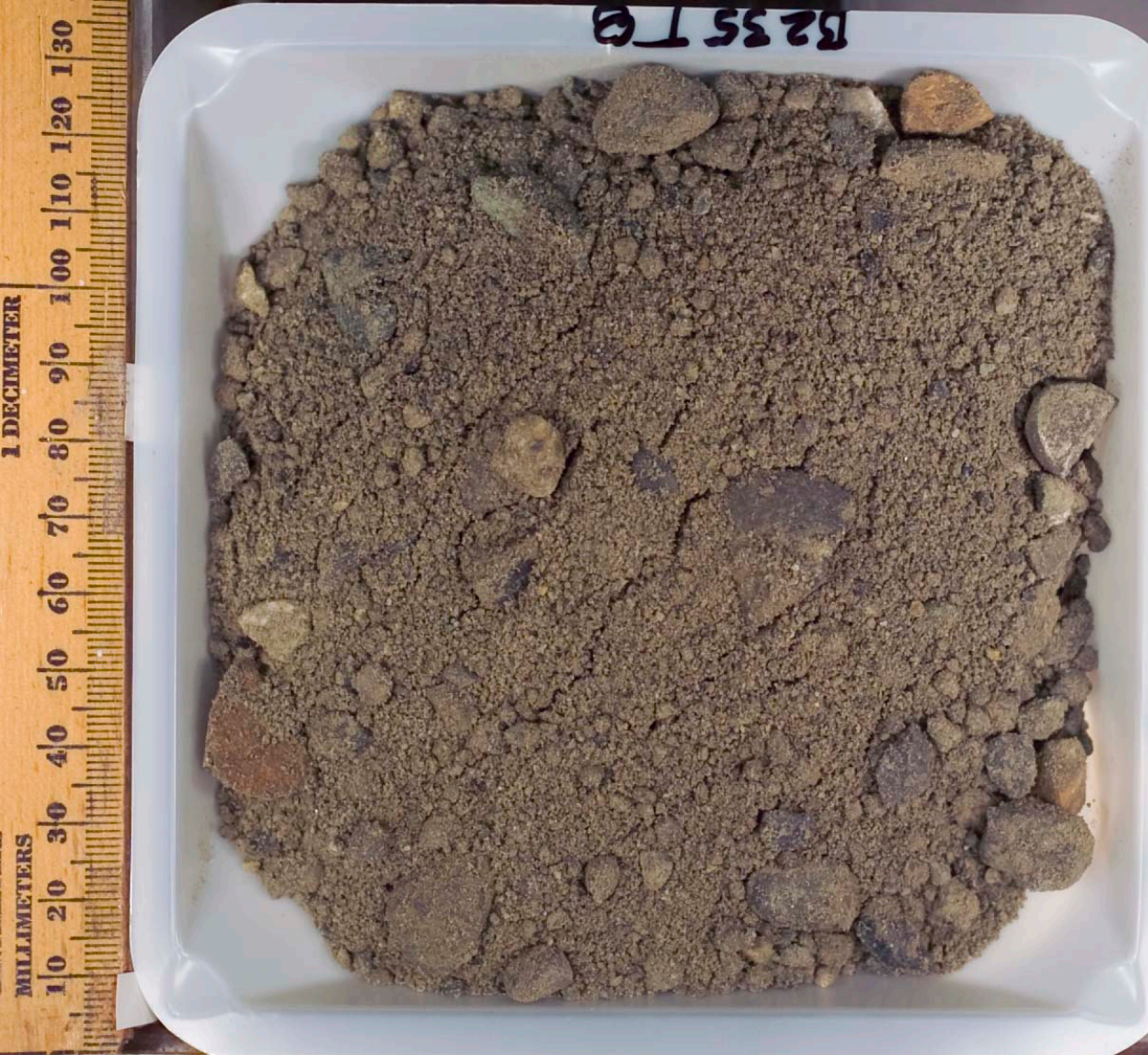




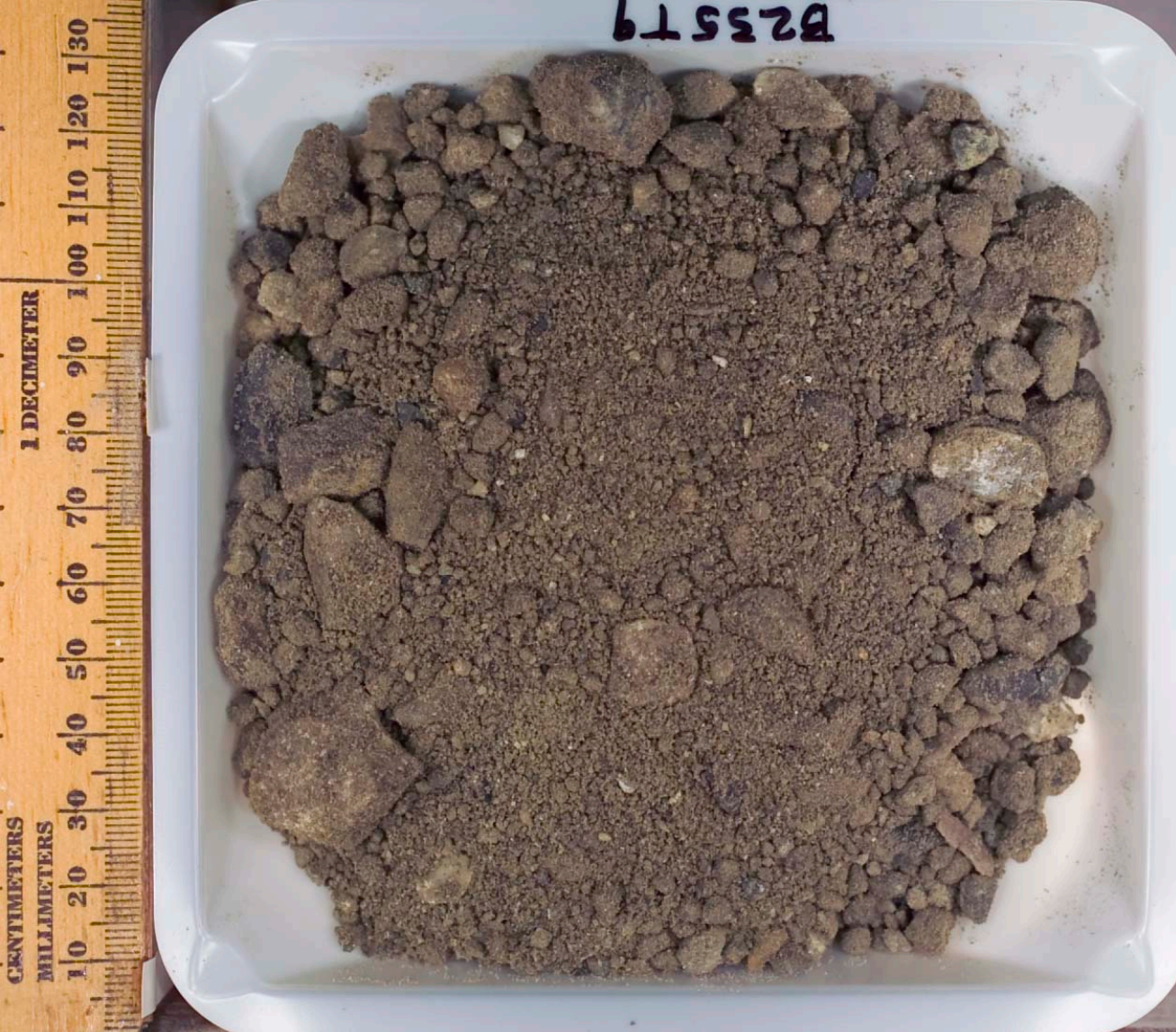




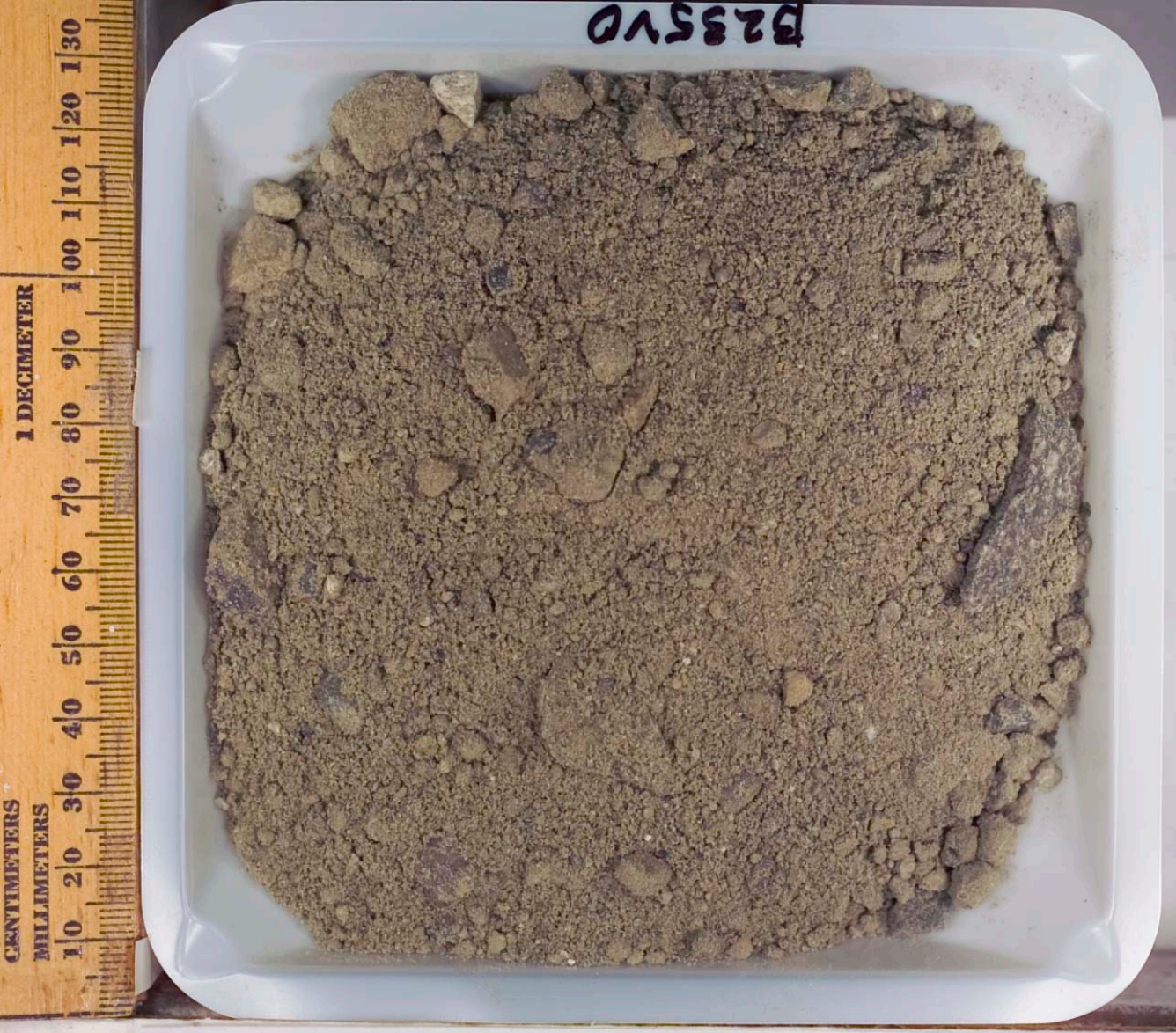




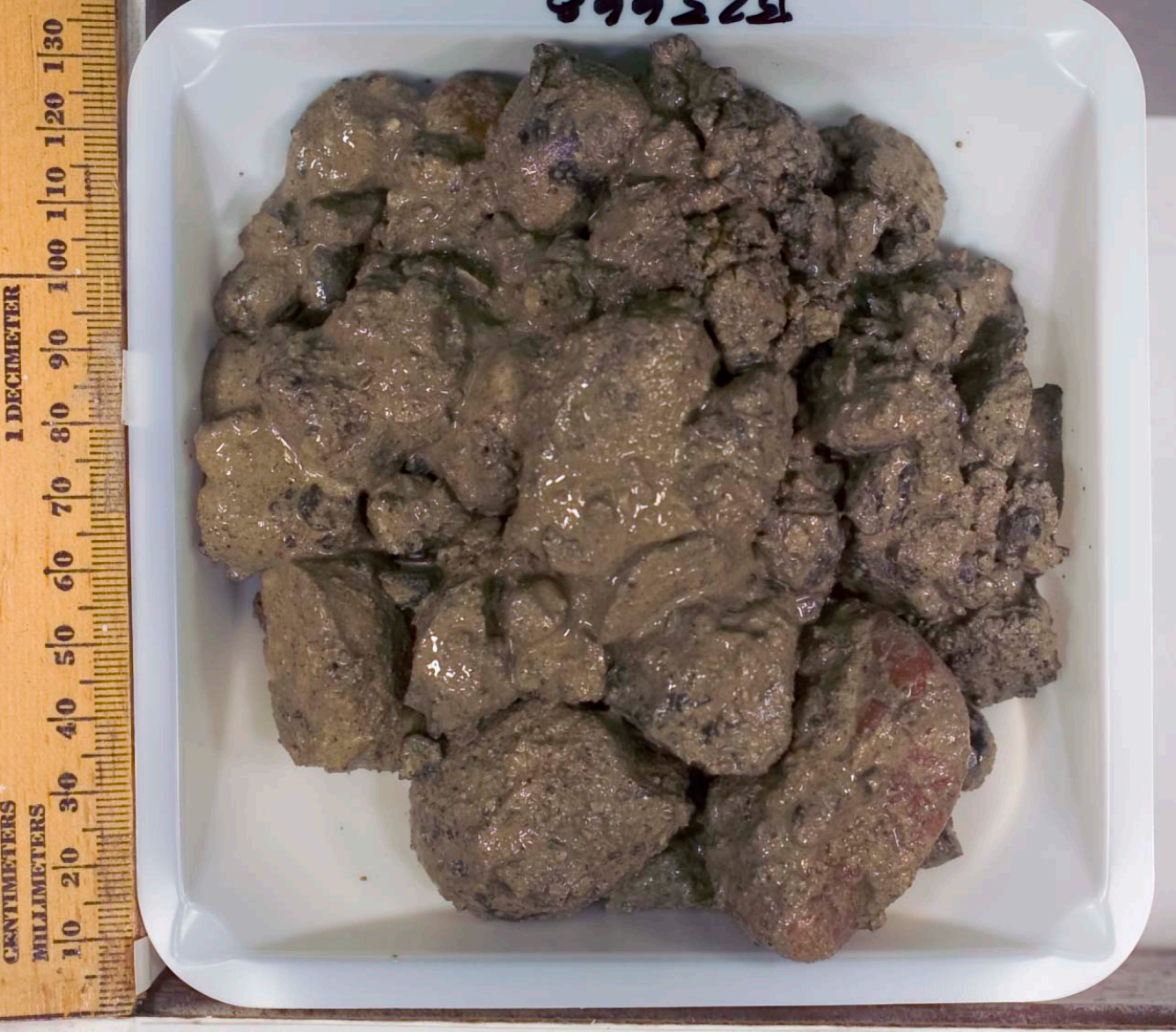

C7515 B23668

Borehole ID Sample ID
$308.5 \mathrm{ft}$
Grab Sample 


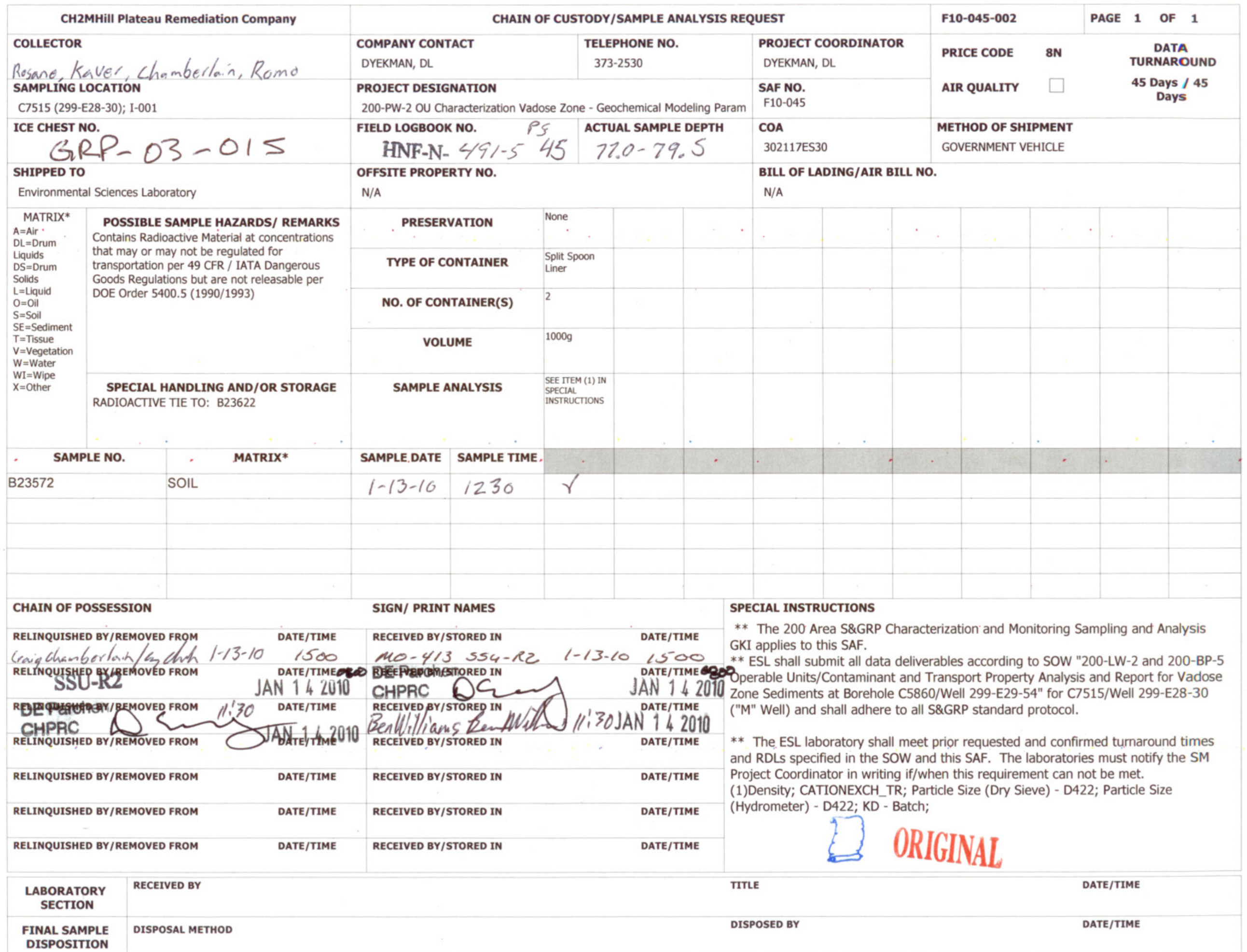




\section{CHAIN OF CUSTODY/SAMPLE ANALYSIS REQUEST}

COLLECTOR

Rosane, Kaver, Chamberlain, Romo SAMPLING LOCATION

C7515 (299-E28-30); I-002

ICE CHEST NO.

$$
\text { GRP-03-015 }
$$

\section{SHIPPED TO}

Environmental Sciences Laboratory

\section{MATRIX* POSSIBLE SAMPLE HAZARDS/ REMARKS \begin{tabular}{l|l}
$A=A i r$ & Contains Radioactive Material at concentrations \\
$D L=D r u m$ &
\end{tabular} Liquids that may or may not be regulated for \\ DS=Drum transportation per 49 CFR / IATA Dangerous \\ Solids Goods Regulations but are not releasable pe \\ $\mathrm{L}=\mathrm{Liquid}$
$\mathrm{O}=$ Oil \\ $\mathrm{S}=$ Soil \\ $\mathrm{SE}=$ Sediment \\ $\mathrm{T}=\mathrm{T}$ Tissue
$\mathrm{V}=$ Vegetation
$\mathrm{W}=$ (n) \\ $\mathrm{W}=$ Water \\ WI $=$ Wipe \\ $\mathrm{X}=\mathrm{Other}$ \\ SPECIAL HANDLING AND/OR STORAGE} RADIOACTIVE TIE TO: B23622
COMPANY CONTACT

DYEKMAN, DL

PROJECT DESIGNATION

200-PW-2 OU Characterization Vadose Zone - Geochemical Modeling Param

FIELD LOGBOOK NO.

$$
\text { HNF-N- } 78,5-45 \quad 79.5
$$

OFFSITE PROPERTY NO.

PRESERVATION

TYPE OF CONTAINER

NO. OF CONTAINER(S)

vOLUME

SAMPLE ANALYSIS

$1 \mathrm{~L}$

$\begin{array}{ll}\text { SEE ITEM (1) IN } & \text { Moisture } \\ \text { SPECIAL } & \text { Content - D2216; }\end{array}$

SPECTAL
INSTRUCTION
F10-045-003

PAGE 1 OF 2

RICE CODE

$8 \mathrm{~N}$

DYEKMAN, DL

SAF NO.

AIR QUALITY

TURNAROUND

45 Days / 45

Days

METHOD OF SHIPMENT

COA

GOVERNMENT VEHICLE

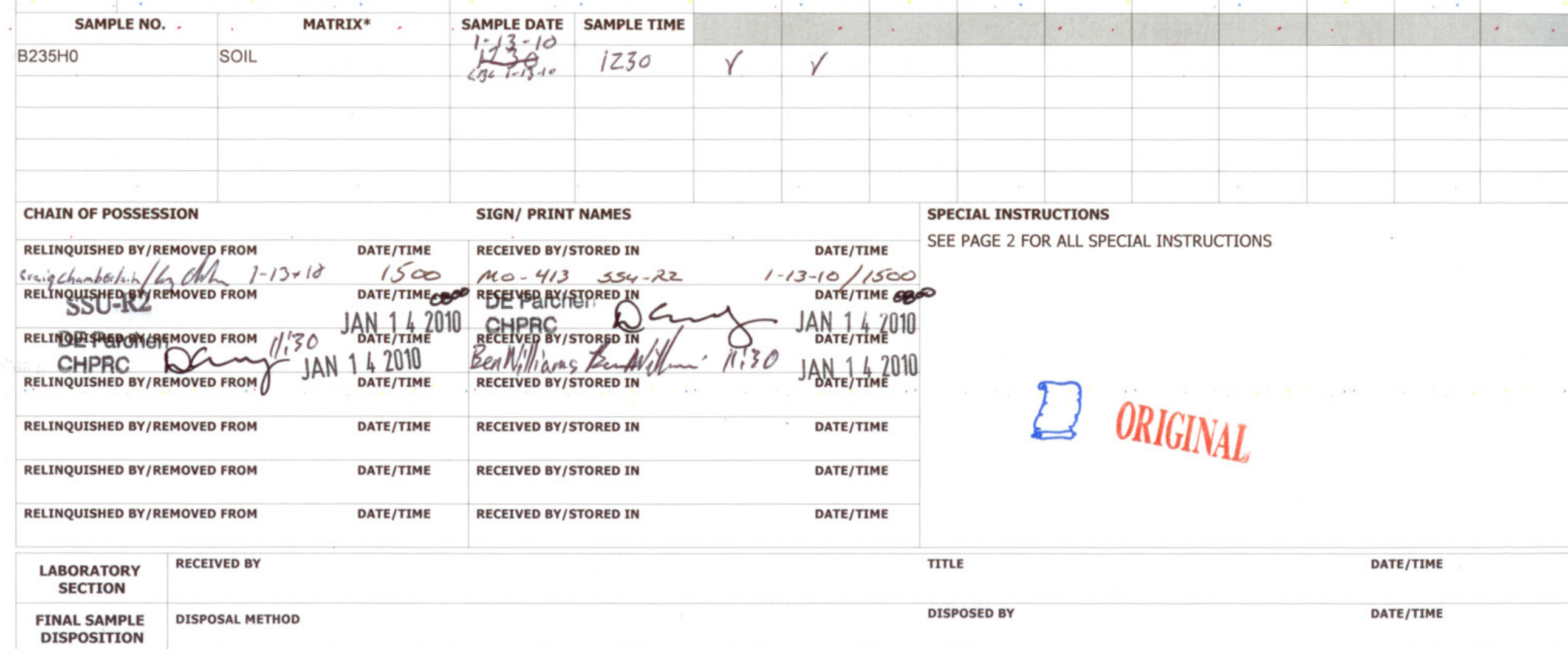




\begin{tabular}{|c|c|c|c|c|c|c|c|}
\hline CH2MHill Plateau Remediation Company & \multicolumn{3}{|c|}{ CHAIN OF CUSTODY/SAMPLE ANALYSIS REQUEST } & F10-045-003 & & PAGE & OF \\
\hline $\begin{array}{l}\text { COLLector } \\
\text { Rogone Kaver, Chamberlain Romo }\end{array}$ & $\begin{array}{l}\text { COMPANY CONTACT } \\
\text { DYEKMAN, DL }\end{array}$ & $\begin{array}{l}\text { TELEPHONE NO. } \\
373-2530\end{array}$ & $\begin{array}{l}\text { PROJECT COORDINATOR } \\
\text { DYEKMAN, DL }\end{array}$ & \multirow{2}{*}{$\begin{array}{l}\text { PRICE CODE } \\
\text { AIR QUALITY }\end{array}$} & $\mathbf{8 N}$ & \multirow{2}{*}{\multicolumn{2}{|c|}{$\begin{array}{c}\text { DATA } \\
\text { TURNAROUND } \\
45 \text { Days / } 45 \\
\text { Days }\end{array}$}} \\
\hline $\begin{array}{l}\text { SAMPLING LOCATION } \\
\text { C7515 (299-E28-30); I-002 }\end{array}$ & \multicolumn{2}{|c|}{$\begin{array}{l}\text { PROJECT DESIGNATION } \\
\text { 200-PW-2 OU Characterization Vadose Zone - Geochemical Modeling Param }\end{array}$} & $\begin{array}{r}\text { SAF NO. } \\
\text { F10-045 }\end{array}$ & & $\square$ & & \\
\hline ICE CHEST NO. & $\begin{array}{l}\text { FIELD LOGBOOK NO. } \\
\text { HNF-N- }>2 /-545\end{array}$ & $\begin{array}{l}\text { ACTUAL SAMPLE DEPTH } \\
79.5\end{array}$ & $\begin{array}{l}\text { COA } \\
302117 \text { ES30 }\end{array}$ & $\begin{array}{l}\text { METHOD OF S! } \\
\text { GOVERNMENT }\end{array}$ & $\begin{array}{l}\text { PMENT } \\
\text { HICLE }\end{array}$ & & \\
\hline $\begin{array}{l}\text { SHIPPED TO } \\
\text { Environmental Sciences Laboratory }\end{array}$ & \multicolumn{2}{|l|}{$\begin{array}{l}\text { OFFSITE PROPERTY NO. } \\
\text { N/A }\end{array}$} & \multicolumn{3}{|c|}{$\begin{array}{l}\text { BILL OF LADING/AIR BILL NO. } \\
\text { N/A }\end{array}$} & & \\
\hline
\end{tabular}

\section{SPECIAL INSTRUCTIONS}

** The 200 Area S\&GRP Characterization and Monitoring Sampling and Analysis GKI applies to this SAF.

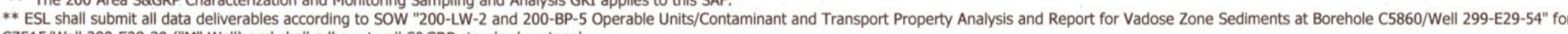
C7515/Well 299-E28-30 ("M" Well) and shall adhere to all S\&GRP standard protocol.

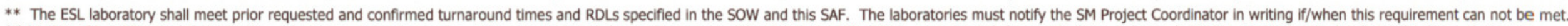

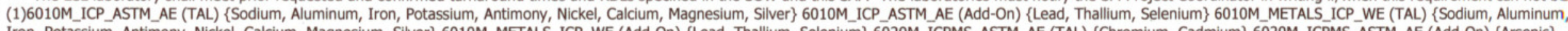

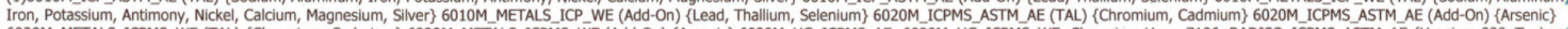

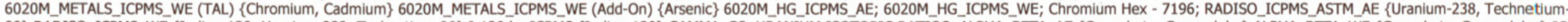

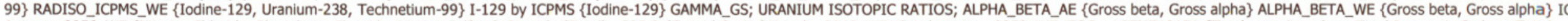

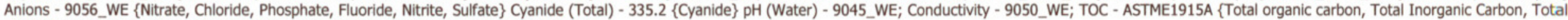
carbon\} 2320_ALKALINITY \{Carbonate ion, Bicarbonate, Alkalinity, Calcium Carbonate\}

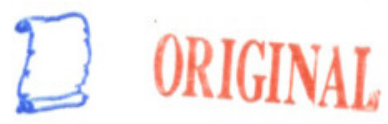


CH2MHill Plateau Remediation Company

COLLECTOR

Rosane, Kaver, Chamborlain, Romo SAMPLING LOCATION

C7515 (299-E28-30); I-003

ICE CHEST NO.

$$
\text { GRP-03-015 }
$$

Environmental Sciences Laboratory

\section{MATRIX* \\ POSSIBLE SAMPLE HAZARDS/ REMARKS}

$A=A$ ir .

$\mathrm{DL}=\mathrm{Drum}$

Liquids

DS $=$ Drum

Solids

$\mathrm{L}=$ =il

$S=S$ oil

$\mathrm{SE}=$ Sediment

$T=$ Tissue
$V=$ Vegetatio

$V=$ Vegetatio

$W=$ Water

$\mathrm{WI}=$ Wipe
$\mathrm{X}=$ Other Contains Radioactive Material at concentrations that may or may not be regulated for

transportation per 49 CFR / IATA Dangerous

Goods Regulations but are not releasable per

DOE Order $5400.5(1990 / 1993)$

SPECIAL HANDLING AND/OR STORAGE RADIOACTIVE TIE TO: B23622

\begin{tabular}{|l|l|}
\hline SAMPLE NO. & MATRIX* \\
\hline B23573 & SOIL \\
\hline
\end{tabular}

\section{CHAIN OF POSSESSION}

RELINQUISHED BY/REMOVED FROM Lraig Chamborlow Ln Wh RELINQUISHED BY/REMOVED FROM

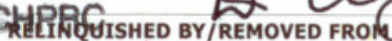

RELINQUISHED BY/REMOVED FROM

RELINQUISHED BY/REMOVED FROM

RELINQUISHED BY/REMOVED FROM

\section{LABORATORY RECEIVED BY}

SECTION

FINAL SAMPLE

DISPOSITION
CHAIN OF CUSTODY/SAMPLE ANALYSIS REQUEST

COMPANY CONTACT

TELEPHONE NO.

$373-2530$

PROJECT DESIGNATION

200-PW-2 OU Characterization Vadose Zone - Geochemical Modeling Param P 5 ACTUAL SAMPLE DEPTH

HNF-N- $491-545 \quad 80.1-82.6$ OFFSITE PROPERTY NO.

N/A

PROJECT COORDINATOR

DYEKMAN, DL

SAF NO.

F10-045

COA

302117ES30

BILL OF LADING/AIR BILL NO.

N/A FIELD LOGBOOK NO.
F10-045-005

PAGE 1 OF 1

PRICE CODE

$8 \mathrm{~N}$

DATA

45 Days / 45

Days

\section{METHOD OF SHIPMEN}

GOVERNMENT VEHICLE
TURNAROUND

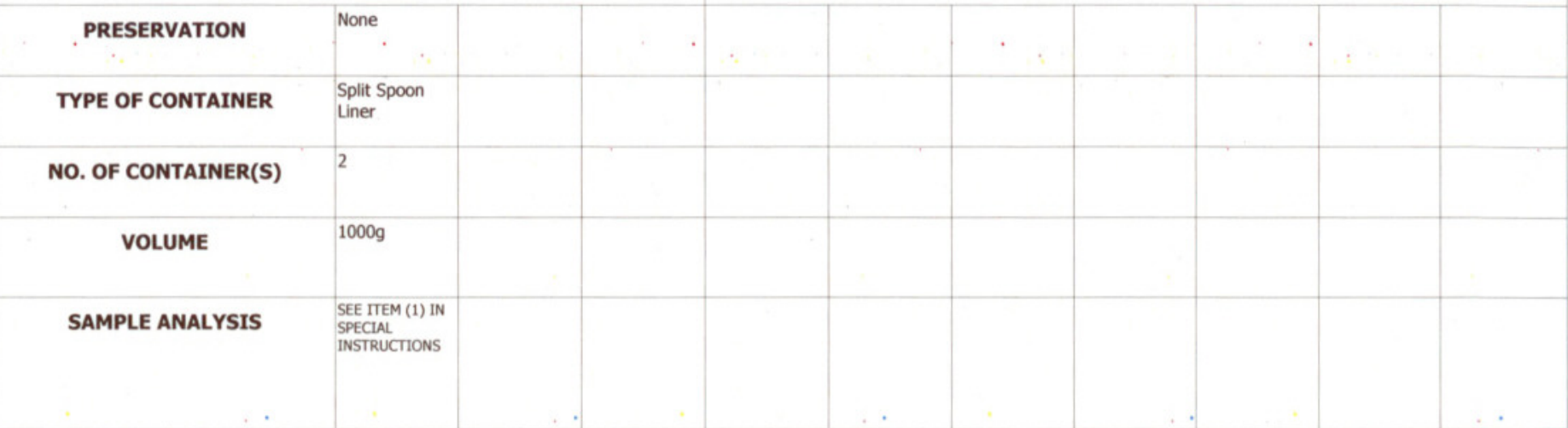

SAMPLE DATE SAMPLE TIME

$1-13-10 \quad 1245 \quad \gamma$

\section{SIGN/ PRINT NAMES}

DATE/TIME RECEIVED BY/STORED IN DATE/TIME

$1-13-10 \quad 1500$ DATE/TIME 200 REFEPER BWISTORED IN

JAN 142010 DATE/TIME

(2) AN 1 the10 EDIN

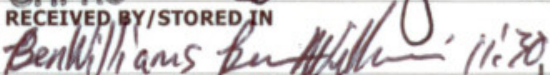

AN 14201 DATE/TIME

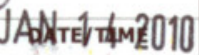

DATE/TIME
DATE/TIME
DATE/TIME
DATE/TIME RECEIVED BY/STORED IN

RECEIVED BY/STORED IN DATE/TIME

RECEIVED BY/STORED IN DATE/TIME

\section{CHPRC} RECEIYEP,BY/STOREDJN

\section{SPECIAL INSTRUCTIONS}

** The 200 Area S\&GRP Characterization and Monitoring Sampling and Analysis GKI applies to this SAF.

** ESL shall submit all data deliverables according to SOW "200-LW-2 and 200-BP-5 Operable Units/Contaminant and Transport Property Analysis and Report for Vadose Zone Sediments at Borehole C5860/Well 299-E29-54" for C7515/Well 299-E28-30 ("M" Well) and shall adhere to all S\&GRP standard protocol.

The ESL laboratory shall meet prior requested and confirmed turnaround times and RDLS specified in the SOW and this SAF. The laboratories must notify the SM Project Coordinator in writing if/when this requirement can not be met.

(1)Density; CATIONEXCH_TR; Particle Size (Dry Sieve) - D422; Particle Size

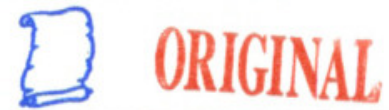

TITLE

DATE/TIME

DISPOSED BY

DATE/TIME (Hydrometer) - D422; KD - Batch; 


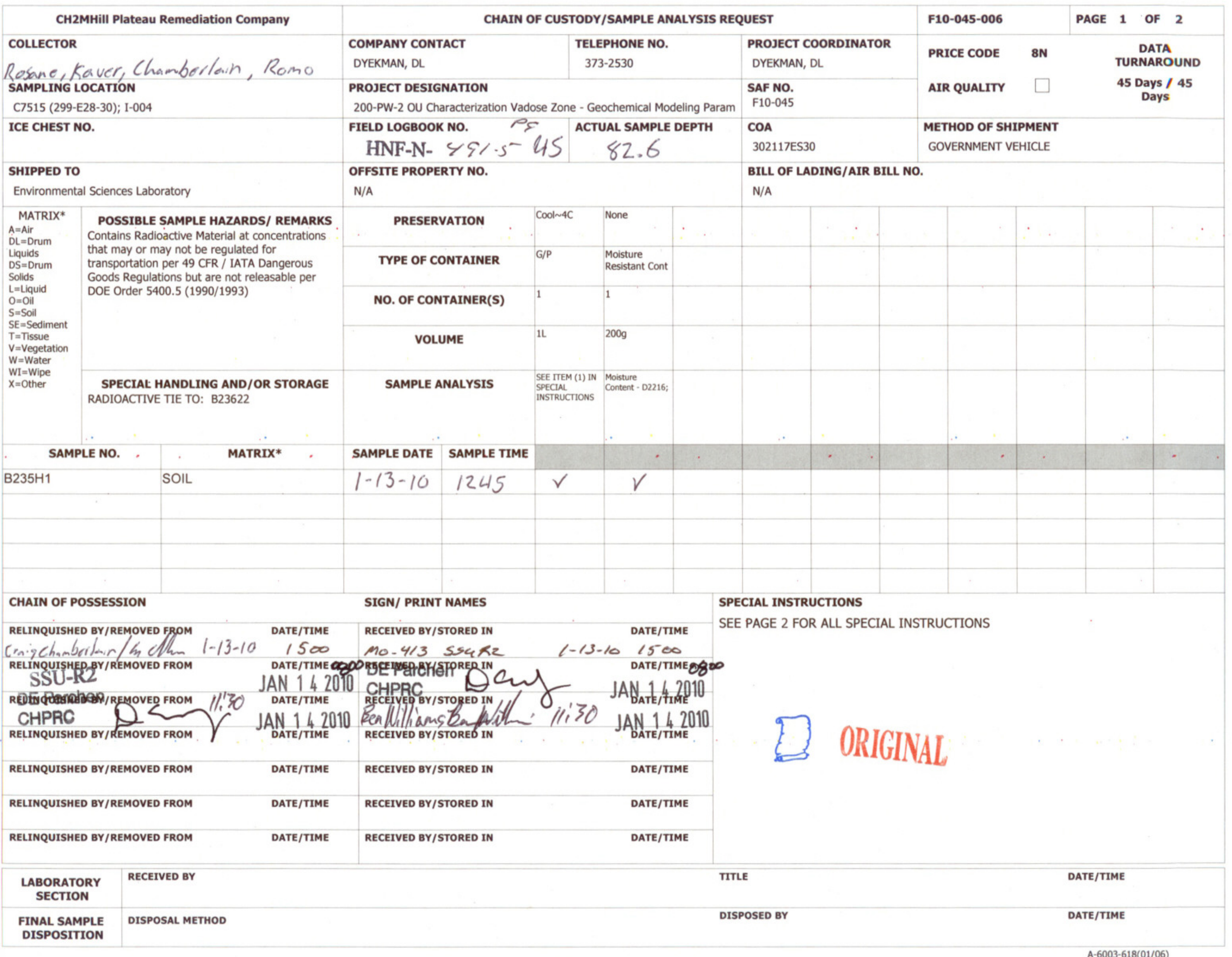




\begin{tabular}{|c|c|c|c|c|c|c|c|}
\hline CH2MHill Plateau Remediation Company & \multicolumn{3}{|c|}{ CHAIN OF CUSTODY/SAMPLE ANALYSIS REQUEST } & \multicolumn{2}{|l|}{ F10-045-006 } & PAGE & OF \\
\hline $\begin{array}{l}\text { COLlector } \\
\text { Rosane Kaver, Chamberlain Romo }\end{array}$ & $\begin{array}{l}\text { COMPANY CONTACT } \\
\text { DYEKMAN, DL }\end{array}$ & $\begin{array}{l}\text { TELEPHONE NO. } \\
373-2530\end{array}$ & $\begin{array}{l}\text { PROJECT COORDINATOR } \\
\text { DYEKMAN, DL }\end{array}$ & \multirow{2}{*}{$\begin{array}{l}\text { PRICE CODE } \\
\text { AIR QUALITY }\end{array}$} & $\mathbf{8 N}$ & \multirow{2}{*}{\multicolumn{2}{|c|}{$\begin{array}{c}\text { DATA } \\
\text { TURNAROUND } \\
45 \text { Days / } 45 \\
\text { Days }\end{array}$}} \\
\hline $\begin{array}{l}\text { SAMPLING LOCATION } \\
\text { C7515 (299-E28-30); I-004 }\end{array}$ & \multicolumn{2}{|c|}{$\begin{array}{l}\text { PROJECT DESIGNATION } \\
\text { 200-PW-2 OU Characterization Vadose Zone - Geochemical Modeling Param }\end{array}$} & $\begin{array}{l}\text { SAF NO. } \\
\text { F10-045 }\end{array}$ & & $\square$ & & \\
\hline ICE CHEST NO. & 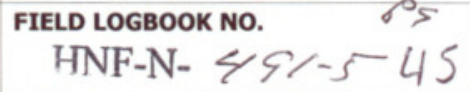 & $\begin{array}{l}\text { ACTUAL SAMPLE DEPTH } \\
82.6\end{array}$ & $\begin{array}{l}\text { COA } \\
302117 E S 30\end{array}$ & $\begin{array}{l}\text { METHOD OF SH } \\
\text { GOVERNMENT }\end{array}$ & $\begin{array}{l}\text { MENT } \\
\text { ICLE }\end{array}$ & & \\
\hline $\begin{array}{l}\text { SHIPPED TO } \\
\text { Environmental Sciences Laboratory }\end{array}$ & \multicolumn{2}{|l|}{$\begin{array}{l}\text { OFFSITE PROPERTY NO. } \\
\text { N/A }\end{array}$} & \multicolumn{3}{|c|}{$\begin{array}{l}\text { BILL OF LADING/AIR BILL NO. } \\
\text { N/A }\end{array}$} & & \\
\hline
\end{tabular}

\section{SPECIAL INSTRUCTIONS}

** The 200 Area S\&GRP Characterization and Monitoring Sampling and Analysis GKI applies to this SAF.

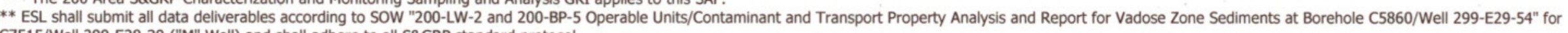
C7515/Well 299-E28-30 ("M" Well) and shall adhere to all S\&GRP standard protocol.

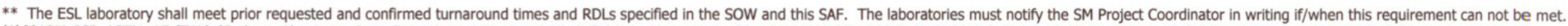

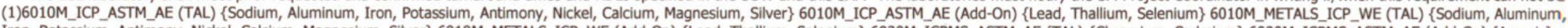

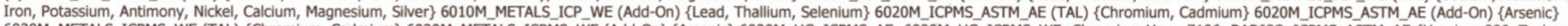

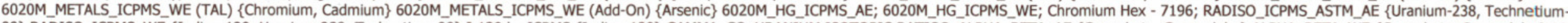

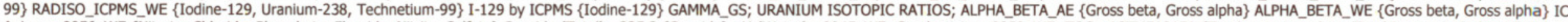

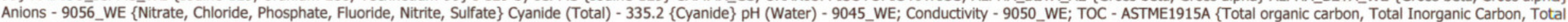
carbon\} 2320_ALKALINITY \{Carbonate ion, Bicarbonate, Alkalinity, Calcium Carbonate\}

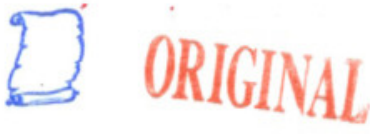


CH2MHill Plateau Remediation Company COLLECTOR

Rosane, Kaver, Chamberla.n, Rome SAMPLING LOCATION

C7515 (299-E28-30); I-005

ICE CHEST NO.

\section{SHIPPED TO}

$$
\text { GRP-03-015 }
$$

Environmental Sciences Laboratory

\begin{tabular}{l|l} 
MATRIX* & POSSIBLE SAMPLE HAZARDS/ REMARKS
\end{tabular} $A=A$ ir

$\mathrm{DL}=$ Drum

DS=Drum

$\mathrm{L}=$ Liquid
$\mathrm{O}=\mathrm{Oil}$

Contains Radioactive Material at concentrations

that may or may not be regulated for

transportation per 49 CFR / IATA Dangerous

Goods Regulations but are not releasable per

DOE Order 5400.5 (1990/1993)

$\mathrm{SE}=$ Sediment

$\mathrm{T}=$ Tissue

$\mathrm{V}=$ Vegetation

$W=$ Water

$X 1=$ Wipe

SPECIAL HANDLING AND/OR STORAGE RADIOACTIVE TIE TO: B23623

SAMPLE NO.

MATRIX*

B23574 SOIL

\section{CHAIN OF POSSESSION}

RELINQUISHED BY/REMOVED FROM

Crig Chambeilan/h then 1-13-10 1500

SSUISHED BY/REMOVED FROM

REEEQRRSChES/REMOVED FROM

\section{CHPRC}

$P^{2}$ JAN 14 ín10

RELINQUISHED BY/REMOVED FRON

RELINQUISHED BY/REMOVED FROM

RELINQUISHED BY/REMOVED FROM

RELINQUISHED BY/REMOVED FROM

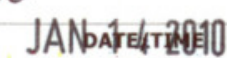

DATE/TIME

DATE/TIME

DATE/TIME

\section{CHAIN OF CUSTODY/SAMPLE ANALYSIS REQUEST}

COMPANY CONTACT

DYEKMAN, DL

\section{TELEPHONE NO.}

373-2530

\section{PROJECT DESIGNATION}

200-PW-2 OU Characterization Vadose Zone - Geochemical Modeling Param

FIELD LOGBOOK NO.

HNF-N- $Y$ I - 5 L $82.3-84.8$ OFFSITE PROPERTY NO.

N/A

COA

N/A

\section{$510-045-008$}

PAGE 1 OF 1

PROJECT COORDINATOR

DYEKMAN, DL

SAF NO.

F10-045

302117 ES30

BILL OF LADING/AIR BILL NO.
PRICE CODE $\quad 8$ N

DATA TURNAROUND 45 Days / 45 Days

\section{METHOD OF SHIPMENT}

GOVERNMENT VEHICLE

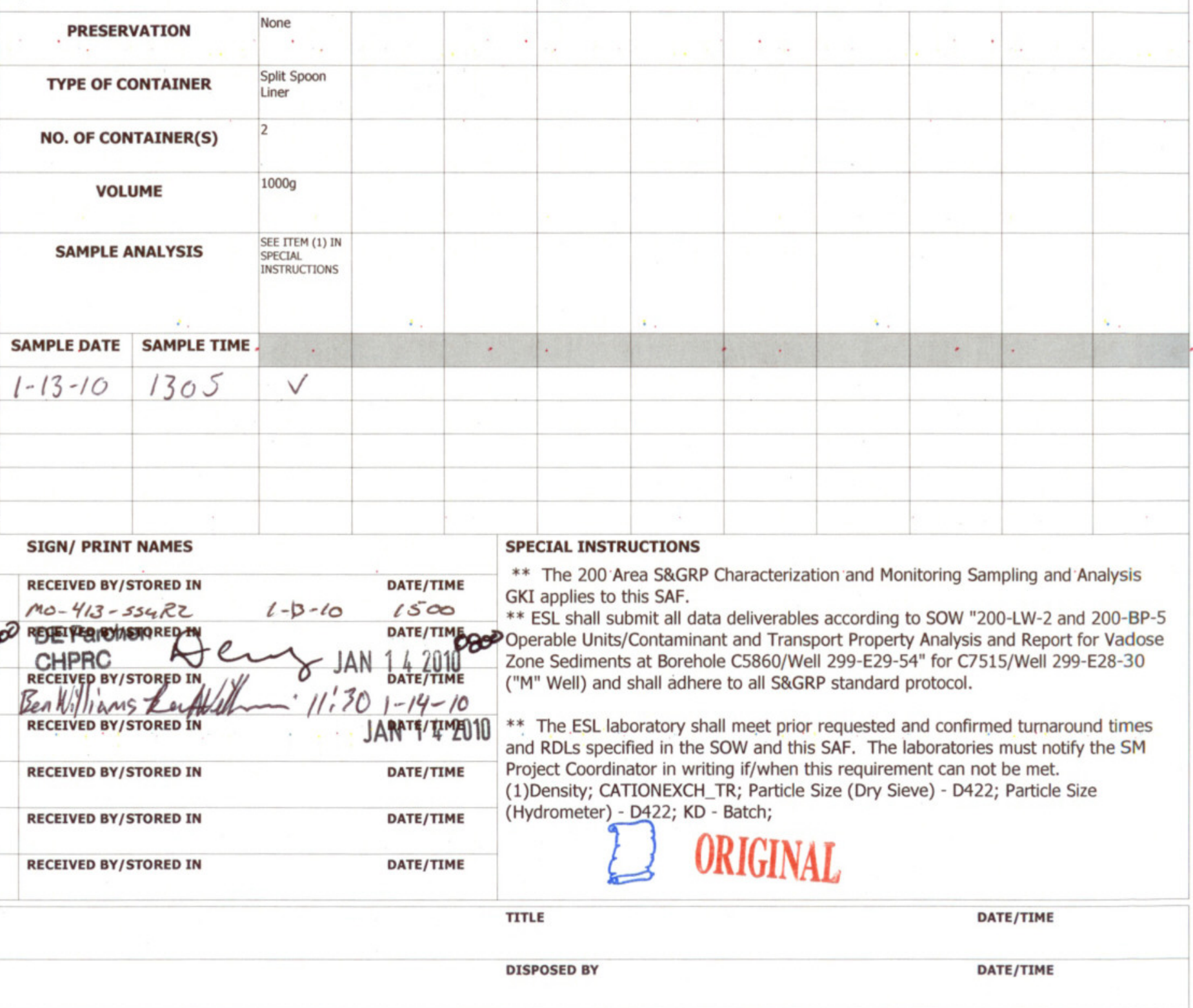




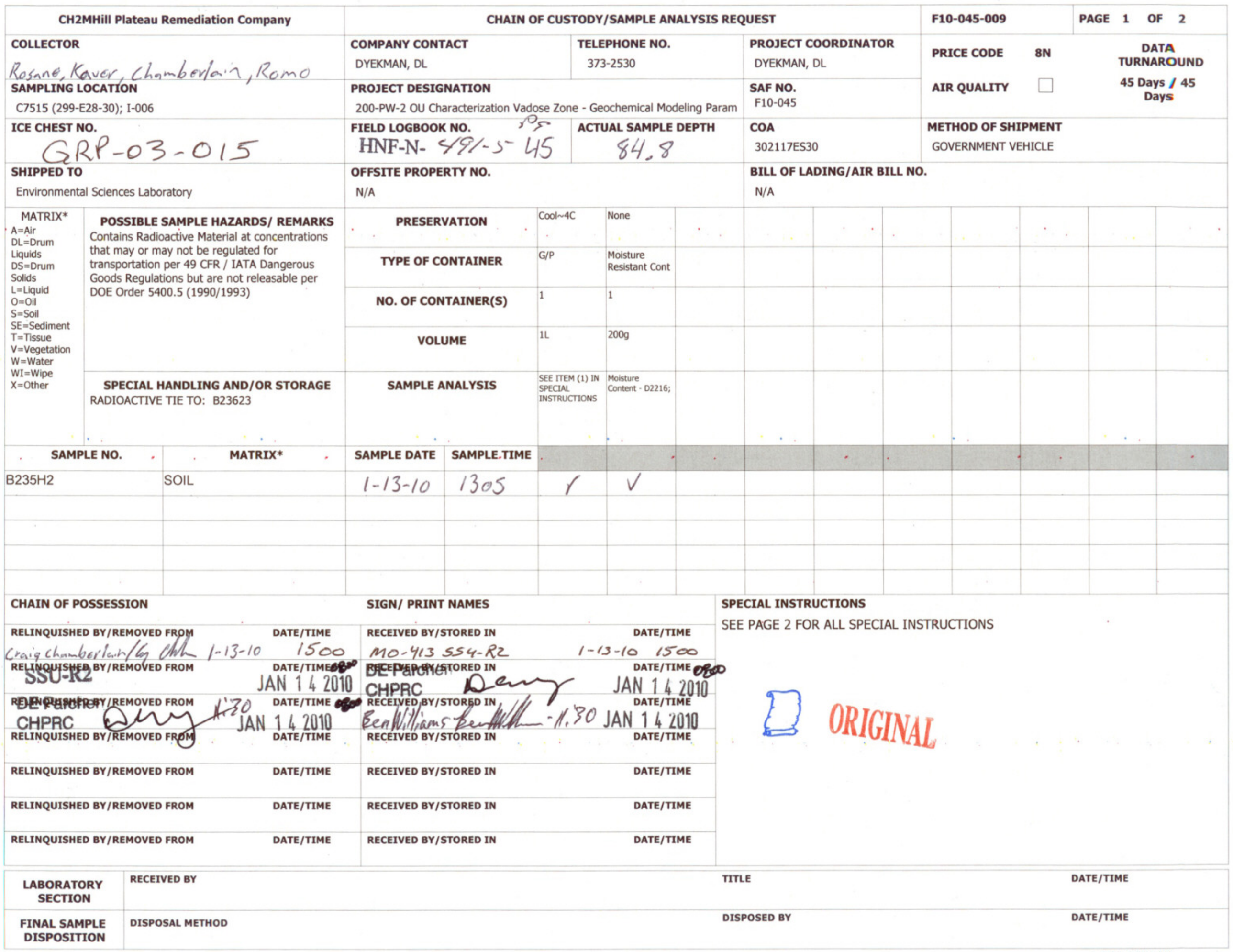




\begin{tabular}{|c|c|c|c|c|c|c|c|}
\hline CH2MHill Plateau Remediation Company & \multicolumn{3}{|c|}{ CHAIN OF CUSTODY/SAMPLE ANALYSIS REQUEST } & \multicolumn{2}{|l|}{ F10-045-009 } & PAGE & OF \\
\hline $\begin{array}{l}\text { COLLECTOR } \\
\text { Resane, Kaver, Chamberlain, Romo }\end{array}$ & $\begin{array}{l}\text { COMPANY CONTACT } \\
\text { DYEKMAN, DL }\end{array}$ & $\begin{array}{l}\text { TELEPHONE NO. } \\
373-2530\end{array}$ & $\begin{array}{l}\text { PROJECT COORDINATOR } \\
\text { DYEKMAN, DL }\end{array}$ & \multirow{2}{*}{$\begin{array}{l}\text { PRICE CODE } \\
\text { AIR QUALITY }\end{array}$} & $8 \mathrm{~N}$ & \multirow{2}{*}{\multicolumn{2}{|c|}{$\begin{array}{c}\text { DATA } \\
\text { TURNAROUND } \\
45 \text { Days / } 45 \\
\text { Days }\end{array}$}} \\
\hline $\begin{array}{l}\text { SAMPLING LOCATION } \\
\text { C7515 (299-E28-30); I-006 }\end{array}$ & \multicolumn{2}{|c|}{$\begin{array}{l}\text { PROJECT DESIGNATION } \\
\text { 200-PW-2 OU Characterization Vadose Zone - Geochemical Modeling Param }\end{array}$} & $\begin{array}{l}\text { SAF NO. } \\
\text { F10-045 }\end{array}$ & & $\square$ & & \\
\hline ICE CHEST NO. & $\begin{array}{l}\text { FIELD LOGBOOK NO. } \\
\text { HNF-N- } 49 /-5-45\end{array}$ & $\begin{array}{l}\text { ACTUAL SAMPLE DEPTH } \\
\qquad 84.8\end{array}$ & $\begin{array}{l}\text { COA } \\
302117 \text { ES30 }\end{array}$ & \multicolumn{4}{|c|}{$\begin{array}{l}\text { METHOD OF SHIPMENT } \\
\text { GOVERNMENT VEHICLE }\end{array}$} \\
\hline $\begin{array}{l}\text { SHIPPED TO } \\
\text { Environmental Sciences Laboratory }\end{array}$ & \multicolumn{2}{|l|}{$\begin{array}{l}\text { OFFSITE PROPERTY NO. } \\
\text { N/A }\end{array}$} & \multicolumn{3}{|c|}{$\begin{array}{l}\text { BILL OF LADING/AIR BILL NO. } \\
\text { N/A }\end{array}$} & & \\
\hline
\end{tabular}

\section{SPECIAL INSTRUCTIONS}

** The 200 Area S\&GRP Characterization and Monitoring Sampling and Analysis GKI applies to this SAF.

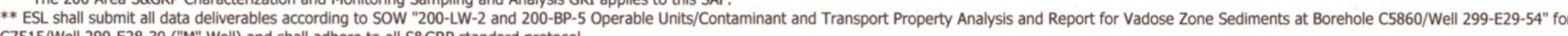
C7515/Well 299-E28-30 ("M" Well) and shall adhere to all S\&GRP standard protocol.

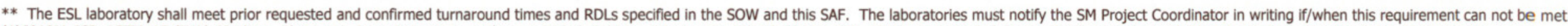

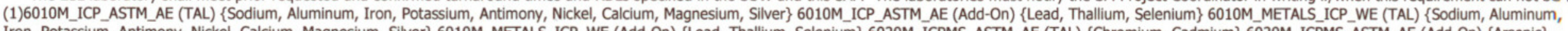

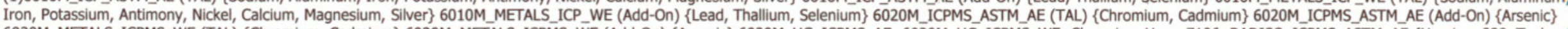

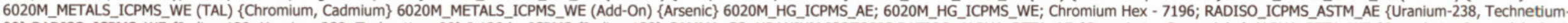

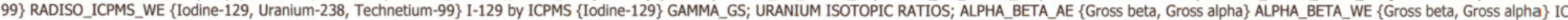

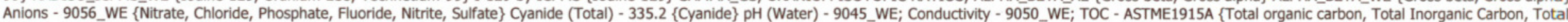
carbon\} 2320_ALKALINITY \{Carbonate ion, Bicarbonate, Alkalinity, Calcium Carbonate\}

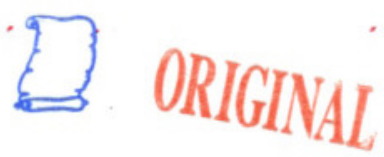




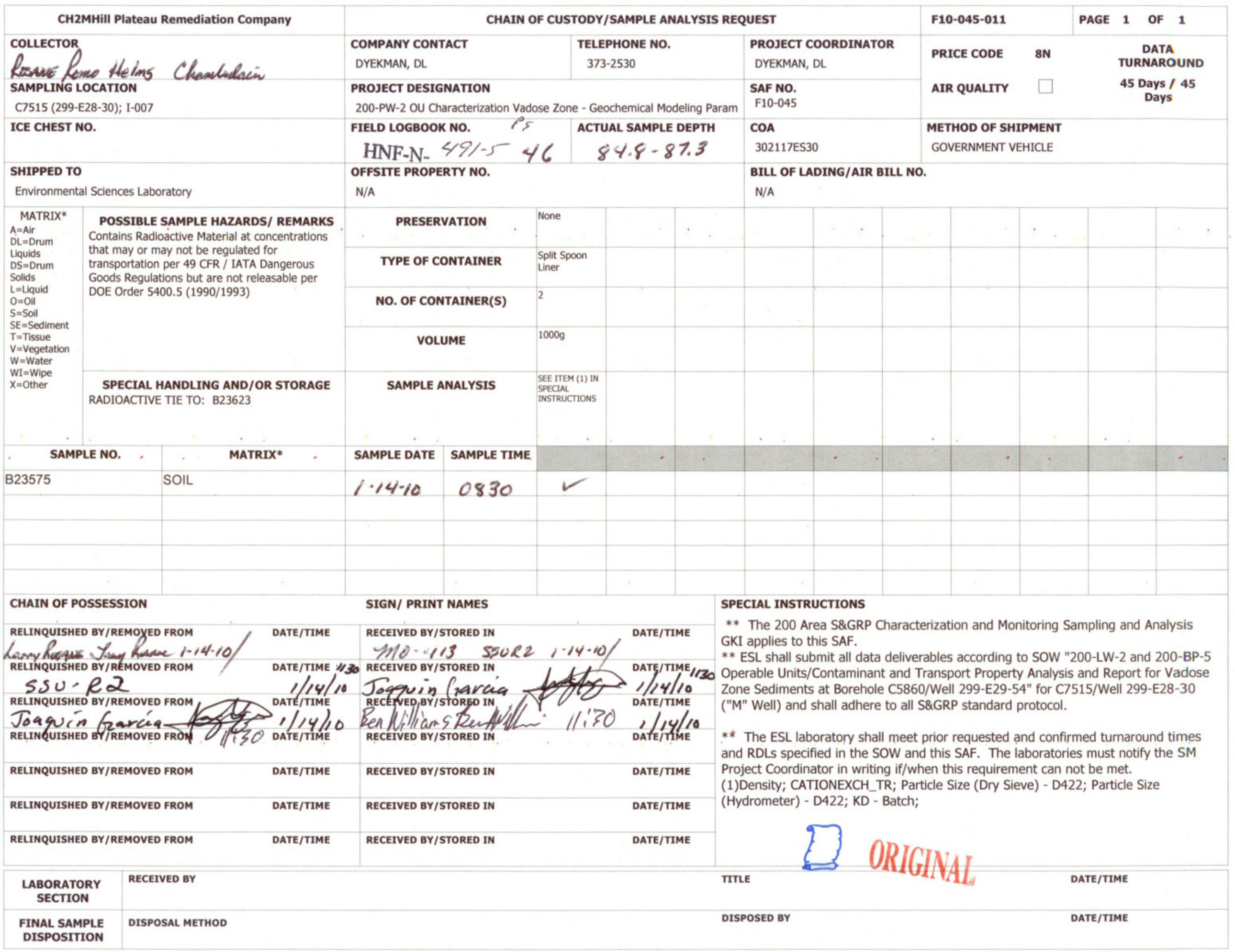




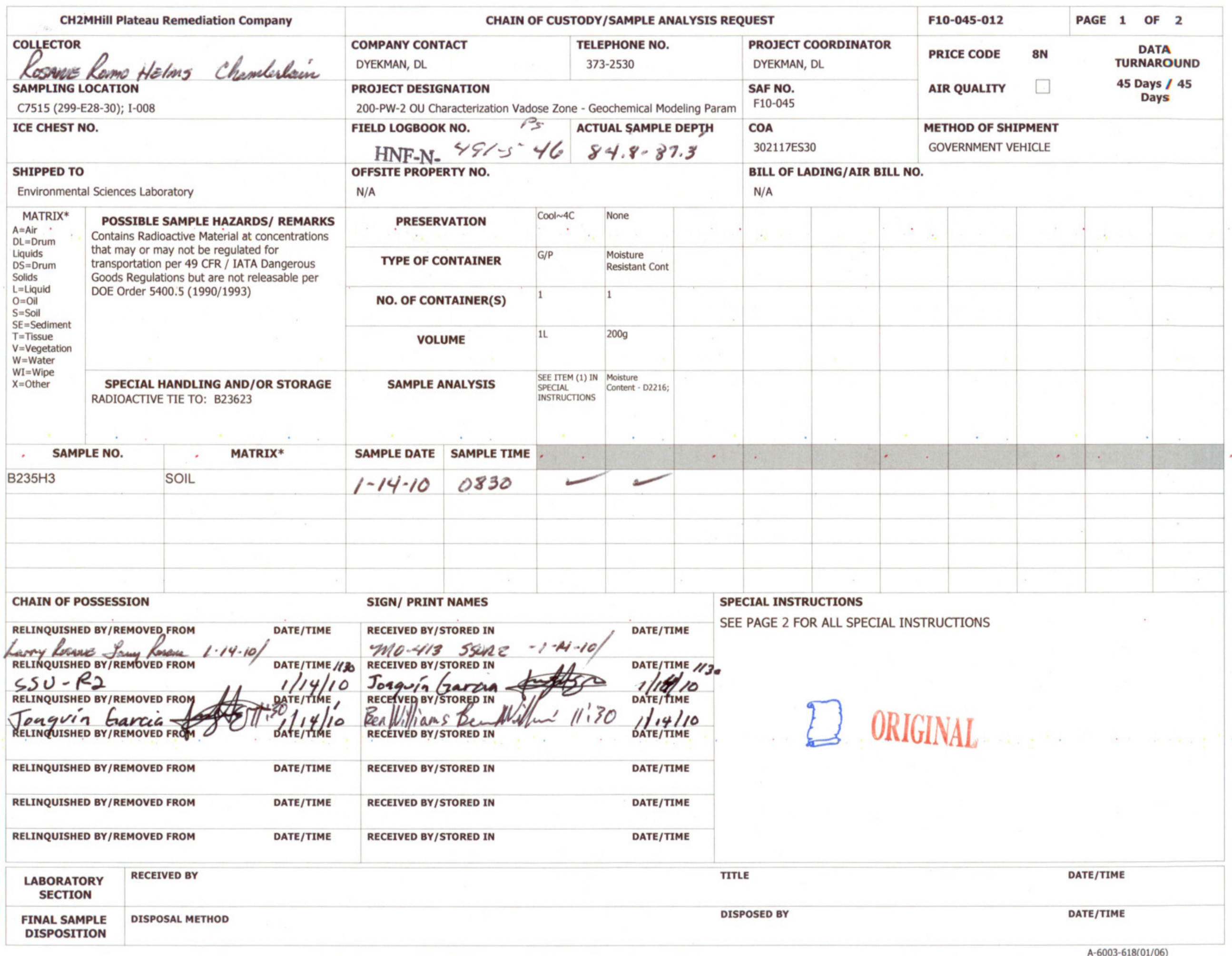




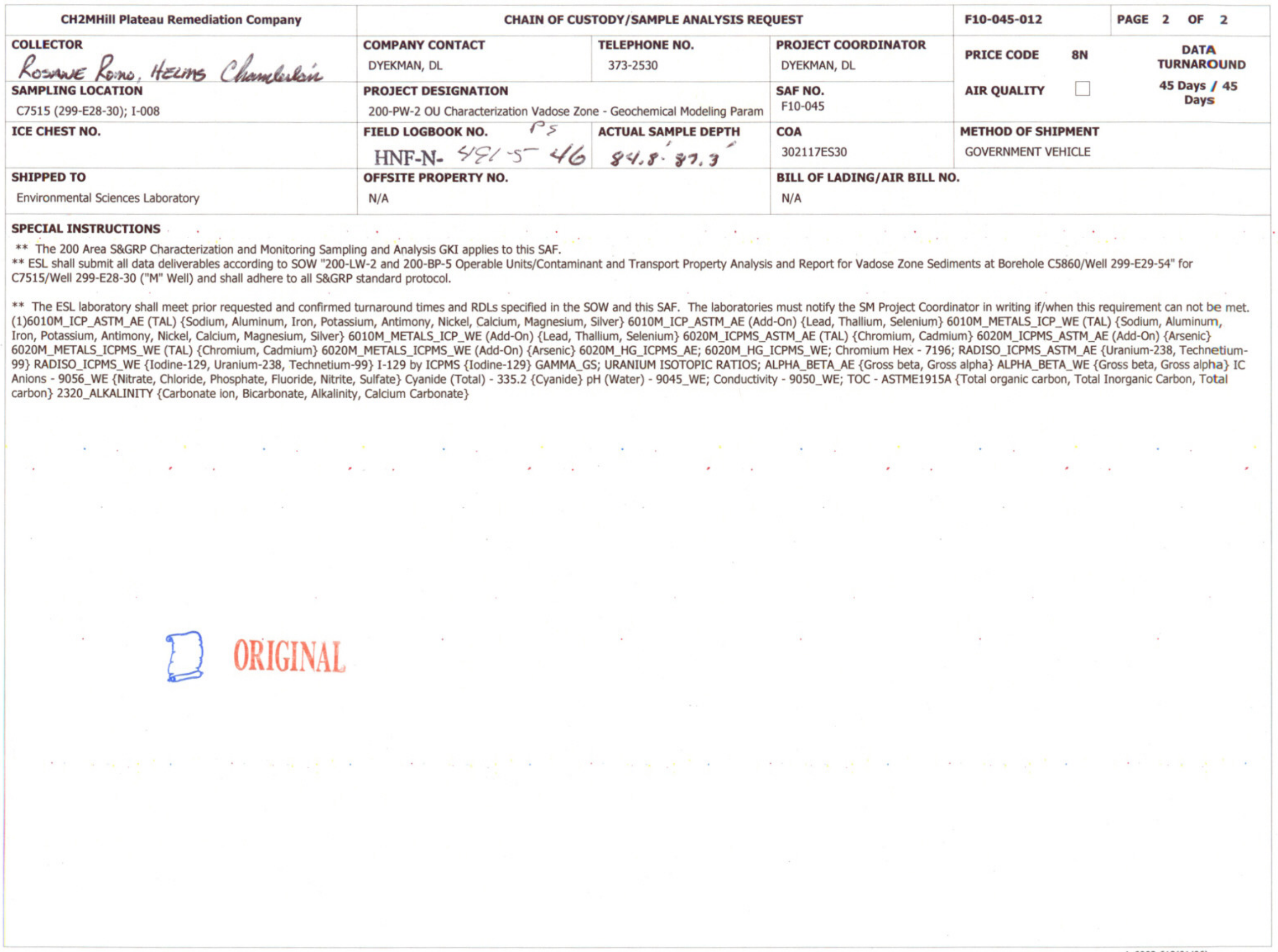




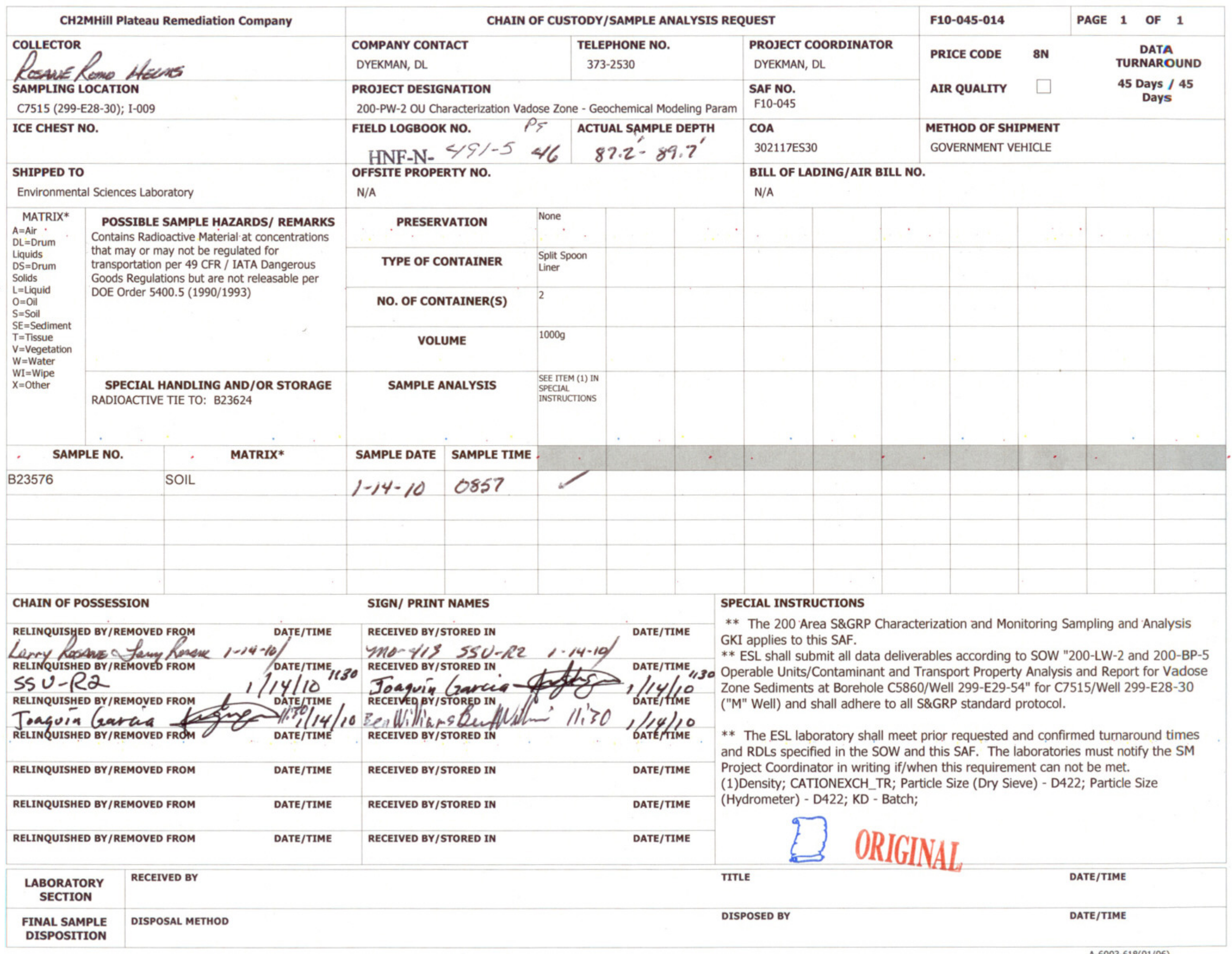




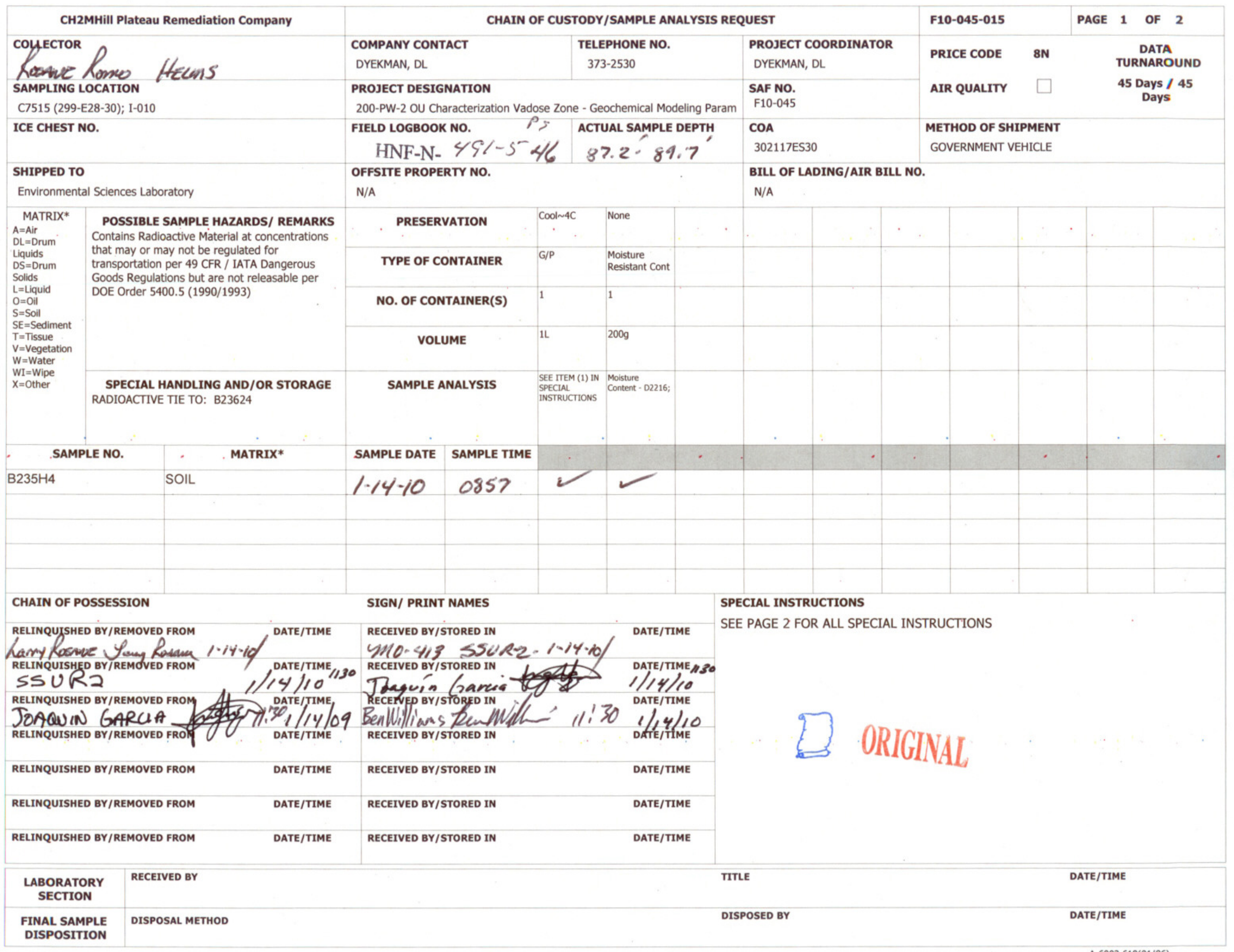




\begin{tabular}{|c|c|c|c|c|c|c|c|}
\hline CH2MHill Plateau Remediation Company & \multicolumn{3}{|c|}{ CHAIN OF CUSTODY/SAMPLE ANALYSIS REQUEST } & \multicolumn{2}{|l|}{ F10-045-015 } & PAGE & 2 OF \\
\hline Colpector & $\begin{array}{l}\text { COMPANY CONTACT } \\
\text { DYEKMAN, DL }\end{array}$ & $\begin{array}{l}\text { TELEPHONE NO. } \\
373-2530\end{array}$ & $\begin{array}{l}\text { PROJECT COORDINATOR } \\
\text { DYEKMAN, DL }\end{array}$ & \multirow{2}{*}{$\begin{array}{l}\text { PRICE CODE } \\
\text { AIR QUALITY }\end{array}$} & $\mathbf{8 N}$ & \multirow{2}{*}{\multicolumn{2}{|c|}{$\begin{array}{c}\text { DATA } \\
\text { TURNAROUND } \\
45 \text { Days / } 45 \\
\text { Days }\end{array}$}} \\
\hline $\begin{array}{l}\text { SAMPLING LOCATION } \\
\text { C7515 (299-E28-30); I-010 }\end{array}$ & \multicolumn{2}{|c|}{$\begin{array}{l}\text { PROJECT DESIGNATION } \\
\text { 200-PW-2 OU Characterization Vadose Zone - Geochemical Modeling Param }\end{array}$} & $\begin{array}{r}\text { SAF NO. } \\
\text { F10-045 }\end{array}$ & & $\square$ & & \\
\hline ICE CHEST NO. & $\begin{array}{l}\text { FIELD LOGBOOK NO. } \\
\text { HNF-N- } 49 /-546\end{array}$ & $\begin{array}{l}\text { ACTUAL SAMPLE DEPTH } \\
87.2-89.7\end{array}$ & $\begin{array}{l}\text { COA } \\
302117 \text { ES30 }\end{array}$ & $\begin{array}{l}\text { METHOD OF SH } \\
\text { GOVERNMENT }\end{array}$ & $\begin{array}{l}\text { PMENT } \\
\text { HICLE }\end{array}$ & & \\
\hline $\begin{array}{l}\text { SHIPPED TO } \\
\text { Environmental Sciences Laboratory }\end{array}$ & $\begin{array}{l}\text { OFFSITE PROPERTY NO. } \\
\text { N/A }\end{array}$ & & $\begin{array}{l}\text { BILL OF LADING/AIR BILI } \\
\text { N/A }\end{array}$ & & & & \\
\hline
\end{tabular}

\section{SPECIAL INSTRUCTIONS}

** The 200 Area S\&GRP Characterization and Monitoring Sampling and Analysis GKI applies to this SAF.

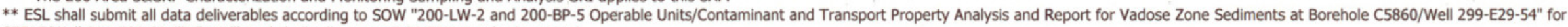
C7515/Well 299-E28-30 ("M" Well) and shall adhere to all S\&GRP standard protocol.

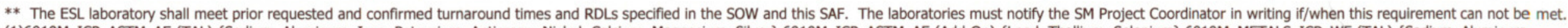

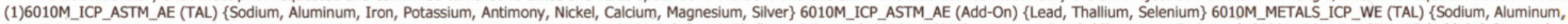

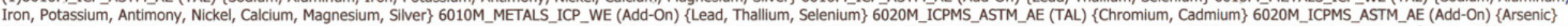

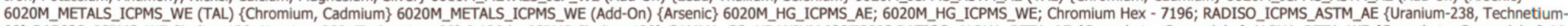

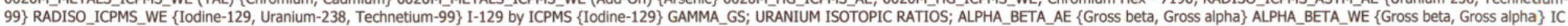

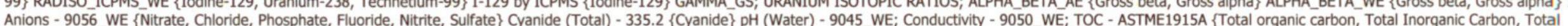
carbon\} 2320_ALKALINITY \{Carbonate ion, Bicarbonate, Alkalinity, Calcium Carbonate 


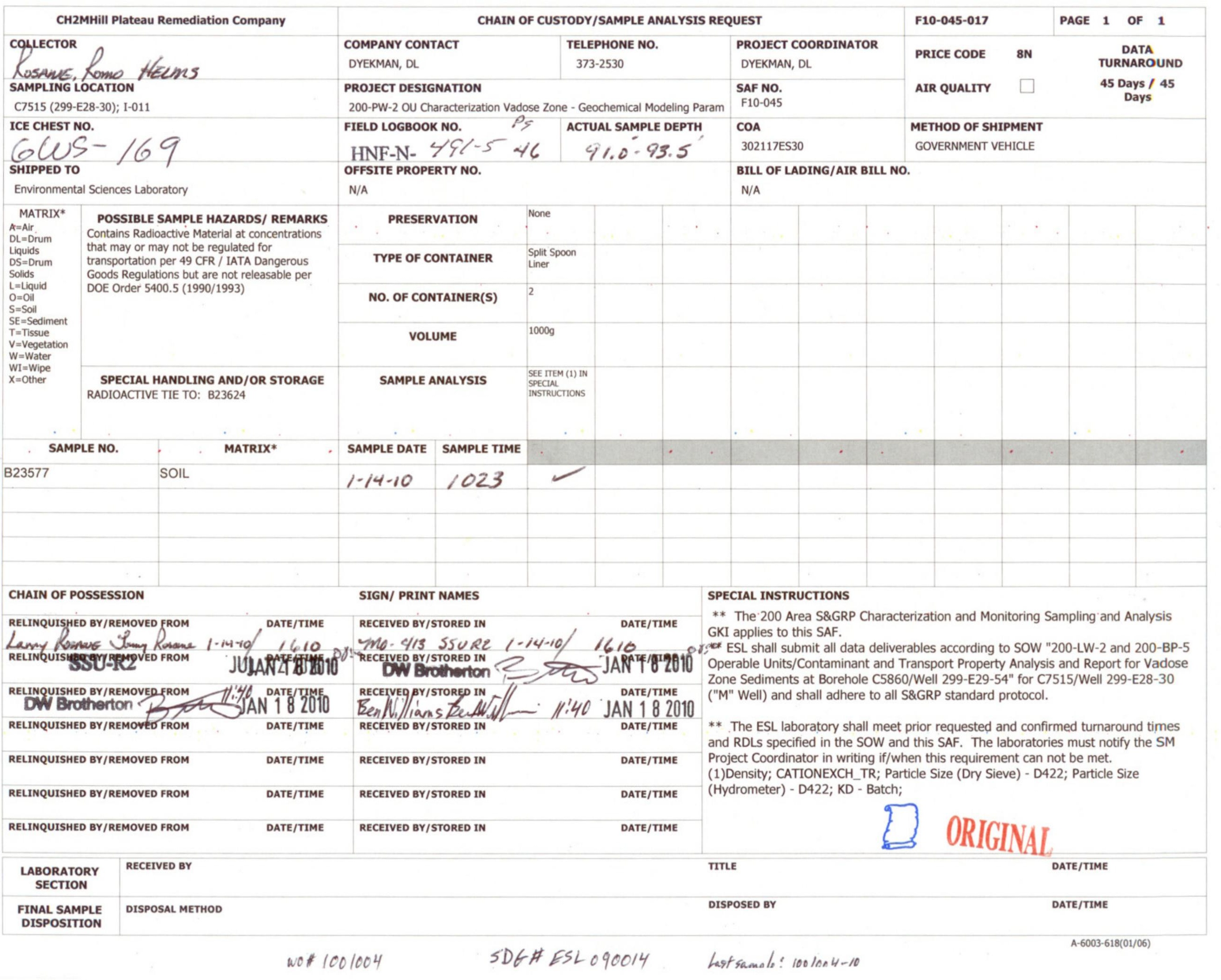




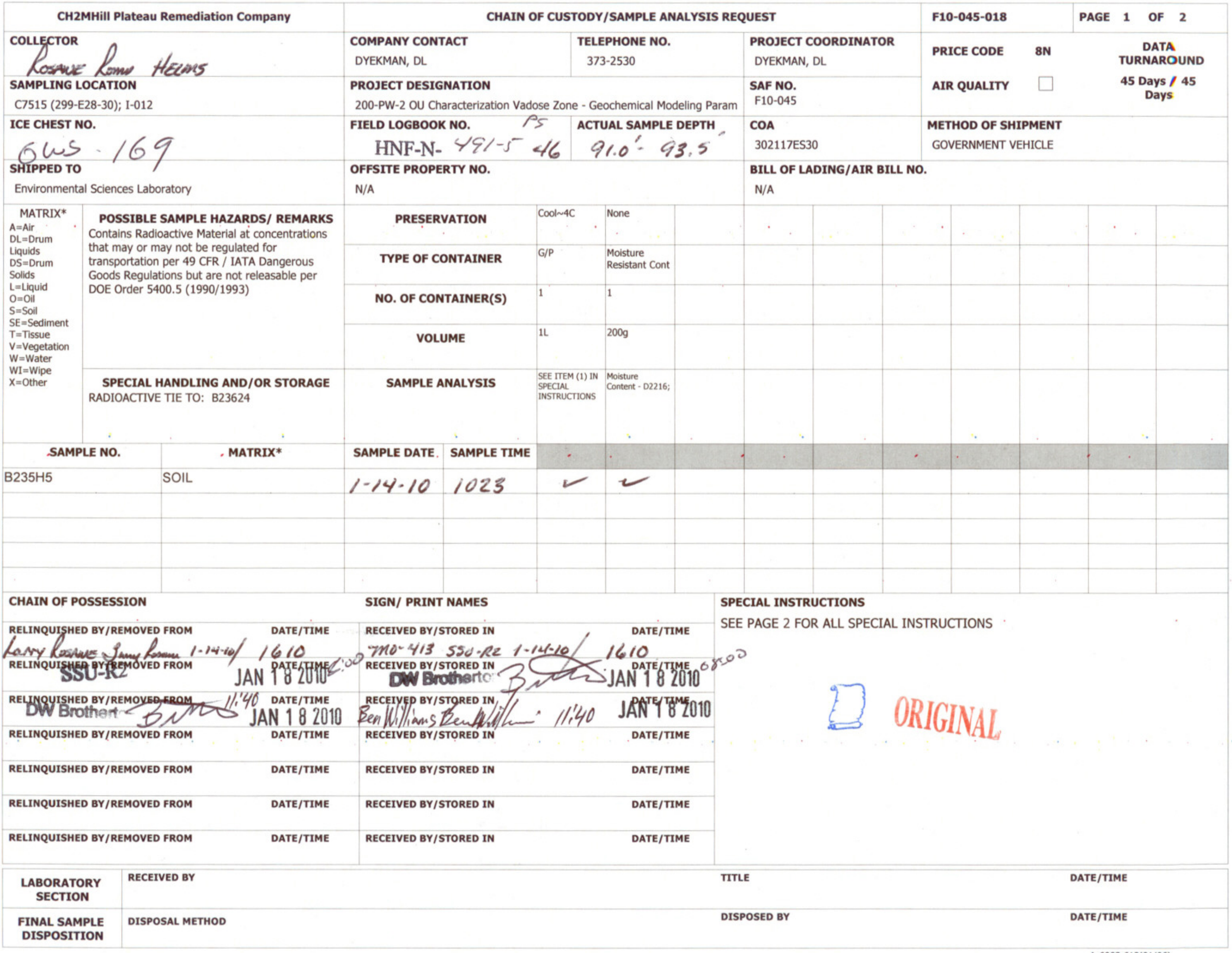




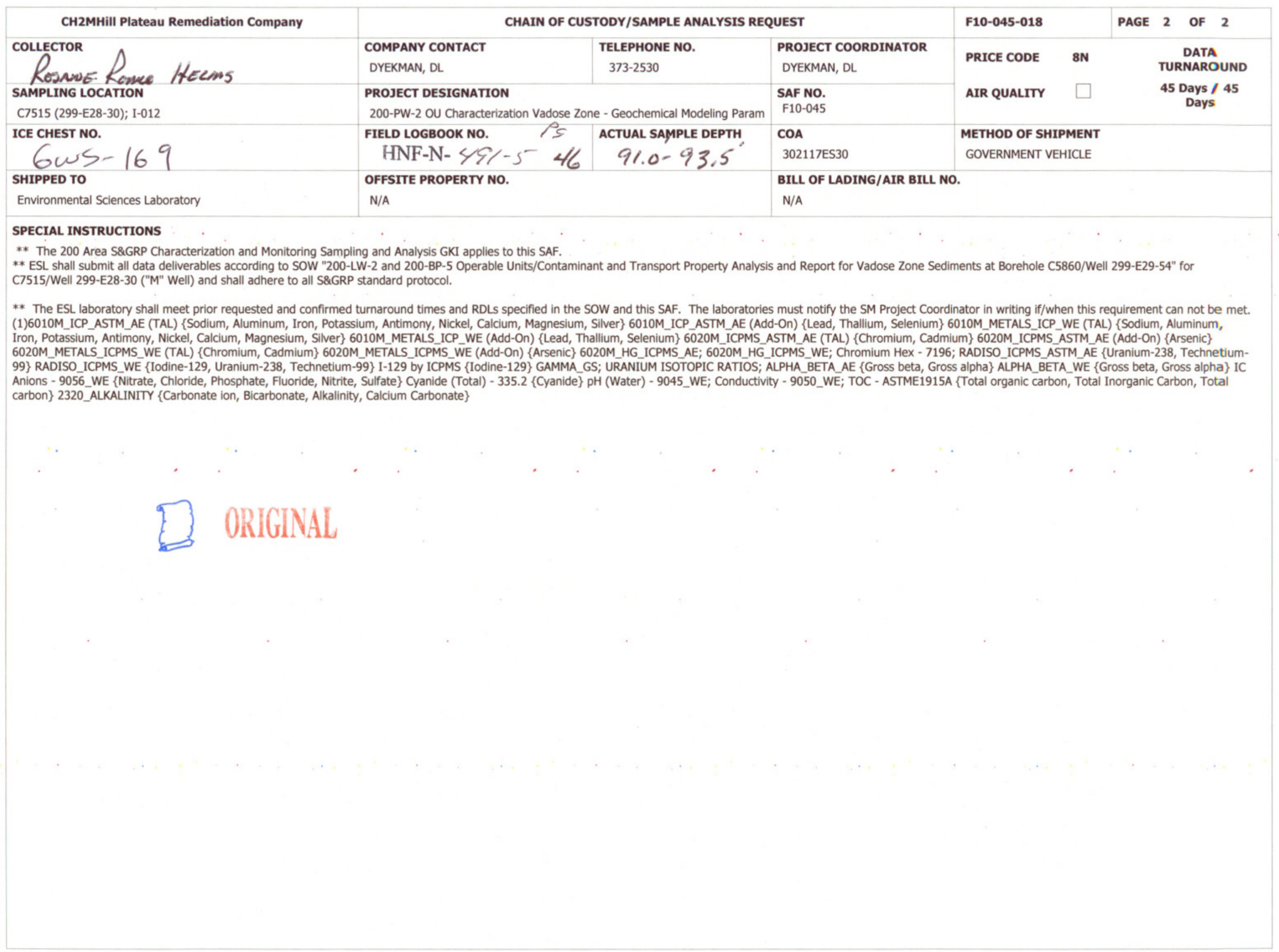




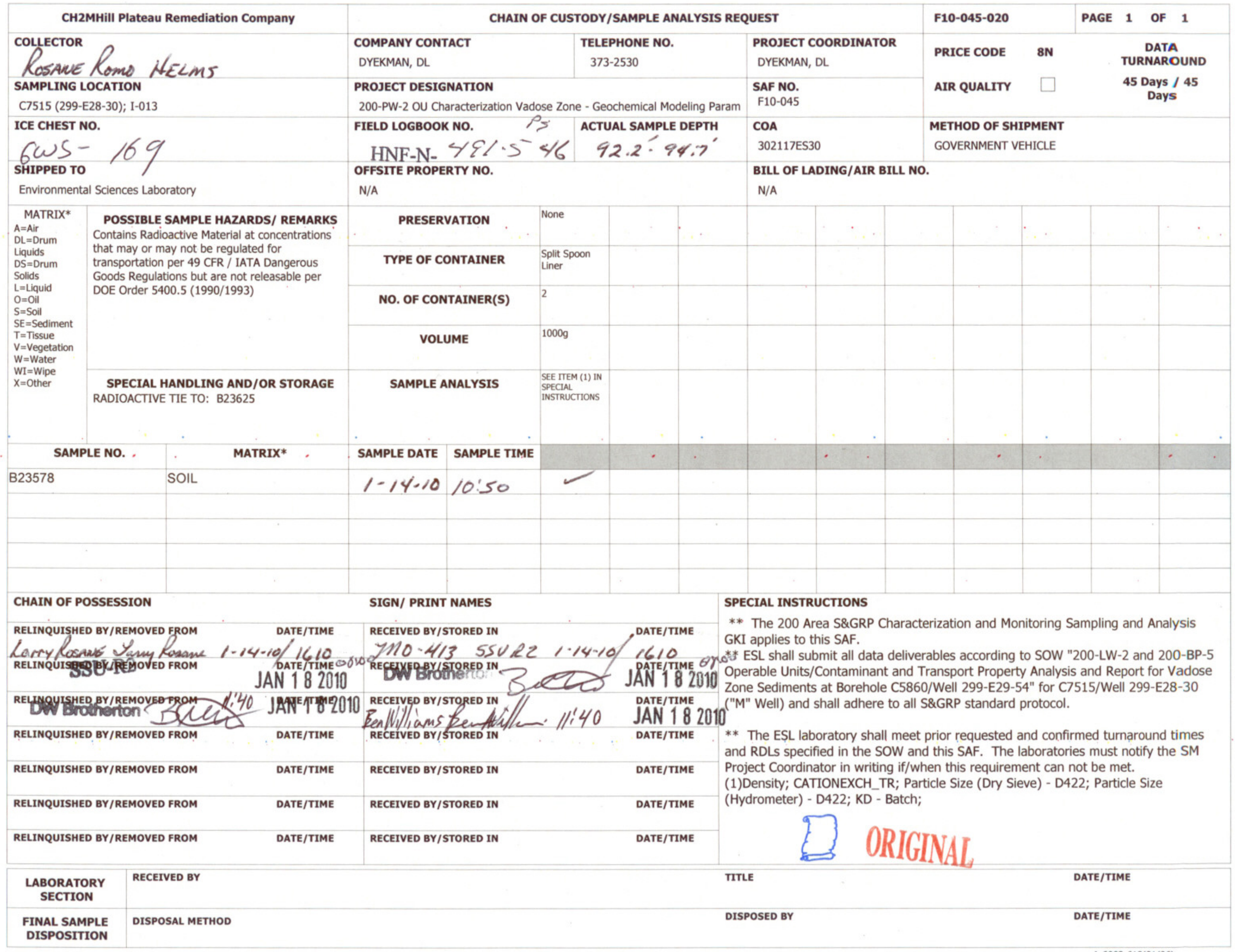




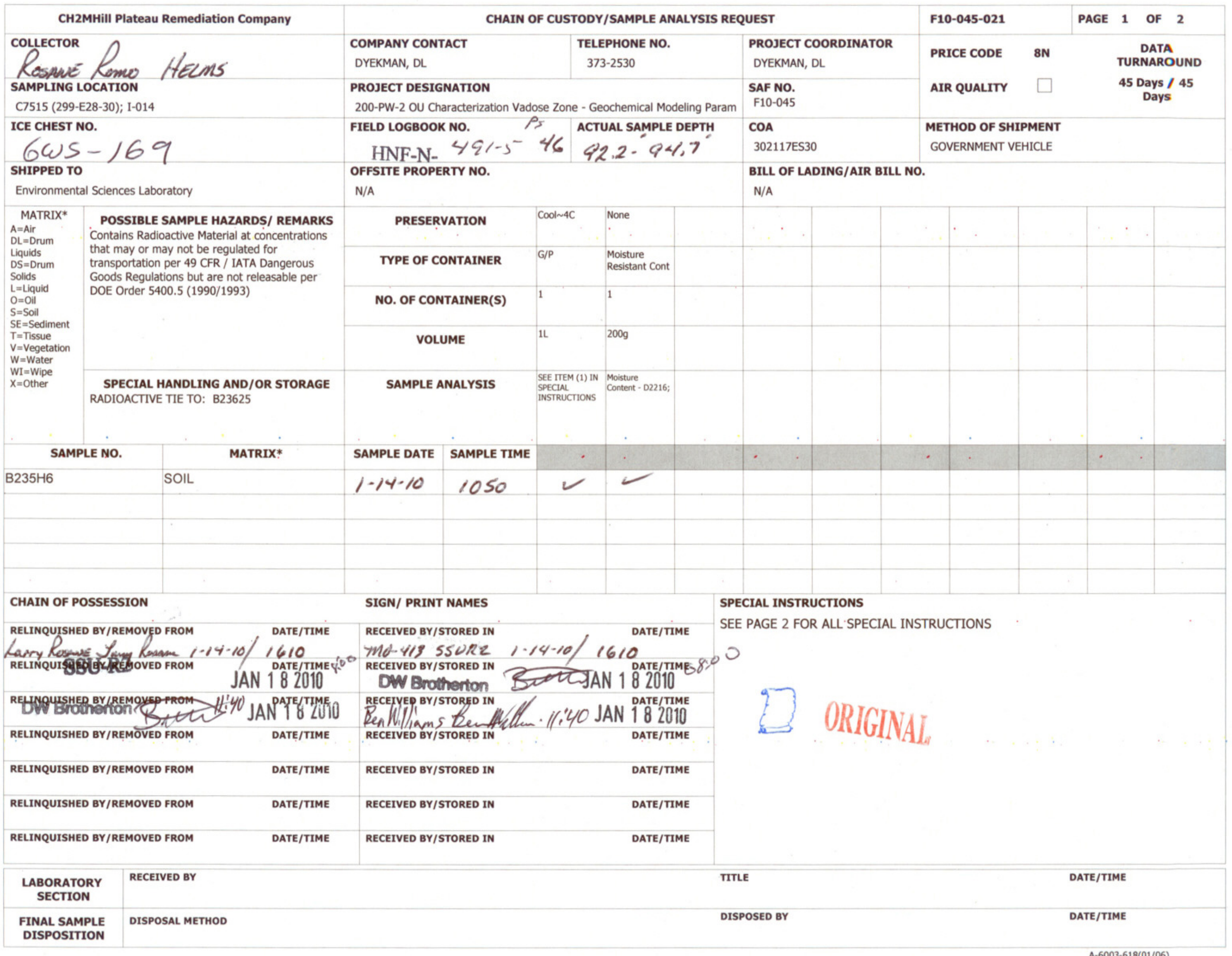




\begin{tabular}{|c|c|c|c|c|c|c|c|}
\hline CH2MHill Plateau Remediation Company & \multicolumn{3}{|c|}{ CHAIN OF CUSTODY/SAMPLE ANALYSIS REQUEST } & \multicolumn{2}{|l|}{ F10-045-021 } & PAGE & OF \\
\hline COLLETOR & $\begin{array}{l}\text { COMPANY CONTACT } \\
\text { DYEKMAN, DL }\end{array}$ & $\begin{array}{l}\text { TELEPHONE NO. } \\
373-2530\end{array}$ & $\begin{array}{l}\text { PROJECT COORDINATOR } \\
\text { DYEKMAN, DL }\end{array}$ & PRICE CODE & $\mathbf{8 N}$ & & $\begin{array}{l}\text { DATA } \\
\text { TURNAROUND }\end{array}$ \\
\hline $\begin{array}{l}\text { SAMPLING LOCATION } \\
\text { C7515 (299-E28-30); I-014 }\end{array}$ & $\begin{array}{l}\text { PROJECT DESIGNATION } \\
\text { 200-PW-2 OU Characterization Vadose Zo }\end{array}$ & e- Geochemical Modeling Param & $\begin{array}{l}\text { SAF NO. } \\
\text { F10-045 }\end{array}$ & AIR QUALITY & $\square$ & & $\begin{array}{l}45 \text { Days / } 45 \\
\text { Days }\end{array}$ \\
\hline $\begin{array}{l}\text { ICE CHEST NO. } \\
\text { GWS }-169\end{array}$ & $\begin{array}{l}\text { FIELD LOGBOOK NO. } P S \\
\text { HNF-N- } Y C / 5_{-}^{-} 4 \mathrm{C}\end{array}$ & $\begin{array}{l}\text { ACTUAL SAMPLE DEPTH } \\
92.2=94,7\end{array}$ & $\begin{array}{l}\text { COA } \\
302117 \text { ES30 }\end{array}$ & $\begin{array}{l}\text { METHOD OF SH } \\
\text { GOVERNMENT V }\end{array}$ & $\begin{array}{l}\text { MENT } \\
\text { HICLE }\end{array}$ & & \\
\hline $\begin{array}{l}\text { SHIPPED TO } \\
\text { Environmental Sciences Laboratory }\end{array}$ & $\begin{array}{l}\text { OFFSITE PROPERTY NO. } \\
\text { N/A }\end{array}$ & & $\begin{array}{l}\text { BILL OF LADING/AIR BIL } \\
\text { N/A }\end{array}$ & & & & \\
\hline
\end{tabular}

\section{SPECIAL INSTRUCTIONS}

** The 200 Area S\&GRP Characterization and Monitoring Sampling and Analysis GKI applies to this SAF.

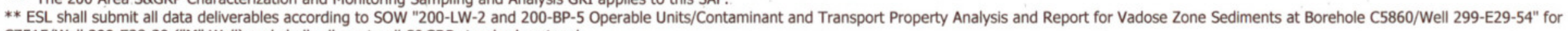
C7515/Well 299-E28-30 ("M" Well) and shall adhere to all S\&GRP standard protocol.

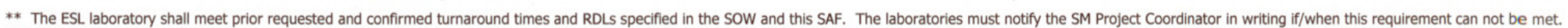

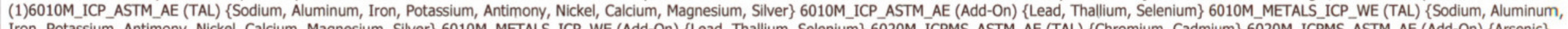

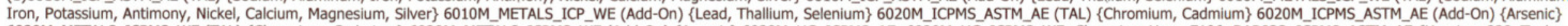

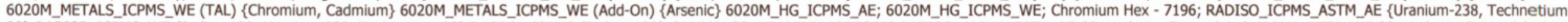

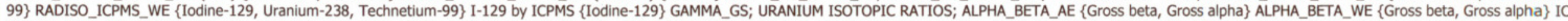

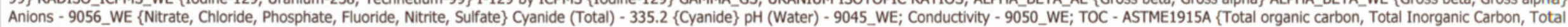
Anions - 9056_WE \{Nitrate, Chloride, Phosphate, Fluoride, Nitrite, Sulfate\} Cyanide (Tota)
carbon\} 2320_ALKALINITY \{Carbonate ion, Bicarbonate, Alkalinity, Calcium Carbonate\}

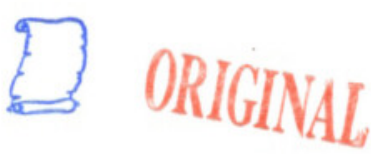




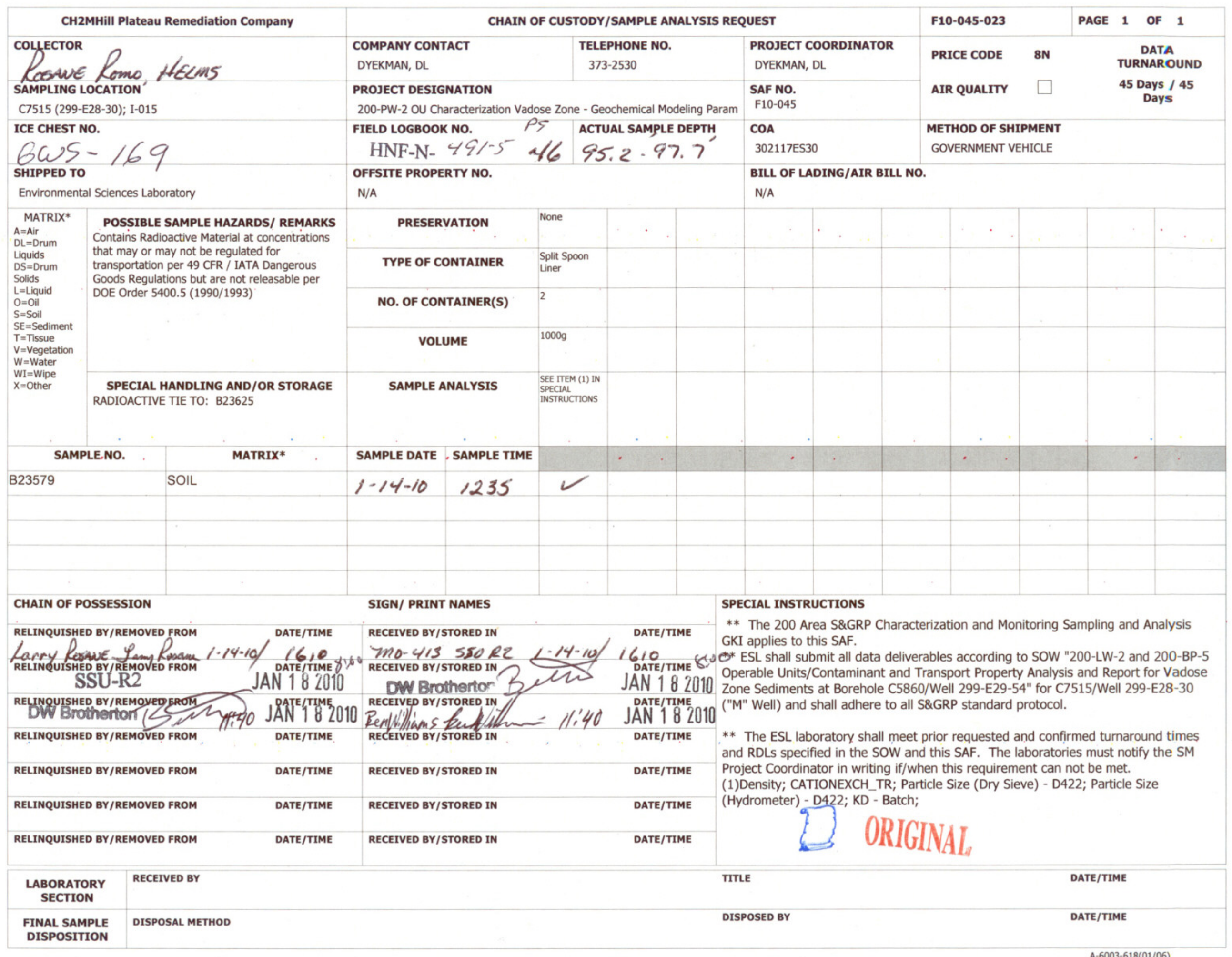




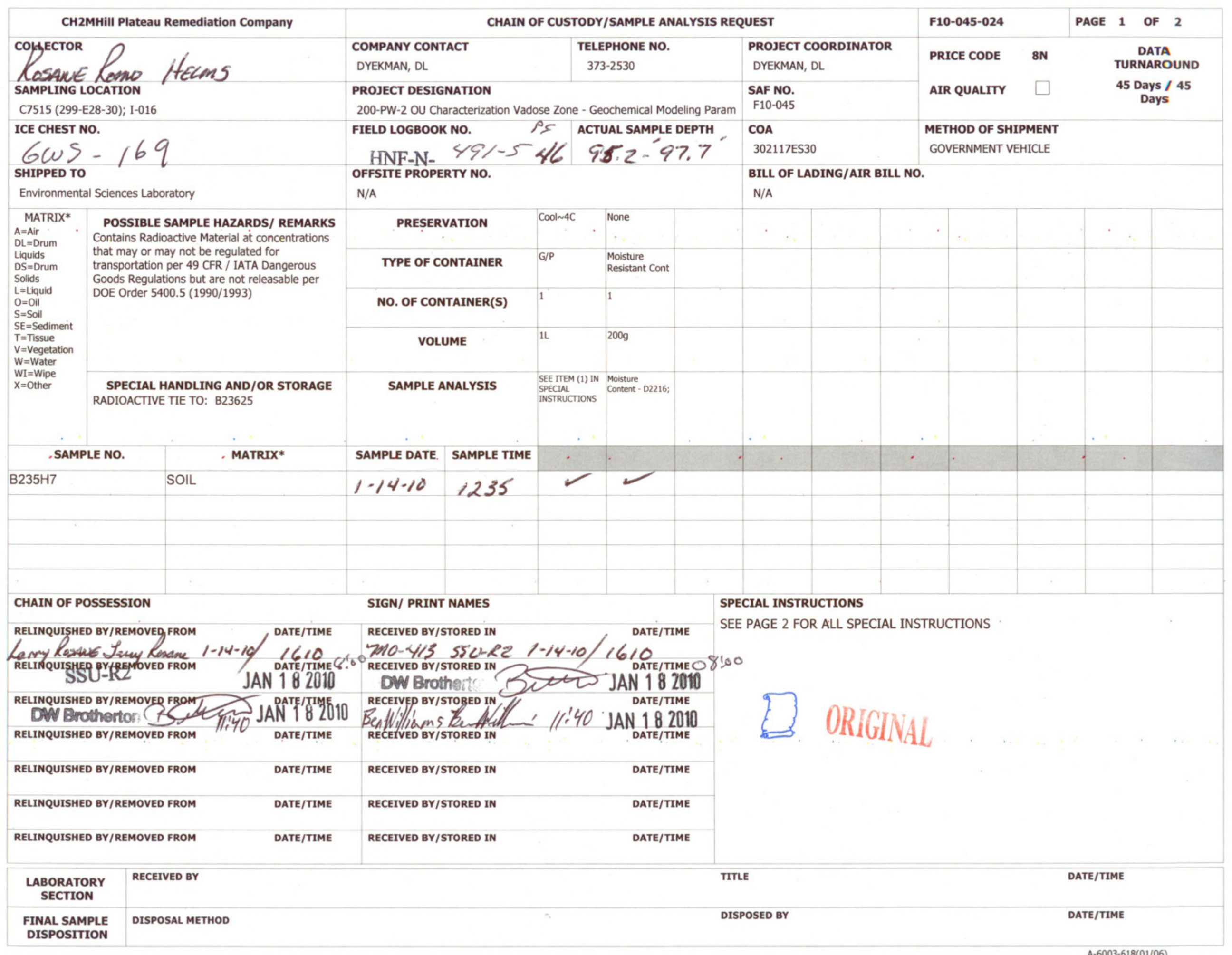




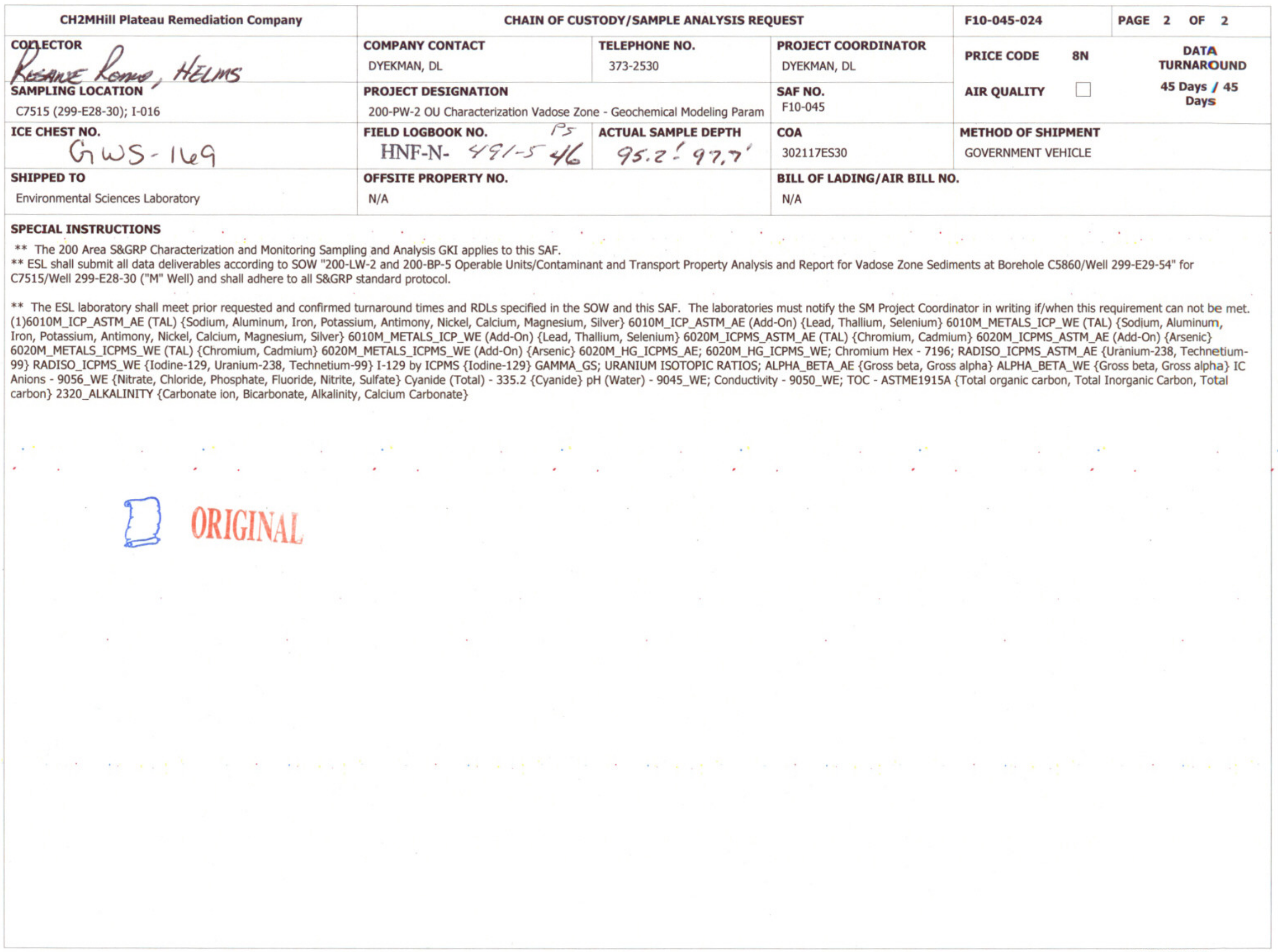


PROJECT DESIGNATION SAF NO.
F10-045

PRICE CODE

200-PW-2 OU Characterization Vadose Zone - Geochemical Modeling Param | F10-045

ICE CHEST NO.

FIELD LOGBOOK NO.

PS ACTUAL SAMPLE DEPTH

AIR QUALITY

\section{METHOD OF SHIPMENT}




\begin{tabular}{|l|l|l}
\hline COMPANY CONTACT & TELEPHONE NO. & PROJECT COORDINATOR \\
\hline DYEKMAN, DL & $373-2530$ & OYEKMAN, DL \\
\hline PROJECT DESIGNATION & & SAF NO. \\
\hline 200-PW-2 OU Characterlzation Vadose Zone - Geochemical Modeling Param & F10-045 \\
\hline
\end{tabular}

PRICE CODE

200-PW-2 OU Characterization Vacose Zone - Geochemical Modeling Param F10-04

AIR QUALITY

ICE CHEST NO.

$6 \underset{\text { SHIPPED TO }}{6}-169$

FIELD LOGBOOK NO.

3 ACTUAL SAMPLE DEPTH COA

METHOD OF SHIPMENT

302117 GS30 GOVERNMENT VEHICLE

HNF-N- $991-5481 / 22.2$

N/A

MATRIX* ${ }^{-r}$ POSSIBLE SAMPLE HAZARDS/ REMARKS

MATRIX* POSSIBLE SAMPLE HAZARDS/ REMARKS
A=Air
Contains Radioactive Material at concentrations Contains Radioactive Material at concentuaron
that may or may not be regulated for transportation per 49 CFR / IATA Dangerous Goods Regulations but are not
DOE Order 5400.5 (1990/1993) N/A

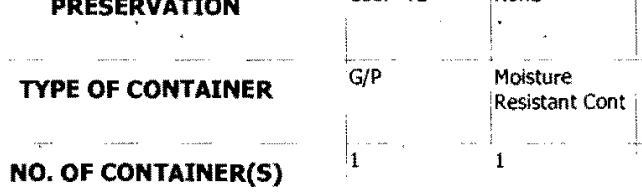

$$
\begin{aligned}
& \text { volume }
\end{aligned}
$$

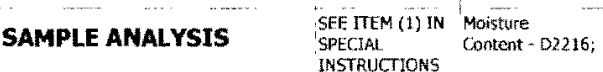

SPECIAL HANDLING AND/OR STORAGE RADIOACTIVE TIE TO: B23630

SAMPLE DATE SAMPLE TIME

$1-15-10$ O 810

\section{SIGN/ PRINT NAMES}

SPECIAL INSTRUCTIONS

CHAIN OF POSSESSION

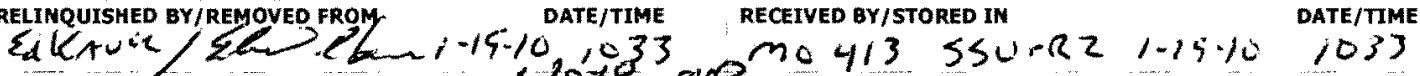
SEE PAGE 2 FOR ALL SPECIAL INSTRUCTIONS

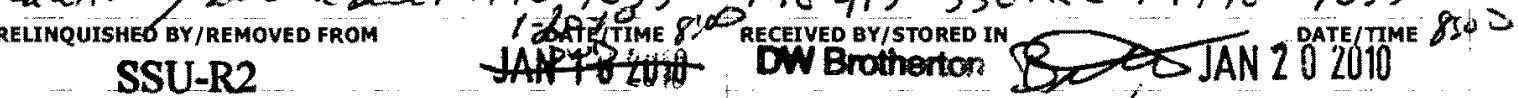

$$
\text { SSU-R2 }
$$

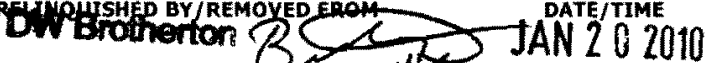

RELINQUISHED BY/REMHOOED FROM I/TSO DATE/TIME

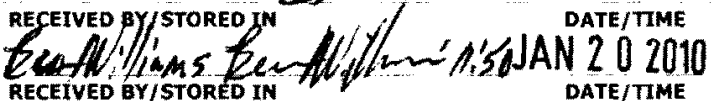

RELINQUISHED BY/REMOVED FROM

DATE/TIME

RECEIVED BY/STORED IN

DATE/TIME

RELINQUUSSHED BY/REMOVED FROM

DATE/TIME

RECEIVED BY/STORED IN

DATE/TIME

RELINQUISHED BY/REMOVED FROM

DATE/TIME

RECEIVED BY/STORED IN 


\section{CH2MHill Plateau Remediation Company}

\section{COLLECTOR}

Kavir, Roino

SAMPLING LOCATION

C7515 (299-E28-30); I-036

iCE CHEST No. -169

SHIPPED TO

Environmental Sclences Laboratory

** The 200 Area S\&GRP Characterization and Monitoring Sampling and Analysis GKI applies to this SAF.

** ESL shall submit all data deliverables according to SOW "200-LW-2 and 200-BP-5

(

** The ESL laboratory shall meet prior requested and confirmed turnaround times and RDLS specified in the SOW and this SAF. The laboratories must notify the SM Project Coordinator in writing iff $/$ hhen this requirement can not be met. (1)6010M_ICP_ASTM_AE (TAL) (Sodum, Aluminum, Iron, Potassium, Antimony, Nickel, Calcium, Magnesium, Silver\} 6010M_ICP_ASTM_AE (Add-On) \{Lead, Thallium, Selenium\} 6010M_METALS ICP_WE (TAL) (SOdum, Aluminum,

6020M_METALS ICPMS WE (TAL) (Chromium, Cadmium\} 6020M_METALS_ICPMS_WE (Add-On) \{Arsenic\} 6020M_HG_ICPMS_AE; 6020M_HG_ICPMS_WE; Chromium Hex - 7196; RADISO_ICPMS_ASTM_AE \{Uranium-238, Technetium-

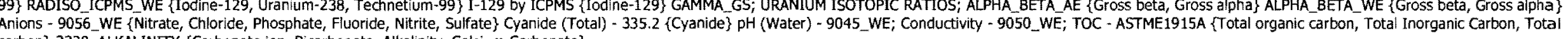
(2) 
Kaver Romo

SAMPLING LOCATION

C7515 (299-E28-30); I-037

ICE CHEST NO.

$6 \cos 5-169$

Environmental Sciences Laboratory

MATRIX

POSSIBLE SAMPLE HAZARDS/ REMARKS

Contains Radioactive Material at concentrations

that may or may not be regulated for
transportation per 49 CFR / IATA Dangerous
Goods Regulations but are not releasable per

DOE Order 5400.5 (1990/1993)

SPECIAL HANDLING AND/OR STORAGE

RADIOACTIVE TIE TO: B23631

SAMPLE NO.

B23590

MATRIX*

SOIL

COMPANY CONTACT

TELEPHONE NO.

PROJECT COORDINATOR

DYEKMAN, DL

\begin{tabular}{r|r|} 
& DAF NO. \\
F10-045
\end{tabular}

PRICE CODE $8 \mathrm{~N}$

DATA

PROJECT DESIGNATION

AIR QUALITY $\square \quad 45$ Days / 45

Days

200-PW-2 ou Characterization Vadose Zone - Geochemical Modeling Pard

HNF-N. $491-548 \mid 122.1$ w 124.6 OFFSTIE PROPERTY NO.

COA METHOD OF SHIPMENT

METHOD OF SHIPMENT

302117 S30 GOVERNMENT VEHICLE

N/A

PRESERVATION

TYPE OF CONTAINER Split Spoon

NO. OF CONTAINER(S)

volume

$1000 \mathrm{~g}$

SAMPLE ANALYSIS

.

$\mathrm{N} / \mathrm{A}$

SAMPLE DATE SAMPLE TIME

$1.19-10 \quad 0920$

SIGN/ PRINT NAMES

\section{CHAIN OF POSSESSION}

RELINQUISHED BY/REMOVED FROM Ed Kovin Sh $1-19.10$

$$
\text { SSU-R2 }
$$

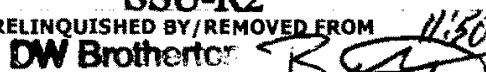

RELINQUISHED BY/REMOVEDFROM

RELINQUISHED BY/REMOVED FROM

RELINQUISHED BY/REMOVED FROM

RELINQUISHED BY/REMOVED FROM

LABORATORY RECEIVED BY

SECTION

FINAL SAMPLE
DISPOSITION

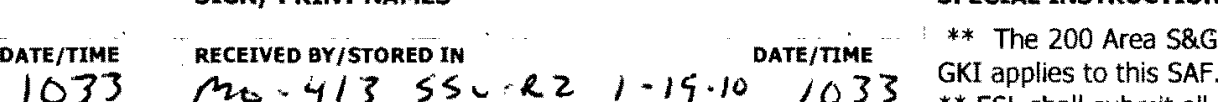

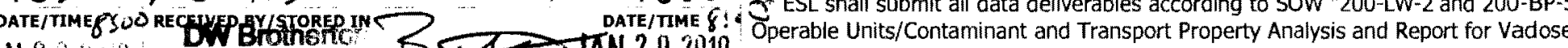
JAN 20 li 1 STAN 20 2010 Zone Sediments at Borehole C5860/Well 299-E29-54" for C7515/Well 299-E28-30 DAN $\{U$ ITIME RECEIVEq BY/STOGEO IN DATE/TIME Re RECENED BY/STORED in

DATE/TTME RECEIVED BY/STORED IN

DATE/TME ("M" Well) and shall adhere to all S\&GRP standard protocol.

** The ESL laboratory shall meet prior requested and confirmed turnaround times and RDLs specified in the SOW and this SAF. The laboratories must notify the SM Project Coordinator in writing if/when this requirement can not be met. (1)Density; CATIONEXCH_TR; Particle Size (Dry Sieve) - D422; Particle Size

DATE/TIME RECEIVED BY/STORED IN

DATE/TME (Hydrometer) - D422; KD - Batch;

DATE/TIME RECEIVED BY/STORED IN

DATE/TIME $\xi 3$ bunan

DATE/TIME 


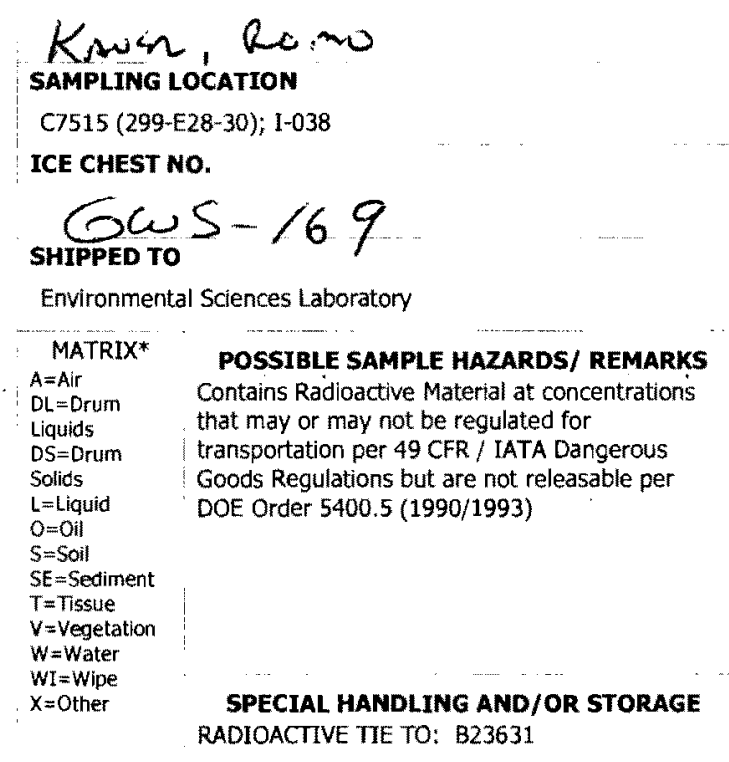

Kowin, Ro no

SAMPLING LOCATION

C7515 (299-E28-30); 1-038

ICE CHEST NO.

$\operatorname{Gou}_{\text {SHIPPED TO }}-169$

Environmental Sciences Laboratory

MATRIX* POSSIBLE SAMPLE HAZARDS/ REMARKS

PAAI
$\mathrm{D}=\mathrm{I}$

CaAr
Dif Drum
Liquids

transportation per 49 CFR / IATA Dangerous
Goods Regulations but are not releasable per

Goods Regulations but are not releasable per

DOE Order 5400.5 (1990/1993)

SPECIAL HANDLING AND/OR STORAGE RADIOACTVE TIE TO: B23631

\begin{tabular}{|c|c|c|}
\hline $\begin{array}{l}\text { COMPANY CONTACT } \\
\text { DYEKMAN, DL }\end{array}$ & $\begin{array}{l}\text { TELEPHONE NO. } \\
373-2530\end{array}$ & $\begin{array}{l}\text { PROJECT COORDINATOR } \\
\text { DYEKMAN, DL }\end{array}$ \\
\hline \multirow{2}{*}{\multicolumn{2}{|c|}{$\begin{array}{l}\text { PROJECT DESIGNATION } \\
\text { 200-PW-2 OU Characterization Vadose Zone - Geochemical Modeling Paran }\end{array}$}} & \\
\hline & & \\
\hline FIELO LOGBOOK NO. & ACTUAL SAMPLE & $\mathrm{COA}$ \\
\hline
\end{tabular}

PRICE CODE

AIR QUALTTY

DATA
TURNAROUND
45 DaYs/ 45

45 Days / 45

METHOD OF SHIPMENT

48 ACTUAL SAMPLE DEPTH COA

OFFSTE PROPERTY NO.

\begin{tabular}{cccc|c} 
TYPE OF CONTAINER & G/P & $\begin{array}{l}\text { Mossture } \\
\text { Resistant Cont }\end{array}$ \\
NO. OF CONTAINER(S) & i & 1 \\
\hline VOLUME & IL & 2009 \\
\hline
\end{tabular}

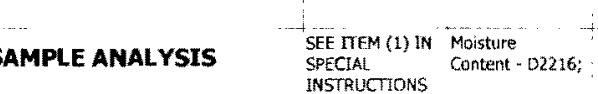

SAMPLE NO.

MATRIX* .

SAMPLE DATE SAMPLE TIME

$1.19 .10: 0922$

N/A

GETHE ORT VEHICLE

SIGN/ PRINT NAMES

SPECIAL INSTRUCTIONS

CHAIN OF POSSESSION DATE/TME

RELINQUISHED BYIREMOVED EROM DATE/TIME $1-19-10 / 033$ MO. 463 SSUR2 115,101033

al xusy zan term

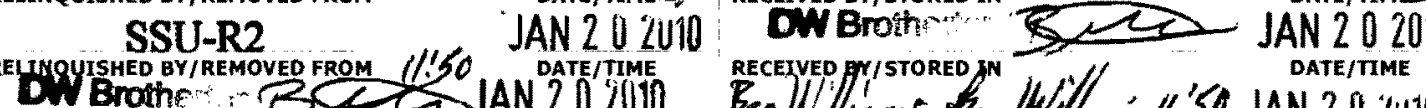

SSU-R2
JAN 20 ZU10 DW DW Broth

RELINOUISHED BY/REMOVED From

RELINQUISHED BY/REMOVED FROM

DATE/TIME

RECEIVED BY/STORED II

DATE/TIME

2 WIGLN

RELINQUISHED BY/REMOVED FROM

Date/Time

RECEIVED BY/STORED IN

DATE/TIME

RELINQUISHED BY/REMOVED FROM

DATE/TIME

RECEIVED BY/STORED IM

DATE/TME 
200-PW-2 OU Characteriza

SAF NO.

CE CHEST NO.

FIEID

F10-045

AIR QUALITY

Cous - cos

HNF-N- 491 -

$12 \% .6$

METHOD OF SHIPMENT

OFFSTE PROPERTY NO.

302117 ES30

GOVERNMENT VEHICLE

Environmental Sciences Laboratory

SPECIAL INSTRUCTIONS

N/A

** The 200 Area S8GRP Characterization and Monitoring Sampling and Analysis GKI applies to this SAF.

** ESL shall submit all data deliverables according to SOW "200-LW-2 and 200-BP-5 Operable Units/Contaminant and Transport Property Analysis and Report for Vadose Zone Sediments at Borehole C5860/Well 299-E29-54" for
C7515/Well 299-E28-30 ("M" Well) and shall adhere to all S\&GRP standard protocol.

** The ESL laboratory shall meet prior requested and confirmed turnaround times and ROLS specifed in the SOW and this SAF. The laboratories must notify the SM Project Coordlnator in writing iff/when this requirement can not be met.

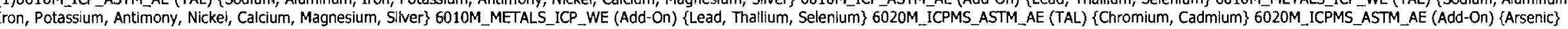

6020M_METAL_ICPMS_WE (TAL) (Chromium, Cadmlum\} 6020M_METALS_ICPMS_WE (Add-On) \{Arsenic\} 6020M_HG_ICPMS_AE; 6020M_HG_ICPMS_WE; Chromium Hex - 7196; RADISO_ICPMS_ASTM_AE \{Uranium-238, TeChnetium-

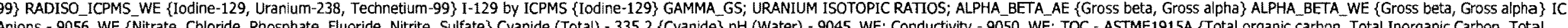
Anions - 9056-WE \{Nitrate, Chloride, Phosphate, Fluoride, Nitrite, Sulfate\} Cyanide (Tota)
Carbon\} 2320 ALKALINITY \{Carbonate ion, Bcarbonate, Alkalinity, Calcium Carbonate\}

D 
CH2MHill Plateau Remediation Company COLLECTOR

\section{Kaver pono}

SAMPLING LOCATION

C7515 (299-E28-30); I-039

ICE CHEST NO.

Govipto $5-169$

Environmental Sciences Laboratory

MATRIX* POSSIBLE SAMPLE HAZARDS/ REMARKS

MAAir

Contains Radioactive Material at concentrations
D $L=0$ rum
that may or may not be regulated for

$\begin{array}{ll}\text { liguids } & \text { that may or may not be regulated for } \\ \text { DS=Drum } & \text { transportation per } 49 \text { CFR / IATA Dangerous }\end{array}$

Goods Regulations but are not releasable per

DOE Order $5400.5(1990 / 1993)$

SPECIAL HANDLING AND/OR STORAGE RADIOACTIVE TIE TO: B2363:

SAMPLE NO.

PAGE 1 OF 1

CHAIN OF CUSTODY/SAMPLE ANALYSIS REQUEST

$\begin{array}{lcc}\text { PRICE CODE } & 8 \mathrm{~N} & \begin{array}{c}\text { DATA } \\ \text { TURNAROUND }\end{array} \\ \text { AIR QUALITY } & \square & \begin{array}{l}45 \text { Days / } 45 \\ \text { Days }\end{array}\end{array}$

PROJECT DESIGNATION

$373-2530$

PROJECT COORDINATOR

DYEKMAN, DL

45 Days / 45
Days

200-PW-2 OU Characterization Vadose Zone - Geochemical Modeling Param F10-045

FIELD LOGBOOK NO. PS 48 ACTUAL SAMPLE DEPTH

HNF-N- Y $91-5$ i25.1 to 127.6 OFFSTTE PROP

COA METHOD OF SHIPMENT

GOVERNMENT VEHICLE

BILL OF LADING/AIR BILL NO.

N/A

PRESERVATION

TYPE OF CONTAINER

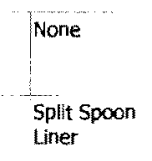

NO. OF CONTAINER(S)

volume

SAMPLE ANALYSIS

SEE TEM (1) IN
SPECALA
INSRUCTIONS

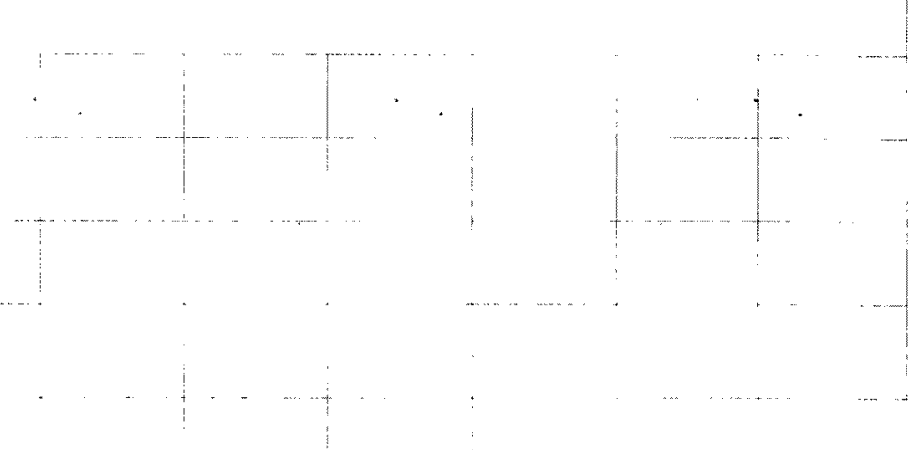

CHAIN OF POSSESSION

RELINQUISHED BYIREMOVED FROA

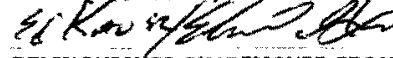
RELINQUISHED BY/REMOVED FROM SSU 2 2

DATE/TIME

RECEIVED BY/STORED IN MO 413 SSU - 22,15 DATE/TIME SPECIAL INSTRUCTIONS

** The 200 Area S\&GRP'Characterization and Monitoring Sampling and Analysis DATE/TMMES. Operable Units/Contaminant and Transport Property Analysis and Report for Vadose DW Brothaton JAN DATETIME DW Brotherton $R$ JAN 202 EELINQUTSHED BY/REMOVEED FROM OATE/TTME

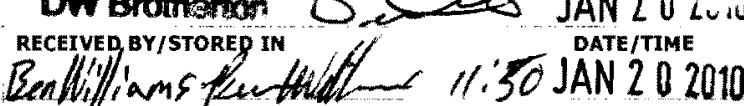
3,50 M" Well) and shall adhere to all S\&GRP standard protocol.

DATE/TME

* The ESL laboratory shall meet prior requested and confirmed turnaround times and RDLS specified in the SOW and this SAF. The laboratories must notify the SM

RELINQUISHED BY/REMOVED FROM

DATE/TIME

RECEIVED BY/STORED IN

DATE/TIME

(1)

DATE/TIME

(1)Density; CATIONEXCH_TR; Particle Size (Dry Sieve) - D422; Partide Size
(Hydrometer) - D422; KD - Batch;

DATE/TIME RECEIVED BY/STORED I

DATE/TIME

RECEIVED BY/STORED IN

DATE/TIME
2 ORIGLL

nTLE

DISPOSED BY
DATE/TIME

DATE/TIME 
COLLECTOR

$$
\text { Kavin Ro.mo }
$$

SAMPLING (299-E28-30); I-040

ICE CHEST NO.

Gevs-169

SHIPPED TO

Environmental Sciences Laboratory

MATRIX* POSSIBLE SAMPLE HAZARDS/ REMARKS

$\mathrm{A}=\mathrm{A} A \mathrm{ir}$
$\mathrm{DL}=\mathrm{Drum}$$\quad$ Contains Radioactive Material at concentrations

$\begin{array}{ll}\text { Liquids } & \text { that may or may not be regulated for } \\ \text { DS }=\text { Drum } & \text { transportation per } 49 \text { CFR / IATA Dangerous }\end{array}$

Goods Regulations but are not releasable per

DOE Order 5400.5 (1990/1993)

SPECIAL HANDLING AND/OR STORAGE SPECIAL HANDLING AND/0
RADIOACTIVE TIE TO: B23631

\section{COMPANY CONTAC}

DYEKMAN, DL

TELEPHONE NO.

PROJECT COORDINATOR

DYEKMAN, DL

SAF NO.
F10-045

200-PW-2 OU Characterization Vadose Zone - Geochemical Modeling Param F10-045

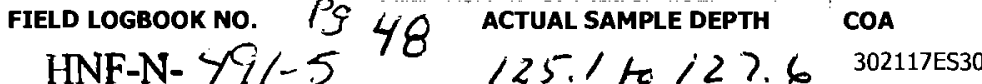
OFFSTIE PROPERTY NO.

$\mathrm{N} / \mathrm{A}$

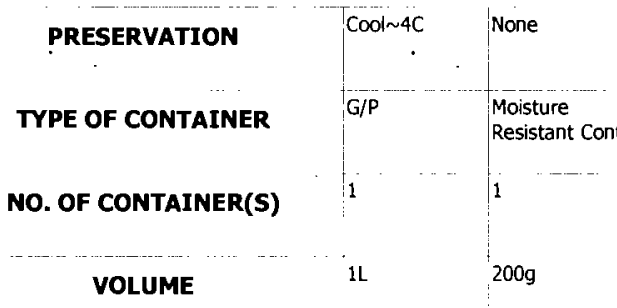

SAMPLE ANALYSIS $\begin{aligned} & \text { SEE TIMM (1) IN Moisture } \\ & \text { SPECLAL } \\ & \text { INSTRUCIONS } \\ & \text { Content - D2216 }\end{aligned}$
BILL OF LADING/AIR BILL NO.

$\mathrm{N} / \mathrm{A}$

\section{TURATA \\ 45 Days / 45}

\section{METHOD OF SHIPMEN}

GOVERNMENT VEHICLE

Comere

SAMPLE DATE SAMPLE TIME -

$1-15-10$ OSSO

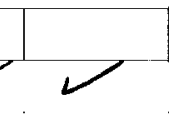

SIGN/ PRINT NAMES

SPECIAL INSTRUCTIONS

CHAIN OF POSSESSION

DE BYTSED IN

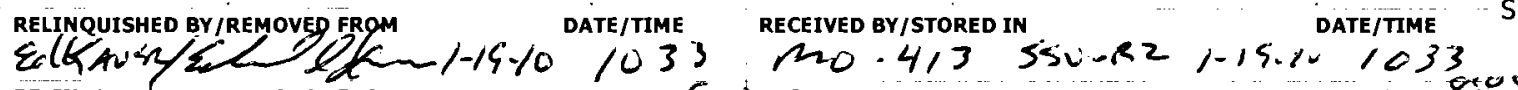

SEE PAGE 2 FOR AL SPECIAL INSTRUCTIONS

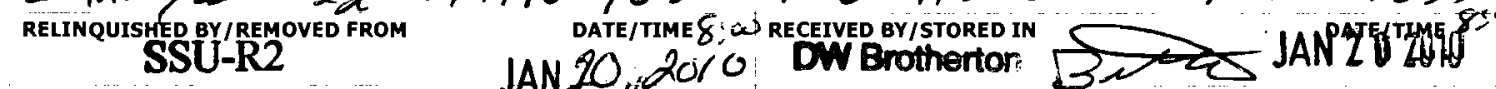

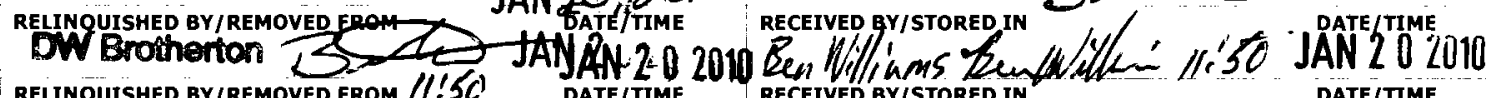
RELINQUiSHE, BY/REMOVED FRom /I!50 . DATE/TIME

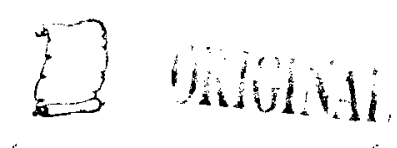

RELINQUISHED BY/REMOVED FROM

DATE/TIME

RECEIVED BV/STORED IN

DATE/TIME

RELINQUISHED BY/REMOVED FROM

DATE/TIME

RECEIVED BY/STORED IN

DATE/TIME

RELINQUISHED BY/REMOVED FROM

DATE/TIME

RECEIVED BY/STORED IN

DATE/TIME 
PROJECT COORDINATOR

PROJECT DESIGNATION

AIR QUALITY $\square$

\begin{tabular}{lll} 
SAF No. \\
\hline
\end{tabular}

FIELD LOGBOOK NO PS 48 ACTUAL SAMPLEDEPTH cOA

CE CHEST NO.

HNF-N- $Y Y \ell-S$

COA

OFFSITE PROPERTY NO.

METHOD OF SHIPMENT

N/A

BILL OF LADING/AIR BILL NO.

SHIPPED TO

Environmental Sciences Laboratory

SPECIAL INSTRUCTIONS

** The 200 Area S\&GRP Characterization and Monitoring Sampling and Analysis GKI applies to this SAF.

** ESL shall submit all data deliverables according to SOW" "200-LW-2 and 200-BP-5 Operable Units/Contaminant and Transport Property Analysis and Report for Vadose Zone Sediments at Borehole C5860/Well 299-E29-54" for

C7515/Well 299-E28-30 ("M" Well) and shall adhere to all S\&GRP standard protocol.

** The ESL laboratory shall meet prior requested and confirmed turnaround times and RDLs specified in the SOW and this SAF. The laboratories must notify the SM Project Coordinator in writing if/when this requirement can not be met.

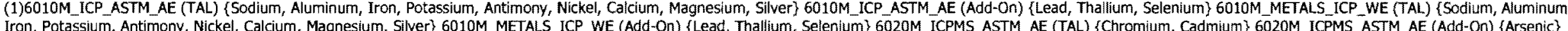

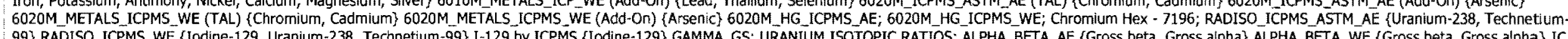
99\} RADISO _. Anions - 9056-WE (Nitrate, Chloride, Phosphate, Flooride, Nitrite, surfate) Cyanide

E) Oll 
COLLECTOR

CHAIN OF CUSTODY/SAMPLE ANALYSIS REQUEST

F10-045-062

PAGE 1 OF 2

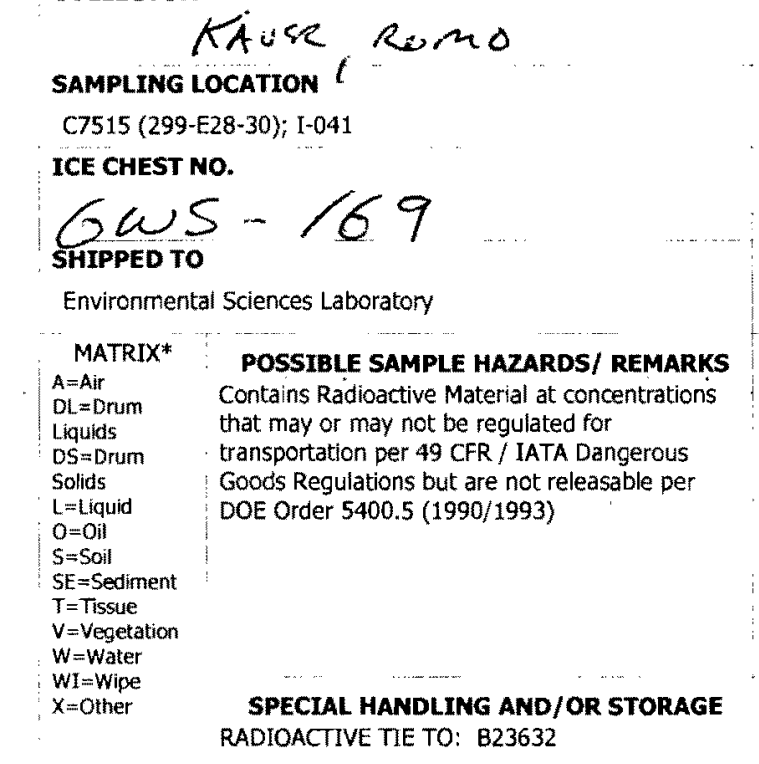

$\begin{array}{lll}\text { COMPANY CONTACT } & \text { TELEPHONE NO. PROJECT COORDINATOR }\end{array}$

DYEKMAN, DL $373-2530 \quad$ DYEKMAN, DL

PRICE CODE

200-PW-2 OU Characterization vadose Zone - Geochemical Modeling Param F10-045

FIELD LOGBOOK NO. $P_{5}$ Y ACTUAL SAMPLE DEPTH COA METHOD OF SHIPMENT

AIR QUALITY $\square$

45 Days $/ 45$
Days

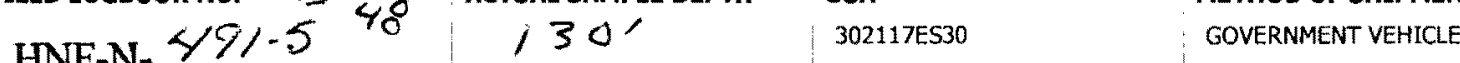

GOVER

BILL OF LADING/AIR BILL NO.

N/A

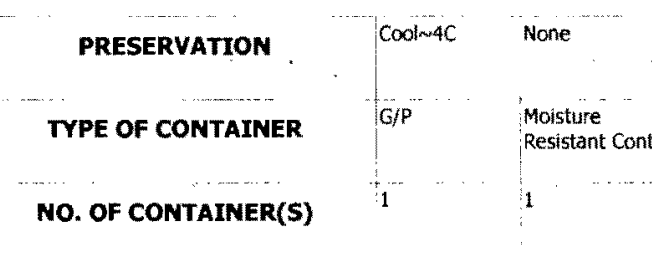

volume

it $\quad 2009$

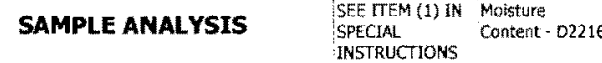

SAMPLE NO.

MATRIX* .

SAMPLE DATE SAMPLE TIME

1.19 .1010 .5

$\mathrm{B} 235 \mathrm{KO}$

SIGN/ PRINT NAMES

SPECIAL INSTRUCTIONS

CHAIN OF POSSESSION

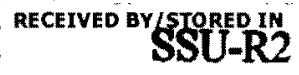

SEE PAGE 2 FOR ALL SPECIAL INSTRUCTIONS

RELINQUISHED BYIREMOVED FROM DATE/TIME

RELINQUISHED BY/REMOVED FROM
SSU-R2 DATE/TIMEj:

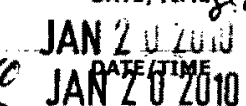

DW Brothert?

RELINQUISHED BY/REM OVED FROM

RELINQUISHED BY/REMOVED FROM

DATE/TIME

DWEPYTSTORE IN DSee JAN

RELINQUISHED BY/REMOVED FROM

DATE/TIME

RECEIVEg/SYSTOREP IN

DATE TMME

RELINQUISHED BY/REMOVED FROM

DATE/TIME

RECEIVED BY/STORED IN

DATE/TME

LABORATORY RECEIVED QY

DATE/TIME

RECEIVED BY/STORED IN

DATE/TIME

$\sum_{2}$ Visuli

SECTION

FINAL SAMPLE DISPOSAL METHO

DATE/TIME

DATE/TIME 
CH2MHill Plateau Remediation Company

\section{COLLECTOR}

$$
\text { Kaush, Remo }
$$

SAMPLING LOCATION

C7515 (299-E28-30); 1-04

ICE CHEST NO.

$6005-169$

Environmental Sciences Laboratory

\section{SPECIAL INSTRUCTIONS}

** The 200 Area S\&GRP Characterzzation and Monitoring Sampling and Analysis GKI applies to this SAF

** ESL shall submit all data deliverables according to SOW "200-LW-2 and 200-BP-5 Operable Units/Contaminant and Transport Property Analysis and Report for Vadose Zone Sediments at Borehole C5860/Well 299-E29-54" for

** The ESL laboratory shall meet prior requested and confirmed turnaround times and RDLS specified in the SOW and this SAF. The laboratories must notify the SM Project coordinator in writing iffwhen this requirement can not be met. (1)6010M_ICP_ASTM_AE (TAL) \{Sodium, Aluminum, Iron, Potasssium, Antimony, Nickel, Calcium, Magnesium, Silver\} 6010M_ICP_ASTM_AE (Add-On) \{Lead, Thallium, Selenium\} 6010M_METALS_ICP_WE (TAL) \{Sodium, Aluminum,

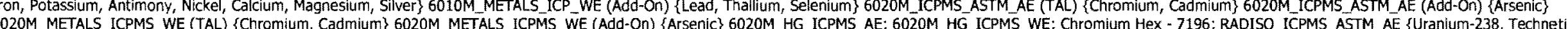

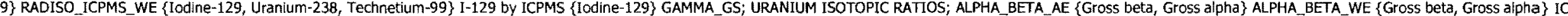
Anions - 9056_WE \{Nitrate, Chloride, Phosphate, Fiuoride, Nitrite, Sulfate\} Cyanide (Total) - 335.2 \{Cyanide\} pH (Water) - 9045_WE; Conductivity - 9050_WE; TOC - ASTME1915A \{Total organic Carbon, Total Inorganic Carbon, Total carbon\} 2320_ALKALINITY \{Carbonate ion, Bicarbonate, Alkalinity, Calcium Carbonate\}

\section{CHAIN OF CUSTODY/SAMPLE ANALYSIS REQUEST}

$373-2530$

PROJECT COORDINATOR

SAF

\section{COA}

302117 ES 30

METHOD OF SHIPMENT

GOVERNMENT VEHICLE

DIUL OF LADING/AIR BILL NO.
PAGE 2 OF 2

DATA
TURNAROUND

45 Days / 45
Days
FIELD LOGBOOK NO. P5 48 ACTUAL SAMPLE DEPTH

HNF-N- $49 \%$

$130^{\circ}$

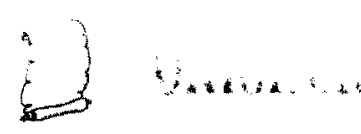




\section{KAUER, ROMo}

SAMPLING LOCATION

C7515 (299-E28-30); I-042

ICE CHEST NO.

$$
\underset{\text { SHIPPED TO }}{2}
$$

Environmental Sciences Laboratory

MATRIX*

POSSIBLE SAMPLE HAZARDS/ REMARKS Contains Radioactive Material at concentrations that may or may not be regulated for
transportation per 49 CFR / IATA Dangerous Goods Regulations but Ro not relengerous DOE Order $5400.5(1990 / 1993)$

SPECIAL HANDLING AND/OR STORAGE RADIOACTIVE TIE TO: B23632 \begin{tabular}{l|l} 
COMPANY CONTACT & TELEPHONE NO.
\end{tabular}

DYeKMaN, DL

TELEPHONE NO.

PROJECT DESIGNATION

SAF NO

FIELD LOGBOOK NO. $P$

- Geochemical Modeling Paran

COA

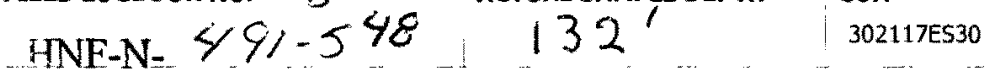

MOOVRNMTT VEICLE

BILL OF LADING / AIR BILL NO.

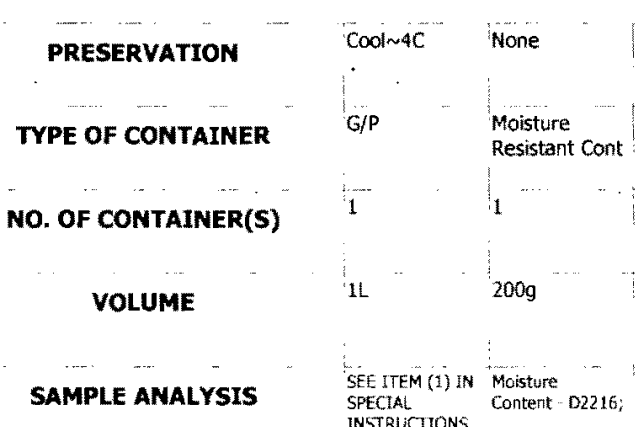

\section{METHOD OF SHIPMENT}

SAMPLE NO.
B235K1

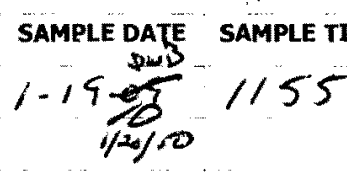

RELinguished By/RemoviebFom

RELINQUISHED BY/REMOVED FROM

RELINQUISHED BY/REMOVED FROM

RELINQUISHED BY/ REMOVED FROM

\section{LABORATORY
SECTION}

DISPOSITION

RECEIVED BY
DATE/TIME

RECEIVED BY/STORED IN

DATE/TIME

RECEIVED BY/STORED IN

DATE/TIME

RECEIVED BY/STORED IN

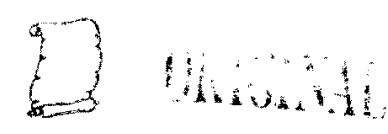

DATE/TME

DATE/TIME

DATE/TIME 
CH2MHill Plateau Remediation Company

COLLECTOR

\section{KaUER, Romo} SAMPLING LOCATION OD15 (299-E28-30); 1-042 ICE CHEST NO.

$6 \cos 5-16$

Environmentał Sciences Laboratory

SPECIAL INSTRUCTIONS

** The 200 Area S\&GRP Characterization and Monitoring Sampling and Analysis GKI applies to this SAF.

** ESL shall submit all data deliverables according to SOW "200-LW-2 and 200-BP-5

to all S\&GRP standard protocol.

** The ESL laboratory shall meet prior requested and confirmed turnaround times and RDLS specified in the SOW and this SAF. The laboratories must notify the SM Project Coordinator in writing iff when this requirement can not be met.
(1)6010M ICP ASTM AE (TAL) \{Sodium, Aluminum, Iron, Potassium, Antimony, Nickel, Calcium, Magnesium, Silver\} 6010M ICP. ASTM AE (Add-On) \{Lead, Thallium, Selenium\} 6010M METALS ICP WE (TAL) USodium, Aluminum,

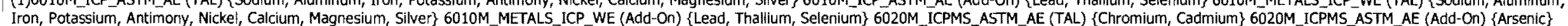
6020M_METALS_ICPMS_WE (TAL) \{Chromium, Cadmium\} 6020M_METAL__ICPMS_WE (Add-On) \{Arsenic\} 6020M_HG_ICPMS_AE; 6020M_HG_ICPMS_WE; Chromium Hex - 7196; RADISO_ICPMS_ASTM_AE \{Uranium-238, Technetium-

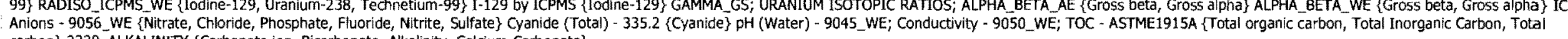

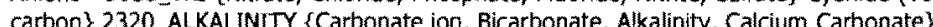

OF CUSTODY/SAMPLE ANALYSIS REQUEST

DOECT COORDINATOR

SAF NO.
200-PW-2 OU Characterization Vadose Zone - Geochemical Modeling Param

FELD LOGBOOK NO. PS 48 ACTUAL SAMPLE DEPTH COA

HNF-N- $49 /-5 \quad 132^{\prime}$ 302117ES30

BILL OF LADING/AIR BILL NO.
45 Days / 45

Days

\section{What}


PROJECT COORDINATOR

PROJECT DESIGNATION

AIR QUALITY

200-PW-2 OU Characterization Vadose Zone - Geochemical Modeling Param F10-045

FIELD LOGBOOK NO. PS 48 ACTUAL SAMPLE DEPTH COA

METHOD OF SHIPMENT

ICE CHEST NO.

$6 \operatorname{lov}_{\text {SAIPPED TO }} 5-16$

Environmental Sciences Laboratory

HNF-N- 491.5 / 136

COA $302117 E S 30$

GOVERNMENT VEHICLE

OFFSTTE PROPERTY NO.

BILL OF LADING/AIR BILL NO.

POSSIBLE SAMPLE HAZARDS/ REMARKS

Contains Radioactive Material at concentrations

that may or may no Be reguated for

Goods Regulations but are not releasable per

DOE Order 5400.5 (1990/1993)

PRESERVATION Col $4 \mathrm{C}$

TYPE OF CONTAINER G/P $\quad \begin{aligned} & \text { Moisture } \\ & \text { Resistant Cont }\end{aligned}$

NO. OF CONTAINER(S)

VOLUME

$\mathrm{N} / \mathrm{A}$

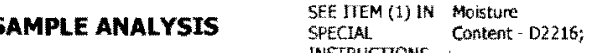

SPECIAL HANDLING AND/OR STOPAGE

RADIOACTIVE TIE TO:

KS $1 / 12 / 10$

- SAMPLE No.

MATRIX*

B235K2

SOIL

SAMPLE DATE SAMPLE TIME

1.19 .101235

\section{SIGN/ PRINT NAMES}

SPECIAL IMSTRUCTIONS

CHAIN OF POSSESSION

SEE PAGE 2 FOR ALL SPECIAL INSTRUCTIONS

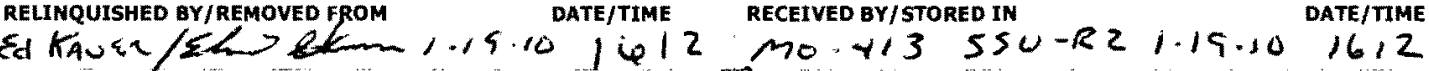

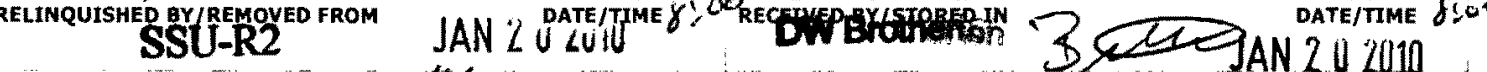

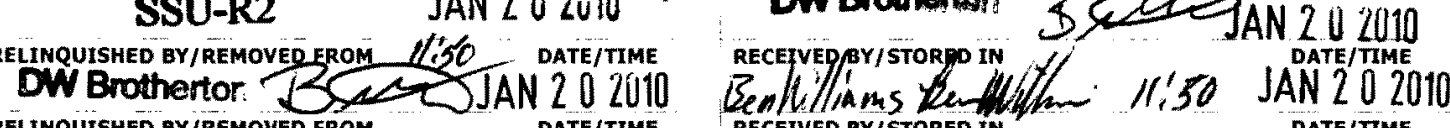

RELINQUISHED QY/REMOVED FROM

DATE/TIME

ReCEIVED BY/STORED IN

RELINQUISHED BY/REMOVED FROM

DATE/TIME

RECEIVED BY/STORED IN

DATE/TME

RELINQUISHED BY/REMOVED FROM

DATE/TIME

RECEIVED BY/STORED IY

DATE/TIME

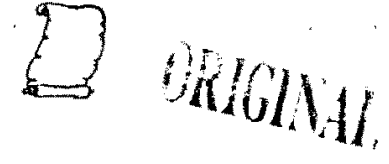

RELINQUISHED BY/REMOVED FROM

DATE/TIME

RECEIVED BY/STORED IN

DATE/TIME

\section{LABORATORY RECEIVED QY}

SECTION 


\section{CH2MHill Plateau Remediation Company} COLLECTOR

$$
\text { Kauer, Remo }
$$

SAMPLING LOCATION

C7515 (299-E28-30); I-04

ICE CHEST NO.

ocus 16

\section{SHIPPED TO}

Environmental Sciences Laboratory

\section{SPECIAL INSTRUCTIONS}

** The 200 Area S\&GRP Characterization and Monitoring Sampling and Analysis GKI applies to this SAF.
*ESLL shall submit all data deliverables according to SOW "200-LW-2 and 200-8P-5 Operable Units/Contaminant and Transport Property Analysis and Report for Vadose Zone Sediments at Borehole C5860/Well 299-E29-54" for ** ESL shall submit all data deliverables according to SOW "200-LW-2 and 200-BP-5
C7515/Well 299-E28-30 ("M" Well) and shall adhere to all SRGRP standard protocol.

** The ESL laboratory shall meet prior requested and confirmed turnaround times and RDLs specified in the SOW and this SAF. The laboratories must notify the SM Project Coordinator in writing iffwhen this requirement can not be met. (1)6010M_ICP_ASTM_AE (TAL) \{Sadium, Aluminum, Iron, Potassium, Antimony, Nickel, Calcium, Magnesium, Silver\} 6010M_ICP_ASTM_AE (Add-On) \{Lead, Thallium, Selenium\} 6010M_METALS.ICP_WE (TAL) \{Sodium, Aluminum, Iron, Potass um, Antimon, 99\} RADISO_ICPMS WE \{Iodine-129, Uranium-238, Technetium-99\} I-129 by ICPMS \{lodine-129\} GAMMA_GS; URANIUM ISOrOPIC RATOS; ALPHA_BETA_AE \{Gross beta, Gross alpha\} ALPHA_BETA_WE \{Gross beta, Gross alpha\} IC

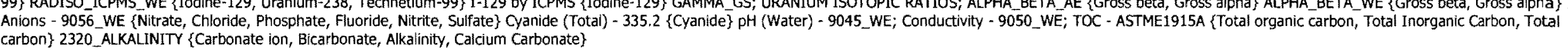

CHAIN OF CUSTODY/SAMPLE ANALYSIS REQUEST

\section{F10-04S-065}

AIR QUALITY

METHOD OF SHIPMENT

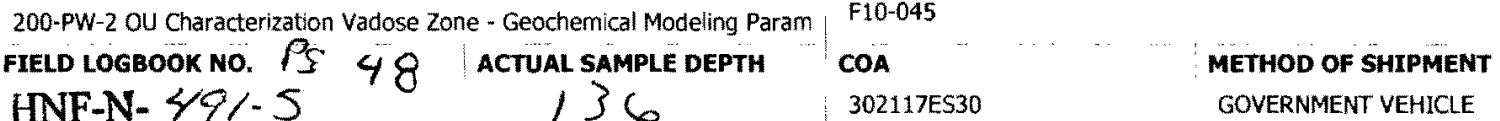

HNF-N- $49 /-5$

BILL OF LADING/AIR BIL NO.
TURNAROUND

45 Days $/ 45$
Days

$$
\sum_{1}^{4} \operatorname{lol} 11
$$




$$
\text { Kau\&r, Reimo }
$$

SAMPLING LOCATION

C7515 (299-E28-30); I-044

ICE CHEST NO.

SHIPPED TO

Environmental Sciences Laboratory

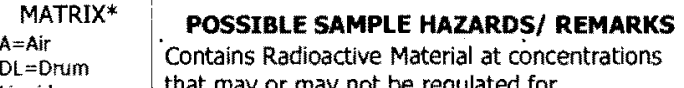

that may or may not be regulated for

Goods Regulations but are not releasable per

DOE Order $5400.5(1990 / 1993)$

PEECIAL HANDLING AND/OR STORAGE SPECIAL HANDLING AND/OR
RADIOACTIVE TIE TO: B23032

$$
B 23665,1 / 2 / 10
$$

SAMPLE NO.

B235K3 $\underset{\text { SHIPPED TO }}{601}-6$

COMPANY CONTACT

$$
\text { TELEPHONE NO. }
$$

373-2530

DYEKMAN, DL

SAF NO.
F10-045
COA

PROJECT DESIGNATION

200-PW-2 OU Characterization Vadose Zone - Geochemical Modeling Param: F10-045

FIELD LOGBOOK NO. IS 48 ACTUAL SAMPLE DEPTH COA

$\begin{array}{ll}\text { HNF-N. } 49 / .5 & 137.5\end{array}$

\begin{tabular}{|c|c|c|}
\hline PRESERVATION & Cool $4 \mathrm{C}$ & None \\
\hline TYPE OF CONTAIMER & G/P & $\begin{array}{l}\text { Moisture } \\
\text { Resistant Cont }\end{array}$ \\
\hline NO. OF CONTAIMER(S) & 1 & i \\
\hline Volume & it & 2009 \\
\hline IMPLE & $\begin{array}{l}\text { SEE TES } \\
\text { SPECTAL }\end{array}$ & $\begin{array}{l}\text { Moisture } \\
\text { Content-D2216; }\end{array}$ \\
\hline
\end{tabular}

\begin{tabular}{l|l} 
COA & METHOD OF SHIPMENT \\
302117 ES30 & GOVERNMENT VEHICLE
\end{tabular}

BILL OF LADING/AIR BILL NO.

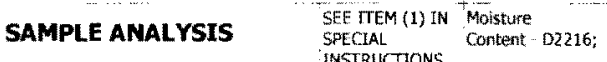

SAMPLE DATE SAMPLE TIME

$1-19.101245$

\section{SIGN/ PRINT NAMES}

RHAIN OF POSSESSION

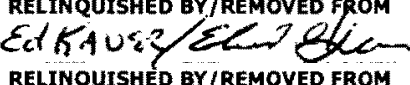
RELINQUISHED BY/REM

JAN 20 ULIO

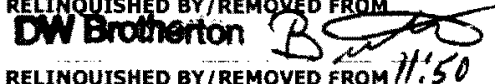

JAN $20 \mathrm{CU}$

RELINQUTSHED BY/REMOVED FROM

DATE/TIME.

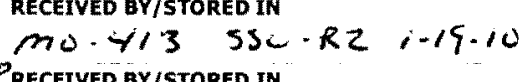

RECEIVED BY/STORED IN
DEW Brothar.

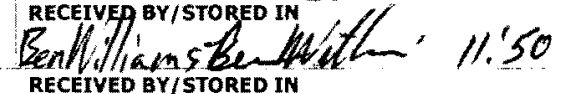

DATE/TTME

RECEIVED BY/STORED IN

DATE/TMME

RELINQUISHED BY/REMOVED FROM

DATE/TIME

RECEIVED BY/STORED IN

DATE/TME

DATE/TIME

RECEIVED BY/STORED IN 
200-PW-2 OU Charadterization Vadose Zone - Geochemical Modeling Param $\begin{aligned} & \text { SAF NO. } \\ & \text { F10-045 }\end{aligned}$

FIELD LOGBOOK NO. PS 48 ACTUAL SAMPLE DEPTH

COA

302117 ES30

\section{SPECIAL INSTRUCTIONS}

** The 200 Area S\&GRP Characterization and Monitoring Sampling and Analysis GKI applles to this SAF.

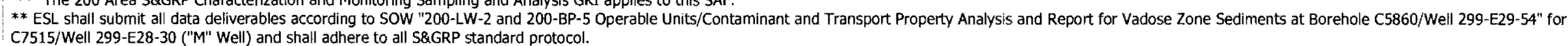

** The ESL laboratory shall meet prior requested and confirmed turnaround times and RDLs specified in the SOW and this SAF. The laboratories must notify the SM Project coordinator in writing if/when this requirement can not be met.

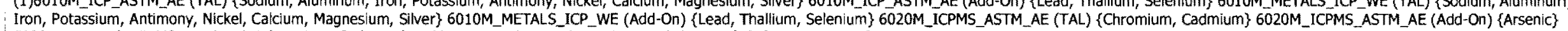
6020M_METALS_ICPMS_WE (TAL) \{Chromium, Cadmium\} 6020M_METALS_ICPMS_WE (Add-On) \{Arsenic\} 6020M_HG_ICPMS_AE; 6020M_HG_ICPMS_WE; Chromium Hex - 7196; RADISO_ICPMS_ASTM_AE \{Uranium-238, Technetium99) RADISO_ICPMS WE \{lodine-129, Uranium-238, Technetum-99\} I-129 by ICPMS \{lodine-129\} GAMMA_GS; URANIUM ISOTOPICRATOS; ALPHA_BETAAAE \{Gross beta, Gross alpha\} ALPHA_BETA_WE \{Gross beta, Gross alpha\} IC

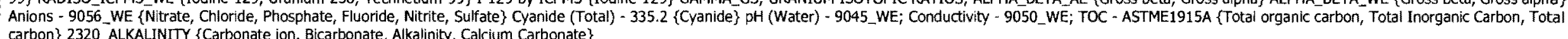

$\xi 3 \ldots+1$ 
PROJECT DESIGNATION

SAF NO.

AIR QUALITY

200-PW-2 OU Characterization Vadose Zone - Geochemical Modeling Param F10-045

C7515 (299-E28-30); I-045

200-PW-2 OU Characterization Vadose Zone - Geochemical Modeling Para
HIFPATGBOoK No. 48 ACTUAL SAMPLE DEPTH

COA METHOD OF SHIPMENT

$6 \cos 5-16$

SHIPPED TO

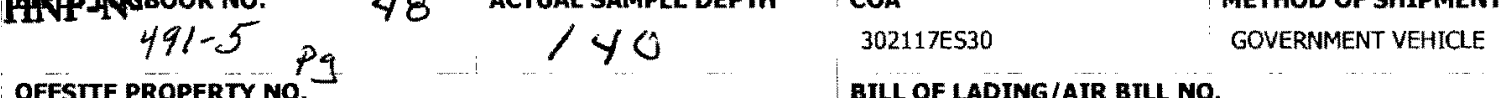

OFFSTTE PROPERTY NO.

BILL OF LADING/AIR BILL NO.

N/A

MATRIX* POSSIBLE SAMPLE HAZARDS/ REMARKS

$A=A i r$
DL=Drum

\begin{tabular}{l|l}
$\begin{array}{l}\text { Liquids } \\
\text { DS=Drum }\end{array}$ & that may or may not be regulated for \\
& transportation per 49 CFR / IATA Dangerous
\end{tabular}

transportation per 49 CFR / IATA Dangerous
Goods Regulations but are not releasable per

PRESERVATION

TYPE OF CONTAINER

NO. OF CONTAINER(S)

volume

SPECIAL HANDLING AND/OR STORAGE RADIOACTIVE TIE TO: B23633

SAMPLE AMALYSTS

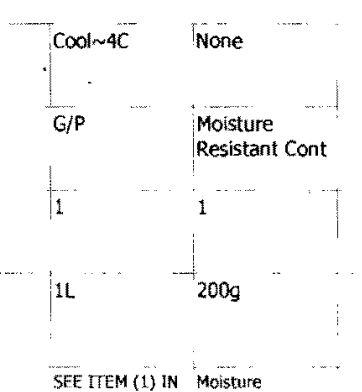

SAMPle date SAMPLE TIME

$1-19-10,3 / 0$

SEE TRMM (1) IN MOisture
SPECLAL
INSTRUCTIONS COntent-D2216

SAMPLE No. - MATRIX*

B235K4

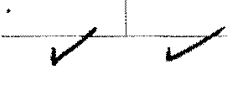

CHAIN OF POSSESSION

SIGN/ PRINT NAMES

RELINQUISHED BYIREMOVED ERQM

RECEIVED BY/STORED IN

SPECIAL INSTRUCTIONS

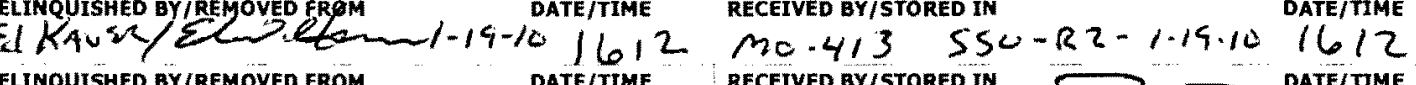

RELINQUISHED BY/REMOVED FROM
SSU-R2

RELINQUISHED BY/REMOÜED FROM

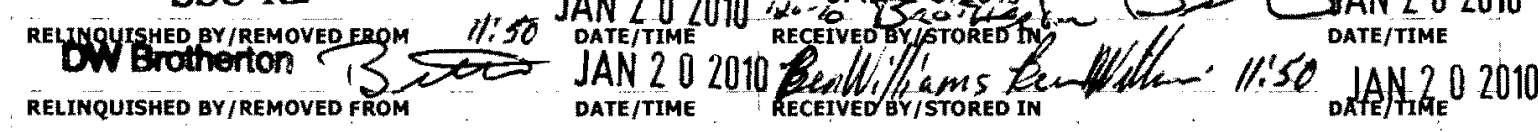

RELINQUISHED BY/REMOVED FROM

DATE/TIME

RECEIVED BY/STORED IN

DATE/TTME

DS ORIGINAI

RELINQUISHED BY/REMOVED FROM

DATE/TIME RECEIVED BY/STORED IN

DATE/TIME

RELINQUISHED BY/REMOVED FROM

DATE/TIME RECEIVED QY/STORED IN

DATE/TIME 


\section{Kauen Romo}

SAMPLING LOCATION

C7515 (299-E28-30); I-045

ICE CHEST NO.

$6055-16$

GIPPED TO

Environmental Sciences Laboratory

\section{SPECIAL INSTRUCTIONS}

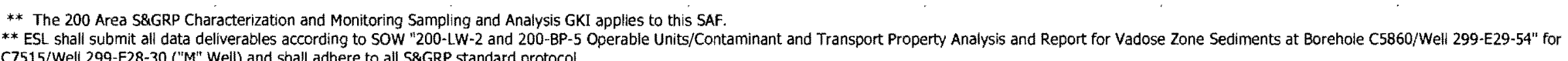
C7515/Well 299-E28-30 ("M" Well) and shall adhere to all 58GRP standard protocol.

** The ESL laboratory shall meet pror requested and conffrmed turnaround times and RDLs specified in the SOW and this SAF. The laboratories must notify the SM Project Coordinator in writing iffwhen this requirement can not be me (1)6010M_ICP_ASTM_AE (TAL) \{Sodium, Aluminum, Iron, Potassium, Antimony, Nickel, Calcium, Magnesium, Silver\} 6010M_ICP_ASTM_AE (Add-On) \{Lead, Thallium, Selenium\} 6010M_METALS_ICP_WE (TAL) \{Sodium, Aluminum, 6020M_METALS_ICPMS_WE (TAL) \{Chromium, Cadmium\} 6020M_METALS_ICPMS_WE (Add-ON) \{Arsenic\}6020M_HG_ICPMS_AE; 6020M_HG_ICPMS_WE; Chromium Hex-7196; RADISO_ICPMS_ASTM_AE \{Uranium-238, Technetium99\} RADISO_ICPMS_WE \{Iodine-129, Uranium-238, Technetium-99\} I-129 by ICPMS \{lodine-129\} GAMMA_GS; URANiUM ISOTOPIC RATOS; ALPHA_BETA AE \{Gross beta, Gross aloha\} ALPHA_BETA_WE \{Gross beta, Gross alpha\} IC
Anions - 9056_WE \{Nitrate, chloride, Phosphate, Fluoride, Nitrite, Sulfate\} Cyanide (Total) - 335.2 \{Cyanide\} pH (Water) - 9045_WE; Conductivity - 9050_WE; TOC - ASTME1915A \{Total organic carbon, Total Inorganic Carbon, Total carbon\} 2320 ALKALINTY (Carbonate ion, Bicarbonate, Alkalinity, Calcium Carbonate)

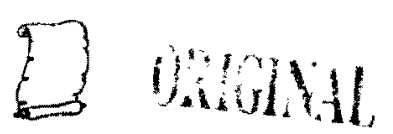


CH2MHill Plateau Remediation Company

COLLECTOR

Káner, Remo

SAMPLING LOCATION

C7515 (299-E28-30); 1-046

ICE CHEST NO.

GimpeD To -16

Environmental Sciences Laboratory

MATRIX

$A=A i r$
$D L=D r u m$

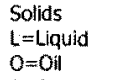

POSSBLE SAMPLE HAZARDS/ REMARKS

Contals Radioactive Material at concentrations

transportation per 49 CFR / IATA Dangerous

Goods Regulations but are not releasable per

DOE Order 5400.5 (1990/1993)

SPECIAL HANDLING AND/OR STORAGE RADIOACTIVE TTE TO: B23633

\section{CHAIN OF CUSTODY/SAMPLE ANALYSIS REQUEST}

F10-045-069

PAGE 1 OF 2

COMPANY CONTACT TELEPHONE NO. PROJECT COORDINATOR

DYTMAMV DL

ROJECT COORDINATOR PRICE CODE

DYEKMAN, DL

SAF NO.
F10-045

FIELD-2 OU Characterization Vadose Zone - Geoc SAMPLE DEPTH COA

HNF-N- $491-5$

OFFSTTE PROPERTY NO.

COA 302117 ES30

aIr Quality

TURNARA

45 Days / 45
Days

METHOD OF SHIPMENT

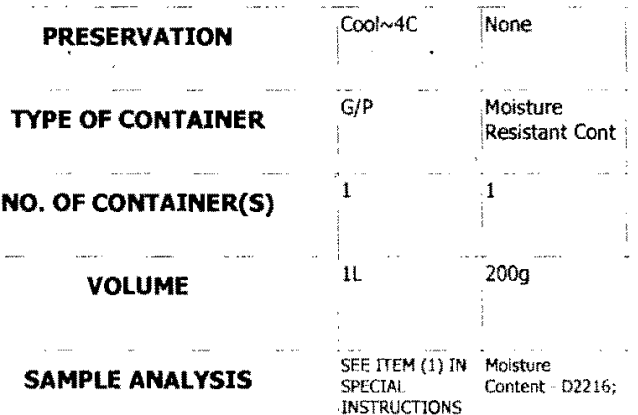

MOVERNME
- SAMPLE No

SOIL

MATRIX*

SAMPLE DATE SAMPLE TIME

$1-15-10 / 420$

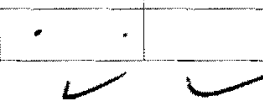

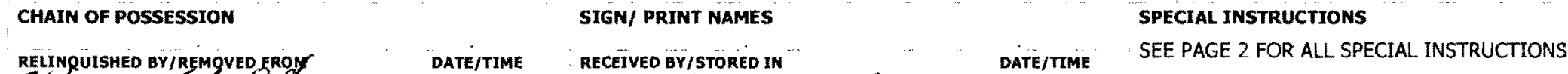

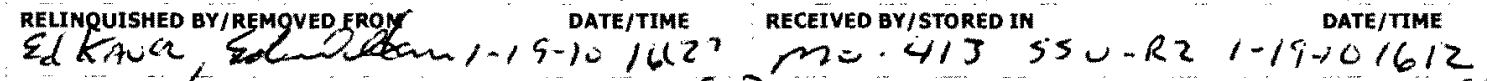

$$
\text { SSU-R2 }
$$

JAN 202010 DW Brotherto: $B S 4$ JAN 202010

\section{RELINQUISHED BY/REM O}

JAN 2020

RECEIVEDPYisToREg IN

DW Brotherton S

RELINQUUISHED BY/REMOVED FROM

DATE/TTME

Received BYs ISTORED IN

JAN? 2 0 ThM 2010

RELINQUISHED BY/REMOVED FROM

DATE/TIME

RECEIVED BY/STORED IN

DATE/TIME

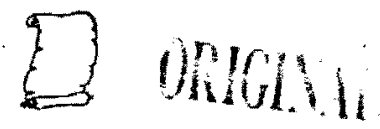

RELINQUISHED BY/REMOVED FROM

DATE/TIME

RECEIVED BY/STOREO IN

DATE/TIME

DATE/TIME 
COLLECTOR

$$
\text { KAUCR Remo }
$$

SAMPLING LOCATION

$\mathrm{C} 7515$ (299-E28-30); I-046

ICE CHEST NO.

$$
\text { ECos }-16
$$

SHIPPED TO

Environmental Sciences Laboratory

\section{SPECIAL INSTRUCTIONS}

** The 200 Area S\&GRP Characterization and Monitoring Sampling and Analysis GKI applies to this SAF.

** ESL shall submit all data deliverables according to SOW "200-LW-2 and 200-BP-5

** The ESL laboratory shall meet prior requested and confirmed turnaround times and RDLL specified in the SOW and this SAF. The laboratories must notify the SM Project Coordinator in writing iffiwhen this requirement can not be met. (1)6010M_ICP_ASTM_AE (TAL) \{Sodium, Alurninum, Iron, Potassium, Antimony, Nickel, Calcium, Magnesium, Silver\} 6010M_ICP_ASTM_AE (Add-On) \{Lead, Thalliurn, Selenium\} 6010M_METALS_ICP.WE (TAL) (Sodium, Aluminum,

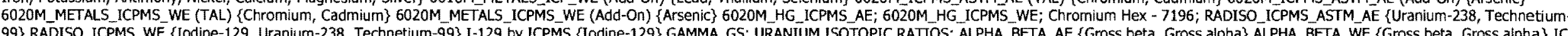
99\} RADISO_ICPMS WE \{Iodine-129, Uranium-238, Technetium-99\} I-129 by ICPMS \{lodine-129\} GAMMA_GS; URANIUM ISOTOPIC RATIOS; ALPHA_BETA_AE \{Gross beta, Gross alpha\} ALPHA_BETA_WE \{Gross beta, Gross alpha\} IC Anions - 9056_WE \{Nitrate, Chloride, Phosphate, Fluoride, Nitrite, Sulfate\} Cyanide (Total) - 335.2 \{Cyanide\} pH (Water) - 9045_WE; Conductivity - 9050_WE; TOC - ASTME1915A (Total organic Carbon, Total Inorganic Carbon, Total
carbon\} 2320_ALKALINITY \{Carbonate ion, Bicarbonate, Alkalinity, Calcium Carbonate\} 
СH2MHill Plateau Remediation Company

COLLECTOR

Kaush, Romo

C7515 (299-E28-30); $1-047$

ICE CHEST NO.

$6 \omega s-16$

SHIPPED TO

Environmental sciences Laboratory

MATRIX* POSSIBLE SAMPLE HAZARDS/ REMARKS

$A=A i r$
$D L=D r u m$$\quad$ Contains Radioactive Material at concentrations

\begin{tabular}{l|l} 
Liquids & that may or may not be regulated for \\
DS $=$ Drum & transportation per 49 CFR I IATA Dangerous \\
Solids & Goods Regulations but are not releasable per
\end{tabular}

DOE Order 5400.5 (1990/1993)

SPECIAL HANDLING AND/OR STORAGE SPECIAL HANDLING AND/OR
RADIOACTIVE TIE TO: B23633
F10-045-071

PAGE 1 OF 1

conouranta

DYEKMAN DI

TELEPHONE NO.

PROJECT COORDIMATOR

DYEKMAN, DL

SAF NO.

$\begin{array}{lll}\text { PRICE CODE } & \text { 8N } & \text { DATA } \\ \text { TURNAROUND }\end{array}$

AIR QUALITY $\square \quad 45$ Days/ 45

200-PW-2 OU Characterization vadose Zone - Geochemical Modeling Param F10-045

HEIFE- 48 ACTUAL SAMPLE DEPTH COA

\section{PRESERVATION}

TYPE OF CONTAINER

NO. OF CONTAINER(S)

volume

BILL OF LADING/AIR BILL NO.

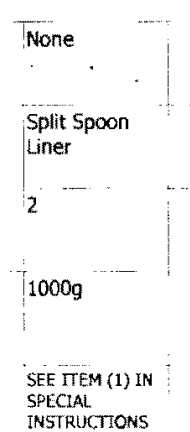

$\begin{array}{ll}\text { SAMPLE ANALYSIS } & \text { SEE TEM } \\ \text { SPCEA } 1 \text { IN } \\ \text { INSTRUCTIONS }\end{array}$

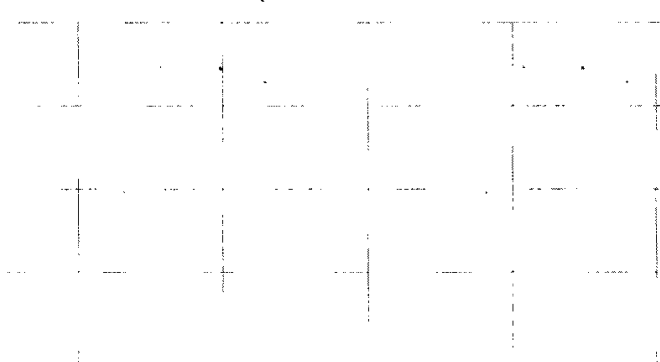

SAMPLE DATE SAMPle TIME -

$1-19.10 / 425$

SIGN/ PRINT NAMES

DATE/TIME RECEIVED BY/STORED IN DATE/TIME

no -413 JAN 202010

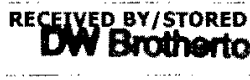

$55 \cup-R 2$ DATE/TME

** The 200 Area S\&GRP Characterization and Monitoring Sampling and Analysis

RELINQUISHED BY/REMOVED FROM RELTOUTH

SSU-R2

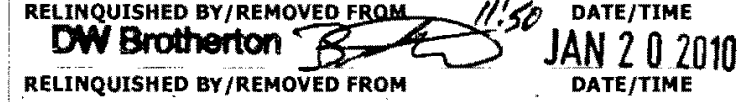

RELINQUISHED BY/REMOVED FROM

DATE/TIMEE in

列 JAN 202848 Zone Sediments at Borehole C5860/Well 299-E29-54" for C7515/Well 299-E28-30 DATE/TMME
("M" Well) and shall adhere to all S\&GRP standard protocol.

RELINQUISHED BY/REMOVED FROM

DATE/TIME

RECEIVED BY/STORED IN

DATE/TIME

RELINQUTSHED BY/REMOVED FROM

DATE/TIME

DATE/TIME

** The ESL laboratory shall meet prior, requested and confirmed tumaround times and ROLS specified in the SOW and this SAF. The laboratories must notify the SM Project Coordinator in writing if/when this requirement can not be met.

RECEIVED BY/STORED IN

DATE/TME

DATE/TIME

RELINQUTSHED BY/REMOVED FROM

LABORATORY
SECTION

FINAL SAMPLE DISPOSAL METHOD

DISPOSITION (Hydrometer) - D422; KD - Batch;

20 ORIGINAI,

TITLE

DISPOSED BY
DATE/TIME

DATE/TIME 


\section{CH2MHill Plateau Remediation Company}

COLECTOR

Kaver Rome

SAMPLING LOCATION

C7515 (299-E28-30); I-048

ICE CHEST NO.

$6 \cos -16$

HIPPED TO

Environmental Sciences Laboratory

A=Air
$\mathrm{DL}=$ Prum
Contains Radioactive Material at concentrations that may or may not be regulated for

Gods Regultions but are nat a Dangerous

DOE Order 5400.5 (1990/1993)

$L=L$ iquid
$0=0$ :
$S=5$ oil

SE=Sediment
T=Tissue
IW

$v=v e g e t a t i o n$
$W==W a t e r$
$x=0$ ter

WI=Wipe
$x=$ other

SPECIAL HANDLING AND/OR STORAGE RADIOACTIV TTE TO: $B 23633$

SAMPLENO

MATRIX*

SOIL
CHAIN OF CUSTODY/SAMPLE ANALYSIS REQUEST

F10-045-072

PAGE 1 OF 2

COMPANY CONTACT

TELEPHONE NO.

PROJECT COORDINATOR

DYEKMAN, DL

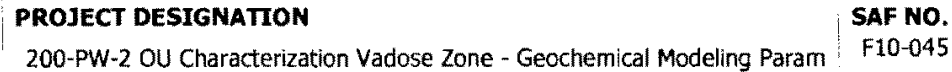

FIELD LOGBOOK No. 48 ACTUAL SAMPLE DEPTH COA

METHOD OF SHIPMENT

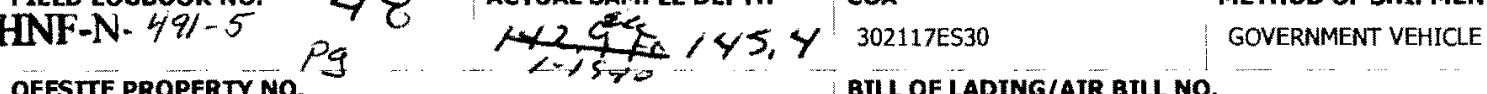

BILL OF LADING/AIR BILL NO.

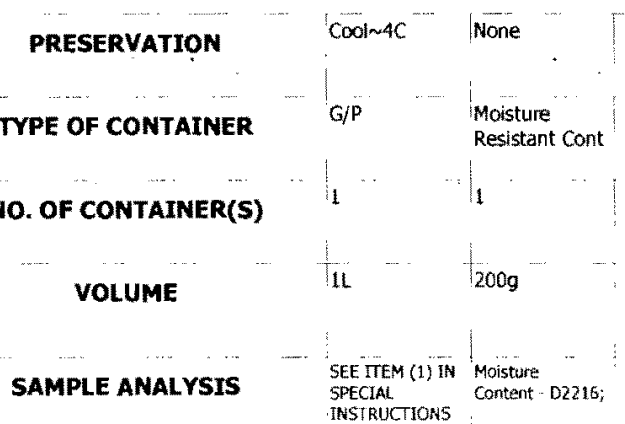

SAMPLE DATE "SAMPLE TIME

$1-19.10,1425$
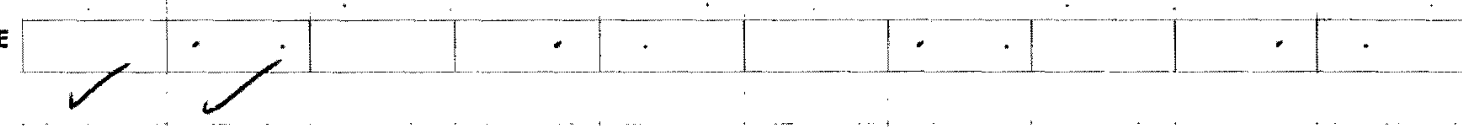

CHAIN OF POSSESSION

SIGN/ PRINT NAMES

SPECIAL INSTRUCTIONS

RELINQUUISHED BY $/$ REMOVED FROM

DATE/TIME RECEIVED BY/STORED IM

SEE PAGE 2 FOR ALL SPECIAL INSTRUCTIONS

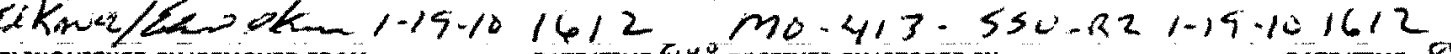

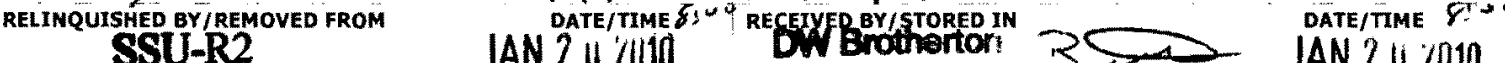

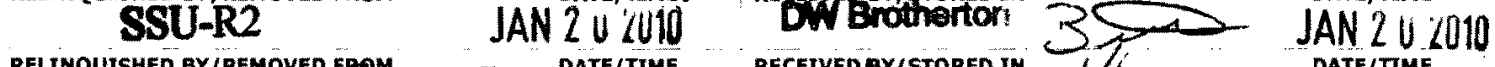

DW Brotherton EROM DATE/TIME

OW Brotherton

RELINQUISHED BY/REMOVED FROM

DATE/TIME

RECEIVED BY/STORED IN

D ORIGINAL

RELINQUUSSHED BY/REMOVED FROM

DATE/TIME

RECEIVED BY/STORED IM

DATE/TIME

RELINQUISHED BY/REMOVED FROM

Date/TIMe

RECEIVED BY/STORED IM

Date/time

SECTION 


\section{CH2MHill Plateau Remediation Company}

\section{COLLECTOR}

Kaver, Romo

SAMPLING LOCATION

C7515 (299-E28-30); $1-04$

ICE CHEST NO.

$6 \omega 5-16$

HIPPED TO

Environmental Sciences Laboratory

\section{SPECIAL INSTRUCTIONS}

** The 200 Area 5\&GRP Characterization and Monitoring Sampling and Analysis GKI applies to this SAF.

** The 20 Area SRGRP Characterization and Monitoring Sampling and Analysis GKI applies to this SAF,
** ESL shall submit all data deliverables according to SOW "200-LW-2 and 200-BP-5 Operabie Units/Contaminant and Transport Property Analysis and Report for Vadose Zone Sediments at Borehole C5860/Well 299 -E29-54" for *ESL shall submit all data deliverables according to SOW "200-LW-2 and 200-BP-5
C7515/Well 299-E28-30 ("M" Well) and shall adhere to all S\&GRP standard protocol.

** The ESL. laboratory shall meet prior requested and confirmed turnaround times and RDLs specified in the SOW and this SAF. The laboratories must notify the SM Project Coordinator in writing Iffiwhen this requirement can not be met. (1)6010M_ICP_ASTM_AE (TAL) \{Sodium, Aluminum, Iron, Potassium, Antimony, Nickel, Calcium, Magnesium, Silver\} 6010M_ICP_ASTM_AE (Add-On) \{Lead, Thallium, Selenium\} 6010M_METALS_ICP_WE (TAL) \{Sodium, Aluminum,

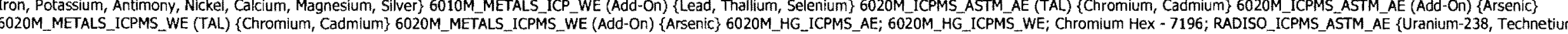
9) RADISO_ICPMS_WE \{lodine-129, Uranium-238, Technetium-99\} I-129 by ICPMS \{lodine-129\} GAMMA_GS; URANIUM ISOTOPIC RATIOS; ALPHA_BETA_AE \{Gross beta, Gross alpha\} ALPHA_BETA_WE \{Gross beta, Gross aipha\} IC

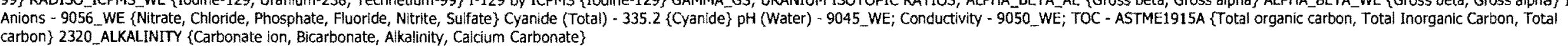

D whithi
TELEPHONE NO.

PRICE CODE

AIR QUALITY

SAF No.

METHOD OF SHIPMEN

200-PW-2 OU Characterization Vadose Zone - Geochemical Modeling Param

FIELD LOGBOOK NO.
HNF-N $-491-5$

OFFSTTE PROPERTY NO

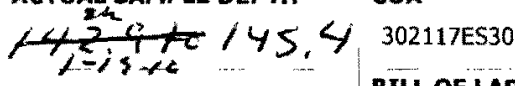

\section{BiLL OF LADING/AIR BILL NO.}

GOVERNMENT VEHICLE
PAGE 2 OF 2 DATA
TURMAROUND

$\underset{\text { Days }}{45 \text { Oays } / 45}$
N/A 
CHzMHill Plateau Remediation Company

COLLECTOR

Kaus pomo

C7515 (299-E28-30): I-049

ICE CHEST NO.

$$
\text { GWS - } 168
$$

SHIPPED TO

Environmental Sclences Laboratory

POSSIBLE SAMPLE HAZARDS/ REMARKS Contains Radioactive Material at concentration

that may or may not be regulated for

Goods Regulations but are not releasable per

DOE Order $5400.5(1990 / 1993)$

SPECIAL HANDLING AND/OR STORAGE SPECIAL HANDLING AND/OR
RADIOACTIVE TIE TO: B23634

SAMPLE No.

MATRIX*

B23593

SOIL
F10-045-074

PAge 1 of 1

CHAIN OF CUSTODY/SAMPLE ANALYSIS REQUEST

DATA
TURNAROUND

AIR QUALITY $\square \quad \underset{\text { Days }}{45 \text { Days / } 45}$

SAF NO.

200-PW-2 OU Characterization Vadose Zone - Geochemical Modeling Param F10-045

FIELD LOGBOOK NO. 48 ACTUAL SAMPLE DEPTH COA METHOD OF SHIPMENT

HNF-N. $491-5$

48 ACTUAL SAMPLE DEPTH

\begin{tabular}{|c|c|}
\hline PRESERVATION & None \\
\hline TYPE OF CONTAINER & $\begin{array}{l}\text { Split Spocon } \\
\text { Liner }\end{array}$ \\
\hline NO. OF CONTAINER(S) & $j^{2}-a+2-a$ \\
\hline yolume & 10009 \\
\hline SAMPLE ANALYSIS & $\begin{array}{l}\text { SEE TEM (1) II } \\
\text { SPECKRL } \\
\text { IISTRCTIONS }\end{array}$ \\
\hline
\end{tabular}

$302117 E 530$

BILL OF LADING/AIR BILL NO.

N/A

GOVERNMENT VEHICLE

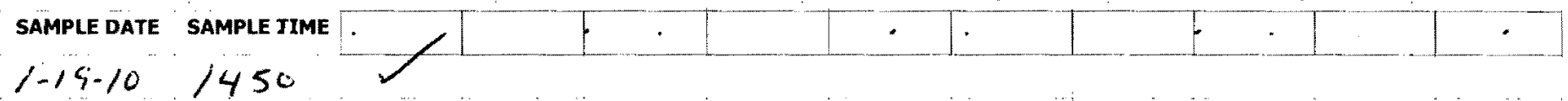

SIGN/ PRINT NAMES

DATE/TIME RECEIVED GY/STORED IN

BAN"

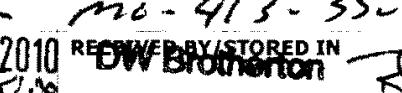

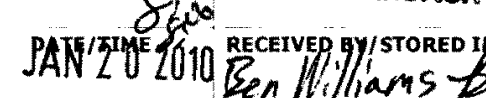

SSU-REY 2 VED FROM

DATE/TIME

DATE/TIME

DATE/TIME

RECEIVED BY/STORED IN

DATE/TIME

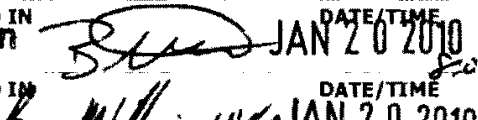

SPECIAL INSTRUCTIONS

** The 200 Area S\&GRP Characterization and Monitoring Sampling and Analysis Operable Units/Contaminant and Transport Property Analysis and Report for Vadose
Zone Sediments at Borehole C5860/Well 299-E29-54" for C7515/Well 299-E28-30 ("M" Well) and shall adhere to all S\&GRP standard protocol.
RELINQUISHED BY/REMOVED FROM

RELINQUISHED BY/REMOVED FROM

RELINQUISHED BY/REMOVED FROM

LABORATORY

FINAL SAMPLE
DISPOSTIION
RECEIVED BY JANTE/TME 202010

** The ESL laboratory shall meet prior requested and confirmed turnaround times and RDLS specified in the SOW and this SAF. The laboratories must notify the SM Project Coordinator in writing if/when this requirement can not be met. (1)Density; CATIONEXCH._R; Particle Size (Dry Sleve) - D422; Particle Size

\section{DATE/TME}

( Hydrometer)-D422;- Batch;

DATE/TME

DATE/TIME

DISPOSED BY 
CH2MHill Plateau Remediation Company

COLLECTOR

\section{Kaus, Romo}

C7515 (299-E28-30); I-050

ICE CHEST NO.

GwS-168

SHIPPED TO

Environmental Sciences Laboratory

MATRIX* POSSTIBLE SAMPLE HAZARDS/ REMARKS

$A=A$ Air
$\mathrm{DL}=$ Drum

transportation per 49 . regulated for

Goods Repulations but are not releasable per

DOE Order $5400.5(1990 / 1993){ }^{\circ}$

SPECIAL HANDLING AND/OR STORAGE RADIOACTIVE TIE TO: 823634

SAMPLENO.
B235K7

CHAIN OF POSSESSION

SIGN/ PRINT NAMES

RELINQUISHED BY/REMOYED FROM

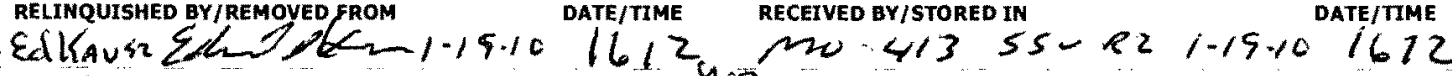

SPECIAL INSTRUCTIONS

RELINQUISHED BY/REMOVED FROM JAN 2020 TO RECEIVED BY/STORED IN

$$
\text { SSU-R2 }
$$

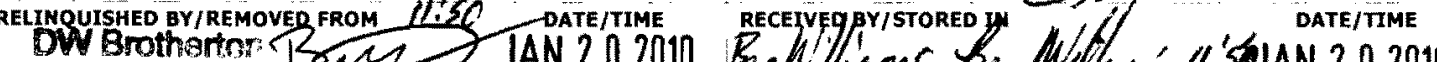

ReINQQISHEO BY/REMOVED From

RELINQUISHED BY/REMOVED FROM

DATE/TIME

RECEIVED BY/STORED IN

DATE/TIME

RELINQUUISHED BY/REMONED FROM

DATE/TIME

RECEIVED BY/STORED IN

DATE/TIME

RELINQUISHED BY/REMOVED FROM

DATE/TIME

RECEIVED BY/STORED IN

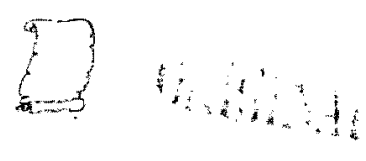

\section{LABORATORY
SECTION}

FINAL SAMPLE DISPOSAL METHOD

FINAL SAMPLE
DISPOSITION
F10-045-075

PAGE 1 OF 2

PRICE CODE

DATA
TRNAROUND

45 Days / 45
Days

METHOD OF SHIPMENT

F10-045

302117 GS30 GOVERNMENT VEHICLE

ILL OF LADIMG/AIR BILL NO.

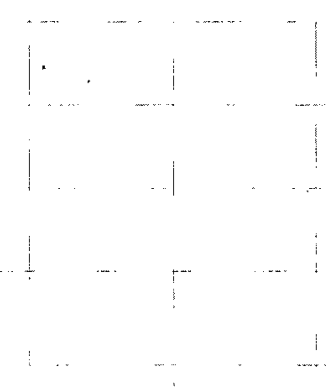


CH2MHill Plateau Remediation Company

\section{COLLECTOR}

$$
\text { Kavis Romo }
$$

SAMPLING LOCATION

C7515 (299-E28-30); I-050

ICE CHEST NO.

$6 \omega s-168$

Environmental Sciences Laboratory

SPECIAL INSTRUCTIONS

** The 200 Area S\&GRP Characterization and Monitoring Sampling and Analysis GKI applies to this SAF.
*E ESL shall submit all data deliverables according to SOW "200-LW-2 and 200-BP-5 Operable Units/Contaminant and Transport Property Analysis and Report for Vadose Zone Sediments at Borehole C5860/Well 299-E29-54" for
C2515/Well C75 ESL shall submit all data deliverables according to SOW "200-LW-2 and 200-BP-5 28 - 28 -30 (" $M$ " Well) and shall adhere to all S\&GRP standard protocol.
C5el

** The ESL laboratory shall meet prior requested and confirmed turnaround times and RDLs specified in the SOW and this SAF. The laboratories must notify the SM Project Coordinator in writing if/when this requirement can not be met. (1)6010M_ICP_ASTM_AE (TAL) \{Sodium, Aluminum, Iron, Potassslum, Antimony, Nickel, Cakcium, Magnesium, Silver\} 6010M_ICP_ASTM_AE (Add-On) \{Lead, Thallium, Selenium\} 6010M_METALS_ICP_WE (TAL) \{Sodium, Aluminum, 6020M METALS ICPMS WE (TAL) \{Chromium, Cadmium\} 6020M METALS ICPMS WE (Add-On) \{Arsenic\} 6020M, HG ICPMS AE; 6020M HG ICPMS WE; Chromium Hex - 7196; RADISO ICPMS ASTM AE \{Uranium-238, Technetium99\} RADISO_ICPMS_WE \{Iodine-129, Uranium-238, Technetium-99\} I-129 by ICPMS \{Iodine-129\} GAMMA_GS; URANIUM ISOTOPIC RATIOS; ALPHA_BETA_AE \{Gross beta, Gross alpha\} ALPHA_BETA_WE \{Gross beta, Gross alpha\} IC Anions - 9056_WE \{Nitrate, Chloride, Phosphate, Fluoride, Nitrite, Sulfate\} Cyanide (Total) - 335.2 \{Cyanide\} pH (Water) - 9045_WE; Conductivity - 9050_WE; TOC - ASTME1915A \{Total organic Carbon, Total Inorganic Carbon, Total carbon\} 2320 ALKALINTTY \{Carbonate ion, Bicarbonate, Alkallinity, Calcium Carbonate\}

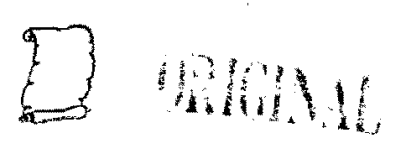


CH2MHill Plateau Remediation Company

COLLECTOR

SAMPLING LOCATION

$$
\text { Katar, Romp }
$$

C7515 (299-E28-30); I-051

ICE CHEST NO.

$\underset{\text { Shipped to }}{6 \cos }-168$

Environmental Sclences Laboratory

MATRIX* POSSIBLE SAMPLE HAZARDS/ REMARKS

$A=A i r$
$\mathrm{DL}=$ Drum

Lquids that may or may not be regulated for

Goods Reguations but are not reangerous

DOE Order 5400.5 (1990/1993)

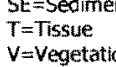

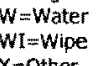

SPECIAL HANDLING AND/OR STORAGE RADIDACTIVE TIE TO: B23634

\section{CHAIN OF CUSTODY/SAMPLE ANALYSIS REQUEST}

F10-045-077

PAGE 1 OF 1

COMPANY CONTACT

TELEPHONE NO.

PROJECT COORDINATOR

DYEKMAN, DL.

SAF NO.
F10-045

PROJECT DESIGNATION

$373-2530$

PRICE CODE

AIR QUALITY

DATA
TURNAROUND
TS DaYs/ 45

45 Days / 45

METHOD OF SHIPMENT

COA 302117 ES30

FIELD LOGBOOK NO.

HNF-N- $491-5$

OFFSTE PROPERTY NO. P马

48 Actual SAMPLE DEPTH

BILL OF LADING/AIR BILL NO.

N/A

None

TYPE OF CONTAINER Split Spoon

NO. OF CONTAINER(S)

volume

2

10009

SAMPLE ANALYSIS SEE TEM (1) IN

SAMPLE DATE SAMPLE TIME

$1-19.101515$

SIGN/ PRINT NAMES

DATE/TIME RECEIVED BY/STORED IN 1612 Mo. 413 AAH 202010

RELINQUTSHED BY/REMOVED FROM RELINQUISHED BY/REMOVED FROM SSU-R2

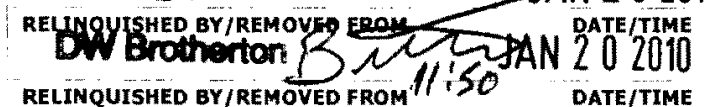

RELINQUISHED BY/REMOVED FROM

DATE/TIME

DW Brothertori $50-R 2,15-10,16 / 2$

** The 200 Area S\&GRP Characterization and Monitoring Sampling and Analysis

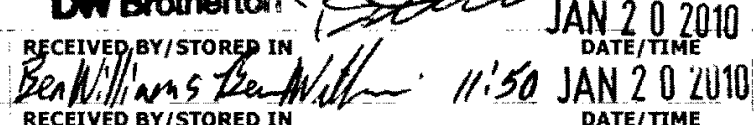

PECIVED QYISTORED IM

DATE/TMME

DATE/TIME

DATE/TIME

DATE/TMME

(200-BP-5 Zone Sediments at Borehole C5860/Well 299-E29-54" for C7515/Well 299-E28-30 ("M" Well) and shall adhere to all S\&GRP standard protocol.

** The ESL laboratory shall meet prior requested and confirmed turnaround times and RDLS specified in the SOW and this SAF. The laboratories must notify the SM Project Coordinator in writing if/when this requirement can not be met.

RELINQUUSHED BY/REMOVED FROM

DATE/TIME

RECEIVED BY/STORED IN (Hydrometer) - D422; KD - Batch;

RELINQUISHED BY/REMOVED FROM

DATE/TIME

RECEIVED BY/STORED IN

$$
2 \text { ORIGIIII }
$$

TTIE
DATE/TIME

DATE/TIME 
CH2MHill Plateau Remediation Company

COLLECTOR

K.4us, Ro.mo

C7515 (299-E28-30); 1-052

ICE CHEST NO.

$6 \omega 5-168$

SHIPPED TO

Environmental Sciences Laboratory

MATRIX* POSSIBLE SAMPLE HAZARDS/ REMARKS

$\begin{aligned} & A=A i r \\ & D L\end{aligned}=$ Drum Contains Radioactive Material at concentrations that may or may not be regulated for

DOE Order $5400.5(1990 / 1993)$

SPECIAL HANDLING AND/OR STORAGE RADIOACTIVE TIE TO: B23634

SAMPLE NO.
B235K8
SAMPLING LOCATION

Goods Regulstions but are nat releangerous

F10-045-078

PAGE 1 OF 2

\begin{tabular}{|c|c|c|c|c|c|}
\hline $\begin{array}{l}\text { COMPANY CONTACT } \\
\text { DYEKMAN, DL }\end{array}$ & $\begin{array}{l}\text { TELEPHONE NO. } \\
373-2530\end{array}$ & $\begin{array}{l}\text { PROJECT COORDINATOR } \\
\text { DYEKMAN, DL }\end{array}$ & PRICE CODE & $\mathbf{8 N}$ & $\begin{array}{c}\text { DATA } \\
\text { TURNAROUND }\end{array}$ \\
\hline OJE & & $\begin{array}{l}\text { SAF NO. } \\
\text { F10-045 }\end{array}$ & AIR QUALITY & $\square$ & $\begin{array}{c}45 \text { Days / } 45 \\
\text { Days }\end{array}$ \\
\hline
\end{tabular}

200-PW-2 Ou characterization Vadose Zone - Geochemical Modeling Param F10-04

\begin{tabular}{lll|l|l|l} 
FIELD LOGBOOK NO. & 48 & ACTUAL SAMPLE DEPTH & COA & METHOD OF SHIPMENT
\end{tabular}

$\begin{array}{lll}\text { HNF-N- } 491-5 \mathrm{pg} & 302117 \text { ES30 } \\ \text { OFFSITE PROPERTY NO. } & \text { BILL OF LADING/AIR BILL NO. }\end{array}$

N/A

PRESERVATION CoOnAC None

TYPE OF CONTAINER G/P $\begin{aligned} & \text { Moisture } \\ & \text { Resistant Cont }\end{aligned}$

NO. OF CONTAINER(S)

volume

SAMPLE ANALYSIS $\quad$ SEE IIEM (1) IN Moisture

SAMPLE DATE SAMPLE TIME

$1.15 .10 \quad 1515$

SIGN/ PRINT NAMES

SPECIAL INSTRUCTIONS

CHAIN OF POSSESSION

DATE/TIME RECEIVED BY/STORED IN

SEE PAGE 2 FOR ALL SPECIAL INSTRUCTIONS

RELINQUISHED GY/REMOVED FROM

DATE/TIME

$\theta, p$

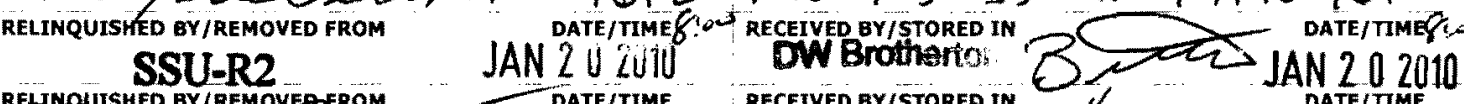

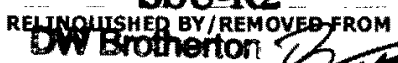

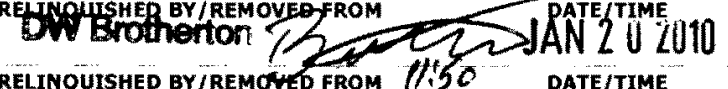

RELINQUISHED BY/REMOVED FROM

DATE/TIME

RECEIVEP BY /STOREP IN

DATE/TME

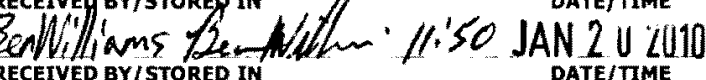

RECEIVED QY/STORED IN

DATE/TME

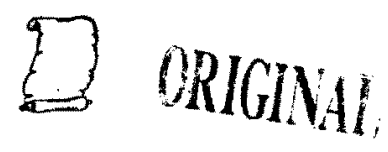

RELINQUISHED BY/REMOVED FROM

DATE/TTME

RECEIVED BY/STORED IN

DATE/TIME

DATE/TIME

RECEIVED BY/STORED IN

DATE/TIME 
C7515 (299-E28-30); I-052

200-PW-2 Ou Characterization Vadose Zone - Geochemical Modeling Param

ICE CHEST NO.

FIELD LOGBOOK NO. 48 ACTUAL SAMPLE DEPTH COA

METHOD OF SHIPMENT

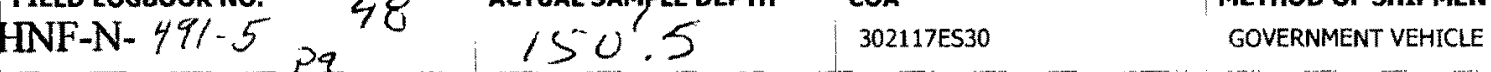

$6005-168$

OFFSITE PROPERTY No.

BILL OF LADING/AIR BILL NO.

N/A

N/A

\section{SPECIAL INSTRUCTIONS}

** The 200 Area S\&GRP Characterization and Monitoring Sampling and Analysis GKI applies to this SAF.

** ESL shall submit all data deliverables according to SOW "200-LW-2 and 200-BP-5 Operable Units/Contaminant and Transport Property Analysis and Report for Vadose Zone Sediments at Borehole C5860/Well 299-E29-54" for
C7515/Well 299-E28-30 ("M" Well) and shall adhere to all S8GRP standard protocol.

** The ESL laboratory shall meet prior requested and confirmed turnaround times and RDLs specifed in the SOW and this SAF. The laboratorles must notify the SM Project Coordinator in writing Iff/when this requirement can not be met. (1)6010M_ICP_ASTM_AE (TAL) \{Sodium, Aluminum, Iron, Potassium, Antimony, Nickel, Calcium, Magnesium, Silver\} 6010M_ICP_ASTM_AE (Add-On) \{Lead, Thallium, Seeenium\} 6010M_METALSIICP_WE (TAL) \{Sodium, Aluminum,

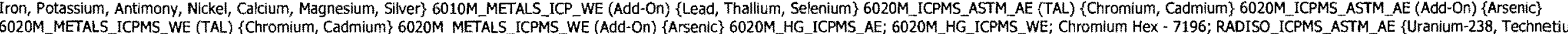

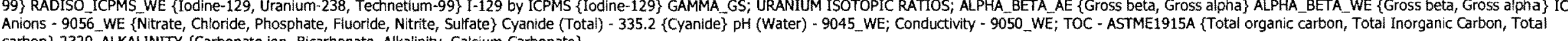
carbon\} 2320_ALKALINITY' \{Carbonate ion, Bicarbonate, Alkalinity, Calcium Carbonate\}

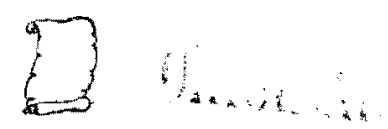


SAMPLING LOCATION

ICE CHEST NO.

$6 \omega S-168$

SHIPPED TO

Environmental Sciences Laboratory

MATRIX* POSSIBLE SAMPLE HAZARDS/ REMARKS

$A=A i r \quad$ Contains Radioactive Material at concentrations

that may or may not be regulated for

Goods Regulations but a / IATA Dangerous

DOE Order 5400.5 (1990/1993)

\section{COMPANY CONTACT}

TELEPHONE NO.
$373-2530$

PROJECT COORDINATOR

DYEKMAN, DL.

PROJECT DESIGNATION

SAF NO.
F10-045

200-PW-2 OU Characterization Vadose Zone - Geochemical Modeling Param F10-045

FIELD LOGBOOK NO.

48 ACTUAL SAMPLE DEPTH

HNF-N $491-5$
OFFSITE PROPERTY NO.

COA

N/A

\begin{tabular}{l|l}
\hline $3021175 S 30$ & METHOD OF SHIPMENT
\end{tabular}

PRESERVATION

BILL OF LADING/AIR BILL NO.

$\mathrm{N} / \mathrm{A}$

TYPE OF CONTAINER Split Spoon

NO. OF CONTAINER(S)

volume

SPECIAL HANDLING AND/OR STORAGE RADIOACTIVE TIE TO: 823635

SAMPLE ANALYSIS

$\begin{array}{lcc}\text { PRICE CODE } & \text { SN } & \begin{array}{c}\text { DATA } \\ \text { TURNAROUND }\end{array} \\ \text { AIR QUALITY } & \square & \text { 45 DaYs / 45 }\end{array}$

METHOD OF SHIPMENT

GOVERNMENT VEMICLE
SAMPLE NO
MATRIX*
SAMPLE DATE SAMPLE TIME
$1-19.10,1525$
B23595
SOIL

SIGN/ PRINT NAMES

RECEIVED BY/STORED IN RELINQUISHED BY/REMQVED FRgM 1. 15. DATE/TIME

\section{SSU-R2}

\section{RELINQUISHED BY/REMOVED FROM}

DW Brothertec Be

RELINQUISHED BY/REMOVED FROM

RELINQUISHED BY/REMOVED FROM

RELINQUISHED BY/REMOVED FROM

RECEIVED BY

SECTION

FINAL SAMPLE
DISPOSITION

DATE/TIME RECEIVED BY/STORED IN

DATE/TIME RECEIVED BY/STORED IN

DATE/TIME
I0 DATE/TIME YUO RECEIVED BY/STORED IN DATE/TIME

\section{SPECIAL INSTRUCTIONS}

** The 200 Area S\&GRP Characterization and Monitoring Sampling and Analysis GKI applies to this SAF.

Operable Units/Contaminant and Transport Property Analysis and Report 200-BP-5 Operable Units/Contaminant and Transport Property Analysis and Report for Vadose
Zone Sediments at Borehole C5860/Well 299-E29-54" for C7515/Well 299-E28-30

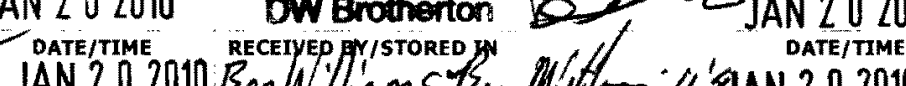
DATE/TIME

DATE/TIME ("M" Well) and shall adhere to all S\&GRP standard protocol.

** The ESL laboratory shall meet prior requested and confirmed turnaround times and RDLs specified in the SOW and this SAF. The laboratories must notify the SM Project Coordinator in writing if/when this requirement can not be met. (1)Density; CATIONEXCH_TR; Particle Size (Dry Sieve) - D422; Particle Size (Hydrometer) - D422; KD - Batch;

$$
\text { E MKUNIL }
$$


ICE CHEST NO.

$$
6005-168
$$

SHIPPED TO

Environmental Sciences Laborator

MATRIX* POSSIBLE SAMPLE HAZARDS/ REMARKS

$\mathrm{DL}=$ = Drum Contains Radioactive Material at concentrations

that may or may not be regulated for
transportation per 49 CFR / IATA Dangerous

Goods Regulations but are not releasable per

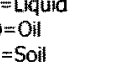

SPECIAL HANDLING AND/OR STORAGE RADTOACTIVE TIE TO: 823635

SAMPLE No.

B235K9

MATRIX*

$$
\text { SOIL }
$$

\section{COMPANY CONTACT}

DYEKMAN, DL.

TELEPHONE NO.
$373-2530$

$$
373-2530
$$

PROJECT COORDINATOR

200-PW-2 OU Characterization Vadose Zone - Geochemical Modeling Para

FIELD LOGBOOK NO.

48 actual SAMPLE DEPTH

$$
151.9
$$

SAF NO.

\begin{tabular}{|c|c|c|}
\hline PRESERVATION & $\operatorname{Coop} \sim 4 \mathrm{C}$ & None \\
\hline TYPE OF CONTAINER & $G / P$ & $\begin{array}{l}\text { Moisture } \\
\text { Resistant Cont }\end{array}$ \\
\hline NO. OF CONTAINER(S) & 1 & 1 \\
\hline Volume & it & 2009 \\
\hline SAMPLE ANALYSIS & $\begin{array}{l}\text { SEE ITEM } \\
\text { SPFCIAL }\end{array}$ & Moisture \\
\hline
\end{tabular}

F10-045

HNF-N $-49 l-5$ PG
OFFSTTE PROPERTY NOI

$\mathrm{N} / \mathrm{A}$

SAMPLE DATE SAMPLE TIME

$1-15-10$

1525

BILL OF LADING/AIR BILL NO.
10-045-081

PAGE 1 OF 2

TURATA

45 Days / 45
Days

302117 ES30 GOVERNMENT VEHICLE

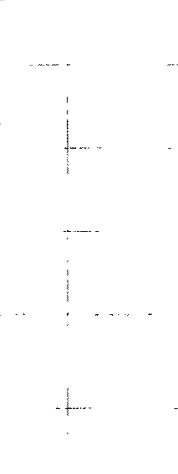

CHAIN OF POSSESSION

SIGN/ PRINT NAMES

SPECIAL INSTRUCTIONS

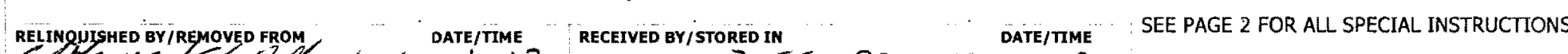

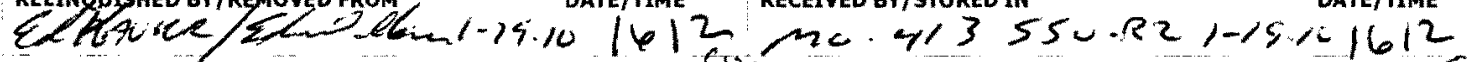

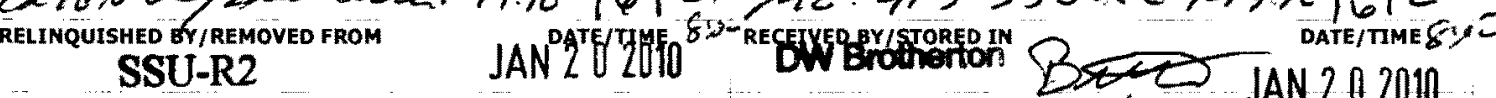
JAN 202010

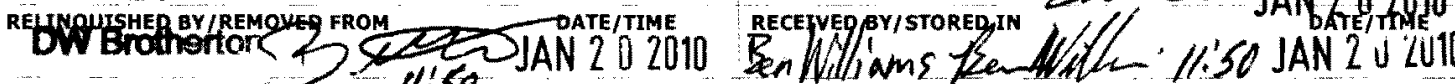

RELINQUISHED BY/REMOVED FROM

RELINQUISHED BY/REMOVED FROM

DATE/TIME

RECEIVED BY/STORED IN

DATE/TIME

RELINQUISHED GY/REMOVED FROM

DATE/TIME

RECEIVED BY/STORED IN

DATE/TIME

DATE/TIME

RECEIVED BY/STORED IN

DATE/TIME

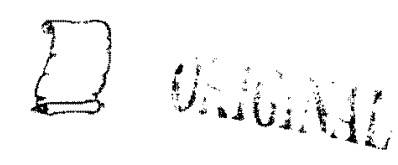

RECEIVED BY

SECTION

FINAL SAMPLE DISPOSAL METHOD

DISPOSITION 
CH2MHill Plateau Remediation Company

COLLECTOR

fatus, Romo

SAMPLING LOCATION

C7515 (299-E28-30); I-054

ICE CHEST NO.

$6 \mathrm{ws}-168$

SHIPPED TO

Environmental Sciences Laboratory

SPECIAL INSTRUCTIONS

** The 200 Area S\&GRP Characterization and Monitoring Sampling and Analysis GKI applies to this SAF.
** ESL Shall submit all data deliverables according to SOW "200-W-2 and 200-BP-5 Operable Units/Contaminant and Transport Property Analysis and Report for Vadose Zone Sediments at Borehole C5860/Well 299-E29-54" for
C7515/Wer ** ESL shall submit all data deliverables according to SOW "200-LW-2 and 200-BP-5
C7515/Well 299-E28-30 (" $M "$ Well) and shall adhere to all S\&GRP standard protocol.

** The ESL laboratory shall meet prior requested and confirmed turnaround times and RDLs specifed in the SOW and this SAF. The laboratories must notify the SM Project coordinator in writing if/when this requirement can not be met. (1)6010M_ICP_ASTM_AE (TAL) \{Sodium, Aluminum, Iron, Potassium, Antimony, Nickel, Calcium, Magnesium, Silver\} 6010M_ICP_ASTM_AE (Add-On) \{Lead, Thallium, Selenium\} 6010M_METALS_ICP_WE (TAL) \{Sodium, Aluminum,

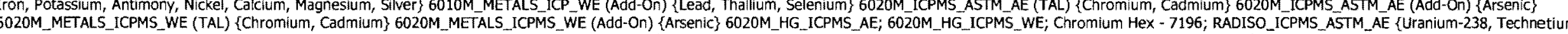
9) RADISO_ICPMS WWE \{Iodine-129, Uranium-238, Technetium-99\} I-129 by ICPMS \{Iodine-129\} GAMMA GS; URAMU Anions - 9056 WE \{Nitrate, Chloride, Phosphate, Fluoride, Nitrite, Sulfate\} Cyanide (Total) - 335.2 \{Cyanide\} pH (Water) - 9045_WE; Conductivity - 9050_WE; TOC - ASTME1915A \{Total organic Carbon, Total Inorganic Carbon, Total carbon\} 2320_ALKALINITY \{Carbonate ion, Bicarbonate, Alkalinity, Calcium Carbonate\}

\section{CHAIN OF CUSTODY/SAMPLE ANALYSIS REQUEST}

TELEPHONE NO.

PROJECT COORDINATOR

DVERMAN, D

PROJECT DESIGNATION

200-PW-2 OU Characterization Vadose Zone - Geochemical Modeling Param

FIELD LOGBOOK NO. 48

151.9

F10-045

$302117 E S 30$

GOVERNMENT VEHICL

G/AIR BILL NO.

INF-N-4q-5 $\frac{\rho}{2}$

N/A N/A
PAGE 2 OF 2 DATA
TURNAROUND

45 Days /
Days

G 
COLLECTOR

Kayex Romo

SAMPLING LOCATION

C7515 (299-E28-30); I-056

ICE CHEST NO.

$$
\text { Cus }=168
$$

Environmental Sciences Laboratory

MATRIX* POSSIBLE SAMPLE HAZARDS/ REMARKS

$A=A i r$
$\mathrm{DL}=$ Drum

that may or may not be regulated for
transportation per 49 CFR / IATA Dang

Goods Regulations but are not releasable per DOE Order $5400.5(1990 / 1993)$

$S=$ Soli
Ex Sediment

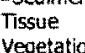

$W=$ water
$W=$ Wipe
$x=0$ ther

SPECIAL HANDLING AND/OR STORAGE SPECIAL HANDLING AND
RADIOACTIVE TIE TO: B23635
SAMPLE NO.

B235LO

SOIL
MATRIX*
COMPANY CONTACT

\begin{tabular}{l|l} 
DYEKMAN, DL & $373-2530$ \\
\hline PROEEKMAN, DL
\end{tabular}

PROJECT DESIGNATION

\begin{tabular}{l|l} 
SAF NO. \\
- Geochemical Modeling Param & F10-045
\end{tabular}

HNF-N-491-5 pg $49: 154.2 \quad 302117$ ES30

METHOD OF SHIPMENT

BILL OF LADING/AIR BILL NO.

N/A

\begin{tabular}{c|c|c|}
\hline PRESERVATION & Coon 4C & None \\
TYPE OF CONTAINER & G/P & $\begin{array}{l}\text { Molsture } \\
\text { Resistant Cont }\end{array}$ \\
\hline NO. OF CONTAINER(S) & 1 & 1 \\
\hline VOLUME & HL & 2009 \\
\hline
\end{tabular}

SAMPLE ANALYSIS SEE TEM (1) IN Moisture -
FIELD LOGBOOK NO ACTUAL SAMPLE DEPTH COA

SAMPLE DATE SAMPLE TIME

$1.20 .10: 0810$

\section{SIGN/ PRINT NAMES}

RELINQUISHED BY/REMOVED FROM Bob homo Rotwhono l/edro

SSU-R2 JAN

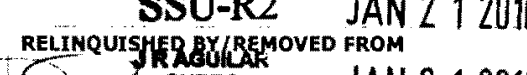
REFINQUISHED BY/REMOVED IANOM? $1 ?^{\text {Nin }}$

RELINQUUSHED BY/REMOVED FROM

RELINQUISHED BY/REMOVED FROM

RELINQUISHED QY/REMOVED FROM

LABORATORY

DISPOSITION
DATE/TIME RECEIVED BY/STORED IN 1035 MO-413 S54-22 $1-20.10,035$

DATE/TIME RECEIVED BY/STORED IN

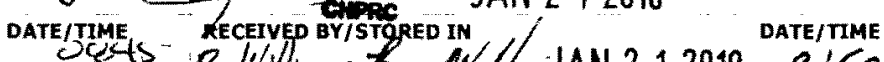

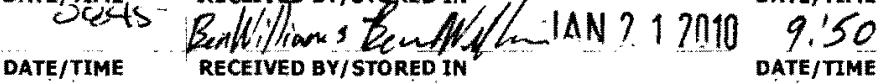

DATE/TTME RECEIVED BY/STORED IN $\cdots$ DATE/TIME

DATE/TIME RECEIVED BY/STORED IN

DATE/TIME RECEIVED BY/STORED IN
SPECIAL INSTRUCTIONS

SEE PAGE 2 FOR ALL-SPECIAL INSTRUCTIONS

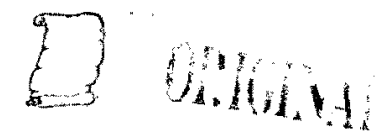




\section{COMPANY CONTACT}

DYEKMAN, DL

TELEPHONE NO.

373-2530

PROJECT COORDINATOR

PROJECT DESIGNATION

$\begin{array}{ll} & \text { SAF NO." } \\ & \text { F10-045 }\end{array}$

FIELD LOGBOOK NO.

HNF-N- $491-5$ ACTUAL SAMPLE DEPTH

pq 44 154.2

COA

$302117 \mathrm{FS} 30$

Environmental Sciences Laboratory

N/A

\section{SPECIAL. INSTRUCTIONS}

** The 200 Area S\&GRP Characterization and Monitoring Sampling and Analysis GKL applies to this SAF.

** ESL shall submit all data deliverables according to SOW "200-LW-2 and 200-BP-5 Operable Units/Contaminant and Transport Property Analysis and Report for Vadose Zone Sediments at Borehole C5860/Well 299-E29-54" for
C515/Well 299-E28-30 ("M" Well) and shall adhere to all S\&GRP standard protocol.

** The ESL laboratory shall meet prior requested and confirmed turnaround times and RDLs specified in the SOW and this SAF. The laboratories must notify the SM Project Coordinator in writing iff/when this requirement can not be me (1)6010M_ICP_ASTM_AE (TAL) \{Sodium, Aluminum, Iron, Potassium, Antimony, Nickel, Calcium, Magnesium, Silver\}6010M_ICP.ASTM_AE (Add-ON) \{Lead, Thallium, Selenium\} 6010M_METALS_ICP_WE (TAL) \{Sodium, Aluminum, 6020M_METALS_ICPMS_WE (TAL) \{Chromium, Cadmlum\} 6020M_METALS_ICPMS_WE (Add-On) \{Arsenic\} 6020M_HG_ICPMS_AE; 6020M_HG_ICPMS_WE; Chromium Hex - 7196; RADISO_ICPMS_ASTM_AE \{Uranium-238, Technetium$99\}$ RADISO_ICPMS_WE \{Iodine-129, Uranium-238, Technetium-99\} I-129 by ICPMS \{Iodine-129\} GAMMA_GS; URANIUM ISOTOPIC RATIOS; ALPHA_BETA_AE \{Gross beta, Gross alpha\} ALPHA_BETA_WE \{Gross beta, Gross alpha\} IC Anions - 9056_WE \{Nitrate, Chloride, Phosphate, Fluoride, Nitrite, Sulfate\} Cyanide (Totai) - 335.2 \{Cyanide\} pH (Water) - 9045_WE; Conductivity - 9050_WE; TOC - ASTME1915A \{Total organic carbon, Total Inorganic Carbon, Total

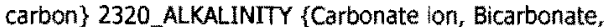

$\left\{\begin{array}{l}1 \\ 2\end{array}\right.$ 


\section{KAUER Romo}

C7515 (299-E28-30); I-057

ICE CHEST NO.

SHIPPED TO

Environmental Sciences Laboratory

MATRIX* POSSIBLE SAMPLE HAZARDS/ REMARKS Contains Radioactive Material at concen
that may or may not be regulated for

transportation per 49 CFR / IATA Dangerous Goods Regulations but are not releasable per DOE Order $5400.5(1990 / 1993)$ RADIOACTIVE TIE TO: B23636
SAMPLE NO.

B23597
MATRIX*
SAMPLING LOĆ́ATION

$$
\text { GwS - } 168
$$
Contains Radioactive Material at concentrations

SPECIAL HANDLING AND/OR STORAGE

\section{COMPANY CONTACT}

DYEKMAN, DL

TELEPHONE NO.

$373-2530$

PROJECT COORDINATOR

DYEKMAN, DL

PRICE CODE $\quad 8 \mathrm{~N}$

DATA
URNAROUND

45 Days / 45

200-PW-2 OU Characterization Vadose Zone - Geochemical Modeling Param F10-045

FIELD LOGBOOK NO. ACTUAL SAMPLE DEPTH

HNF-N 491-5 Pg49 156.9

COA

OFFSTIE PROPERTY NO.

$302117 E S 30$

AIR QUALITY

METHOD OF SHIPMENT

GOVERNMENT VEHICLE
PRESERVATION None

Split spoon

NO. OF CONTAINER(S)

volume

$1000 \mathrm{~g}$

\section{SEE TTEM (1) IN}

BILL OF LADING/AIR BILL NO.

N/A
SAMPLE DATE SAMPLE TIME

$1-20-10 \quad 0845$

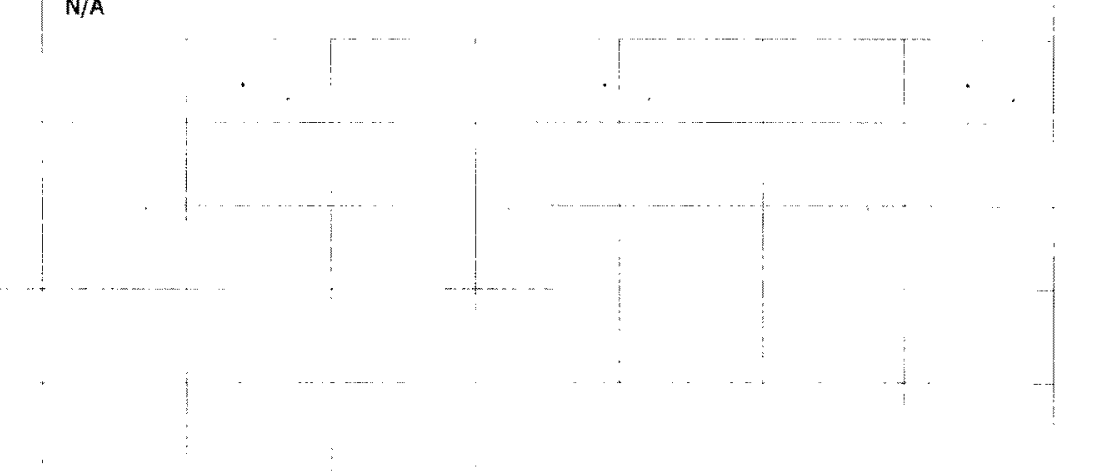

ELINQUISHED BY/REMOVED FROM SSU-R2 JAN 212010 DATE/TIME RELINQYISHEDBRLREMOVED FROM QELINQUISHED BY/REMGUED TROM AN 212010 OR 0845
DATE/TIME

\section{RELINQUISHED BY/REMOVED FROM}

RELINQUISHED BY/REMOVED FROM

RELINQUISHED BY/REMOVED FROM DATE/TIME DATE/TIME DATE/TIME

\section{CHAIN OF POSSESSION}

\section{SIGN/ PRINT NAMES}

RECEIVED BY/STORED IN

Mo-4/3 $554-22 \quad 1-20-10 \quad 1035$

RECEVERBYSTSORED IN $S$ JAN 212010 DATE/TME

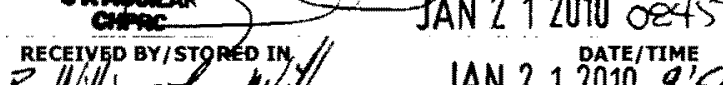
BeAllilians Zenthill- JAN 2120109,50

DATE/TIME

RECEIVED BY/STORED IN

RECEIVED BY/STORED IM

RECEIVED BY/STORED IM Robronofolezino $1-2010$ DATE/TME
DATE/TTME

DATE/TIME

DATE/TME
SPECIAL INSTRUCTIONS

** The 200 Area S\&GRP Characterization and Monitoring Sampling and Analysis

ESL shall submit all data deliverables according to SOW "200-LW-2 and 200-8P-5 Zone Sediments at Borehont and Transport Property Analysis and Report for Vados Well) and shall adhere to all S\&GRP standard protocol.

* The ESL laboratory shall meet prior requested and confirmed turnaround times and RDLS specified in the SOW and this SAF. The laboratories must notify the SM

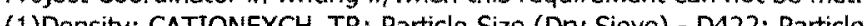
(1)Density; CATONEXCH_TR; Particle Size (Dry Sieve) - D422; Particle Size (Hydrometer) - D422; KD - Batch

\section{LABORATORY
SECTION}

RECEIVED BY

\section{DATE/TIME}


CH2MHill Plateau Remediation Company

CHAIN OF CUSTODY/SAMPLE ANALYSIS REQUEST

F10-045-087

PAGE 1 OF 2

COLLECTOR

KaUER ROMO

SAMPLING LOCATION
C7515 (299-E28-30); I-058

ICE CHEST NO.

$$
\text { G6is - } 168
$$

SHIPPED TO

Environmental Sciences Laboratory

MATRIX POSSIBle SAMPLE HAZARdS/ REMARKS

Contains Radioactive Material at concentrations

that may or may not be regulated for

Goods Regulations but are not releasable per

DOE Order 5400.5 (1990/1993)

SPECIAL HANDLING AND/OR STORAGE RADIOACTIVE TIE TO: B23636

SAMPLE NO.

MATRIX*

B235L 1

SOIL

\begin{tabular}{|c|c|c|c|c|}
\hline $\begin{array}{l}\text { COMPANY CONTACT } \\
\text { DYEKMAN, DL }\end{array}$ & $\begin{array}{l}\text { TELEPHONE NO. } \\
373-2530\end{array}$ & $\begin{array}{l}\text { PROJECT COORDINATOR } \\
\text { DYEKMAN, DL }\end{array}$ & PRICE CODE & $\mathbf{8 N}$ \\
\hline $\begin{array}{l}\text { PROJECT DESIGNATION } \\
200 \text {-PW-2 OU Characterization Yadd }\end{array}$ & 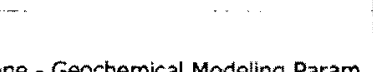 & $\begin{array}{l}\text { SAF NO. } \\
\text { F10-045 }\end{array}$ & AIR QUALITY & $\square$ \\
\hline $\begin{array}{l}\text { FELD LOGBOOK NO. } \\
\text { HNF-N. } 491-5\end{array}$ & ACTUAL SAMPLE DEPTH & $\begin{array}{l}\text { COA } \\
302117 E S 30\end{array}$ & $\begin{array}{l}\text { METHOD OF SHI } \\
\text { GOVERNMENT VE }\end{array}$ & $\begin{array}{l}\text { MENT } \\
\text { ITCEF }\end{array}$ \\
\hline
\end{tabular}

DATA

45 Days 145

Days / 45
Days

HNF-N. $491-5$ p $49154.4-15 \% .9 \quad 302117$ ES30
OFFSITE PROPERTY NO. N/A

N/A

\begin{tabular}{|c|c|c|} 
PRESERVATION & Cool 4C & None \\
TYPE OF CONTAINER & Gip & $\begin{array}{l}\text { Moisture } \\
\text { Resistant Cont }\end{array}$ \\
NO. OF CONTAINER(S) & 1 & 1 \\
\hline
\end{tabular}

VOLUME

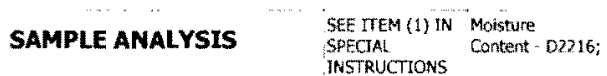

SAMPLE DATE SAMPLE TIME

$1-20-10 \quad 0845$

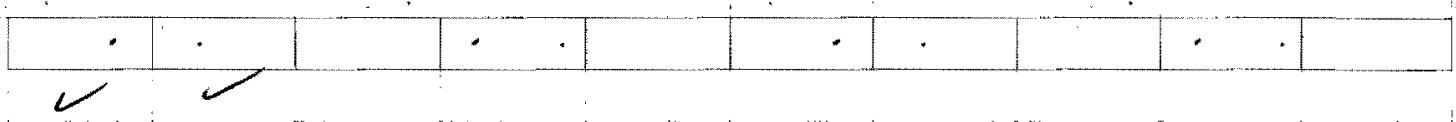

SIGN/ PRINT NAMES

DATE/TIME RECEIVED QY/STORED IN $1 / 20 / 10$ DaTE/TMME JAN 212 Da

JAN 212010 DAE/TIME

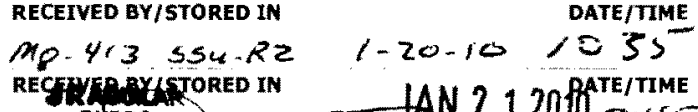

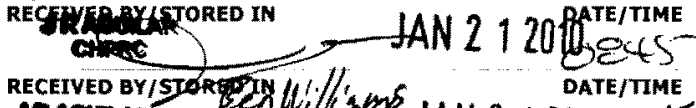

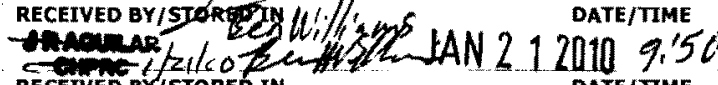
DATE/TIME

DATE/TIME

DATE/TIME

RECEIVED BY/STORED IN

DATE/TIME

DATE/TIME

DATE/TTME

TAE/TTME

RECEIVED BY/STORED IN

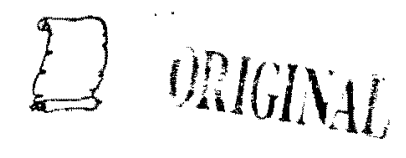

SPECIAL INSTRUCTIONS

SEE PAGE 2 FOR ALL SPECIAL INSTRUCTIONS

DATE/TIME

DATE/TIME

LABORATORY

RECEIVED BY 


\section{SPECIAL INSTRUCTIONS}

** The 200 Area S\&GRP Characterization and Monitoring Sampling and Analysis GKI applies to this SAF.

** ESL shall submit all data deliverables according to SOW "200-LW-2 and $200-\mathrm{BP}-5$ Op
$\mathrm{C} 7515 / \mathrm{Well}$
$299-\mathrm{E} 28-30$ ("M" Well) and shall adhere to all S8GGP standard protocol.

** The ESL laboratory shall meet prior requested and confirmed turnaround times and RDLs specified in the SOW and this SAF. The laboratories must notify the SM Project Coordinator in writing iffwhen this requirement can not be met.

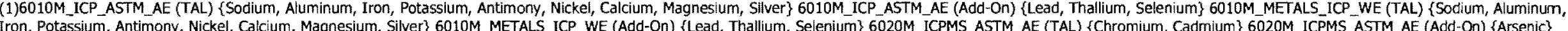

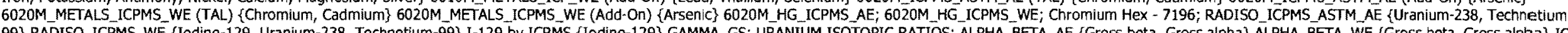
Anions - 9056_WE \{Nitrate, Chloride, Phosphate, Fluoride, Nitrite, Sulfate\} Cyanide (Total) - 335.2 \{Cyanide\} pH (Water) - 9045_WE; Conductivity - 9050_WE; TOC - ASTME1915A \{Total organic Carbon, Total Inorganic Carbon, Total (a)

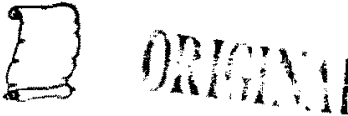


C7515 (299-E28-30); I-059

ICE CHEST NO.

SHIPPED TO

$$
G w 5-168
$$

Environmental Sciences Laboratory

MATRIX* POSSIBLE SAMPLE HAZARDS/ REMARKS

$A=A$ Air
$D=D$ Drum that may or may not be regulated for Goods Regultions but Goods Regulations but are not
DOE Order 5400.5 (1990/1993)

PROJECT DESIGNATION

200-PW-2 OU Chacterization Vadose Zone - Geochemical Modeling Param $\begin{gathered}\text { SAF NO } \\ \text { F10-045 }\end{gathered}$

METHOD OF SHIPMENT

FIELD LOGBOOK NO. ACTUAL SAMPLE DEPTH COA
HNF-N. $491-5$
COA GOVERNMENT VEHICLE

OFFSTTE PROPERTY NO. BILL OF LADING/AIR BILL NO.

\section{PRESERVATION}

TYPE OF CONTAINER Split Spoon

NO. OF CONTAINER(S)

volume

10009

SAMPLE ANALYSIS

\section{SEE ITEM (1) IN
SPECCAM
INSTRUCTONS}

\section{SAMPLE DATE SAMPLE TIME}


200-PW-2 OU Characterization Vadose Zone - Geochemical Modeling Param $\mathrm{F} 10-045$
FIELD LOGBOOK NO. ACTUAL SAMPLE DEPTH

SHIPPED TO

$$
6005-168
$$

Environmental Sciences Laboratory

MATRIX * POSSIBLE SAMPLE HAZARDS/ REMARKS

MATRIX
A=Ar
Consumins Radicactive Material at concentrations

that may or may not be regulated for
tiquids

$\begin{array}{lll}\mathrm{S} S=\text { Drum } & \text { transportation per } 49 \text { CFR / IATA Dangerous } \\ \text { Solids } & \text { Goods Regulations but are not releasable per }\end{array}$

DOE Order 5400.5 (1990/1993)

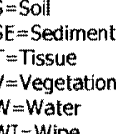

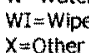

SPECIAL HANDLING AND/OR STORAGE RADIOACTIVE TIE TO: B23636
HNF-N-491-5 pg 49 158.9
302117 ES30

COA

OFFSTTE PROPERTY NO.

METHOD OF SHIPMENT

45 Days / 45
Days

N/A

PRESERVATION COON $4 \mathrm{C}$ None

TYPE OF CONTAINER G/P $\quad \begin{aligned} & \text { Moisture } \\ & \text { Resistant Cont }\end{aligned}$

NO. OF CONTAINER(S) 1

VOLUME IL 2009

SAMPLE ANALYSIS $\begin{aligned} & \text { SEE TrEM (1) IN Meisture } \\ & \text { SFCTAL } \\ & \text { ENSTRCTIONS }\end{aligned}$

ING/AIR BILL NO.

N/A

GOVERNMENT VEHICLE

SAMPLENO. MATRIX*

B235L2 SOIL

SAMPLE DATE , SAMPLE TIME

$1-20.10 \quad 0915$

SIGN/ PRINT NAMES

DATE/TIME

RELINQUISHED BY/REMOVED FROM Rob Rorro Rot chomo RELINQUISHED BY/REMOVED FROM
SSU-R2 SELINQUISHED BY/REMOVED FROM JAN 2120100245

SR COUGAR $\sum_{\text {CATC }}$ JAN 212 Date/RIME

RELINQUISHED BY/REMOVED FROM

RELINQUISHED BY/REMOVEO FROM

RELINQUISHED BY/REMOVED FROM

DATE/TIME

DATE/TIME

DATE/TIME MO-4/3 S54.RZ 1-20-10 1035

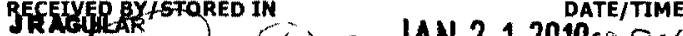

chpac - JAN 120100845

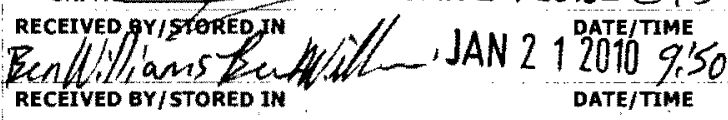

RECEIVED BY/STORED IN

RECEIVED BY/STORED IN

RECEIVED BY/STORED IN
DATE/TIME

DATE/TIME

DATE/TIME
SEE PAGE 2 FOR ALL SPECIAL INSTRUCTIONS

\section{LABORATOR
SECTION}

FINAL SAMPLE DISPOSAL METHOD
DISPOSITION

\section{RECEIVED BY}

TITLE

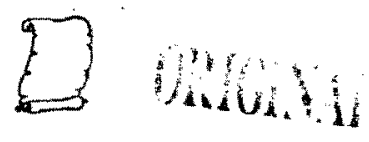

DATE/TIME

DATE/TIME 
CH2MHill Plateau Remediation Company KOLLETTOR Romo SAMPLING LOCATION C7515 (299-E28-30) ICE CHEST NO.

\section{SHIPPED TO}

Environmental Sciences Laboratory

\section{SPECIAL INSTRUCTIONS}

** The 200 Area S\&GRP Characterization and Monitoring Sampling and Analysis GKI applies to this SAF.

** ESL shall submit all data deliverables according to SOW "200-LW-2 and 200-BP-5 Operable Units/Contaminant and Transport Property Analysis and Report for Vadose Zone Sediments at Borehole C5860/Well 299-E29-54" for

** The ESL laboratory shall meet prior requested and confirmed turnaround times and RDLS specified in the SOW and this SAF. The laboratories must notify the SM Project Coordinator in writing iff/when this requirement can not be me. (1)6010M_ICP_ASTM_AE (TAL) \{Sodium, Aluminum, Iron, Potassium, Antmony, Nickel, Cakcium, Magnesium, Silver\} 6010M_ICP_ASTM_AE (Add-On) \{Lead, Thallium, Selenium\} 6010M_METALS_ICP. WE (TAL) \{Sodium, Aluminum,

6020M_METALS_ICPMS_WE (TAL) \{Chromium, Cadmium\} 6020M_METALS_ICPMS_WE (Add-On) \{Arsenic\} 6020M_HG_ICPMS_AE; 6020M_HG_ICPMS_WE; Chromium Hex - 7196; RADISO_ICPMS_ASTM_AE \{Uranium-238, Technetium99\} RADISO_LPMS_WE \{lodine-129, Uranium-238, Technetium-99\} 1-129 by ICPMS (lodne-129\} GAMMA_GS; URANUM Anions - 9056 - WE \{Nitrate, Chloride, Phosphate, Fluoride, Nitrite, Sulfate\} Cyanide (Total) - 335.2 \{Cyanide\} PH (Water) - 9045_WE; Conductivity - 9050_WE; TOC - ASTME1915A \{Total organic carbon, Total Inorganic Carbon, Total
DYEKMAN, DL

SAF NO

PROJECT DESIGNATION

FIELD LOGBOOK NO. ACTUAL SAMPLE DEPTH

HNF-N- 49/-5

pg $49,158.9$

COA

302117 ES30

OF LADING/AIR BILL NO. LLE

\section{DATA \\ 45 Days / 45 \\ Days}

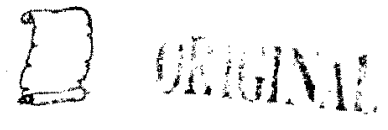


CH2MHill Plateau Remediation Company

COLLECTOR

SAMPLING LOCATION

C7515 (299-E28-30); I-06

ICE CHEST NO.

Gos - 168

SHIPPED TO

Environmental Sciences Laboratory

MATRIX

$A=A i r$
$\mathrm{DL}=$ Drum

\begin{tabular}{l|l} 
liquids & that may or may not be regulated for \\
DS = & transportation per 49 CFR / IATA Dangerous \\
Solds & Goods Regulations but are not releasable per
\end{tabular}

DOE Order 5400.5 (1990/1993)

\section{SPECTAL HANDIING AND/OR STORAGE} RADIOACTIVE TIE TO: $B 23637$
CHAIN OF CUSTODY/SAMPLE ANALYSIS REQUEST

F10-045-092

PAGE 1 OF 1

COMPANY CONTACT

TELEPHONE NO.

PROJECT COORDINATOR

DYEKMAN, DL.

\begin{tabular}{l|l} 
SAF NO. \\
F10-045
\end{tabular}

PROJECT DESIGNATION

\begin{tabular}{ll|l} 
200-PW-2 ou Characterization Vadose Zone - Geochemical Modeling Param & F10-045 \\
ACTUAL SAMPLE DEPTH & COA
\end{tabular}

COA

PRICE CODE

DATA
URNAROUND

AIR QUALITY $\square \quad \underset{\text { Days } / 45}{45}$

PRESERVATIO

TYPE OF CONTAINER

NO. OF CONTAINER(S)

voLUME

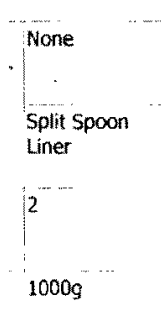

SAMPLE ANALYSIS SEE TIEM (1) IN

\section{METHOD OF SHIPMEN}

\section{SAMPLE DATE SAMPLE TIME}

$1-20.10 \quad 1005$

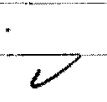

SIGN/ PRINT NAMES

CHAIN OF POSSESSION

RELINQUISHED BY/REMOVED FROM

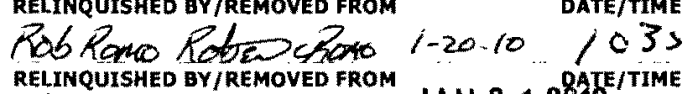
SSU-R2

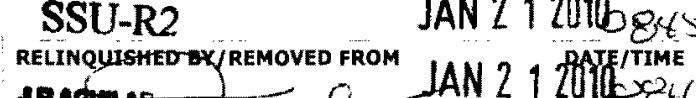
IRACGuL

RELINQUISHED BY/REMOVED FROM

RELINQUISHED QY/REMOVED FROM

RELINQUISHED BY/REMOVED FROM

RECEIVED GY

SECTION

FINAL SAMPLE
DISPOSTION
Mo. $413554-52$

$21-20-101035$

RACVEAR ARTRED II

DATE/TIME

DATE/TIME

RECEIVED BY/STORED IN

DATE/TIME

RECEIVED BY/STORED IM

DATE/TIME

DATE/TMEE

\section{SPECIAL INSTRUCTIONS}

** The 200 Area S\&GRP Characterization and Monitoring Sampling and Analysis GKI applies to this SAF.

作 Zone Sediments at Borehole C5860/Well 299-E29-54" for C7515/Well 299-E28-30

**. The ESL laboratory shall meet prior requested and confirmed turnaround times and RDLS specified in the SOW and this SAF. The laboratories must notify the SM Project Coordinator in writing if/when this requirement can not be met. (Hydrometer) - D422; KD - Batch;
DATE/TME
DATE/TIME 
Сн2мніll Plateau Remediation Company

COLLECTOR

sampling location

C7515 (299-E28-30); 1062

ICE CHEST NO.

$$
\text { Gus-ller }
$$

SHIPPED TO

Environmental Sciences Laboratory

$\underset{\text { Matrix }}{\text { Mair }}$ POSSIBLE SAMPLE HAZARDS/ REMARKS

$A=A$ Air $\quad$ Contains Radioactive Material at concentrations

that may or may not be regulated for

Goods Regulations but are not releasble per

DOE Order 5400.5 (1990/1993)

SPECIAL HANDLING AND/OR STORAGE RADIOACTIVE TIE TO: B23637
SAMPLE NO.

B235L3
MATRIX*

\section{CHAIN OF CUSTODY/SAMPLE ANALYSIS REQUEST}

F10-045-093

PAGE 1 OF 2

COMPANY CONTACT

TELEPHONE NO.

PROJECT COORDINATOR

DYEKMAN, DL

SAF NO.

PRICE CODE $8 N$ DATA
TURNAROUND

AIR QUALITY $\square \quad \underset{\text { Days }}{\mathbf{4 5} \text { Days / } 45}$

PROJECT DESIGNATION

200-PW-2 OU Characterization Vadose Zone - Geochemical Modeling Para

COA

FIELD LOGBOOK NO.

ACTUAL SAMPLE DEPTH

METHOD OF SHIPMENT

\begin{tabular}{ll|l|l|l|l} 
HNF-N. $49 /-5 P G$ GOVERNMENT VEHICLE & 162 & $302117 E 530$ &
\end{tabular}

OFFSITE PROPERTY NO. 3 BILL OF LADING/AIR BILL NO.

PRESERVATION

TYPE OF CONTAINER

NO. OF CONTAINER(S)

voLUME

Coot 4 AC None

SAMPLE DATE , SAMPLE TIME

$1-20-10 \quad 1005$

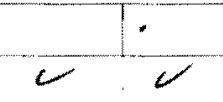

SIGN/ PRINT NAMES

RECEIVED BY/STORED IN

RECEIVED BY/STORED IN
MO- $4 / 3$ SSU-RE
RECEIVD BY/STORED IN

JAN 21 भUT TIME

RObRomo RotWRORO

SSU-R2.

RELWRYLSUER BYLREMOVED FROM

CELINCUTSED BY TEMOVED FROM

RECENE

DATE/TIME

SPECIAL INSTRUCTIONS

SEE PAGE 2 FOR ALL SPECIAL INSTRUCTIONS

IN 21200 DATEITIME

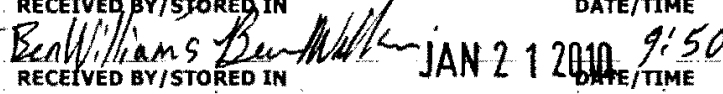

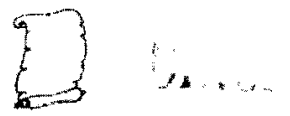

RELINQUISHED BY/REMOVED FROM

RECEIVED BY/STORED IN

DATE/TME

RELINQUISHED BY/REMOVED FROM

DATE/TIME

RECEIVED BY/STORED IN

DATE/TIME

RELINQUISHED BY/REMOVED FROM

DATE/TIME

RECEIVED BY/STORED IN

DATE/TIME 


\section{CHAIN OF CUSTODY/SAMPLE ANALYSIS REQUEST}

\section{F10-045-093}

COLLCTOR

sampling location

C7515 (2998

ICE CHEST NO.

$$
\text { GwS-168 }
$$

\section{SHIPPED TO}

Environmental Sciences Laboratory

\section{SPECIAL INSTRUCTIONS}

** The 200 Area S\&GRP Characterization and Monitoring Sampling and Analysis GKI applies to this SAF.
** ESL shall submit all data deliverables according to SOW "200-LW-2 and 200-BP-5 Operable Units/Contaminant and Transport Property Analysis and Report for Vadose Zone Sediments at Borehole C5860/Well 299-E29-54" for ** ESL shall submit all data deliverables according to SOW "200-LW-2 and 200-BP-5 0 -
C7515/Well 299-E28-30 ("M" Well) and shall adhere to all S\&GRP standard protocol.

** The ESL laboratory shall meet prior requested and confirmed turnaround times and RDLS specified in the SOW and this SAF. The laboratories must notify the SM Project Coordinator in writing if/when this requirement can not be met. (1)6010M_ICP_ASTM_AE (TAL) \{Sodium, Aluminum, Iron, Potassium, Antimony, Nickel, Calcium, Magnesium, Silver\} 6010M_ICP_ASTM_AE (Add-On) \{Lead, Thallium, Selenium\} 6010M_METALS_ICP_WE (TAL) \{Sodium, Aluminum, 6020M METALS ICPMS WE (TAL) \{Chromium, Cadmium\} 6020M METALS ICPMS WE (Add-On) \{Arsenic\} 6020M. HG ICPMS AE; 6020M HG ICPMS WE; Chromium Hex - 7196; RADISO IC̄PMS ASTM AF \{Uranium-238, Techetiu 99\} RADISO_ICPMS_WE \{lodine-129, Uranium-238, Technetium-99\} I-129 by ICPMS \{lodine-129\} GAMMA_GS; URANIUM ISOTOPIC RATIOS; ALPHA_BETA_AE \{Gross beta, Gross alpha\} ALPHA_BETA_WE \{Gross beta, Gross alpha\} IC

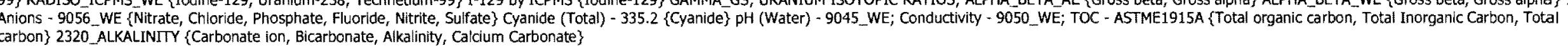

D. 
CH2MHill Plateau Remediation Company

COLLECTOR

$$
\text { Ka GER, Roño }
$$

SAMPLING

ICE CHEST NO.

$$
\text { Gins }-168
$$

SHIPPED TO

Environmental Sciences Laboratory

MATRIX* POSSIBLE SAMPLE HAZARDS/ REMARKS

$A=A i r$
$\mathrm{DL}=$ Drum Contains Radloactive Material at concentrations

liquids that may or may not be regulated for

Goods Regulations but are not releasable per

DOE Order 5400.5 (1990/1993)

SPECIAL HANDLING AND/OR STORAGE SPECIAL HANDLING AND/OR
RADIOACTIVE TIE TO: B23637
CHAIN OF CUSTODY/SAMPLE ANALYSIS REQUEST

F10-045-095

PAge 1 OF 1

COMPANY CONTACT $\quad$ TELEPHONE NO. PROJECT COORDINATOR

DYEKMAN, DL $373-2530 \quad$ DYEKMAN, DL

PROJECT DESIGNATION
SAF NO.
200-PW-2 OU Characterization Vadose Zone - Geochemical Modeling Param $\quad$ F10-045

FIELD LOGBOOK NO. ACTUAL SAMPLE DEPTH

\begin{tabular}{ll|l} 
HNF-N- 491-5 Pg 49 & $161.7-164.2$
\end{tabular}

OFFSITE PROPERTY NO.

$\mathrm{N} / \mathrm{A}$

\section{PReservation}

\begin{tabular}{lll} 
& Split Spoon \\
\hline TYPE OF CONTAINER & Liner
\end{tabular}

NO. OF CONTAINER(S)

voLUME

COA

PRICE CODE

AIR QUALITY

DATA
TURNAROUND

45 Days / 45

METHOD OF SHIPMENT

GOVERNMENT VEHICLE

BILL OF LADING/AIR BILL NO.

\section{SAMPLE ANALYSIS $\begin{gathered}\text { SEE TEM (1) IN } \\ \text { SPEETILL } \\ \text { INSTRUCTONS }\end{gathered}$}

SAMPLE.DATE SAMPLE TIME

$1 / 20 / 101205$

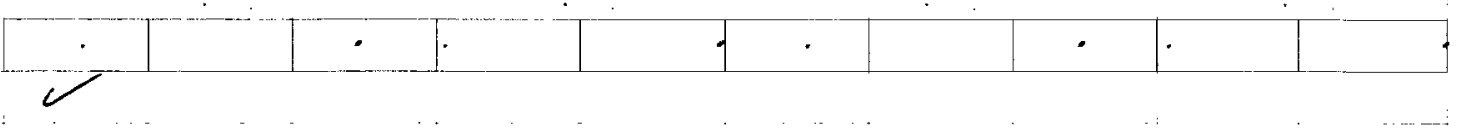

Sign/ PRINT names

DATE/TIME RECEIVED BY/STORED IN

$1330 \mathrm{MO}-4 / 3 \mathrm{ss4} \cdot \mathrm{Rz}_{2}$

RELINQUISHED BYYREMOVED FROM RobRomo Robechoromo SSU-R2

REYHOUUSHED BY/REMOVED FROM RELINQUISHED BY /REMOVIED FROM

RELINQUISHED QY/REMOVED FROM

RELINQUISHED BY/REMOVED FROM

RELINQUISHED BY/REMOVED FROM

LABORATORY RECEIVED BY SECTION

FINAL SAMPLE DISPOSAL METHOD

DISPOSITION DATE/TIME

RECEIVER BVISTORE

DATE/TIME RECEIVED BY/STORED IN

DATE/TIME RECEIVED BY/STORED IN

DATE/TIME

RECEIVED BY/STORED IN

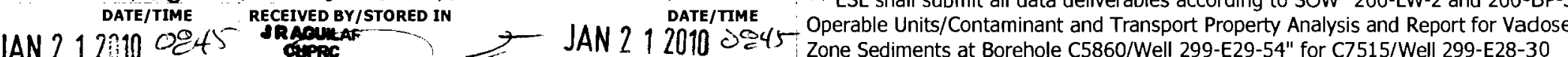

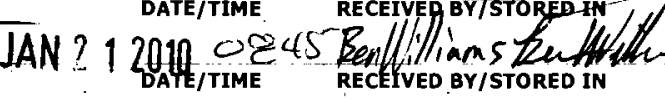

JAN 2 DATE/TUME 9 :'Sd

DATE/TIME ** The ESL laboratory shall meet prior requested and confirmed turnaround times and RDLS specified in the SOW and this SAF. The laboratories must notify the SM

DATE/TIME

Project Coordinator in writing if/when this requirement can not be met.
DATE/TME

DATE/TIME
DATE/TIME

DATE/TIME 
CH2MHill Plateau Remediation Company

COLIECTOR

Kayer Rono

C7515 (299-E28-30); I-064

ICE CHEST NO.

$$
\text { GWS-16\% }
$$

SHIPPED TO

Environmental Sciences Laboratory

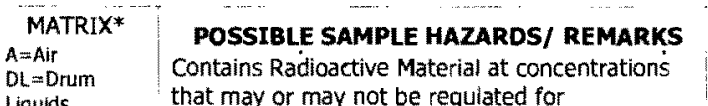

liquds
L that may or may not be regulated for
transportation per $49 \mathrm{CFR} /$ IATA Dange

transportation per 49 CFR / IATA Dangerous

Gods Regulations
DOE Order $5400.5(1990 / 1993)$

SPECIAL HANDLING AND/OR STORAGE RADTOACTIV TIE TO: B23637

SAMPLE NO.

MATRIX*
F10-045-096

PAGE 1 OF 2

PRICE CODE

DATA
TURNAROUND

45 Days / 45
Days

PROJECT DESIGNATION

PROJECT COORDINATOR

DYEKMAN, DL

SAF NO.

AIR QUALITY

COA

METHOD OF SHIPMENT

FIELD LOGBOOK NO.

ACTUAL SAMPLE DEPTH

HNF-N- 491-5 pg 49 164.2

302117 ES30

GOVERNMENT VEHICLE

OFFSTTE PROPERTY NO. $\quad$ BILL OF LADING/AIR BILL NO.

PRESERVATIO

TYPE OF CONTAINER G/P $\begin{gathered}\text { Moisture } \\ \text { Resistant Cont }\end{gathered}$

NO. OF CONTAINER(S)

volume

N/A

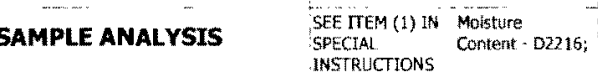

SAMPLE DATE SAMPLE TIME

$1-20-101205^{-}$

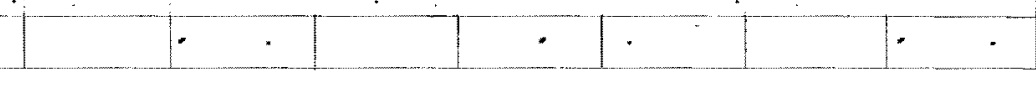

SIGN/ PRINT NAMES

CHAIN OF POSSESSION

REINQUISHED BY/REMOVED FROM

Toblomo hotomomo 1-20-10 1330

RELINQUISHED BY/REMOVED FROM SSU-R2 JAN 212010 OATE/MME RELTQUISHED BY/REMOVED FROM RELINQUE

RELINQUISHED BY/REMOVED FROM

RELUNQUISHED BY/REMOVED FROM

RELINQUISHED QY/REMOVED FROM

RECEIVED aY

SECTION

FINAL SAMPLE
DISPOSITION
010 Se4

RECEIVED BY/STORED IN

$\operatorname{mo-4/3} 554-R^{2}$

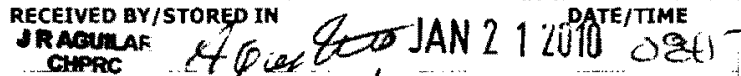

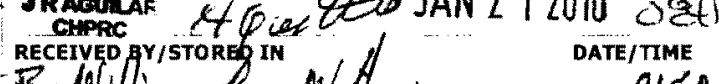

Ren Wi li ams Be Matho'JAN 212019 9:50

DATE/TIME

RECEIVED BY/STORED IN

DATE/TIME

DATE/TIME RECEIVED BY/STORED IN DATE/TIME

DATE/TIME RECETYEO BY/STORED IM

DATE/TIME
SPECIAL INSTRUCTIONS

SEE PAGE 2 FOR ALL SPECIAL INSTRUCTIONS 
CH2MHill Plateau Remediation Company

COLLECTOR

KAMELING LOCATION, ROMO

ICE CHEST NO.

$$
\text { Gus - } 168
$$

SHIPPED TO

Environmental Sciences Laboratory

SPECIAL INSTRUCTIONS

** The 200 Area S\&GRP Characterization and Monitoring Sampling and Analysis GKI applies to this SAF.

** ESL shall submit all data deliverables according to SOW "200-LW-2 and 200-BP-5 Operable Units/Contaminant and Transport Property Analysis and Report for Vadose Zone Sediments at Borehole C5860/Well 299-E29-54" for

C7515/Well 299-E28-30 ("M" Well) and shall achere to all S\&GRP standard protocol.

** The ESL laboratory shall meet prior requested and confirmed turnaround times and RDLS specified in the SOW and this SAF. The laboratories must notify the SM Project coordinator in writing iff/when thls requirement can not be met. Aluminum, Iron, Potassium, Calcium, Nickel, Magnesium, Silver\} 6010M_METALS_ICP_WE (Add-On) \{Lead, Thallium, Selenium\} 6020M_ICPMS_ASTM_AE (TAL) \{Chromium, Cadmium\} 6020M ICPMS_ASTM_AE (Add-On) \{Arsenic\}

6020M_METALS_ICPMS_WE (TAL) \{Chromium, Cadmium\} 6020M_METALS_ICPMS_WE (Add-On) \{Arsenic\}6020M_HG_ICPMS_AE; 6020M_HG_ICPMS_WE; Chromium Hex-7196; RADISO_ICPMS_ASTM_AE \{Uranium-238, Technetium99\} RADISO_ICPMS WE \{lodine-129, Uranium-238, Technetium-99\} I-129 by ICPMS \{lodine-129\} GAMMA_GS; URANIUM ISOTOPIC RATIOS; ALPHA_BETA_AE \{Gross beta, GrOSS alpha\} ALPHA_BETA_WE \{Gross beta, Gross alpha\} IC

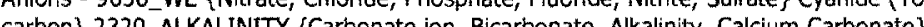

$\{3 \quad n+11$ 
CH2MHill Plateau Remediation Company

COLLECTOR

Kay 2 , Romo
SAmpling Location'

C7515 (299-E28-30); I-065

ICE CHEST NO.

$$
\text { Givs-168 }
$$

SHIPPED TO

Environmental Sciences Laboratory

MATRIX* | POSSIBLE SAMPLE HAZARDS/ REMARKS

$A=A \mathrm{~A}$,
$\mathrm{D}=\mathrm{Drum}$ Contains Radioactive Material at concentrations that may or may not be regulated for
transportation per 49 CFR / IATA Danger Goods Regulations but are not releasable per DOE Order 5400.5 (1990/1993)

SPECLAL HANDLING AND/OR STORAGE RADIOACTIVE TIE TO: B23638

SAMPLE NO. , MATRIX*

B235L5 - SOIL
F10-045-098

PAGE 1 OF 2

CHAIN OF CUSTODY/SAMPLE ANALYSIS REQUEST

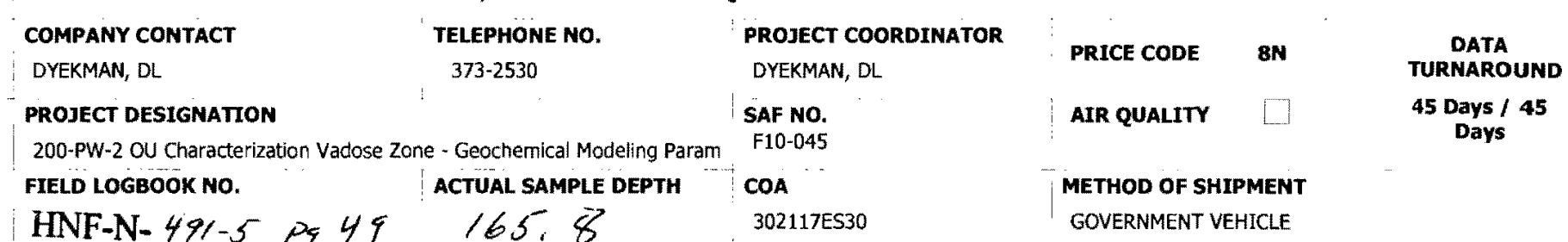

HNF-N-491-5 PG $49 \quad 1658$ 302117ES30

OFFSTIE PROPERTY NO.

$\mathrm{N} / \mathrm{A}$

PRESERVATION

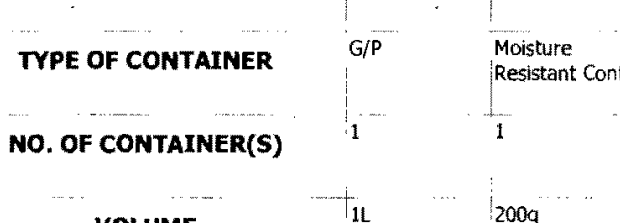

VOLUME

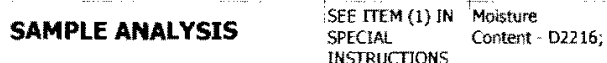

SAMPLE DATE SAMPLE TIME.

$1-20-10 \quad 1240$

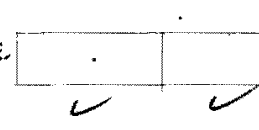

CHAIN OF POSSESSION

RELINQUISHED GY/REMOVED FROM Robrow Rokn Romo

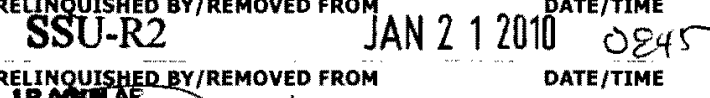
TRALERE

RELINQUISHED BY/REMOVED FROM

RELINQUISHED BY/REMOVED FROM

RELINQUISHED QY/REMOVED FROM

LABORATORY

FINAL SAMPLE
DISPOSITION

\section{SIGN/ PRINT NAMES}

RECEIVED BY/STORED IN

$1-20-10$ Date/time

mo.4/3 ssu-R2 1-20-10 1330

RECEIEED BYTSTQRED IN JAAZ 14 LUIV 0845

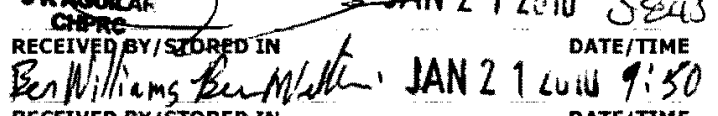

RECEVED BY/STORED IN

DATE/TIME RECEIVED BY/STORED IN $\cdots$ DATE/TIME

DATE/TIME

RECEIVED BY/STORED IN

DATE/TIME

RECEIVED BY/STORED IN

DATE/TIME

DATE/TIME

\section{SPECIAL INSTRUCTIONS}

SEE PAGE 2 FOR ALL SPECIAL INSTRUCTIONS

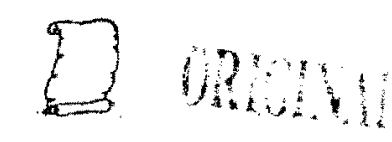


PROJECT COORDINATOR

SAF NO
F10-045

C7515 (299-E28-30); 1-065

FIELD LOGBOOK NO.

ACTUAL SAMPLE DEPTH

AIR QUALITY D

FIELD LOGBOOK NO.

\section{COA}

HNF-N- $491-5$ PG 49 AFFITE PROPERT No.
RCR. 1.20 .10

$$
\text { Givs-16r }
$$

SHIPPED TO

OFFSTTE PROPERTY NO.

COA

METHOD OF SHIPMENT

Environmental Sciences Laboratory

N/A

BILL OF LADING/AIR BILL NO.

$\mathrm{N} / \mathrm{A}$

\section{INTRUCTIONS}

*** The 200 Area S\&GRP Characterization and Monitoring Sampling and Analysis GKI applies to this SAF,

** ESL shall submit all data deliverables according to SOW "200-LW-2 and 200-BP-5
C7515/Well 299-E28-30 ("M" Well) and shall adhere to all S\&GRP standard protocol.

** The ESL laboratory shall meet prior requested and confirmed turnaround times and RDLs specified in the SOW and this SAF. The laboratories must notify the SM Project Coordinator in writing if/when this requirement can not be met. (1)6010M_ICP_ASTM_AE (TAL) \{Sodium, Iron, Potassium, Antimony, Aluminum, Nickel, Calcium, Magnesium, Silver\} 6010M_ICP ASTM AE (Add-On) \{Lead, Thallium, Selenium\} 6010M_METALS ICP_WE (TAL) \{Sodium, Aluminum,

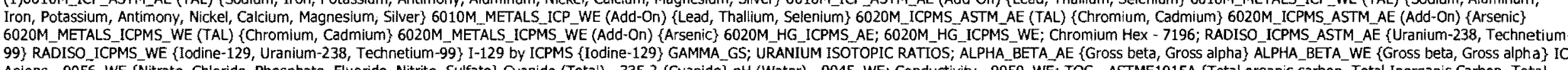

Anions - 9056_WE \{Nitrate, Chloride, Phosphate, Fluoride, Nitrite, Sulfate\} Cyanide (Tota) - 335.2 \{Cyanide\} PH (Water) - 9045_WE; Conductivity - 9050_WE; TOC - ASTME1915A \{Total organic Carbon, Total Inor
carbon\} 2320_ALKALINITY \{Carbonate ion, Bicarbonate, Alkalinity, Calcium Carbonate\}

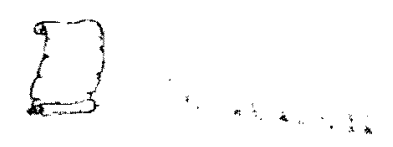


CH2MHill Plateau Remediation Company

COLLECTOR

$$
\text { KaYER, ROMO }
$$

SAMPLING

C7515 (299-E28-30); I-066

ICE CHEST NO

SHIPPED TO

$$
\text { Gus -168 }
$$

Environmental Sciences Laboratory

MATRIX* POSSIBLE SAMPLE HAZARDS/ REMARKS

$A=A$ Air
Di Drum Contains Radioactive Material at concentrations that may or may not be regulated for
transportation per 49 CFR / IATA Dangerous transportation per 49 CFR / IATA Dangerous Goods Regulations but are not
DOE Order $5400.5(1990 / 1993)$

SPECIAL HANDLING AND/OR STORAGE RADIOACTIVE TIE TO: B23638
- SAMPLE NO
MATRIX

B235L6

SOIL

\section{CHAIN OF POSSESSION}

RELINQUISHED GY/REMOVED FROM Rob Romo Rob C Romo

\section{SSU-R2}

BETXOURARE BY/REMOVED FROM

CHPRC

RELINQUISHED BYJREMOVED FROM

RELINQUISHED BY/REMOVED FROM

RELINQUISHED BY/REMOVED FROM

RELINQUISHED BY/REMOVED FROM

LABORATORY RECEIVED B

SECTION

FINAL SAMPLE DISPOSAL METHOD

CHAIN OF CUSTODY/SAMPLE ANALYSIS REQUEST

F10-045-099

PRICE CODE $\quad 8 \mathrm{~N}$

TURNAROUND

Days

PROJECT DESIGNATION

373-2530 DYEKMAN, DL

SAF NO.

200-PW-2 OU Characterization Vadose Zone - Geochemical Modeling Param F10-045
FIELD LOGBOOK NO.
ACTUAL SAMPLE DEPTH

METHOD OF SHIPMENT

HNF-N-491.5Pg $49 \quad 169.5$

PRESERVATION

TYPE OF CONTAINER

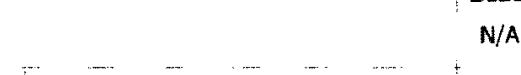

ADING/AIR BILL NO.

N/A

VOLUME

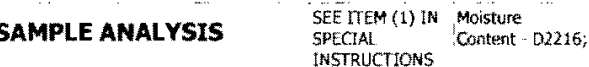

$1-20-10 \quad 1300$

\section{SIGN/ PRINT NAMES}

DATE/TIME $-20.10 \quad 1330$ JAN 21 WU10 OEY JAN 21 2U10 OQ

RECEIVED BY/STORED IN MO- $4 / 3554-R 2$ RECERAG BYTSTORED

RECEIVEqRG

JAN 212010 DATEL

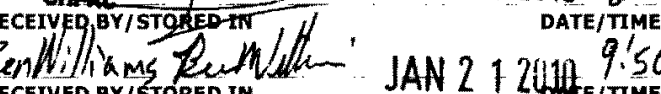

RECEIVED BY/STORED IN

DATE/TIME

DATE/TIME

RECEIVED BY/STORED IN

DATE/TIME

DATE/TIME
RECEIVEO BY/STORED IN
SPECIAL INSTRUCTIONS

SEE PAGE 2 FOR ALL SPECIAL INSTRUCTIONS

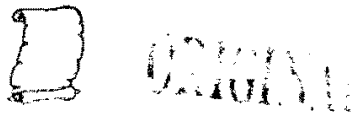




\section{KaMpING KaER, ROMO}

C7515 (299-E28-30); I-066 ICE CHEST NO

$$
\text { Givs-168 }
$$

\section{SHIPPED TO}

Environmental Sciences Laboratory

\section{SPECIAL INSTRUCTIONS}

** The 200 Area S\&GRP Characterization and Monitoring Sampling and Analysis GKI applies to this SAF.

** ESL shall submit all data deliverables according to SOW "200-LW-2 and 200-BP-5 Operable Units/Contaminant and Transport Property Analysis and Report for Vadose Zone Sediments at Borehole C5860/Well 299-E29-54" for

** The ESL laboratory shall meet prior requested and confirmed turnaround times and RDLS specified in the SOW and this SAF. The laboratories must notify the SM Project Coordinator in writing iffwhen this requirement can not be met. (1)6010M_ICP_ASTM_AE (TAL) \{Sodium, Aluminum, Iron, Potassium, Antimony, Nickel, Calcium, Magnesium, Silver\} 6010M_LCP_ASTM_AE (Add-On) \{Lead, Thallium, Selenium\} 6010M_METALSICP_WE (TAL) (SOdium, Aluminum,

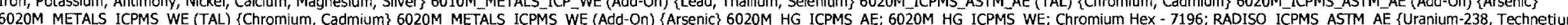
9\} RADISO ICPMS WE \{lodine-129, Uranium-238, Technetium-99\} I-129 by ICPMS \{lodine-129\} GAMMA GS; URANIUM ISOTOPIC RATIOS; ALPHA BETA AE \{Gross beta, Gross alpha\} ALPHA BETA WE \{Gross beta, Gross alpha\} IC Anions - 9056_WE \{Nitrate, Chloride, Phosphate, Fluoride, Nitrte, Sulfate\} Cyanide (Total) - 335.2 \{Cyanide\} pH (Water) - 9045_WE; Conductivity - 9050_WE; TOC - ASTME1915A \{Total organic carbon, Total Inorganic Carbon, Total carbon\} 2320_ALKALINITY \{Carbonate ion, Bicarbonate, Aikalinity, Calcium Carbonate

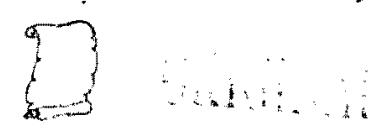


PROJECT COORDINATOR

C7515 (299-E28-30): I-067

\begin{tabular}{|l|l|}
\hline PROJECT DESIGNATION & SAF NO. \\
\hline 200-PW-2 OU Characterization Vadose ZOne - Geochemical Modeling Param F10-045 &
\end{tabular}

PRICE CODE

C7515 (299-E28-30

FIELD LOGBOOK NO.

$5 \operatorname{los}-169$

FTELD LOGBOOK NO.

ACTUAL SAMPLE DEPTH

AIR QUALITY

45 Days $/ \cdot 45$
Days

Environmental Sciences Laboratory

OFFSTEE PROPERTY NO.

COA

N/A

MATRIX * POSSIBLE SAMPLE HAZARDS/ REMARKS

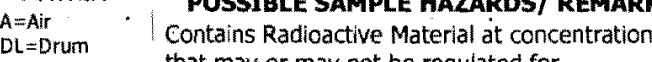
that may or may not be regulated for Goods Regulations but are nat re Dangerous DOE Order 5400.5 (1990/1993)

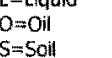

SPECIAL HANDLING AND/OR STORAGE RADIOACTIVE TIE TO: B23657

\section{PRESERVATION}

171.9

302117ES30

METHOD OF SHIPMENT

BILL OF LADING/AIR BILL NO.

GOVERNMENT VEHICLE

TYPE OF CONTAINER G/P $\quad \begin{aligned} & \text { Maisture } \\ & \text { Resistant Cont }\end{aligned}$

NO. OF CONTAINER(S)

VOLuME

SAMPLE ANALYSIS

${ }_{1} \cdots \cdots$

1

2009

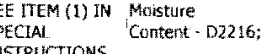

SAMPLE DATE SAMPLE TIME

$1 / 25 / 10$

$12: 40$

SIGN/ PRINT NAMES

SPECIAL INSTRUCTIONS

CHAIN OF POSSESSION

RECEIVED BY/STORED IN

SEE PAGE 2 FOR ALL SPECIAL INSTRUCTIONS

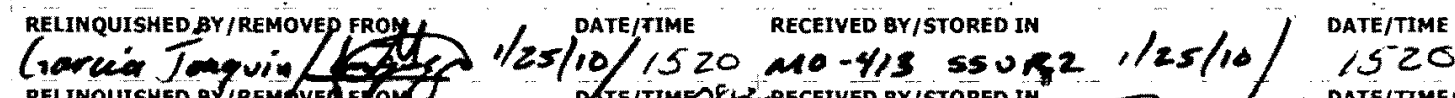

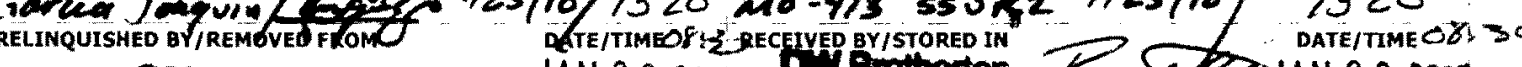

SSU-R2

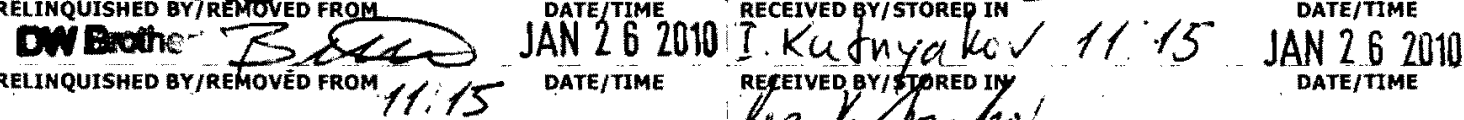

RELINQUISHED QY/REMOVED FROM

DÄTE/TIME -

lur

DATE/TIME

53901

RELINQUISHED BY/REMOVED FROM

DATE/TIME

RECEIVED BY/STORED IN

DATE/TIME

RELINQUISHED BY/REMOVED FROM

DATE/TIME

RECEIVED BY/STORED IN

DATE/TIME 
C7515 (299-E28-30); 1-067

ICE CHEST NO.

\begin{tabular}{l} 
200-PW-2 OU Characterization Vadose Zone - Geochemical Modeling Param \\
FIELD LOGBOoK No. \\
\hline ACTUAL SAMPLE DEPTH
\end{tabular}

6us - 169

HNF-N- 49l-S

171.9

COA

METHOD OF SHIPMENT

OFFSTTE PROPERTY NO.

302117ES30

GOVERNMENT VEHICLE

GILL OF LADING/AIR BILL NO.

N/A

\section{SPECIAL INSTRUCTIONS}

N/A

** The 200 Area S\&GRP Characterization and Monitoring Sampling and Analysis GKI applies to this SAF.

** ESL shall submit all data deliverables according to SOW "200-LW-2 and 200-BP-5 Operable Units/Contaminant and Transport Property Analysis and Report for Vadose Zone 5ediments at Borehole C5860/Well 299-E29-54" for C7515/Well 299-E28-30 ("M" Well) and shall adhere to all S\&GRP standard protocol.

** The ESL laboration shall meet prior requested and confirmed turnaround times and RDLs specified in the SOW and this SAF. The laboratories must notify the SM Project Coordinator in writing iff/when this requirement can not be met. (1)6010M_ICP_ASTM_AE (TAL) \{Sodium, Aluminum, Iron, Potassium, Antimony, Nickel, Calcium, Magnesium, Silver\} 6010M_ICP_ASTM_AE (Add-On) \{Lead, Thailium, Selenium\} 6010M_METALS_ICP_WEE (TAL) \{Sodium, Aluminum,

6020M METALS ICPMS WE (TAL) \{Chromium, Cadmium\} 6020M METALS ICPMS WE (Add-On) \{Arsenic\} 6020M HG ICPMS AE; 6020M HG ICPMS WE; Chromium Hex - 7196; RADISO ICPMS ASTM_AE \{Uranium-238, Technetium99\} RADISO_ICPMS WE \{Iodine-129, Uranium-238, Technetium-99\} I-129 by ICPMS \{Iodine-129\} GAMMA_GS; URANIUM ISOTOPIC RATIOS; ALPHA_BETA_AE \{Gross beta, Gross alpha\} ALPHA_BETA_WE \{Gross beta, Gross alpha\} IC

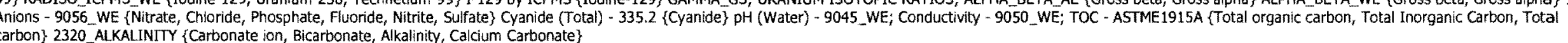

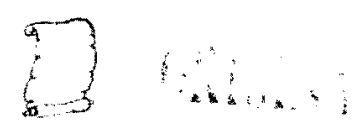


COLleCtOR COMPANY CONTACT

\section{Rust, Rorame, Chamberhme, kaver, Carciug DYEKMAN, DL}

PROJECT DESIGNATION
PYEKMAN, DL

TELEPHONE NO.

PROJECT COORDINATOR

DYEKMAN, DL

SAF NO.

200-PW-2 OU Characterization Vadose Zone - Geochemical Modeling Param F10-045

C7515 (299-E28-30); 1-068

FIELD LOGBOOK NO.

ACTUAL SAMPLE DEPTH

COA
$302117 E S 30$

PRICE CODE

DATA
TURNAROUND
4S Days/

HNF-N- $491-5 \mathrm{pg} 50 \quad 173 \mathrm{ft}$.

$302117 E 530$ METHOD OF SHIPMENT

$6 \cos 5-169$

OFFSTTE PROPERTY NO.

BILL OF LADING/AIR BILL NO.

Environmental Sciences Laboratory

POSSIBLE SAMPLE HAZARDS/ REMARKS Contains Radioactive Material at concentrations
that may or may not be regulated for

transportation per 49 CFR / IATA Dangerous

Goods Regulations but are not releasable per

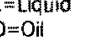

\section{N/A}

SAMPLE NO.
B235L8

TYPE OF CONTAINER G/P Mosture

NO. OF CONTAINER(S)

volume

SAMPLE ANALYSIS

2000

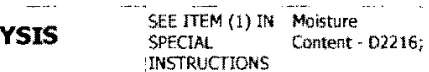

SAMPLE DATE SAMPLE TIME

$1 / 25 / 10 \quad 1246$

CHAIN OF POSSESSION

SIGN/ PRINT NAMES

SPECIAL INSTRUCTIONS

RELINQUished aY/REMOVED Froy, DATE/TIME RECEIVED aY/STORED IN IN DATE/TTME SEE PAGE 2 FOR ALL SPECIAL INSTRUCTIONS

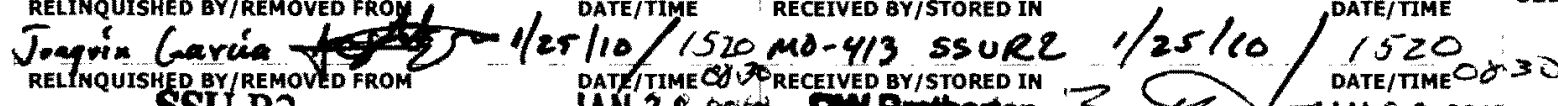

\section{SSU-R2}

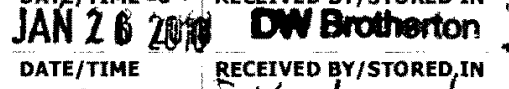

RLINQUISHED BY/REMOVEDEE ER

DATE/TTME RECEIVED QY/STOREDIN

RELINQUISHED BYTREMOVEDFROM $11: 15$ JATE/TIME 20101 RECEVED GYYGORED IN

RELINQUISHED BY/REMOVED FROM

DATE/TIME

$\lim _{\text {REdEIVD }}$ BY

11.15

JAN 262010

RELINQUISHED BY/REMOVED FROM

DATE/TIME

RECEIVED BY/STORED IN

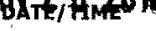

DATE/TIME

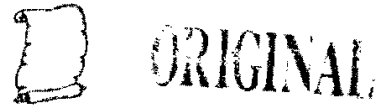

RELINQUISHED BY/REMOVED FROM

DATE/TIME

RECEIVED BY/STORED IN 
200-PW-2 OU Characterization Vadose Zone - Geochemicai Modeling Param F10-045

302117 ES30

GOVERNMENT VEHICLE

SHIPPED TO

N/A

BILL OF LADING/AIR BILL NO.

N/A

\section{SPECIAL INSTRUCTIONS}

** The 200 Area S\&GRP Characterization and Monitoring Sampling and Analysis GKI applies to this SAF.
** ESL shall submit all data deliverables according to SOW "200-LW-2 and 200-BP-5 Operable Units/Contaminant and Transport Property Analysis and Report for Vadose Zone Sediments at Borehole C5860/Well 299-E29-54" for C7515/Well 299-E28-30 ("M" Well) and shail adhere to all S\&GRP standard protocol.

** The ESL laboratory shall meet prlor requested and confirmed turnaround times and RDLs specified in the SOW and this SAF. The laboratories must notify the SM Project Coordinator in writing iffwhen this requirement can not be met. (1)6010M_ICP_ASTM_AE (TAL) \{Sodium, Aluminum, Iron, Potassium, Antimony, Nickel, Calcium, Magnesium, Silver\} 6010M_ICP_ASTM_AE (Add-On) \{Lead, Thailium, Selenium\} 6010M_METALS_ICP_WE (TAL) \{Sodium, Aluminum,

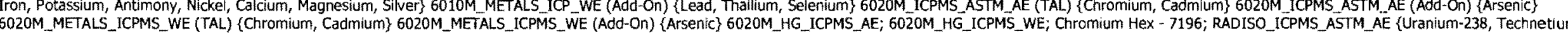
93\} RADISO_ICPMS_WE \{lodine-129, Uranium-238, Teennetium-99\} I-129 by ICPMS \{Iodine-129\} GAMMA_GS; URANIUM ISOTOPIC RATIOS; ALPHA_BETA_AE \{Gross beta, Gross alpha\} ALPHA_BETA_WE \{Gross beta, Gross alpha\} IC

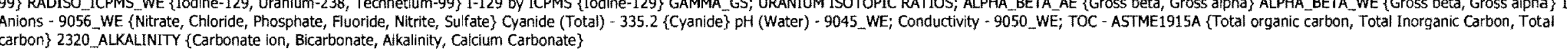

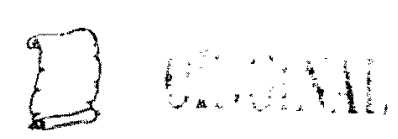




\section{fust. Chamberline, kaver, fosane, Gavia DYEKMAN, DL}

F10-045-104

PAGE 1 OF 2

C7515 (299-E28-30): 1069

ICE CHEST NO.

PROJECT DESIGNATION

TELEPHONE NO.

PROJECT COORDINATOR

SAF NO.
200-PW-2 OU CNaTION

FIELD LOGBOOK NO.

Fiono

ows - 169

SHIPPED TO

OFFSTTE PROPERTY NO.

$177 \cdot 0^{\prime}$

COA 302117 ES30

PRICE CODE

AIR QUALIT

DATA
TURNAROUND
45 DaYS/ 45

$\mathrm{N} / \mathrm{A}$

Environmental Sciences Laboratory

MATRIX* POSSIBLE SAMPLE HAZARDS/ REMARKS

$A=A i r$
$D L=D r u m$ Contains Radioactive Material at concentrations.

$\begin{array}{ll}\text { Liquids } & \text { that may or may not be regulated for } \\ \text { DS =Drum } & \text { transportation per } 49 \text { CFR / IATA Dangerous }\end{array}$ Goods Regulations but are not releasable per
DOE Order $5400.5(1990 / 1993)$

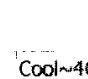

IILL OF LADING/AIR BILL NO.

METHOD OF SHIPMENT

\section{TYPE OF CONTAINER G Gip $\begin{aligned} & \text { Moisture } \\ & \text { Resistant cont }\end{aligned}$}

NO. OF CONTAINER(S)

volume

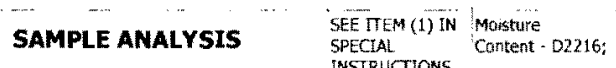

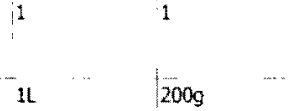

SPECIAL HANDLING AND/OR STORAGE RADIOACTIVE TIE TO: B23639

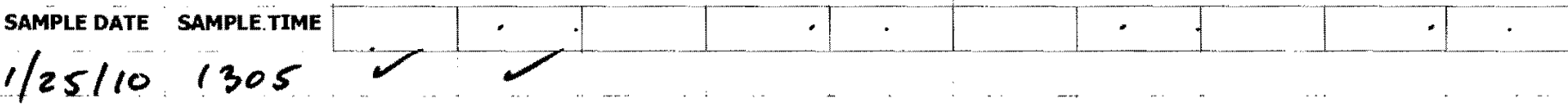

CHAIN OF POSSESSION

SIGN/ PRINT NAMES

SPECIAL INSTRUCTIONS

RELINQUISHED BY/REMOVEP FROM DATE/TIME RECEIVED BY/STORED IN PATE/TIME SEE PAGE 2 FOR ALL SPECIAL INSTRUCTIONS

Jomuin Coria $1 / 25110 / 1520$ M0-413 SSU-R2 $1 / 25110 / 520$

RE RE Brothentor B. SEC

\section{RELINQUISHED BY/REM}

JAN 262010

DW Brotherton B B J DATE/TIME 202010

RELINQUUTSHED BY/REMOVED FROM

DATE/TIME

RECEIVEDY KutsTORED IN $11: 15$

JAN 26 DATE 6010

RELINOUISHED BY/REMOVED FRÖM

DATE/TIME

Regerved gystgRed IV

JAN TRGE $_{\text {G }}$ W

CEIVED BYISTRED IN

$\{$ minili

RELINQUISHED BY/REMOVED FROM

DATE/TIME

RECEIVED BY/STORED IN

DATE/TIME

DATE/TME 
CH2MHill Plateau Remediation Company

$302117 E S 30$

\section{METHOD OF SHIPMENT}

BILL OF LADING/AIR BILL NO. $\mathrm{N} / \mathrm{A}$

N/A

\section{SPECTAL INSTRUCTIONS}

** The 200 Area S\&GRP Characterization and Monitoring Sampling and Analysis GKI applies to this SAF.

** ESL shall submit all data deliverables according to SOW "200-LW-2 and 200-BP-5 Operable Units/Contaminant and Transport Property Analysis and Report for Vadose Zone Sediments at Borehole C5860/Well 299-E29-54" for

** The ESL laboratory shall meet prior requested and confirmed turnaround times and RDLs specified in the SOW and this SAF. The laboratories must notify the SM Project Coordinator in writing iff when this requirement can not be met. (1)6010M_ICP_ASTM_AE (TAL) \{Sodium, Aluminum, Iron, Potassium, Antimony, Nickel, Calcium, Magnesium, Silver\} 6010M_ICP_ASTM_AE (Add-On) \{Lead, Thallium, Selenium\} 6010M_METALSIICP_WE (TAL) \{Sodium, Aluminum, Iron, Potassium, Antimony, NIckel, Calcium, Magnesium, Siver) 6010M_METALS_ICP.WE (Add-On) \{Lead, Thallium, Selenium\} 6020M__CPMS_ASTM_AE (TAL) \{Chromium, Cadmium\} 6020M_ICPMS_ASTM_AE (Add-On) \{Arsenic\}, 99\} RADISO ICPMS WE \{Iodine-129, Uranium-238, Technet um-99\} I-129 by ICPMS \{lodine-129; GAMMA GS; URANIUM ISOTOPIC RATIOS; ALPHA BETA AE \{Gross beta, GrosS alpha\} ALPHA BETA WE \{Gross beta, Gross alpha\} IC Anions - 9056_WE \{Nitrate, Chloride, Phosphate, Fluoride, Nitrite, Sulfate\} Cyanide (Total) - 335.2 \{Cyanide\} pH (Water) - 9045 WE; Conductivity - 9050_WE; TOC - ASTME1915A (Total organic Carbon, Total Inorganic Carbon, Total carbon\} 2320_ALKALINITY \{Carbonate ion, Bicarbonate, Akalinity, Calcium Carbonate\}

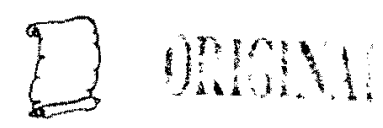




$$
\text { TELEPHONE NO. }
$$

Rust, Rosame, keneer, Chametrer line, Garci DYEKMAN, DL

C7515 (299-E28-30): I-070

ICE CHEST NO.

PROJECT DESIGNATION

$373-2530$

PROJECT COORDINATOR

\begin{tabular}{l|l} 
SAF NO. \\
F10-045
\end{tabular}

PRICE CODE

DATA
TRNAROUND

FIELD LOGBOOK NO.

ACTUAL SAMPLE DEPTH

COA

HNF-N- $491-5 \rho 50,179.15$

302117ES30

AIR QUALITY

45 Days / 45
Days

$6 \underset{\text { Hipped To }}{0}$

OFFSITE PROPERTY NO.

METHOD OF SHIPMENT

N/A

Environmental Sciences Laboratory

PRESERVATION

MATRIX* POSSIBLE SAMPLE HAZARDS/ REMARKS

$\mid \begin{aligned} & A=A i r \\ & \text { DL=Drum }\end{aligned}$

that may or may not be regulated for
transportation per 49 CFR / IATA Dangerous

transportation per 49 CrR/ IATA Dangerous
Goods Regulations but are not releasable per
DOE Order 5400.5 (1990/1993)

TYPE OF CONTAINER /G/P $\quad \begin{aligned} & \text { Moisture } \\ & \text { Resistant Cont }\end{aligned}$

NO. OF CONTAINER(S)

volume

SAMPLE ANALYSIS SEF TEM (1) IN Molsture -

SPECIAL HANDLING AND/OR STORAGE
RADIOACTVE TIE TO: B23639

- Sample No

MATRIX*

SAMPLE dATE SAMPLE TIME :

$1 / 25 / 10$ 1405

SIGN/ PRINT NAMES

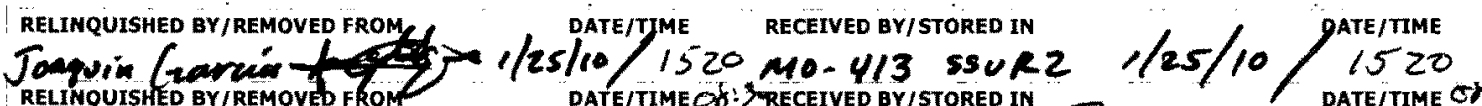
SSU-R2

JAN 262010 DW Brothertor: FDCZ JAN 26.2010

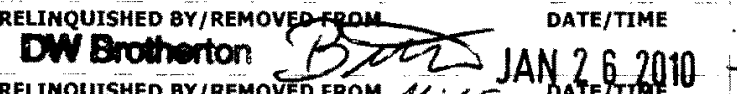

DW Erotherton 372

RELINQUISHED BY/REMOVED FROM

DATE/TIME

RECEIYED BVISTORED WO $11: 15$

Regived ayd fopeo In

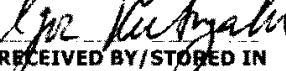

JAN 262010

DATE/TIME

E? IRoflit

RELINQUISHED BY/REMOVEO FROM

DATE/TIME

RECEIVED BY/STORED IN

DATE/TIME

RECEIVED BY/STORED IN

RELIMQUISHED BY/REMOVED FROM
SPECIAL INSTRUCTIONS

EA PAGE 2 FOR ALL SPECIAL INSTRUCTIONS

RECEIVED BY

SECTION
SERATORY

FINAL SAMPLE DISPOSAL METHOD 


\section{$6005-169$}

\section{HNF-N- $491-5$ OFFSTIE PROPERTY NO.} $\mathrm{N} / \mathrm{A}$

302117 ES30

METHOD OF SHIPMENT

BILL OF LADING/AIR BILL NO

SPECIAL INSTRUCTIONS

$\mathrm{N} / \mathrm{A}$

** The 200 Area S\&GRP Characterization and Monitoring Sampling and Analysis GKI applies to this SAF.

** ESL shall submit all data deliverables according to SOW "200-LW-2 and 200-BP-5 Operable Units/Contaminant and Transport Property Analysis and Report for Vadose Zone Sediments at Borehole C5860/Well 299-E29-54" for 299-E28-30 ("M" Well) and shall adhere to all S\&GRP standard protocol.

** The ESL laboratory shall meet prior requested and confirmed turnaround times and RDLs specified in the SOW and this SAF. The laboratories must notify the SM Project Coordinator in writing if/when this requirement can not be met. (1)6010M_ICP_ASTM_AE (TAL) \{Sodium, Aluminum, Iron, Potassium, Antimony, Nickel, Calcium, Magnesium, Silver\} 6010M_ICP_ASTM_AE (Add-ON) \{Lead, Thallium, Selenium\} 6010M_METALS_ICP_WE (TAL) \{Sodium, Aluminum, 6020M_METALS_ICPMS WWE (TAL) \{Chromium, Cadmlum\} 6020M_MEIALS ICPMS WE (Add-ON) \{Arsenic\}6020M_HG_ICPMS_AE; 6020M_HG_ICPMS_WE; Chromium Hex-7196; RADISO_ICPMS_ASTM_AAE \{Uranium-238, Technetium99\} RADISO_ICPMS_WE \{lodine-129, Uranium-238, Technetium-99\} I-129 by ICPMS \{Iodine-129\} GAMMA_GS; URANIUM ISOTOPIC RATIOS; ALPHA_BETA_AE \{Gross beta, Gross alpha\} ALPHA_BETA_WE \{Gross beta, Gross alpha\} IC Anions - 9056 WE _Nitrate, Chloride, Phosphate, Fuoride, Nitrite, Sulfate\} Cyanide (Total) - 335.2 \{Cyanide\} PH (Water) - 9045_WE; Conductivity - 9050_WE; TOC - ASTME1915A \{Total organic carbon, Total Inorganic Carbon, Total
carbon\} 2320_ALKALINITY \{Carbonate ion, Bicarbonate, Alkalinity, Calcium Carbonate\}

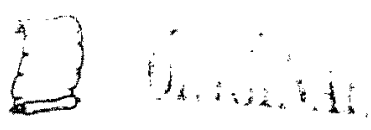


PROJECT COORDINATOR

\begin{tabular}{|l|l|l|}
\hline DYEKMAN, DL & $373-2530$ & DYEKMAN, DL \\
\hline
\end{tabular}
SAF NO.
F10-045

SAMPLING LOCATION

PROJECT DESIGNATION

200-PW-2 Ou Characte

COA

6 LCS -168

IELD LOGBOOK NO.

ACTUAL SAMPLE DEPTH

PRICE CODE $\quad 8 \mathrm{~N}$

HNF-N- 491-5pg $50 \quad i 79.15^{\prime}-181.65^{\prime}$

COA

AIR QUALITY

\section{METHOD OF SHIPMENT} BILL OF LADING/AIR BILL NO.

Environmental Sciences Laboratory

N/A

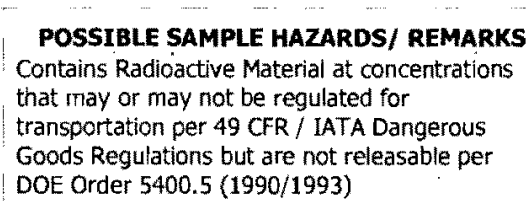

POSSIBLE SAMPLE HAZARDS/ REMARKS Contains Radioactive Material at concentrations that may or may not be regulated for

Goods Regulations but are not releasable per DOE Order 5400.5 (1990/1993)

PRESERVATION

TYPE OF CONTAINER Split Spoon

12

volume

10009

SPECIAL HANDLING AND/OR STORAGE RADIOACTIVE TIE TO: 823639

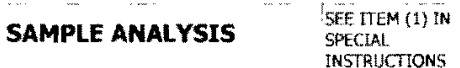$$
\text { GOVERNMENT VEHICLE }
$$

SAMPLE DATE SAMPLE TIME

$1 / 25 / 10 \quad \ldots 1405$

B235B1 SOIL

MATRIX*

\section{SIGN/ PRINT NAMES}

\section{CHAIN OF POSSESSION}

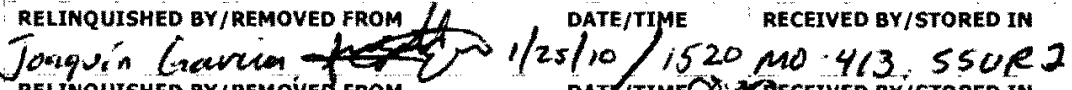

\section{SSOY-R2 \\ JAN 2 TIT 6010

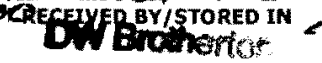

RELINQUISHED BY/REM

DW Brotherton
EELINQUISHED BY/REMOVED POOM $/ 1: 15$ DATE/TME

RELINQUUISHED BY/REMOYED FROM

DATE/TIME

T.Kutryater

REREIVED M STORED WW

RELINQUISHED BY/REMOVED FROM

DATE/TIME

RECEIVED QY/STORED IN

RELINQUISHED BY/REMOVED FROM

Date/TIME

RECEIVED BY/STORED IN

\section{SPECIAL INSTRUCTIONS}

** The 200 Area S\&GRP Characterization and Monitoring Sampling and Analysis *

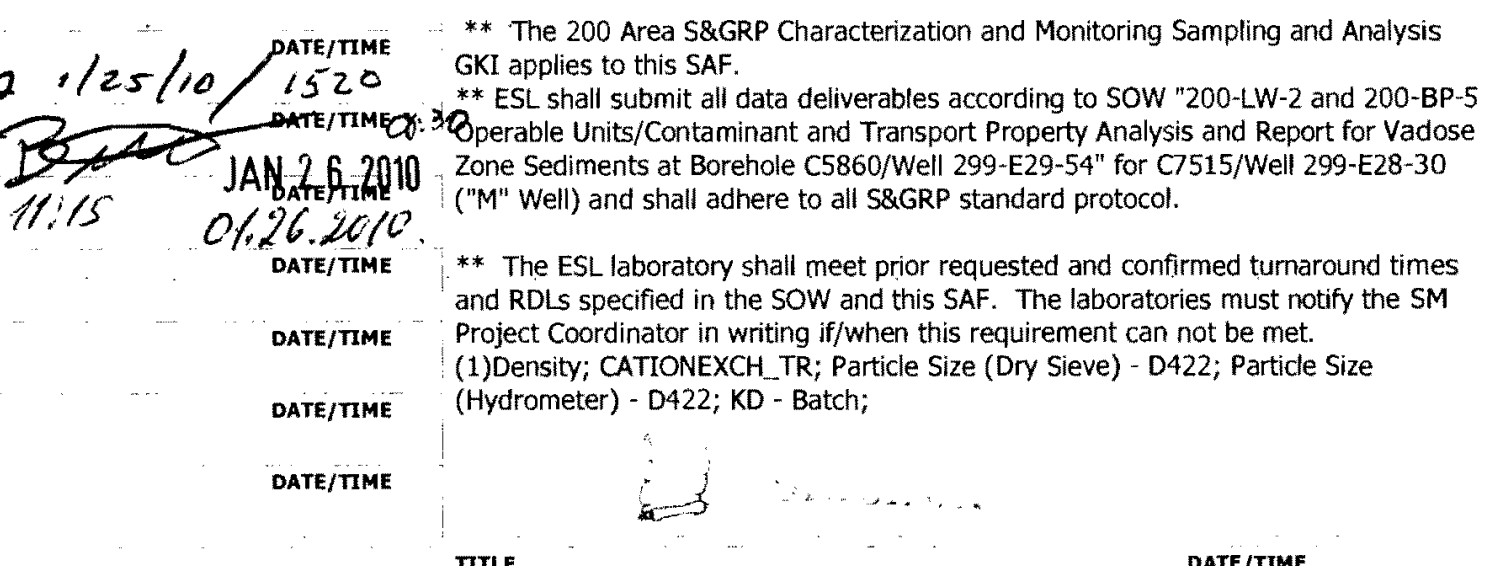

TITL
SECTION
LAORATORY

FINAL SAMPLE
DISPOSITION
DATE/TIME

DISPOSED BY

A-6003-6188(01/06) 
200-PW-2 OU Characterization Vadose Zone - Geochemical Modeling Param $\begin{aligned} & \text { SAF NO- } \\ & \text { F10-045 }\end{aligned}$

AIR QUALITY ACTUAL SAMPLE DEPTH COA

METHOD OF SHIPMENT FIELD LOGBOOK NO.

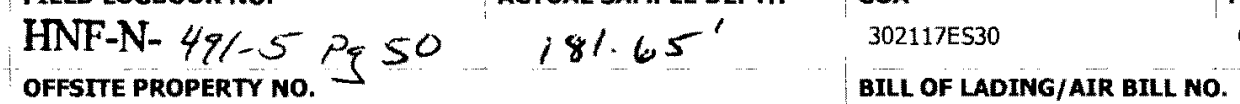

COA

SHIPPED TO

Environmental Sciences Laboratory N/A

MATRIX* POSSIBLE SAMPLE HAZARDS/ REMARKS

$A=A i r$
$\mathrm{DL}=$ Drum

Liquids
DS $=$ Drum that may or may not be regulated for
transportation per 49 CFR / IATA Dangerous

transportation per 49 CFR / IATA Dangerous
Goods Regulations but are not releasable per
DOE Order 5400.5 (1990/1993)

SPECIAL HANDLING AND/OR STORAGE RADIOACTIVE TIE TO: B23639

\section{PRESERVATION}

TYPE OF CONTAINER G/P $\quad \begin{aligned} & \text { Moisture } \\ & \text { Resistant cont }\end{aligned}$

NO. OF CONTAINER(S)

VOLUME

N/A

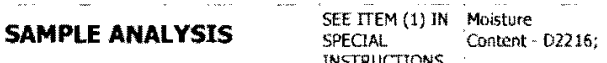

- SAMPLE No

MATRIX*

SAMPLE DATE SAMPLE TIME $1 / 25-10$

\section{SIGN/ PRINT NAMES}

RELINQUISHED BY/REMOVED FROM/ DATE/TIME

DATE/TIME RECEIVED BY/STORED IN

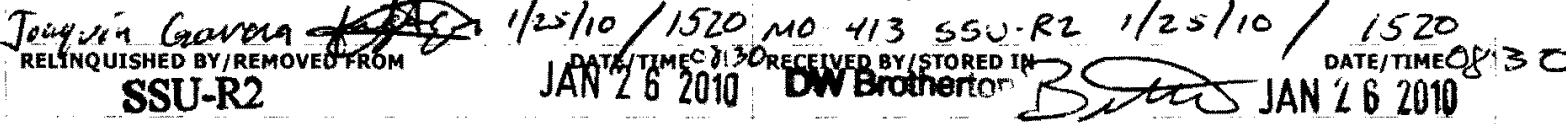

JAAT 2 G 6010

RECEIvED BY/STOREP IN

RELINQUISHED BY/REMOVED FROM $11: 15$ J JAN 262010

RELINQUUISHED BY/REMOVED FROM

DATE/TIME

REGEIVEQ BYis JORED

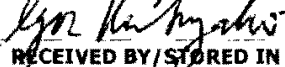

DATE/TIME

RECEIVED BY/STORED IN

DATE/TIME

RECEIVED BY/STORED IN
JAND? 26,2010

DATE/TIME

DATE/TIME

Date/time
RELINQUUISHED BY/REMOVED FROM
SPECIAL INSTRUCTIONS

EE PAGE 2 FOR ALL SPECIAL INSTRUCTIONS 


\title{
SPECIAL INSTRUCTIONS
}

\author{
N/A
}

** The 200 Area S\&GRP Characterization and Monitoring Sampling and Analysis GKI applies to this SAF.

** ESL shall submit all data deliverables according to SOW "200-LW-2 and 200-BP-5 Operable Units/Contaminant and Transport Property Analysis and Report for Vadose Zone Sediments at Borehole C5860/Well 299-E29-54" for

C7515/Well 299-E28-30 ("M" Well) and shall adhere to all S\&GRP standard protocol.

** The ESL laboratory shail meet prior requested and confirmed turnaround times and RDLs specified in the SOW and this SAF. The laboratories must notify the SM Project Coordinator in writing iff/when this requirement can not be met. Iron, Potassium, Antimony, Nickel, Calcium, Magnesium, Silver\} 6010M_METALS_ICP_WE (Add-On) \{Lead, Thallium, Selenium\} 6020M_ICPMS_ASTM_AE (TAL) \{Chromium, Cadmium\} 6020M_ICPMS_ASTM_AE (Add-On) \{Arsenic\}

6020M_METALS ICPMS_WE (TAL) \{Chromium, Cadmium\} 6020M_METALS_ICPMS_WE (Add-ON) \{Arsenic\} 6020M_HG_ICPMS_AE; 6020M_HG_ICPMS_WE; Chromium Hex - 7196; RADISO_ICPMS ASTM_AE \{Uranium-238, Technetium99\} RADISO_ICPMS WE \{Lodine-129, Uranium-238, Technetium-99\} I-129 by ICPMS \{Iodine-129\} GAMMA_GS; URANIUM ISOTOPIC RATIOS; ALPHA_BETA_AE \{Gross beta, Gross alpha\} ALPHA_BETA_WE \{Gross beta, Gross alpha\} IC Anions - 9056-WE \{Nitrate, Chloride, Phosphate, Fluorlde, Nitrite, Suffate\} Cyanide (Total) - 335.2 \{Cyanide\} pH (Water) - 9045_WE; Conductivity - 9050_WE; TOC - ASTME1915A \{Total organic carbon, Total Inorganic Carbon, Total
carbon\} 2320 ALKALINITY \{Carbonate ion, Bicarbonate, Alkalinity, Calclum Carbonate\}

$\{310.11$ 
PROJECT COORDINATOR

\begin{tabular}{l|l|l}
\hline DYEKMAN, DL & $373-2530$ & DYEKMAN, DL \\
\hline
\end{tabular}

PROJECT DESIGNATION SAF NO.

PRICE CODE

200-PW-2 Ou Characterization Vadose Zone - Geochemical Modeling Param F10-045

FIELD LOG

ACTUAL SAMPLE DEPTH

AIR QUALITY

Gus - 169

Environmental Sciences Laboratory

MATRIX*

HNF-N- $491-5$ Pg 5

$181.5^{\prime}-184.0^{\circ}$

COA

METHOD OF SHIPMEN

N/A

BILL OF LADING/AIR BILL NO.

N/A

POSSIBL SAMPLE HAZARDS/ REMARIS

Contains Radioactive Material at concentrations

that may or may not be regulated for

Goods Regulations but are not releasable per

DOE Order 5400.5 (1990/1993)

TYPE OF CONTAINER Split SPOON

Do comtaimer(s)

volume

10009

SPECIAL HANDLING AND/OR STORAGE

SPECIAL HANDLING AND/OR
RADIOACTTVE TIE TO: B23640

SAMPLE ANALYSIS SEE TEM (1) IN

SAMPLE NO.

B235B2

MATRIX*

$$
\text { SOIL" }
$$

SAMPLE DAJE SAMPLE TIME

$1 / 25 / 10: 1440$

\section{SIGN/ PRINT NAMES}

\section{CHAIN OF POSSESSION}

REINQUISHED BY/REMOVED FROM Johquín Carvia SSU-R2

RECEIVED BY/STORED IN $1 / 25 / 10^{\text {DATE/TIME }} / 1520$ DATE/TIMESS : JAN 2620101 DW Brotherton S.en 25 JAN 262010 RELINQUISHED BY/REM OVEB FROM 11.15 DATE/TIME

RELINQUISHED BY/REMOVED FROM

DATE/TIME

GWFProtherto IN

\section{RECEIVED BY/STOREQ IN}

I Ku tnea kis 1.1.15

REgIVED QY/PORED IN/T-

Rg CEIVED BY/SEGRED IN

RELINQUISHED BY/REMOVED FROM

DATE/TIME

RECEIVED BY/STORED IN

DATE/TIME

RECEIVED BY/STORED IN

RECEIVED BY

LABORATORY
SECTION

INAL SAMPLE

\section{SPECIAL INSTRUCTIONS}

** The 200 Area S\&GRP Characterization and Monitoring Sampling and Analysis DATE/TIME ** The 200 Area S\&GRP

1520 ** ESL Shall this sAF. DATe/TIMer: 3 Operable Units/Contaminant and Transport Property Analysis and Report for Vadose JAN 26 2010 Zone Sediments at Borehole C5860/Well 299-E29-54" for C7515/Well 299-E28-30 ("M" Well) and shall adhere to all S\&GRP standard protocol.

JAN 262010

DATE/TIME

** The ESL laboratory shall meet prior requested and confirmed tumaround times and RDLS specified in the SOW and this SAF. The laboratories must notify the SM Project Coordinator in writing if/when this requirement can not be met.

DATEITME (1)Density; CATIONEXCH_TR; Particle Size (Dry Sieve) - D422; Particle Size

DATE/TME (Hydrometer) - D422: KD - Batch; 
PROJECT DESIGNATION

PRICE CODE

8N

200-PW-2 Ou Characterization Vadose Zone - Geochemical Modeling Param F10-045

ICE CHEST NO.

200-PW-2 OU Characte
FIELD LOGBOOK NO.

ACTUAL SAMPLE DEPTH COA

AIR QUALITY

45 Days $/ 45$
Days

$605-168$

HNF-N- 49/-5 PG 50 184.

METHOD OF SHIPMENT

OFFSTIE PROPERTY NO. BILL OF LADING/AIR BILL NO.

Environmental Sciences Laboratory

MATRIX* POSSIBLE SAMPLE HAZARDS/ REMARKS

\begin{tabular}{l|l}
$A=A i r$ \\
DL=Drum
\end{tabular}

that may or may not be regulated for

Goods Regulations but are not releasioto

Goods Regulations but are not
DOE Order 5400.5 (1990/1993)

SPECIAL HANDLING AND/OR STORAGE RADIOACTIVE TE TO: B23640

PRESERVATION

TYPE OF CONTAINER IG $\quad \begin{aligned} & \text { Moisture } \\ & \text { Resstant Cont }\end{aligned}$

No. OF CONTAINER(S)

volume

$1 \mathrm{~L} \quad 200 \mathrm{~g}$

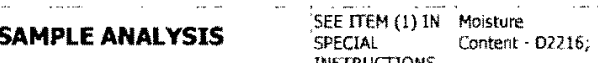

N/A

SAMPLENO. MATRIX* SAMPLE DaTE 'SAMPLE TIME

$1 / 25110,1440$

CHAIN OF POSSESSION

SIGN/ PRINT NAMES

SPECIAL INSTRUCTIONS

RELINQUISHED BY/REMOVED FRQY DATE/TIME RECEIVED BY/STORED IN

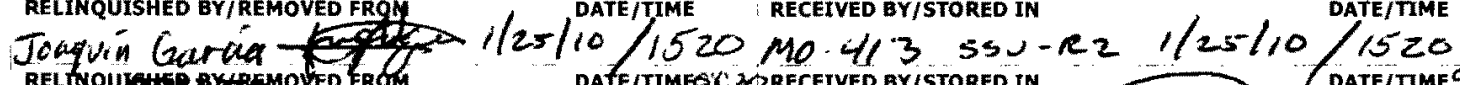

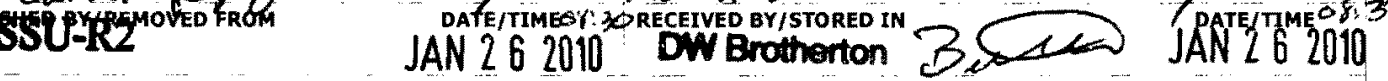

RELINQUISHED BY/REMdVEDFROM

$\$$ Plifun

RELINQUISHED BY/REMOVED FROM

DATE/TIME

lape

DATE/TMME

RELINQÜISHED BY/REMOVED FROM

DATE/TIME

RECEIVED BY/STORED IN

DATE/TIME

RELINQUUISHED BY/REMOVED FROM

DATE/TIME

RECEIVED BY/STORED IN

DATE/TMEE 
BILL OF LADING/AIR BILL NO.

\section{SPECIAL INSTRUCTIONS}

** The 200 Area S\&GRP Characterization and Monitoring Sampling and Analysis GKI applies to this SAF.
** ESL shall Submit all datat deliverables according to SOW "200-LW-2 and 200-BP-5 Operable Units/Contaminant and Transport Property Analysis and Report for Vadose Zone Sediments at Borehole C5860/Well 299-E29-54" for ** ESL shall submit all data deliverables according to SOW "200-LW-2 and 200-BP-5
C7515/Well 299-E28-30 ("M" Well) and shall adhere to all S\&GRP standard protocol.

** The ESL laboratory shall meet prior requested and confirmed turnaround times and RDLs specified in the SOW and this SAF. The laboratories must notify the SM Project Coordinator in writing iffwhen this requirement can not be met. (1)6010M_ICP_ASTM_AE (TAL) (Sodium, Aluminum, Iron, Potassium, Antimony, Nickel, Calcium, Magnesium, Silver\} 6010M_ICP.ASTM_AE (Add-On) \{Lead, Thallium, Selenium\} 6010M_METALS_ICP_WE (TAL) \{Sodium, Aluminum, 6020M_METALS ICPMS WE (TAL) \{Chromium, Cadmium\} 6020M METALS ICPMS_WE (Add-On) \{Arsenic\}6020M_HG IICPMS_AE; 6020M_HG_ICPMS. WE; Chromium Hex - 7196; RADISO_ICPMS_ASTM_AE \{Uranium-238, Technetium9\} RADISO_ICPMS_WE \{Iodine-129, Uranium-238, Technetium-99\} I-129 by ICPMS \{Iodine-129\} GAMMA_GS; URANIUM ISOTOPIC RATIOS; ALPHA_BETA_AE \{Gross beta, Gross alpha\} ALPHA_BETA_WE \{Gross beta; Gross alpha\} IC Anions - 9056_WE \{Nitrate, Chloride, Phosphate, Fluoride, Nitrite, Sulfate\} Cyanide (Total) - 335.2 \{Cyanide\} PH (Water) - 9045_WE; Conductivity - 9050_WE; TOC - ASTME1915A \{Total Organic Carbon, Total Inorganic Carbon, Total carbon\} 2320_ALKALINITY \{Carbonate ion, Bicarbonate, Alkalinity, Calcium Carbonate\}

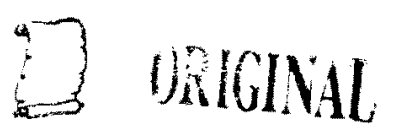


CHAIN OF CUSTODY/SAMPLE ANALYSIS REQUEST

COJLECTOR

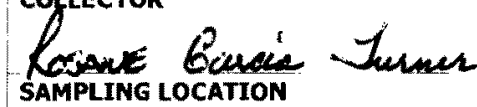

C7515 (299-E28-30); I-075

C7515 (299-E28-

sin $\angle-100$

SHIPPED TO

Environmental Sciences Laboratory

MATRIX* POSSIBLE SAMPLE HAZARDS/ REMARKS Contains Radioactive Material at concentrations that may or may not be regulated for

transportation per 49 CFR / IATA Dangerous Goods Regulatons but are not releasable per

SPECIAL HANDLING AND/OR STORAGE RADIOACTIVE TIE TO: B23640

SAMPLE NO. MATRIX* B235B3 SOIL MATRIX

\section{COMPANY CONTACT}

DYEKMAN, DL

TELEPHONE NO.

$373-2530$

PROJECT DESIGNATION

200-PW-2 OU Character
FIELD LOGBOOK NO.

HNF-N- $49 /-5$ pg $5 /$

N/A

PReservation

TYPE OF CONTAIMER

NO. OF CONTAINER(S)

volume

SAMPLE ANALYSIS

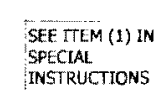
N/A

BILL OF LADING/AIR BILL NO.

$\cdots$

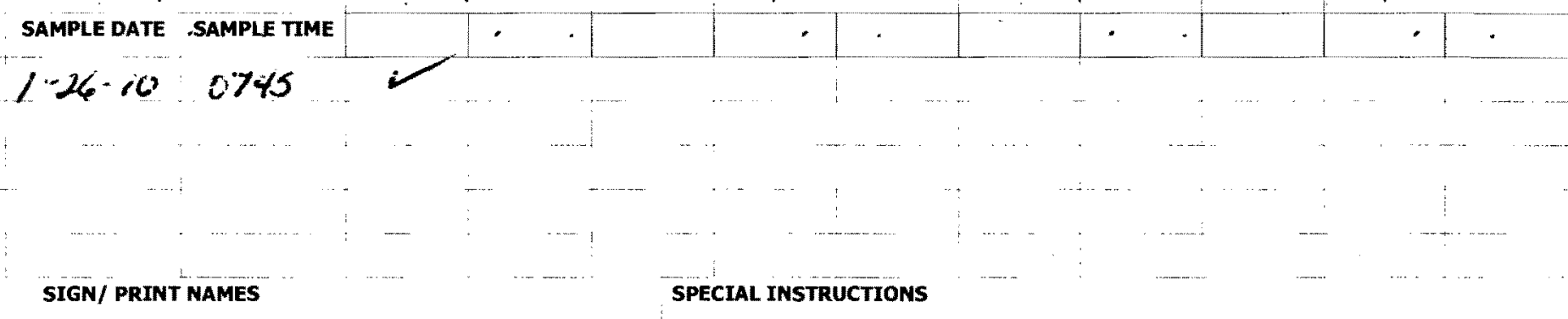

\section{CHAIN OF POSSESSION}

\section{SIGN/ PRINT NAMES}

RELINQUSSHED BY/REMOVER FROM DATE/TIME RECEIVED BY/STORED IN

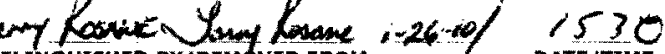
Mo -3 (3)

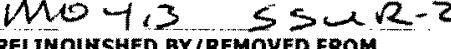
PELINQUISHED BY/REMOVED FROM

RELINQUISHED BY/REMOVED FROM 11,00

RELINQUISHED BY/REMOVED FROM DATE/TIME $1-270$ $1-2 D_{1} / 0$ i- 2 DATE/TIME.

CEEIVEO gY/STORED IN

DATE/TME

** The 200 Area S\&GRP Characterization and Monitoring Sampling and Analysis
GKI applies to this SAF.

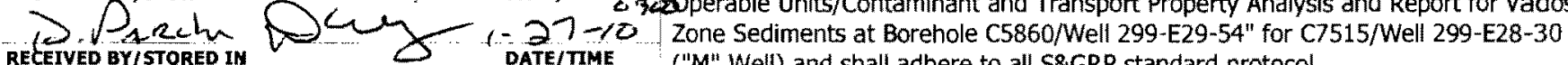
T.Ku huga kor $1-27-10$

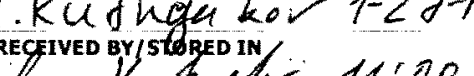

DATE/TMME in thatar 11:00

** The ESL laboratory șhall meet prior requested and confirmed tumaround times. DATE/TIME REgEVED BY/ST GDED IN

DATE/TME and RDLS specified in the SOW and this SAF. The laboratories must notify the SM

RELINQUISHED BY/REMOVED FROM

DATE/TIME

RECEIVED BY/STORED IN

DATE/TMME (1)Density; CATIONEXCH TR; Particle Size (Dry Sieve) - D422; Particle Size

RELIMQUTSHED BY/REMOVED FROM

DATE/TIME

PECEIVED BY/STORED IN

DATE/TTME

2 ORIGINAL

DISPOSED BY
DATE/TIME

DATE/TIME 
CH2MHill Plateau Remediation Company

CHAIN OF CUSTODY/SAMPLE ANALYSIS REQUEST

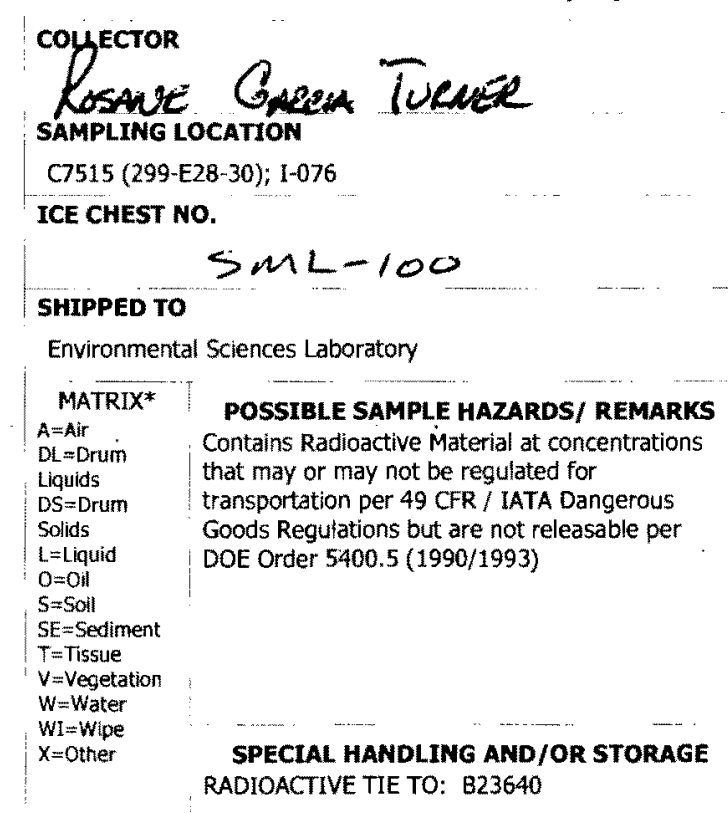

F10-04S-114

PAGE 1 OF 2

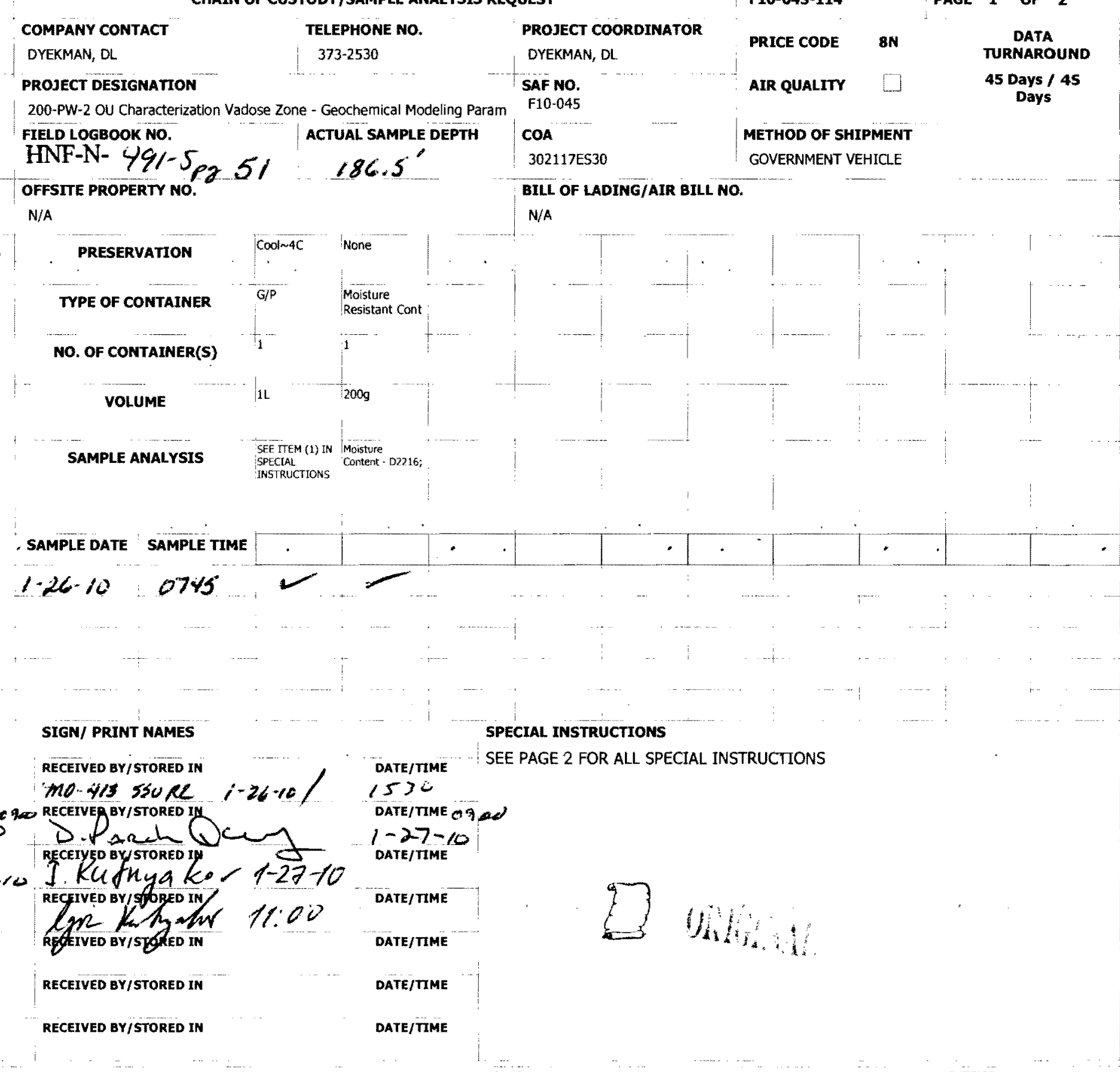


SHIPPED TO

HNF-N- $4 \%$ ACTUAL SAMPLE DEPTH

Environmental Sciences Laboratory

186.5

COA

METHOD OF SHIPMENT

TiLL OF LADING/AIR BILL No.

\section{SPECIAL INSTRUCTIONS}

*** The 200 Area S\&GRP Characterization and Monitoring Sampling and Analysis GKI applies to this SAF.

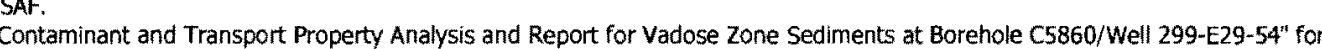

** The ESL laboratory shall meet prior requested and confirmed turmaround times and RDLs specified in the SOW and this SAF. The laboratories must notify the SM Project Coordinator in writing iff/when thils requirement can not be met. (1)6010M_ICP_ASTM_AE (TAL) \{Sodium, Aluminum, Iron, Potassium, Antimony, Nickel, Calcium, Magnesium, Silver\} 6010M_ICP_ASTM_AE (Add-On) \{Lead, Thallium, Selenium\} 6010M_METALSIICP_WE (TAL) \{Sodium, Aluminum,

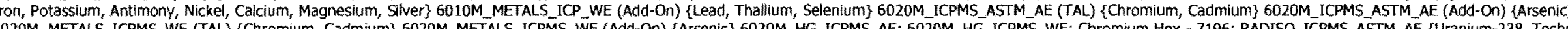

9) RADISO IIPMS WE \{IDdine-129, Uranium-238, Technetium-99\} I-129 by ICPMS \{Iodine-129\} GAMMA GS; URANIUM ISOTOPIC RATDOS- ALPHA BETA AE \{Gross beta, Gross alpha\} ALPHA BETA WE \{Gross beta, Gross alpha\} IC

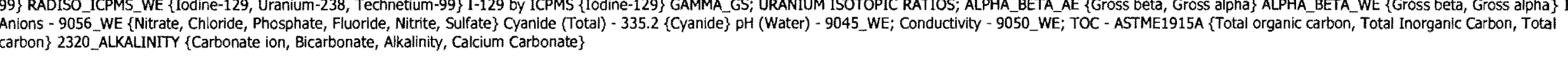


CH2MHill Plateau Remediation Company

\section{collecto}

Costue Garien Tinmel

C7515 (299-E28-30)7

ICE CHEST NO.

$\operatorname{son}<-100$

HIPPED TO

Environmental Sciences Laborator

MATRIX* POSSIBLE SAMPLE HAZARDS/ REMARKS

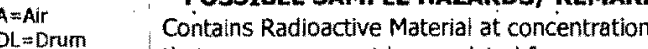

that may or may not be regulated for

Dansportar ma per 49 crim / IATA Dangerous

Goods Regulations but are not releasable per
DOE Order $5400.5(1990 / 1993)$

$S=S a l l$
$S E=$ Sediment
$T=$ Tissue
$V=$ Vegetation

$W=$ Wate
$W I=W i p e$

SPECIAL HANDLING AND/OR STORAGE RADIOACTIVE TIE TO: B23641
SAMPLE NO

B235B4
MATRIX*

\section{CHAIN OF CUSTODY/SAMPLE ANALYSIS REQUEST}

F10-045-116

PAgE 1 OF 1

TELEPHONE NO.

PROJECT COORDINATOR

373-2530

DYEKMAN, DL

$\begin{array}{ll}\text { SAF NO. } & \\ \text { F10.045 }\end{array}$

PROJECT DESIGNATION

200-PW-2 OU Characterization Vadose Zone - Geochemical Modeling Param
FIELD LOGBOOK NO. ACTUAL SAMPLE DEPTH

ACTUAL SAMPLE DEPTH
$182.7^{\prime} \cdot 189.2$

HNF-N- $49 \%-5$

PRESERVATION

TYPE OF CONTAINER

NO. OF CONTAINER(S)

VOLUME

COA
$302117 E S 30$

PRICE CODE $\quad 8 \mathrm{~N}$

AIR QUALITY

DATA

Days

METHOD OF SHIPMENT

GOVERNMENT VEHICLE

BILL OF LADING/AIR BILL NO.

N/A

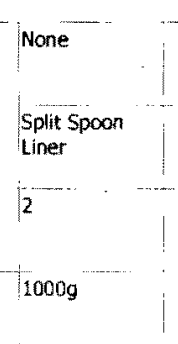

SAMPLE ANALYSIS

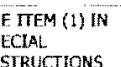

SAMPLE DATE SAMPLE TIME

$1-2610 \quad 0835$

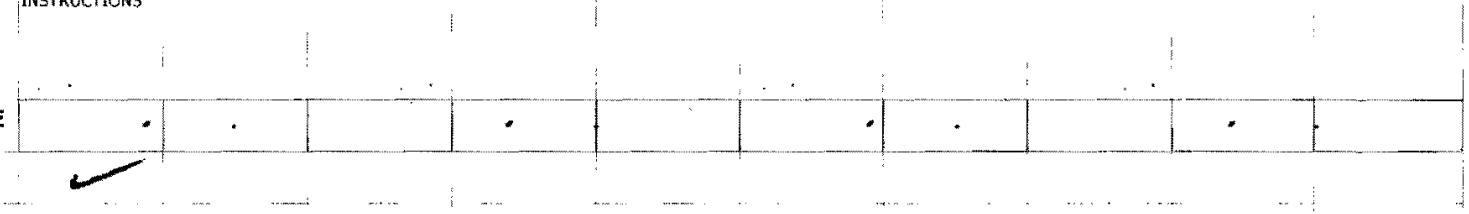

SIGN/ PRINT NAMES

CHAIN OF POSSESSION

DATE/TIME RECEIVED BY/STORED IM

RELINQUISHED BY/REMOVED FROM

$\begin{array}{ll}\text { MO } 413 \text { SSUR2 } & 1-27-10 \\ \text { RELINQYSSED BY/REMOYED FROM } & \text { DATE/TTME }\end{array}$

$1-2) \times 10$

DELINQUISHED GY/REMOVED FROM 61 .00 DATE/TTME

RELINQUISHED BY/REMOYED FROM

DATE/TIME

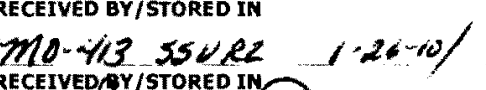

D.PARCL

r. Ku fug a kor $1-27-10$

RECENED BYISTORED IN $11: 00$

in thing

1.00

DATE/TTME

RECEIVED BY/STORED IN

DATE/TIME

RECEIVED BY/STORED IN
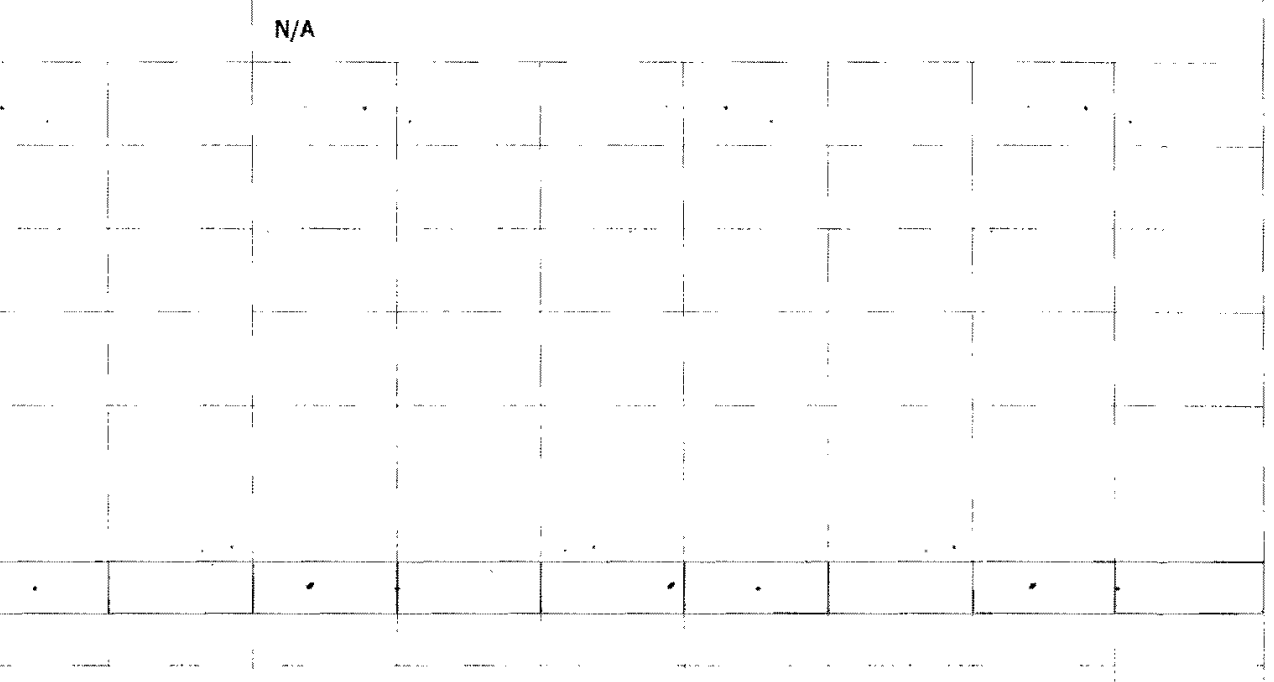

\begin{tabular}{l} 
LABORATORY RECEIVED BY \\
SECTION \\
FINAL SAMPLE \\
\hline
\end{tabular}

FINAL SAMPL
DISPOSITION

ISPOSAL METHOD

DISPOSED BY

DATE/TIME

DATE/TTM 


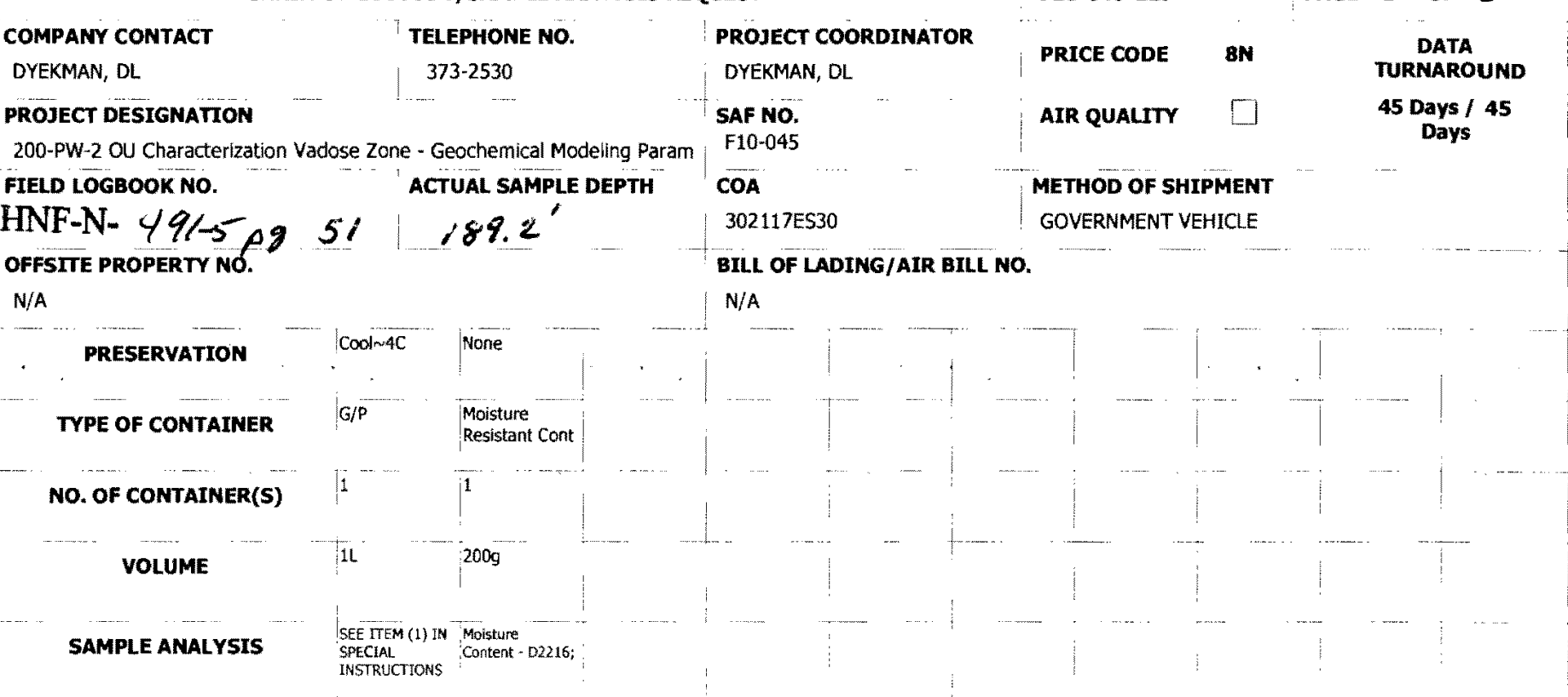
B235M
SAMPLE NO.
MATRIX*
SAMPLE DATE SAMPLE TIME
$i 26.10 \quad 0835$
SOIL

\section{CHAIN OF POSSESSION}

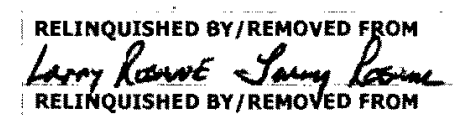
mo4l 3 SSU R2 RELINQYUTHED BY/REMOVED FROM D. ARch

RELINQUISHED QY/REMOVED FROM

RELINQUISHED BY/REMOVED FROM

RELINQUISHED BY/REMOVED FROM

SIGN/ PRINT NAMES

\author{
SPECIAL INSTRUCTIONS \\ DATE /TIME SEE PAGE 2 FOR ALL SPECIAL INSTRUCTIONS \\ 1530 \\ DATE/TTMEGSia \\ $1.27-10$
DATE/TIME \\ DATE/TIME \\ DATE/TIME \\ DATE/TIME \\ DATE/TME

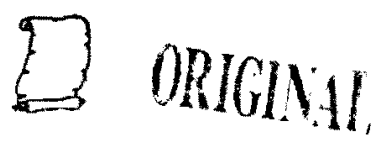

\section{LABORATORY RECEIVED OY \\ SECTION \\ FINAL SAMPLE DISPOSAL METHOD \\ DISPOSITION}


CH2MHill Plateau Remediation Company

\section{COLLLECTOR}

Roshex Guria Tumer

CHAIN OF CUSTODY/SAMPLE ANALYSIS REQUEST

SAMPLING LOCATION

TELEPHONE NO.

F10-045-117

PAGE 2 OF 2

C7515 (299-E28-30); I-078

DYEKMAN, DL

$373 \cdot 2530$

PROJECT COORDINATOR

PROJECT DESIGNATION

PRICE CODE

8N

SAF NO.

AIR QUALTTY

DATA
TURNAROUND
TS DaYS / 45

$\underset{\text { Days }}{45 \text { Days } / 45}$

FIELD LOGBOOK NO ACTUAL SAMPLE DEPTH

HNF-N- $491-5 p$

COA

METHOD OF SHIPMENT

-5 py $51+189.2^{\prime} \quad 302117$ ES30

\section{SHIPPED TO}

OFFSTIE PROPERTY NO.

$\mathrm{N} / \mathrm{A}$

BILL OF LADING/AIR BILL NO.

N/A

\section{SPECIAL INSTRUCTIONS}

** The 200 Area S\&GRP Characterization and Monitoring Sampling and Analysis GKI applies to this SAF.

** ESL shall submit all data deliverables according to SOW "200-LW-2 and 200-8P-5 Operable Units/Contaminant and Transport Property Analysis and Report for Vadose Zone Sediments at Borehole C5860/Well 299-E29-54" for

** The ESL laboratory shall meet prior requested and confirmed turnaround times and RDLs specified in the SOW and this SAF. The laboratories must notify the SM Project Coordinator in writing iffwhen this requirement can not be me (1)6010M_LP_ASTM. AE (TAL) \{Sodtum, Aluminum, Iron, Potassium, Antimony, Nickel, Calcium, Magnesium, Silver\} 6010M_ICP_ASTM_AE (Add-On) \{Lead, Thallium, Selenium\} 6010M METALS_ICP_WE (TAL) (Sodium, Aluminum,

6020M METALS_ICPMS WE (TAL) \{Chromium, Cadmium\} 6020M_METALS ICPMS WW (Add-On) \{Arsenic\} 6020M_HG_ICPMS_AE; 6020M_HG_ICPMS WE; Chromium Hex - 7196; RADISO_ICPMS_ASTM_AE \{Uranium-238, Technetium-

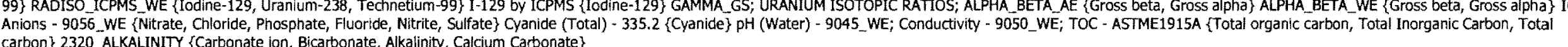

D Dilginti 
CH2MHill Plateau Remediation Company

\section{cgLLECTOR}

Rosane Gaveia Tueuce Rust

C7515 (299-E28-30)

ICE CHEST NO

$$
\sin <100
$$

SHIPPED TO

Environmental Sciences Laboratory

MATRIX* 7 POSSIBLE SAMPLE HAZARDS/ REMARKS Contains Radioactive Material at concentrations that may or may not be regulated for Doe DOE Order 5400.5 (1990/1993)

SPECIAL HANDLING AND/OR STORAGE RDIOACTVE TIE TO: B2364

SAMPLE NO.

B235B5

MATRIX*

$$
\text { SOIL }
$$

\section{CHAIN OF CUSTODY/SAMPLE ANALYSIS REQUEST}

F10-045-119

PAGE 1 OF 1

COMPANY CONTAC

TELEPHONE NO

PROJECT COORDINATOR

DYEKMAN, DL

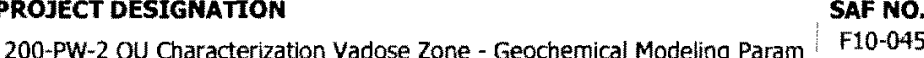

PROJECT DESIGNATION

PRICE CODE

FIELD LOGBOOK NO

COA

AIR QUALITY

TURNAROUND

Days

HNF-N $491-5 \rho g, 51,90.0^{\circ} \cdot 192,5^{\circ}$

OFFSITE PROPERTY NO.

METHOD OF SHIPMENT

GOVERNMENT VEHICLE

$\mathrm{N} / \mathrm{A}$

PRESERVATION

TYPE OF CONTAINER

NO. OF CONTAINER(S)

VOLUME

BILL OF LADING/ATR BILL NO.

N/A

SAMPLE ANALYSIS

\begin{tabular}{lll} 
& & \\
1 & $\cdots$ & \\
1 & & \\
1 & & \\
1 & & \\
1 & & \\
1 & $\cdots$ & \\
\hline
\end{tabular}

\section{SAMPLE DATE SAMPLE TIME}

$1-26 \cdot 10 \quad 0935$

\section{SIGN/ PRINT NAMES}

CHAIN OF POSSESSION

RELINQUISHED BY/REMOUED FROM Lanay Rusiwe Sanglosone $1.26 .0 \%$ DATE/TIME DATE/TIME

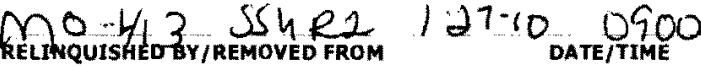

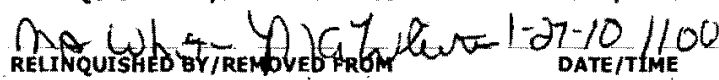
RELINQUISHED BY/REMOVED FROM - - DATE/TIME RELINQUISHED QY/REMOVED FROM - - DATE/TIME RELINQUISHED BY/REMOVED FROM DATE/TIME
RECEIVED BY/STORED IN

Mo.4/3 sSvez $1-26$ of 1530

Mabitimathin 1-27-10 0500

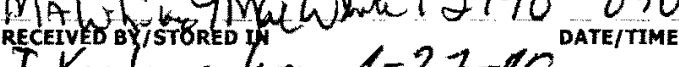
I. Kufnya kov $1-27-10$ Regeived BY/GoRED IN// M:OO DATE/TTME

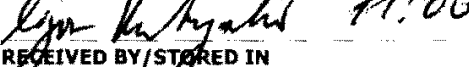

DATE/TME DATE/TTME DATE/TIME

RECEIVED BY/STORED IN
SPECIAL INSTRUCTIONS

** The 200 Area S\&GRP Characterization and Monitoring Sampling and Analysis

** ESL shall submit all data deliverables according to SOW "200-LW-2 and 200-BP-5 perable Units/Contaminant and Transport Property Analysis and Report for Vadose ("M" Well) and shall adhere to all S\&GRP standard protocol.

** The ESL laboratory shall meet prior requested and confirmed turnaround times and RDLS Specifed in the SOW and this SAF. The laboratories must notify the SM Project Coordinator in writing if/when this requirement can not be met. (1)Density; CATIONEXCH_TR; Particle Size (Dry Sieve) - D422; Particle Size

$$
\text { f Jilulin }
$$




$$
\sin 2100
$$

SHIPPED TO

al Sciences Laboratory

-

POSSIBLE SAMPLE HAZARDS/ REMARKS Contains Radioactive Material at concentrations transportation per be regulated for Goods Regulations but are not releasable per DOE Order 5400.5 (1990/1993)

SPECLAL HANDLING AND/OR STORAGE SPDEACTIVE TE TO: B23641

- SAMPLE no.

MATRIX
SOIL
COMPANY CONTACT

DYEKMAN, DL TELEPHONE NO.

$373-2530$

PROJECT COORDINATOR

DYEKMAN, DL

PRICE CODE

SAF NO.

PROJECT DESIGNATION

200-PW-2 OU Characterzation Vadose Zone - Geochemical Modeling Param F10-045

FIELD LOGBOOK NO. ACTUAL SAMPLE

COA

HNF-N- $49-5 \mathrm{Pg} 51 \quad 190.00 .92 .5^{\prime \prime}$

COA

DATA
TURNAROUND

45 Days / 45
Days

N/A

OF LADING/AIR BILL NO.

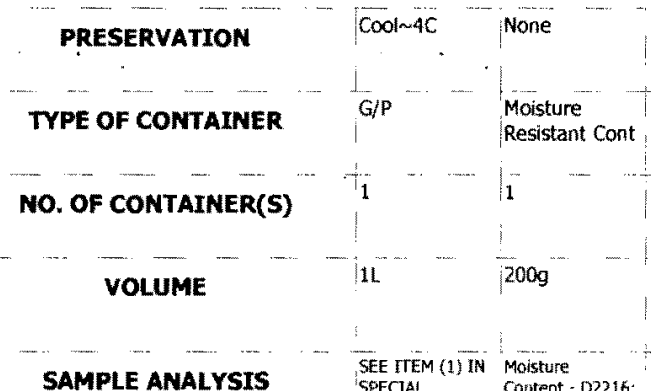

PRESERVATION

TYPE OF CONTAINER G/P Moisture

NO. OF CONTAINER(S)$$
\text { VoLume }
$$

SAMPLE ANALYSTS

\section{CHAIN OF POSSESSION}

RELINQUIGHED QY/REMOVED FROM

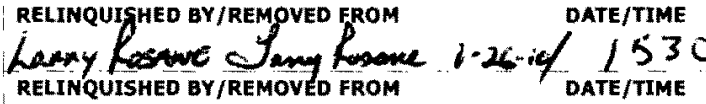

inO 413 SS S - R

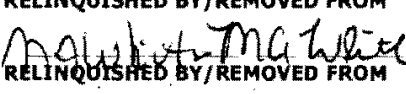

RELINQUISHED QY/REMOVED FROM

RELINQUISHED BY/REMOVED FROM

RELINQUISHED BY/REMOVED FROM

$$
1 \text {-27\% } 10 \text { Date/Time } 090
$$

Date/TIME

DATE/TIME

DATE/TIME

DATE/TIME

\section{SIGN/ PRINT NAMES}

RECEIVED QY/STORED IN ME-2/3 5SUReZ $1-26.0 / 1530$

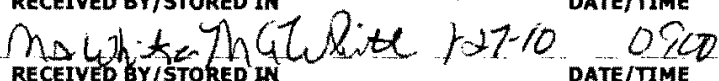
I. Ku fryaleor $1-2 z-10$ Regued ay forón In $11: 00$ RELEIVED BY/STquO IN

DATE/TMME

DATE/TMME

RECEIVED BY/STORED IN

RECEIVED BY/STORED IN

DATE/TIME

DATE/TIME

\section{SPECIAL INSTRUCTIONS}

SEE PAGE 2 FOR ALL SPECIAL INSTRUCTIONS

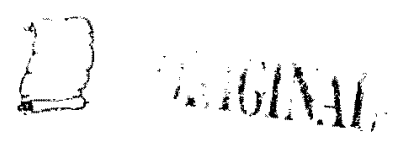

DATE/TIME 
loshwe Giscia Turmer
samping LOCATION

C7515 (299-E28-30); 1-080

TELEPHONE NO.

PROJECT COORDINATOR

PRICE CODE

DATA
TURNAROUN
T5 DaYs/ 45

PROJECT DESTGMATION

SAF NO.

AIR QUALITY $\square$

8N

PROJECT DESIGNATION

45 Days $/ 45$
Days

ICE CHEST NO.

FIELD LOGBOOK NO.

COA

METHOD OF SHTPMENT

HNF-N- $491-5 P_{q} 51$

SHIPPED TO

OFFSTIE PROPERTY NO.

302117ES30

GOVERNMENT VEHICLE

N/A

BILL OF LADING/AIR BILL NO.

Environmental Sciences Laboratory

$N / A$

\section{SPECIAL INSTRUCTIONS}

** The 200 Area S\&GRP Characterization and Monitoring Sampling and Analysis GKI applies to this SAF. Q** ESL shall submit all data deliverables according to SOW "200-LW-2 and 200-BP-5 Operable Units/Contaminant and Transport (1)6010M_LCP_ASTM_AE (TAL) (Sodium, Aluminum, Iron, Potassium, Antimony, Nickel, Calcium; Magnesium, Silver\} 6010M_ICP.ASTM_AE (Add-OOn) \{Lead, Thalllum, Selenium\}6010M_METALS_ICP_WE (TAL) \{Sodium, Aluminum,

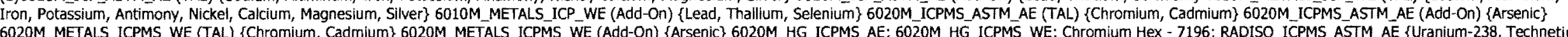
90) RADISO ICPMS WE ( Anions - 9056 WE \{Nitrate, Chloride, Phosphate, Fluoride, Nitrite, Sulfate\} Cyanide (Total) - 335.2 \{Cyanide\} PH (Water) - 9045_WE; Conductivity - 9050_WE; TOC - ASTME1915A \{Total organic Carbon, Total Inorgenilc Carbon, Total carbon\} 2320_ALKALINITY \{Carbonate ion, Bicarbonate, Alkalinity, Calcium Carbonate\}

INIDIYO 
CH2MHill Plateau Remediation Company

CHAIN OF CUSTODY/SAMPLE ANALYSIS REQUEST

F10-045-122

PAGE 1 OF

COLLECTOR

Roswli, Rust, Turezis, Gavesia

C7515 (299-E28-30); I-081

ICE CHEST NO.

$5 m L 100$

SHIPPED TO

$$
\text { mL } 100
$$

Environmental Sciences Laboratory

POSSIBLE SAMPLE HAZARDS/ REMARKS Contains Radloactive Material at concentrations

that may or may not be regulated for Goods Regr 5400.5 (

DOE Order 5400.5 (1990/1993)

SPECIAL HANDLING AND/OR STORAGE RADIOACTIVE TTE TO: B23642

$\mathrm{B} 235 \mathrm{M}$

SAMPLE NO.

Soll

MATRIX*

COMPANY CONTACT

TELEPHONE NO

PROJECT COORDINATOR

DYEKMAN, DL

SAF NO.
F10-045

PROJECT DESIGNATION

200-PW-2 Ou Characteriz ACTUAL SAMPLE DEPTH

PRICE CODE

DATA

FIELD LOGBOOK NO.

195:

AIR QUALITY

TURNAROUND

Days / 45
Days

BILL OF LADING/AIR BILL NO.

N/A

PRESERVATION

TYPE OF CONTAINER

NO. OF CONTAINER(S)

VOLUME

SAMPLE ANALYSIS

SAMPLE DATE, SAMPLE TIME

$1-26-10 \quad 1053$

CHAIN OF POSSESSION

REINQUISHED BY/REMOVED FROM

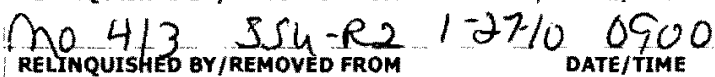

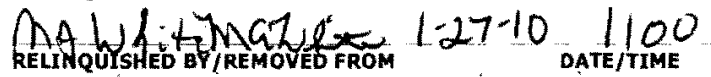

RELINQUISHED BY/REMOVED FROM

RELINQUISHED BY/REMOVED FROM

DATE/TIME

DATE/TIME
SIGN/ PRINT NAMES

RECEIVED BY/STORED IN
Mo. $1 / 3$ SSU-CZ $1-26-10 /$ DATE/TIME
RECEIVED BY/STORE IN

Ma Whitughawht 1-27-10 o500

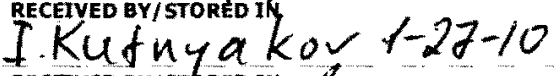

RECTIVED BY/STTRED IN

yo thatyent 11100

DATE/TMME

DATE/TIME

DATE/TIME

\section{SPECIAL INSTRUCTIONS}

SEE PAGE 2 FOR ALL SPECIAL INSTRUCTIONS

\section{\&) Minutiti}




\section{Rosine Rust Tutuer Grecia}

COMPANY CONTACT

DYEKMAN, OL

TELEPHONE NO.

PROJECT COORDINATOR

PROJECT DESIGNATION

$$
373-2530
$$

OYEKMAN, DL

PRICE CODE

DATA

SAMPLING LOCATION

SAF NO.

AIR QUALITY

METHOD OF SHIPMENT

45 Days / 45

FIELD LOGBOOK NO.

ACTUAL SAMPLE DEPTH

HNF-N-4/9/-5 $5 /, 45.0^{\circ}$

302117 ES30

GOVERNMENT VEHICLE

SHIPPED TO

OFFSTIE PROPERTY N $\delta$

BIIL OF LADING/ATR BILL NO.

Environmental Sciences Laboratory

N/A

N/A

\section{SPECIAL.INSTRUCTIONS}

** The 200 Area S\&GRP Characterization and Monitoring Sampling and Analysis GKI applies to this SAF

"* ESL shall submit all data deliverables according to SOW "200-LW-2 and 200-BP-5 Operable Units/Contaminant and Transport Property Analysis and Report for Vadose Zone Sediments at Borehole C5860/Well 299-E29-54" for

** The ESL laboratory shall meet prior requested and confirmed turnaround times and RDLs specified in the SOW and this SAF. The laboratories must notify the SM Project Coordinator in writing iff when this requirement can not be met. (1)6010M_ICP_ASTM AE (TAL) \{Sodium, Aluminum, Iron, Potassium, Antimony, Nickel, Calcium, Magnesium, Siver\})6010M_ICP_ASTM_AE (Add-On) (Lead, Thallium, Selenium\}6010M_METALS_ICP_WE (TAL) \{Sodium, Aluminum,

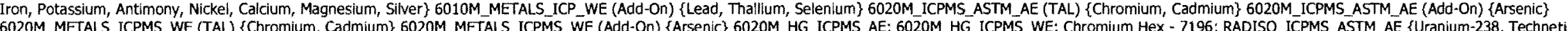

99\} RADISO ICPMS WE \{Iodine-129, Uranium-238, Technetium-99\} I-129 by ICPMS \{Iodine-129\} GAMMA GS; URANIUM ISOTOPIC RATIOS; ALPHA BETA AE \{Gross beta, Gross alpha\} ALPHA BETA WE \{Gross beta, Gross alpha\} IC Anions - 9056_WE \{Nitrate, Chloride, Phosphate, Fluoride, Nitrite, Sulfate\} Cyanide (Total) - 335.2 \{Cyanide\} pH (Water) - 9045_WE; Conductivity - 9050_WE; TOC - ASTME1915A \{Total organic carbon, Total Inorganic Carbon, Total carbon\} 2320 ALKALINITY \{Carbonate ion, Bicarbonate, Alkalinity, Calcium Carbonate\}

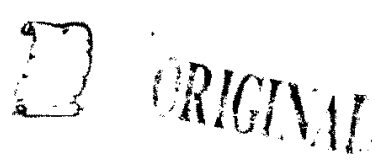


$\sum_{\text {SHIPPED TO }} m L$

00

Environmental

Sciences Laboratory

MATRIX* POSSIBLE SAMPLE HAZARDS/ REMARKS

$A=$ Air
$\mathrm{D} \mathrm{L}=\mathrm{Drum}$

that may or may not be regulated for

Goods Re

Goods Regulations but are not $r$ D
DOE Order 5400.5 (1990/1993)

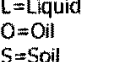

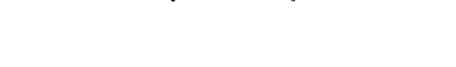

SPECIAL HANDLING AND/OR STORAGE RADIOACTIVE TE TO: B23642
SAMPLe No.
MATRIX*
COMPANY CONTACT

DYEKMAN, DL

PROJECT DESIGNATATION

200-PW-2 Ou Characterizato

TELEPHONE NO.

373-2530

PROJECT COORDINATOR

DYEKMAN, DL

SAF NO.

FIELD LOGBOOK NO.

- Geochemical Modeling Pa

HNF-N- $491-5 \mathrm{pg} 51 \quad 197.0^{\circ}$

N/A

PRESERVATION

TYPE OF CONTAINER G/P $\quad \begin{aligned} & \text { Moisture } \\ & \text { Resistant Cont }\end{aligned}$

NO. OF CONTAINER(S)

volume

SAMPLE ANALYSIS

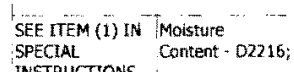

SAMPLE DATE SAMPLE TIME

$\therefore-26-10$

1108

\section{SIGN/ PRINT NAMES}

CHAIN OF POSSESSION

RELINQUUSHED BY/REMOVED FROM

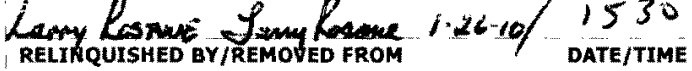

MO 413 SS4-R2 $1-27-10$ O900

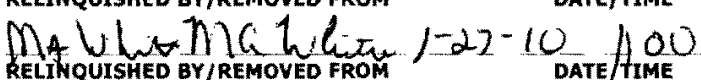

RELINQUiSHED BY/REMOVED From

DATE/TMEE

RELINQUISHED BY/REMOVED FROM

DATE/TIME

RELINQUISHED BY/REMOVED FROM

DATE/TIME

\section{RECETVED BY/STORED IN}

MO 413 SSW RE

- 15 30

na whichatulide $1 \cdot 27: 00900$

RECEIVED BY/STORED IY Ku $01-27-10$

REgIVED BY/STORED IM/ 11.00 DATE/TIME

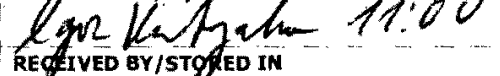

RECEIVED BY/STORED IN

DATE/TIME

DATE/TIME

RECEIVED BY/STORED IN

DATE/TIME

\section{SPECIAL INSTRUCTIONS}

METHOD OF SHIPMENT
N/A

GOVERNMENT VEHICLF 
CH2MHill Plateau Remediation Company

Coluector
sampling location

SAMPLING LOCATION

ICE CHEST NO.

\section{SHIPPED TO}

Environmental Sciences Laboratory

SPECIAL INSTRUCTIONS

** The 200 Area S\&GRP Characterization and Monitoring Sampling and Analysis GKI applies to this SAF. ** The 200 Area S\&GRP Characterization and Monitoring Sampling and Analysis GKI applies to this SAF.
** ESL shall submit all data deliverables according to SOW "200--W-2 and 200-BPP-5 Operable Units/Contaminant and Transport Property Analysis and Report for Vadose Zone Sediments at Borehole C5860/Well 299-E29-54" for
C7515/Well 299-E28-30 ("M" Well) and shall adhere to all S\&GRP standard protocol.

** The ESL Iaboratory shall meet prior requested and confirmed turnaround times and RDLS specified in the SOW and this SAF, The laboratories must notify the SM Project Coordinator in writing iff/when this requirement can not be met.

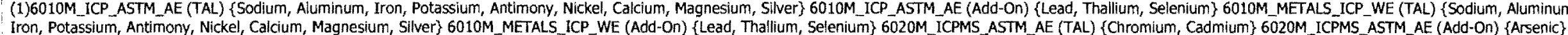
6020M_METALS_ICPMS_WE (TAL) \{Chromium, Cadmium\} 6020M_METALS_ICPMS_WE (Add-On) \{Arsenic\} 6020M_HG_ICPMS.AE; 6020M_HG_ICPMS_WE; Chromium Hex - 7196; RADISO_ICPMS_ASTM_AE \{Uranlum-238, TeChnetiumAnions - 9056 WE _Nitrate, Chloride, Phosphate, Fluoride, Nitrite, Sulfate\} Cyanide (Total) - 335.2 (Cyanide\} pH (Water) - 9045_WE; Conductivity - 9050_WE; TOC - ASTME1915A \{Total organic carbon, Total Inorganic Carbon, Total

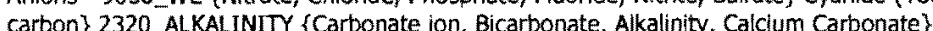

\section{CHAIN OF CUSTODY/SAMPLE ANALYSIS REQUEST}

\section{TELEPHOME NO.}

PROJECT COORDINATOR

PROJECT DESIGNATION

SAF NO.

FIELD LOGBOOK NO. ACTUAL SAMPLE DEPTH

COA
TURNAROUND

Days

\section{OFFSTTE PROPERTY NO $9 g$ SI $\ldots 197.0 \ldots \ldots$.}

METHOD OF SHIPMEN

TVEHICLE

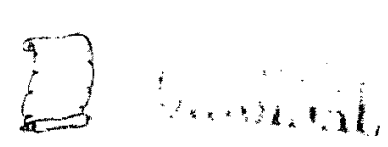


CH2MHill Plateau Remediation Company

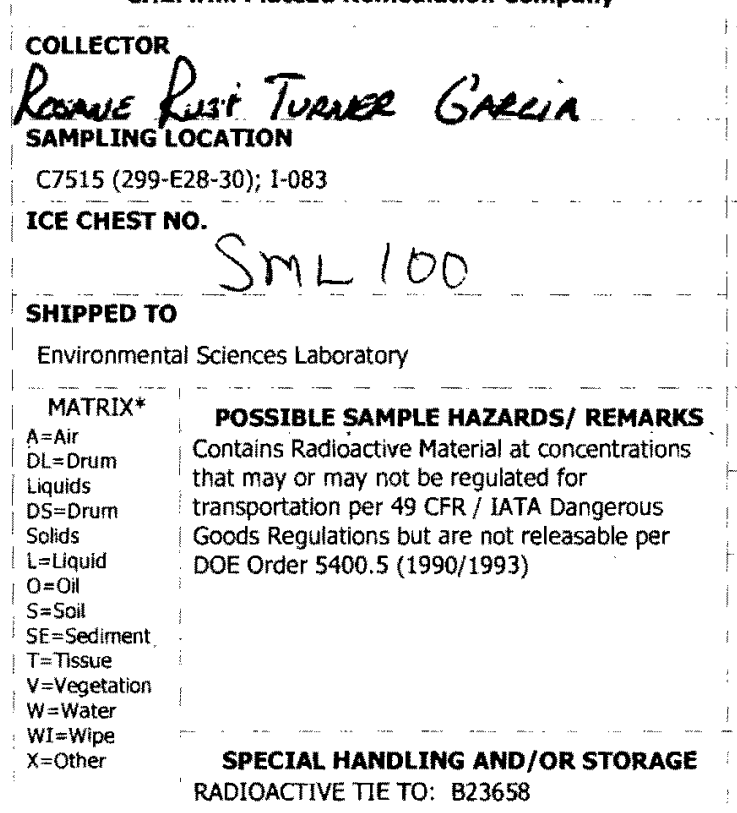

$$
\text { SAMPLE No. }
$$

B235M8
SOIL

MATRIX

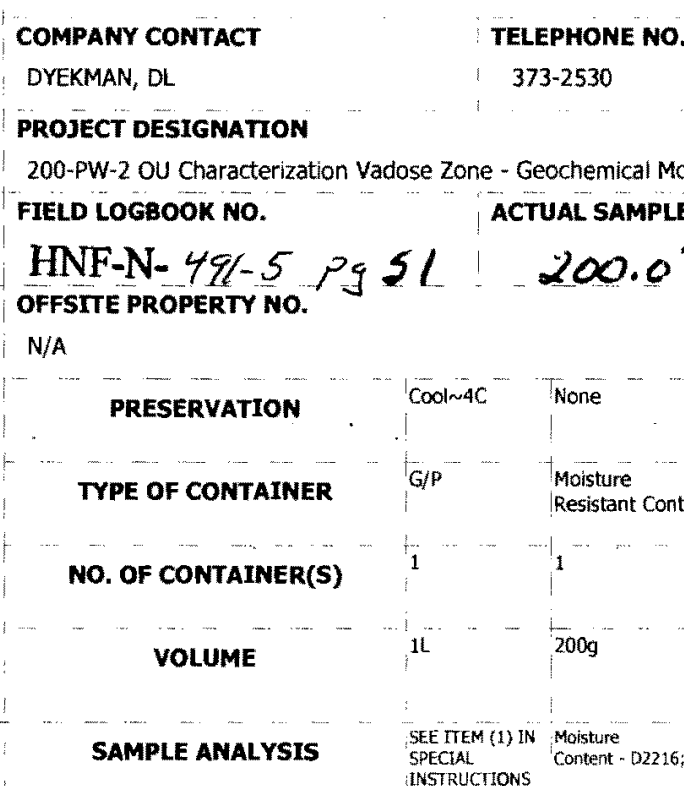

F10-045-125

PAGE 1 OF 2

PRICE CODE

AIR QUALITY $\square$

MAN, D

SAF NO.

AIR QUALITY $\square$

SAMPLE DATE SAMPLE TIME

$1-26-10 \quad 1325$

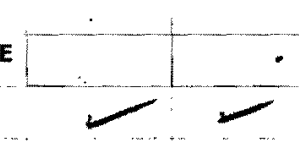

CHAIN OF POSSESSION

REIINQUISHED BY/REMOVEO FROM

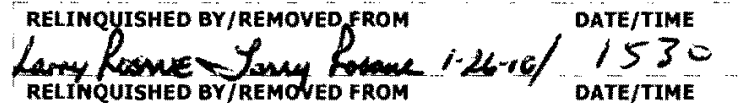

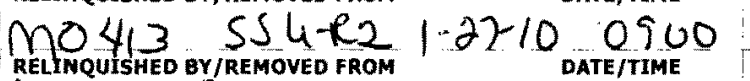

SIGN/ PRINT NAMES

RECEIVED BY/STORED IN

MO. 413 S5U.R2 $1-26 \% / 1530$

Mow h toma hitin 1-27-10 O900

Tha wh Ma White $1-22-10$ DATE/TIME 00

RELINQUISHED BY/REMOVED FROM

DATE/TIME

RefeIVED BY IsToRE IN/

lar valazilo 11:00

DATE/TIME

DATE/TMME

DATE/TIME

DATE/TIME

DATE/TIME

RELINQUISHED BY/REMOVED FROM

DATE/TIME
SPECIAL INSTRUCTIONS

SEE PAGE 2 FOR ALL SPECIAL INSTRUCTIONS

\& ORIOI: 


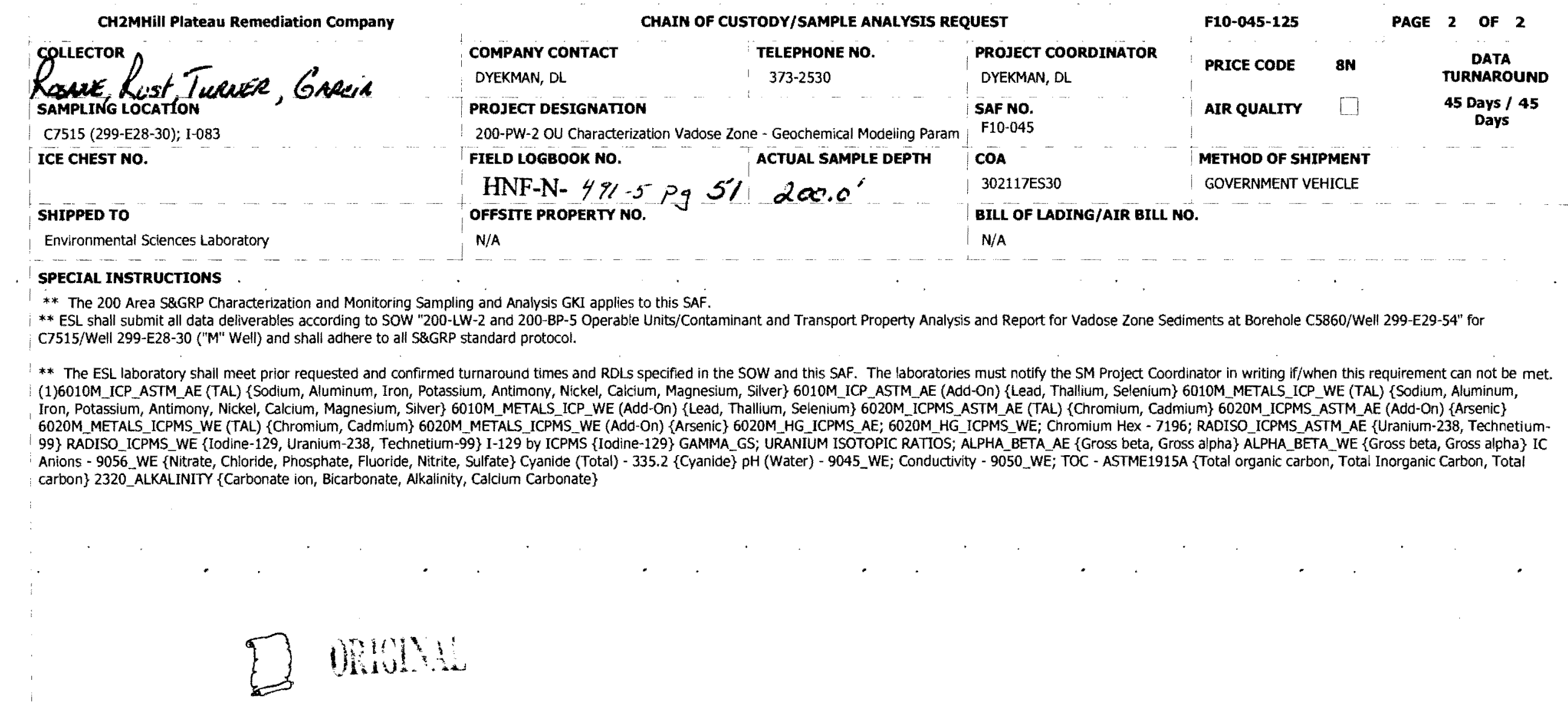




\section{CHAIN OF CUSTODY/SAMPLE ANALYSIS REQUEST}

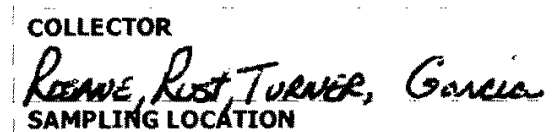

C7515 (299-E28-30): I-0B

ICE CHEST NO.

$$
\sin L-100
$$

SHIPPED TO

Environmental Sciences Laboratory

$\underset{A=A i r}{\text { MATRIX }}$ POSSIBLE SAMPLE HAZARDS/ REMARKS

$A=A i r$
$\mathrm{DL}=\mathrm{Drum}$

that may or may not be regulated for
transportation per 49 CFR / IATA Dangerous

Goops Regulations but are not releasable per
DOE Order $5400.5(1990 / 1993)$

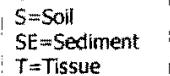

$S E=$ Sedime
$T=$ Tissue
$v=$ Vesetation
$W=$ Water
$W=W$
$X=0$ iner

, $W_{x=\text { In }}=$ Wiper

SPECIAL HANDLING AND/OR STORAGE RADIOACTIVE TE TO: B23658

MATRIX*

B235M9

DYEKMAN, DL

PROJECT DESIGMATION

TELEPHONE NO.
$373-2530$

FIELD LOGBOOK NO.

HNF-N-49/.5 pg $5 /$. 202.0

OFFSITE PROPERTY NO.

PRESERVATION

TYPE OF CONTAINER

NO. OF CONTAIMER(S)

volume

SAMPLE ANALYSTS

\section{ANALYSIS}

SAMPLE. DATE SAMPLE TIME

con

\section{- SAMple no}

COMPANY CONTACT

200-PW-2 OU Chaterization Vadose Zone - Geochemical Modeling Parm F10-045 ACTUAL SAMPLE DEPTH

$1-26 \cdot 10$

1425

SIGN/ PRINT NAMES

CHAIN OF POSSESSION

DATE/TIME RECEIVED BV/STORED IN

mo- 413 sSuRe

RELUQUISHED BY/REMOVED FRgM 1 DATE/TIME

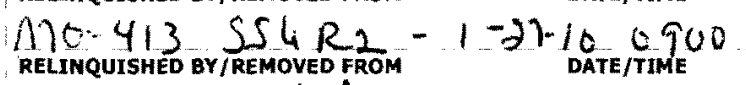

MAWh tema lultit

RELINQUISHED BY/REMOVED FROM

RELINQUISHED BY/REMOVED FROM

RELINQUISHED BY /REMOVED FROM

$10 \quad 1100$

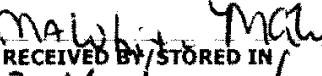

I Ku STER IN

REGEIVED BVISTged IN

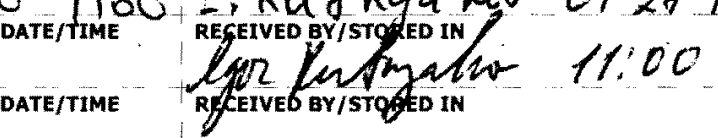

DATE/TIME

RECEIVED BY /STORED IN

DATE/TIME

Date/time-

DATE/TIME

RECEIVED BY/STORED IN

DATE/TMME
PROJECT COORDINATOR

DYEKMAN, DL

SAF NO.

F10-045-126

PAGE 1 OF 2

302117 ES30

BILL OF LADING/AIR BILL NO

\section{SPECIAL INSTRUCTIONS}

SEE PAGE 2 FOR ALL SPECIAL INSTRUCTIONS

PRICE CODE

URMAROUND

45 Days / 45

Days

METHOD OF SHIPMEN

GOVERNMENT VEHICLE

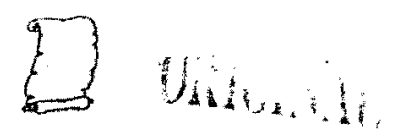

\section{LABORATORY
SECTION}

FINAL SAMPLE DISPOSAL METHOD 
CH2MHill Plateau Remediation Company

\section{COjLECTOR}

Rosave Rust Turner, Gascue

C7515 (299-E28-30); I-084

ICE CHEST NO.

\section{SHIPPED TO}

Environmental Sciences Laboratory

\section{SPECIAL INSTRUCTIONS.}

** The 200 Area S\&GRP Characterization and Monitoring Sampling and Analysis GKI applies to this SAF.

** ESL shall submit all data deliverables according to SOW "200-LW-2 and 200-BP-5 Operable Units/Contaminant and Transport Property Analysis and Report for Vadose Zone Sediments at Borehole C5860/Well 299-E29-54" for
C7515/Well 299-E28-30 ("M" Well) and shall adhere to all S80-CP standard protoc-

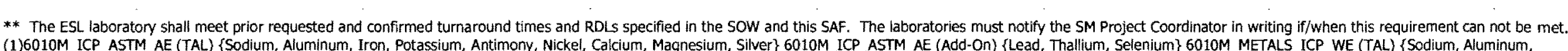
(1)6010M_ICP_ASTM AE (TAL) \{Sodium, Aluminum, Iron, Potassium, Antimony, Nickel, Cakiciurn, Magnesium, Silver\} 6010M_ICP.ASTM.AAE (AAd-On) \{Lead, Thallium, Selenium\} 6010M_METALS.ICP. WE (TAL) \{Sodium, Aluminum,

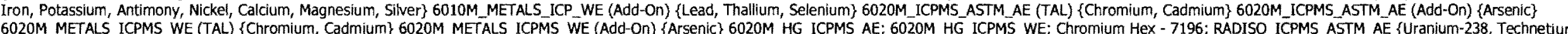
99\} RADISO ICPMS WE \{Iodine-129, Uranium-238, Technetium-99\} I-129 by ICPMS \{Iodine-129\} GAMMA_GS; URANIUM ISOTOPIC RATIOS; ALPHA BETA AE \{Gross beta, Gross alpha\} ALPHA_BETA_WE \{Gross beta, Gross alpha\} IC

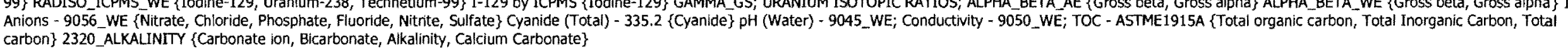

$\{3$ Vin. 1
F10-045-126

PAGE 2 OF 2

PROJECT COORDINATOR

DYEKMAN, DL

DYEKMAN, DL

DATA
TURNAROUND

$\underset{\substack{\text { Days } \\ \text { Days }}}{45}$

200-PW-2 OU Characterization Vadose Zone - Geochemical Modeling Param F10-045

COA

METHOD OF SHIPMENT

HNF-N- 49

ACTUAL SAMPLE DEPTH

$302117 E 530$

GOVERNMENT VEHICLE

OIL OF LADING/AIR BILL NO.

Days
OFFSTE PROPERTY NO.

N/A i 
PROJECT DESIGNATION SAF No.

AIR QUALTYY

200-PW-2 OU Characterization Vadose Zone - Geochemical Modeling Param $/ F 10-045$

SAMPLING LOCATION

COA

ICE CHEST NO

FIELD LOGBOOK NO. ACTUAL SAMPLE DEPTH

SHIPPED TO

Environmental Sciences Laboratory
HNF-N- $491-5 p a 52$ $205^{\prime}$
302117 ES30 GERNMENT VEHICLE

METHOD OF SHIPMEN

POSSIBLE SAMPLE HAZARDS/ REMARKS Contars Radoactive Material at concentrations

transportation por 49 Ce / ited for

Goods Regulations but are not releasable per

PRESERVATION

TYPE OF CONTAINER

NO. OF CONTAIMER(S)

volume

SPECIAL HANDLING AND/OR STORAG RADIOACTIVE TIE TO: B23643

SAMPLE ANALYSIS

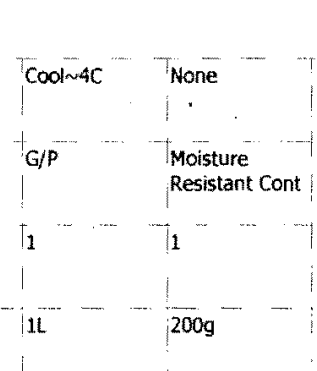

SAMPLE NO.
MATRIX*

\section{SAMPLE DATE SAMPLE TIME}

$1-27-12 \quad 0810$

\section{CHAIN OF POSSESSION}

REIINQUISHED BY/REMOVED FROM

Rob Roruo Robaramo

REINQUISHEP BY /REMOKEO FROM J.R.AGuila: 12010

REHRQC

RELIMQUISHED BY/REMOVED FROM

RELIMQUISHED BY/REMOVED FROM

RELINQUISHED BY/REMOYED FROM

RELINQUISHED GY/REMOVED FROM

RECEIVED By

\section{SECTION}

FINAL SAMPLE DISPOSAL METHOD

DISPOSITION

\section{SIGN/ PRINT NAMES}

DATE/TIME RECEIVED BY/STORED IN DATE/TIME 200 PE $4 / 3$ SSG -R2

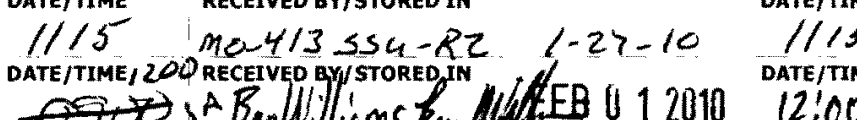
A DATE/TMME DATE/TIME

DATE/TIME

RECEIVED BY/STORED IN

DATE/TIME

DATE/TME

DATE/TIME

DATE/TME
BILL OF LADING/AIR BILL NO. $\mathrm{N} / \mathrm{A}$

\section{SPECIAL INSTRUCTIONS}

SEE PAGE 2 FOR ALL SPECIAL INSTRUCTIONS

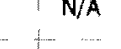




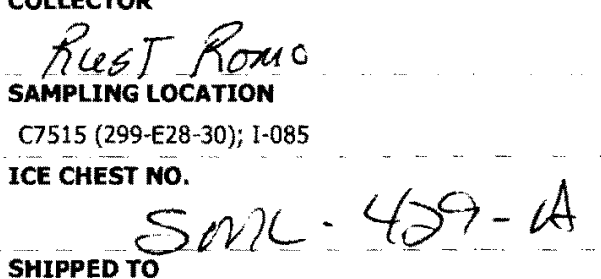

SHIPPED TO

Environmental Sciences Laboratory

\section{SPECIAL INSTRUCTIONS}

** The 200 Area S\&GRP Characterization and Monitoring Sampling and Analysis GKI applies to this SAF.

** ESL shall submit all data deliverables according to SOW "200-LW-2 and 200-BP-5 Operable Units/Contaminant and Transport Property Analysis and Report for Vadose Zone Sediments at Borehole C5860/Well 299-E29-54" for

** The ESL laboratory shall meet prior requested and confirmed turnaround times and RDLs specified in the SOW and this SAF. The laboratories must notify the SM Project Coordinator in writing iff when this requirement can not be met (1)6010M_ICP_ASTM AE (TAL) \{Sodium, Aluminum, Iron, Potassium, Antimony, Nickel, Calcium, Magnesium, Silver\} 6010M_ICP_ASTM_AE (Add-On)) (Lead, Thallium, Selenium\} 6010M_METALS ICP_WE (TAL) (SOdium, Aluminum,

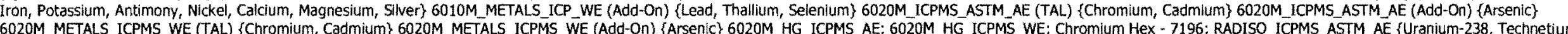
99\} RADISO ICPMS WE \{lodine-129, Uranium-238, Technetium-99\} I-129 by ICPMS \{Iodine-129\} GAMMA GS; URANIUM ISOTOPIC RATIOS; ALPHA BETA AE \{Gross beta, Gross alpha\} ALPHA BETA WE \{Gross beta, Gross alpha\} IC Anions - 9056_WE \{Nitrate, Chloride, Phosphate, Fluoride, Nitrite, Sulfate\} Cyanide (Total) - 335.2 \{Cyanide\} pH (Water) - 9045_WE; Conductivity - 9050_WE; TOC - ASTME1915A (Total organic carbon, Total Inorganic Carbon, Total Aarbon\} 2320_ALKALINITY \{Carbonate ion, Bicarbonate, Alkalinity, Cakium Carbonate

93 ind

\section{DYEKMAN, DL}

$373-2530$

DRETECT COORDINATOR

PROJECT DESIGMATION

200-PW-2 OU Characterization Vadose Zone - Geochemical Modeling Param

FIELD LOGBOOK NO. ACTUAL SAMPLE DEPTH

HNF-N. 49/-5 gg $52 \quad 205$

\section{SAF NO.}

AIR QUALITY

DATA
TURNAROUND
TUR

45 Days / 45
Days

METHOD OF SHIPMENT

GOVERNMENT VEHICLE

ADING/AIR BIIL NO. 
PROJECT COORDINATOR

PRICE CODE

PROJECT DESIGNATION SAF NO."

AIR QUALITY

200-PW-2 OU Characterization Vadose Zone - Geochemical Modeling Param $\quad$ F10-045

FIELD LOGBOOK NO.

ACTUAL SAMPLE DEPTH

COA

METHOD OF SHIPMENT

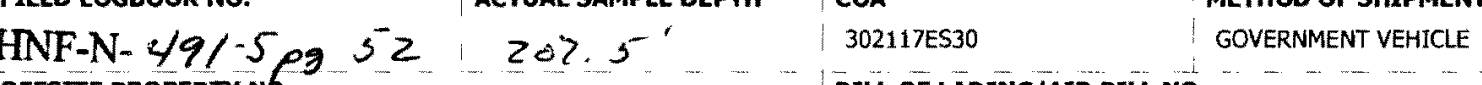

OFFSITE PROPERTY N6.

N/A

SAMPLE NO."
B235N1

PRESERVATIOA

TYPE OF CONTAINER

NO. OF CONTAINER(S)

VOLUME

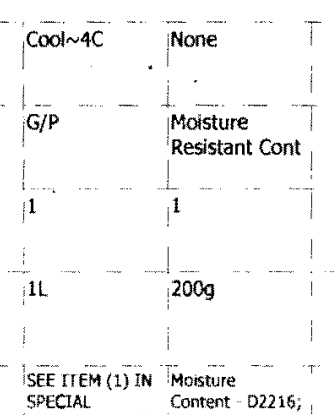

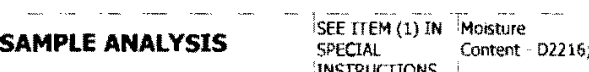

\section{SAMPLE DATE., SAMPLE TIME}

$1-22-10 \quad 0838$

\section{CHAIN OF POSSESSION}

REIINQUISHED BY/REMOVED FROM

SIGN/ PRINT NAMES

RobRono Rothcharo

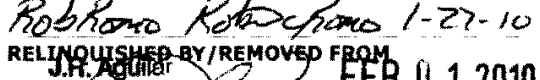

CHRAC

RELINQUISHED BY/REMOVED FROM

RELINQUISHED BY/REMOVED FROM

RELINQUISHED BY/REMOVED FROM

RELINQUISHED BY/REMOVED FROM

RECEIVED BY

LABORATORY
SECTION

EINAL SAMPLE DISPOSAL METHOD

DISPOSITION

RECEIVED BY/STORED IN
RECEIVED BY/STORED IN

1115 Mo $4 / 3$ ss4-A2, $1-27-10$

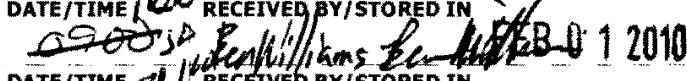

DATE/TIME ZT. RECENVD BY/STORLD IN

date/time

RECEIVED BY/STORED IN

RECEIVED BY/STORED IN

DATE/TIME

RECEIVED BY/STORED IN

DATE/TIME 
\begin{tabular}{l|l|l}
\hline PROJECT DESIGNATION & SAF NO. \\
\hline 200-PW-2 OU Characterization Vadose Zone - Geochemical Modeling Param & F10-045 \\
\hline I & COA
\end{tabular}

\section{SHIPPED TO}

FIELD LOGBOOK NO.

COA

METHOD OF SHIPMEN

Environmental Sciences Laboratory

ERTYNo.

BILL OF LADING/AIR BILL No.

\section{SPECIAL INSTRUCTIONS}

*** The 200 Area 58GRP Characterization and Monitoring Sampling and Analysis GKI applies to this SAF.

** ESL shall submit all data deliverables according to SOW "200-LW-2 and 200-BP-5 Operable Units/Contaminant and Transport Property Analysis and Report for Vadose Zone Sediments at Borehole C5860/Well 299-E29-54" for

** The ESL laboratory shall meet prior requested and confirmed turnaround times and RDLs spectied in the SOW and this SAF. The laboratories must notify the SM Project coordinator in writing if/ when this requirement can not be me. (1)6010M_ICP_ASTM_AE (TAL) \{Sodium, Aluminum, Iron, Potassium, Antimony, Nickel, Calcium, Magnesium, Silver\} 6010M_ICP_ASTM_AE (Add-On) \{Lead, Thallium, Selenium\} 6010M_METALS,ICP.WEE (TAL) \{Sodium, Aluminum,

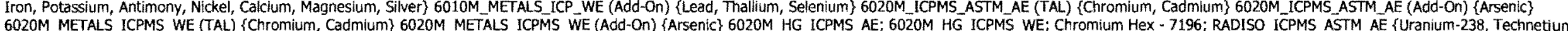
99\} RADISO_ICPMS_WE \{Lodine-129, Uranium-238, Technetium-99\} I-129 by ICPMS \{lodine-129\} GAMMA_GS; URANiUM ISOTOPIC RATIOS; ALPHA_BETA_AE \{Gross beta, Gross alpha\} ALPHA_BETA_WE \{Gross beta, Gross alpha\} carbon\} 2320_ALKALINITY \{Carbonate ion, Bicarbonate, Alkalinity, Cakium Carbonate

D IRrew 
C7515 (299-E28-30); I-087

ICE CHEST NO.

COMPANY CONTACT

TELEPHONE NO.

PRICE CODE

PROJECT DESIGNATION

373-2530

PROJECT COORDINATOR

DYEKMAN, DL

AIR QUALITY

45 Days / 45

\begin{tabular}{l:lll} 
& 200-PW-2 OU Characterization Vadose Zone - Geochemical Modeling Param & F10-045 \\
\hline & COA & \\
\hline
\end{tabular}

\begin{tabular}{|l|l|l|l|l} 
FIELD LOGBOOK NO. & ACTUAL SAMPLE DEPTH & COA & METHOD OF SHIPMENT \\
HNF-N- $491-5 \mathrm{Pg}^{-2}$ & $2 / 0^{\prime}$ & $302117 E S 30$ & GOVERNMENT VEHICLE
\end{tabular}

SHIPPED TO

$$
510 x-429-A
$$

Environmental Sciences Laboratory OFFSTTE PROPERTY NO.

302117 ES30

Days

MATRIX* POSSIBLE SAMPLE HAZARDS/ REMARKS

$A=A i r$
$\mathrm{DL}=0$ rum

liquids Chat may or may not be regulated for

Gansportation per 49 CFR / IATA Dangerous

DOE Order 5400.5 (1990/1993)

N/A

BILL OF LADING/AIR BILL NO.

\begin{tabular}{l|l} 
MTPE OF CONTAINER \\
Resistant Cont
\end{tabular}

NO. OF CONTAINER(S)

volume

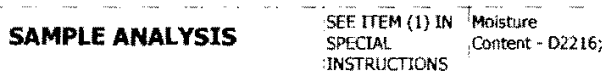
SPECIAL HANDLING AND/OR STORAG
RADIOACTIVE TIE TO: B23659

- SAMPLE No

B235N2

MATRIX*

SAMPLE DATE SAMPLE TIME

$1-27-10,0928$

CHAIN OF POSSESSION

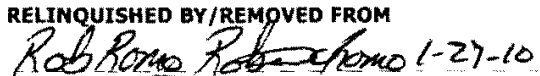

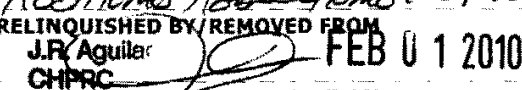

RELINQUUSSHED DF TREMOVED FROM

RELUNQUISHED BY/REMOVED FROM

RELINQUISHED BY/REMOVED FROM

RELINQUUISHED BY/REMOVED FROM

RELINQUISHED BY/REMOVED FROM

\section{REceived ar \\ SECTION}

FINAL SAMPLE DISPOSAL METHOD

\section{SIGN/ PRINT NAMES} ATE/TIMEI 2 Q M RECE-4/3 SSL-R2 $>-27-10$

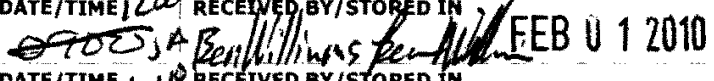

DATE/TIME

RECEIVED BY/STTORED IN

DATE/TIME

RECEIVED BY/STORED IN

DATE/TIME

RECEIVED BY/STORED IN

DATE/TIME

RECEEIVED BY GSTORED IN 
CH2MHill Plateau Remediation Company COLLECTOR

\section{SAMPLING LOCATION}

C7515 (299-E28-30); 1-087

ICE CHEST NO.

SMIPPED TO

$$
\text { SML }-429-\Delta
$$

Environmental Sciences Laboraton

SPECIAL INSTRUCTIONS

** The 200 Area S\&GRP Characterization and Monitoring Sampling and Analysis GKI applles to this SAF.

** ESL shall submit all data deliverables according to SOW "200-LW-2 and 200-BP-5
C7515/Well 299-E28-30 ("M" Well) and shall adhere to all S\&GRP standard protocol.

** The ESL laboratory shall meet prior requested and confirmed turnaround times and RDLs specified in the SOW and this SAF. The laboratorles must notify the SM Project Coordinator in writing if/when this requirement can not be met

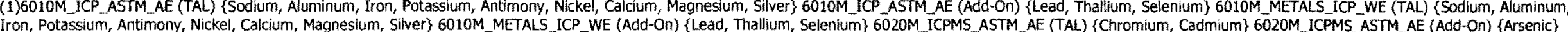
6020M_METALS_ICPMS WWE (TAL) (Chromium, Cadmium\} 6020M_METALS_ICPMS_WE (Add-On) \{Arsenic\}6020M_HG_ICPMS_AE; 6020M_HG_ICPMS_WE; Chromium Hex - 7196; RADISO_ICPMS_ASTM_AE \{Uranium-238, TeChnetium -

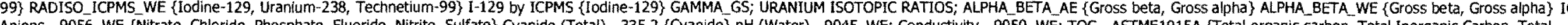
Anions - 250 _

$$
\text { \&3 Hif? }
$$


C7515 (299-E28-30)
ICE CHEST NO.

\begin{tabular}{|l|c|c|}
\hline COMPANY CONTACT & TELEPHONE NO. & PROJECT COORDINATOR \\
\hline DYEKMAN, DL & $373-2530$ & DYEKMAN, DL \\
\hline PROJECT DESIGNATION & & SAF NO. \\
\hline 200-PW-2 OU Characterization Vadose Zone - Geochemical Modeling Param & F10-045 \\
\hline
\end{tabular}

PRICE CODE

SI $) C-429-A$

200-PW-2 OU Characterizato

AIR QUALITY

SHIPPED TO

FIELD LOGBOOK NO. ACTUAL SAMPLE DEPTH COA

METHOD OF SHIPMENT

\begin{tabular}{ll|c|c|c|} 
HNF-N- $49 /-5 p g$ & 52 & $2 / 2.5$ & $302117 E S 30$ & GOVERNMENT VEHICLE \\
OFFSITE PROPERTY NO. & & BILL OF LADING/AIR BILL NO.
\end{tabular}

Environmental Sciences Laboratory

MATRIX* POSSIBLE SAMPLE HAZARDS/ REMARKS

$A=A i r$
$\mathrm{DL}=\mathrm{Drum}$

Liquids i that may or may not be regulated for

transportation per 49 CFR / IATA Dangerous

Goods Regulations but are not releasable per

SPECIAL HANDLING AND/OR STORAGE RADIOACTIVE TIE TO: 823659

$\mathrm{N} / \mathrm{A}$

N/A

\section{PRESERVATION}

TYPE OF CONTAINER

No. OF CONTAINER(S)

volume

SAMPLE ANALY'YSIS

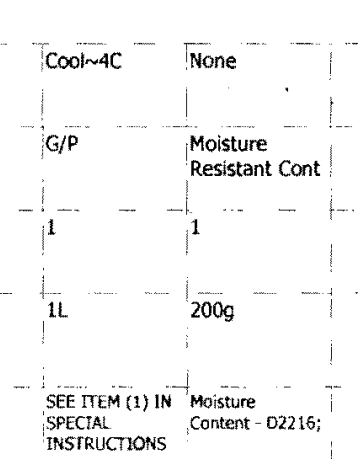

SAMPLE NO.

B235N3

MATRIX:

SAMPLE DATE SAMPLE TIME

$1-27-10 \quad 1002$

\section{CHAIN OF POSSESSION}

RELINQUISHED BY/REMOVED FROM

RobRomo Roberte Romo $1-27-10$

J.R. Aguilar /REMOYEOFRM EU 12010

RELINOQ PISTED BY REMOVED FROM

RELINQUISHED BY/REMOVED FROM

RELINQUISHED BY/REMOVED FROM

RELUNQUISHED BY/REMOVED FROM

RELINQUISHED BY/REMOVED FROM

LABORATORY RECEIVED BY

SECTION

FINAL SAMPLE DISPOSAL METHOD

\section{SIGN/ PRINT NAMES}

DATE/TTME RECEIVED BY/STORED IN $1 / 15$ mo-4/3 s54R2 $\{2 \geq-10$

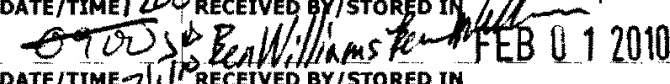
DATE/TIME DATE/TIME DATE/TIME DATE/TIME

RECEIVED BY/STORED IN

RECEIVED BY/STORED IN

RECEIVED BY/STORED IN

RECEIVED BY/STORED IN

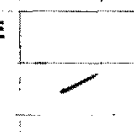
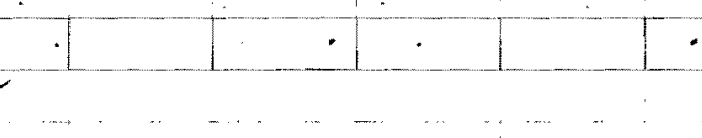

SPECIAL INSTRUCTIONS

DATE/TIME SEE PAGE 2 FOR ALL SPECIAL INSTRUCTIONS

1115

12.00

12,00

DATE/TIME

DATE/TTME

DATE/TIME

2 ORIGNI

DATE/TIME

DATE/TMME 
302117 ES30

\section{SPECIAL INSTRUCTIONS}


CH2MHill Plateau Remediation Company

CHAIN OF CUSTODY/SAMPLE ANALYSIS REQUEST

F10-045-134 PAGE 1 of 2

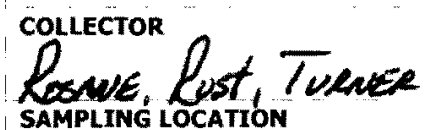

C7515 (209-628-30); I

ICE CHEST NO.

Gws - 169

SHIPPED TO

Environmental sciences Laboratory

MATRIX* POSSTIBLE SAMple HAZARDS/ REMARKS

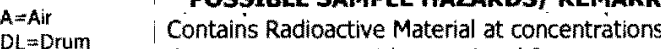

that may or may not be regulated for

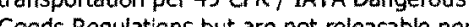

Goods Regulations but are not releasable per
DOE Order $5400.5(1990 / 1993)$

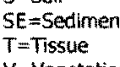

$\mathrm{V}=$ Vegetation
$W=$ Water
WT

$\mathrm{m}=\mathrm{W}$
$\mathrm{x}=\mathrm{Oth}$

SPECIAL HANDLING AND/OR STORAGE RADIOACTIVE TEE TO: B23644

B235N4

SAMPLEÑO.

$$
\text { soll }
$$

MATRIX*

COMPANY CONTACT

DYEKMAN, DL

TELEPHONE NO.

373-2530

PROJECT COORDIMATOR

DYEKMAN, DL

SAF NO.

OJECT DESIGNATION

200-PW-2 OU Charderzation Vadose Zone - Geochemical Modeling Paran F10-045

FIELD LOGBOOK NO. - ACTUAL SAMPLE DEPTH COA

HNF-N- 49/-5

OFFSITE PROPERTY NO?

53

215,5

302117ES30

N/A

PRESERVATION

TYPE OF CONTAINER

NO. OF CONTAINER(S)

vOLUME

SAMPLE ANALYSIS

215.5

ILL OF LADING/AIR BILL NO.

N/A

CHAIN OF POSSESSION

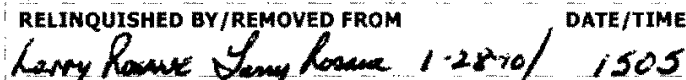

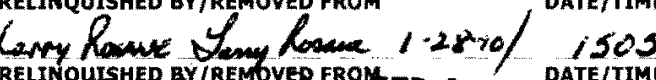
URNAROUND

Days / 45
Days
RELINQUISHED BY/TEG OUED FROM

RELINQUUTSHED BY/REMOVED FROM

RELINQUISHED BY/REMOVED FROM

RELINQUISHED BY/REMOVED FROM

RELINQUISHED BY/REMOVED FROM

\section{LABORATORY RECEIVED BY} SECTION

FINAL SAMPLE DISPOSAL METHOD

DISPOSTTION
SIGN/ PRINT NAMES

RECEIVED BY/STORED IN

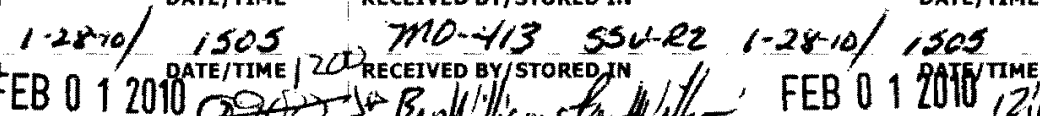

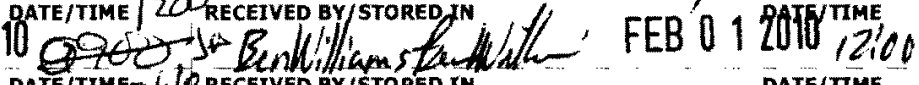

DATE/TTME TRECEIVED BY/STORED IN

DATE/TIME

DATE/TMM

DATE/TIME

RECEIVED BY/STORED IN

DATE/TMM

DATE/TIME

DATE/TIME

RECEIVED BY/STORED IN

DATE/TIME

\section{SPECIAL INSTRUCTIONS}

SEE PAGE 2 FOR ALL SPECIAL INSTRUCTIONS
RECEIVED BY/STORED IN 


\section{CH2Mrill Plateau Remediation Company}

CHAIN OF CUSTODY/SAMPLE ANALYSIS REQUEST

F10-045-134

\section{COLLECTOR}

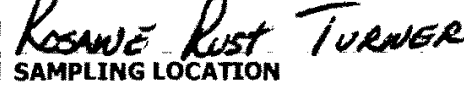

C7515 (299-E28-30): I-089

ICE CHEST NO.

$$
6,055-169
$$

\section{SHIPPED TO}

Environmental Sciences Laboratory

\section{TELEPHONE NO.}

PROJECT COORDINATOR

DYEKMAN, DL

PRICE CODE

\section{DreTha, OL}

SAF NO.

AIR QUALITY

200-PW-2 OU Characterization Vadose Zone - Geochemical Modeling Param F10-045

FIELD LOGBOoK NO. ACTUAL SAMPLE DEPTH

HNF-N- 44/-5

$53 \quad 215,5^{\prime}$

302117 ES30

METHOD OF SHIPMENT

OFFSTIE PROPERTY HOO

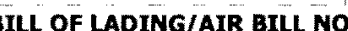

\section{SPECIAL INSTRUCTIONS}

** The 200 Area S\&GRP Characterization and Monitoring Sampling and Analysis GKI applies to this SAF.

** ESL shall submit all data deliverables according to SOW "200-LW-2 and 200-BP-5 Operable Units/Contaminant and Transport Property Analysis and Report for Vadose Zone Sediments at Borehole C5860/Well 299-E29-54" for

** The ESL laboratory shall meet prior requested and confirmed turnaround times and RDLs specified in the SOW and this SAF. The laboratories must notify the SM Project Coordinator in writing iffwhen this requirement can not be met. (1)6010M_ICP_ASTM_AE (TAL) \{Sodium, Aluminum, Iron, Potassium, Antimony, Nickel, Calcium, Magnesium, Sllver\} 6010M_ICP_ASTM_AE (Add-On) \{Lead, Thallium, Selenium\} 6010M_METALS_ICP.WE (TAL) \{Sodium, Aluminum,

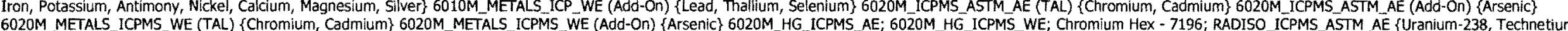

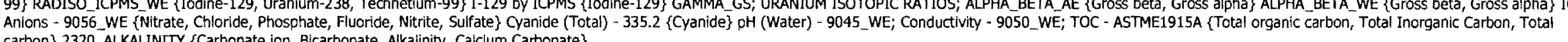
carbon\} 2320_ALKALINITY \{Carbonate Ion, Bicarbonate, Alkailinity, Caiclum Carbonate\}

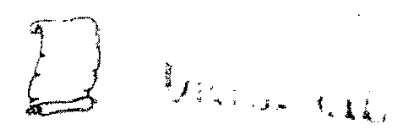


PROJECT DESIGNATION

PRICE CODE

\begin{tabular}{l|l|l} 
SAF NO. & AIR QUALITY \\
\hline Fin
\end{tabular}

200-PW-2 OU Characterization Vadose Zone - Geochemical Modeling Param

FIELD LOGBOOK NO.

COA METHOD OF SHIPMENT

HNF-N-491-5 Pg 53 2 2/7.5

OFFSTTE PROPERTY NO.

MATRIX* ;

B235N5

SAMPLE DATE SAMPLE TIME

CHAIN OF POSSESSION

SIGN/ PRINT NAMES

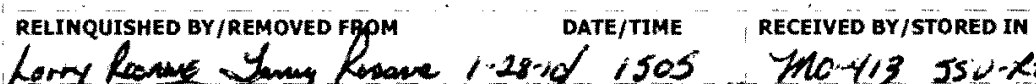

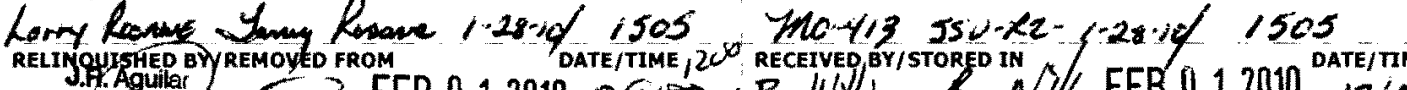

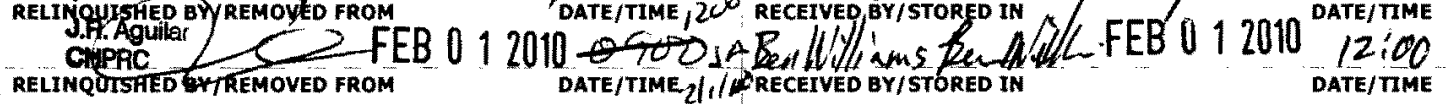

RELINQUISHED BY/REMOVED FROM

RELINQUISHED BY/REMOVED FROM

RELINQUISHED BY/REMOVED FROM

RELINQUISHED BY/REMOVED FROM

RECEIVED BY

SECTION

EINAL SAMPLE DISPOSAL METHOD

DISPOSITION
DATE/TTME RECEIVED BY/STORED IM

DATE/TIME RECEIVED BY/STORED IN

DATE/TIME RECEIVED BY/STORED IN

DATE/TIME RECEIVED BY/STORED IN
DATE/TIME

DATE/TIMEE

DATE/TIME

DATE/TIME

\section{SPECTAL INSTRUCTIONS}

SEE PAGE 2 FOR ALL SPECIAL INSTRUCTIONS

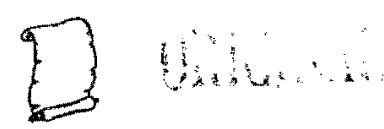


CH2MHill Plateau Remediation Company

\section{COLLECTOR}

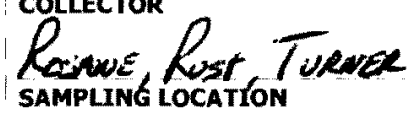

SAMPLINGLOCATION

ICE CHEST NO.

SHIPPED TO

Environmental Sciences Laboraton

\section{SPECIAL INSTRUCTIONS}

** The 200 Area S\&GRP Characterization and Monitoring Sampling and Analysis GKI applies to this SAF

** ESL shall submit all data deliverables according to SOW "200-LW-2 and 200-BP-5 Operable Units/Contaminant and Transport Property Analysis and Report for Vadose Zone Sediments at Borehole C5860/Well 299-E29-54" for

** The ESL laboratory shall meet prior requested and confirmed turnaround times and RDLs specified in the SOW and this SAF. The laboratories must notify the SM Project coordinator in writing if/when this requirement can not be met.

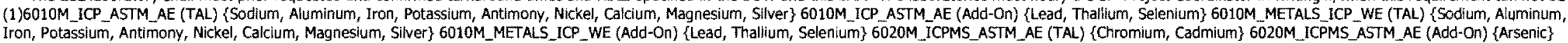

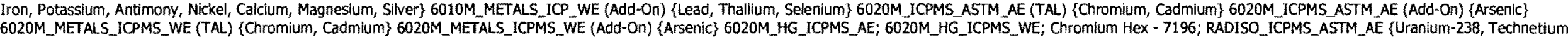
99\} RADISO_ICPMS WE \{Iodine-129, Uranium-238, Technetium-99\} 1-129 by ICPMS \{Iodine-129\} GAMMA_GS; URANIUM ISOTOPIC RATIOS; ALPHA_BETA_AE \{Gross beta, Gross alpha\} ALPHA_BETA_WE \{Gross beta, Gross alpha\} IC Anions - 9056 WE \{Nitrate, Chloride, Phosphate, Fluoride, Nitrite, Sulfate\} Cyanide (Total) - 335.2 \{Cyanide\} PH (Water) - 9045_WE; Conductivity - 9050_WE; TOC - ASTME1915A \{Total organic Carbon, Total Inorganic Carbon, Totai carbon\} 2320_ALKALINITY \{Carbonate ion, Bicarbonate, Alkainity, Calcium Carbonate 


\begin{tabular}{|c|c|c|c|c|c|}
\hline $\begin{array}{l}\text { COMPANY CONTACT } \\
\text { DVEKMAN, DL }\end{array}$ & $\begin{array}{l}\text { TELEPHONE NO. } \\
373-2530\end{array}$ & $\begin{array}{l}\text { PROJECT COORDINATOR } \\
\text { DYEKMAN, DL }\end{array}$ & PRICE CODE & $\mathbf{8 N}$ & $\begin{array}{c}\text { DATA } \\
\text { TURNAROUND }\end{array}$ \\
\hline PROJECT DESIGNATION & & $\begin{array}{l}\text { SAF NO. } \\
\text { SF10-045 }\end{array}$ & AIR QUALITY & $\square$ & $\begin{array}{l}45 \text { Days / } 45 \\
\text { Days }\end{array}$ \\
\hline
\end{tabular}

SAMPLE No.

MATRIX*

B235N6

PROJECT DESIGNATIO

SAF NO.
F10-045

200-PW-2 OU Characte ACTUAL SAMPLE DEPTH COA

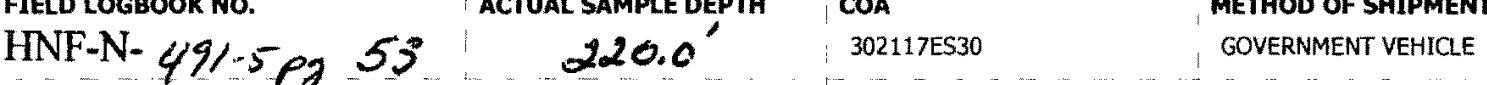

OFFSITE PROPERTY NO. T' BILL OF LADING/AIR BILL NO.

N/A

N/A

PRESERVATION

TYPE OF CONTAINER

NO. OF CONTAINER(S)

volume

SAMPLE ANALYSIS

SAMPLE DATE SAMPLE TIME

$1.28-10 \quad 0920$

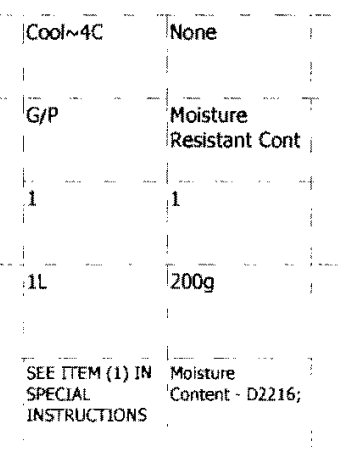

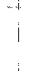

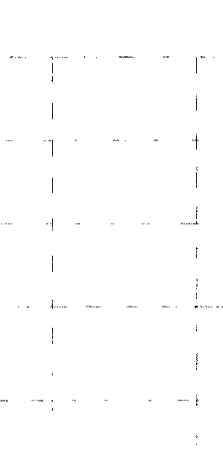

CHAIN OF POSSESSION

RELINQUISHED BY/REMOVED FROM

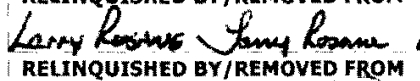
J.R. Agenar ar

RELINQUISHED BY/REMOVED FROM

RELINQUISHED BY/REMOVED FROM

RELINQUISHED BY/REMOVED FROM

RELINQUUSHED BY/REMOVED FROM
SIGN/ PRINT NAMES

DATE/TIME RECEIVED BY/STORED IN

1505 MO. 413 5SU.Re 1.28.i4 DATE/TM

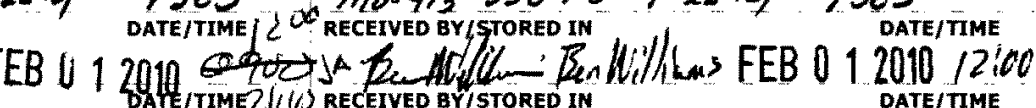

DATE/TIME

RECEIVED BY/STORED IN

DATE/TIME

RECEIVED BY/STORED IN

Date/Time

RECEIVED BY/STORED IN

DATE/TIME

RECEIVED BY/STORED iN

DATE/TIME

DATE/TIME

DATE/TIME

date/time

SPECIAL INSTRUCTIONS

SEE PAGE 2 FOR ALL SPECIAL INSTRUCTIONS 
CH2MHill Plateau Remediation Company

\section{caucrone \\ Remes lost Trence}

C7515 (299-E28-30); 1091

ICE CHEST NO.

\section{SHIPPED TO}

Environmental Sciences Laboraton

\section{SPECIAL INSTRUCTIONS}

** The 200 Area S\&GRP Characterization and Monitoring Sampling and Analysis GKI applies to this SAF.
** ES shall subnit all Iata delliverables according to SOW "200-LW-2 and 200-PP-5 Operable Units/Contaminant and Transport Property Analysis and Report for Vadose Zone Sediments at Borehole C5860/Well 299-E29-54" for C7515/Well 299-E28-30 ("M" Well) and shall adhere to all SRGRP standard protocol.

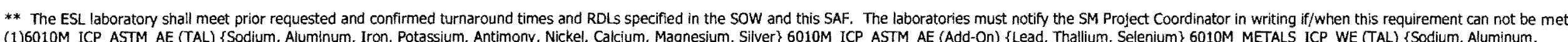
(1)6010M_ICP_ASTM_AE (TAL) \{Sodium, Aluminum, Iron, Potassium, Antimony, Nickel, Calcium, Magnesium, Silver\} 6010M_ICP_ASTM_AE (Add-On) \{Lead, Thallium, Selenium\} 6010M_METALS_ICP_WE (TAL) \{Sodium, Aluminum, 6020M_METALS_ICPMS_WE (TAL) \{Chromium, Cadmium\} 6020M_METALS_ICPMS_WE (Add-On) \{Arsen_ic\} 6020M_HG_ICPMS_AE; 6020M_HG_ICPMS_WE; Chromium Hex-7196; RADISO_ICPMS_ASTM_AE \{Uranium-238, Technetium99\} RAD - 9056_WE [Nitrate, Chloride, Phosphate, Fluoride, Nitrite, Sulfate\} Cyanide (Total) - 335.2 \{Cyanide\} pH (Water) - 9045 _WE; Conductivity - 9050_WE; TOC - ASTME1915A \{Total organic carbon, Total Inorganic Carbon, Total
Anlons
PROJECT DESIGNATION

TELEPHONE NO.

TATOR

DYEKMAN, DL

SAF NO.
F10-045

FIELD LOGBOOK NO.

ACTUAL SAMPLE DEPTH

HNF-N- 49/-5

COA

\section{METHOD OF SHIPMEN}

$$
\text { D }
$$


PROJECT DESIGNATION

373-2530

PRICE CODE

DYEKMAN, D

\begin{tabular}{l:l} 
& SAF NO. \\
200-PW-2 OU Characterization Vadose Zone - Geochemical Modeling Param & F10-045 \\
\hline
\end{tabular}

FIELD LOGBOOK NO. ACTUAL SAMPLE DEPTH

COA-

\begin{tabular}{l|c|c|c|c|} 
HNF-N- $491-5 \rho 253$ & $22.0^{\prime}$ & $302117 E S 30$ & MEVERNMENT VEHICLE
\end{tabular}

BILL OF LADING/AIR BILL NO.

N/A

SAMPLE NO.

MATRIX*

PRESERVATION

TYPE OF CONTAINER

NO. OF CONTAINER(S)

voLume

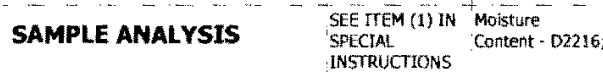

SAMPLE DATE SAMPLE TIME

$1-28-10 \quad 09+0$

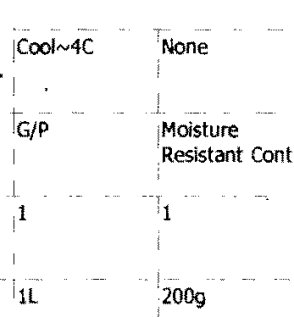

Days

CHAIN OF POSSESSION

SIGN/ PRINT NAMES

SPECIAL INSTRUCTIONS

RELINQUISHED BY/REMOVED FROM DATE/TIME "RECEVED BY/STORED IN

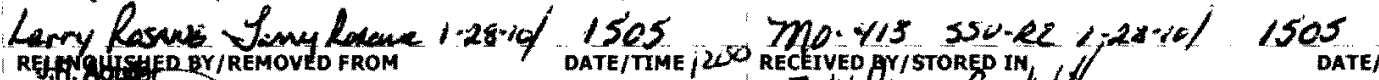
OHPR

RELINQUUISHED BY /REMOVED FROM

RELINQUTSHED BY/REMOVED FROM

RELINQUTSHED BY/REMOVED FROM

RELINQUISHED QY/REMOVED FROM

LABORATORY RECEIVED BY

SECTION

FINAL SAMPLE DISPOSAL METHOD

DISPOSITION
DATE/TIME RECEIVED BY/STORED IN

DATETTIME" " RECEIVED BY/STORED IN

DATE/TIME RECEIVED BY/STORED IN

DATE/TIME RECEIVED BY/STORED IN
DATE/TTME

DATE/TIME

DATE/TME

Date/time
\& Num? 
CH2MHill Plateau Remediation Company

CHAIN OF CUSTODY/SAMPLE ANALYSIS REQUEST

F10-045-138

PAGE 2 OF 2

\section{COLlector \\ Saswive Rust Tucation TuRuER \\ SAMRLINE tocation \\ ICE CHEST NÖ. \\ SHIPPED TO}

TELEPHONE NO.

PROJECT COORDINATOR

PRICE CODE

DATA

TURNAROUND

DROJEAN, DL

SAF NO.

AIR OUALITY

45 Days / 45
Days

200-PW-2 OU Charaterizon Vafose Zone - Geochemical Modeling Param F10-045

FIELD LOGBOOK NO. ACTUAL SAMPLE DEPTH COA

METHOD OF SHIPMEN

HNF-N-49/-5 pz 53 222.0' 302117ES30

GOVERNMENT VEHICLE

OFFSTE PROPERTY NG

N/A

N/A

\section{SPECIAL INSTRUCTIONS.}

** The 200 Area S\&GRP Characterization and Monitoring Sampling and Analysis GKI applies to this SAF.

*** ESL shall submit all data deliverables according to SOW "200-LW-2 and 200-BP-5 Operable Units/Contaminant and Transport Property Analysis and Report for Vadose Zone Sediments at Borehole C5860/Well 299-E29-54" for
$\mathrm{C} 5515$ / Well 299-E28-30 ("M" Well) and shall adhere to ail S\$GRP standard protocol.

** The ESL laboratory shall meet pror requested and confirmed turnaround times and RDLs Specifed in the SOW and this SAF. The laboratories must notify the SM Project Coordinator in writing iffiwhen this requirement can not be met

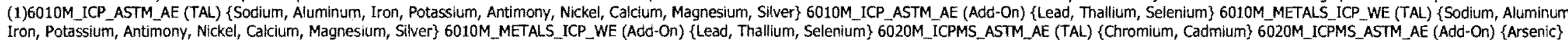
6020M_METALS_ICPMS_WE (TAL) \{Chromium, Cadmium\} 6020M_METALS_ICPMS_WE (Add-On) \{Arsenic\} 6020M_HG_ICPMS_AE; 6020M_HE_ICPMS_WE; Chromium Hex-7196; RADISO_ICPMS_ASTM_AE \{Uranilum-238, TeChnetiumAnions - 9056 WE (Nitrate, Chloride, Phosphate, Fluoride, Nitrite, Suifate\} Cyanide (Total) - 335.2 \{Cyanide\} pH (Water) - 9045_WE; Conductivity - 9050_WE; TOC - ASTME1915A (Total organic carbon, Total Inorganic Carbon, Total

D DRGALA 


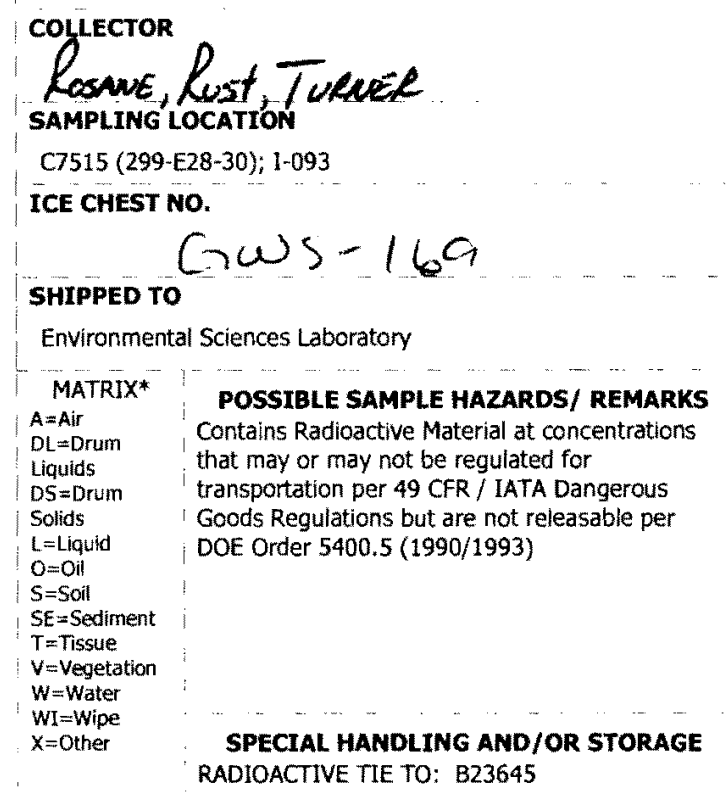
$\begin{array}{lll}373-2530 & \text { DYEKMAN, } \\ & \text { SAF NO. } \\ & \text { F10-045 }\end{array}$

PRICE CODE DATA

PROJECT DESIGNATION

AIR QUALITY

200-PW-2 OU Characterization Vadose Zone - Geochentical Modeling Param
FIEL LOGBOOK NO. PS 33 ACTUAL SAMPLE DEPTH

HNF-N- $/ 9 / 95$ S'3 ACTUAL SAMPLE DEFTH COA METHOD OF SHIPMENT

Days

OFFSTTE PROPERTY NO.

302117 S30 GOVERMMENT VEHIOE

N/A

PRESERVATION

TYPE OF CONTAINER

NO. OF CONTAINER(S)

VOLUME

SAMPLE ANALYSIS

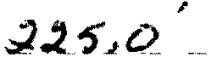

BILL OF LADING/AIR BILL NO.

N/A

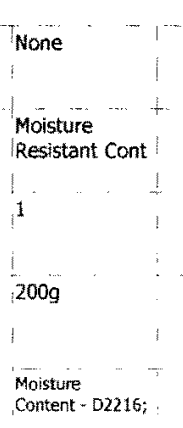

$\begin{array}{lllll}\text { SAMPLE NO. } & \text { MAMPLE Date SAMPLE TIME } \\ \text { B235N8 } & \text { SOIL } & 1-28 \% 10 & 1025\end{array}$

CHAIN OF POSSESSION

RELINQUUSHED BY/REMOVED FROM

Lermy hesue Yom heme

CHPRC

RELINQUISHED QY REMOVEO FROM

RELINQUUISHED BY/REMOVED FROM

RELINQUUTSHED BY/REMOYED FROM

RELINQUUISHED BY/REMOVED FROM

RELINQUISHED BY/REMOVED FROM

RECEIVED SECTION

inAl SAMPLE DIsposal Method

DISPOSITION
SIGN/ PRINT NAMES

DATE/TIME RECEIVED BY/STORED IN

DATE/TIME RECEIVED BY/STORED IN
1505 DO.
DATE/TTME 200 RECEIVED GY/STORED IN

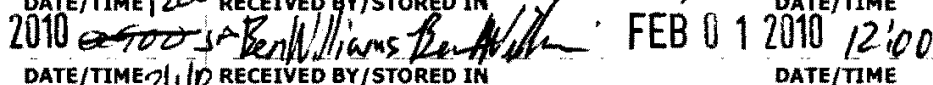

DATE/TIME RECEIVED BY/STORED IN

DATE/TIME RECEIVED BY/STORED IN

DATE/TIME RECEIVED BY/STORED IN

DATE/TIME
DATE/TTME

DATE/TIME

DATE/TIME

DATE/TIME
SPECIAL INSTRUCTIONS

SEE PAGE 2 FOR ALL SPECIAL INSTRUCTIONS 
COLLCTOR

\section{Rossue Rust, Tuenter}

ICE CHEST NO.

Givs - 169

Envircnmental Sciences Laboraton

\section{SPECIAL INSTRUCTIONS}

*** The 200 Area S\&GRP Characterization and Monitoring Sampling and Analysis GKI applles to this SAF.

** ESL shall submit all data deliverables according to SOW "200-LW-2 and 200-BP-5 Operable Units/Contaminant and Transport Property Analysis and Report for Vacose Zone Sediments at Borehole C5860/Well 299-E29-54" for

** The ESL laboratory shall meet prior requested and confirmed turnaround times and ROLs specified in the SOW and this SAF. The laboratories must notify the SM Project Coordinator in writing iff/when this requirement can not be met (1)6010M_ICP_ASTM_AE (TAL) \{Sodium, Alumirum, Iron, Potassium, Antimony, Nickel, Calcium, Magnesium, Silver\} 6010M_ICP_ASTM_AE (Add-On) \{Lead, Thallium, Selenium\} 6010M_METALS_ICP_WEE (TAL) \{Sodium, Aluminum,

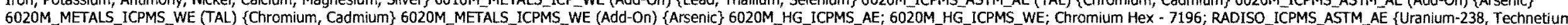
Anions - 9056_WE \{Nitrate, Chloride, Phosphate, Fluoride, Nitrite, Sulfate\} Cyanide (Total) - 335.2 \{Cyanide\} PH (Water) - 9045_WE; Conductivity - 9050_WE; TOC - ASTME1915A \{Total organic carbon, Total Inorganic Carbon, Total carbon\} 2320_ALKALINITY \{Carbonate ion, Bicarbonate, Alkalinity, Calcium Carbonate\}
ROJECT COORDINATOR

F10-045

$302117 E S 30$

METHOD OF SHIPMEN

HNF-N- 49/

$225.0^{\circ}$

IADING/AIR BIL NO.
PS 53 actUAL SAMPLE DEP
DATA
URNAROUND

45 Days / 45
Days

\section{D. orlGitil}




$$
\text { Gow } 5 \text { - 14? }
$$

SHIPPED TO

Environmental Sciences Laboratory

MATRIX*
A=Air. Contains Radicactive Material at concentrations that may or may not be regulated for
transportation per 49 CFR / IATA Dangerous Goods Reguations but are not releasabie per Goods Regulations but are not re
DOE Order 5400.5 (1990/1993)

SPECIAL HANDLING AND/OR STORAGE RADIOACTIVE TIE TO: B23645

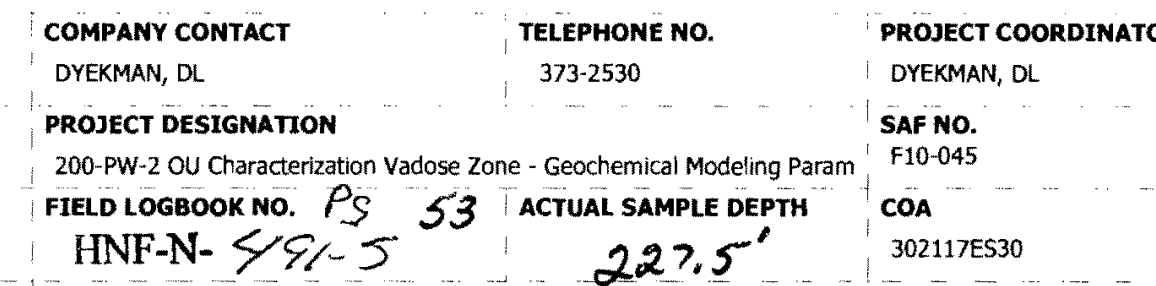

PRICE CODE

DATA

AIR QuALIT

8N

OFFSTTE PROPERTY NO.

BILL OF LADING/AIR BILL NO.

N/A

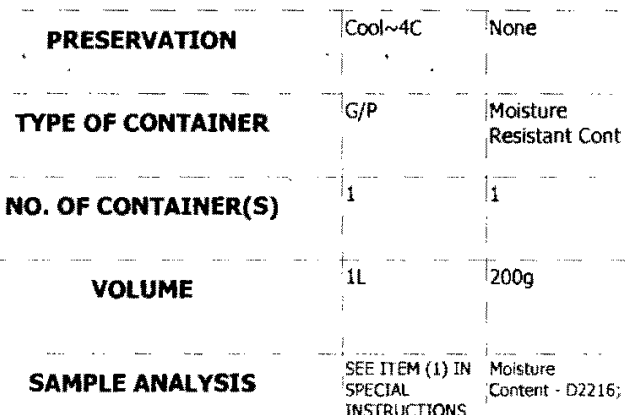

N/A

SAMpleno.

MATRIX*

SAMPLE DATE SAMPLE TIME.

$1-28 \cdot 10 \quad 12.48$

${ }_{2}$

CHAIN OF POSSESSION

SIGN/ PRINT NAMES

RELINQUISHED BY/REMOVED FROM DATE/TTME RECEIVED BY/STORED IN

DATE/TIME RECEIVRED BY/STORED IN

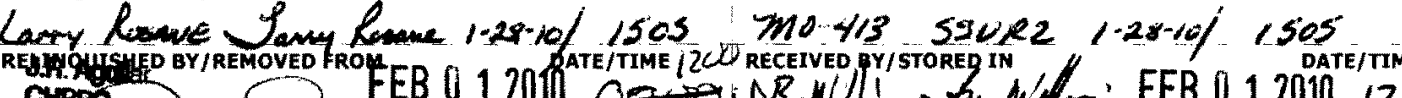

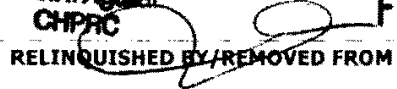

RELINQUUSSHED BY/REMOVED FROM

RELINQUISHED BY/REMOVED FROM

RELINQUISHED BY/REMOYED FROM

RELINQUISHED BY / REMOVED FROM

RECEIVED B

SECTION

FINAL SAMPLE DISPOSAL METHOD

Of

DATE/TME

RECEIVED BY/STORED IN

Date/TIME

DATE/TIME

RECEIVED BY/STORED IN

DATE/TIME

DATE/TIME

DATE/TME

DATE/TIME

RECEIVED BY/STORED IM

DATE/TIME
SPECIAL INSTRUCTIONS

SEE PAGE 2 FOR ALL SPECIAL INSTRUCTIONS 


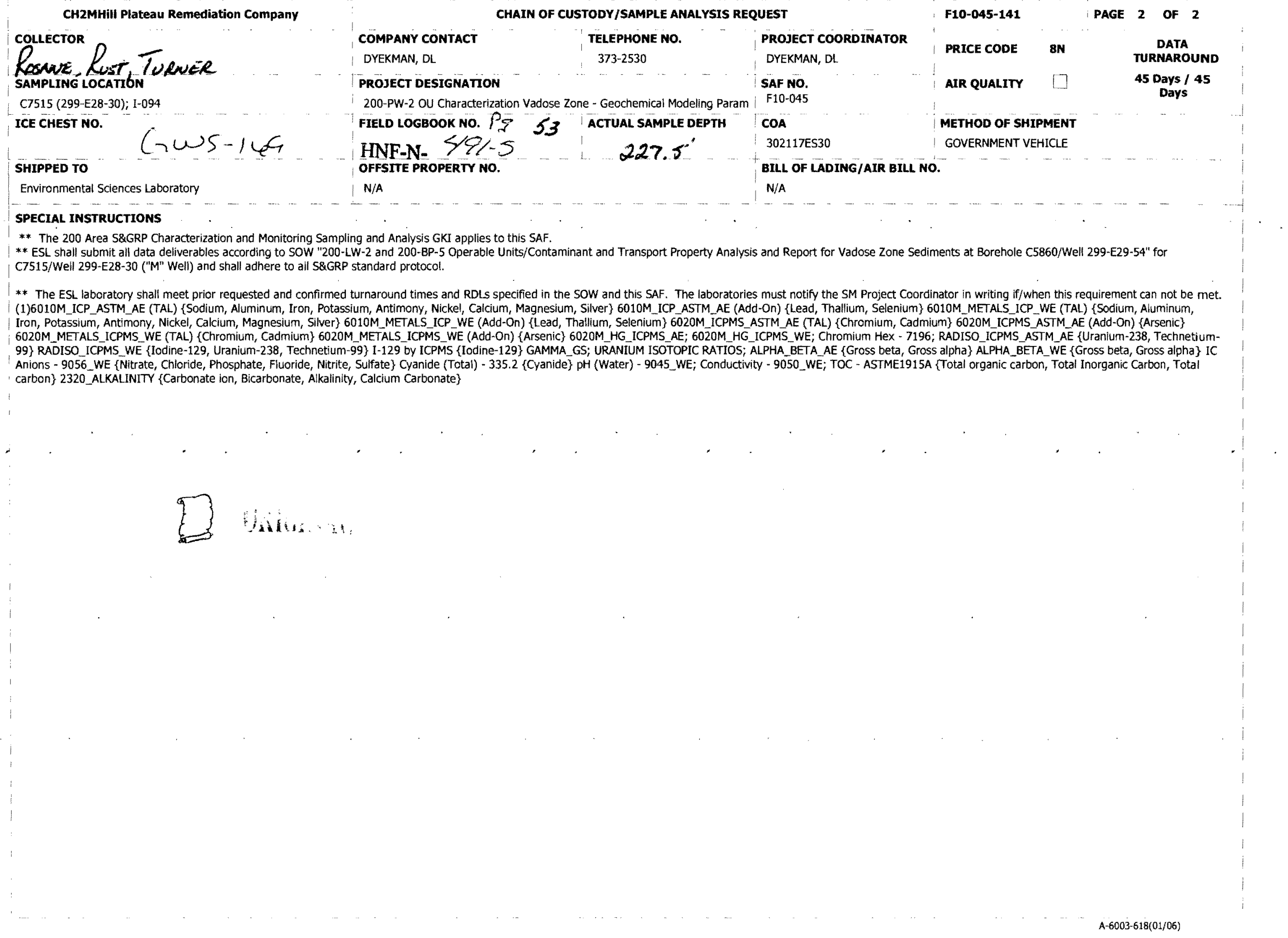


373-2530

PROJECT COORDINATOR

PROJECT DESIGNATION

AIR QUALITY $\square$

TURNAROUND

200-PW-2 OU Characteration Vadose Zone - Gecchemical Modeling Param F10-045

cows - 169

FIELD LOGBOOK NO. PS 53 TACTUAL SAMPLE DEPTH

COA METHOD OF SHIPMENT

\begin{tabular}{l|l|l|l|l|} 
HNF-N- & $29 / .5$ & $302117 E S 30$ & GOVERNMENT VEHICLE
\end{tabular}

Environmental Sciences Laboratory

OFFSTIE PROPERTY NO.

BILL OF LADING/AIR BILL NO

N/A

TAZTES/ REMARKS

that may or may not be regulated for
than

transportation per 49 CFR / IATA Dangerous

Goods Regulations but are not releasable per
DOE Order $5400.5(1990 / 1993)$

SPECIAL HANDLING AND/OR STORAGE RADIOACTIVE TIE TO: B23661

TYPE OF CONTAINER G/P Meisture

NO. OF CONTANER

[1 $\quad \cdots \quad-1$

VOLUME

il. 2009

SAMPIE AMALYSIS

$$
\begin{aligned}
& \text { SEE ITEM (1) IN MOistare } \\
& \text { 'PSECIA } \\
& \text { JNSTRUCTONS }
\end{aligned}
$$

SAMPLE NO.

MATRIX*
SOIL
SAMPLE DATE SAMPLE TIME

$128-10 \quad 1245$

\section{CHAIN OF POSSESSION}

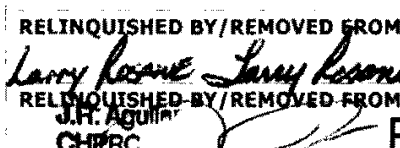
CHRBC

RELINQUISHED BY/REMOVED FROM

RELINQUISHED BY/REMOVED FROM

REIINQUISHED BY/REMOVED FROM

RELINQUISHED BY/REMOVED FROM

\section{LABORATORY
SECTION}

FINAL SAMPLE DISPOSAL METHOD

\section{SIGN/ PRINT NAMES}

RECEIVED BY/STORED IN $1505 \quad m 0-4135502 z$

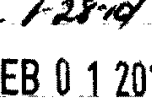

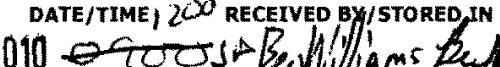

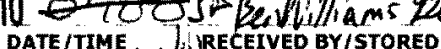
DATE/TIME

DATE/TIME

RECEIVED BY/STORED IN

DATE/TIME

RECEIVED BY/STORED IN

DATE/TIME
RECEIVED BY/STORED IN

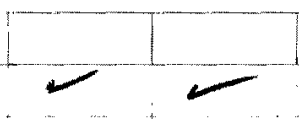
$1-28-101$ sos W'FEB O $12010^{12: 00}$ DATE/TIME DATE/TIM DATE/TIME DATE/TTME DATE/TIME
SPECIAL INSTRUCTIONS

SEE PAGE 2 FOR ALL SPECIAL INSTRUCTIONS

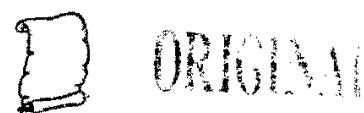




\section{COLLECTOR}

Fin-045

\section{SPECIAL INSTRUCTIONS}

\section{HNF-N- $Y Q /-2$}

$302117 E S 30$

\section{METHOD OF SHIPMENT}

GOVERNMENT VEHICLE
OFFSTE PROPERTY NO.
N/A

** The 200 Area S\&GRP Characterization and Monitoring Sampling and Analysis GKI applies to this SAF.

** ESL shall submit all data deliverables according to SOW "200-LW-2 and 200-BP-5 Operable Units/Contaminant and Transport Property Analysis and Report for Vadose Zone Sediments at Borehole C5860/Weil 299-E29-54" for

** The ESL laboratory shall meet prior requested and confirmed turnaround times and RDLs specifiled in the SOW and this SAF. The laboratories must notify the SM Project Coordinator in writing if /when this requirement can not be met. (1)6010M_ICP_ASTM_AE (TAL) \{Sodium, Aluminum, Iron, Potassium, Antimony, Nickel, Calcium, Magnesium, Silver\} 6010M_ICP_ASTM.AE (Add-On) \{Lead, Thallium, Selenium\} 6010M_METALS_ICP.WEE (TAL) \{Sodium, Aluminum, 60201_METALS_ICPMS WE (TAL) \{Chromium, Cadmium\} 6020M_METALS_ICPMS_WE (Add-On) \{ATSenic\} 6020M,HG_ICPMS_AE; 6020M_HG_ICPMS_WE; Chromium Hex-7196; RADISO_ICPMS_ASTM_AEE \{Uranium-238, Technetium -

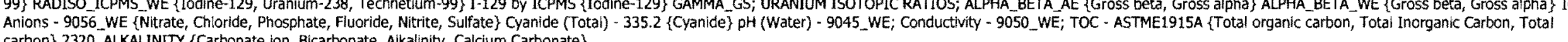
Carbon\} 2320_ALKALINITY \{Carbonate ion, Bicarbonate, Akalinity, Calcium Carbonate\}

\section{B DRGINII}




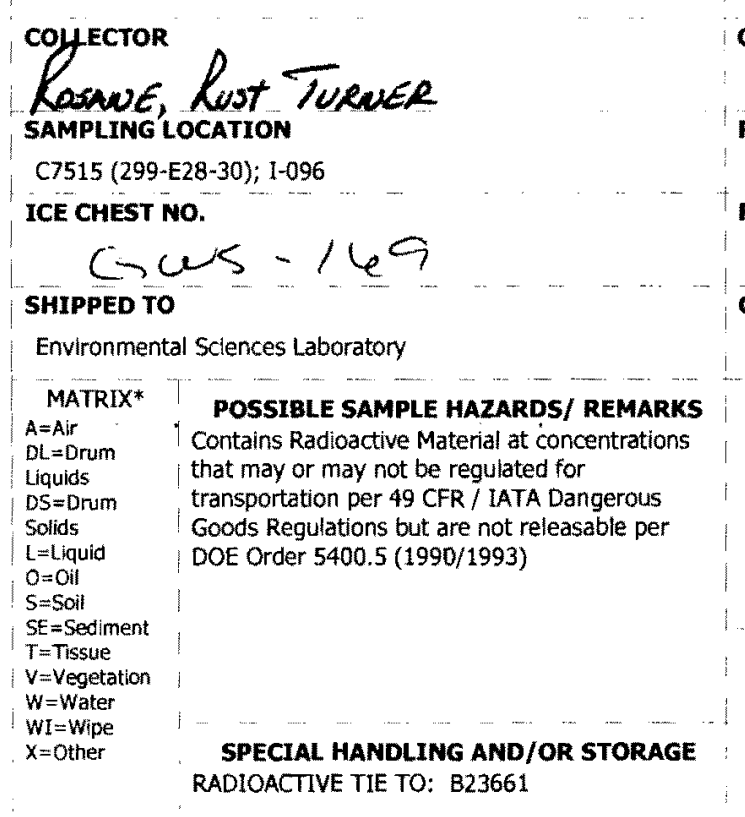

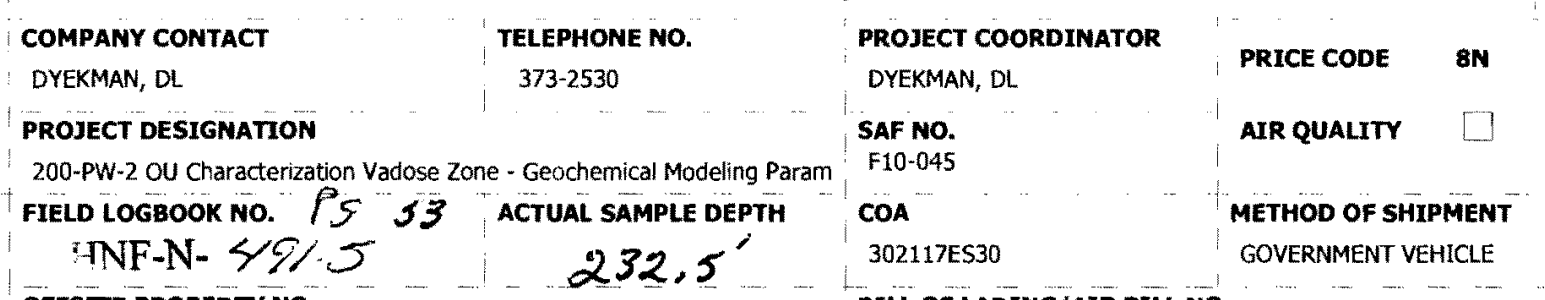




\section{SPECIAL INSTRUCTIONS}

** The 200 Area S\&GRP Characterization and Monitoring Sampling and Analysis GKI applies to this SAF.

** ESL shall submit all data deliverables according to SOW "200-LW-2 and 200-BP-5 Operable Units/Contaminant and Transport Property Analysis and Report for Vadose Zone Sediments at Borehole C5860/Well 299-E29-54" for

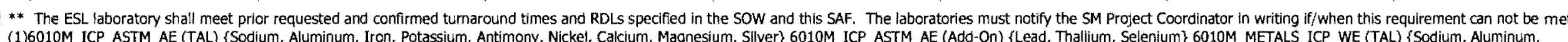
(1)6010M_ICP_ASTM_AE (TAL) \{Sodium, Aluminum, Iron, Potassium, Antimony, Nickel, Calcium, Magnesium, Sllver\} 6010M_ICP_ASTM_AE (Add-On) \{Lead, Thallium, Seenium\} 6010M_METALSIICP_WE (TAL) \{Sodium, Aluminum,

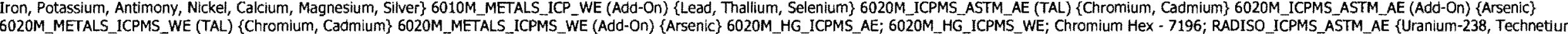
99\} RADISO ICPMS WE \{lodine-129, Uranium-238, Technetium-99\} I-129 by ICPMS \{lodine-129\} GAMMA GS; URANIUM ISOTOPIC RATIOS; ALPHA BETA AE \{Gross beta, Gross alpha\} ALPHA BETA_WE \{Gross beta, Gross alpha\} IC Anions - 9056_WE \{Nitrate, Chiloride, Phosphate, Fluoride, Nitrite, Sulfate\} Cyanide (Total) - 335.2 \{Cyanide\} pH (Water) - 9045_WE; Conductivity - 9050_WE; TOC - ASTME1915A (Total organic Carbon, Total Inorganic Carbon, Total carbon\} 2320_ALKALINITY \{ Carbonate ion, Bicarbonate, Alkalinity, Calcium Carbonate 
MATRIX*

$\mathrm{B} 235 \mathrm{P} 2$

SAMPLE DATE SAMPLE TIME

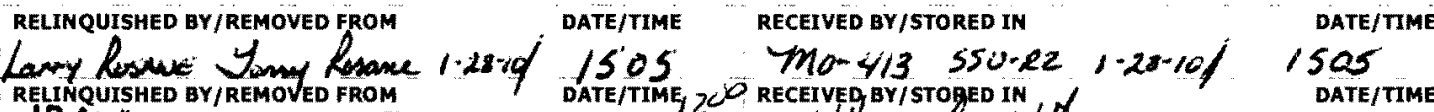

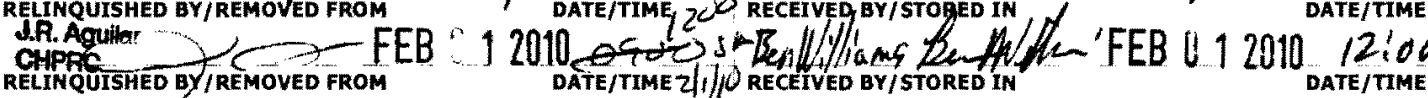

RELLNQUISHED BY/REMOVED FROM

RELINQUISHED BY/REMOVED FROM

RELINQUISHED BY/REMOVED FROM

RELINQUUISHED BY/REMOVED FROM

DATE/TIME

DATE/TIME

DAte/time

DATE/TIME

Date/time

DATE/TIME

DATE/TIME

DATE/TIME

\section{LABORATORY
SECTION}

FINAL SAMPLE DISPOSAL METHOD

\section{SPECIAL INSTRUCTIONS}

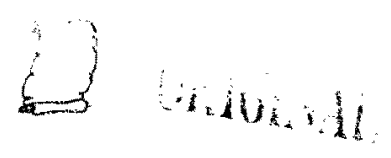

SEE PAGE 2 FOR ALL SPECIAL INSTRUCTIONS 


\section{SPECIAL INSTRUCTIONS}


\begin{tabular}{l|l}
$373-2530$ & DYEKMAN, DL
\end{tabular}

SAT NO.

RROJECT DESIGNATION

200-PW-2 OU Characterization vadose Zone - Geochemical Modeling Param
FIELD LOGBOOK NO. PS S3 ACTUAL SAMPLE DEPTH

HNF-N- $591-5$

53 ACTUAL SAMPLE DEPTH

AIR QUALTY $\square$

DATA

OFFSTE PROPERTY NO.

$237.0^{\prime}{ }^{302117 E 530}$

$\underset{\text { Days }}{45 \text { Days }} 45$

N/A

PRESERVATION

TYPE OF CONTAINER

NO. OF CONTAINER(S)

voLume

SAMPLE ANALYSIS

METHOD OF SHIPMENT

SAMPLENO

B235P3

SOIL

MATRIX*

\section{SAMPLE DATE SAMPLE TIME}

BILL OF LADING/AIR BILL NO.

GOVERNMENT VEHICLE

$1.28 \cdot 10: 1435$

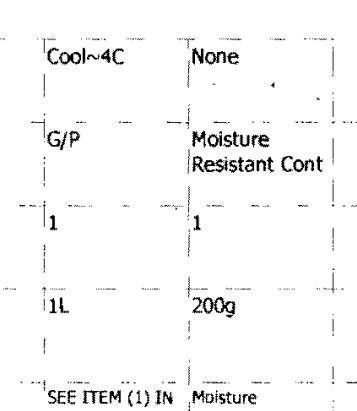

$N / A$

CHAIN OF POSSESSION

RELINQUISHED BY/REMOVED FROM

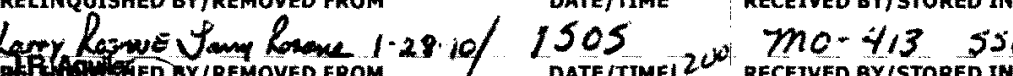

CHPDC

RELINQUISHED GXYREMOVED FROM

RELINQUISHED BY/REMOVED FROM

RELINQUISHED BY/REMOVED FROM

RELINQUISHED BY/REMOYED FROM

RELINQUISHED BY/REMOVED FROM

RECEIVED B

SECTION

FINAL SAMPLE DISPOSAL METHOD

DATE/TTME RECEIVED BY/STORED IN

DATE/TIME

RECEIVED BY/STORED IN

DATE/TIME

RECEIVED BY/STORED IN

DATE/TIME

DATE/TIME

DATE/TIME
DATE/TIME Zl/ IDORECEIVED BY/STORED IM

9 Uinivin.

\section{SPECIAL INSTRUCTIONS}

SEE PAGE 2 FOR ALL SPECIAL INSTRUCTIONS 


\section{CH2MHill Plateau Remediation Company}

\section{COjLECTO \\ SAMPING LOCAT TURUER}

c7515 (299-E28-3

ICE CHEST NO.

$6015-164$

SHIPPED TO

Environmental Sciences Laboratory

COMPANY CONTACT

TELEPHONE NO.

DYEKMAN, DL

$373-2530$

PROJECT COORDINATOR

PROJECT DESIGNATION

200-PW-2 OU Characterization Vadose Zone - Geochemical Modeling Param

FIELD LOGBOOK NO. PS

INF-N- $49 /-5$

53 ACTUAL SAMPLE DEPTH

237.0

OFFSITE PROPERTY NO.

N/A

DYEKMAN, DL

SAF NO

PRICE CODE

AIR QUALITY

DATA
TURNAROUND

$\underset{\text { Days }}{45 \text { Days } / 45}$

COA

METHOD OF SHIPMENT

GOVERNMENT VEHICLE

\section{SPECIAL INSTRUCTIONS}

*** The 200 Area S\&GRP Characterization and Monitoring Sampling and Analysis GKI applies to this SAF.

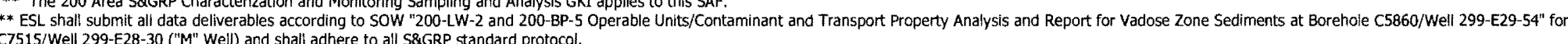

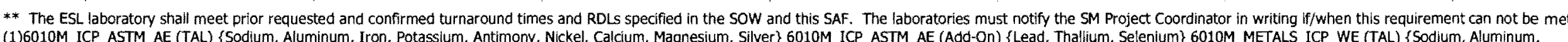
(1)6010M_ICP_ASTM_AE (TAL) \{Sodium, Aluminum, Iron, Potassium, Antimony, Nickel, Calcium, Magnesium, Silver\} 6010M_ICP.ASTM_AE (Add-On) \{Lead, Thallium, Selenium\} 6010M_METALS_ICP_WE (TAL) \{Sodium, Aluminum,

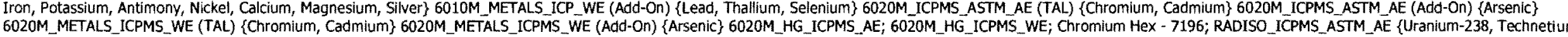

99\} RADISO ICPMS WE \{Todine-129, Uranium-238, Technetium-99\} I-129 by ICPMS \{lodine-129\} GAMMA GS. URANIUM ISOTOPIC RATIOS. ALPHA BETA AE \{Gross beta, Gross alpha\} ALPHA BETA WE \{Gross beta, Gross alpha\} IC Anions - 9056_WE \{Nitrate, Chloride, Phosphate, Fluoride, Nitrite, Sulfate\} Cyanide (Total) - 335.2 \{Cyanide\} PH (Water) - 9045_WE; Conductivity - 9050_WE; TOC - ASTME1915A \{Total organic Carbon, Total Inorganic Carbon, Total carbon\} 2320_ALKALINITY \{Carbonate ion, Bicarbonate, Alkalinity, Cakcium Carbonate\}

Bonine 
CH2MHill Plateau Remediation Company

couteron

\begin{tabular}{|c|c|}
\hline \multicolumn{2}{|c|}{$\begin{array}{l}\text { C7515 (299-E28-30); 1-099 } \\
\text { ICE CHEST NO. } \\
\text { SHIPPED TO } \\
\text { Environmental Sciences Laboratory }\end{array}$} \\
\hline 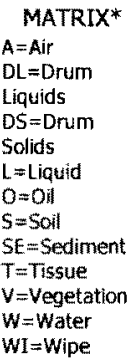 & $\begin{array}{l}\text { POSSIBLE SAMPLE HAZARDS/ REMARKS } \\
\text { Contains Radioactive Material at concentrations } \\
\text { that may or may not be regulated for } \\
\text { transportation per } 49 \text { CRR / IATA Dangerous } \\
\text { Goods Regulations but are not releasable per } \\
\text { DOE Order } 5400.5 \text { (1990/1993) }\end{array}$ \\
\hline $\mathrm{x}=$ other & $\begin{array}{l}\text { SPECIAL HANDLING AND/OR STORAGE } \\
\text { RADIOACTIVE TE TO: B23666 }\end{array}$ \\
\hline
\end{tabular}

CHAIN OF CUSTODY/SAMPLE ANALYSIS REQUEST

F10-045-149

PRICE CODE $\quad$ 8N $\quad \begin{gathered}\text { DATA } \\ \text { TURNAROUND }\end{gathered}$

AIR QUALTYY $\quad \square \quad \underset{\text { Days }}{\mathbf{4} \text { Days }} \mathbf{4 5}$

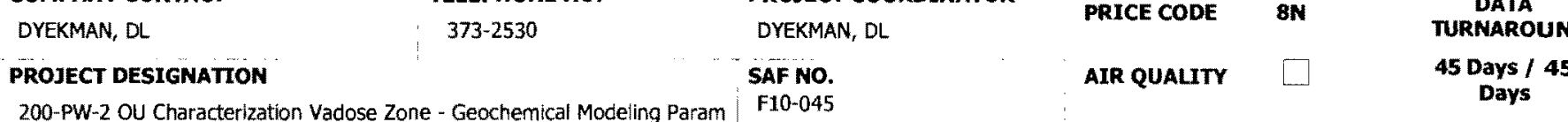

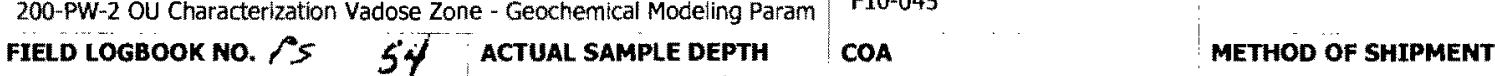

HNF-N- $49 / 5$ S $240.0^{\prime} \quad 302117$ GS30 GOVERNMENT VEHICLE

OFFSTTE PROPERTY NO. BILL OF LADING/AIR BILL NO.

N/A N/A

\begin{tabular}{|c|c|c|}
\hline PRESERVATION & Cool $44 C$ & None \\
\hline TYPE OF CONTAINER & Gip & $\begin{array}{l}\text { Molsture } \\
\text { Resistant Cont }\end{array}$ \\
\hline NO. OF CONTAINER(S) & 1 & 1 \\
\hline & IL & 2009
\end{tabular}

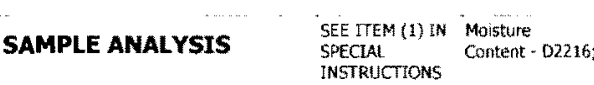

.

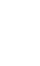
SAMPLE NO.
MATRIX*

soll

\section{SAMPLE DATE SAMPLE TIME}

$1.24=10 \quad 0748$

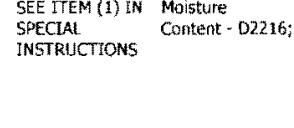

\section{CHAIN OF POSSESSION}

RELINQYSSHED BY/REMOVED FROM

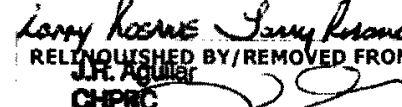
RELINQUISHED BY I REMOVED FROM

RELINQUISHED BY / $/$ KEMOVED FROM

RELINQUISHED BY/REMOVED FROM

RELINQUISHED BY/REMOVED FROM

RELINQUISHED BY/REMOVED FROM

RELINQUISHED BY/REMOVED FROM

LABORATORY RECEIVED BY SECTION

FINAL SAMPLE DISPOSAL METHOD
DISPOSITION

\section{SIGN/ PRINT NAMES}

DATE/TIME RECEIVED BY/STORED IN

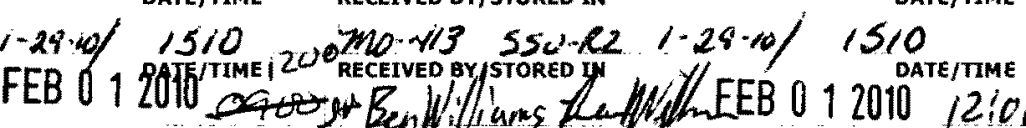

DATE/TIME-21, io RECENED BY/STORED IN

\section{DATE/TIME}

RECEIVED BY/STORED IN

DATE/TIME

RECEIVED BY/STORED IN

DATE/TIME

RECEIVED BY /STORED IN

DATE/TIME

RECEIVED BY/STORED IN

DATE/TIME

DATE/TME

DATE/TIME

\section{SPECLAL INSTRUCTIONS}

SEE PAGE 2 FOR ALL SPECIAL INSTRUCTIONS
DATE/TIME

gonguili 

COLLECTOR Rasane Rust Rono C7515 (299-E28-30); 1 -099 ICE CHEST NO. ICE CHEST NO. Environmental Sciences Laboratory

\section{SPECIAL INSTRUCTIONS}

** The 200 Area S\&GRP Characterization and Monitoring Sampling and Analysis GKI applies to this SAF.

"ESL shall submit all data deliverables according to SOW "200-LW-2 and 200-BP-5 Operable Units/Contaminant and Transport Property Analysis and Report for vadose Zone Sediments at Borehole C5860/Well 299-E29-54" for

** The ESL laboratory shall meet prior requested and confirmed turnaround times and RDLs specified in the SOW and this SAF. The laboratories must natify the SM Project Coordinator in writing iffwhen this requirement can not be me (1)6010M_ICP_ASTM_AE (TAL) \{Sodlum, Aluminum, Iron, Potassium, Antimony, Nickel, Calcium, Magnesium, Silver\} 6010M_ICP_ASTM_AE (Add-On) \{Lead, Thallium, Selenium\}6010M_METALS_ICP_WE (TAL) \{Sodium, Aluminum,

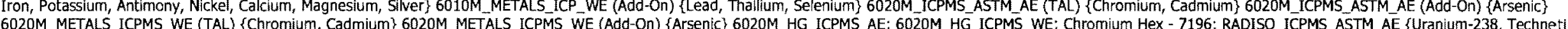

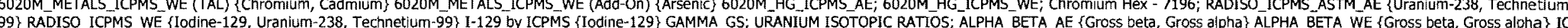
Anions - 9056_WE \{Nitrate, Chloride, Phosphate, Fluoride, Nitrte, Sulfate\} Cyanide (Total) - 335.2 \{Cyanide\} pH (Water) - 9045_WE; Conductivity - 9050_WE; TOC - ASTME1915A \{Total organic carbon, Total Inorganic Carbon, Total carbon\} 2320_ALKALINITY \{Carbonate ion, Bicarbonate, Alkalinity, Calcium Carbonate\}

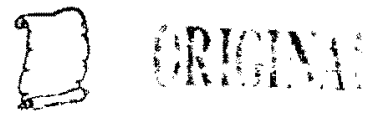

FIEL LOGBOOK No. PS ST ACTUAL SAMPLE DEPTH

INTF

HNF-N-

240.0

\section{COA}

302117 ES3

BILL OF LADING/AIR BILL NO.
METHOD OF SHIPMENT

GOVERNMENT VEHICL
PAGE 2 OF 2

DATA
TURNAROUND

45 Days / 45
Days 


\section{PAGE 1 OF 2}

Roluector

SAMPLING LOCATION

C7515 (299-E28-30)
ICE CHEST NO.

COMPANY CONTACT

DYEKMAN, DL

TELEPHONE NO.

PROJECT COORDINATOR

PRICE CODE

PROJECT DESIGNATION SAF NO.

200-PW-2 OU Characterization Vadose Zone - Geochemical Modeling Param F10-045

FiELD LOGBOOK NO. PS 54 actual SAMPLE DEPTH

$\mathrm{COA}$

AIR QUALITY

DATA
TURNAROUN

45 Days / 45
Days
HNF-N- $S / 9 /-5$
$241.9^{\circ}$
302117 ES30
GOVERNMENT VEHICLE

METHOD OF SHIPMENT

OFFSTTE PROPERTY NO.

OILL OF LADING/AIR BILL NO

N/A

\begin{tabular}{l|ll} 
PRESERVATION & Cool $4 \mathrm{C}$ & |None \\
TYPE OF CONTAINER & G/P & $\begin{array}{l}\text { Moisture } \\
\text { Resistant Cont }\end{array}$ \\
\hline O. OF CONTAINER(S) & 1 & 1 \\
\hline
\end{tabular}

VOLUME

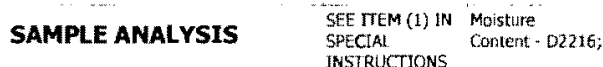

N/A
MATRIX* T POSSIBLE SAMPLE HAZARDS/ REMARKS Contains Radioactive Material at concentrations transportation per 49 CFR / IATA Dangerous Goods Regulations but are not releasable per

\section{SPECIAL HANDLING AND/OR STORAGE

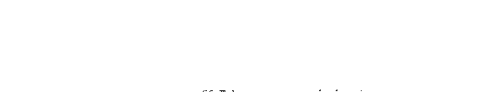
RADIOACTIVE TIE TO: B23666 SAMPLE NO.

MATRIX* B235P5 SOIL

CHAIN OF POSSESSION

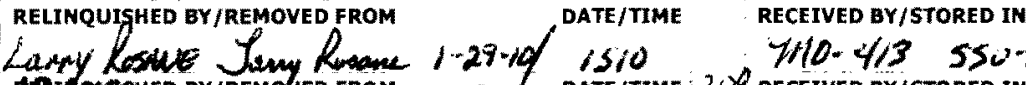
CUPRC

RELINUTSHED GY REMOVED FROM

PELINOUISHED BY /REMOVED FROM

REIMQUISHED QY/REMOVIO TROM

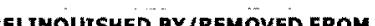

ELINQUISHED BY/REMOVED TROM

\section{LABORATORY RECEIVED BY} SECTION

FINAL SAMPLE
DISPOSITION
$1 \cdot 29 \cdot 10 \% 1510$ DATE/TIMEZ / hIORECEIVED BY/STORED IN

DATE/TIME RECEIVED BY/STOREO IN

DATE/TIME RECEIVED QY/STORED IN

Daterning

DATE/TME

DATE/TIME

DATE/TIME RECEIVED BY/STORED IN

DATE/TIME

DATE/TIME

RECEIVED BY/STORED IN
SPECIAL INSTRUCTIONS

SEE PAGE 2 FOR ALL SPECIAL INSTRUCTIONS 
DYEKMAN, DL

200-PW-2 OU Characterization Vadose Zone - Geochemical Modeling Param F10-045

302117ES30

METHOD OF SHIPMENT OFFSITE PROPERTY NO.

BILL OF LADING/AIR BILL NO.

** The 200 Area S\&GRP Characterization and Monitoring Sampling and Analysis GKI applies to this SAF.

** ESL shall submit all data deliverables according to SOW "200-LW-2 and 200-BP-5 Operable Units/Contaminant and Transport Property Analysis and Report for Vadose Zone Sediments at Borehole C5860/Well 299-E29-54" for C7515/Well 299-E28-30 ("M" Well) and shall adhere

** The ESL laboratory shall meet prior requested and confirmed turnaround times and RDLs specified in the SOW and this SAF. The laboratories must notify the SM Project Coordinator in writing if/when this requirement can not be met. (1)6010M_ICP_ASTM_AE (TAL) \{Sodiurm, Aluminum, Iron, Potassium, Antimony, Nickel, Calcium, Magnesium, Silver\} 6010M_ICP_ASTM_AE (Add-On) \{Lead, Thallium, Selenium\} 6010M_METALS_ICP.WEE (TAL) \{Sodium, Aluminum,

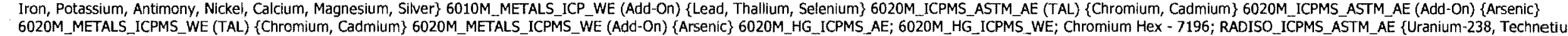

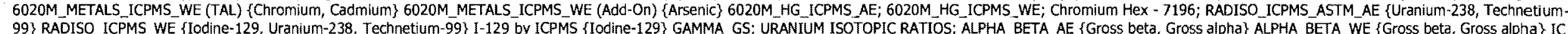
Anions - 9056_WE \{Nitrate, Chloride, Phosphate, Fluoride, Nitrite, Sulfate\} Cyanide (Total) - 335.2 (Cyanide\} pH (Water) - 9045_WE; Conductivity - 9050..WE; TOC - ASTME1915A (Total organic carbon, Total Inorganic Carbon, Total carbon\} 2320 ALKALINITY \{Carbonate ion, Bicarbonate, Alkalinity, Cakcium Carbonate\} 
CH2MHill Plateau Remediation Company

quatered

\section{Rasue Rest Romo \\ C7515 (299-E28-30); I-101}

ICE CHEST NO. $42^{2}>$

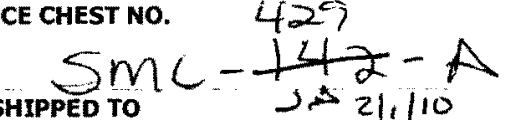

Environmental Sciences Laboratory

MATRIX* POSSIBLE SAMPLE HAZARDS/ REMARKS

$A=A i r \quad$ Contains Radloactive Material at concentrations

that may or may not be regulated for

transportation per 49 CFR / IATA Dangerous

Goods Regulations but are not releasable per

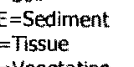

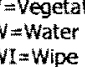

SPECIAL HANDLING AND/OR STORAGE RADIOACTIVE TIE TO: 823647
SAMPLE NO

$\mathrm{B} 235 \mathrm{~B} 6$
MATRIX*
TELEPHONE NO.

PROJECT COORDINATOR

DYEKMAN, DL

SAF NO.

PROJECT DESIGNATION

$373-2530$

PRICE CODE $\quad 8$ N

AR QUALITY $\square \quad \underset{\text { Days }}{45 \text { Days / } 45}$
PAGE 1 OF 1

\begin{tabular}{|l|l|l|l|l} 
200-PW-2 OU Characterization vadose Zone - Geochemical Modeling Param F10-045 & METHOD OF SHIPMENT \\
FIELD LOGBOOK NO. PS 54 & ACTUAL SAMPLE DEPTH COA
\end{tabular}

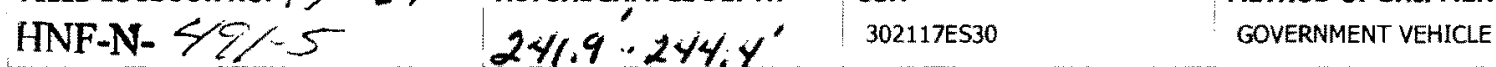

OFFSITE PROPERTY

$241.9 \cdot 244: y^{\prime}$ BILL OF LADING/AIR BILL NO.

N/A

\section{PRESERVATION}

TYPE OF CONTAINER

No. OF CONTAINER(S)

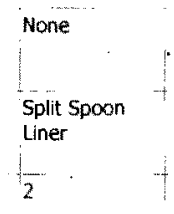

volume

10009

SAMPLE ANALYSIS SEE ITEM (1) IN

N/A

SAMPLE DATE SAMPLE TIME

$1.29 \cdot 10 \quad 0820$

\section{ECIRALTIONS}

\section{CHAIN OF POSSESSION}

RELINQUISYED BY/REMOYED FROM

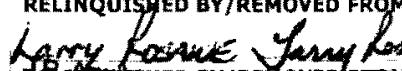

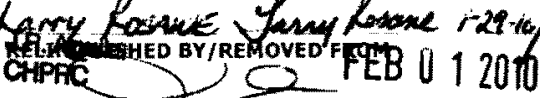

RELINQUISHEQ BY RREMOVED FROM

RELINQUISHED BY/REMOVED FROM

RELINQUISHED BYIREMOVED FROM

RELINOUISHED QYIREMOVED FROM

REUNQUISHED BY/REMOVED FROM

\section{LABORATORY RECEIVED BY}

SECTION

FINAL SAMPLE
DISPOSITION
SIGN/ PRINT NAMES

DATE/TIME RECEIVED BY/STORED IN

DATETIME 200 RECEIVED BYISTORED

DATE/TTMEZ/I IO RECEIVED BY / STORED IN

DATE/TIME RECEIVED BY/STORED IN

DATE/TIME RECEIVED BY/STORED IN

DATE/TIME RECEIVED BY/STORED IN

DATE/TIME 


\begin{tabular}{|c|c|c|}
\hline DYEKMAN, DL & 373-2530 & DYEKMAN, DL \\
\hline PROJECT DESIGMATION & & SAF NO. \\
\hline $\begin{array}{l}\text { 200-PW-2 OU Characterization } \\
\text { FIELD LOGBOOK NO. PS }\end{array}$ & $\begin{array}{l}\text { Vadose Zone - Geochemical Modeling Param } \\
5 \% \text { ACTUAL SAMPLE DEPTH }\end{array}$ & $C O A$ \\
\hline
\end{tabular}

SHIPPED TO

200-PW-2 OU Characterization Vadose Zone - Geochemical Modeling Para
FIELD LOGBOOK NO. PS $5 \neq$ ACTUAL SAMPLE DEPTH

METHOD OF SHIPMENT
HNF-N- $Y 9 / \cdot 5$
$244, Y$
302117ES30
GOVERNMENT VEHICLE

FSITE PROPERTY NO.

BILL OF LADING/AIR BILL NO.

Environmental Sciences Laboratory

MATRIX* POSSIBLE SAMPLE HAZARDS/ REMARKS

$A=A i r$
$\mathrm{DL}=$ Drum $\quad$ Contains Radloactive Material at concentrations

that may or may not be regulated for

transportation per 4 S CFR / IATA Dangerous

Goods Regulations but are not releasable per

PRESERVATION

Cool $4 \mathrm{C}$ None

$\mathrm{N} / \mathrm{A}$

TYPE OF CONTAINER G/P $\quad \begin{aligned} & \text { Misture } \\ & \text { Resistant Cont }\end{aligned}$

NO. OF CONTAINER(S)

VOLUME

1

2009

SPECIAL HAMDLING AND/OR STORAGE RADIOACTIVE TIE TO: B23647

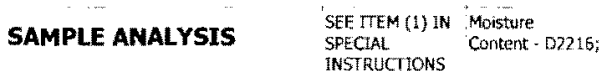

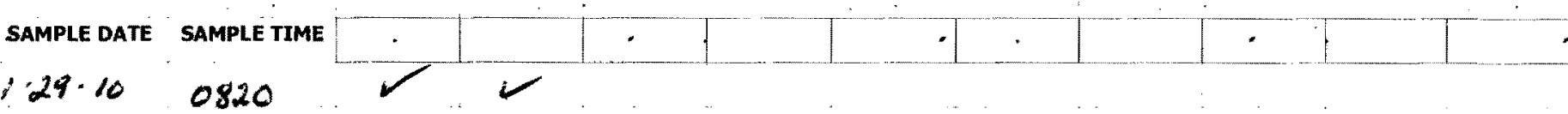

B235P6

MATRIX*

SOIL

$129 \cdot 10$

0820

SIGN/ PRINT NAMES

CHAIN OF POSSESSION

RELINQUISHED BY/REMOVEDFROM

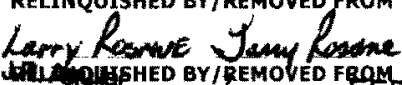
CHPAC

RELTNQUUSHED GYTREMOVED FROM

RELINQUISTED BY/REMOVED FROM

RELINQUISHED BY/REMOVED FROM

RELINQUISHED BY/REMOVED FROM

RELINQUISHED BY/REMOVED FROM

RECEIVED BY

SECTION

FINAL SAMPLE
RECEIVED BY/STORED IN

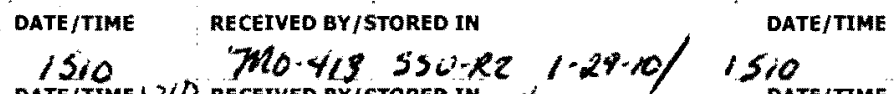

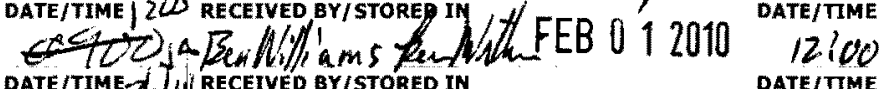

DATETME- T. IJ RECEIVED BY/STORED IN DATE/TME

DATE/TIME RECEIVED BY/STORED IN

DATE/TME

DATE/TIME

L3 andilli

DATE/TIME RECEIVED BY/STORED IN DATE/TIME

DATE/TIME RECEIVED BY/STORED IN
SPECIAL INSTRUCTIONS

SEE PAGE 2 FOR ALL SPECIAL INSTRUCTIONS 


$$
\text { COA } 302117 \text { ES30 }
$$

\section{METHOD OF SHIPMENT} OFFSTE PROPERTY NO.

BILL OF LADING/ATR BTLL NO.

$$
\text { Environmental Sciences Laboratory }
$$$$
\text { N/A }
$$$$
\text { N/A }
$$

\section{SPECIAL INSTRUCTIONS}

** The 200 Area S\&GRP Characterization and Monitoring Sampling and Analysis GKI applies to this SAF

" ESL shall submit all data delive rables according to SOW "200-LW-2 and 200-BP-5 Operable Units/Contaminant and Transport Property Analysis and Report for vadose Zone Sediments at Borehole C5860/Well 299-E29-54" for

** The ESL laboratory shall meet prior requested and confirmed turnaround times and RDLs specified in the SOW and this SAF. The laboratories must notify the SM Project Coordinator in writing iff when this requirement can not be met (1)6010M_ICP_ASTM_AE (TAL) \{Sodium, Aluminum, Iron, Potassium, Antimony, Nickel, Calcium, Magnesium, Silver\} 6010M_ICP.ASTM_AE (Add-ON) \{Lead, Thallium, Selenium\} 6010M_METALS ICP_WE (TAL) \{Sodium, Alluminum,

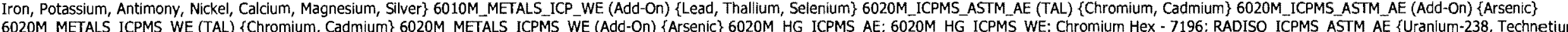
99\} RADISO ICPMS WE \{Iodine-129, Uranium-238, Technetium-99\} I-129 by ICPMS \{Iodine-129\} GAMMA GS: URANIUM ISOTOPIC RATIOS. ALPHA BETA AE \{Gross beta, Gross alpha\} AIPHA BETA WE \{Gross beta, Gross alpha\} IC Anions - 9056_WE \{Nitrate, Chloride, Phosphate, Fluorice, Nitrte, Sulfate\} Cyanide (Total) - 335.2 \{Cyanide\} pH (Water) - 9045_WE; Conductivity - 9050_WE; TOC - ASTME1915A \{Total organic Carbon, Total Inorganic Carbon, Total carbon\} 2320_ALKALNITY \{Carbonate ion, Bicarbonate, Aikalinity, Calcium Carbonate\}

Hivivin 


$$
\sin L-420-A
$$

Environmental Sciences Laboratory

"MATRIX* POSSIBLE SAMPLE HAZARDS/ REMARKS Contans Radioactive Material at concentrations that may or may not be regulated for Goods Regu Goods Regulations but are not releasable por

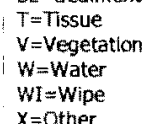

SAF NO.

AIR QUALITY

200-PW-2 OU Characterization Vadose Zone - Geochemical Modeling Param F10

FIELD LOGBOoK No. PS 54 actuá SAMPLE DEPTH

COA METHOD OF SHIPMENT

INF-N- $48 / .5 \quad 244.6 \div 247.1$

OFFSTIE PROPERTY NO.

302117 ES30

METHOD OF SHIPMEN

N/A

PRESERVATION

TYPE OF CONTAINER Split SpOoOn

NO. OF CONTAINER(S)

VOLUME

10009

SAMPLE ANALYSIS

SEE TEM (1) IN
SPLCLA
SWSTELTTONS

FF LADING/AIR BILL NO

N/A
B235B

SAMPLE NO.

MATRIX*

\section{SAMPLE DATE SAMPLE,TIME \\ $1 \cdot 28 \cdot 10 \quad 1045$}

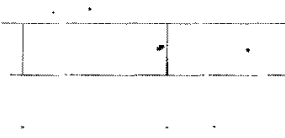

\section{SIGN/ PRINT NAMES}

RELINQUished BY/REMOVED FRO

Q.0.

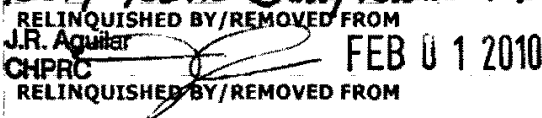

RELINQUISHED BY/REMOVED FROM

RELINQUISHED BY/REMOVED FROM

RELIMQUISHED BY/REMOVED FROM

RELINQUISHED BY/REMOVED FROM

\section{LABORATORY RECEIVED BY \\ SECTION}

FINAL SAMPLE DISPOSAL METHOD

DISPOSITION
DATE/TIME RECEIVED BY/STORED IN

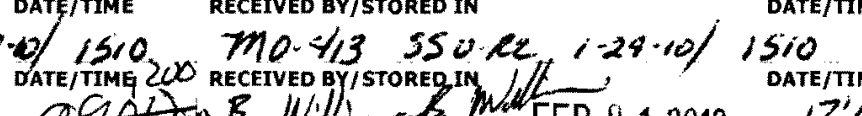

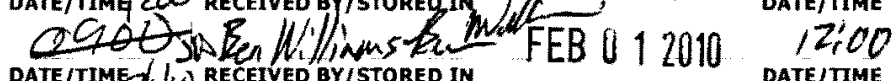
DATE/TTME DATE/TIME RECEIVED BY/STORED IN DATE/TME DATE/TMME DATE/TIME DATE/TTME DATE/TIME RECEIVED BY/STORED IN

\section{DATE/TIM}

\section{SPECIAL INSTRUCTIONS}

** The 200 Area S\&GRP Characterization and Monitoring Sampling and Analysis GKI applies to this SAF.

** ESL shall submit all data deliverables according to SOW "200-LW-2 and 200-BP-5 Operable Units/Contaminant and Transport Property Analysis and Report for Vadose

** The ESL laboratory shall meet prior requested and confirmed turnaround times and RDLS Specified in the SOW and this SAF. The laboratories must notify the SM Project Coordinator in writing if/when this requirement can not be met. (1)Density; CATIONEXCH_TR; Particle Size (Dry Sieve) - D422; Partide Size (Hydrometer) - D422; KD - Batch;

TIRE

ORIGINA

DISPOSED BY 
COMPANY CONTACT

DYEKMAN, DL

TELEPHONE NO.

PROJECT COORDINATOR

DYEKMAN, DL

\begin{tabular}{ll} 
PROJECT DESIGNATION & SAF NO. \\
\hline 200 -PW-2 OU Characterization Vadose ZONe - Geachemical Modeling Param F10-045
\end{tabular}

FIELD LOGBOOK No. PS SU ACTUAL SAMPLE DEPTH COA

FIELD LOGBOOK NO. PS 54 ACTUAL SAMPLE DEPTH
HNF-N- $4 / 9 / .5$

METHOD OF SHIPMENT

BILL OF LADING/AIR BILL NO.

N/A

Environmental Sciences Laboratory

MATRIX* POSSIBLE SAMPLE HAZARDS/ REMARKS

$A=$ Air
$\mathrm{DL}=$ Drum Contains Radioactive Material at concentrations that may or may not be reguiated for

Goods Regon

GOE Order 5400.5 (1990/1993)

PRESERVATION COONAC None

TYPE OF CONTAINER G/P $\quad \begin{aligned} & \text { Moisture } \\ & \text { Resistant Cont }\end{aligned}$

NO. OF CONTAINER(S) 1

volume

SPECIAL HANDLING AND/OR STORAGE RADIDACTIVE TIE TO: 823647

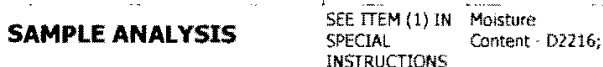

N/A

SAMPLE NO.

MATRIX*

SAMPLE DATE SAMPLE TIME

$1 \cdot 29-10 \quad 1045$

SOIL

\section{CHAIN OF POSSESSION} RELINQUSGHED BY/REMOVED EROM

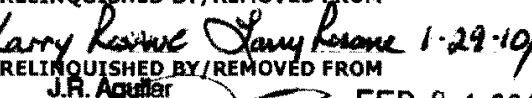

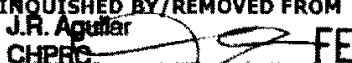

RELINQUISHED BY/REMOVED FROM

RELINQUISHED BY/REMOVED FROM

RELINQUISHED BY/REMOVED FROM

RELINQUUSHED BY/REMOVED FROM

RELINQUISHED BY/REMOVED FROM

RECEIVED BY

SECTION

FINAL SAMPLE
DISPOSITION

\section{IGN/ PRINT NAMES}

DATE/TTME RECEIVED BY/STORED IN

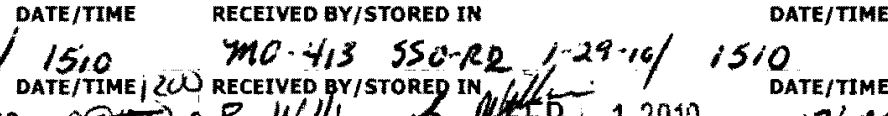
12:00 DATE/TIME 2/, |W RECEIVED BY/STORED IM

DATE/TIME

RECEIVED BY/STORED IN

DATE/TIME

RECEIVED BY/STORED IN

DATE/TIME

RECEIVED BY/STORED IN

DATE/TTME
12,00
DATE/TIME

DATE/TIME

DATE/TIME

DATE/TIME

DATE/TIME
SPECIAL INSTRUCTIONS

SEE PAGE 2 FOR ALL SPECIAL INSTRUCTIONS 


\section{COLLECTOR \\ Ranue lust Romo}

SAMPLING LOCATION

C7515 (299-E28-30); I-104

ICE CHEST NO.

$$
\sin -429-A
$$

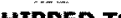

Environmental Sciences Laboratory

\section{SPECIAL INSTRUCTIONS}

** The 200 Area S\&GRP Characterization and Monitoring Sampling and Analysis GKI applies to this SAF.

* ESL shall submit all data deliverables according to SOW "200-LW-2 and 200-BP-5 Operable Units/Contaminant and Transport Property Analysis and Report for Vadose Zone Sediments at Borehole C5860/Well 299-E29-54" fo C7515/Well 299-E28-30 (" $M$ " Well) and shall adhere to all S\&GRP standard protocol.

** The ESL laboratory shall meet prior requested and confirmed turnaround times and RDLs Speclfied in the SOW and this SAF. The laboratories must notify the SM Project Coordinator in writing if/when this requirement can not be me(1)6010M_ICP_ASTM_AE (TAL) \{Sodium, Aluminum, Iron, Potassium, Antimony, Nickel, Calcium, Magnesium, Silver\} 6010M_ICP_ASTM_AE (Add-On); \{Lead, Thallium, Selenium\} 6010M_METALS ICP_WE (TAL) \{Sodium, Aluminum,

ron, Potassium, Antimony, Nickel, Caldium, Magnesium, Slvers,

99\} RADISO ICPMS WE \{lodine-129, Uranium-238, Technetium-99\} I-129 by ICPMS \{Iodine-129\} GAMMA GS; URANIUM ISOTOPIC RATIOS; ALPHA BETA AE \{Gross beta, Gross alpha\} ALPHA BETA WE \{Gross beta, Gross alpha\} IC Anions - 9056_WE \{Nitrate, Chioride, Phosphate, Fuoride, Nitrite, Sulfate\} Cyanide (Total) - 335.2 \{Cyanide\} PH (Water) - 9045_WE; Conductivity - 9050_WE; TOC - ASTME1915A \{Total organic Carbon, Total Inorganic Carbon, Total carbon\} 2320_ALKALINITYY \{Carbonate ion, Bicarbonate, Alkalinity, Calcium Carbonate\}

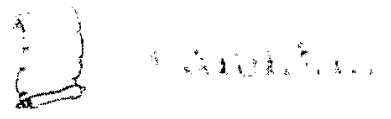



COLLECTOR Rosave Rust Rome

C7515 (299-E28-30); I-105

CE CHEST NO.

$$
5914 \cdot 429 \cdot A
$$

SHIPPED TO

Environmental Sciences Laboratory

MATRIX* POSSIBLE SAMPLE HAZARDS/ REMARKS

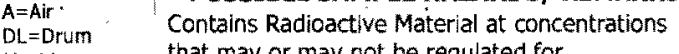

that may or may not be regulated for
Liquids
S $=$ transportation per 49 CFR I IATA Dangerous

transportation per 49 CFR / IATA Dangercus GOE Order 5400.5 (1990/1993)

SPECIAL HANDLING AND/OR STORAGE RADIOACTIVE TIE TO: B23648

SAMPLE NO.
SOIL MATRIX*

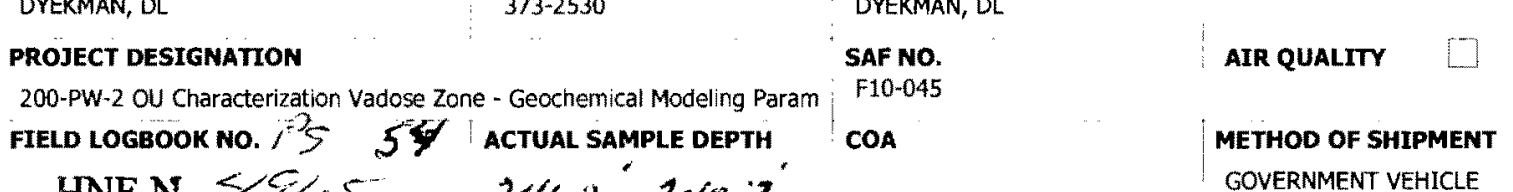

$$
\text { HNF-N- } 5 \% 5^{\circ} 24 \% .2{ }^{\circ} 248.7^{\circ} \text { GOVERNMENT VEHICLE }
$$

\begin{tabular}{|c|c|}
\hline PRESERVATION & 'None \\
\hline YPE OF CONTAINER & $\begin{array}{l}\text { Split Spoon } \\
\text { Liner }\end{array}$ \\
\hline 10. OF CONTAINER(S) & 2 \\
\hline VOLUME & $1000 \mathrm{~g}$ \\
\hline SAMPLE ANALYSIS & 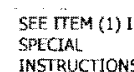 \\
\hline
\end{tabular}

\section{BILL OF LADING/AIR BILL NO.}

OFFSTIE PROPERTY NO. N/A

SAMPLE DATE SAMPLE TIME.

$1 \cdot 29 \cdot 10 \quad 1222$

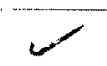

\section{SIGN/ PRINT NAMES} DATE/TIME RECEIVED BY/STORED IN

RELINQUTSHED BY/REMOVED FROM

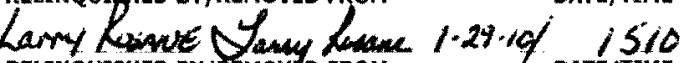
$710 \cdot$ - Y13 $550 \cdot 02$ CHPRE 2 FEB 12010 RELINQUISHEDG TTREMOVED FROM DATE/TIME II $_{1}$. SATE/TIME izion DATE/TTME

DATE/TIME DATE/TIME

DATE/TIME

RECETVED BY/STORED IN

DATE/TIME

RECEIVED BY/STORED IN

DATE/TIME

RECEIVED BY/STORED IN
DATE/TIME

DATE/TTME

DATE/TIME
SPECIAL INSTRUCTIONS

** The 200 Area S\&GRP Characterization' and Monitoring Sampling and Analysis GKI applies to this SAF.

ESL shall submit all data deliverables according to SOW "200-LW-2 and 200-BP-5 Operable Units/Contaminant and Transport Property Analysis and Report for Vadose ("M" Well) and shall adhere to all S\&GRP standard protocol.

**. The ESL laboratory shall meet prior requested and confirmed turnaround times and RDLS specified in the SOW and this SAF. The laboratories must notify the SM (1)Density; CATIONEXCH TR; Particle Size (Dry Sieve) - D422; Particle Size

(Hydrometer) - D422; KD - Batch

DATE/TIME

\section{FINAL SAMPLE}


CH2MHill Plateau Remediation Company

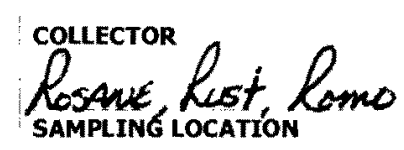

C7515 (299-E28-30); I-106

ICE CHEST NO.

$$
5+2 x-409-A
$$

SHIPPED TO

Environmental Sciences Laboratory

MATRIX* POSSIBLE SAMPLE HAZARDS/ REMARKS

$A=A$ ir
$D L=$ Drum Contains Radioactive Material at concentrations

that may or may not be regulated for transportation per 49 CFR / IATA Dangerous Goods Regulations but are not releasable per
DOE Order 5400.5 (1990/1993)

$S E=$ Sediment
$T=$ Tissue
$V=$ Vegetation

$W=$ Water

SPECIAL HANDLING AND/OR STORAGE RADIOACTIVE TIE TO: B23648

$$
\text { SAMPLE NO. . }
$$

B235P8

SOIL

MATRIX* -
CHAIN OF CUSTODY/SAMPLE ANALYSIS REQUEST

F10-045-159

PRICE CODE $\quad 8 \mathrm{~N}$

AIR QUALITY $\square$

Days

METHOD OF SHIPMENT

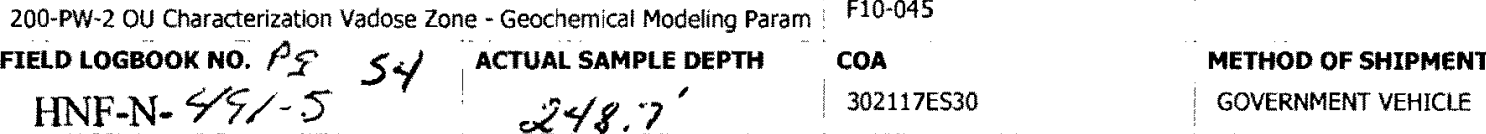

200-PW-2 ou Charaterization Vadose Zone - Geochemical Modeling Para F10-045

OFFSTTE PROPERTY NO. BILL OF LADING/AIR BILL NO.

N/A

\begin{tabular}{c|c|c|} 
PRESERVATION & $\left.\right|_{\text {G/P }} ^{\text {Cool } 4 C}$ & Mone \\
TYisture \\
Resistant Cont
\end{tabular}

N/A

SEE ITEM (1) iN MOisture
SPECALI
INSTRUCHONS

SAMPLE DATE SAMPLE TIME

$1 \cdot 29 \cdot 10 \quad 1222$

\section{CHAIN OF POSSESSION}

RELINQUISHED BY/REMOVED FROM

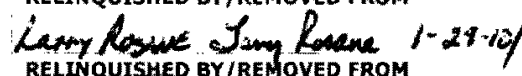

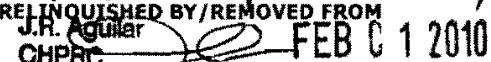
CHPAC

REINQUTSHED BY/PEMOVED FROM

RELINQUISHED BY/REMOVED FROM

RELINQUISHED BY/REMOVED FROM

RELINQUISHED BY/REMOVED FROM

\section{LABORATORY RECEIVED BY}

SECTION

FINAL SAMPLE
DISPOSITION

\section{SIGN/ PRINT NAMES}

DATE/TIME RECEIVED BY/STORED IN

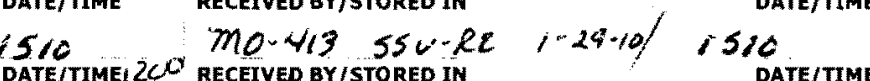

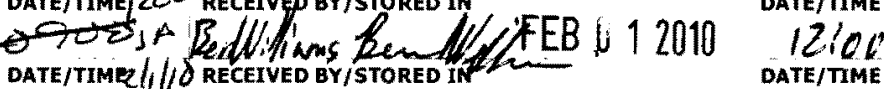

Date/TMM

DATE/TIME RECEIVED GY/STORED IN

DATE/TIME RECEIVED BY/STORED IN

DATE/TIME RECEIVED BY/STORED IN

DATE/TIME

PECEIVED BY/STORED IN

DATE/TIME

DaTe/TIME

DATE/TME

DATE/TME

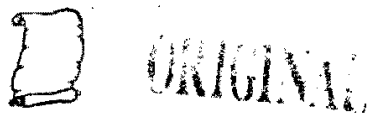

SEE PAGE 2 FOR ALL SPECIAL INSTRUCTIONS 


\section{Rasme Rust Ramo $_{\text {sampling location }}^{\text {comortor }}$ \\ C7515 (299-E28-30); I-10}

ICE CHEST NO.

SHIPPED TO

$$
S_{\text {To }} M c-427-A
$$

\section{COMPANY CONTACT}

DYEKMAN, DL

TELEPHONE NO.

373-2530

PROJECT COORDINATOR

DYEKMAN, DL

SAF NO.

200-PW-2 OU Characterization Vadose Zone - Geochemical Modeling Param

FIELD LOGBOOK NO. 3

ACTUAL SAMPLE DEPTH

HNF-N- $48 /-5$

248.7

OFFSTE PROPERTY NO.

N/A

\section{SPECIAL INSTRUCTIONS}

** The 200 Area S\&GRP Characterization and Monitoring Sampling and Analysis GKI applies to this SAF.

"Units/Contaminant and Transport Property Analysis and Report for Vadose Zone Sediments at Borehole C5860/Well 299-E29-54" for

N* The ESL laboratory shall meet prior requested and confirmed turnaround times and RDLs specifed in the SOW and this SAF. The laboratories must notify the SM Project Coordinator in writing if/When this requirement can not be met. (1)6010M_ICP_ASTM_AE (TAL) (Sodium, Aluminum, Iron, Potasssium, Antimony, Nickel, Calcium, Magnesium, Siver\} 6010M_ICP_ASTM_AE (Add-On) \{Lead, Thallium, Selenium\} 6010M_METALSICP_WE (TAL) \{Sodium, Aluminum,

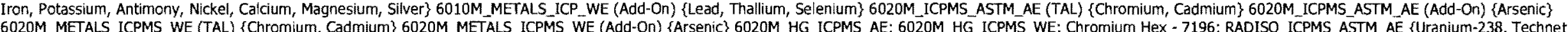
99 \}ADISO ICPMS WE \{Iodine-129, Uranium-238, Technetium-99\} I-129 by ICPMS \{Iodine-129\} GAMMA_GS; URANIUM ISOTOPIC RATIOS: ALPHA_BETA_AE \{Gross beta, Gross alpha\} ALPHA BETIA WE \{Gross beta, Gross alpha\} IC Anions - 9056_WE \{Nitrate, Chloride, Phosphate, Fluoride, Nitrite, Sulfate\} Cyanide (Tota) - 335.2 \{Cyanide\} pH (Water) - 9045_WE; Conductivity - 9050_WE; TOC - ASTME1915A \{Total organic Carbon, Total Inorganic Carbon, Total carbon\} 2320_ALKALINITY \{Carbonate ion, Bicarbonate, Aikalinity, Calcium Carbonate\}

D

is $\%$ 


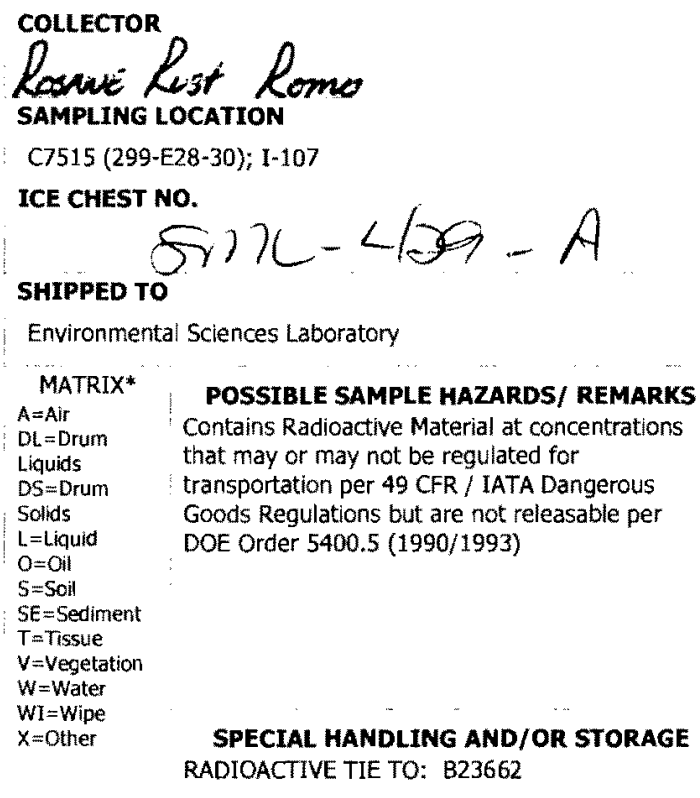

Environmental Sciences Laboratory

MATRIX* POSSIBLE SAMPLE HAZARDS/ REMARKS that may or may not be regulated for

Gaspo Regulations but CATA Dangerous

Goods Regulations but are not releasable pe

SPECIAL HAMDLING AND/OR STORAGE RADIOACTIVE TIE TO: B23662

\begin{tabular}{|c|c|c|c|c|}
\hline $\begin{array}{l}\text { COMPANY CONTACT } \\
\text { DYEKMAN, DL }\end{array}$ & $\begin{array}{l}\text { TELEPHONE NO. } \\
373-2530\end{array}$ & $\begin{array}{l}\text { PROJECT COORDINATOR } \\
\text { DYEKMAN, DL. }\end{array}$ & PRICE CODE & $8 \mathrm{~N}$ \\
\hline PROJECT DESIGNATION & & SAF $N$ & AIR QUALITY & $\square$ \\
\hline $\begin{array}{l}\text { :00-PW-2 OU Characterization vadose } 2 \\
\text { IELD LOGBOOK NO. PS } 54\end{array}$ & $\begin{array}{l}\text { e - Geochemical Modeling Param } \\
\text { ACTUAL SAMPLE DEPTH }\end{array}$ & $\begin{array}{l}F 10-045 \\
\text { COA } \\
302117 F S 30\end{array}$ & METHOD OF SH & MENT \\
\hline
\end{tabular}

TURNAROUND

Days

OFFSTTE PROPERTY NO.

BILL OF LADING/AIR BILL NO.

N/A

PRESERVATION COOI AC NONE

TYPE OF CONTAINER G/P $\quad \begin{aligned} & \text { Moisture } \\ & \text { Resistant cont }\end{aligned}$

NO. OF CONTAINER(S) 1

VOLUME $\quad$ H. 2009

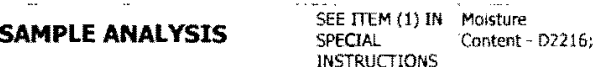

SAMPLE NO.

MATRIX

B235P9

SOIL

SAMPLE DATE SAMPLE TIME

$1 \cdot 29 \cdot 10$

1355

\section{SIGN/ PRINT NAMES}

CHAIN OF POSSESSION

RELIMQUSSHED BY/REMOVED FROM

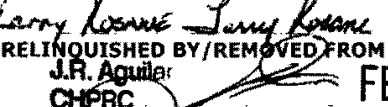

RELINQURC

RELINQUISHED BY/REMOVED FROM

RELINQUUSHED BY/REMOVED FROM

RELINQUISHED BY/REMOVED FROM

RELINQUISHED BY/REMOVED FROM

LABORATORY RECEIVED B

SECTION

FINAL SAMPLE DISPOSAL METHOD
DATE/TIME RECEIVED BY/STORED IN

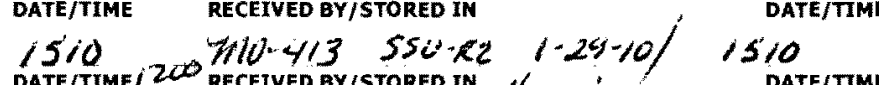
10 DATE/TME DATE/TIME 2 h IIDRECEIVED BY/STORED IN DATE/TIME

DATE/TIME

DATE/TIME

DATE/TIME
RECEIVED BY/STORED IN

DATE/TIME

DATE/TIME

RECEIVED BY/STORED IN

RECEIVED BY/STORED IN
SPECIAL INSTRÜCTIONS

SEE PAGE 2 FOR ALL SPECIAL INSTRUCTIONS 


\section{CQLLECTOR} Kasine fust Romo

SAMPLING LOCATION

C7515 (299-E28-30); I-107

ICE CHEST NO.

$$
\operatorname{smL}_{\mathrm{C}}-42 C_{7}-A
$$

SHIPPED TO

Environmental Sciences Laboratory

CHAIN OF CUSTODY/SAMPLE ANALYSIS REQUEST

TELEPHONE NO.

$373-2530$

PROJECT COORDINATOR

DYEKMAN, DL

PROJECT DESIGNATION

200-PW-2 OU Characteriz

FIELD LOGBOOK NO. 15

HNF-N- $8 \% /-$

54 Actual SAMPLE DEPTH

252.5

OFFSITE PROPERTY NO.

DYEKMAN, DL

SAF NO.

$\mathrm{N} / \mathrm{A}$
DATA

$\underset{\text { Days }}{45 \text { Days / } 4 S}$

\section{SPECIAL INSTRUCTIONS}

** The 200 Area S\&GRP Characterization and Monitoring Sampling and Analysis GKI applies to this SAF.

* ESL shall submit all data deliverables according to SOW "200-LW-2 and 200-BP-5 Operable Units/Contaminant and Transport Property Analysis and Report for Vadose Zone Sediments at Borehole C5860/Well 299-E29-54" for

** The ESL laboratory shall meet prior requested and confirmed turnaround times and RDLs specified in the SOW and this SAF. The laboratories must notify the SM Project Coordinator in writing iff when this requirement can not be met. (1)6010M_ICP_ASTM_AE (TAL) \{Sodium, Aluminum, Iron, Potassium, Antimony, Nickel, Calcium, Magnesium, Silver\} 6010M_ICP_ASTM_AE (Add-On) \{Lead, Thallium, Selenium\} 6010M_METALSIICP_WE (TAL) (Sodium, Aluminum,

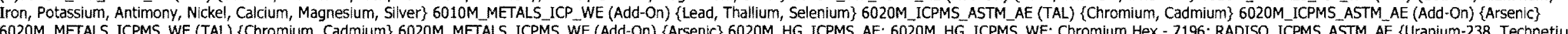

99\} RADISO ICPMS WE \{Iodine-129, Uranium-238, Technetium-99\} I-129 by ICPMS \{Iodine-129\} GAMMA GS: URANTUM ISOTOPIC RATIOS: ALPHA BETA AE \{Gross beta, Gross alpha\} ALPHA BETA WE \{Gross beta, Gross alpha\} IC Anions - 9056_WE \{Nitrate, Chloride, Phosphate, Fluoride, Nitrite, Sulfate\} Cyanide (Total) - 335.2 \{Cyanide\} pH (Water) - 9045_WE; Conductivity - 9050_WE; TOC - ASTME1915A \{Total organic Carbon, Total Inorganic Carbon, Total carbon\} 2320_ALKALINITY \{Carbonate Ion, Bicarbonate, Alkalinity, Calcium Carbonate\}

$\{3$ Mng 


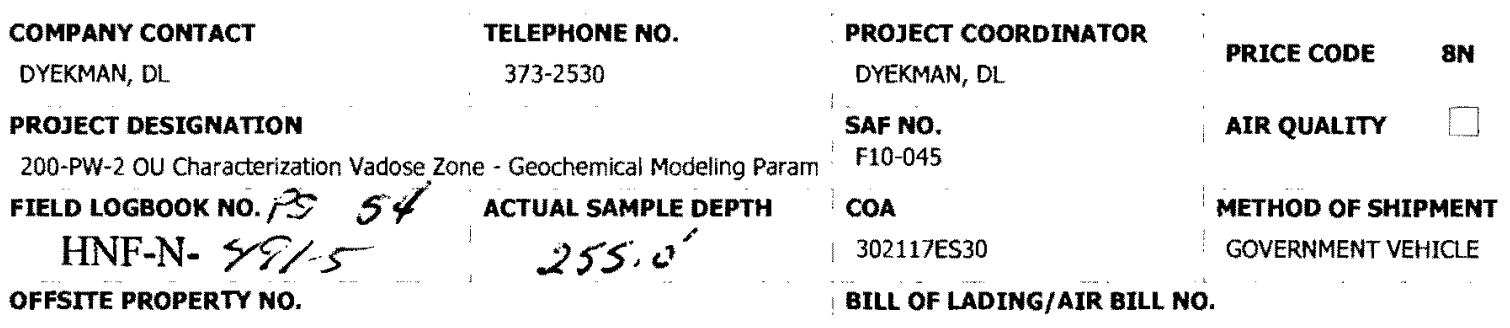

FIELD LogBook No. $P S 54$ ACTUAL SAMPLE DEPTH COA $5 \%$ METHOD OF SHIPMENT

N/A

N/A

\begin{tabular}{|c|c|c|}
\hline PRESERVATION & cool $4 \mathrm{C}$ & Wone \\
\hline TYPE OF CONTAINER & $G / \bar{P}$ & $\begin{array}{l}\text { Molsture } \\
\text { Resistant Con }\end{array}$ \\
\hline NO. OF CONTAIMER(S) & 1 & 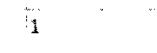 \\
\hline VOLUME & 11 & 2009 \\
\hline SAMPLE ANALYSIS & $\begin{array}{l}\text { 'SEE ITEM } \\
\text { SPRECIAL }\end{array}$ & $\begin{array}{l}\text { Mocisture } \\
\text { Content-D221 }\end{array}$ \\
\hline
\end{tabular}

- sample no.
MATRIX*
SAMPLE DATE SAMPLE TIME

$1-29-10$
SIGN/ PRINT NAMES

CHAIN OF POSSESSION

RELINQUISHED BY/REMOVED FROM DATE/TIME RECEIVED BY/STORED IN

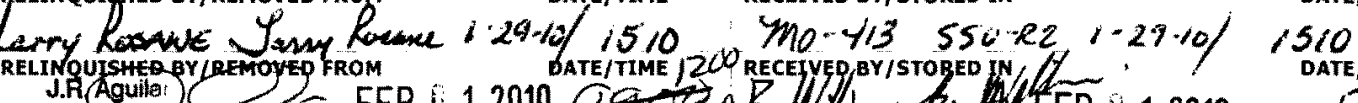

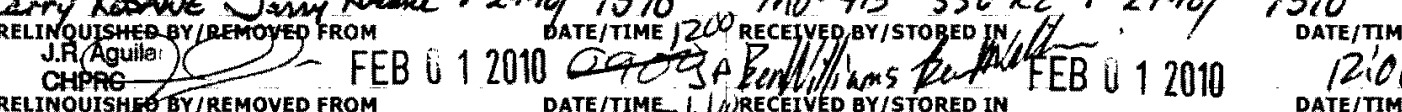

RELINQUISHED BY/REMOVED FROM

RELINQUISHED BY/REMOVED FROM

RELINQUISHED BY/REMOVED FROM

RELINQUISHED GY/REMOVED FROM

\section{LABORATORY RECEIVED BY \\ SECTION}

FINAL SAMPLE DISPOSAL METHOD

DISPOSTTION

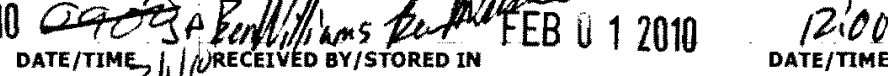

DATE/TIME

DATE/TIME

RECEIVED BY/STORED IN

DATE/TIME

DATE/TIME
12,00

Date/time

DATE/TIME

DATE/TIME

DATE/TIME

\section{SPECIAL INSTRUCTIONS}

\section{SEE PAGE 2 FOR ALL SPECIAL INSTRUCTIONS}

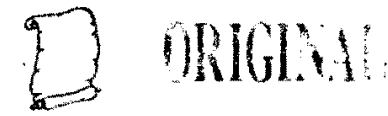




$$
S_{10} M-409-A
$$

Environmental Sciences Laboratory

COMPANY CONTACT

DYEKMAN, DL

TELEPHONE NO.

PROJECT COORDINATOR

PRICE CODE $8 \mathrm{~N}$

PROJECT DESIGNATION

200-PW-2 OU Characterization Vadose Zone - Geochemical Modeling Paran

FIELD LOGBOOK NO. PS

DMMAN, DL

DATA

TURNAROUND

AR QUALITY $\square \quad 45$ Days / 45

HNF-N. $45 /-5$

F10-045

Days

\section{SPECIAL INSTRUCTIONS}

** The 200 Area S\&GRP Characterization and Monitoring Sampling and Analysis GKI applies to this SAF.

"ESL shall submit all data deliverables according to SOW "200-LW-2 and 200-BP-5 Operable Units/Contaminant and Transport Property Analysis and Report for Vadose Zone Sediments at Borehole C5860/Well 299-E29-54" for

** The ESL laboratory shall meet prior requested and confirmed turnaround times and RDLs specified in the SOW and this SAF. The laboratories must notify the SM Project Coordinator in writing iff/when this requirement can not be met. (1)6010M_ICP_ASTM_AE (TAL) \{Sodium, Aluminum, Iron, Potassium, Antimony, Nickel, Calcium, Magnesium, Silver\} 6010M_ICP_ASTM_AE (Add-On) \{Lead, Thallium, Selenium\} 6010M_METALS_ICP_WE (TAL) \{Sodium, Aluminum, (T)

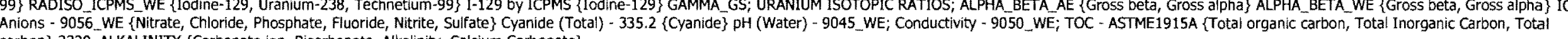
carbon\} 2320_ALKALINITY' \{Carbonate ion, Bicarbonate, Alkalinity, Calcium Carbonate\}

$\{3$ 
CH2MHill Plateau Remediation Company

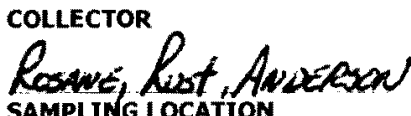

C7515 (299-E28-30); I-109

C7515 (299-E28-30).

ICE CHEST $\mathrm{NO}$

$$
\text { Gus - } 169
$$

SHIPPED TO

Environmental Sciences Laboratory

MATRI ${ }^{*}$ POSSIBLE SAMPLE HAZARDS/ REMARKS

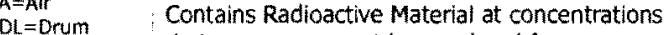
that may or may not be regulated for Gansportation per 49 CR / IATA Dangerous Goods Regulations but are not releasable per
DOE Order $5400.5(1990 / 1993)$

SPECIAL HANDLING AND/OR STORAGE RADIOACTIVE TIE TO: B23649

\section{SAMPLE NO.}

MATRIX*

B235R1

SOIL
CHAIN OF CUSTODY/SAMPLE ANALYSIS REQUEST

F10-045-164

PAGE 1 OF 2

PRICE CODE $\quad$ BN

TURNAROUND

Days

PROJECT DESIGNATION
\begin{tabular}{|l|l|l|} 
& SAF NO. & AIR QUALITY \\
\hline 200-PW-2 OU Characterization Vadose ZOne - Geochemical Modeling Param & F10-045 & \\
\hline FIELD LOGBOOK NO. PS SS ACTUAL SAMPLE DEPTH & COA & METHOD OF SHIPMENT
\end{tabular}

\begin{tabular}{l|l|l|l|l}
\hline FIELD LOGBOOK NO. PS SS ACTUAL SAMPLE DEPTH & COA & METHOD OF SHIPMENT \\
\hline
\end{tabular}

HNF-N- $4 / 9 /-5$
OFFSITE PROPERTY NO.

N/A

N/A

PRESERVATION

TYPE OF CONTAINER G/P $\quad \begin{aligned} & \text { Mosture } \\ & \text { Resistant Cont }\end{aligned}$

NO. OF CONTAINER(S) i i i

VOLUME it $\quad$ it

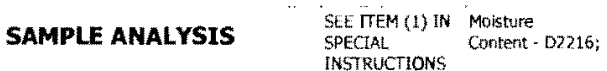

SAMPLE DATE SAMPLE TIME

$2-1-10.0805$

\section{CHAIN OF POSSESSION}

RELINQUISHED BY/REMOVED FROM

Larry Roswe Somy forme $2-1-10 \%$ DATE/TIME 1452 RELINGSSUPOK2 2 RMO JED FROM

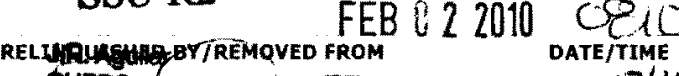
QHPAC RELINQUISHED QYYREMOVED RROM DATE/TIME

RELINQUISHED BY/REMOVED FROM

DATE/TIME

DATE/TIME

DATE/TIME

\section{SIGN/ PRINT NAMES}

RECEIVED BY/STORED IN

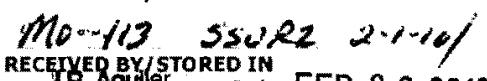

CHPAG 5 EEB 022010

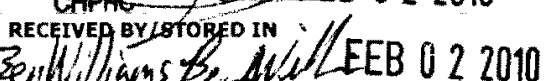

Renlilians Be A

RECEIVED BY/STORED IN

RECEIVED BY/STORED IN

RECEIVED BY/STORED IN
SPECIAL INSTRUCTIONS

DATE/TIME SEE PAGE 2 FOR ALL SPECIAL INSTRUCTIONS

1452

DATE/TMME

OPATE/TIME

12,10

DATE/TMME

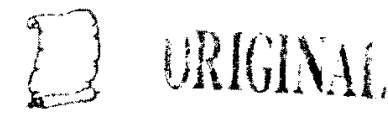

RELINQUISHED BY/REMOVED FROM

LABORATORY
SECTION

$\underset{\substack{\text { FINAL SAMPLE } \\ \text { DISPOSITION }}}{ }$
RECEIVED BY

DPOSITION
DATE/TIME

DATE/TIME

DATE/TME 


\section{COLLECTOR}

\section{Rasue hot Anocescou}

SAmpLng Location

ICE CHEST NO.

$605-169$

SHIPPED TO

Environmental Sciences Laboratory

\section{SPECTAL INSTRUCTIONS}

** The 200 Area S\&GRP Characterization and Monitoring Sampling and Analysis GKI applies to this SAF.

** ESL shall submit ail data deliverables according to SOW "200-LW-2 and 200-BP-5 Operable Units/Contaminant and Transport Property Analysis and Report for Vadose Zone Sediments at Borehole C5860/Well 299-E29-54" for

** The ESL laboratory shall meet prior requested and confirmed turnaround times and RDLS specified in the SOW and this SAF. The laboratories must notify the SM Project Coordinator in writing iffwhen this requirement can not be met. (1)6010M_ICP_ASTM_AE (TAL) \{Sodium, Aluminum, Iron, Potassium, Antimony, Nickel, Calcium, Magnesium, Silver\} 6010M_ICP_ASTM_AE (Add-ON) \{Lead, Thallium, Selenium\} 6010M_METALS ICP_WE (TAL) \{Sodium, Aluminum, ron, Potassium, Antimony, Nickel, Calcium, Magnesium, Silver\} 6010M_METALSIICP_WE (Add-On) \{Lead, Thallium, Selenium\} 6020M_ICPMS_ASTM_AE (TAL) \{Chromium, Cadmlum\} 6020M_ICPMS_ASTM_AE (Add-On) \{Arsenic\} 99\} RADISO ICPMS WE \{Iodine-129, Uranium-238, Technetium-99\} I-129 by ICPMS \{Iodine-129\} GAMMA GS; URANIUM ISOTOPIC RATIOS; ALPHA BETA AE \{Gross beta, Gross alpha\} ALPHA BETA WE \{Gross beta, Gross alpha\} IC Anions - 9056_WE \{Nitrate, Chloride, Phosphate, Fluoride, Nitrite, sulfate\} Cyanide (Total) - 335.2 \{Cyanide\} pH (Water) - 9045_WE; Conductivity - 9050_WE; TOC - ASTME1915A (Total organic carbon, Total Inorganic Carbon, Total carbon\} 2320_ALKALINITY \{Carbonate ion, Bicarbonate, Akalinity, Calcium Carbonate\}

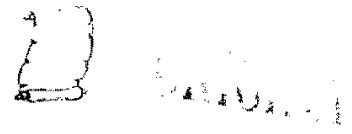


SHIPPED TO

Environmental Sciences Laboratory

MATRIX* POSSIBLE SAMPLE HAZARDS/ REMARKS

$A=A i r$
$D L=D r u m$
$C$ that may or may not be regulated for transportation per 49 CFR / IATA Dangerous
Goods Regulations but are not releasable per
DOE Order 5400.5 (1990/1993)

\section{SAMPLE No.} SPECIAL HANDLING AND/OR STORAGE
RADIOACTIVE TIE TO: B23649 RADIOACTIVE TIE TO: B23649

SOIL \\ CHAIN OF CUSTODY/SAMPLE ANALYSIS REQUEST}

COMPANY CONTACT

TELEPHONE NO.

$373-2530$

F10.045-165

$\begin{array}{ll}\text { PROJECT DESIGNATION } & \text { SAF NO. } \\ \text { 200-PW-2 OU Characterization Vadose Zone - Geochemical Modeling Param F10-045 }\end{array}$

200-PW-2 OU Characterization Vadose Zone - Geochemical Mode

FIELD LOGBOOK NO. PS
HNF-N- $Y C \% .5$

OFFSTTE PROPERTY NO.$$
25 \% .5^{\circ}
$$

TETHOD OF SHIPMENT

N/A

PRESERVATION ICOON 4 C Non

TYPE OF CONTAINER G/P Moisture

NO. OF CONTAIMER(S)

VOLUME

ii. $\quad 200 \mathrm{~g}$

$\begin{array}{ll}\text { SAMPLE ANALYSIS } & \text { SEF TEM (1) IN Moisture } \\ \text { SPFCCAL } & \text { C21 } \\ \text { COAtent - D2216 }\end{array}$
COA 302117 IES30

MIL OF LADING/AIR BILL NO.

PRICE CODE

AIR QUALITY

$\mathrm{N} / \mathrm{A}$
CHAIN OF POSSESSION

RELINQUISHED BY/REMOVED FROM

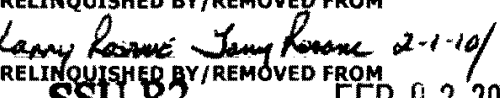
SSU-R2

RELJYPXISHED BY/REMOVED FROM DEB 022010 DaTE/TIME

RETTNQYUSHED BY/REMOVED FROM

RELINQUISHED BY/REMOVED FROM

RELINQUISHED BY/REMOVED FROM

RELINQUISHED BY/REMOVED FROM

RECEIVED BY

SECTION

IINAL SAMPLE DISPOSAL METHOD

\section{SIGN/ PRINT NAMES}

DATE/TIME DATE/TIME

DATE/TIME

RECEIVED BY/STORED IN

DATE/TIME

DATE/TIME

DATE/TIME

RECEIYED BY/STORED IN

RECEIVED BY/STORED IN

RECEIVED BY/STORED IN
SAMPLE DATE SAMPLE TIME

$2-1-10$

0408
DATE/TMME

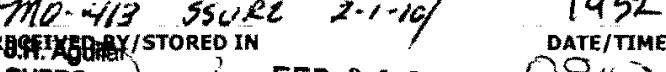

CHPAC

accenvep istoped IN 2010 DATE/TIME

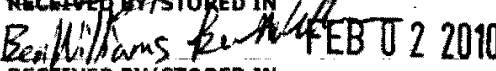

$121 / 0$
DATE/TIME

DATE/TIME

DATE/TIME

DATE/TIME
SPECIAL INSTRUCTIONS

SEE PAGE 2 FOR ALL SPECIAL INSTRUCTIONS

$$
\text { E) IPIOYia }
$$


BILL OF LADING/AIR BILL NO

\section{SPECIAL INSTRUCTIONS}


PROJECT DESIGNATION

\begin{tabular}{ll} 
PROJECT DESIGNATION & SAF NO. \\
\hline 200-PW-2 OU Characterization Vadose Zone - Geochemical Modeling Param & F10-045 \\
\hline
\end{tabular}

AIR QUALITY

$$
\text { Geos-169 }
$$

SHIPPED TO

Environmental Sciences Laboratory

MATRIX* POSSIBLE SAMPLE HAZARDS/ REMARKS

$A=A$ Ai
$D L=$ Com that may or may not be regulated for Goods Regultions but a DOE Order 5400.5 (1990/1993)

SPECIAL HANDLING AND/OR STORAGE RADIOACTIVE TIE TO: B23663

B235R3

MATRIX*

FIEID LOGBOOK NO PS SS ACTUAL SAMPIE DEPTH

HNF-N- $49 /-5$ OFFSTIE PROPERTY NO.

PRESERVATION

TYPE OF CONTAINER

NO. OF CONTAINER(S)

volume

SAMPLE ANALYSIS

$$
262.5^{\circ}
$$

COA
$302117 E S 30$

METHOD OF SHIPMENT
BILL OF LADING/AIR BILL NO.

N/A

SOIL

SAMPLE DATE SAMPLE TIME

$2 \cdot 1-10,0943$

\section{SIGN/ PRINT NAMES}

CHAIN OF POSSESSION

RELINQUISHED BY/REMOVED FROM Larny hosiue Jamy fuane 2-1\% 1452 FEB \& 22010 OATE/TIME

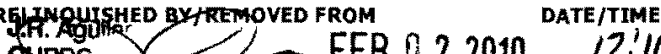
RELIRQC - FEB O 22010 12illo

RELINOUISHED BY/REMOVED FROM

RELINQUISHED BY/REMOVED FROM

RELINQUISHED BY/REMOVED FROM

\section{AABORATORY RECEIVED BY}

SECTION

INAL SAMPLE DISPOSAL METHOD

FINAL SAMPLE
DISPOSITION
Mo. $\% 13$ ssuee

REGEVERGM

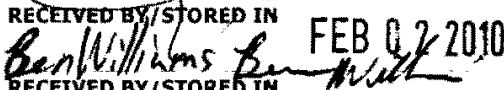

RECEIVED BY/STORED IN

DATE/TIME

DATE/TIME
RECEIVED BY/STORED IN

RECEIVED BY/STORED IN 
TIF-N- SY/4

262.5

COA 302117 ES30

GOVERNMENT VEHICLE

OFFSTTE PROPERTY NO

BILL OF LADING/AIR BILL NO. 


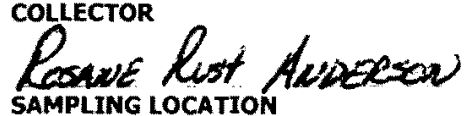

C7515 (299-E28-30): I-112

ICE CHEST NO.

$$
6005-169
$$

SHIPPED TO

Environmental Sciences Laboratory

MATRIX* POSSIBLE SAMPLE HAZARDS/ REMARKS Contains Radioactive Material at concentrations that may or may not be regulated for Goods Regulations but are not releasable per DOE Order $5400.5(1990 / 1993)$

SPECIAL HANDLING AND/OR STORAGE RADIOACTIVE TIE TO: B23663

\section{COMPANY CONTACT}

DYEKMAN, DL

TELEPHONE NO

$373-2530$

PROJECT COORDINATOR

DYEKMAN, DL

SAF NO.
F10-045
COA

200-PW-2 OU Characterzation Vadose Zone - Geochemical Modeling Param F10-045

FIELD LOGBOOK NO. IS $55^{\circ}$ ACTUAL SAMPLE DEPTH

HNF-N- $49 /-$

$265,0^{\circ}$

$\begin{array}{ll}\text { COA } & \text { METHOD OF SHIPMENT } \\ 302117 \text { ES30 } & \text { GOVERNMENT VEHICLE }\end{array}$

BILL OF LADING/AIR BILL NO.

PRESERVATION

TYPE OF CONTAINER G/P Mesisture

NO. OF CONTAINER(S)

VOLUME

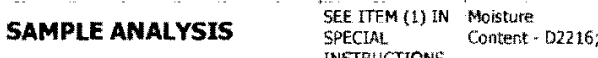

$\begin{array}{lll}\text { PRICE CODE } & \text { SN DATA } \\ \text { TURNAROUND }\end{array}$

AIR QUALITY $\square \quad 45$ Days / 45

MATRIX*

$B 235 R 4$

SAMPLE DATE SAMPLE TIME

\section{CHAIN OF POSSESSION}

RELINQUISHED BY/REMOVED FROM

Lamy Cosane Fam fosme 2-1-10\% 1452

RELIRUUSHED BY/REMOVED FROM

SSU-R2

FEB 022010 OQ10

BELLLSOUISHED BY/REMOVED FROM CHPPC.

RELINQUISHED BY/REMOVED FROM

RELINQUISHED BY/REMOVED FROM

RELUNQUISHED BY/REMOVED FROM

LABORATORY
SECTION

TINAL SAMPLE

DISPOSITION

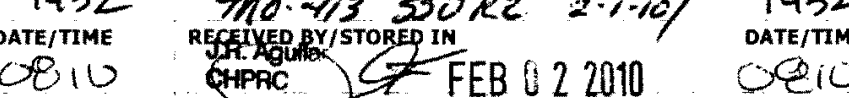

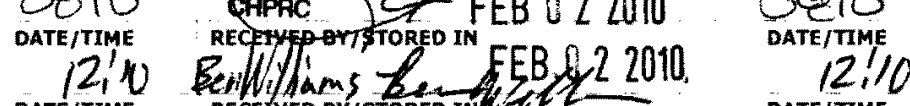

DATE/TIME

\section{SPECIAL INSTRUCTIONS}

SEE PAGE 2 FOR ALL SPECIAL INSTRUCTIONS

\section{Z ORIGINI}

DATE/TIME 'RECEIVED BY/STORED IN $\cdots$

DATE/TIME RECEIVED BY/STORED IN DATE/TIME

DATE/TIME REEEIVED BY/STORED IN DATE/TIME 


\section{Couscror hest hoosesw}

SAMPLING LOCATION

CE CHEST NO.

$$
\text { Crus. } 169
$$

SHIPPED TO

Environmental Sciences Laboratory

\section{SPECIAL INSTRUCTIONS}

** The 200 Area S\&GRP Characterization and Monitoring Sampling and Analyssls GKI applies to this SAF.

** ESL shall submit all data deliverables according to SOW "200-LW-2 and 200-8P-5 Operable Units/Contaminant and Transport Property Analysis and Report for Vadose Zone Sedlments at Borehole C5860/Well 299-E29-54" for

** The ESL laboratory shall meet prior requested and confirmed turnaround times and RDLs specified in the SOW and this SAF. The laboratories must notify the SM Project Coordinator in writting iff when this requirement can not be met. (1)6010M_ICP_ASTM_AE (TAL) \{Sodium, Aluminum, Iron, Potassium, Antimony, Nickel, Calcium, Magnesium, Silver\} 6010M_ICP_ASTM_AE (Add-On) \{Lead, Thallium, Selenium\} 6010M_METALSICP_WE (TAL) \{Sodium, Potassium, 6020M MEALS ICPMS WE (TAL) YChro 9) RADISO ICPMS WE \{lodine-129, Uranium-238, Technetium-99\} I-129 by ICPMS \{lodine-129\} GAMMA GS: URANIUM ISOTOPIC RATIOS: ALPHA BETA AE \{Gross beta, Gross alpha\} ALPHA BETA WE \{Gross beta, Gross alpha\} IC Anions - 9056_WE (Nitrate, Chloride, Phosphate, Fluoride, Nitrite, Sulfate\} Cyanide (Total) - 335.2 \{Cyanide) pH (Water) - 9045_WE; Conductivity - 9050_WE; TOC - ASTME1915A (Total organic carbon, Total Inorganic Carbon, Total
carbon\} 2320_ALKALINITY \{Carbonate ion, Bicarbonate, Alkalinity, Calcium Carbonate\}

\section{Projer Desteraton}

TELEPHONE NO.

PROJECT COORDINATOR

SAF NO.

AIR QUALITY

FIELD LOGBOOK NO. PS 55 ACTUAL SAMPLE DEPTH

HNF-N- $2 / \% /-5$

265.0

BILL OF LADING/AIR BILL NO.

PROPERTY NO.
DATA
TURNAROUND

45 Days / 45

\& molu! 


$$
\text { tws }-16 \%
$$

SHIPPED TO

Environmental Sciences Laboratory

MATRIX* POSSIBLE SAMPLE HAZARDS/ REMARKS Contains Radioactive Material at concentrations hat may or may not be regulated for Goods Regulations but are not releasable per DOE Order $5400.5(1990 / 1993)$

SPECIAL HANDLING AND/OR STORAGE RADTOACTIVE TIE TO: B23650

SAMPLE NO.
B235R5

COMPANY CONTACT

DYEKMAN, DL

TELEPHONE NO.

PROJECT COORDINATOR

DYEKMAN, DL

PRICE CODE

DATA

200-PW-2 OU Characterization Vadose Zone - Geochemical Modeling Param $\begin{array}{r}\text { SAF NO. } \\ \text { F10-045 }\end{array}$

FIELD LOGBOOK NO. PS $55^{\circ}$ ACTUAL SAMPLE DEPTH COA

HNF-N- $5 / 9 /-5$ 2C7.5' 302117 S 30

AIR QUALTTY

TURNAROUND

45 Days / 45
Days

METHOD OF SHIPMENT
PREservation

TYPE OF CONTAINER G/P $\quad \begin{aligned} & \text { Moisture } \\ & \text { Resistant Cont }\end{aligned}$

NO. OF CONTAINER(S)

volume

SAMPLE ANALYSIS $\quad \begin{gathered}\text { SEE ITEM (1) IN Moisture } \\ \text { SPECIA } \\ \text { SNSTRLTIONS }\end{gathered}$
SAMPLE DATE SAMPLE TIME

$2-1-10$

1215

\section{CHAIN OF POSSESSION}

RELINQUSSHED BY/REMOVED FROM hany fotre ling farce 2-1.10/ 1452 FEB 022010 OE 10

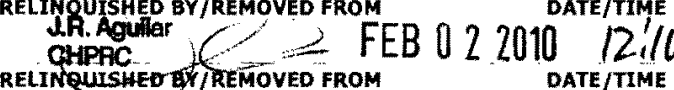

RELINQUISHED BY/REMOVED FROM

RELINQUISHED BY/REMOVED FROM

RELINQUISHED BY/REMOVED FROM

\section{LABORATORY RECEIVED BY \\ SECTION}

DINAL SAMPLE DPSAL METHOD

\section{SIGN/ PRINT NAMES}

RECEIVED BY/STORED IN

mo-413 550

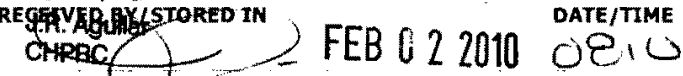

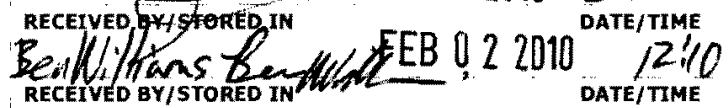

RECEIVED BY/STORED IN DATE/TME

DATE/TIME

DATE/TIME

DATE/TIME

RECEIVED BY/STORED IN

DATE/TTME
SPECIAL INSTRUCTIONS

SEE PAGE 2 FOR ALL SPECIAL INSTRUCTIONS

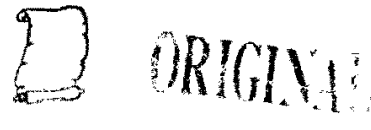


$302117 E S 30$

BILL OF LADING/AIR BILL NO.

SPECIAL INSTRUCTIONS

** The 200 Area S\&GRP Characterization and Monitoring Sampling and Analysis GKI applies to this SAF.
** ESL shall submit all data deliverables according to SOW "200-LW-2 and 200-BP-5 Operable Units/Contaminant and Transport Property Analysis and Report for Vadose Zone Sediments at Borehole C5860/Well 299-E29-54" for C7515/Well 299-E28-30 ("M" Well) and shall adhere to all S\&GRP standard protocol.

** The ESL laboratory shall meet prior requested and confirmed turnaround times and RDLs specified in the SOW and this SAF. The laboratories must notify the SM Project Coordinator in writing iffiwhen this requirement can not be met. (1)6010M_ICP_ASTM_AE (TAL) \{Sodium, Aluminum, Iron, Potassium, Antimony, Nickel, Calcium, Magnesium, Silver\} 6010M_ICP.ASTM_AE (Add-On) \{Lead, Thallium, SElenium\} 6010M_METALS_ICP_WE (TAL) \{Sodium, Aluminum,

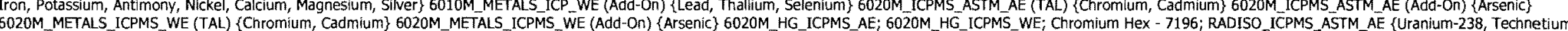

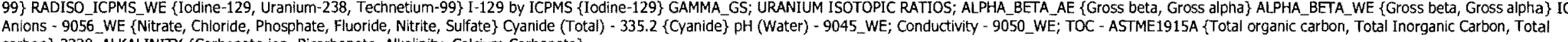
carbon\} 2320_ALKALINITY \{Carbonate ion, Bicarbonate, Alkalinity, Calcium Carbonate\}

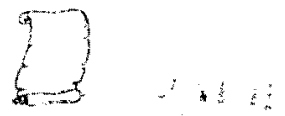


DYEKMAN, DL

TELEPHONE NO.

PRICE CODE

DATA
TURNAROUND
TS Days

PROJECT DESIGNATION

AIR QUALTTY

200-PW-2 OU Garaterization Vadose Zone - Geochemical Modeling Param F10-045

FIELD LOGBOOK NO. $P 5$ SS ACTUAL SAMPLE DEPTH COA

Days / 45
Days

ICE CHEST NO.

$$
\text { Cows - } 16 c_{1}
$$

SHIPPED TO

UNF-N- $49 /-5$ $270.0^{\circ}$

302117 ES30

METHOD OF SHIPMENT

OFFSTTE PROPERTY NO.

N/A

POSSIBLE SAMPLE HAZARDS/ REMARKS Contains Radioactive Material at concentrations that may or may not be regulated for Coods Regulations but are not Dangerous DOE Order 5400.5 (1990/1993)
Dosable per

SPECIAL HANDLING AND/OR STORAGE RADIOACTIVE TIE TO: B23650

SAMPLE.NO. MATRIX

PRESERVATION

TYPE OF CONTAINER G/P $\quad \begin{aligned} & \text { Moisture } \\ & \text { Resistant cont }\end{aligned}$

NO. OF CONTAINER(S)

VoLUME

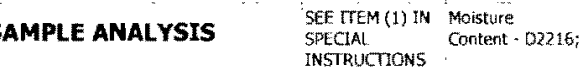

L OF LADING/AIR BILL NO.

GOVERNMENT VEHICE

CHAIN OF POSSESSION

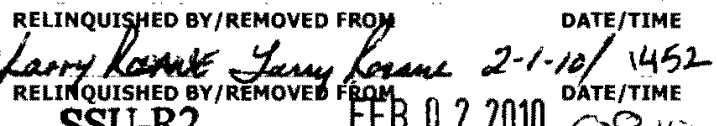
SSU-R2 FEB 0220100810

RELINQUUISHED BYIREMOVED FROM

DATE/TIME

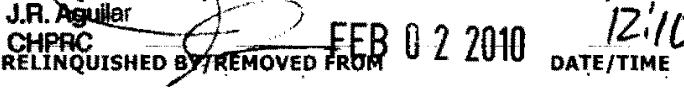

RELINQUISHED BY/REMOVED FROM

DATE/TIME

DATE/TIME

SIGN/ PRINT NAMES

RECEIVED BY/STORED IN

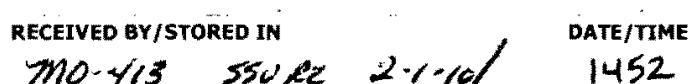

1452
RECEIVEDEYSTOREDIN

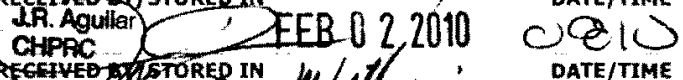

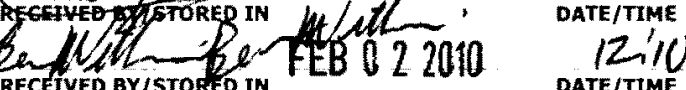

RECEIVED BY/STORED IN $\cdot$ DATE/TMME

RELINQUISHED BY/REMOVED FROM

DATE/TIME

DATE/TIME

RECEIVED QY/STORED IN

DATE/TIME

\section{SPECTAL INSTRUCTIONS}

SEE PAGE 2 FOR ALL SPECIAL INSTRUCTIONS

D ORIGI:

DISPOSED BY

DATE/TIME 
SHIPPED TO

FIELD LogBook No. $\% 955$ actual SAMPLE DEPTH

COA

METHOD OF SHIPMENT

HNF-N- $S / Y$ -

270.0

$302117 E 530$

GOVERNMENT VEHICLE

BILL OF LADING/AIR BILL NO.

N/A

\section{SPECIAL INSTRUCTIONS}

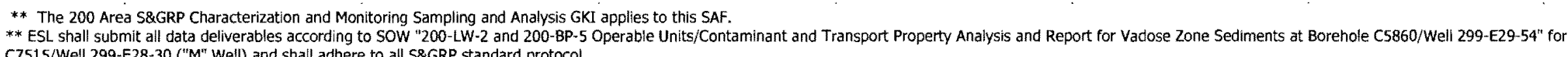

** The ESL laboratory shall meet prior requested and confirmed turnaround times and RDLs specified in the SOW and this SAF. The laboratories must notify the SM Project Coordinator in writing if/when this requirement can not be me (1)6010M_ICP_ASTM_AE (TAL) \{Sodium, Aluminum, Iron, Potassium, Antimony, Nickel, Calcium, Magnesium, Silver\} 6010M_ICP.ASTM_AE (Add-On) \{Lead, Thallium, Selenium\} 6010M_METALS_ICP_WE (TAL) \{Sodium, Aluminum, 6020M_METALS_ICPMS WE (TAL) \{Chromium, Cadmlum\} 6020M_METALS_ICPMS_WE (Add-On) \{Arsenic\} 6020M_HG_ICPMS_AE; 6020M_HG ICPMS_WE; Chromium Hex - 7196; RADISO_ICPMS_ASTM_AE \{Uranium-238, Technetium-

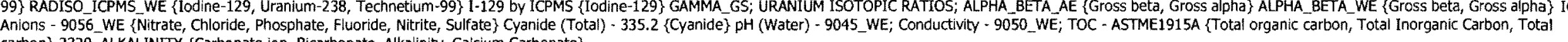
carbon\} 2320 AlKA INTYY \{Carbonate ion, Bicartonate, Aklinity, aicium Carbonate\}

$\{3$ 
SHIPPED TO

Environmental Sciences Laboratory

MATRIX* POSSIBLE SAMPLE HAZARDS/ REMARKS Contains Radioactive Materlal at concentrations that may or may not be regulated for Goods Regulations but ar $/$ IATA Dangerous Goods Regulations but are not red
DOE Order 5400.5 (1990/1993)

SPECIAL HANDLING AND/OR STORAGE RADIOACTIVE TIE TO: B23664
SOIL
SAMPLE NO.

MATRIX*

\section{COMPANY CONTACT}

DYEKMAN, DL TELEPHONE NO.

$373-2530$

PROJECT DESIGNATION

200-PW-2 Ou Characterization Vadose Zone - Geochemical Modeling Param

FIELD LOGBOOK NO. PS SS aCTUAL SAMPLE DEPTH

HNF-N- $4 / 2 /-5$

$272.5^{\prime}$

N/A

PRESERVATIO

\begin{tabular}{ll|l} 
TYPE OF CONTAINER & Moisture \\
Resistant cont
\end{tabular}

NO. OF CONTAINER(S) 1

volume

$1 \mathrm{~L} \quad 2009$

SAMPIE ANALYSIS

$$
\begin{aligned}
& \text { SEE IEM (1) IN Moisture } \\
& \text { SPCEAL } \\
& \text { INTRUCTONS }
\end{aligned}
$$

SAMPLE DATE SAMPLE TIME
SIGN/ PRINT NAMES

CHAIN OF POSSESSION

RELINQUISHED BY/REMOVED FROM

DATE/TIME RECEIVED BY/STORED IN

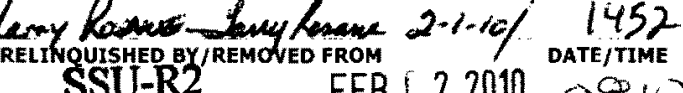
FEB

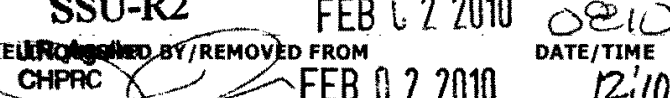
RELINQUISHEDPG/REMOVED FROM 22010

RELINQUISHED BY/REMOVED FROM

RELINQUISHED BY/REMOVED FROM

RELINQUISHED BY/REMOVED FROM

RECEIVED BY

LABORATORY

FINAL SAMPLE
DISPOSITION
Date/TIO

DATE/TIME

DATE/TIME

DATE/TIME

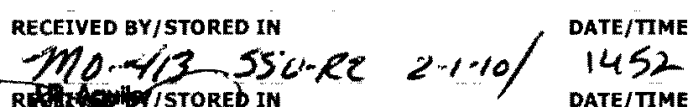

CHPAC L ISTORED IN FEB \& 2010 OQTE/TIME

RECEIVEEgorTSTOREP IN

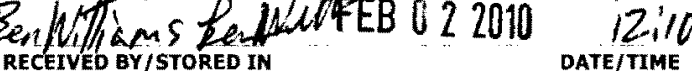

RECEIVED BY/STORED IN

DATE/TIME

DATE/TIME

RECEIVED BY/STORED IN
PROJECT COORDINATOR

DYEKMAN, DL

SAF NO.

COA

$302117 E S 30$
PRICE CODE

DATA
URNAROUND

45 Days / 45
Days

\section{METHOD OF SHIPMENT}

N/A GOVERNMENT VEHICLE

\section{SPECIAL INSTRUCTIONS}

SEE PAGE 2 FOR ALL SPECIAL INSTRUCTIONS

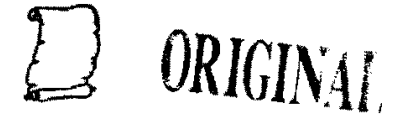




\section{SHIPPED TO}

HNF-N $4 \%$

COA

METHOD OF SHIPMENT

GOVERNMENT VEHICLE

OF LADING/AIR BIUL NO.

SPECIAL INSTRUCTIONS

** The 200 Area S\&GRP Characterization and Monitoring Sampling and Analysis GKI applies to this SAF.
** ESL shall submit all data deliverables according to SOW "200-LW-2 and 200-8P-5 Operable Units/Contaminant and Transport Property Analysis and Report for Vadose Zone Sediments at Borehole C5860/Well 299-E29-54" for C7515/Well 299-E28-30 ("M" Well) and shall adhere to all S\&GRP standard protocol.

** The ESL laboratory shall meet pror requested and confirmed turnaround times and RDLs specified in the SOW and this SAF. The laboratories must notify the SM Project Coordinator in writing if/when this requirement can not be met. (1)6010M_ICP_ASTM_AE (TAL) \{Sodium, Aluminum, Iron, Potassium, Antimony, Nickel, Calcium, Magnesium, Silver\} 6010M_ICP_ASTM_AE (Add-On) \{Lead, Thallum, Selenium\} 6010M_METALS_ICP_WE (TAL) \{Sodium, Aluminum, 6020M_METALS_ICPMS_WE (TAL) \{Chromium, Cadmium\} 6020M_METALS_ICPMS_WE (Add-On) \{Arsenic\} 6020M_HG_ICPMS_AE; 6020M_HG_ICPMS_WE; Chromium Hex-7196; RADISO_ICPMS_ASTM_AE \{Uranium-238, Technetium-

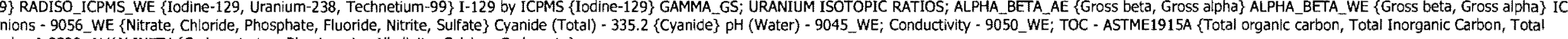
carbon\} 2320_ALKALNITY \{Carbonate ion, Bicarbonate, Akalinity, Calcium Carbonate\}

In) 
CH2MHill Plateau Remediation Company

COLLECTOR

Kays $\Omega_{\text {Rono }}$

C7515 (299-E28-30); I-116

C7515 (299-E28-30)
ICE CHEST NO.

SHIPPED TO

Gws - 166

Environmental

A=Air POSSIBLE SAMPLE HAZARDS/ REMARKS Contains Radioactive Material at concentrations

that may or may not be regulated for

Goods Regulations but are not releasable per
DOE Order 5400.5 (1990/1993)

SPECIAL HANDLING AND/OR STORAGE RADIOACTIVE TIE TO: B23664
SAMPLE NO

B235R8
MATRIX*.

SOIL

CHAIN OF CUSTODY/SAMPLE ANALYSIS REQUEST

F10-045-174

PAGE 1 OF 2

DATA
TURMAROUND

45 Days / 45
Days
PRICE CODE

PROJECT COORDINATOR

SAF NO.

AIR QUALITY

F10-045

200-PW-2 OU Characterization

$\operatorname{COA}$

302117 ES30

METHOD OF SHIPMENT

HNF-N- $4 \% /-5=56: 275^{\prime}$

BILL OF LADING/AIR BILL NO.

PRESERVATION COOI 4C N' Non

$\begin{array}{lll}\text { TYPE OF CONTAINER G/P } & \begin{array}{l}\text { Moisture } \\ \text { Resistant Cont }\end{array} \\ & \end{array}$

NO. OF CONTAINER(S)

i
FIELD LOGBOOK NO. $\rho_{c}$

$2-3-10$

0845
SIGN/ PRINT NAMES

RECEIVED BY/STORED IN

MO-4/3,55L-R2 2/3/10/1550

CHPPC $+F E Q+20100700$

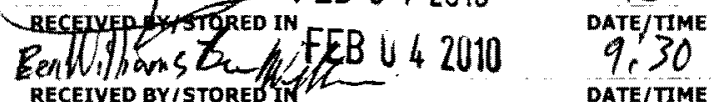

DATEmine

RECEIVED BY/STORED IN

DATE/TIME

DATE/TIME

RECEIVED BY/STORED IN

DATE/TIME
RECEIVED BY/STORED IN

\section{SPECLAL INSTRUCTIONS}

SEE PAGE 2 FOR ALL SPECIAL INSTRUCTIONS

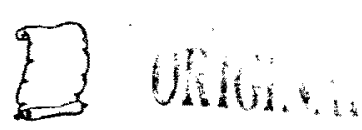

RELINQUISHED BY/REMOVED FROM

LABORATORY
SECTION

FINAL SAMPLE DISPOSAL METHOD

DISPOSITION
DATE/TIME

DATE/TIME 
Plateau Remediation Company COLLCTOR

KausR Ronco

SAMPLING LOCATION

C7515 (299-E28-30); I-116

ICE CHEST NO.

SHIPPED TO

Environmental Sciences Laboratory

$\cos 16-16$

SPECIAL INSTRUCTIONS

** The 200 Area S\&GRP Characterization and Monitoring Sampling and Analysis GKI applies to this SAF.

"* ESL shall submit all data deliverables according to SOW "200-LW-2 and 200-BP-5 Operable Units/Contaminant and Transport Property Analysis and Report for Vadose Zone Sediments at Borehole C5860/Well 299-E29-54" for

** The ESL laboratory shall meet prior requested and confrrmed turnaround times and RDLs specified in the SOW and this SAF. The laboratories must notify the SM Project Coordinator in writing iff/when this requirement can not be met. (1)6010M_ICP_ASTM_AE (TAL) \{Sodium, Aluminum, Iron, Potassium, Antimony, Nickel, Calcium, Magnesium, Silver\} 6010M_ICP_ASTM_AE (Add-On) \{Lead, Thallium, Selenium\} 6010M_METALS ICP_WE (TAL) \{Sodium, Aluminum, Iron, Potassium, Antimony, Nickel, Calcium, Magnesium, Silver\} 6010M_METAL_SICP _WE (Add-On) \{Lead, Thallium, Selenium\} 6020M_ICPMS_ASTM_AE (TAL) \{Chromium, Cadmium\} 6020M_ICPMS_ASTM_AE (Add-On) \{Arsenic\},

99\} RADISO_ICPMS WE \{Iodine-129, Uranium-238, Technetium-99\} I-129 by ICPMS \{Iodine-129\} GAMMA_GS; URANIUM ISOTOPIC RATIOS; ALPHA BETA AE \{Gross beta, Gross alpha\} ALPHA BETA WE \{Gross beta, Gross alpha\} IC Anions - 9056_WE \{Nitrate, Chloride, Phosphate, Fluoride, Nitrite, Suffate\} Cyanide (Total) - 335.2 \{Cyanide\} pH (Water) - 9045_WE; Conductivity - 9050_WE; TOC - ASTME1915A \{Total organic carbon, Total Inorganic Carbon, Total

carbon\} 2320_ALKALINITY \{Carbonate ion, Bicarbonate, Alkalinity, Calcium Carbonate\}

E)
DATA

45 Days $/ 45$

Days

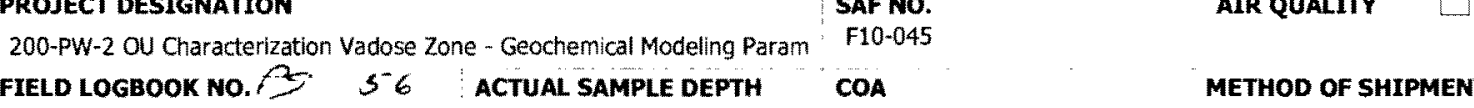

$302117 E 530$

TUL OF LADING/AIR BIL NO.

N/A 
C7515 (299-E28-30); I-117

ICE CHEST NO.

$$
\text { Gows - } 166
$$

SHIPPED TO

Environmental Sciences Laboratory

MATRIX* POSSIBLE SAMPLE HAZARDS/ REMARKS

$A=A i r$
$D L=D r u m$ that may or may not be regulated for transportation per 49 CrR / IATA Dangerous
Goods Regulations but are not releasable per
DOE Order 5400.5 (1990/1993)

SPECIAL HANDLING AND/OR STORAGE RADIOACTIVE TIE TO: B23651
SAMPLE NO.

\section{COMPANY CONTACT}

DYEKMAN, DL

TELEPHONE NO
$373-2530$

200-PW-2 OU Characterization Vadose Zone - Geochemical Modeling Param

FIELD LOGBOOK NO. PS 56 ACTUAL SAMPLE DEPTH

HNF-N-47/-5 $\quad 278.0$

OFFSTIE PROPERTY NO.

PRESERVATION

TYPE OF CONTAINER G/P $\quad \begin{aligned} & \text { Molsture } \\ & \text { Resistant Cont }\end{aligned}$

NO. OF CONTAINER(S)

VOLUME

ii 200

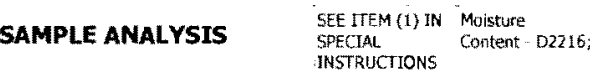

SAMPLe daTe SAMPLE TIME

$2-3-10 \quad 1035$

SIGN/ PRINT NAMES

OATE/TIME RECEIVED BY/STORED IN

Mo- $413,554-22$

RERAYGAGM/STORED II

\section{CHAIN OF POSSESSION}

Ro

Robromoxhoze eno 2/310 / 1550

SSU-R2 FEB U 42010 O 200

RELIYRUISH

EEBQ 42010 DATE/TTME

CHABC $=$ PFEO $+30109,30$

RELINQUTSHED STREMOVED FROM $4.4 U 10$ DUTE/TIM

RELINQUISHED BY/REMOVED FROM

DATE/TIME

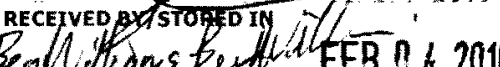

CIn

RECEIVED BY/STORED IN

DATE/TIME

RECEIVED BY/STORED IN

DATE/TIME

RELINQUISHED BY/REMOVED FROM

RECEIVED BY/STORED IN
Dect coordimator

DYEKMAN, DL

SAF NO.
F10-045

PRICE CODE
SN
TURNAROUND

AIR QUALTTY $\square \quad \underset{\text { Days }}{45 \text { Days } / 45}$

COA METHOD OF SHIPMENT

BILL OF LADING / AIR BILL NO.

N/A

LABORATORY
SECTION 


\begin{tabular}{|c|}
\hline $\begin{array}{l}\text { COLLECTOR } \\
\text { KAUER ROMU } \\
\text { SAMPLING ĹOCATION }\end{array}$ \\
\hline $\begin{array}{l}\text { C7515 (299-E28-30); I-117 } \\
\text { ICE CHEST NO. }\end{array}$ \\
\hline
\end{tabular}

SHIPPED TO

Environmental Sciences Laboratory

SPECIAL INSTRUCTIONS

** The 200 Area S\&GRP Characterlzation and Monitoring Sampling and Analysis GKI applies to this SAF.

** ESL shall submit all data deliverables according to SOW "200-LW-2 and 200-BP-5 Operable Units/Contaminant and Transport Property Analysis and Report for Vadose Zone Sediments at Borehole C5860/Well 299-E29-54" for

C7515/Well 299-E28-30 ("M" Well) and shall adhere to all S\&GRP standard protocol.

** The ESL laboratory shall meet prior requested and confirmed turnaround times and RDLs specified in the SOW and this SAF. The laboratories must notify the SM Project Coordinator in writing iffwhen this requirement can not be met. (1)6010M_ICP_ASTM_AE (TAL) \{Sodium, Aluminum, Iron, Potassium, Antimony, Nickel, Cakium, Magnesium, Silver\} 6010M_.ICP_ASTM_AE (Add-On) \{Lead, Thallium, Selenium\} 6010M_METALSICPP_WE (TAL) \{Sodium, Aluminum,
Iron, Potassium, Antimony, Nickel, Calcium, Magnesium, Silver\} 6010M METALS ICP WE (Add-On) \{Lead, Thallium, Selenium\} 6020M ICPMS ASTM AE (TAL) \{Chromium, Cadmium\}6020M ICPMS ASTM AE (Add-On) \{Arsenic\}

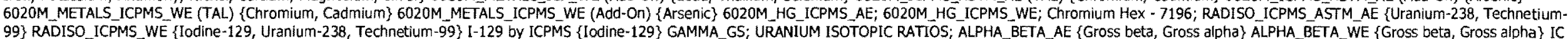
$99\}$ RADISO_ICPMS WE \{Lodine-129, Uraniun-238, Technetium-99\} I-129 by ICPMS \{Iodine-129\} GAMMA_GS; URANIUM ISOTOPIC RATIOS; ALPHA_BETA_AE \{Gross beta, Gross alpha\} ALPHA_BETA_WE \{Gross beta, Gross alpha\} IC
Anions - 9056 WE \{Nitrate, Chloride, Phosphate, Fluoride, Nitrite, Sulfate\} Cyanide (Total) - 335.2 \{Cyanide\} pH (Water) - 9045_WE; Conductivity - 9050_WE; TOC - ASTME1915A \{Total organic carbon, Total Inorganic Carbon, Total Anions - 9056-WE \{Nitrate, Chloride, Phosphate, Fluoride, Nitrite, Sulfate\} Cyanide (Totol
Carbon\} 2320_ALKALINITY \{Carbonate ion, Bicarbonate, Alkalinity, Calcium Carbonate\}

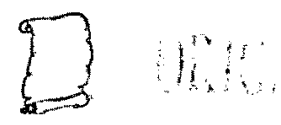


KaLER, Romo

SAMPLING LOCATION

C7515 (299-E28-30); I-118

ICE CHEST NO.

$$
\text { Goss - 146 }
$$

SHIPPED TO

Environmental Sciences Laboratory

MATRIX* I POSSIBLE SAMPLE HAZARDS/ REMARKS Contains Radioactive Material at concentrations transportation per 49 CFR / IATA Dong Goods Regulations but are not releasable per DOE Order $5400.5(1990 / 1993)$

SPECIAL HANDLING AND/OR STORAGE RADIOACTIVE TIE TO: B2365
- SAMPLE No.

B235TO
MATRIX*

\begin{tabular}{l|l|l} 
COMPANY CONTACT & TELEPHONE NO. & PROJECT COORDINATOR \\
DYEKMAN, DL & $373-2530$ & DYEKMAN, DL \\
\hline PROJECT DESIGNATION & & SAF NO. \\
\hline 200-PW-2 OU Characterization Vadose Zone - Geochemical Modeling Param & F10-045
\end{tabular}

200-PW-2 OU Characterization Vadose Zone - Genchemical Modeling Param F10-045

FIELD LOGBOOK NO. $7^{2} 5,56$ ACTUAL SAMPLE DEPTH IOA
HNF-N- 48,5
$280^{\circ}$
$302117 E S 30$

PRICE CODE

\begin{tabular}{l} 
DATA \\
TURNAROUND \\
\hline
\end{tabular}

45 Days / 45

AIR QUALITY

\section{METHOD OF SHIPMENT} BILL OF LADING/AIR BILL NO N/A

$$
\begin{aligned}
& \text { PREsERVATION } \\
& \text { TYPE OF CONTAINER }\left.\quad\right|^{G / P} \quad \begin{array}{l}
\text { Moisture } \\
\text { Resistant Cont }
\end{array} \\
& \text { NO. OF CONTAINER(S) }\left.\quad\right|_{1} \cdots{ }^{\prime} \\
& \text { VOLUME } \\
& \begin{array}{ll}
\text { SAMPLE ANALYSIS } & \text { SEE TEM (1) IN Moisture } \\
\text { SPECIIA } \\
\text { iNSTRECTIONS }
\end{array}
\end{aligned}
$$

\section{CHAIN OF POSSESSION}

RELINQUISHED BY/REMOVED FROM Ro6 Romo Rolax Romo $2 / 3 / 10$ Date/TIME

$\begin{array}{lll}\text { REUNRUSHED BY/REMOVD FROM } & \text { DATE/TIME } \\ \text { SSU-R } & \text { FEB \& } 42010 \text { O>00 }\end{array}$

RELUQUTSUF BY/REMGVED FROM

CHPRC CTEC FEB i 42010

RELINQUISHED GT TKEMOVED FROM

RELINQUISHED BY/REMOVED FROM

RELINQUISHED GY/REMOVED FROM

RELINQUUSHED BY/REMOVED FROM

RECEIVED B

SECTION

FINAL SAMPLE
DISPOSITION
SIGN/ PRINT NAMES

RECEIVED BY/STORED IN RECEIVED BY/STORED IN 1 HO $413,554-R 2 \quad 2 / 3 / 101550$ CHPRC $9 ! 30$ DATE/TIME

DATE/TMME

DATE/TIME

DATE/TIME Beally RECEIVED QYY/STORED IN

RECEIVED BY/STORED IN

9.30 DATE/TIME

DATE/TIME

DATE/TIME

DATE/TTME
SPECIAL INSTRUCTIONS

SEE PAGE 2 FOR ALL SPECIAL INSTRUCTIONS 


\section{METHOD OF SHIPMENT} OFFSITE PROPERTY NO.

302117ES30

COVERTMENT VEHICIE

$$
\text { N/A }
$$

ING/AIR BILL NO.

$\mathrm{N} / \mathrm{A}$

\section{SPECIAL INSTRUCTIONS}

** The 200 Area S\&GRP Characterization and Monitoring Sampling and Analysis GKI applies to this SAF. C7515/Well 209mit all data deliverables according to SOW "200-LW-2 and 200-BP-5 OPE

** The ESL laboratory shall meet prior requested and confirmed turnaround times and RDLs specified in the SOW and this SAF. The laboratories must notify the SM Project Coordinator in writing iffwhen this requirement can not be met. (1)6010M_LCPASTM_AE (TAL) (Sodium, Aluminum, Iron, Potassium, Antimony, Nickel, Calciurn, Magnesium, Silver\} 6010M_ICP_ASTM_AE (Add-On) \{Lead, Thallium, Seeerium\} 6010M_METALS ICP_WE (TAL) \{Sodium, Aluminum, 6020M_METALS_ICPMS_WE (TAL) \{Chromium, Cadmium\} 6020M_METALS_ICPMS_WE (Add-ON) \{Arsenic\} 6020M_HG_ICPMS_AE; 6020M_HG_ICPMS_WE; Chromium Hex - 7196; RADISO_ICPMS_ASTM_AE \{Uranium-238, Technetiurn99\} RADISO_ICPMS WWE \{lodine-129, Uranium-238, Technetium-99\} I-129 by ICPMS \{lodine-129\} GAMMA_GS; URANIUM ISOTOPIC RATIOS; ALPHA BEETA_AE \{Gross beta, Gross alpha\} ALPHA_BETA_WE \{Gross beta, Gross a'pha\} IC

Anions - 9056_WE \{Nitrate, Chloride, Phosphate, Fiuoride, Nitrite, Sulfate\} Cyanide (Total) - 335.2 \{Cyanide\} PH (Water) - 9045_WE; Conductivity - 9050_WE;
carbon\} 2320_ALKALINITY \{Carbonate ion, Bicarbonate, Alkalinity, Calcium Carbonate\}

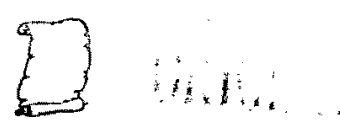


COLLECTOR

\section{KAY $2 R$}

SAMPLING LOCATION

C7515 (299-E28-30); I-119

ICE CHEST NO.

Givs - 166

SHIPPED TO

Environmental Sciences Laboratory

MATRIX* POSSIBLE SAMPLE HAZARDS/ REMARKS

$A=A i r$
$D L=D r u m$ that may or may not be regulated for Goods Regulations but are not releasable per DOE Order 5400.5 (1990/1993)

SPECIAL HANDLING AND/OR STORAGE RADIOACTIVE TIE TO: B2365

SAMPLE NO. . MATRIX*

B235B9

\begin{tabular}{|c|c|c|c|c|c|}
\hline $\begin{array}{l}\text { COMPANY CONTACT } \\
\text { DYEKMAN, DL }\end{array}$ & $\begin{array}{l}\text { TELEPHONE NO. } \\
373-2530\end{array}$ & $\begin{array}{l}\text { PROJECT COORDINATOR } \\
\text { DYEKMAN, DL. }\end{array}$ & PRICE CODE & $8 \mathrm{~N}$ & $\begin{array}{c}\text { DATA } \\
\text { TURNAROUND }\end{array}$ \\
\hline PROJECT DESIGNATION & & $\begin{array}{l}\text { SAF NO. } \\
\text { F10-045 }\end{array}$ & AIR QUALITY & $\square$ & $\begin{array}{l}45 \text { Days / } 45 \\
\text { Days }\end{array}$ \\
\hline
\end{tabular}

FIELD LOGBOOK NO. $P S S C$ ACTUAL SAMPLE DEPTH COA
HNF-N- $4 \% 15$
302117 ES30

OFFSTTE PROPERTY NO.

BILL OF LADING/AIR BILL NO.

N/A

N/A

\begin{tabular}{|c|c|}
\hline PRESERVATION & None \\
\hline TYPE OF CONTAINER & $\begin{array}{l}\text { Split Spoon } \\
\text { Liner }\end{array}$ \\
\hline
\end{tabular}

NO. OF CONTAINER(S)

volume

10009

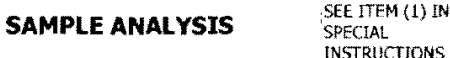

\section{CHAIN OF POSSESSION}

RELINQUISHED BY/REMQUED FROM

Ro b

SSU-R2 PEB 242010 DATE/TIME

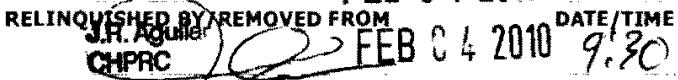

RELINQUISHED QY LREMOVED FROM

RELINQUISHED BY/REMOVED FROM

DATE/TIME

RELINQUISHED BY/REMOVED FROM

DATE/TIME

RELINQUISHED BY/REMOVED FROM

DATE/TIME

DATE/TIME

\section{SIGN/ PRINT NAMES}

RECEIVED BY/STORED IN

Mo- $4 / 3,554-82$

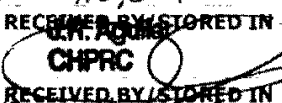

Benllillitims for

$2-30 \quad$ is 50

DATE/TMM

042010 FEB $\cup 42010$

\section{OTUT}

$9 ! 30$

RECEIVED BY/STORED IN

RECEIVED BY/STORED IN

RECEIVED BY/STORED IN

\section{Dare}

DATE/TIME

DATE/TIME

DATE/TIME

\section{SPECIAL INSTRUCTIONS}

** The 200 Area S\&GRP Characterization and Monitoring Sampling and Analysis applies to this SAF.

** ESL shall Submit all data deliverables according to SOW "200-LW-2 and 200-8P-5 Operable Units/Contaminant and Transport Property Analysis and Report for Vadose Zone Sediments at Borehole C5860/Well 299-E29-54" for C7515/Well 299-E28-30 (Mell) and shall adhere to all S\&GRP standard protocol.

The ESL laboratory shall meet prior requested and confirmed turnaround times and RDLS specified in the SOW and this SAF. The laboratories must notify the SM

Project Coordinator in writing iff when this requirement can not be met.

(Hydromite - D422; KD _ Barth;
TITLE Uition
DATE/TIME
DISPOSED BY
DATE/TIME

\section{SECTION}

FINAL SAMPLE DISPOSAL METHOD
DISPOSITION 
CH2MHill Plateau Remediation Company

\section{COLLECTOR}

\section{KAYSR Romo}

SAMPLING LOCATION

C7515 (299-E28-

ICE CHEST

$$
\text { Gous }-166
$$

SHIPPED TO

Environmental Sciences Laboratory

MATRIX* POSSIBLE SAMPLE HAZARDS/ REMARKS

$A=A i r$
$D=D r u m$

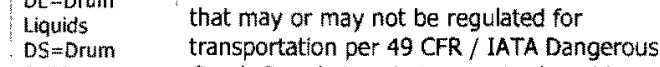

transportation per 49 CFR / IATA Dangerous
Goods Regulations but are not releasable per
DOE Order 5400.5 (1990/1993)

SPECIAL HANDLING AND/OR STORAGE RADIOACTIVE TIE TO: 823651
SAMPLE NO.

B235T1
MATRIX* .
TELEPHONE NO.

PROJECT COORDINATOR

DYEKMAN, DL

SAF NO.

PROJECT DESIGNATION

373-2530

PAGE 1 OF 2

PRICE CODE

45 Days / 45
Days

200-PW-2 OU Characterization Vadose Zone - Geochemical Modeling Param F10-045

\begin{tabular}{|l|l|l|} 
FIELD LOGBOOK NO. $\rho_{S}$ : 56 & ACTUAL SAMPLE DEPTH
\end{tabular}
HNF-N- $\angle / S /-S$
282.1
COA
302117 ES30
GOVERNMENT VEHICLE

METHOD OF SHIPMEN

BILL OF LADING/AIR BILL NO.

N/A

PRESERVATION

'Cool ac None

TYPE OF CONTAINER

NO. OF CONTAINER(S)

VOLUME

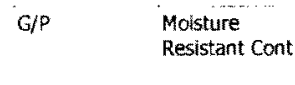

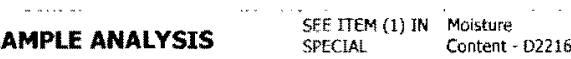

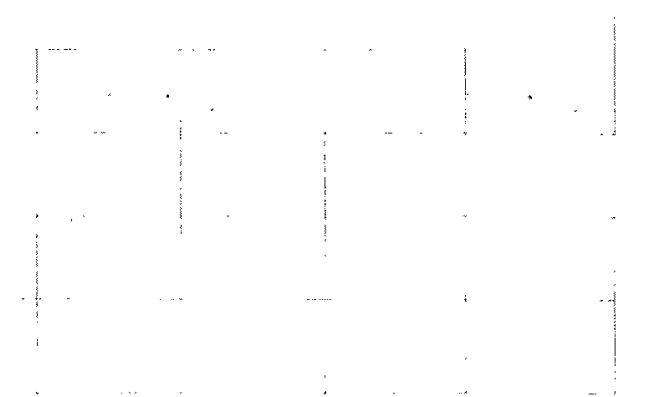

\section{CHAIN OF POSSESSION}

RELINQUISHED BY/REMOVED EROM

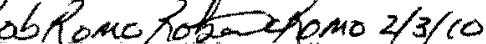

SSUS-R2

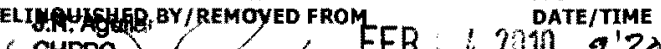

CHPRC

RELTHQUISHED BY TKEMOVED FROM

DATE/TIME

RELINQUISHED BY/REMOVED FROM

RELINQUISHED BY/REMOVED FROM

RELINOUISHED BY/REMOVED FROM

DATE/TIME

DATE/TIME

DATE/TIME
RECEIUED BY/STORED IN

\section{SIGN/ PRINT NAMES}

RECEIVED BY/STORED IN

mo-413 55a-Rz

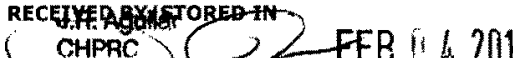

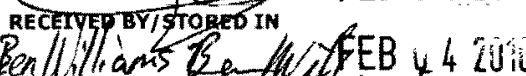

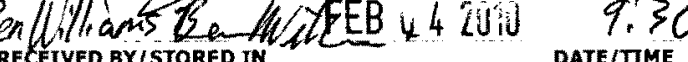

DATE/TIME

DATE/TIME

DATE/TIME
SPECIAL INSTRUCTIONS

SEE PAGE 2 FOR ALL SPECIAL INSTRUCTIONS

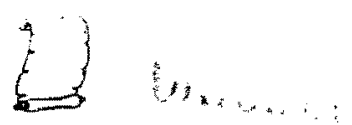



COLLECTOR

$$
\text { KACUCR }
$$

SAMPLING LOCATION

C7515 (299-E28-30); I-120

ICE CHEST NO.

SHIPPED To COWS -164

Environmental Sciences Laborator

\section{SPECIAL INSTRUCTIONS}

** The 200 Area S8GRP Characterization and Monitoring Sampling and Analysis GKI applies to this SAF.

"* ESL shall submit all data deliverables according to SOW "200-LW-2 and 200-8P-5 Operable Units/Contaminant and Transport Property Analysis and Report for Vadose Zone Sediments at Borehole C5860/Well 299-E29-54" for

** The ESL laboratory shall meet prior requested and confirmed turnaround times and RDLs specified in the Sow and this SAF. The laboratories must notify the SM Project Coordinator in writing if/when this requirement can not be met.
(1)6010M_ICP ASTM AE (TAL) \{Sodium, Aluminum, Iron, Potassium, Antimony, Nickei Calcium, Magnesium, Silver\} 6010M ICP ASTM AE (Add-On) \{Lead Thallium, Selenium\}6010M METALS ICP WE (TAL) (Sodium, Aluminum, (1)6010M_ICP_ASTM_AE (TAL) \{Sodium, Aluminum, Iron, Potassium, Antimony, Nickel, Calcium, Magnesium, Silver\} 6010M_ICP_ASTM_AE (Add-On) \{Lead, Thallium, Selenium\} 6010M_METALS_ICPPWWE (TAL) \{Sodium, Aluminum,

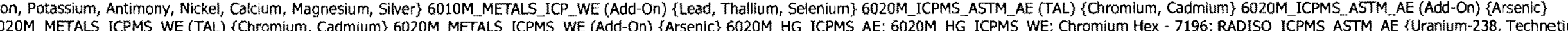
99\} RADISO ICPMS WE \{Iodine-129, Uranium-238, Technetium-99\} I-129 by ICPMS \{Iodine-129\} GAMMA GS; URANIUM ISOTOPIC RATIOS; ALPHA BETA AE \{Gross beta, Gross alpha\} ALPHA BETA WE \{Gross beta, Gross alpha\} IC Anions - 9056_WE \{Nitrate, Chioride, Phosphate, Fluoride, Nitrite, Sulfate\} Cyanide (Total) - 335.2 \{Cyanide\} pH (Water) - 9045_WE; Conductivity - 9050_WE; TOC - ASTME1915A \{Total organic Carbon, Total Inorganic Carbon, Total carbon\} 2320_ALKALINITY \{Carbonate ion, Bicarbonate, Alkalinity, Calcium Cartonate\}

Thina

$\begin{array}{ll:ll}\text { 200-PW-2 ou Characterization vadose Zone - Geochemical Modeling Param } & \text { F10-045 } & \text { COA } & \text { METHOD OF SHIPMENT }\end{array}$
HNF-N- 48 -
282.1
302117 ES30
GOVERNMENT VEHICLE

BILL OF LADING/AIR BILL NO.

BILL OF
TELEPHONE NO.

$373-2530$

PROJECT COORDINATOR

AIR QUALITY

45 Days / 45

Days/
Days 
CH2MHill Plateau Remediation Company

COLLECTOR

Krover, Romo, Boytes

SAMPLING LOCATION

ICE CHEST NO.

$$
6102-166
$$

SHIPPED TO

MATRIX*

POSSIBLE SAMPLE HAZARDS/ REMARKS Contains Radioactive Material at concentrations that may or may not be regulated for DOE Order 5400.5 (1990/1993)

SPECIAL HANDLING AND/OR STORAGE RADIOACTIVE TIE TO: B23652
Environmental Sciences Laboratory Goods Regulations but are not releasable per

TELEPHONE NO. PROJECT COORDINATOR

$$
373-2530
$$

DYEKMAN, DL

SAF No.

PROJECT DESIGNATTON

200-PW-2 OU Characterization Vadose Zone - Geochemical Modeling Param F10-045

FIELD LOGBOOK NO. PS 57 ACTUAL SAMPLE DEPTH COA

HNF-N- $Y Q / 5$ 284.0 to $286 .{ }^{-302117 E S 30}$

\section{METHOD OF SHIPMENT} BILL OF LADING/AIR BILL NO.

\section{PRESERVATION}

TYPE OF CONTAINER

NO. OF CONTAINER(S)

voLUME

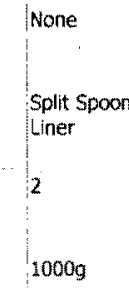

SAMPLE ANALYSIS
$\mathrm{N} / \mathrm{A}$

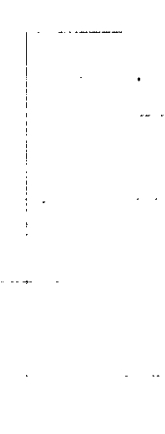

SOIL
MATRIX*
SAMPLE DATE SAMPLE TIME

$2 \cdot 4-1045$
SIGN/ PRINT NAMES

\section{CHAIN OF POSSESSION}

DATE/TIME

RECEIVED BY/STORED IN RELINQUISHED BY/REMOVED FROM

RELINQUTSHED BY/REMOVED FROM

RELINQUISHED BY/REMOVED FROM

RELINQUISHED BY/REMOVED FROM

\section{LABORATORY RECEIVED BY}

\section{SECTION}

DISPOSITION EdKAver/Ehasen 2.4\% 1540 MO-413 SSU1-R2 RELINQUHFPR日 QY NREMOVED FROM

\section{DATE/TIMEO $7{ }^{2}$}

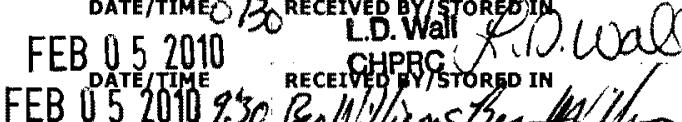

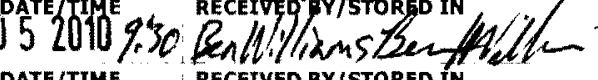

DATE/TIME

DATE/TIME

CCEVED BVISTORED

DATE/TIME

RECEIVED BY/STORED IN

DATE/TTME
EECEIVED BY/STORED IN
Days 
PROJECT COORDINATOR

SAMPLING LOCATION

DYEKMAN, DL

$373-2530$

DYEKMAN, DL

PRICE CODE

DATA
TURNAROUND
45 DaYS/ 45

PROJECT DESIGNATION SAF NO.

AIR QUALITY

45 Days $/ 45$

200-PW-2 Ou Characterization Vadose Zone - Geochemical Modeling Param F10-045

FIELD LOGBOOK NO. A 57 ACTUAL SAMPLE DEPTH COA

$$
602-166
$$

SHIPPED TO

Environmental Sciences Laboratory

\begin{tabular}{l|l} 
HNF-N- $491-5$ & $2 \otimes 6.5$
\end{tabular}

302117 ES 30

OFFSITE PROPERTY NO.

N/A

POSSIBLE SAMPLE HAZARDS/ REMARKS
Contains Radioactive Material at concentrations
that may or may not be regulated for

that may or may not be regulated for

Goods Regulations but are not releasable pe

DOE Order $5400.5(1990 / 1993)$

SPECIAL HANDLING AND/OR STORAGE RADIOACTIVE TRE TO: B23652

PRESERVATION

TYPE OF CONTAINER G/P Moisture

volume

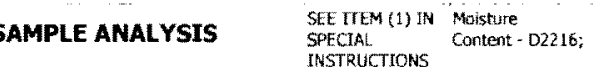

N/A
SAMPLE NO.

B235T2

\section{CHAIN OF POSSESSION}

RELINQUISHED BY/REMOVED FROM

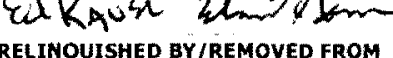
mo 413 SSH-R2

RELINQUISHED QY LYREMOVED FROM

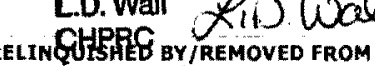

RELINQUISHED BY/REMOVED FROM

RELINQUISHED BY/REMOVED FROM

RELINQUUISHED BY/REMOVED FROM

\section{RECEIVED BY}

SECTION

FINAL SAMPLE DISPOSAL METHOD DISPOSITION

\section{SIGN/ PRINT NAMES}

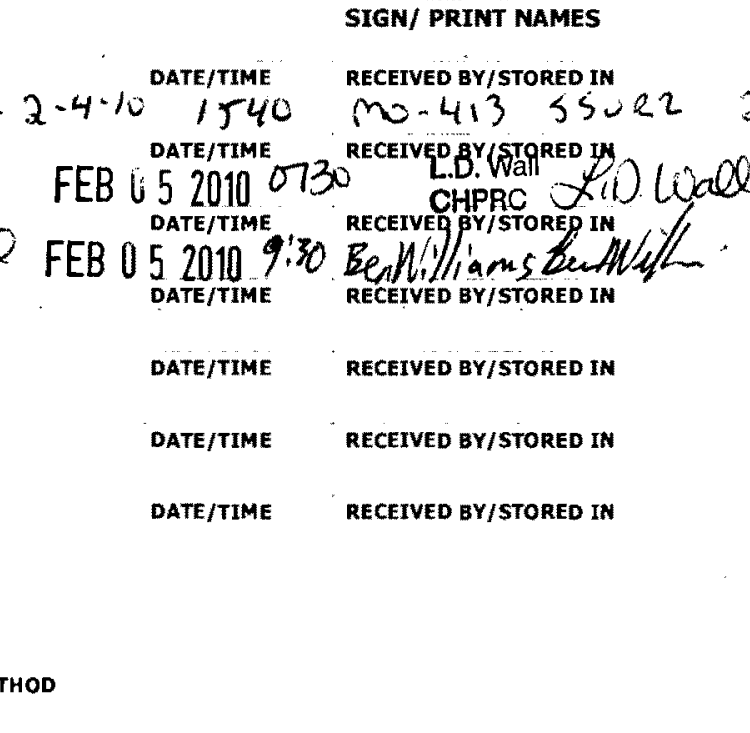

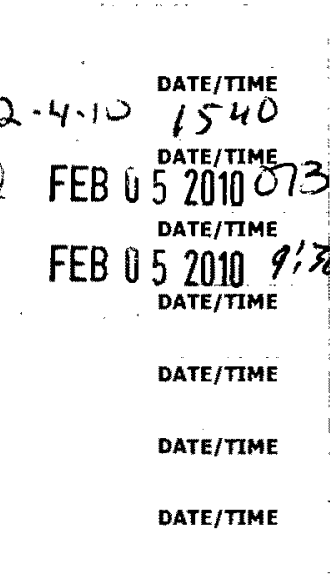

TILE

SPECIAL INSTRUCTIONS

SEE PAGE 2 FOR ALL SPECIAL INSTRUCTIONS

$X j L$ pothle only $1 / 2$ full Due to

97 Amiont of matrait

bais in splet spons

DISPOSED BY

DATE/TIME

DATE/TIME 
CH2MHill Plateau Remediation Company

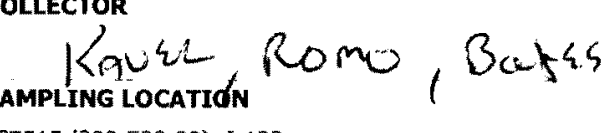

C7515 (299-E28-30); I-122

ICE CHEST NO.

SHIPPED TO

$$
602-166
$$

Environmental Sciences Laboratory

\section{SPECIAL INSTRUCTIONS}

* The 200 Area SRGPP Characterizztion and Monitoring Sampling and Analysis $C \mathrm{Kl}$ ap

"ESL shall submit all data deliverables according to SOW "200-LW-2 and 200-BP-5 Operable Units/Contaminant and Transport Property Analysis and Report for Vadose Zone Sediments at Borehole C5860/Well 299-E29-54" for

** The ESL laboratory shall meet prior requested and confirmed turnaround times and RDLs specified in the SOW and this SAF. The laboratories must notify the SM Project Coordinator in writing if/when this requirement can not be met. (1)6010M_ICP_ASTM_AE (TAL) \{Sodium, Aluminum, Iron, Potassium, Antimony, Nickel, Calcium, Magnesium, Silver\} 6010M_ICP_ASTM_AE (Add-On) \{Lead, Thallium, Selenium\} 6010M_METALS_ICP_WEE(TAL) \{Sodium, Aluminum,

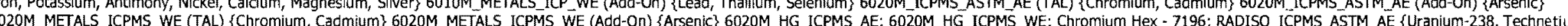
99\} RADISO_ICPMS WE \{Iodine-129, Uranium-238, Technetium-99\} [1-129 by ICPMS \{Iodine-129\} GAMMA_GS; URANIUM ISOTOPIC RATIOS; ALPHA_BETA_AE \{Gross beta, Gross alpha\} ALPHA_BETA_WE \{Gross beta, Gross alpha\} IC Anions - 9056_WE \{Nitrate, Chloride, Phosphate, Fluoride, Nitrite, Suffate\} Cyanide (Total) - 335.2 \{Cyanide\} PH (Water) - 9045_WE; Conductivity - 9050_WE; TOC - ASTME1915A \{Total organic Carbon, Total Inorganic Carbon, Total arbon\} 2320_ALKALINITY \{Carbonate ion, Bicarbonate, Alkalinity, Calcium Carbonate\}

\section{DRGIH}




\section{CH2MHill Plateau Remediation Company}

COLLECTOR

Kunke, Romo Bates SAMPLING LOCATION

C7515 (299-E28-30); I-123

ICE CHEST NO.

$602-166$

SHIPPED TO

Environmental Sciences Laboratory

MATRIX* ${ }^{-1}$ POSSIBLE SAMPLE HAZARDS/ REMARKS

$\begin{aligned} & A=A i r \\ & D\end{aligned}=0$ rum $\quad$ Contains Radioactive Material at concentrations

that may or may not be regulated for

Grods Regultions buf

Goods Regulations but are not releasable per
DOE Order $5400.5(1990 / 1993)$

$\mathrm{L}=$ Liquid
$\mathrm{O}=\mathrm{Oil}$

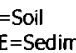

$T=$ Tissue
$V=$ Vegetation
$W=$ Water

$W=$ Water
$W=W(i p e$

SPECIAL HANDLING AND/OR STORAGE RADIOACTIVE TIE TO: B23652
CHAIN OF CUSTODY/SAMPLE ANALYSIS REQUEST

F10-045-185

PAGE 1 OF 1

PRICE CODE

PROJECT COORDINATOR

DYEKMAN, DL

SAF NO.
F10-045

AIR QUALITY

TURNAROUND

$\underset{\substack{\text { Days } \\ \text { Days }}}{45}$

PROJECT DESIGNATION

373-2530

200-PW-2 OU Characterization Vadose Zone - Geochemical Modeling Param
FIELD LOGBOOK NO. $r^{2} 57$ ACTUAL SAMPLE DEPTH COA

NF-N- 4/O/-S

288.1 to 288.6302117 ES30

METHOD OF SHIPMENT

GOVERNMENT VEHICLE

BILL OF LADING/AIR BILL NO.

\begin{tabular}{|c|l|}
\hline PRESERVATION & None \\
\hline TYPE OF CONTAINER & Split Spoon \\
\hline liner
\end{tabular}

SAMPLE NO

B235C 1
MATRIX*
CHAIN OF POSSESSION

RELINQUISHED BY/REMGVED FRgM RELINQUISHED BY/REMOVED FROM MO. 413 SSU-R2 RELINQUISHED BY /REMOVED FROM

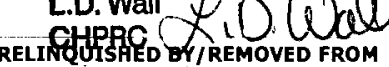

RELINQUISHED BY/REMOVED FROM

RELINQUISHED BY/REMOVED FROM

RELINQUISHED BY/REMOVED FROM

RECEIVED BY SECTION

FINAL SAMPLE
DISPOSITION
SOIL

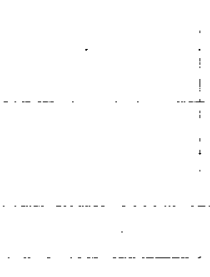

SAMPLE DATE SAMPLE TIME $2 \cdot 4 \cdot 10: 1215$

SIGN/ PRINT NAMES

1540

RECEIVED BY/STORED IN

mo-4/3 5s

SPECIAL INSTRUCTIONS

DATE/TME - ** The 200 Area S\&GRP Characterization and Monitoring Sampling and Analysis GKI applies to this SAF.

DATE/TIME Z RECETEEQPWETPREDIN DATE'TME FEB 05281073

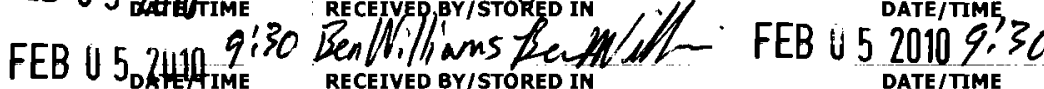

\section{DATE/TIME RECEIVED BY/STORED IM \\ DATE/TME}

** The ESL laboratory shall meet prior requested and confirmed tumaround times and RDLS specified in the SOW and this SAF. The laboratories must notify the SM Project Coordinator in writing if/when this requirement can not be met.

DATE/TIME RECEIVED BY/STOPED IN

DATE/TIME (1)Density; CATIONEXCH_TR; Particle Size (Dry Sieve) - D422; Particle Size

DATE/TIME " RECEIVED BY/STORED IM
DATE/TIME
DATE/TIME 
CH2MHill Plateau Remediation Company

COLLECTOR

Kaus, Remo, Bates

ICE CHEST NO.

ICE CHEST NO. $602-166$

SHIPPED TO

Environmental Sciences Laboratory

MATRIX*

POSSIBLE SAMPLE HAZARDS/ REMARKS Contains Radioactlve Materlal at concentrations

transportation per 49 CFR / IATA Dangerous

Goods Regulations but are not reieasable per
DOE Order 54005 (1990/1993)

=Liquid

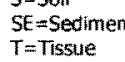

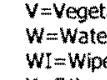

SPECIAL HANDLING AND/OR STORAGE RADIOACTVE TIE TO: B23652

SAMPLE NO

B235T3

SOIL

MATRIX*

\section{SAMPLE DATE SAMPLE IIME}

$2-4-10 \cdot 1215$

SIGN/ PRINT NAMES

RECEIVED BY/STORED IN
$M O .413$ SSU

CHAIN OF POSSESSION

DATE/TMME 40

SSU.R2 2.4-10 1540

Ed Kavis, Eldur. De tama

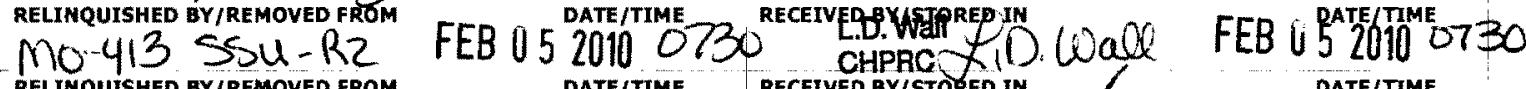

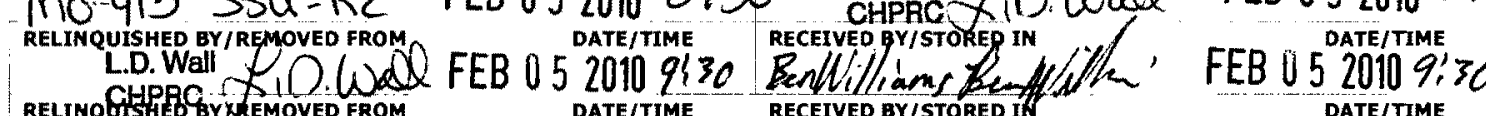

RELINQUISHED BY/REMOVED FROM

RELINQUISHED BY/REMOVED FROM

RELINQUISHED BY/REMOVED FROM

DATE/TIME

RECEIVED BY/STORED IN

RECEIVED BY/STORED IN

DATE/TIME

RECEIVED BY/STORED IN
DATE/TTME

DATE/TME

DATE/TIME

DATE/TIME
F10-045-186

PAGE 1 OF 2

PRICE CODE $\quad$ BN

Data

AIR QUALITY $\square \quad \underset{\text { Days }}{45 \text { Days / } 45}$

COA
302117 MES30
GOVERNMENT VEHICLE

BILL OF LADING/AIR BILL NO.

$\mathrm{N} / \mathrm{A}$

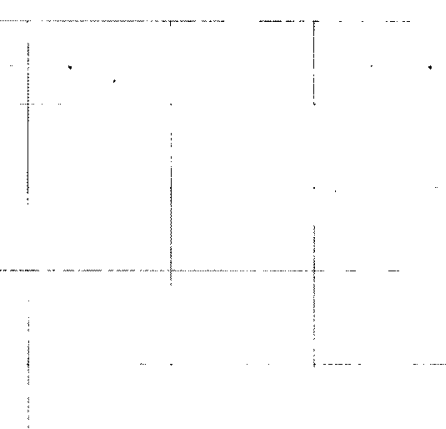

SPECIAL INSTRUCTIONS

SEE PAGE 2 FOR ALL SPECIAL INSTRUCTIONS
LABORATORY
SECTION

FINAL SAMPLE DISPOSAL METHOD

FINAL SAMPLE
DISPOSITION 
CH2MHill Plateau Remediation Company

Kaver, Remo, Bates

SAMPLING LOCATION

C7515 (299-E28-30); I-124

ICE CHEST NO. $602-166$

SHIPPED TO

Environmental Sciences Laboratory

\section{SPECIAL INSTRUCTIONS}

** The 200 Area S\&GRP Characterization and Monitoring Sampling and Analysis GKI apples io this SAF.

** ESL shall submit all datad deliverables according to SOW "200-LW-2 and 200-BP-5 Operable Units/Contaminant and Transport Property Analysis and Report for Vadose Zone Sediments at Borehole C5860/Well 299-E29-54" for

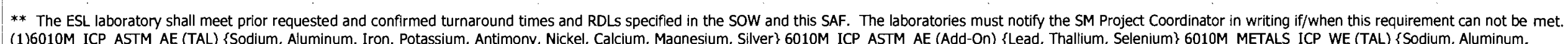
(1)6010M_ICP_ASTM_AE (TAL) \{Sodium, Aluminum, Iron, Potassium, Antimony, Nickel, Calcium, Magnesium, silver\} 6010M_ICP_ASTM_AC (Add-ON) \{Lead, Thallium, Selenium\} 6010M_METALL_IIPP_WE (TAL) \{Sodium, Aluminum,

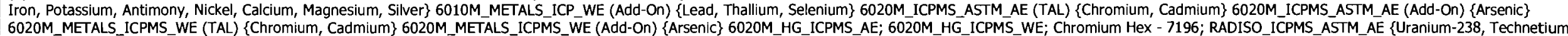
99\} RADISO ICPMS WE \{Iodine-129, Uranium-238, Technetium-99\} I-129 by ICPMS \{Iodine-129\} GAMMA GS; URANIUM ISOTOPIC RATIOS; ALPHA BETA AE \{Gross beta, Gross alpha\} ALPHA BETA_WE \{Gross beta, Gross alpha\} IC Anions - 9056_WE \{Nitrate, Chloride, Phosphate, Fluoride, Nitrite, Sulfate\} Cyanide (Total) - 335.2 \{Cyanide\} PH (Water) - 9045_WE; Conductivity - 9050_WE; TOC - ASTME1915A (Total organic carbon, Total Inorganic Carbon, Total carbon\} 2320_ALKALINITY \{Carbonate ion, Bicarbonate, Alkalinity, Calcium Carbonate
TELEPHONE NO.

PROJECT COORDINATOR

SAF NO.

200-PW-2 OU Characterization Vadose Zone - Geochemical Modeling Param

FIELD LOGBOOK NO. PS

HNF-N- YG/-

57 ACTUAL SAMPLE DEPTH

COA

302117ES30

GOVERNMENT VEHICLE

\section{CHAIN OF CUSTODY/SAMPLE ANALYSIS REQUEST}

E) Habu: 
CH2MHill Plateau Remediation Company

COLLECTOR

I Kaven Roma; Butss SAMPLING LOCATION

C7515 (299-E28-30); I-125

ICE CHEST NO.

$602-164$

SHIPPED TO

Environmental Sciences Laboratory

MATRIX*

POSSIBLE SAMPLE HAZARDS/ REMARKS Contans Radioactive Material at concentrations transportation per 49 be regulated for Goods Regulations but are not releasable per

DLliquid DOE Order $5400.5(1990 / 1993)$

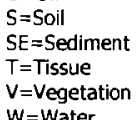

$\mathrm{W}=$ Water

SPECIAL HANDLING AND/OR STORAGE RADIOACTVE TIE TO: B23653

- SAMPLE NO

B235C2

MATRIX
CHAIN OF CUSTODY/SAMPLE ANALYSIS REQUEST

F10-045-188

PAGE 1 OF 1

\section{COMPANY CONTACT}

DYEKMAN, DL

TELEPHONE NO.
$373-2530$

200-PW-2 OU Characteriza

SAF NO.

PROJECT COORDINATOR

FIELD LOGBOOK NO $P$. 57 Zane - Geochemical Modeling Param

HNF-N- $496-537287.0$ to 289.5

OFFSITE PROPERTY NO.

$\mathrm{N} / \mathrm{A}$

F10-045

COA

302117ES30

PRICE CODE

AIR QUALTT

DATA

TURNAROUND

Days / 45
Days

METHOD OF SHIPMENT

GOVERNMENT VEHICLE

BILL OF LADING/AIR BILL NO.

$\mathrm{N} / \mathrm{A}$

PRESERVATION

TYPE OF CONTAINER

NO. OF CONTAINER(S)

volume

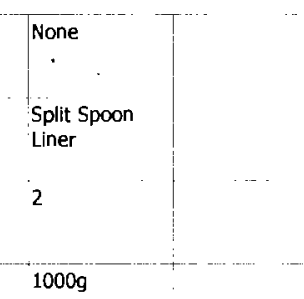

SAMPLE ANALYSIS

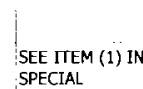

SAMPLE DATE SAMPLE TIME

$2-4-10$

1245

RECEIVED BY/STORED IN

MO- 413 STS

** The 200 Area S\&GRP Characterization and Monitoring Sampling and Analysis Eikaus/ klunele RELINQUISHED BY/REMOVED FROM MNO- 413 SSU-RZ

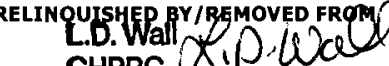
RELINQHISPAB B B REMOVED FROM

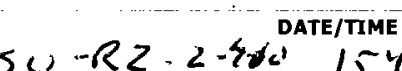
** ESL shall submit all

DATE/TIME 73 RECEIYEPBYSTPRED HW

FEB 0520100730

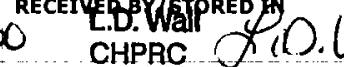

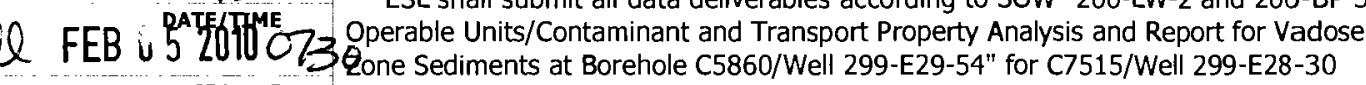

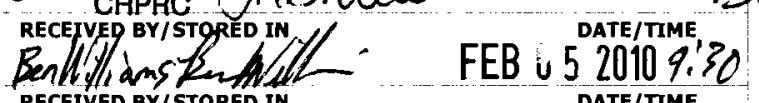

RELINOUISHED BY/REMOVED FROM DATE/TIME

DATE/TIME

DATE/TIME (M" Well) and shall adhere to all S\&GRP standard protocol.

RELINOUISHED BY/REMOYED FROM

DATE/TIME

RECEIVED BY/STORED IN

DATE/TIME

** The ESL laboratory shall meet prior requested and confirmed turnaround times

DATE/TIME

RECEIVED BY/STORED IN

RELINQUISHED BY/REMOVED FROM

DATE/TIME

RECEIVED BY/STORED IN

DATE/TIME

and RDLS specified in the SOW and this SAF. The laboratories must notify the SM

Project Coordinator in writing if/when this requirement can not be met.
(1)Density; CATIONEXCH TR; Particle Size (Dry Sieve) - D422; Particle Size

DATE/TIME (Hydrometer) - D422; KD - Batch;

$$
\vdots 3 \text { anili }
$$


C7515 (299-E28-30); I-126

ICE CHEST NO.

$$
6 w 2-166
$$

SHIPPED TO

Environmental Sciences Laboratory

MATRIX*

POSSIBLE SAMPLE HAZARDS/ REMARKS

Contalins Radioactive Material at concentrations
that may or may not be regulated for

transportation per 49 CFR / IATA Dangerous

Goods Regulations but are not releasable per

SPECIAL HANDLING AND/OR STORAGE RADIOACTIVE TIE TO: B23653

\section{SAMPLE NO}

B235T4
MATRIX*

\section{COMPANY CONTACT}

YEKMAN, DL

PROJECT DESIGNATION

200-PW-2 OU Characterization Vadose Zone - Geochemical Modeling Param

FIELD LOGBOOK NO. is 57 ACTUAL SAMPLE DEPTH

HNF-N- $49,-5$

FFSTTE PROPERTY NO.

PRESERVATION

TYPE OF CONTAINER

NO. OF CONTAINER(S)

VOLUME

SAMPLE ANALYSIS

SAMPLE DATE SAMPLE TIME

$2-4 \cdot 10$

1245

SIGN/ PRINT NAMES

CHAIN OF POSSESSION

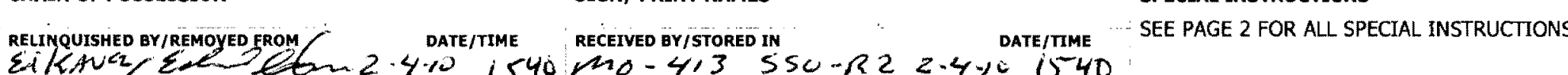
289,5

PROJECT COORDINATOR

DYEKMAN, D

SAF NO.

COA

302117 ES30

ILL OF LADING/AIR BILL NO.
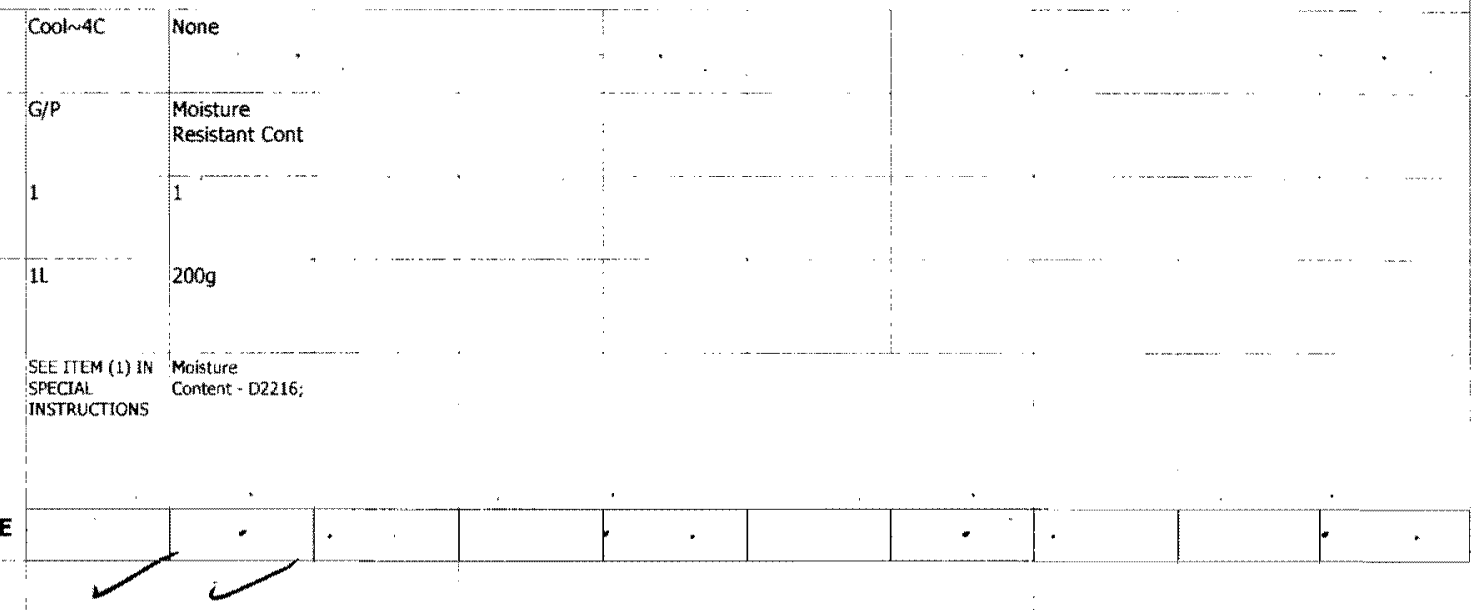
DATA
TURNAROUND 45 Days / 45
Days

AIR QUALTTY

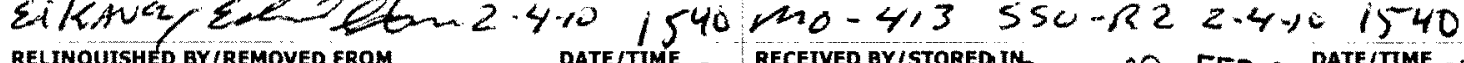

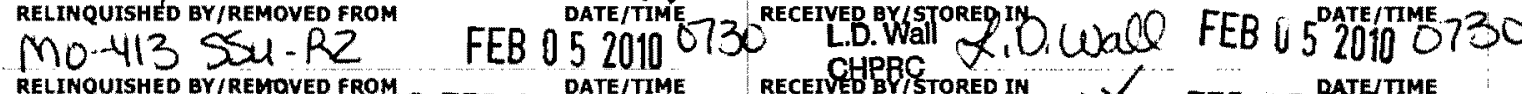

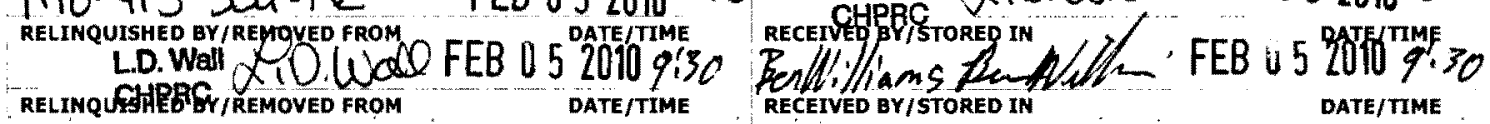

RELINQUISHED BY/REMOVED FROM

DATE/TIME

DATE/TIME

RECEIVED BY/STORED IN

DATE/TIME

RELINQUISHED BY/REMOVED FROM

DATE/TIME

RECEIVED QY/STORED IN

DATE/TMME

RELINQUISHED BY/REMOVED FROM

DATE/TIME

RECEIVED BY/STORED IN

DATE/TIME 


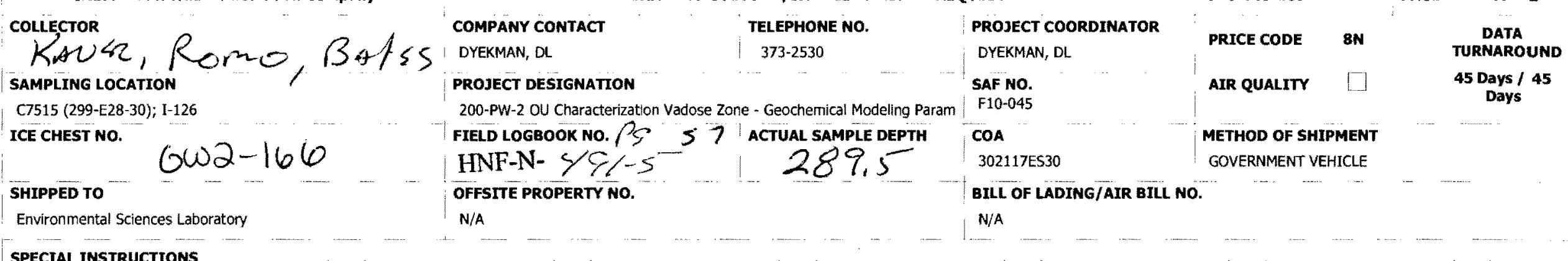

\section{SPECIAL INSTRUCTIONS}

** The 200 Area S\&GRP Characterization and Monitoring Sampling and Analysis GKI applies to this SAF. ** ESL shall submit all data deliverables according to SOW "200-LW-2 and 200-BP-5
C7515/Well 299-E28-30 (" $M$ " Well) and shall adhere to all S\&GRP standard protocol.

** The ESL laboratory shall meet prior requested and confirmed turnaround times and RDLs specified in the SOW and this SAF. The laboratories must notify the SM Project Coordinator in writing iff/when this requirement can not be met.

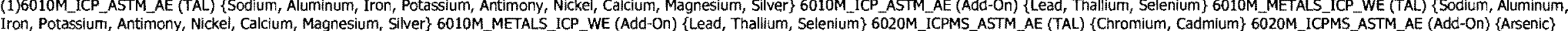
6020M_METALS_ICPMS WW (TAL) \{Chromium, Cadmium\} 6020M_METALS_ICPMS WEE (Add-On) \{Arsenic\} 6020M_HG_ICPMS AE; 6020M_HG_ICPMS_WE; Chromium HeX - 7196; RADISO_ICPMS_ASTM_AE \{Uranium-238, Technetium99\} RADISO_LCPMS_WE \{lodine-129, Uranium-238, Technetium-99\} 1-129 by ICPMS \{lodine-129\} GAMMA_GS; URANIUM ISOTOPIC RATTOS; ALPHA_BETA_AE \{Gross beta, Gross alpha\} ALPHA_BETA_WE \{Gross beta, Gross alpha\} 1 IC Anions - 9056-WE \{Nitrate, Chloride, Phosphate, Fluoride, Nitrite, Sulfate\} Cyanide (Total) - 335.2 \{Cyanide\} pH (Water) - 9045_WE; Conductivity - 9050_WE; TOC - ASTME1915A \{Total organic carbon, Total Inorganic Carbon, Total
carbon\} 2320_ALALINTYY \{Carbonate ion, Bicarbonate, Alkalinity, Calcium Carbonate\}

$\{3$ illitio 
Kauer Romo, Bates

SAMPLING LOCAfION

C7515 (299-E28-30); I-127

ICE CHEST NO.

$$
6002-166
$$

SHPPED TO

Environmental Sciences Laboratory

A=Air
DL=Drum

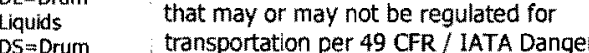

transportation per 49 CFR / IATA Dangerous

DOE Order $5400.5(1990 / 1993)$

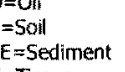

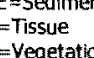

$W=$ Water
WI $=$ Wipe
$X=$ int

SPECIAL HANDLING AND/OR STORAGE

PROJECT DESIGNATION

AIR QUALITY

SAF NO
P10-045

FIELD LOGBOOK NO. 3557 ACTUALSAMPLEDEPTH

\section{METHOD OF SHIPMENT}

\begin{tabular}{l|l|l} 
HNF-N $-491-5$ & 289.8 to 292,3302117 ES30 & GOVERNMENT VEHICLE
\end{tabular} OFFSTTE PROPERTY NO.

BILL OF LADING/AIR BILL NO.

\section{PRESERVATION}

TYPE OF CONTAINER

NO. OF CONTAINER(S)

vOLUME

SAMPLE AMALYSIS

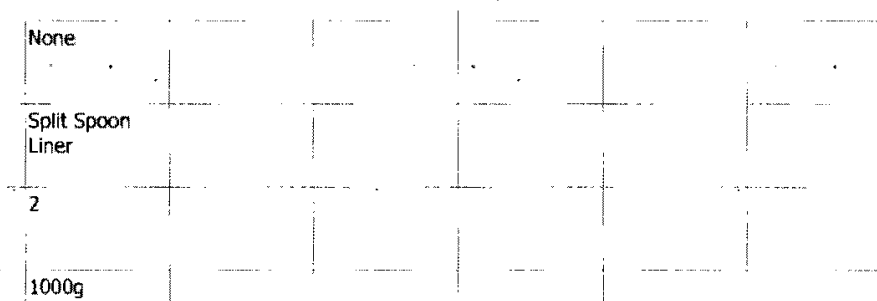
PADIOACTIVE TIE TO: B23653
B235C3
- SAMPLE nO.

MATRIX*

SAMPLE DATE SAMPLE TIME

$2-4.10 / 400$

\section{SIGN/ PRINT NAMES} $4 \% 1540$ mo.413 MO 413 SSU-R2

RECEIVED BYTSTOBRD EY.t.Wand ry

MTRUCTONS
TQUISHED BY/REMQVED FROM

L.D. Wall \& W. COa

RELINQUISHED BY/REMOVED FROM

RELINQUISHED BY/REMOVED FROM

RELINQUISHED BY/REMOVED FROM

\section{TECEIVED BY}

SECTION

INAL SAMPLE DISPOSAL METHOD

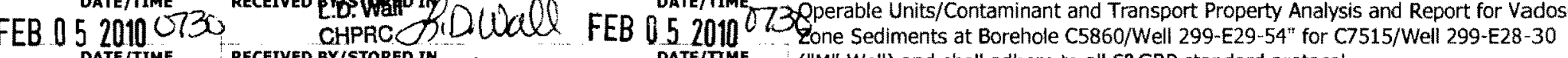

\section{FEB $0520109: 30$ Benlifl(lians Zen till \\ FEB $052010 \%$ 'ं́d}

DATE/TIME RECEIVED BY/STORED IN

DATE/TIME RECEIVED BY/STORED IN

DATE/TIME RECEIVED BY/STORED IN

DATE/TME

DATE/TIME Well) and shall adhere to all S\&GRP standard protocol.

** The ESL laboratory shall meet prior requested and confirmed turnaround times and RDLS specified in the SOW and this SAF. The laboratories must notify the SM Project Coordinator in writing if/when this requirement can not be met. DATE/TIME (Hydrometer) - D422; KD-Batch;

$$
\text { Unibi: }
$$

DATE/TIME

\section{DATE/TIME}


PROJECT DESIGNATION

SAF NO.
F10-045

ICE CHEST NO.

200-PW-2 OU Characteriza

AIR QUALITY

\section{SHIPPED TO}

$$
602-166
$$

Fif

\begin{tabular}{l|c|l|l} 
HNF-N- $49 /-5$ & 292,3 & MA & METHOD OF SHIPMENT \\
GOVERNMNT VEHICLE
\end{tabular}

OFFSTTE PROPERTY NO.

BILL OF LADING/AIR BILL NO.

$$
\text { N/A }
$$

Contains Radioàctive Material at concentrations that may or may not be regulated for transportation per 49 CFR / IATA Dangerous GOof Order 5400.5 (1990/1993)

PRESERVATION

TYPE OF CONTAINER

NO. OF CONTAINER(S)

volume

SAMPLE ANALYSIS

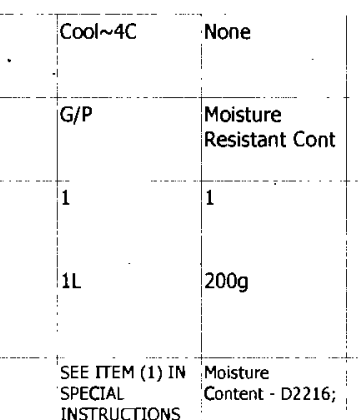
RADIOACTVE TIE TO: 823653

SAMPLE NO.

MATRIX*

B235T5

SOIL

SAMPLE DATE SAMPLE TIME

$2-4 \cdot 10 / 400$

\section{SIGN/ PRINT NAMES}

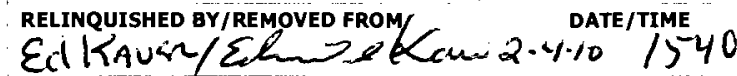

RECEIVED BY/STORED IN STRUCTIONS

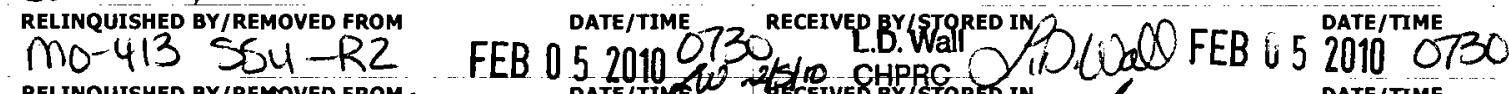

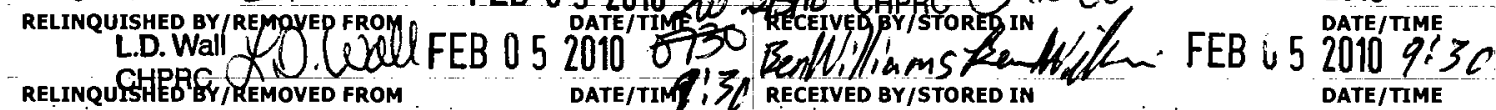

RELINQUYSHARES GY TREMOYED FROM

RELINQUISHED BY/REMOVED FROM

DATE/TIME

RECEIVED BY/STORED IN

DATE/TIME

RELINQUISHED BY/REMOVED FROM

DATE/TIME

RECEIVED BY/STORED IN

DATE/TIME

RELINQUISHED QY/REMOVED FROM

DATE/TIME

RECEIVED BY/STORED IN

DATE/TIME

\section{SPECIAL INSTRUCTIONS}

\section{SEE PAGE 2 FOR ALL SPECIAL INSTRUCTIONS}

$$
\{3.11
$$


KausR Remo, Bates SAMPLING LOCATION

C7515 (299-E28-30): I-128

ICE CHEST NO.

SHIPPED TO

$$
\text { Ge2-166 }
$$

Environmental Sciences Laboratory

\section{SPECIAL INSTRUCTIONS}

** The 200 Area S\&GRP Characterization and Monitoring Sampling and Analysis GKI applies to this SAF.
** ESL shall submit all data deliverables accordlng to SOW "200-LW-2 and 200-BP-5 Operable Units/Contaminant and Transport Property Analysis and Report for Vadose Zone Sediments at Borehole C5860/Well 299-E29-54" for C75L shall submit all data deliverables according to SOW "200-LW-2 and 200-BP-5
C7515/Well 299-E28-30 (" $\mathrm{M}$ " Well) and shall adhere to all S\&GRP standard protocol.

** The ESL laboratory shall meet prior requested and confirmed turnaround times and RDLs specified in the SOW and this SAF. The laboratories must notify the SM Project Coordinator in writing if/when this requirement can not be met. (1)6010M_ICP_ASTM_AE (TAL) \{Sodium, Aluminum, Iron, Potassium, Antimony, Nickel, Calcium, Magnesium, Silver\} 6010M_ICP_ASTM_AE (Add-On) \{Lead, Thallium, Selenium\} 6010M_METALS_ICP_WE (TAL) \{Sodium, Aluminum,

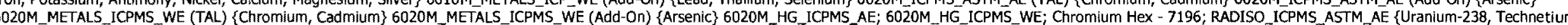

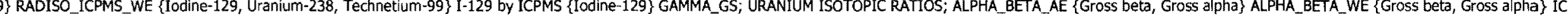
Anions - 9056_WE \{Nitrate, Chloride, Phosphate, Fluoride, Nitrite, Sulfate\} Cyanide (Total) - 335.2 \{Cyanide\} pH (Water) - 9045_WE; Conductivity - 9050_WE; TOC - ASTME1915A (Total organic Carbon, Total Inorganic Carbon, Total carbon\} 2320_ALKALINITY \{Carbonate ion, Bicarbonate, Alkalinity, Calcium Carbonate\}

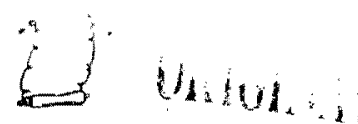


PROJECT COORDINATOR

C7515 (299-E28-30); I-129

PROJECT DESIGNATION

$\begin{array}{ll}\text { 200-PW-2 OU Characterization Vadose Zone - Geochemical Modeling Param } & \text { SAF No. } \\ \text { F10-045 }\end{array}$

ICE CHEST NŌ.

FIELD LOGBOOK NO. $P S S$ ACTUAL SAMPLE DEPTH COA

HNF-N-49/-S 292.3 to $\left.294.8\right|^{302117 E S 30}$

PRICE CODE

SHIPPED TO

$$
602-166
$$

OFFSTTE PROPERTY NO.

Environmental Sciences Laboratory

BILL OF LADING/AIR BILL NO.

A=Air POSSIBLE SAMPLE HAZARDS/ REMARKS

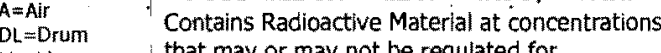
that may or may not be regulated for

Goods Regulations but are not releasabte per DOE Order $5400.5(1990 / 1993)$

SPECIAL HANDLING AND/OR STORAGE SPECIAL HANDLING AND
RADIOACTIVE TIE TO: B23653

\begin{tabular}{|c|c|}
\hline PRESERVATION & None \\
\hline TYPE OF CONTAINER & $\begin{array}{l}\text { Solt Spoon } \\
\text { Liner }\end{array}$ \\
\hline 0. OF CONTAINER(S) & \\
\hline
\end{tabular}

\section{SAMPLE ANALYSIS SEE SFEM (1)}

AIR QUALITY

\section{METHOD OF SHIPMENT}

SAMPLE NO.
B235C4

SAMPLEDATE , SAMPLE TIME

$2-4.10: 1500$

CHAIN OF POSSESSION

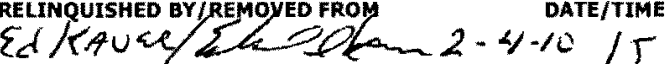
RELINQUISHED BY/REMOVED FROM $\operatorname{Mo}-413$ SSH-R2 L.D. Wall $\%$ BY EEOHORAED BY/REMOVED FROM

RELINQUISHED BY/REMOVED FROM RELINQUISHED BY/REMOVED FROM RELINQUISHED BY /REMOVED FROM

\section{LABORATORY RECEIVED BY}

SECTION

FINAL SAMPLE
DISPOSITION

DATE/TIME

\section{SIGN/ PRINT NAMES}

RECEIVED BY/STORED

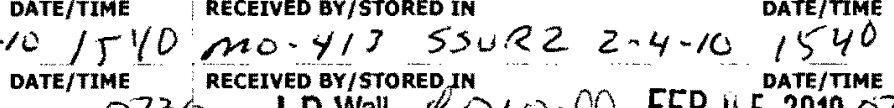

FEB 05 2010 073 L.D. Wall Q ${ }^{2}$ Q W W

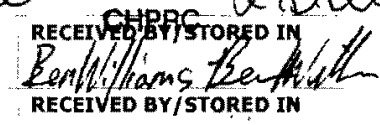

DATE/TIME

DATE/TIME

RECEIVED BY/STORED IN

RECEIVED BY/STORED IN

DATE/TIME

RECEIVED BY/STORED IN

\section{SPECIAL INSTRUCTIONS}

** The 200 Area S\&GRP Characterization and Monitoring Sampling and Analysis

** ESL shall submit all data deliverables according to SOW "200-LW-2 and 200-BP-5 (" $M$ " Well) and shall adhere to all S\&GRP standard protocol.

FEB \& 520109,30

** The ESL laboratory shall meet prior requested and confirmed turnaround times and RDLS specified in the SOW and this SAF. The laboratories must notify the SM Project Coordinator in writing if/ when this requirement can not be met. (1)Density; CATIONEXCH_TR; Particle Size (Dry Sieve) - D422; Particle Size (Hydrometer)-D422; KD-Batch; * NO Recovery $25 \%$

$\Leftrightarrow 3 b_{1+i}$ 


$$
\text { ICE CHEST NO. }
$$

$$
\text { Gurs - } 036
$$

COMPANY CONTACT

DYEKMAN, DL

TELEPHONE NO.

PROJECT COORDINATOR

PROJECT DESIGNATION

\begin{tabular}{l|l}
$373-2530$ & DYEKMAN, DL \\
\hline
\end{tabular}

SAF NO.

$\begin{array}{lll}\text { PRICE CODE } & \text { 8N } & \text { DATA } \\ \text { TURNAROUND }\end{array}$

200-PW-2 OU Characterization Vadose Zone - Geochemical Modeling Param F10-045

FIELD LOGBOOK NO. PS S \& ACTUAL SAMPLE DEPTH COA

\begin{tabular}{l|ll} 
ItNF-N-491-5 & 294.0 & 302117 ES30
\end{tabular}

AIR QUALTTY $\square \quad \underset{\text { Days }}{45 \text { Days }} \mathbf{4 5}$

OFFSITE PROPERTY NO.

N/A

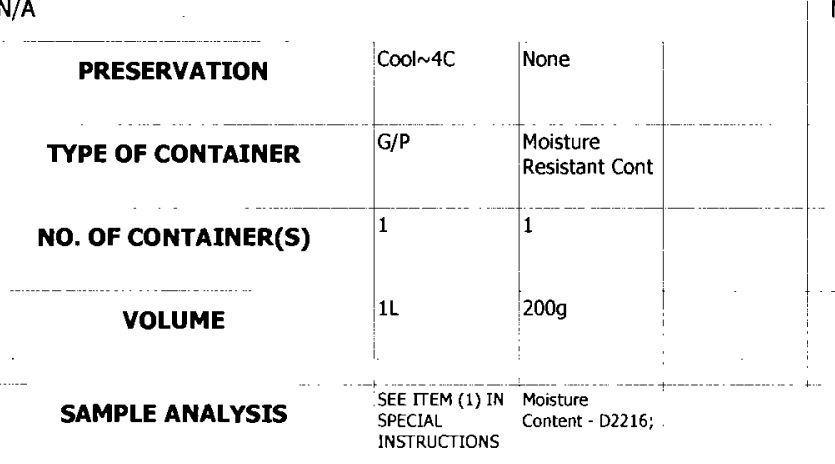

GOVERNMENT VEHICLE

SAMPLE DATE SAMPLE TIME

$2.8 \% 0750$

BILL OF LADING / AIR BILL NO.

BILL

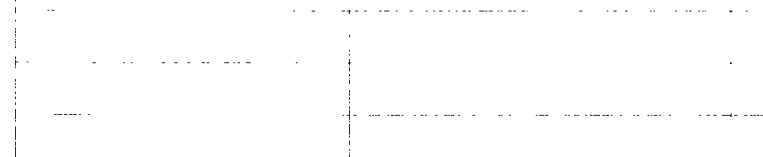

CHAIN OF POSSESSION

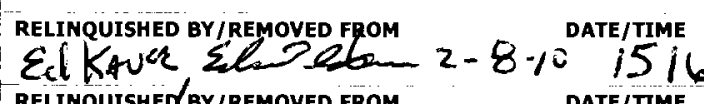
SSU-R2 FEB O 92010 DATE/TME

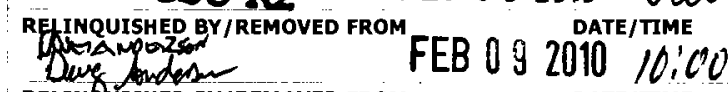
RELINGUISHED BY/REMOVED FROM

RELINQUUSHED BY/REMOVED FROM

RELINQUISHED QY/REMOVED FROM

RELINQUISHED QY/REMOVED FROM

DATE/TIME

\section{SIGN/ PRINT NAMES}

RECEIVED BY/STORED IN
$m 0-413$ SSU - R2 $2-8-0 \quad 15 / 6$

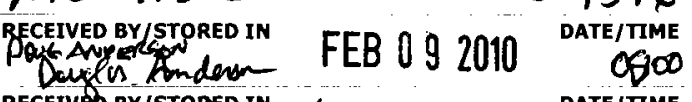
RECEIVEg BY/STOQED IN/f/FEB 092010 DATE/TMME Benllilians teutllthe FEB $92010 \quad 10^{\circ} 00$ RECEIVED BY/STORED IN RECEIVED BY/STORED IN

DATE/TIME

DATE/TIME

RECEIVED BY/STORED IN

DATE/TIME

DATE/TMME
SPECIAL INSTRUCTIONS

SEE PAGE 2 FOR ALL SPECIAL INSTRUCTIONS

\section{ORIGINAL}


PROJECT COORDINATOR

$$
G \omega S-036
$$

COA

METHOD OF SHIPMENT

SPECIAL INSTRUCTIONS

N/A

** The 200 Area S\&GRP Characterization and Monitoring Sampling and Analysis GKI applies to this SAF.

** ESL shall submit all data deliverables according to SOW "200-LW-2 and 200-BP-5 Operable Units/Contaminant and Transport Property Analysis and Report for Vadose Zone Sediments at Borehole C5860/Well 299-E29-54" for C7515/Welt 209-E28-30 " $M$ " Well) and shall adhere to all S\&GRP standard protocol.

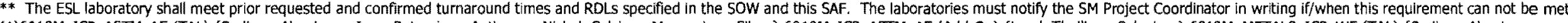

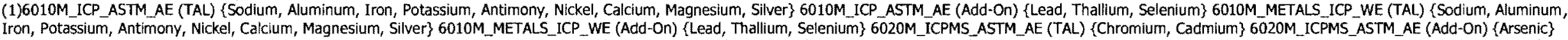

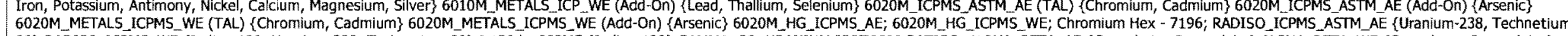
99\} RADISO ICPMS WE \{Iodine-129, Uranium-238, Technetium-99\} I-129 by ICPMS \{Iodine-129\} GAMMA GS; URANIUM ISOTOPIC RATIOS: ALPHA BETA AE \{Gross beta, Gross alpha\} ALPHA BETA WE \{GGoss beta, Gross alpha\} IC Anions - 9056_WE (Nitrate, Chloride, Phosphate, Fluoride, Nitrite, Sulfate\} Cyanide (Total) - 335.2 \{Cyanide\} pH (Water) - 9045_WE; Conductivity - 9050_WE; TOC - ASTME1915A \{Total organic Carbon, Total Inorganic Carbon, Total carbon\} 2320_ALKALNITY \{Carbonate ion, Bicarbonate, Alkalinity, Calcium Carbonate\}

\section{ORIGINAL}


Kampling Location' Gatic: a, Afjuilor

C7515 (299-E28-30): I-131

TELEPHONE NO.

PRICE CODE

DATA

OYEKMAN DL

373-2530

PROJECT COORDINATOR

DYEKMAN, DL

TURNAROUND

PROJECT DESIGNATION

SAF NO
F10-045
COA

AIR QUALITY

Days

ICE CHEST NO.

\section{SHIPPED TO}

Environmental Sciences Laboratory

MATRIX* POSSIBLE SAMPLE HAZARDS/ REMARKS

\begin{tabular}{l|l}
$A=A i r$ \\
$D L=D r u m$
\end{tabular},$\quad$ Contains Radioactive Material at concentrations that may or may not be regulated for Goods Regulations but are not releasable per DOE Order 5400.5 (1990/1993)

200-PW-2 OU Characterization Vadose Zone - Geochemical Modeling Paran

FIELD LOGBOOK NO. $\rho_{S}$ S है ACTUAL SAMPLE DEPTH

COA - METHOD OF SHIPMENT

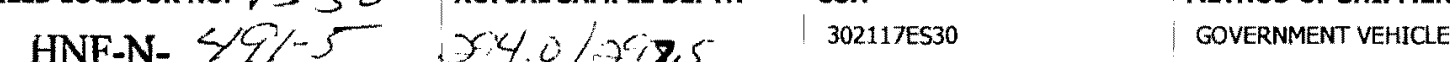
OFFSITE PROPERTY NO.

N/A

TYPE OF CONTAINER $\begin{gathered}\text { Split Spoon } \\ \text { Liner }\end{gathered}$

NO. OF CONTAINER(S)

volume

$2^{-}$

10009

SAMPIE ANALYSIS

SGEF IEM (1) IN
SPECIIA
INSTUUCIONS

SPECIAL HANDLING ANDIOR STORAGE

SPECIAL MANDLING AND
RADIOACTIVE TIE TO: B23654

SAMPLE NO

B235C5

MATRIX*

\section{SAMPLE.DATE SAMPLE TIME}

$2 \cdot \varepsilon \cdot N$ OQL

\section{SIGN/ PRINT NAMES}

CHAIN OF POSSESSION

REUNGUISHED BY/REMOVEDFROM

RELINQUISHED W

SSU-R2

J) Je/ Date/TIME 15

RECEIVED BY/STORED IN FEB 092010

EEP BYISTORERIN

\section{SPECIAL INSTRUCTIONS}

** The 200 Area S\&GRP Characterization and Monitoring Sampling and Analysis

E ofs, ** ESL shall submit all data deliverables according to SOW "200-LW-2 and 200-BP-5 DW Brotherion RELINQUISHED BY/REM OVVEDFROM FEB 09201

\section{EECEIVD BY/STOPED i}

E FEB 092010

DATE/TIME

RECEIVED BY/STORED IN

10.100

EBBAt:42010

Zone Sediments at Borehole C5860/Well 299-E29-54" for C7515
("M" Well) and shall adhere to all S\&GRP standard protocol.

RELINQUISHED BY/REMOVED FROM

DATE/TIME

RECEIVED BY/STORED IN

DATE/TIME and RDLs specified in the SOW and this SAF. The laboratories must notify the SM Project Coordinator in writing if/when this requirement can not be met.

RELINQUISHED GY/REMOVED FROM

DATE/TIME

RECEIVED BY/STORED IN

DATE/TIME

(Hydrometer) - D422; KD - Batch;
(Henty; CATONEXCH_TR; Patten

RELINQUISHED BY/REMOVED FROM

DATE/TIME

RECETVED BY/STORED IN

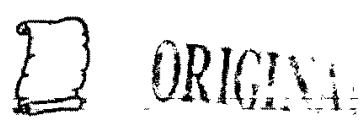

DISPOSED BY
DATE/TIME

\section{(1)}




$$
\text { GWS - } 036
$$

SHIPPED TO

Environmental Sciences Laboratoy

MATRIX* T POSSIBLE SAMPLE HAZARDS/ REMARKS

A=Air
$\mathrm{DL}=$ Drum

\begin{tabular}{l|l} 
Liquids & that may or may not be regulated for \\
\hline
\end{tabular} Goods Regulations but are nat relengetre Goods Regulations but are not releasable per
DOE Order $5400.5(1990 / 1993)$

SPECIAL HANDLING AND/OR STORAGE RADIOACTVE TE TO: 323654

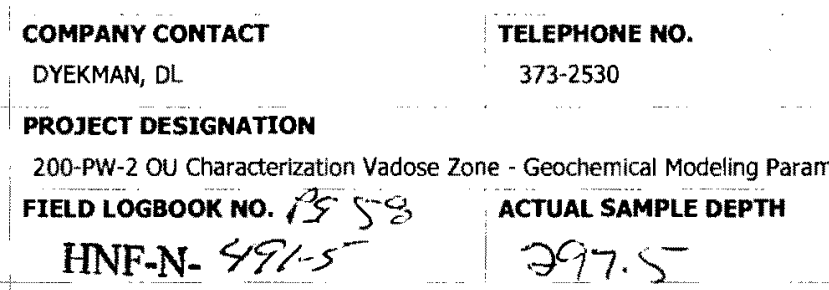

PROJECT COORDINATOR

DYEKMAN, DL

SAF NO.
F10-045

PRICE CODE $\quad 8 \mathrm{~N}$

DATA

F10-045

AIR QUALITY

OFFSITE PROPERTY NO.

BILL OF LADING/AIR BILL NO

PRESERVATIOO

TYPE OF CONTAINER

NO. OF CONTAINER(S)

volume

N/A GOVERNMENT VEHICLE

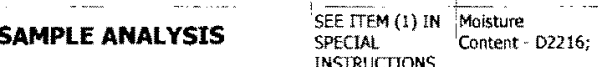

\section{SAMPLE NO.}

B235T7
MATRIX* . SAMPLE dATE SAMPLE TIME

$2 \cdot 8 \cdot 10: 0810$

SIGN/ PRINT NAMES

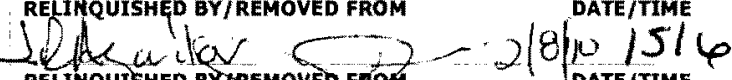

RECEIVED BY/STORED IN

RELINQUISHED BY/REMOVED FROM

\section{SSU-R2}

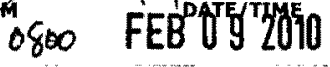

RE HNQUISHED BY/REMOVED FROM

DDuxl An dens 10.00

RELINQUISHED BY/REMOVED FROM

RELINQUISHED BY/REMOYED FROM

RELINQUISHED BY/REMOVED FROM

FINAL SAMPLE
DISPOSITION

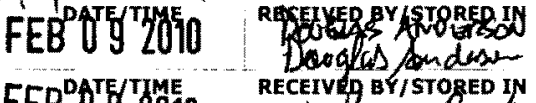

\section{DATE/TIME}

DATE/TIME

DATE/TIME

DATE/TIME

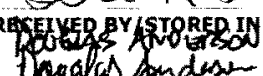

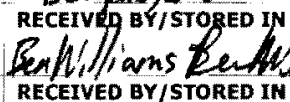

RECEIVED BY / STORED IN

RECEIVED BY/STORED IM
SPECIAL INSTRUCTIONS

DATE/TIME SEE PAGE 2 FOR ALL SPECIAL INSTRUCTIONS

D|elio $15 / 6$

6880 FEB 092010

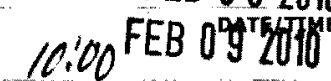

Date/TME

DATE/TIME

ITilit: 
BILL OF LADING/AIR BILL NO.

Environmental Sciences Laboratory

$$
\mathrm{N} / \mathrm{A}
$$

N/A

SPECIAL INSTRUCTIONS

** The 200 Area S\&GRP Characterization and Monitoring Sampling and Analysis GKI applies to this SAF.

** ESL shall submit all data deliverables according to SOW "200-LW-2 and 200-BP-5 Operable Units/Contaminant and Transport Property Analysis and Report for Vadose Zone Sediments at Borehole C5860/Well 299-E29-54" for

** The ESL Laboratory shall meet prior requested and confirmed turnaround times and RDLS Specifed in the SOW and this SAF. The laboratories must notify the SM Project Coordinator in writing iff/when this requirement can not be met.

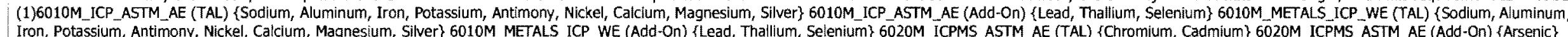

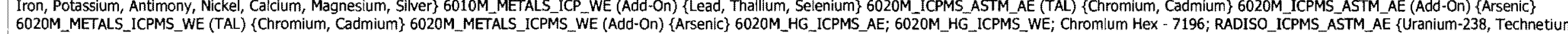
99\} RADISO_ICPMS WE \{Iodine-129, Uranium-238, Technetium-99\} I-129 by ICPMS \{Iodine-129\} GAMMA_GS; URANIUM ISOTOPIC RATIOS; ALPHA_BETA_AE \{Gross beta, Gross alpha\} ALPHA_BETA_WE \{Gross beta, Gross alpha\} IC Anions - 9056_WE (Nitrate, Chloride, Phosphate, Fluoride, Nitrite, Sulfate\} Cyanide (Total) - 335.2 \{Cyanlde\} PH (Water) - 9045_WE; Conductivity - 9050_WE; TOC - ASTME1915A (Total organic Carbon, Total Inorganic Carbon, Total carbon\} 2320_ALKALNITY \{Carbonate ion, Bicarbonate, Alkalinity, Cakcium Carbonate\} 
PROJECT DESIGNATION

C7515 (299-E28-30); I-133

ICE CHEST NO.

$$
\text { Gws - } 036
$$

SHIPPED TO

Environmental Sciences Laboratory

MATRIX* ${ }^{*}$ POSSIBLE SAMPLE HAZARDS/ REMARKS

$A=A$ ir
$D=$ Drum

liquids that may or may not be regulated for

Goods Re

Goods Regulations but are not releasable per
DOE Order $5400.5(1990 / 1993)$

200-PW-2 OU Characterization Vadose Zone - Geochemical Modeling Param

HNF-N $19 /-5$ actUAL SAMPLE DEPTH COA

METHOD OF SHIPMENT TITE PROPERTY NO. 302117ES30 GOVERNMENT VEHICLE

BILL OF LADING/AIR BILL NO.

PRESERVATION

TYPE OF CONTAINER

NO. OF CONTAINER(S)

VOLUME

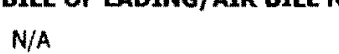

SPECIAL MANDLING AND/OR STORAGE RADIOACTIVE TIE TO: B23654

\section{SAMPLE ANALYSIS $\quad$ SEETTEM (1) IN}

SAMPLE DATE. SAMPLE TIME
SAMPLE NO.
$\supset / \varepsilon / \infty$
1015
SOI
$+\cdots$

- MATRIX*

B235C6

SIGN/ PRINT NAMES

CHAIN OF POSSESSION

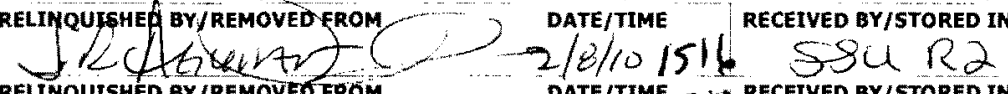

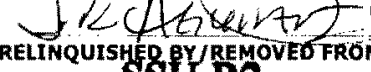

\section{SSU $-\mathrm{R}^{2}$}

$2 / 0 / 10$ DATE/TIME

SPECIAL INSTRUCTIONS

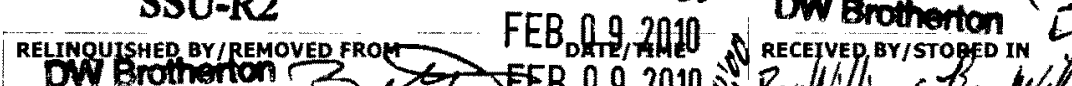

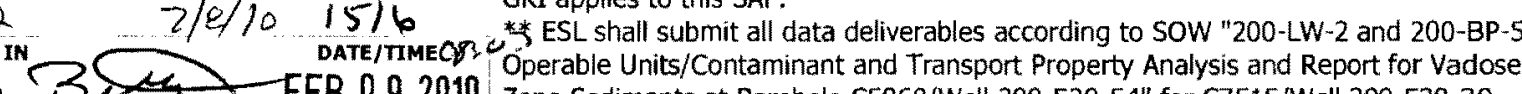

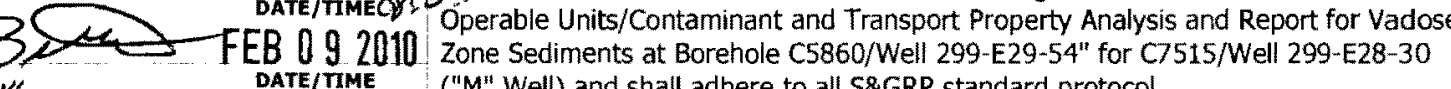

RELINQUISHED BY/REMOVEOFROM

RELINQUISHED BY/REMOVED FROM

RELINQUISHED BY/REMOVED FROM DATE/TIME

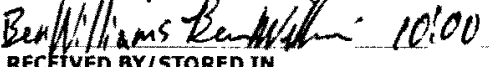

FEB 092010

DATE/TIME

RECEIVED BY/STORED IN

DATE/TTME

RECEIVED BY/STORED IN

RELINQUISHED BY/REMOVED FROM

DAte/TIME

RECEIVED BY/STORED IN

\section{DATE/TIME}

DATE/TIME

** The ESL laboratory shall meet prior, requested and confirmed turnaround times

and RDLS Specified in the SOW and this SAF. The laboratores

Project Coordinator in writing if/when this requirement can not be met.

(1)Density: CATIONEXCH TR; Particle Size (Dry Sieve) - D422; Particle Size

DATE/TME (Hydrometer) - D422; KD - Batch;

DATE/TIME

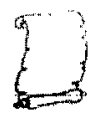

DATE/TIME 
CH2MHill Plateau Remediation Company

COLLECTOR

C7515 (299-E28-30); $1-434$

ICE CHEST NO.

$$
\text { Gws- } 036
$$

SHIPPED TO

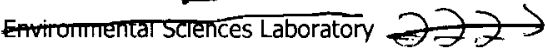

MATRIX* POSSIBLE SAMPLE HAZARDS/ REMARKS

Contains Radioactive Material at concentrations that may or may not be regulated for

transportarion per 49 CFR / IATA Dangerous

Goods Regulations but are not releasable per
DOE Order $5400.5(1990 / 1993)$

SPECIAL HANDLING AND/OR STORAGE RADIOACTIVE TIE TO: B23655

SAMPLE NO

MATRIX*
LAMPLING Location thuinar

CHAIN OF CUSTODY/SAMPLE ANALYSIS REQUEST

F10-045-201

PRICE CODE $\quad$ SN

AIR QUALITY $\square$

\begin{tabular}{l|l|l|l} 
200-PW-2 OU Characterization Vadose Zone - Geochemical Modeling Param & F10-045 \\
FIELD LOGBOOK N0. 9 S 53 & ACTUAL SAMPLE DEPTH & COA & METHOD OF SHIPMENT
\end{tabular}

PROJECT DESIGNATION

TELEPHONE NO.

PROJECT COORDINATOR

DYEKMAN, DL

GE 1 OF 2

TURNAROUND

Days

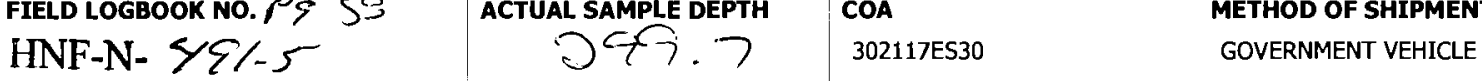

OFFSITE PROPERTY NO. BILL OF LADING/AIR BILL NO.

N/A

N/A

\begin{tabular}{|c|c|c|}
\hline PRESERVATION & Cool $4 \mathrm{C}$ & None \\
\hline TYPE OF CONTAINER & $G / P$ & $\begin{array}{l}\text { Moisture } \\
\text { Resistant Cont }\end{array}$ \\
\hline No. OF CONTAINER(S) & 1 & 1 \\
\hline VOLUME & it & $200 \mathrm{~g}$ \\
\hline 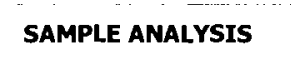 & $\begin{array}{l}\text { SEE MEMC } \\
\text { SPECIAL }\end{array}$ & $\begin{array}{l}\text { Moisture } \\
\text { Content-D2216; }\end{array}$ \\
\hline
\end{tabular}

SAMPLE DATE SAMPLE TIME

$3 / 8 / 10 \quad 1015$

SIGN/ PRINT NAMES

SPECIAL INSTRUCTIONS

CHAIN OF POSSESSION

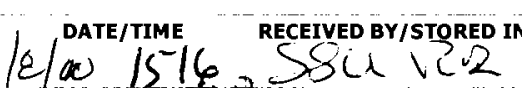

RqLIMQUISSHED BYTREMOVEQEROM

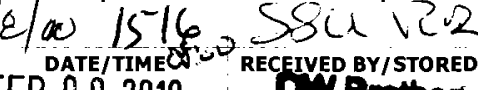

SEE PAGE 2 FOR ALL SPECIAL INSTRUCIIONS

12utevirar

SSU-R2

FEB 092010

DWEP BY/STORED IN

$2 / 8 / 10$ i 1516 DATE/TMEOS:O

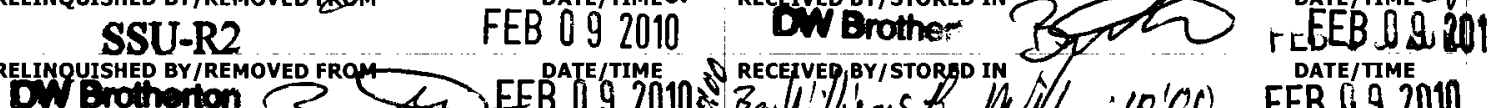

RELINQUISHED BY/REMODEDFROM

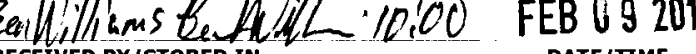

DATE/TIME

DATE/TIME - RECEIVED BY/STORED IN $\cdots \cdots \cdots$ DATE/TME

B ORIGIN!

RELINQUISHED BY/REMOVED FROM

DATE/TIME

RECEIVED BY/STORED IN

DATE/TIME
DATE/TIME

RECEIVED BY/STORED IN

DATE/TIME

RELINQUISHED BY/REMOVED FROM

LABORATORY
SECTION

FINAL SAMPLE
DISPOSITION

DISPOSAL METHOD 


\section{CH2MHill Plateau Remediation Company}

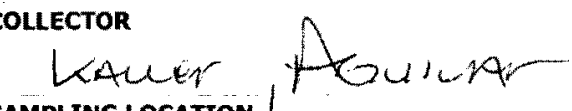

SAMPLINC

C7515 (299-E28-30); I-134

ICE CHEST NO.

$\operatorname{sic} 3-3$

SHIPPED TO

Environmental Sciences Laboratory

SPECIAL INSTRUCTIONS

** The 200 Area S\&GRP Characterization and Monitoring Sampling and Analysis GKI applies to this SAF.

** ESL shall submit all data deliverables according to SOW "200-LW-2 and 200-BP-5 Operable Units/Contaminant and Transport Property Analysis and Report for Vadose Zone Sediments at Borehole C5860/Well 299-E29-54" for

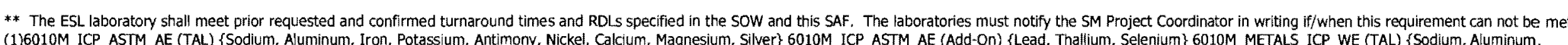
(1)6010M_ICP_ASTM_AE (TAL) \{Sodium, Aluminum, Iron, Potassium, Antimony, Nickel, Calcium, Magnesium, Silver\} 6010M_ICP_ASTM_AE (Add-On) \{Lead, Thallium, Selenium) 6010M_METALSIICP_WE (TAL) \{Sodium, Aluminum,

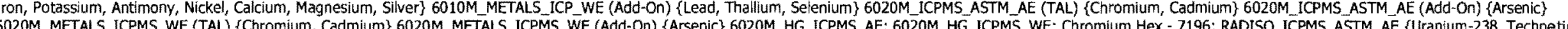
99\} RADISO 1CPMS WE \{lodine-129, Uranium-238, Technetium-99\} I-129 by ICPMS \{Iodine-129\} GAMMA GS; URANIUM ISOTOPIC RATIOS; ALPHA BETA_AE \{Gross beta, Gross alpha\} ALPHA BETA WE \{Gross beta, Gross alpha\} IC

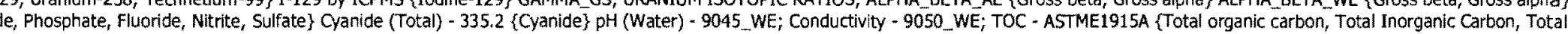
carbon\} 2320_ALKALINITY \{Carbonate ion, Bicarbonate, Alkalinity, Calcium Carbonate\}
F10-045-201

\section{PAGE 2 OF 2 \\ DATA \\ Days}

\section{METHOD OF SHIPMENT}

302117 ES3O GOVERNMENT VEHIOL

BILL OF LADING/AIR BILL NO.
N/A

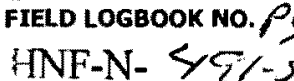

OFFSITE PROPERTY NO.

N/A

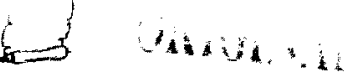


CH2MHill Plateau Remediation Company

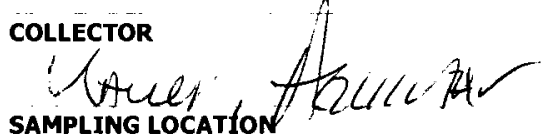

C7515 (299-E28-30); I-135

ICE CHEST NO.

Gus - 036

SHIPPED TO

Environmental Sciences Laboratory

MATRIX* POSSIBLE SAMPLE HAZARDS/ REMARKS

Contains Radioactive Material at concentrations

that may or may not be regulated for

transportation per 49 CFR / IATA Dangerous

Goods Regulations but are not $r$
DOE Order $5400.5(1990 / 1993)$

SPECIAL HANDLING AND/OR STORAGE RADIOACTIVE TIE TO: B23655

$$
\text { SAMPLE NO. }
$$

MATRIX*
SOIL

\section{SIGN/ PRINT NAMES} DATE/TIME RECEIVED BY/STORED IN DATE/TIMEO FEB 0.2040

EIVED BY/STORED IN

DW Brotherton 2010 S Bendlilins Re Mulf

DATE/TIME expcellins kuthly

DATE/TIME

RECEIVED BY/STORED IN

DATE/TIME

RECEIVED BY/STORED IN

DATE/TIME

RECEIVED BY/STORED IN

$2 / 5$

\section{OUSTODY/SAMPLE ANALYSIS REQUEST}

\section{TELEPHONE NO.}

$373-2530$

PROJECT COORDINATOR

DYEKMAN, DL

SAF NO.

cOA

302117ES30

ACTUAL SAMPLE DEPTH

$300,5 / 3 \times 3.0$

BILL OF LADING/AIR BILL NO

N/A

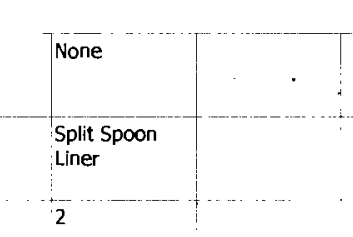

1000

SAMPLE ANALYSIS

SAMPLE TIM
GOVERNMENT VEHICLE

PAGE 1 OF 1

\section{TURNAROUND}

Days / 45
Days
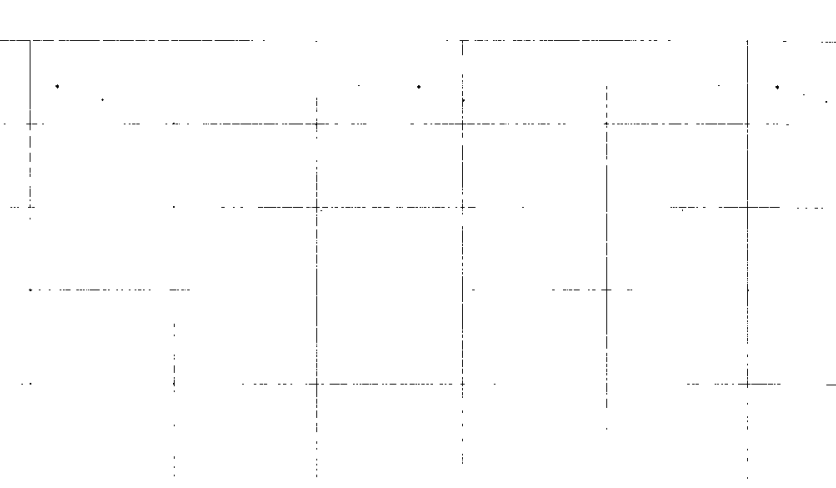

\section{CHAIN OF POSSESSION}

OW Brotherton

RELINQUISHED BY/REMOVED FROM

RELINQUISHED BY/REMOVED FROM

RELINQUISHED BY/REMOVED FROM

RECEIVED BY

LABORATORY
SECTION

FINAL SAMPLE
DISPOSITION

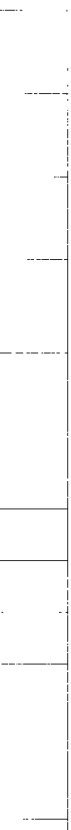

SPECIAL INSTRUCTIONS

\begin{tabular}{l|l} 
DATE/TIME & G* The 200 Area S\&GRP \\
\hline GRplies to this SAF.
\end{tabular}

FEBAT=9T 1010 Operable Units/Contaminant and Tres according to SOW "200-LW-2 and 200-BP-5 Zone Sediments at Borehole C5860/Well 299-E29-54" for C7515/Well 299-E28-30

\section{FEB OO9 2010}

DATE/TIME

DATE/TMME

DATE/TIME

DATE/TIME

** The ESL laboratory shall meet prior requested and confirmed turnaround times. and RDLS specified in the SOW and this SAF. The laboratories must notify the SM Project Coordinator in writing if/when this requirement can not be met. (Hydrometer) - D422; KD - Batch; 
CH2MHill Plateau Remediation Company

COLLETTOR

LAwer dtavina

C7515 (299-E28-30); $1-136$

ICE CHEST NO.

$$
\text { GWS }-036
$$

Environmental Sciences laboraty

MATRIX* POSSTBLE SAMPLE HAZARDS/ REMARKS Contains Radioactive Material at concentrations that may or may not be regulated for transportaton per 49 CFR / IATA Dangerous Goods Regulations but are not releasable per

SPECIAL HANDLING AND/OR STORAGE RADIOACTIVE TIE TO: B23655
SAMPLE NO.
MATRIX*

SOIL

$$
\text { 373-2530 }
$$

PROJECT COORDINATOR

......... DYERMAN, OL

PROJECT DESIGNATION

SAF NO.
F10-045

200-PW-2 OU Characterization Vadose Zone - Geochemical Modeling Para

FIELD LOGBOOK NO. $P_{S},-8$ ACTUAL SAMPLE DEPTH

COA

303.0302117 ES30

TLL OF LADING/AIR BILL NO.

OFFSITE PROPERTY NO.

$\mathrm{N} / \mathrm{A}$

PRESERVATION

TYPE OF CONTAINER

NO. OF CONTAINER(S)

volume

SAMPLE ANALYSIS

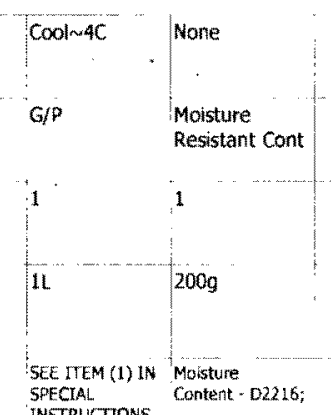

SAMPLE DATE SAMPLE TIME

Ple/ 1215

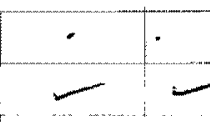

F10-045-204

PAGE 1 OF 2

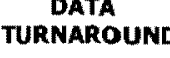

AIR QUALITY $\square \quad \underset{\text { Days }}{45 \text { Days } / 45}$

METHOD OF SHIPMEN

GOVERNMENT VEHICL
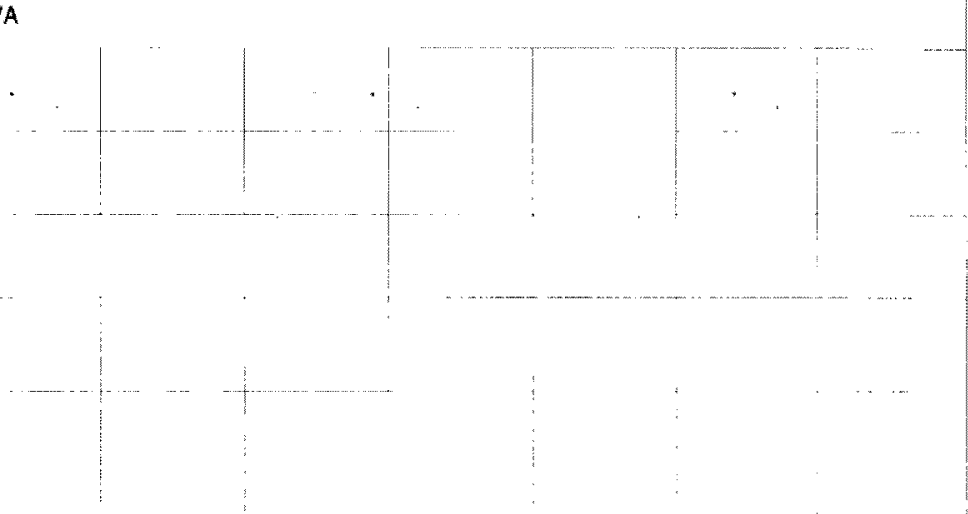

\section{CHAIN OF POSSESSION}

SIGN/ PRINT NAMES

SPECIAL INSTRUCTIONS

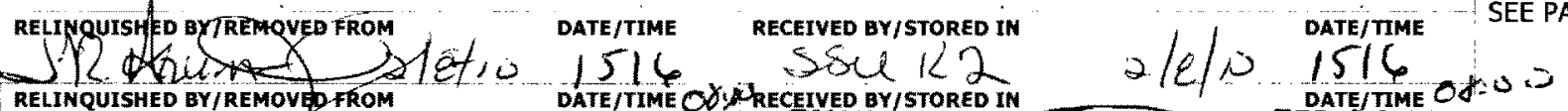

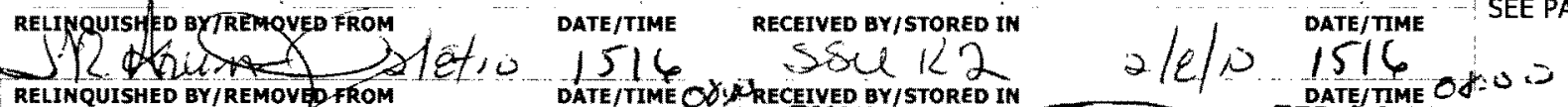

SEE PAGE 2 FOR ALL SPECIAL INSTRUCTIONS

\section{SSU-R2}

FEB 092010 DW Brotherton F FEB O 92010

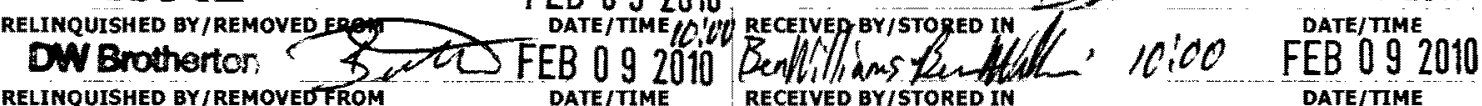

RELINQUISHED BY/REMOVETTFROM

RELINQUTSHED BY/REMOVED FROM

DATE/TIME

RECEIVED BY/STORED IN

DATE/TIME

RELINQUISHED BY/REMOVED FROM

DATE/TIME

RECEIVED BY/STORED IN

DATE/TIME

RELINQUISHED BY/REMOVED FROM

DATE/TIME

RECEIVED BY/STORED IN

DATE/TMME 
CH2MHill Plateau Remediation Company

\section{COLLECTOR}

Laver Htinnar-

MPLING LOCATION

C7515 (299-E28-30); I-136

ICE CHEST NO.

$$
\text { GWS }-036
$$

\section{SHIPPED TO}

Environmental Sciences Laboratory

\section{SPECIAL INSTRUCTIONS}

* The 200 Area S\&GRP Characterization and Monitoring Sampling and Analysis GKI applies to this SAF.

Units/Contaminant and Transport Property Analysis and Report for Vadose Zone Sediments at Borehole C5860/Well 299-E29-54" for

* The ESL laboratory shall meet prior requested and confirmed turnaround times and RDLs specified in the SOW and this SAF. The laboratories must notify the SM Project Coordinator in writing if/when this requirement can not be met. (1)6010M_ICP_ASTM_AE (TAL) \{Sodium, Aluminum, Iron, Potassium, Antimony, Nickel, Calcium, Magnesium, Silver\} 6010M_ICP_ASTM_AE (AAd-On) \{Lead, Thallium, Selenium\} 6010M_METALS_ICP_WE (TAL) \{Sodium, Aluminum,

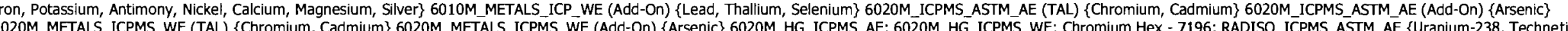

99\} RADISO ICPMS WE \{Iodine-129, Uranium-238, Technetium-99\} I-129 by ICPMS \{Iodine-129\} GAMMA GS; URANIUM ISOTOPIC RATIOS; ALPHA BETA AE \{Gross beta, Gross alpha\} ALPHA BETA WE \{Gross beta, Gross alpha\} IC Anions - 9056_WE \{Nitrate, Chloride, Phosphate, Fluoride, Nitrite, Sulfate\} Cyanide (Total) - 335.2 \{Cyanide\} pH (Water) - 9045_WE; Conductivity - 9050_WË; TOC - ASTME1915A \{Total organic Carbon, Total Inorganic Carbon, Total carbon\} 2320_ALKALINITY \{Carbonate ion, Bicarbonate, Alkalinity, Calcium Carbonate\}

D IRIGHA
AIR QUALIT

302117 ES30

BILL OF LADING/AIR BILL NO.

N/A

VERNMENT VEHICLE
METHOD OF SHIPMEN

\section{Days /
Days

HNF-N- $491-5 \quad 303.0$

OFFSITE PROPERTY NO.

$$
303.0
$$


CH2MHill Plateau Remediation Company

COLLECTOR

Kaver Rmo, ftumit

SAMPLING LOCATION

ICE CHEST NO.

GWS -036
GWEST

SHIPPED TO

Environmental Sciences Laboratory

MATRIX* [ POSSIBLE S.AMPLE HAZARDS/ REMARKS Contains Radioactive Material at concentrations

that may or may not be regulated for

Goods Regulations but are not releasable per
DOE Order 5400.5 (1990/1993)

SPECIAL HANDLING AND/OR STORAGE RADIOACTIVE TIE TO: B23655
CHAIN OF CUSTODY/SAMPLE ANALYSIS REQUEST

F10-045-205

PAGE 1 OF 1

PRICE CODE

DATA
TURNAROUND
45 Days $/ 45$

45 Days / 45

AIR QUALTY

Days

PROJECT DESIGNATION
200-PW-2 OU Characterization Vadose Zone - Geochemical Modeling Param
F10-045

200-PW-2 OU Characterization Vadose Zone - Geochemical Modeling Param
FIELD LOGBOOK NO. F SE ACTUAL SAMPLE DEPTH COA

METHOD OF SHIPMENT

HNF-N- $491-5 \quad 300.1 / 305.6 \quad 302117$ ES30

GOVERNMENT VEHICLE

OFFSITE PROPERTY NO.

BILL OF LADING/AIR BILL NO.

N/A

PRESERVATION

TYPE OF CONTAINER

NO. OF CONTAINER(S)

volume

SAMPLE ANALYSIS
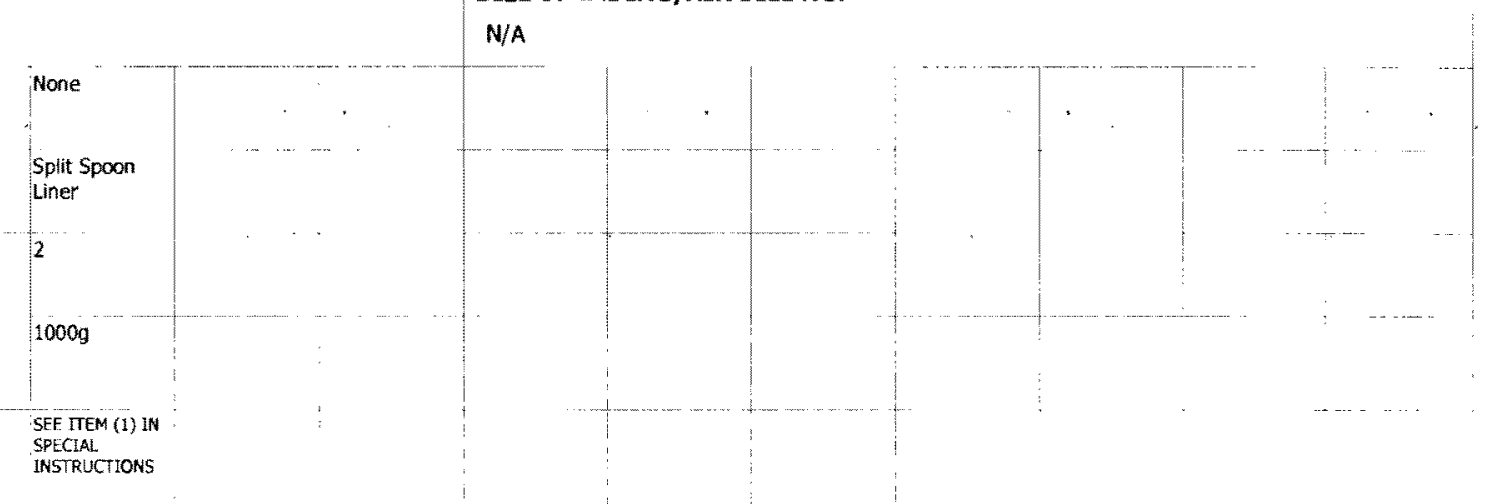

MATRIX* SAMPLE DATE SAMPLE TIME

$\mathrm{B} 235 \mathrm{C} 8$

soll

$2 / \varepsilon / 10$

1320

$\leftarrow$

CHAIN OF POSSESSION

RELINQUISHED BY/REMOVED FROM

I.R.thuprar 2

\section{SSU-R2}

RELINQUISHED BY/REMOVED FRET

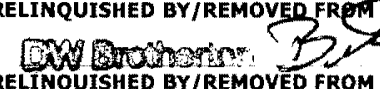

RELINQUISHED BY/REMOVED FROM

RELINQUISHED BY/REMOVED FROM

RELINQUISHED BY/REMOVED FROM

\begin{abstract}
LABORATORY RECEIVED B
\end{abstract}
SECTION

FINAL SAMPLE
DISPOSITION
SIGN/ PRINT NAMES

DATE/TTME RECEIVED BY/STORED IM DATE/TIME SOI RECEIVED BY/STORED IN FEB 092010

DW Brotherton EB 092010

RECEIVED BY/STORED IN

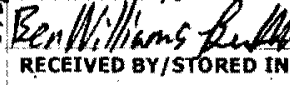

DATE/TIME RE REIVED BY/STORED IN

DATE/TIME RECEIVED BY/STORED IN

DATE/TIME

RECEIVED BY/STORED IN

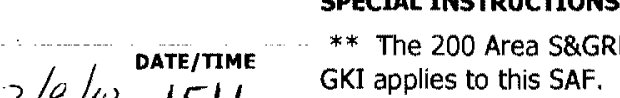

GKI applies to this SAFRP Characterization and Monitoring Sampling and Analysis

ESL Shall subn all

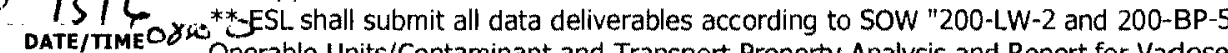
FEB O 92010 Zone Sediments at Borehole C5860/Well 299-E29-54" for C7515/Well 299-E28-30 DATE/TIME ("M" Well) and shall adhere to all S\&GRP standard protocol.

\section{FEB 092010 MATEO}

** The ESL laboratory shall meet prior requested and confirmed turnaround times and RDLs Specified in the SOW and this SAF. The laboratories must notify the SM Project Coordinator in writing if/when this requirement can not be met. (1)Density; CATIONEXCH_TR; Particle Size (Dry Sieve) - D422; Particle Size

DATE/TIME

DATE/TIME

(1)

DATE/TIME

กTLE

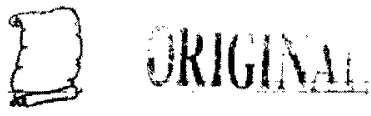

DATE/TTME
DATE/TIME 
CH2MHill Plateau Remediation Company

\section{COLLECTOR}

LXaver Rano, thuimor

C7515 (299-E28
ICE CHEST NO.

GWS-036

SHIPPED TO

Environmental Sciences Laboraton

MATRIX* POSSIBLE SAMPLE HAZARDS/ REMARKS

Contains Radioactive Material at concentrations

that may or may not be regulated for

Dans Regun pers b a $/$ IATA Dangerous

Goods Regulations but are not releasable per
DOE Order $5400.5(1990 / 1993)$

SPECIAL HANDLING AND/OR STORAGE ADIOACTIVE TIE TO: B23656
SAMPLE NO
MATRIX*

B235VO

SOI

CHAIN OF CUSTODY/SAMPLE ANALYSIS REQUEST

F10-045-207

PAGE 1 OF 2

\begin{tabular}{|c|c|c|}
\hline $\begin{array}{l}\text { COMPANY CONTACT } \\
\text { DYEKMAN, DL }\end{array}$ & & $\begin{array}{l}\text { TELEPHONE NO. } \\
373-2530\end{array}$ \\
\hline PROJECT DESIGNATION & & \\
\hline 200-PW-2 OU Characterization $V$ & dose zont & e - Geochemical MC \\
\hline FIELD LOGBOOK NO. PS 58 & & ACTUAL SAMPLE \\
\hline $\begin{array}{l}\text { HNF-N- } 49 /-5 \\
\text { OFFSTE PROPERTY NO. }\end{array}$ & & 305.6 \\
\hline N/A & & \\
\hline PRESERVATION & $\cos \sim 4 \mathrm{C}$ & None \\
\hline TYPE OF CONTAINER & G & $\begin{array}{l}\text { Moisture } \\
\text { Resistant Cont }\end{array}$ \\
\hline NO. OF CONTAINER(S) & 1 & 1 \\
\hline VOLUME & it & $200 \mathrm{~g}$ \\
\hline SAMPLE ANALYS & 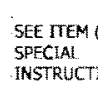 & $\begin{array}{l}\text { (1) I IN Molisture } \\
\text { Trons } \\
\text { Content D2216; }\end{array}$ \\
\hline
\end{tabular}

PRICE CODE

DATA
TURNAROUND

45 Days /
Days

AIR QUALITY

SAF NO.

COA METHOD OF SHIPMENT

GOVERNMENT VEHICLE

302117 ES30

SAMPLE DATE SAMPLE TIME

D/e/ 1320

SIGN/ PRINT NAMES

DATE/TIME RECEIVED BY/STORED IN

Zle/lo $1516 \quad$ OSCL R2 $2 / 2 / 201516$

CHAIN OF POSSESSION

dinakiner of

RELINQUISHED BY/REMOYYOF FROM
SSU-R2 FEB U 902010

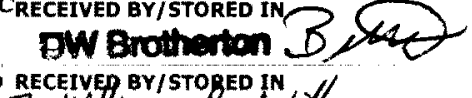

$2 / 2 / 101516$ DATE/TMEOS.0

RELINQUISHED BY/REMOVEDEROM DATE/TTME

FEB IFTHAO

EB O 092010

BenWilliLms bu Whit

100 FEB 0.92010

DATE/TIME RECEIVED GY/STORED IN

DATE/TMME

DATE/TIME

RECEIVED BY/STORED IN

DATE/TIME

DATE/TIME

RECEIVED BY/STORED IN

DATE/TIME
SPECIAL INSTRUCTIONS

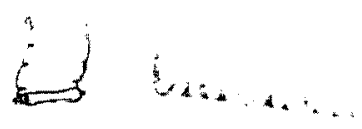

DATE/TIME

\section{SECTION}

FINAL SAMPLE 
Waver Vlomio, Atzinar SAMPLING LOCATION

C7515 (299-E28-30)

$$
\text { GWS - } 036
$$

\section{SHIPPED TO}

Environmental Sciences Laboratory

\section{SPECIAL INSTRUCTIONS}

** The 200 Area S\&GRP Characterization and Monitoring Sampling and Analysis GKI applies to this SAF,
** ESL shall submit all data deliverables according to SOW "200-LW-2 and 200-BP-5 Operable Units/Contaminant and Transport Property Analysis and Report for vadose Zone Sediments at Borehole C5860/Well 299-E29-54" for C7515/Well 299-E28-30 ("M" Well) and shall adhere to all S\&GRP standard protocol.

** The ESL laboratory shall meet prior requested and confirmed turnaround times and RDLs specified in the SOW and this SAF. The laboratories must notify the SM Project Coordinator in writing if/when this requirement can not be met. (1)6010M_ICP_ASTM_AE (TAL) \{Sodium, Aluminum, Iron, Potassium, Antimony, Nickel, Calcium, Magnesium, Silver\} 6010M_ICP_ASTM_AE (Add-On) \{Lead, Thallium, Selenium\} 6010M_METALS_ICP_WE (TAL) \{Sodium, Aluminum,

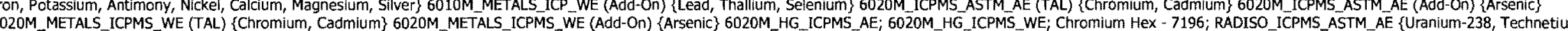
9) RADISO_ICPMS_WE \{Iodine-129, Uranium-238, Technetium-99\} I-129 by ICPMS \{Iodine-129\} GAMMA_GS; URANIUM ISOTOPIC RATIOS, ALPHA_BETA_AE \{Gross beta, Gross alpha\} ALPHA_BETTA_WE \{Gross beta, Gross apha\} IC Anions - 9056_WE \{Nitrate, Chloride, Phosphate, Fluoride, Nitrite, Sulfate\} Cyanide (Total) - 335.2 \{Cyanide\} pH (Water) - 9045_WE; Conductivity - 9050_WE; TOC - ASTME1915A (Total Organic Carbon, Total Inorganic Carbon, Total carbon\} 2320_ALKALINITY \{Carbonate ion, Bicarbonate, Alkalinity, Calcium Carbonate\}

\& vintuh 
CH2MHill Plateau Remediation Company

COLLECTOR

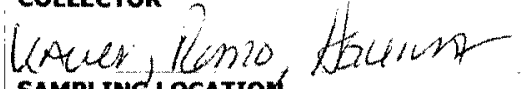

SAMPLING LOCATION

C7515 (299-E28-30); I-139

CHEST NO.

$$
\text { GWS }-036
$$

Environmental Sciences Laboratory

MATRIX* POSSIBLE SAMPLE HAZARDS/ REMARKS
A=Air

Contains Radioactive Material at concentrations

that may or may not be regulated for

Goods Regulations but are IATA Dangerous

DOE Order 5400.5 (1990/1993)

SPECIAL HANDUING AND/OR STORAGE RADPACTIVE TIE TO: 823656

\begin{tabular}{cccc}
\multicolumn{1}{c}{ SAMPLE NO. } & MATRIX* \\
$235 C 9$ & $\cdots$ & SOIL
\end{tabular}

CHAIN OF CUSTODY/SAMPLE ANALYSIS REQUEST

F10-045-208

PAGE 1 OF 1

COMPANY CONTACT

TELEPHONE NO.

PROJECT COORDINATOR

DYEKMAN, DL

PROJECT DESIGNATION

$373-2530$

SAF NO.

200-PW-2 OU Chacterizan Vadose Zone-Geochemical Modeling Pan F10-045

FIELD LOGBOOK NO. PS SE ACTUAL SAMPLE DEPTH COA

HNF-N- $49 /-5 \quad 306.0 / 300.5 \quad 302 / 17$ ES30

PRICE CODE BN

TURNAROUND

45 Days / 45

Days

OFFSITE PROPERTY NO.

BILL OF LADING/AIR BILL NO.

PREservation

TYPE OF CONTAINER

NO. OF CONTAINER(S)

volume

SAMPLE ANALYSIS

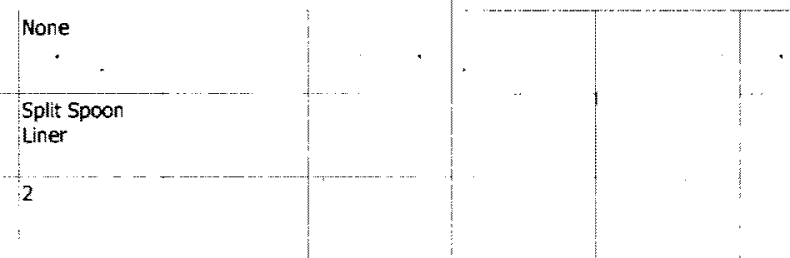

SAMPLE DATE SAMPLE TIME

$2 / 0 / 0$ : 140
1000

SEE TEM (1) IN
SECAAL
INSTRUCTONS
METHOD OF SHIPMEN .

\begin{tabular}{l|l|l|l|}
$\cdot$ & $\cdot$ & $\cdot$ & \\
\hline &
\end{tabular}
CHAIN OF POSSESSION

RELIMQUISHED BY/REMOVED EROM Dileuvia SSU-R2

\section{DLINQUISHED BV REMOVED ROM}

DELINQUTSHED BY/REMOYED FROM

RELINOUISHED BY/REMOYED FROM

RELINQUISHED BY/REMOVED FROM

RELINQUISHED BY/REMOVED FROM

ABORATORY

SECTION

FINAL SAMPLE DISPOSAL METHOD

DISPOSITION
SIGN/ PRINT NAMES

DATE/TTME RECEIVED BY/STORED IN 12/3 1514 S3u RQ

DW Brothention

FEB 092010

FEB 092010

DATE/TIME.

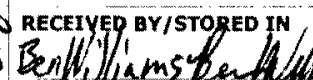

Benllilliams peu hill

DATE/TIME

RECEIVED QY/STORED IN

DATE/TTME

RECEIVED BY/STORED IN

DATE/TIME
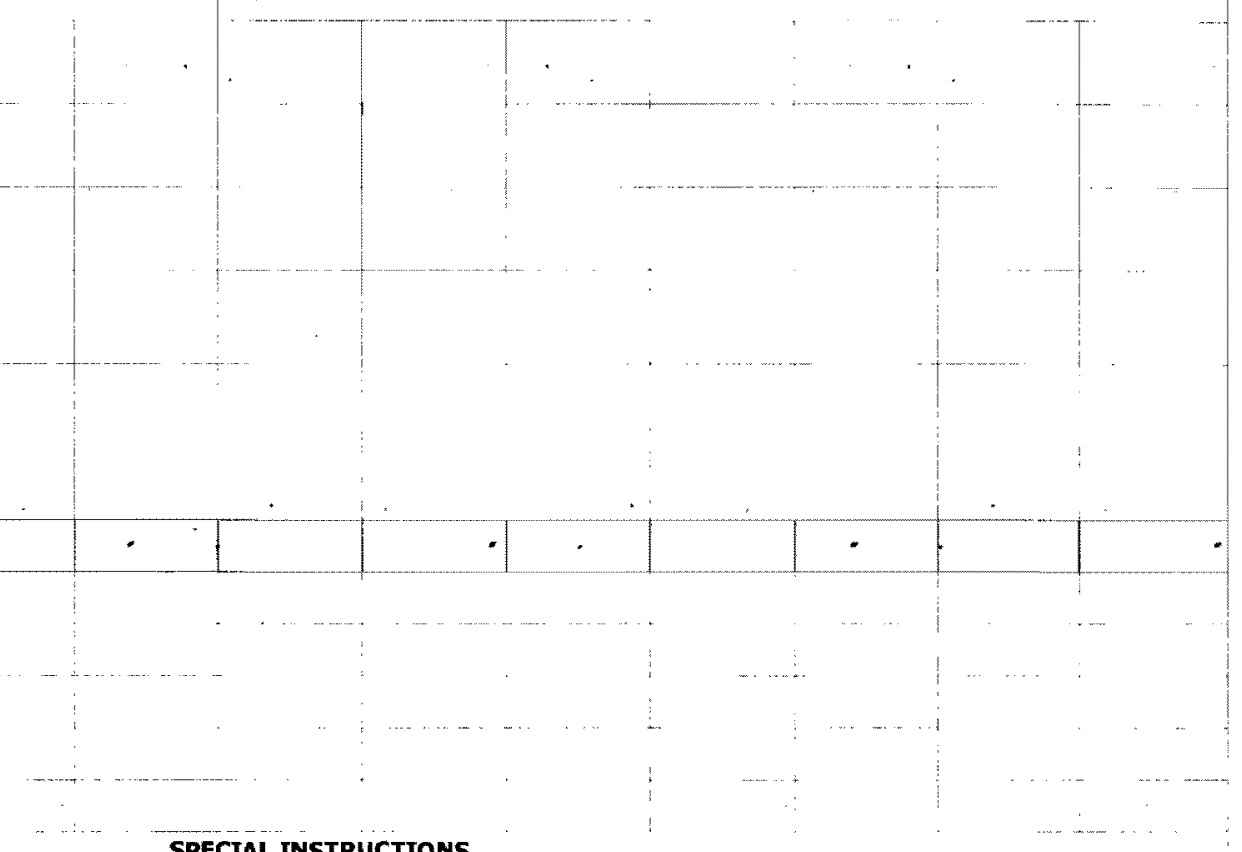

\section{SPECIAL INSTRUCTIONS}

a lefp DATE/TME $\quad$ G* The 200 Area S\&GRP Characterization and Monitoring Sampling and Analysis

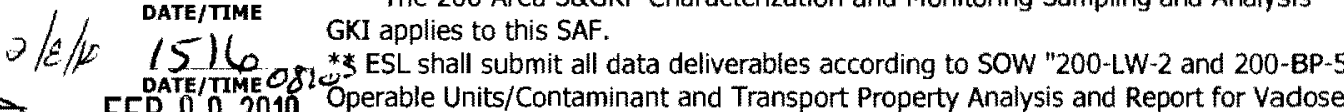
FEB 092010 Operable Units/Contaminant and Transport Property Analysis and Report for Vadose
Zone Sediments at Borehole C5860/Well 299-E29-54" for C7515/Well 299-E28-30 FEB 092010 ("M" Well) and shall adhere to all S\&GRP standard protocol.

DATE/ TIME ** The ESL laboratory shall meet prior requested and confirmed turnaround times and RDLs specified in the SOW and this SAF. The laboratories must notify the SM Project Coordinator in writing if/when this requirement can not be met.
(1)Density; CATONNECH TR; Particle Size (Dry Sieve) - D422; Particle Size

DATE/TME (Hydrometer) - D422; KD- Batch;

DATE/TMME

$$
\vdots \rightarrow \text { RIGINAL }
$$


CH2MHill Plateau Remediation Company

COLLECTOR

Konn- Augher

SAMPLING LOCATION

C7515 (299-E28-30); ADD ON 1

ICE CHEST NO.

$$
\text { GWS - } 036
$$

SHIPPED TO

Environmental Sciences Laborator

$\underset{A=A i r}{\text { MATRIX* POSSIBLE SAMPLE HAZARDS/ REMARKS }}$

$A=A i r$
$D L=D r u m$

Liquids that may or may not be regulated for

transportation per 49 CR/ IATA Dangerous

SPECIAL HANDLING AND/OR STORAG RADIOACTIVE TIE TO: B23667

$$
\text { SAMPLE NO. }
$$

MATRIX*

B23668
Goods Regulations but are not releasable per

CHAIN OF CUSTODY/SAMPLE ANALYSIS REQUEST

$F 10-045-220$

PRICE CODE $\quad$ SN

AIR QUALITY D

DYEKMAN, DD

$\begin{array}{ll}\text { PROJECT DESIGNATION } & \text { SAF NO. } \\ \text { 200-PW-2 OU Characterization Vadose Zone - Geochemical Modeling Param } & \text { F10-045 }\end{array}$

FIELD LOGBOOK NO. Fo $5^{\circ}$ ACTUAL SAMPLE DEPTH

HXIF.N.491-5

OFFSITE PROPERTY NO.

308.5

302117 ES30

METHOD OF SHIPMENT

GOVERNMENT VEHICLE

N/A

N/A

TYPE OF CONTAINER

NO. OF CONTAINER(S)

VOLUME

SAMPLE ANALYSIS

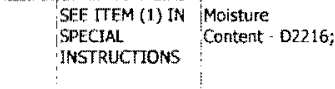

SAMPLE DATE SAMPLE TIME

यथ/०

1410

SIGN/ PRINI NAMES

RECEIVED BY/STORED IN

$5 \& R 2$

RECEIVED BY/STORED IN

REUMRQUYG RED BY REMOVED FROM $-2 / 8 / 01516$

SELINQUISHED BYTREM
SSUTR2

FEB TYY 20 亿O

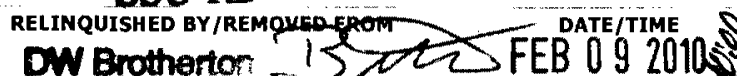

DELINQUISHED BY/REMOVED FROM DATE/TIME

DW Brothartos:

22 FEB O 092010

Bendilfiams Deathith-10.00 FEB D 92010

RELINQUUISHED BY/REMOVED FROM

DATETITIME

RECEIVED BY/STORED IN

DATE/TTME

RELINQUISHED BY/REMOVED FROM

DATE/TIME

RECEIVED BY/STORED IN

DATE/TTME

B ORIGINA

RELINQUISHED BY/REMOVED FROM

DATE/TIME

RECEIVED BY/STORED IN

DATE/TTME

PAGE 1 OF 2

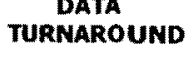

45 Days / 45
Days

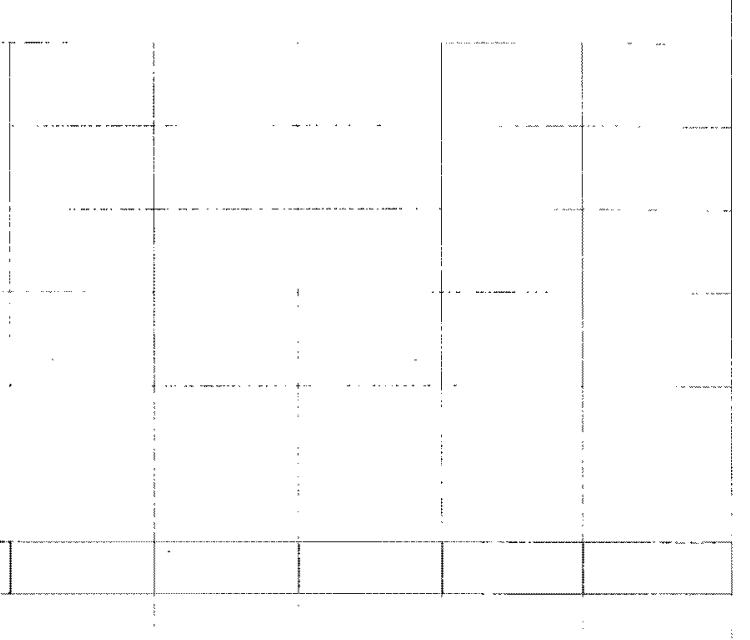

SECTION

init

DATE/TIME

DISPOSED BY

DATE/TIME 


\section{CH2MHill Plateau Remediation Company} COLLECTOR

$$
\text { Kavir, AUäirar }
$$

AMPLING LOCATION

C7515 (299-E28-30); ADD ON 1

CE CHEST NO.

$$
\text { GWS- } 036
$$

\section{SHIPPED TO}

Environmental Sciences Laboraton

\section{SPECIAL INSTRUCTIONS}

** The 200 Area S\&GRP Characterization and Monitoring Sampling and Analysis GKI applies to this SAF.

Contaminant and Transport Property Analysis and Report for Vadose Zone Sediments at Borehole C5860/Weli 299-E29-54" for

** The ESL laboratory shall meet prior requested and confirmed turnaround times and RDLs specified in the SOW and this SAF. The laboratories must notify the SM Project Coordinator in writing iff when this requirement can not be met. (1)6010M_ICP_ASTM_AE (TAL) \{Sodium, Aluminum, Iron, Potassium, Antimony, Nickel, Calcium, Magnesium, Silver\} 6010M_ICP_ASTM_AE (Add-On) \{Lead, Thallium, Selenium\} 6010M_METALSICPP_WE (TAL) \{Sodium, Aluminum,

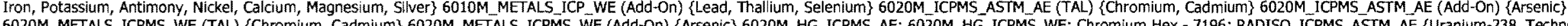

99\} RADISO ICPMS WE \{Iodine-129, Uranium-238, Technetium-99\} I-129 by ICPMS \{lodine-129\} GAMMA GS; URANIUM ISOTOPIC RATIOS; ALPHA BETA AE \{Gross beta, Gross alpha\} ALPHA BETA WE \{Gross beta, Gross alpha\} IC Anions - 9056_WE \{Nitrate, Chloride, Phosphate, Fluoride, Nitrite, Sulfate\} Cyanide (Total) - 335.2 \{Cyanide\} pH (Water) - 9045_WE; Conductivity - 9050_WE; TOC - ASTME1915A \{Total organic carbon, Total Inorganic Carbon, Total carbon\} 2320_ALKALINITY \{Carbonate ion, Bicarbonate, Akalinity, Calcium Carbonate\}

\section{B ORIGH}


COLLECTOR

KaucR, Romo

SAMPLING LOCATION

C7515 (299-E28-30); I-055

ICE CHEST NO.

$$
(-6)=168
$$

SHIPPED TO

Environmental Sciences Laboratory

MATRIX*
PAir
Contains Radioactive Material at concentrations Contains Radioactive Material at concentration
that may or may not be regulated for that may or may not be regulated for Goods Regulations but are not releasable per DOE Order 5400.5 (1990/1993)

SPECTAL HANDLING AND/OR STORAGE RADIOACTIVE TIE TO: B23635

SAMPLE NO.

B23596

soll
MATRIX*

SAMPLE DATE SAMPLE TIME

$1-20-10 \quad 0910$
PROJECT COORDINATOR

DYEKMAN, DL

$\begin{array}{ll}\text { PROJECT DESIGNATION } & \text { SAF NO. } \\ \text { 200-PW-2 OU Characterization Vadose Zone - Geochemical Modeling Param F10-045 } & \end{array}$

FIELD LOGBOOK NO. ACTUAL SAMPLE DEPTH COA

HNF-N-491-5 $p q 49$ (5\%)-154.2 3021175530

PRICE CODE
SN
TURNAROUND

AIR QUALITY $\square \quad 45$ Days $/ 45$

METHOD OF SHIPMENT

GOVERNMENT VEHICIE

BILL OF LADING/AIR BILL NO

N/A

\section{PRESERVATION}

TYPE OF CONTAINER

NO. OF CONTAINER(S)

volume

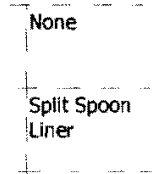

$\frac{1}{2}$

SAMPIE ANALYSIS

10009
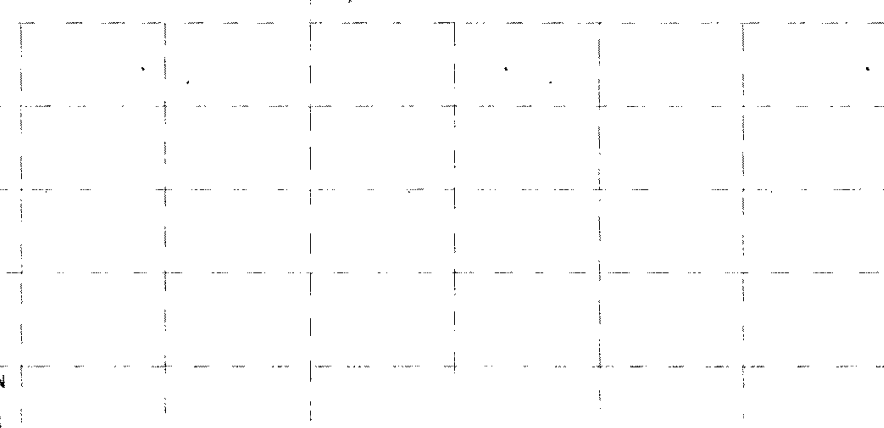

\section{CHAIN OF POSSESSION}

RELIMQUISHED BY/REMOVED FROM

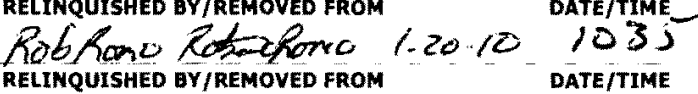
SSU-R2 JAN 212010 DESTS RELINQUISHER MYGEMGVEDTROM 212010 DATE/TIME RELINQUISHED QY/REMOTED FROM RELINQUISHED QY/REMOVED FROM - - - DATE/TIME RELINQUUSHED BY/REMOUED FROM DATE/TIME RELINQUISHED QY/REMOVED FROM DATE/TIME

$$
\text { DATE/TIME }
$$

\section{SIGN/ PRINT NAMES}

Ho. 41355422

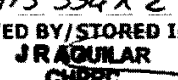

LUAN $212010^{\text {DATE/TME }} 024 \mathrm{~s}$

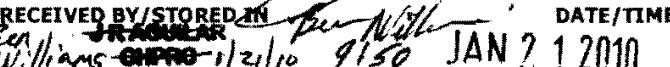

RECEIVED QY/STORED IN

RECEIVED QY /STORED IN

RECEIVED BY/STORED IN

RECEIVED BY/STORED IN

\section{RECEIVED or}

\section{SPECIAL INSTRUCTIONS}

** The 200 Area S\&GRP Characterization and Monitoring Sampling and Analysis

** ESL shall submit all data deliverables according to SOW "200-LW-2 and 200-BP-5 Operable Units/Contaminant and Transport Property Analysis and Report for Vadose Zone Sediments at Borehole C5860/Well 299-E29-54" for C7515/Well 299-E28-30 ("M" Well) and shall adhere to all S\&GRP standard protocol.

** The ESL laboratory shall meet prior requested and confirmed turnaround times and RDLS specified in the SOW and this SAF. The laboratories must notify the SM Project Coordinator in witing it/When this requirement can not be met. (1)Density; CATONEXCH_TR; Particle Size (Dry Sieve) - D422; Particle Size ( (Hydrometer) - D42; K K - Batch

TITLE

DATE/TIME

Disposed ay

DATE/TIME 